\title{
Late Transition Metal Complexes of Pentafluorophenylphosphino- Pincer Ligands
}

\author{
by \\ Bradley George Anderson
}

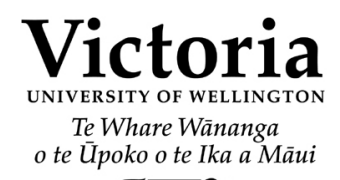

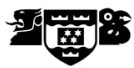

\author{
A thesis \\ submitted to Victoria University of Wellington \\ in fulfilment of the \\ requirements for the degree of \\ Doctor of Philosophy \\ in Chemistry
}

Victoria University of Wellington 


\section{Abstract}

This thesis details the synthesis of new examples of electron-poor pincer ligands, featuring bis(pentafluorophenyl)phosphine donors attached to 1,3-substituted phenylene or 2,6-substituted pyridine backbones, to create tridentate PCP and PNP ligands. The effect of the ligands' electronic nature on the coordination chemistry and ease of pincer complex synthesis with late transition metals is discussed, as is the catalytic activity of the resultant palladium pincer complexes in the Heck and Suzuki reactions.

Symmetric PCP and PNP ligands possessing bis(pentafluorophenyl)phosphinite and bis(pentafluorophenyl)phosphoramine functionalities were synthesised by reaction of bis(pentafluorophenyl)phosphine bromide with resorcinol, 3-hydroxybenzyl-ditert-butylphosphine, 2,6-diaminopyridine, or 2,6-dihydroxypyridine, affording 1,3$\left[\left(\mathrm{C}_{6} \mathrm{~F}_{5}\right)_{2} \mathrm{PO}\right]_{2} \mathrm{C}_{6} \mathrm{H}_{4}(\mathrm{POCOPH}, \mathbf{1}), 1-\left[\left(\mathrm{C}_{6} \mathrm{~F}_{5}\right)_{2} \mathrm{PO}\right]-3-\left({ }^{t} \mathrm{Bu}_{2} \mathrm{PCH}_{2}\right)_{2} \mathrm{C}_{6} \mathrm{H}_{4}$ (POCCPH, 3), 2,6- $\left[\left(\mathrm{C}_{6} \mathrm{~F}_{5}\right)_{2} \mathrm{PNH}\right]_{2} \mathrm{C}_{6} \mathrm{H}_{3} \mathrm{~N}(\mathrm{PNNNP}, \mathbf{1 0})$, and 2,6- $\left[\left(\mathrm{C}_{6} \mathrm{~F}_{5}\right)_{2} \mathrm{PO}\right]_{2} \mathrm{C}_{6} \mathrm{H}_{3} \mathrm{~N}$ (PONOP, 11) respectively. The previously reported $1,3-\left[\left(\mathrm{C}_{6} \mathrm{~F}_{5}\right)_{2} \mathrm{PCH}_{2}\right]_{2} \mathrm{C}_{6} \mathrm{H}_{4}(\mathrm{PCCCPH}$, 2) was also synthesised, with the literature yield improved upon by the use of magnesium-anthracene to generate the required Grignard reagent.

The coordination chemistry of the POCOPH ligand 1 with platinum(0) alkene and platinum(II) dimethyl precursors revealed an affinity for the formation of cis-bridged oligomeric structures. The dimer $[(\mathrm{POCOPH}) \mathrm{Pt}(\mathrm{nb})]_{2}(\mathbf{1 4}, \mathrm{nb}=$ norbornene $)$ was isolated and crystallographically characterised from the reaction between $\mathbf{1}$ and $\left[\mathrm{Pt}(\mathrm{nb})_{3}\right]$. The solid state structure revealed the presence of stabilising $\pi-\pi$ interactions between the aromatic ligand backbones, which were also observed in solution by ${ }^{1} \mathrm{H}$ NMR spectroscopy. Reactions of ligand 1 with platinum and palladium dichloride or chloromethyl starting materials led to rare examples of cis,trans-dimers of the type cis,trans- $[(\mathrm{POCOPH}) \mathrm{MClX}]_{2}(\mathrm{M}=\mathrm{Pd}, \mathrm{Pt} ; \mathrm{X}=\mathrm{Cl}, \mathrm{Me})$. In part due to facile dimer formation with $\mathbf{1}$, metallation of the ligand backbone to form the tridentate pincer complex [(POCOP) $\mathrm{PtCl}](\mathbf{2 5})$ required long reaction times and high temperatures. It was observed that platinum dichloride starting materials with 
more strongly binding ancillary ligands were less prone to oligomer formation, and could facilitate more rapid metallation to from 25. More facile pincer complex formation was also observed for more electron-rich ligands with both PCP and PNP pincer ligands.

The electron poor platinum and palladium POCOP, PCCCP, and POCCP pincer complexes (where the free ligand had been deprotonated upon metallation) were synthesised and subsequently converted into the metal carbonyl species $[(\mathrm{PCP}) \mathrm{M}(\mathrm{CO})]^{+}$. Analysis of $\mathrm{C}-\mathrm{O}$ stretching frequencies by infrared spectroscopy confirmed complexes of POCOP ligand $\mathbf{1}$ were the most electron poor, while those of POCCP ligand 3 were the most electron rich. Decarbonylation of the palladium pincer complexes was observed in solution and in the solid state, and was more facile for complexes with a higher wavenumber $\mathrm{C}-\mathrm{O}$ stretch.

Reaction of the $[(\mathrm{PCP}) \mathrm{PtCl}]$ pincer complexes with methyl nucleophiles revealed that treatment with methylmagnesium iodide resulted in halide exchange, while methyllithium promoted nucleophilic attack at phosphorus. Spectroscopic data indicated that in one instance this led to pentafluorophenyl migration to the metal centre to form a $\left[(\mathrm{PCP}) \operatorname{Pt}\left(\mathrm{C}_{6} \mathrm{~F}_{5}\right)\right]$ complex. Dimethylzinc was successful in methylating the platinum PCP complexes; however, it was observed to degrade the palladium PCP pincer complexes. Treatment of the rhodium PNP pincer complex [(PNNNP)RhCl] (49) with dimethylzinc also resulted in degradation, which spectroscopic evidence indicated proceeded via ligand deprotonation and the formation of a zinc adduct of 49. Low temperature protonolysis of the [(PCP)PtMe] species did not reveal any information about possible interactions between the metal and liberated methane.

The catalytic activity of the electron-poor $[(\mathrm{PCP}) \mathrm{PdCl}]$ complexes were assessed in the Heck and Suzuki cross-coupling reactions. The complexes of $\mathbf{1}, \mathbf{2}$, and $\mathbf{3}$ were all found to possess only modest activity in the Heck reaction, functioning as precatalysts which decomposed to give catalytically-active $\operatorname{Pd}(0)$ colloids. Under milder Suzuki reaction conditions, the most electron-poor complex, [(POCOP $) \mathrm{PdCl}]$ (28) proved to be one of the most active pincer catalysts known for this reaction, able to achieve a turnover number of 176,000 for the coupling of electronically-deactivated aryl bromides and phenylboronic acid. Mercury poisoning tests revealed that Suzuki reactions catalysed by $\mathbf{2 8}$ proceeded via a homogeneous active species. 


\section{Acknowledgments}

Thanks to the boss, Prof. John L. Spencer. Your support, guidance, and experience has been much appreciated during this interesting, challenging, tough and testing time. Thanks to Mum, Dad, and Gran; your enthusiastic support for me in my scholastic endeavours has always been a source of motivation. Cheers to all of the Spencer group members, past and present; Almas, Chris, David, Jacqui, Kathryn, Melanie, Sarah, Teresa. I am especially grateful to Almas and Kathryn for their assistance in developing some half-decent lab skills during my formative years in chemical research.

Thanks to Dr. John Ryan, Ian Vorster, Dr. Robert Keyzers, and Dr. Jono Singh for their assistance with NMR spectroscopy and mass spectrometry. Cheers to Emma for being a better rhodium chemist than me. Thanks to the SPCS administrative and technical stuff; you guys keep the School running smoothly and have made all the little surprises that come with doing research a bit easier to deal with.

Thanks to the CMFC for an increased appreciation of appropriately aged distillates (especially their rheological properties). Thanks those to whom I've talked to about work, your advice and feedback was appreciated. Finally, cheers to the jokers who I've called mates, you've helped keep me sane. It can't have been easy. 


\section{Table of Contents}

Abstract

Acknowledgments

Table of Contents $\quad$ v

List of Figures viii

List of Schemes $\quad$ X

List of Tables $\quad$ xii

Glossary xiii

1 Introduction $\quad 1$

1.1 Metal-Ligand Coordination Complexes . . . . . . . . . . . . . . 1

1.2 Pincer Complexes . . . . . . . . . . . . . . . . . 2

1.2.1 Uses of Pincer Complexes With Phosphorus Donors . . . . . . 5

1.3 Electron-Poor Ligands . . . . . . . . . . . . . . . . . . . . . . 7

1.3.1 Electron-Poor Pincer Complexes . . . . . . . . . . . . . . 9

1.4 Synthesis of PCP and PNP Pincer Complexes . . . . . . . . . . . . . 10

1.5 Palladium-Catalysed Cross-Coupling . . . . . . . . . . . . . . . . 14

1.6 Research Objectives . . . . . . . . . . . . . . . . . . 15

1.7 Concluding Remarks . . . . . . . . . . . . . . . . 15

$\begin{array}{lll}2 & \text { Ligand Synthesis } & 17\end{array}$

2.1 Synthesis of Pentafluorophenyl-Substituted PCP Pincer Ligands . . . 19

2.2 Synthesis of Pentafluorophenyl-Substituted PNP Pincer Ligands . . . 25

2.3 Attempted Synthesis of Trifluoromethylaryl-Substituted Ligands . . . 26

2.4 Concluding Remarks . . . . . . . . . . . . . . . . . . . 27

3 Coordination Chemistry of PCP Pincer Ligands 29

3.1 Historical Overview . . . . . . . . . . . . . . . . . . . . . . 29

3.2 Coordination to $\mathrm{Pt}(0) \quad \ldots \ldots \ldots \ldots$

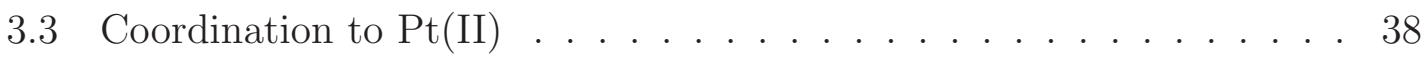


3.3.1 Reactions with $\left[\mathrm{PtMe}_{2}(1,5\right.$-hexadiene $\left.)\right] \ldots \ldots$. . . . . . 38

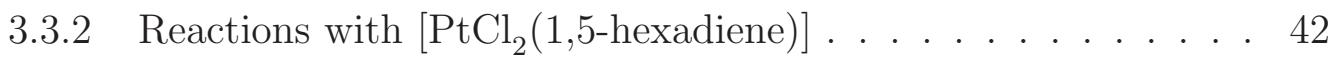

3.3.3 Reactions with $[\mathrm{PtClMe}(1,5$-hexadiene $)] \ldots . . . . . . . .449$

3.3.4 Reactions with $\left[\mathrm{PdCl}_{2}(\mathrm{NCMe})_{2}\right] \ldots \ldots . \ldots . . . . . . .53$

3.3.5 Literature Precedent for cis,trans-Dimers . . . . . . . . . . . . 57

3.4 Concluding Remarks . . . . . . . . . . . . . . . . . . 58

4 Synthesis and Reactivity of PCP Pincer Complexes 61

4.1 Synthesis of PCP Pincer Complexes . . . . . . . . . . . . . . . . . . 61

4.1.1 Effect of Metal Precursor on PCP Pincer Synthesis . . . . . . 62

4.1.2 Effect of the Ligand on PCP Pincer Synthesis . . . . . . . . . 66

4.2 Reactions of Pt/Pd Pincer Compounds . . . . . . . . . . . . . 68

4.2.1 Synthesis of Pincer Metal-Carbonyl Compounds . . . . . . . . 68

4.2.2 Methylation of Pincer Chloride Complexes . . . . . . . . . . . 80

4.2.3 Protonation of Platinum Methyl Complexes . . . . . . . . . . 87

4.3 Concluding Remarks . . . . . . . . . . . . . . . . . . . . . . . 88

5 Synthesis and Reactivity of PNP Pincer Complexes $\quad 90$

5.1 Synthesis of Platinum PNP Complexes . . . . . . . . . . . . . . . 91

5.2 Synthesis of Rhodium PNP Species . . . . . . . . . . . . . . . . 94

5.3 Concluding Remarks . . . . . . . . . . . . . . . . . . . . . . . 104

6 Catalytic Activity of Palladium Pincer Complexes 106

6.1 Palladium Pincer Complexes in Catalysis . . . . . . . . . . . . . . 106

6.2 Mechanistic Implications of Catalysis with PCP Pincer Complexes . . 107

6.3 Performance of $[(\mathrm{PCP}) \mathrm{PdCl}]$ Species in the Heck Reaction . . . . . . 110

6.3.1 Results of Heck Reactions . . . . . . . . . . . . . . . . . 111

6.3.2 Active Species Generation and Reaction Mechanism . . . . . . 115

6.4 Performance of $[(\mathrm{PCP}) \mathrm{PdCl}]$ Species in the Suzuki Reaction . . . . . 120

6.4.1 Results of Suzuki Reactions . . . . . . . . . . . . . . . . . 121

6.4.2 Active Species Generation and Reaction Mechanism . . . . . . 129

6.5 Concluding remarks . . . . . . . . . . . . . . . . . . . . . 140

$\begin{array}{lll}7 & \text { Conclusions } & 143\end{array}$

8 Experimental $\quad 147$

8.1 General Procedures . . . . . . . . . . . . . . . . . . . . . . 147

8.2 Transition Metal Precursors . . . . . . . . . . . . . . . . . 148

8.3 Ligands . . . . . . . . . . . . . . . . . . . . . . . 150

8.4 Platinum Complexes . . . . . . . . . . . . . . . . 153

8.5 Palladium Complexes . . . . . . . . . . . . . . . . . . 165

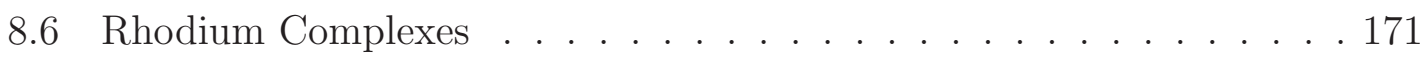


8.7 Catalytic Testing . . . . . . . . . . . . . . . . . 174

$\begin{array}{ll}\text { References } & 177\end{array}$ 


\section{List of Figures}

1.1 Examples of pincer ligand structures. . . . . . . . . . . . 3

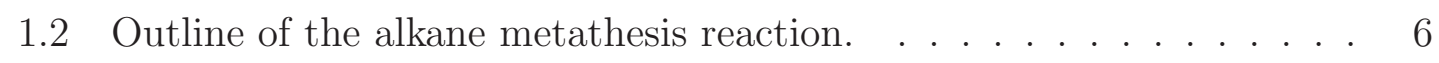

1.3 Outline of water splitting by pincer complexes. . . . . . . . 7

1.4 Electronic effects of phosphorus substituents. . . . . . . . . 8

1.5 Electronic influence on transfer-dehydrogenation catalysis. . . . . . 9

1.6 Facially-coordinated, electron-poor PCP pincer complexes . . . . . 10

3.1 ORTEP diagram of $[(\mathrm{POCOPH}) \mathrm{Pt}(\mathrm{nb})]_{2} \cdot 2 \mathrm{CH}_{2} \mathrm{Cl}_{2}(\mathbf{1 4}) \ldots \ldots 33$

3.2 ORTEP diagram of $[(\mathrm{POCOPH}) \mathrm{Pt}(\mathrm{nb})]_{2} \cdot 2 \mathrm{CH}_{2} \mathrm{Cl}_{2}(\mathbf{1 4}) \ldots \ldots .36$

3.3 Effect of $\pi-\pi$ stacking on the ${ }^{1} \mathrm{H}$ NMR data of compounds 14-16. . . 40

3.4 Two possible structural isomers of $\left[(\mathrm{POCCPH}) \mathrm{PtMe}_{2}\right]_{\mathrm{x}} \ldots \ldots \ldots . .42$

3.5 Formation of $[(\mathrm{POCOP}) \mathrm{PtCl}]$ from $\left[\mathrm{PtCl}_{2}(\right.$ hex $\left.)\right] \ldots \ldots \ldots \ldots . . .47$

3.6 Potential structures for the metallation intermediate $20 \ldots \ldots 48$

3.7 Stacked plot showing cis,trans dimers 19 and $21 \ldots \ldots . \ldots . . . .52$

3.8 Formation of $[(\mathrm{POCOP}) \mathrm{PtCl}]$ from $[\mathrm{PtClMe}($ hex $)] \ldots \ldots \ldots . . .54$

3.9 Previously reported cis,trans-dimers. . . . . . . . . . . . 57

4.1 Effect of ancillary ligand binding strength on dimer formation. . . . . 64

4.2 Molecular orbital diagram of carbon monoxide. . . . . . . . . 71

4.3 ORTEP diagram of $[(\mathrm{POCOP}) \mathrm{Pt}(\mathrm{CO})]\left[\mathrm{SbF}_{6}\right](\mathbf{3 1}) \ldots \ldots \ldots . \ldots 73$

4.4 Anion packing of $[(\mathrm{POCOP}) \mathrm{Pt}(\mathrm{CO})]\left[\mathrm{SbF}_{6}\right](\mathbf{3 1}) \ldots \ldots \ldots \ldots .75$

4.5 IR spectra showing carbonylation of 29 and decarbonylation of 35.77

4.6 Decarbonylation of compounds $\mathbf{3 4}, \mathbf{3 5}$, and $\mathbf{3 6} \ldots \ldots \ldots$. . . . 79

4.7 Spectroscopic data for byproduct $46 \ldots \ldots \ldots \ldots$

4.8 Protonation of $\mathbf{4 3}$ and $\mathbf{4 5}$ at low temperature. . . . . . . 87

5.1 Synthesis of the PNNNP rhodium complex 49. . . . . . . . 96

5.2 NMR spectra for the rhodium-methylzinc adduct $51 \ldots \ldots 99$

6.1 Outline of the classic "textbook" Heck catalytic cycle. . . . . . . . . 108

6.2 Representative examples of cyclometallated P-C chelates. . . . . . 109

6.3 Heck reactions of 28 and 29 . . . . . . . . . . . . . 114

6.4 Suzuki reactions of $\mathbf{2 8}, \mathbf{2 9}, \mathbf{3 0}$ and (DPPF) $\mathrm{PdCl}_{2} \ldots \ldots \ldots \ldots$

6.5 Raw data for Suzuki reactions at $100{ }^{\circ} \mathrm{C} \ldots \ldots \ldots \ldots . \ldots . \ldots 125$ 
6.6 Suzuki reactions of $\mathbf{2 8}$ and $\mathbf{3 0}$ at 80 and $60{ }^{\circ} \mathrm{C}$. . . . . . . . . . . 127

6.7 Suzuki reactions of $\mathbf{2 8}$ with and without the addition of mercury. . . 128

6.8 Outline of the classic "textbook" Suzuki catalytic cycle. . . . . . . . . 130

6.9 Effect of sterics on aryl coordination. . . . . . . . . . . . . 131

6.10 Redox-free mechanism for the Suzuki reaction. . . . . . . . . . . . . 132

6.11 Resonance stabilisation in the Suzuki reaction. . . . . . . . . . . . . . 134

6.12 Plots of decomposition products from palladium pincer complexes. . . 138 


\section{List of Schemes}

1.1 Synthetic methodology for PCP and PNP pincers. . . . . . . . . . . 11

1.2 Synthesis of "click" PCP pincer ligands. . . . . . . . . . . . . . . . . 12

1.3 Synthesis of pincer ligands via "ligand introduction". . . . . . . . . . 13

1.4 Outline of the Heck and Suzuki cross-coupling reactions. . . . . . . . 14

2.1 Synthesis of the phosphinite pincer ligand 1. . . . . . . . . . . . . . 19

2.2 Synthesis of the phosphine pincer ligand $2 \ldots \ldots$. . . . . . . . 20

2.3 Conventional phosphine syntheses unable to produce 2 . . . . . . . . 21

2.4 Synthesis of the phosphine-phosphinite pincer ligand 3. . . . . . . . 23

2.5 Synthesis of the PNP pincer ligands $\mathbf{1 0}$ and 11. . . . . . . . . . 25

2.6 Attempted synthesis of PCP pincer ligand 13. . . . . . . . . . . . . 27

3.1 Formation of a pincer complex and oligomeric byproduct. . . . . . . . 29

3.2 Oligmer formation from the reaction of 1 with $\left[\mathrm{Pt}(\mathrm{COD})_{2}\right] \ldots \ldots$. . . 31

3.3 Formation of $[(\mathrm{POCOPH}) \mathrm{Pt}(\mathrm{nb})]_{2}(\mathbf{1 4}) \ldots \ldots \ldots$

3.4 Formation of $\left[(\mathrm{POCOPH}) \mathrm{PtMe}_{2}\right]_{\mathrm{x}}$ species 15 and 16. . . . . . . . 39

3.5 Formation of $\left[(\mathrm{POCOPH}) \mathrm{PtCl}_{2}\right]_{\mathrm{x}}$ species. . . . . . . . . . . . . 44

3.6 Formation of cis, trans- $[(\mathrm{POCOPH}) \mathrm{PtClMe}]_{2}$ species $(\mathbf{2 1}) . \ldots . . .51$

3.7 Formation of cis, trans $-\left[(\mathrm{POCOPH}) \mathrm{PdCl}_{2}\right]_{2}$ species $(\mathbf{2 3}) \ldots \ldots \ldots 6$

4.1 Effect of starting material on the metallation reaction of ligand 1. . . 62

4.2 Synthesis of the pincer complex 1 from different starting materials. . 66

4.3 Metallation of the pincer backbone to form 25. . . . . . . . . . 67

4.4 Attempted methylations of 25 . . . . . . . . . . . . . . . . 82

4.5 Synthesis of $\mathbf{4 4}$ and conversion back to 26 by ambient $\mathrm{H}_{2} \mathrm{O}$. . . . . 82

5.1 Synthesis of the PNNNP pincer complex 47. . . . . . . . . . . . . . . 92

5.2 Formation of the oligomer 48. . . . . . . . . . . . . . . . 93

5.3 Non-innocent behaviour of PCNCP complexes. . . . . . . . . . . . . . 95

5.4 N-Methylation of the PNNNP ligand. . . . . . . . . . . . . . . . . . . 101

5.5 Rhodium iodide chemistry . . . . . . . . . . . . . . . 103

$6.1 \mathrm{Pd}(\mathrm{IV})$ intermediates in the Heck reaction. . . . . . . . . . . . . . . . 109

6.2 Chelate-assisted oxidative addition to form a $\mathrm{Pd}(\mathrm{IV})$ complex. . . . . 110

6.3 Amine-promoted decomposition path for PCP pincer complexes. . . . 115 
6.4 Alternate decomposition path for PCP pincer complexes. . . . . . . 116

6.5 $\mathrm{P}-\mathrm{O}$ bond hydrolysis in a PCP pincer complex. . . . . . . . . . . . . 118

6.6 NMR-scale Suzuki reaction of 28. . . . . . . . . . . . . . . . . 135

6.7 Pathway for $\mathrm{Pd}(0)$ active catalyst formation in $\mathrm{P}-\mathrm{C}$ chelates. . . . 136 


\section{List of Tables}

1.1 Variable parameters of pincer ligands. . . . . . . . . . . . . . . 4

2.1 Effect of phosphine substituents on the TEP. . . . . . . . . . . . 18

2.2 NMR data of degradation product 9. . . . . . . . . . . . . . . . 24

3.1 Bond distances and angles of $[(\mathrm{POCOPH}) \mathrm{Pt}(\mathrm{nb})]_{2} \cdot 2 \mathrm{CH}_{2} \mathrm{Cl}_{2}$ (14). . 34

3.2 Crystallographic data of $[(\mathrm{POCOP}) \mathrm{Pt}(\mathrm{nb})]_{2} \cdot 2 \mathrm{CH}_{2} \mathrm{Cl}_{2}(\mathbf{1 4})$. . . . . 34

$3.3{ }^{31} \mathrm{P}$ NMR data of dimeric compounds containing ligand $1 . \quad$. . . . . 59

4.1 Infrared C-O stretches of pincer carbonyl complexes. . . . . . . . . . 70

4.2 Bond distances and angles for $[(\mathrm{POCOP}) \mathrm{Pt}(\mathrm{CO})]\left[\mathrm{SbF}_{6}\right](\mathbf{3 1}) . \quad$. . . . 74

4.3 Crystallographic data for complex $[(\mathrm{POCOP}) \mathrm{Pt}(\mathrm{CO})]\left[\mathrm{SbF}_{6}\right](\mathbf{3 1})$. . 74

6.1 Results of the Heck reaction with 28, 29, and 30. . . . . . . . . 112

6.2 Results of the Suzuki reaction with $\mathbf{2 8}$, 29, and $\mathbf{3 0}$. . . . . . . . 122 


\section{Glossary}

COD 1,5-cyclooctadiene

dba trans,trans-dibenzylideneacetone

FWHM full width at half maximum

GCMS gas chromatography mass spectrometry

hex 1,5-hexadiene

HMBC heteronuclear mutiple-bond correlation

HOMO highest occupied molecular orbital

HRMS high resolution mass spectrometry

LUMO lowest unoccupied molecular orbital

$\boldsymbol{m} / \boldsymbol{z} \quad$ mass to charge ratio

nb norbornene

TEP Tolman Electronic Parameter

TOF turnover frequency, moles of product produced per mole of catalyst per hour

TON turnover number, moles of product produced per mole of catalyst 


\section{Notes on terminology}

PCP, PNP, ECE, etc. - specifies generic tridentate pincer ligands that coordinate through the donor atoms specified (where E represents any donor atom).

POCOP, POCCP, PNNNP, etc. - represents pincer ligands possessing substituted 1,3-phenylene or 2,6-pyridyl backbones. The first, third, and fifth letters denote the ligating atoms, while the second and fourth letters indicate the linkages between donor groups and the aromatic backbone.

POCOPH, PCCCPH, POCCPH, etc. - denotes that the aromatic backbone of the particular PCP pincer ligand is unmetallated and therefore possesses a proton on the potentially ligating carbon atom.

$[$ (pincer $\left.) \mathbf{M L}_{\mathrm{n}}\right]$ - it has become a literature convention to present pincer complexes with the pincer ligand appearing in brackets before the metal atom. 


\section{Chapter 1}

\section{Introduction}

This thesis provides an account of research into the synthesis and coordination chemistry of pincer ligands possessing electron-withdrawing substituents. Particular attention was paid to the nature of intermediate species formed during the synthesis of pincer complexes, as well as the effect of ligand electronics on the ease of pincer complex formation and reactivity. The performance of the palladium complexes of these electron-poor pincer ligands was also evaluated in the Heck and Suzuki crosscoupling reactions.

\subsection{Metal-Ligand Coordination Complexes}

The realm of metal-ligand coordination chemistry is invariably a vast and diverse one. With the abundance of metals, donor atoms, and ligand architectures at the chemists' disposal, there are a multitude of potential complexes that can be formed. Each ligand bound to the metal centre will affect both the physical space around the metal centre, and the electron density on the metal itself. While each metal atom possesses its own intrinsic electronic character, it is the manipulation of the metal's steric and electronic environment by the ligand that goes a long way to determining the reactivity of metal complexes.

One of the foundations of coordination chemistry, and in particular organometallic chemistry, is the principle that with the appropriate choice of ligand the electronic and steric parameters of a metal centre can be manipulated. This allows the rational design of coordination complexes with specific objectives in mind. For example, 
cisplatin, cis-[ $\left[\mathrm{PtCl}_{2}\left(\mathrm{NH}_{3}\right)_{2}\right]$, is effective at binding to DNA and killing cancer cells via apoptosis. With accrued knowledge about how the modification of the amine and chloride ligands affect the structure and reactivity of these platinum complexes, more effective anti-cancer agents have been and continue to be developed. ${ }^{1}$ This parable demonstrates the foundation that underpins coordination chemistry: the more that is known about how the electronic and steric modification of ligands affect the reactivity of their metal complexes, the greater the degree to which the reactivity of the complexes can be optimised.

Multidentate ligands further expand upon this foundation. By incorporating more than one donor group into a single ligand, the distance between groups can be manipulated and constrained, impacting the geometries available to coordination complexes, as well as introducing the ability of ligands to bridge and connect multiple metal centres. These properties can be exploited to allow a high degree of finetuning; in catalysis the chelate effect is particularly useful, as it helps to increase the stability of metal complexes with multidentate ligands. ${ }^{2}$ The presence of different types of donor group can also direct reactions to occur preferentially at specific sites, while hemilabile binding of donor groups can afford additional coordination sites if required, while still stabilising the metal in the absence of competition for the coordination site. ${ }^{3}$ The ability of these multidentate ligands to adopt bridging coordination modes also allows for the formation of large, highly-ordered structures. These multinuclear structures can then expand upon the existing properties of a complex, potentially transforming homogeneous catalysts into heterogeneous catalysts, ${ }^{4}$ and also giving rise to new properties that depend on long-range order, such as luminescence. ${ }^{5}$

As multidentate ligands can offer considerable advantages over monodentate ligands, the importance of understanding the effects of ligand modification becomes increasingly important. To be able to design metal complexes with specific objectives or properties in mind, it is crucial to understand how the steric and electronic character of the ligand affects the structure and chemistry of the resultant metal complexes.

\subsection{Pincer Complexes}

One particular type of coordination complex that has demonstrated considerable versatility, applicability, and durability in the scientific literature is that of the pincer ligand. These are defined as being tridentate chelating ligands which co- 
ordinate to metal centres predominantly in a meridional fashion. While this definition of what comprises a pincer ligand is very broad, one trait that is often present in pincer ligands is a rigid backbone, to constrain the degrees of freedom and favour planar coordination geometries. Common motifs that have been employed in pincer ligands are: bisoxazolinyl-phenyls, ${ }^{6}$ diphenylamine-derivatives, ${ }^{7}$ diazaborolidines, ${ }^{8}$ 1,8-substituted anthracyls, ${ }^{9}$ N-heterocyclic carbene-derivatives, ${ }^{10}$ 1,3-substituted ferrocenyls, ${ }^{11}$ and 1,3,5-substituted aromatics containing pendant chains, ${ }^{12}$ as shown in Figure 1.1. Pincer ligands are typically named according to the ligating atoms present, and where the deprotonation of a donor group is required to achieve tridentate pincer coordination, the presence of the proton on the free ligand is usually denoted with an 'H'. Figure 1.1 describes, from top left to bottom right, NCNH, PNPH, PBPH, PCPH, CNC, PCPH, and SCSH pincer ligands.
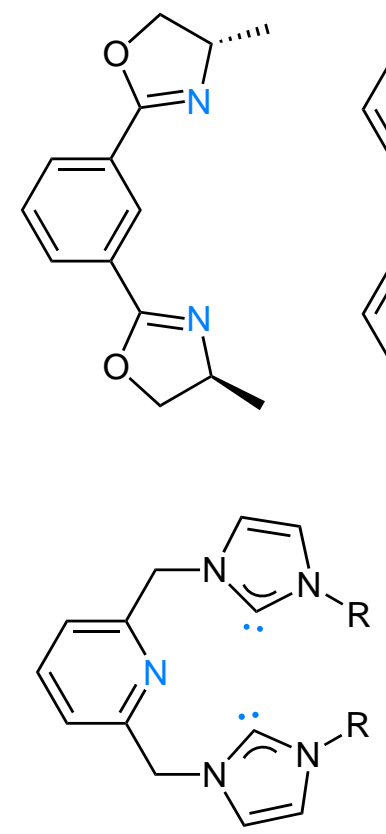
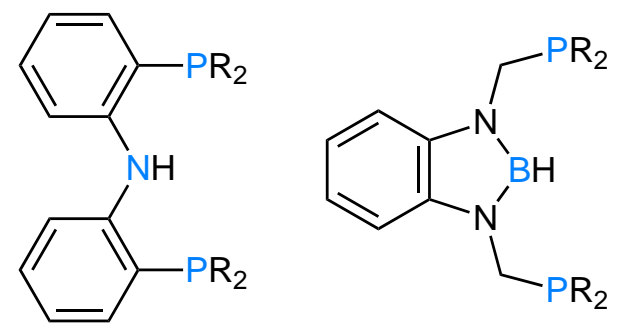
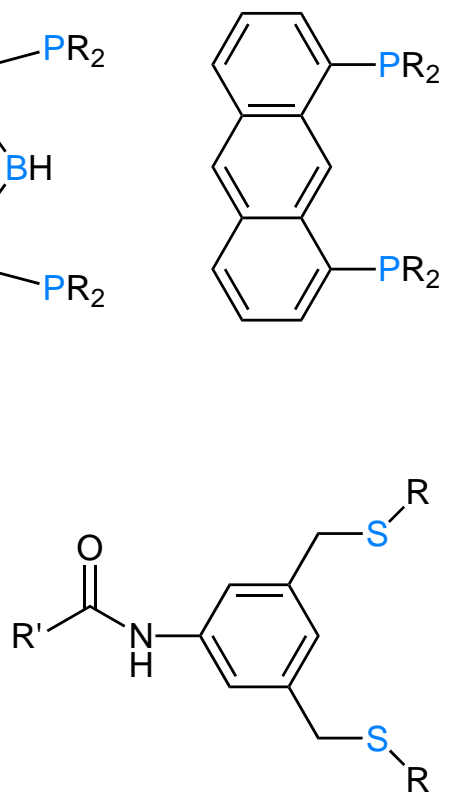

Figure 1.1 Representative examples demonstrating a range of pincer ligand structures.

This range of structural motifs used as pincer ligands serves to highlight their versatility. While maintaining a tridentate, meridional coordination mode, the ligand can possess a range of donor atoms, both anionic and neutral, as light as boron or as heavy as arsenic. While the ligands highlighted in Figure 1.1 generally have $C_{2}$ symmetry, asymmetric pincer ligands can be readily generated by varying either the spacer groups between the donor atom and ligand backbone (often referred to as the "arms" of the pincer ligand), ${ }^{13}$ or by variation of these donor groups themselves (with PNN pincers being a typical example). ${ }^{14}$

The rigid, planar framework provided by pincer ligands is desirable from a chemical point of view as it inhibits cyclometallation of the substituents on donor groups, 
which engenders a high thermal stability to pincer complexes. This makes pincer compounds particularly suited for catalytic processes that operate at high temperatures. Another feature of pincer ligands is the fact that upon coordination to a metal centre the donor groups on the "arms" of the pincer ligand adopt a mutually trans configuration, while the backbone is coordinated trans to an ancillary ligand on the metal. This provides an optimal configuration for catalysis, in which the trans-influence (the labilisation of ligands trans to a specific ligand) is utilised to good effect. Ligands with a low or moderate trans-influence (such as phosphines) can be placed on the arms, where they will be coordinated trans to each other, and therefore undergo minimal mutual destabilisation. With the placement of a donor group with a high trans-influence on the ligand backbone, ligand coordination trans to pincer backbone will be labilised. This helps to prevent inhibition of pincer catalysts by solvent or ancillary ligand coordination.

Table 1.1 Variable parameters of pincer ligands and their control over the steric and electronic properties of complexes. Table adapted from the review by Choi, MacArthur, Brookhart, and Goldman. ${ }^{15}$

\begin{tabular}{|c|c|c|}
\hline \multirow{3}{*}{$Z-\left.\right|_{/ /} ^{Y-E R_{n}}$} & Group & Major Effect(s) of Variation \\
\hline & $\mathbf{E R}_{\mathrm{n}}$ & $\begin{array}{l}\text { Steric control by varying substituents; } \\
\text { control of electron density; lability. }\end{array}$ \\
\hline & $\mathbf{Y}$ & $\begin{array}{l}\text { Control over electron density; } \\
\text { indirect control on steric properties. }\end{array}$ \\
\hline $\begin{array}{l}\text { Most common elements } \\
\text { E: P, N, S, O, As }\end{array}$ & $\mathrm{X}$ & Electronic effects, particularly trans-influence. \\
\hline $\begin{array}{l}\mathrm{X}: \mathrm{C}, \mathrm{N}, \mathrm{B} \\
\mathrm{Y}: \mathrm{CH}_{2}, \mathrm{O}, \mathrm{NH}\end{array}$ & $\mathrm{Z}$ & $\begin{array}{l}\text { Remote control of electron density; } \\
\text { solubility control. }\end{array}$ \\
\hline
\end{tabular}

Despite the range of complicated ligand backbones that can adopt a pincer coordination mode, the most common structural motifs for pincers are those bearing 1,3-substituted phenyl or 2,6-substituted pyridyl groups. However, the simplicity of these aromatic backbones belies the intricacies that can be introduced into the ligands due to their modular nature. Table 1.1 summarises the modification of these backbones and the effect of each component on the ligand as a whole. The ability of pincer ligands to be highly amenable to structural diversity, while still maintaining a reliable and predictable coordination mode, makes them ideal substrates for the investigation of how ligand modifications affect the reactivity of the resultant metal complexes. 


\subsubsection{Uses of Pincer Complexes With Phosphorus Donors}

Of the multitude of pincer complexes that have been reported, arguably, those that have demonstrated the greatest utility are ligands with at least one phosphorus donor atom. The advantage of incorporating phosphorus donors within the pincer coordination motif is that the soft electronic nature and $\pi$-acceptor character of phosphines are ideal for stabilising late transition metals, especially those in low oxidation states. Late transition metal complexes are renowned for their performance in homogeneous catalysis and small molecule activation, with pincer complexes of late transition metals being particularly effective. They have been shown to facilitate organic transformations such as the Heck, Suzuki, Sonogashira, Stille, and Negishi cross-coupling reactions, as well as asymmetric allylic alkylations, hydroaminations, transfer hydrogenations, and aldol-type reactions. Summaries of this work have been detailed in a number of review papers. ${ }^{16-20}$

While pincer complexes are able to assist the chemist with transformations in organic synthesis, they are also at the forefront of research into some of the major developments in organometallic chemistry. One of the most intriguing reactions facilitated by phosphine-based pincer complexes is that of alkane dehydrogenation. In 1996, Jensen, Kaska, and co-workers discovered an iridium PCP pincer species capable of promoting catalytic dehydrogenation of cyclooctane in the presence of a hydrogen acceptor species. ${ }^{21}$ This pincer catalyst offered an advantage over other homogeneous dehydrogenation catalysts as it was stable in solution for prolonged periods under the reaction conditions, and outperformed heterogeneous dehydrogenation catalysts by dehydrogenating a much larger range of substrates and operating at a considerably lower temperature $\left(150{ }^{\circ} \mathrm{C}\right.$ as opposed to $500-900{ }^{\circ} \mathrm{C}$ for heterogeneous species). ${ }^{15}$ This transfer-dehydrogenation has been shown to be selective for the formation of terminal alkenes, with the catalyst undergoing minimal inhibition or degradation.

This facile dehydrogenation of alkanes catalysed by iridium PCP pincer complexes has lead to the development of a system for alkane metathesis, which may in the future aid the production of synthetic fuels from low-cost feedstocks. In this tandem catalytic system (Figure 1.2), an alkane undergoes pincer-catalysed transferdehydrogenation, with the resultant alkene subjected to metathesis using Schrocktype molybdenum or tungsten carbene catalysts. ${ }^{22}$ These alkene metathesis products then act as the hydrogen acceptor species for the alkene transfer-dehydrogenation step, generating new alkane products from the original alkane starting materials. ${ }^{23}$ Currently, problems exist with alkene isomerisation leading to a distribution of products being obtained, ${ }^{22}$ nonetheless this proof of concept is surely an exciting one. 


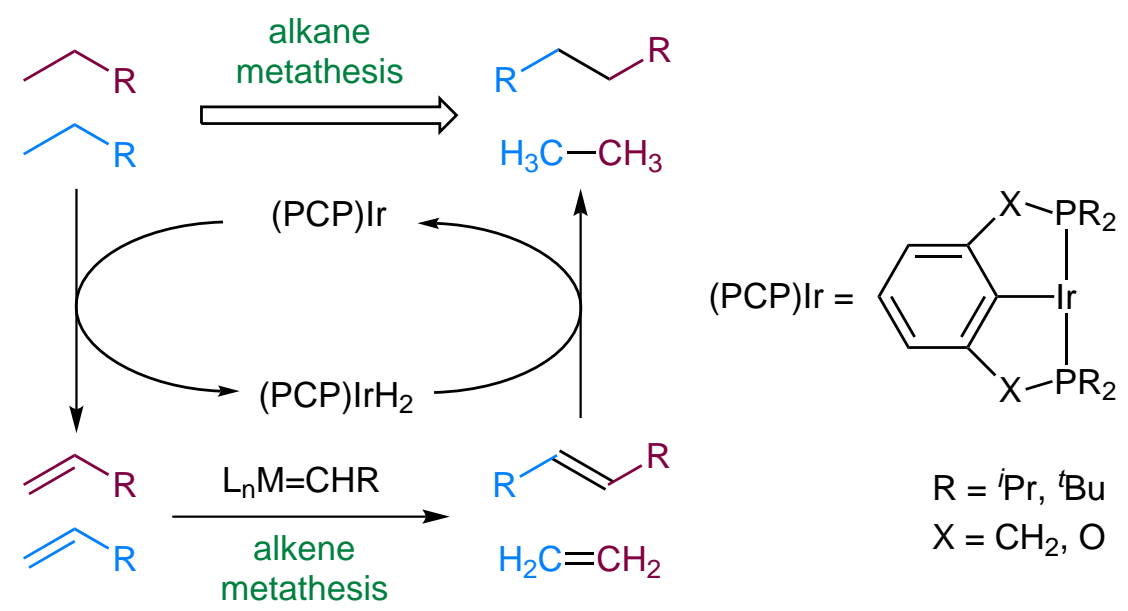

Figure 1.2 Outline of the alkane metathesis reaction. Figure adapted from Haibach et $a .^{22}$

Catalytic alkane dehydrogenation is an example of the selective cleavage and subsequent reaction of carbon-hydrogen bonds, otherwise known as $\mathrm{C}-\mathrm{H}$ activation. This has been a topic of major intrigue and focus, with the March 1995 issue of Accounts of Chemical Research denoting it one of the "Holy Grails" of chemistry. ${ }^{24} \mathrm{~A}$ further breakthrough in this field has been achieved by the Brookhart research group, with the first full characterisation in solution of a methane $\sigma$-complex, $\left[(\mathrm{PNP}) \mathrm{Rh}\left(\mathrm{CH}_{4}\right)\right] \cdot{ }^{25 *}$ This complex, in which a methane molecule is bound to a PNP pincer-ligated rhodium centre through the interaction between a $\mathrm{C}-\mathrm{H}$ bond and the metal, provides a basis for research into how the strength of this metal-methane interaction can be manipulated and ultimately exploited to achieve $\mathrm{C}-\mathrm{H}$ bond functionalisation.

Phosphorus-containing pincer complexes have also shown promise in water splitting - the separation of water into molecular hydrogen and oxygen. Cheap and ready access to this technology is heralded as reducing dependence on fossil fuels and bringing about a hydrogen economy, and hence is a major drawcard for chemical research. ${ }^{27}$ Investigations undertaken with PCN platinum ${ }^{28}$ and $\mathrm{PNN}$ ruthenium ${ }^{29}$ pincer complexes have confirmed key underlying principles that water splitting technology will need to exploit (Figure 1.3). ${ }^{27}$ While these reactions have not been incorporated into a complete catalytic cycle, they indicate that such complexes can offer viable inroads into achieving organometallic water splitting.

${ }^{*}$ The first evidence for $\sigma$-coordination of methane to metal centres was provided by Perutz and Turner, by photolysis of metal carbonyl complexes in a methane matrix at $20 \mathrm{~K} .{ }^{26}$ Due to the low temperatures required for reactions carried out in liquid methane, reaction mixtures were analysed by infrared and visible spectroscopy. The generation of a $\sigma$-methane ligand in the inner coordination sphere of the metal complex by the Brookhart group allowed the reaction to be carried out in a suitable solvent for NMR spectroscopy $\left(\mathrm{CDCl}_{2} \mathrm{~F}\right)$, which provided a greater amount of data on the structure of the methane complex than either infrared or visible spectroscopy could. 


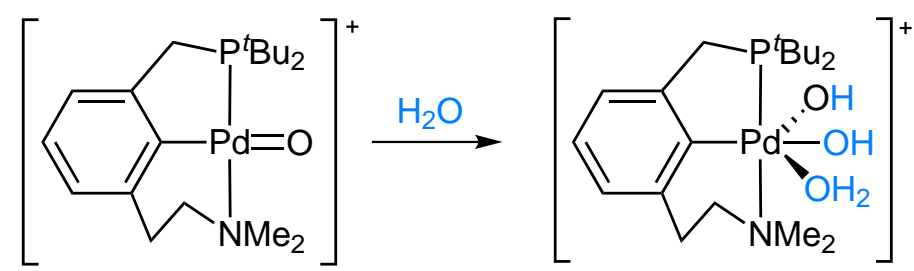

First example of oxidative addition of water to a metal oxo complex.
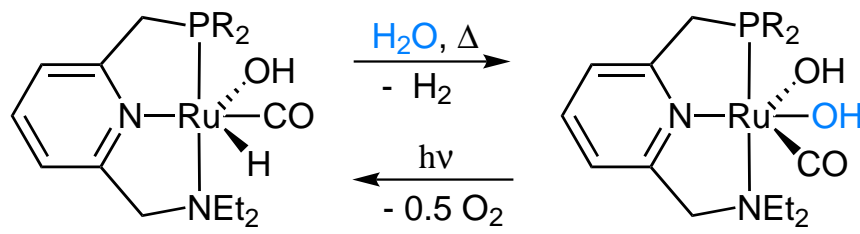

Evolution of hydrogen and oxygen from water, albeit slowly ( 3 days, $100^{\circ} \mathrm{C}$ for the forward reaction).

Figure 1.3 Outline of water splitting by pincer complexes.

\subsection{Electron-Poor Ligands}

Whilst PCP and PNP pincer ligands have been well studied and successfully employed in a number of intriguing catalytic processes, previous work has predominantly focussed on complexes possessing electron-rich phosphorus donors, such as di-tert-butyl or diisopropyl phosphines. Substantially less work has been undertaken to investigate the effect of incorporating electron-poor donor groups into the pincer framework.

There have been a number of reports in which electron-withdrawing groups have been integrated into established ligand motifs and resulted in a significant increase in catalytic performance of the resultant complexes. ${ }^{30-32}$ This effect may appear counterintuitive, as many of the mechanisms prevalent in organometallic transformations are dependent on reactions proceeding through the conventional oxidative addition and reductive elimination pathways. Since oxidative addition is predominantly reliant on electron donation from the metal centre into the $\sigma^{*}$-antibonding orbital of the bond being cleaved, reducing the electron density on the metal should disfavour oxidative addition and reduce the activity of the catalyst. However, electron-deficient metal centres will be poorer at stabilising high oxidation states than electron-rich metals, and consequently reductive elimination steps will be enhanced. Moreover, for important reactions such as $\mathrm{C}-\mathrm{H}$ activation, calculations have shown a number of reaction pathways may be possible (electrophilic, ambiphilic, and nucleophilic), ${ }^{33}$ and that the introduction of electron-withdrawing ligands should generate favourable charge transfer interactions in some instances. ${ }^{34}$ 


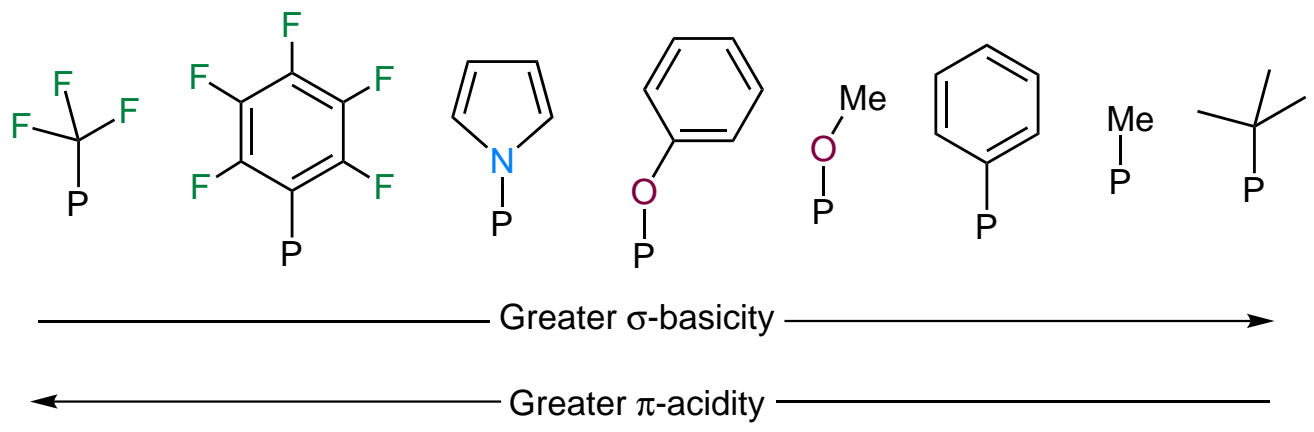

Figure 1.4 Electronic effects of phosphorus substituents.

Numerous studies, both computational and experimental, have been undertaken to develop a comprehensive hierarchy of the degree to which substituents affect the electron density on the phosphorus centre. ${ }^{35-37}$ As phosphorus-metal bonding has both a $\sigma$-donor and a $\pi$-acceptor component, in principle, as the substituents on the phosphorus become more electronegative, the $\sigma$-donor ability of the phosphorus is reduced, while its $\pi$-acceptor ability is increased (Figure 1.4). In practice, the two components are difficult to distinguish; a case in point being the pentafluorophenyl substituent, which had previously been regarded as increasing the $\pi$-acceptor ability of the phosphorus donor, but may now be regarded as predominantly decreasing the $\sigma$-donor ability phosphorus ligands. ${ }^{38}$ Regardless of the exact nature of this electronic effect, fluoroalkyl and fluoroaryl substituents will typically produce very electron-poor metal centres upon coordination; together with phosphites they represent a widely used means of generating electron-withdrawing phosphorus-donor ligands. Fluoroarylphosphine ligands in particular have demonstrated unique reactivities and enhanced catalytic activity over their perprotio analogues, ${ }^{39}$ making them ideal candidates for incorporation into the pincer ligand motif.

The effect imparted by electron-withdrawing groups on the metal centre can also be quantified via the synthesis of metal-carbonyl derivatives of pincer complexes. The frequency of the $\mathrm{C}-\mathrm{O}$ stretch in the infrared spectrum will reflect the amount of electron density on the metal centre; a more electron-rich metal complex will be able to contribute a greater amount of electron density to the carbonyl through $\pi$-backbonding, so will display a $\mathrm{C}-\mathrm{O}$ stretching frequency at a lower wavenumber than for a more electron-poor metal complex. 


\subsubsection{Electron-Poor Pincer Complexes}

Complexes of electron-poor PCP and PNP pincer ligands, while relatively rare, have shown a number of interesting traits worthy of further investigation. The Brookhart group has examined the effect that substitution of the PCP pincer backbone para to the coordinated metal centre (carbon C-5 of the ligand) has on the reactivity of these complexes in iridium-catalysed transfer-dehydrogenation. ${ }^{40}$ It was found that the turnover numbers obtained showed a rough correlation with the electronwithdrawing ability of the substituent: the more withdrawing the substituent, the more active the catalyst (Figure 1.5). ${ }^{\dagger}$ Similarly, replacement of methylene groups on the arms of the ligand with oxygen atoms was observed to increase the activity by one order of magnitude, due to lower $\sigma$-donor ability and greater $\pi$-acceptor ability of phosphinites compared to phosphine ligands. The higher activity of the more electron-poor catalysts was thought to be due to their propensity for $\pi$-coordination of alkenes over $\mathrm{C}-\mathrm{H}$ oxidative addition, reducing catalyst inhibition by the tertbutylethylene starting material. ${ }^{42}$
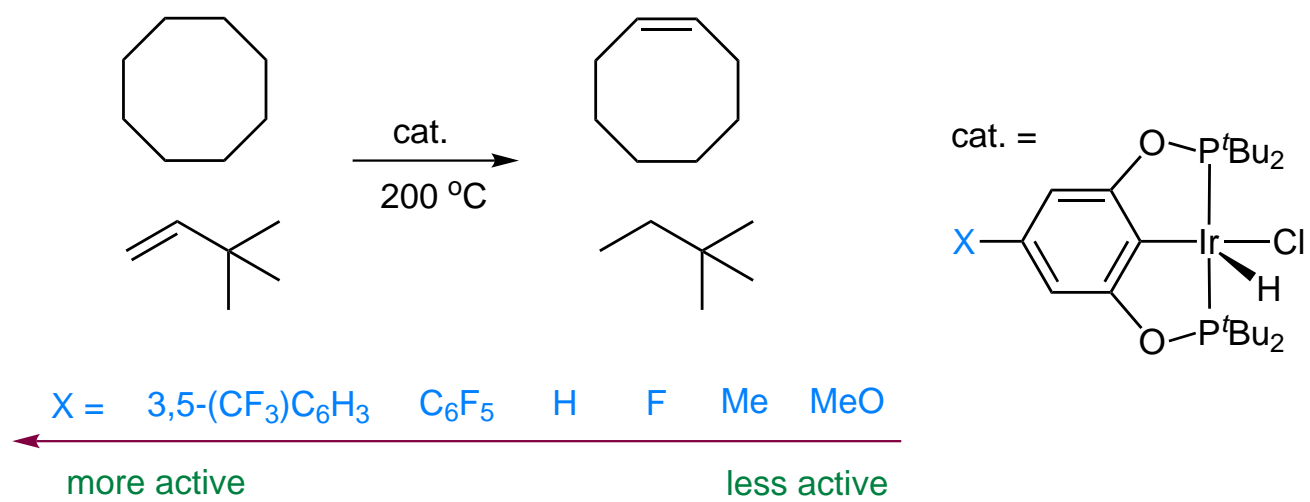

Figure 1.5 Effect of electronic modification of iridium pincer catalysts for the transfer dehydrogenation reaction.

To try to enhance this electron-withdrawing effect, a small number of pincer complexes have been reported whereby the electron-withdrawing substituents are located on the phosphorus donors, putting them in close proximity to the metal centre. An interesting consequence of increasing the electron-withdrawing nature of the ligands is that the mutually trans coordination of phosphorus donors becomes unfavourable. This forces some five- and six-coordinate pincer complexes to adopt a facial (rather than meridional) coordination geometry of the pincer ligand (Figure 1.6). ${ }^{43-45}$ As

\footnotetext{
${ }^{\dagger}$ It is important to note that the introduction of the same substituent at C-5 of the aromatic backbone may cause a different effect to substitution at the phosphorus. A good example of this occurs for alkoxy groups. Compared to alkyl substituents, alkoxy substituents produce more electron-poor phosphorus donors, as they lower the energy of the LUMO on the phosphorus, ${ }^{41}$ increasing $\pi$-acidity. However, when attached directly to the ligand backbone, they produce more electron-rich aromatic rings due to resonance effects.
} 
well as indicating that electron-deficient pincer ligands may provide access to unusual coordination geometries, the five-coordinate complexes possess ancillary ligands separated by $90^{\circ}$ instead of the usual $120^{\circ}$ for meridionally coordinated pincer ligands, a property that may favour reductive elimination steps in catalysis.
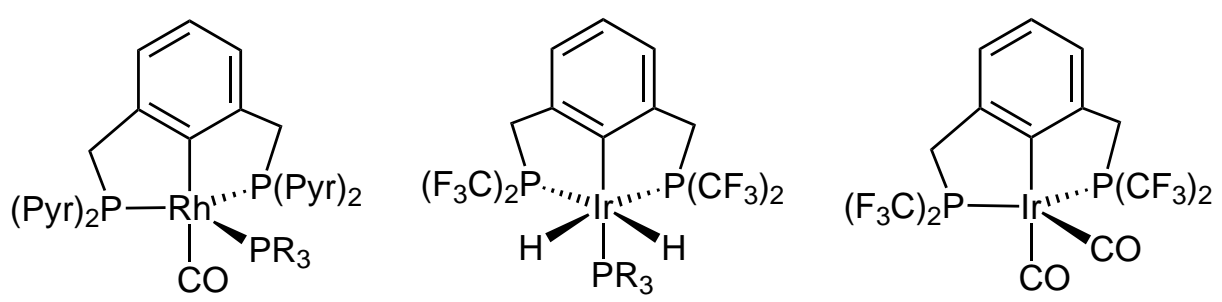

Figure 1.6 Pincer complexes with electron-poor PCP ligands, displaying unusual facial coordination geometry.

A further electron-deficient PCP pincer complex that has demonstrated an unusual reactivity is the 2,4,6- $\left(\mathrm{CF}_{3}\right)_{3} \mathrm{C}_{6} \mathrm{H}_{2}$-substituted [(POCOP) $\left.\operatorname{Ir}\left(\mathrm{N}_{2}\right)\right]$ pincer complex synthesised by the Brookhart group. ${ }^{46}$ In the crystalline form, this complex has demonstrated the ability to undergo exchange of the nitrogen ligand for oxygen, hydrogen, carbon monoxide, ammonia, and ethene, while maintaining its crystallinity. Furthermore, single crystals of this iridium pincer complex were shown to catalyse the selective hydrogenation of ethene in the presence of propene, indicating that catalysis occurred to some extent within the confines of the crystal lattice. As such, this electron-deficient pincer complex appears to be a step towards bringing the selectivity and fine tuning demonstrated by homogeneous catalysts into the realm of heterogeneous catalysis.

\subsection{Synthesis of PCP and PNP Pincer Complexes}

Owing to the interesting possibilities presented by pincer ligands possessing phosphorus donors, this research focusses on PCPH and PNP pincer ligands. The synthesis of such ligands and their complexes have been accomplished utilising a number of different methodologies.

The most common synthetic method for the formation of pincer complexes involves two general steps: first the installation of the donor arms of the ligand on to the backbone, then reaction of the ligand with an appropriate metal precursor to yield the tridentate pincer complex (Scheme 1.1). The methods used to attach the phosphorus donor groups to the ligand backbone generally rely on established organophosphorus 
chemistry, with separate donor groups able to be installed sequentially on the backbone to generate asymmetric $\mathrm{PCP}^{\prime} \mathrm{H}$ or $\mathrm{PNP}^{\prime}$ ligands. ${ }^{13}$ From the outset there is the opportunity to introduce diversity into the structure; as the reaction conditions required to attach the phosphorus donor groups are relatively general and occur under mild conditions (with the exception of the lithiation step in Scheme 1.1) they are tolerant to other functionalities present on the ligand backbone.
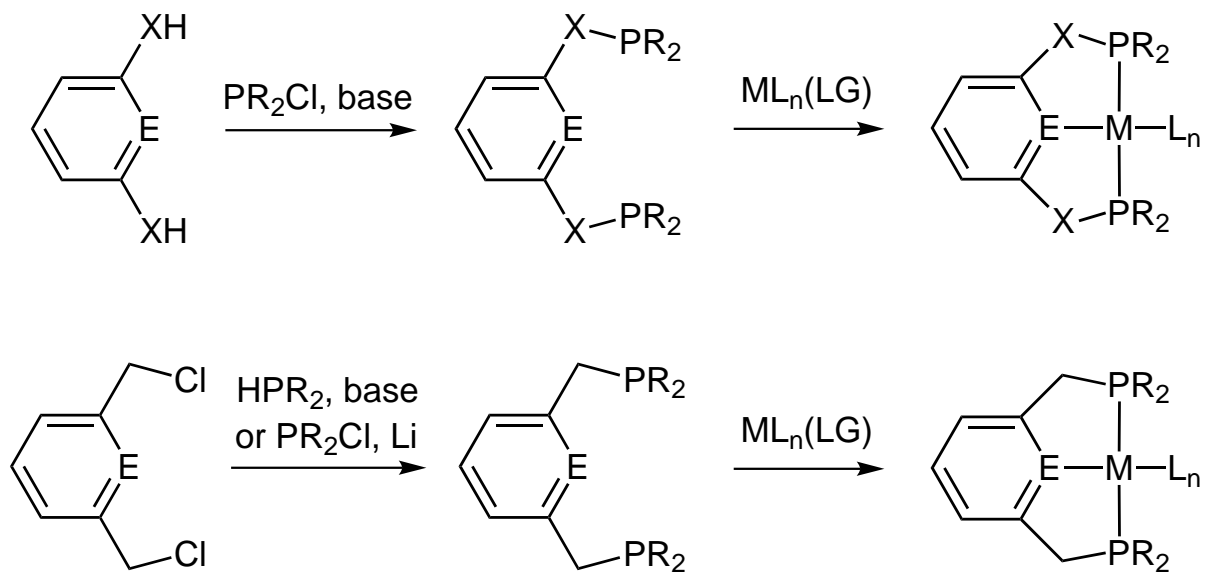

$\mathrm{E}=\mathrm{C}, \mathrm{N}$
$\mathrm{X}=\mathrm{NH}, \mathrm{O}$

Scheme 1.1 Common synthetic methodology employed for PCP and PNP pincer complexes. Lithiation is only required where the secondary phosphine is not sufficiently nucleophilic to react directly with the benzylic chloride. Typically triethylamine is used as a base. LG represents a neutral leaving group.

The complexation of the ligand to the metal is rather more simple for PNP pincers than for PCP pincers. In both cases the metal precursor is usually in the oxidation state desired for the resultant pincer complex, is stabilised by readily displaced neutral ancillary ligands (commonly acetonitrile or cyclooctadiene), and often contains an anionic leaving group (LG). Coordination of phosphorus donors in place of the ancillary ligands occurs first, followed by displacement of the leaving group by the donor group on the ligand backbone to achieve the tridentate pincer coordination mode. This backbone coordination is significantly more facile for PNP ligands than for PCPH ligands, as the former only requires ligand displacement from the metal, while the latter also requires the cleavage of the strong $\mathrm{C}-\mathrm{H}$ bond at the carbon on the between the ligand arms on the backbone (carbon $\mathrm{C}-2$, denoted $\mathrm{E}$ in Scheme 1.1). This increased energy requirement for tridentate coordination of PCPH pincer ligands can lead to their adopting diphosphine-like bridging coordination modes when reactions are carried out under conditions where $\mathrm{C}-\mathrm{H}$ cleavage is not facile. ${ }^{47}$

To overcome the difficulty involved in breaking this strong $\mathrm{C}-\mathrm{H}$ bond to achieve 
ligand metallation, the pincer ligands can alternatively be prepared with bromide or iodide substituents on C-2. This approach is typically combined with a starting material in the zero oxidation state ( $\mathrm{such}$ as $\left[\mathrm{Pd}_{2}(\mathrm{dba})_{3}\right]$ ), as oxidative addition of the carbon-halide bond will yield the pincer halide complex [(PCP)MX]. In reactions with palladium precursors, the presence of the reactive $\mathrm{C}-\mathrm{Br}$ or $\mathrm{C}-\mathrm{I}$ bonds results in oxidative addition at temperatures of around $75^{\circ} \mathrm{C}$ for bromides ${ }^{48}$ and as low as $20{ }^{\circ} \mathrm{C}$ for iodides, ${ }^{49}$ whereas temperatures in excess of $100{ }^{\circ} \mathrm{C}$ are often required for metallation via $\mathrm{C}-\mathrm{H}$ cleavage.

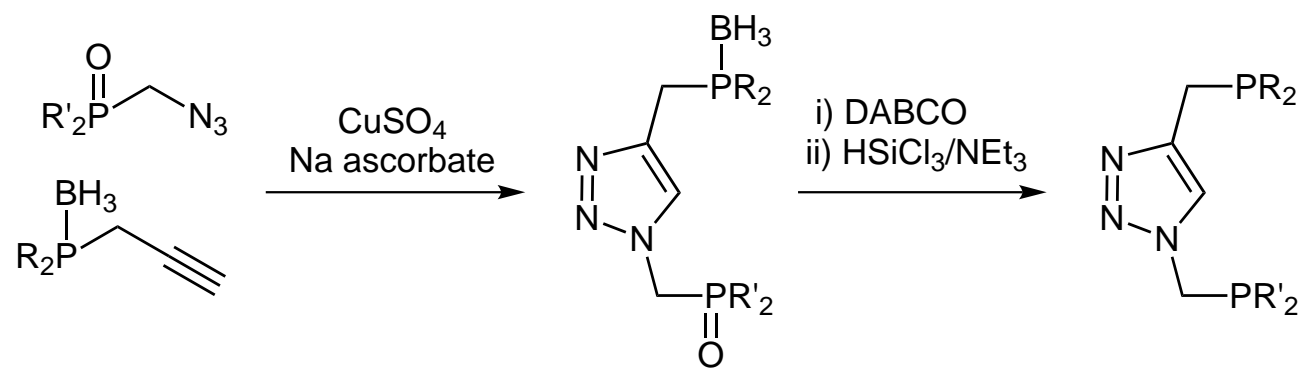

Scheme 1.2 Synthesis of PCP pincer ligands using the azide-alkyne Huisgen cycloaddition.

An alternative approach to the synthesis of $\mathrm{PCPH}$ pincer ligands has been reported using click chemistry (copper-catalysed azide-alkyne Huisgen cycloaddition). Rather than begin with an intact ligand backbone and install functionality upon this, the click methodology focusses on the synthesis of various donor groups attached to alkyne and azide moieties (Scheme 1.2). These donor groups can then be "clicked" together, with the triazole unit formed in the cycloaddition reaction becoming the ligand backbone. ${ }^{50}$ This elegant scheme allows different combinations of donor groups to be readily incorporated into PCPH pincer ligands. However, the triazole ligand backbone is not as inert as phenyl or pyridyl backbones, potentially detracting from the traditionally robust nature of pincer complexes.

A further synthetic method that has been used for the synthesis of palladium PCP complexes is the "ligand introduction route". ${ }^{51}$ In this method (Scheme 1.3, top scheme), Kimura and associates first attach the 1,3-dihydroxy-substituted aromatic backbone to the tetrakis-triphenylphosphine palladium metal precursor through oxidative addition of a $\mathrm{C}-\mathrm{I}$ bond. The phosphorus donors of the pincer ligand are then installed in a condensation reaction between the hydroxyls of the metalled species and a disubstituted phosphine chloride. Finally, tridentate pincer coordination is achieved by displacement of the remaining triphenylphosphine ligands from the metal by the phosphorus donor groups, utilising the chelate effect. In what can be seen as a "reverse ligand introduction route", Bolliger and colleagues first introduce the phosphorus donor groups on to the metal centre through simple coor- 
dination chemistry (Scheme 1.3, bottom scheme), then install the ligand backbone via $\mathrm{P}-\mathrm{N}$ cleavage of a piperidyl substituent on the phosphorus and subsequent elimination of piperidinium hydrochloride. ${ }^{52}$ Heating to $100{ }^{\circ} \mathrm{C}$ is then required to promote metallation of the aromatic backbone. However, reaction times are remarkably short, with reactions reaching completion after 45 minutes at the most.

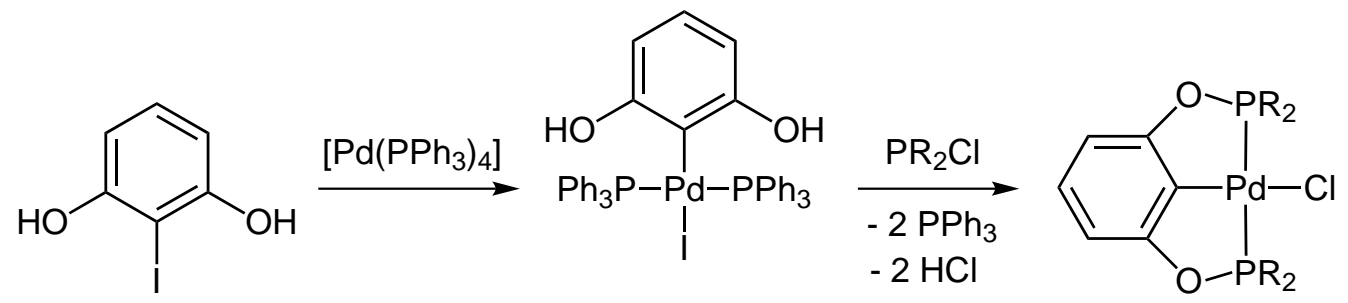

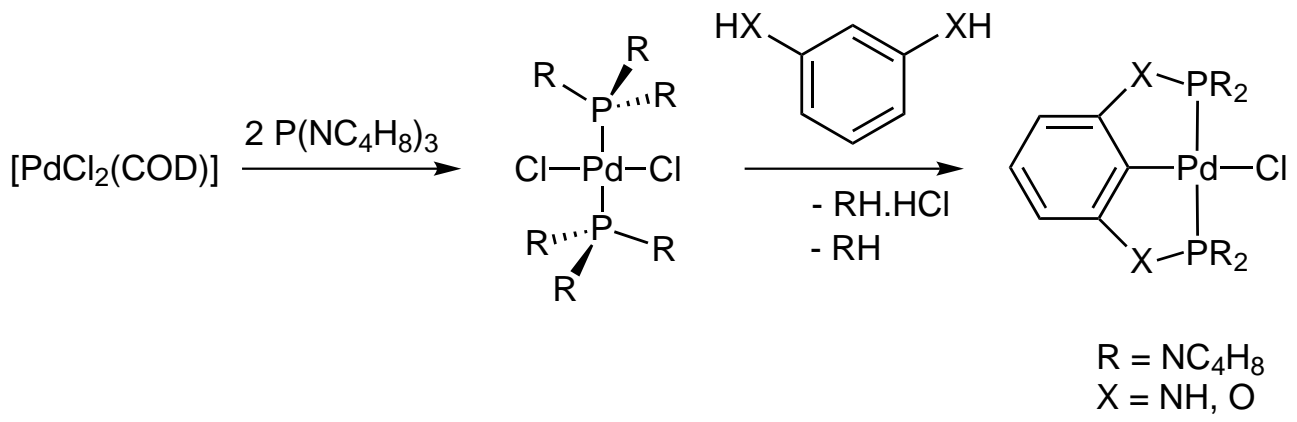

Scheme 1.3 Synthesis of PCP pincer ligands via "ligand introduction" methods.

While these "ligand introduction" methodologies are elegant routes to PCP pincer complexes, they can only be used in certain circumstances. The method of Kimura and associates (Scheme 1.3, top scheme) becomes less effective with increasing ligand bulk; reactions with $\mathrm{PPh}_{2} \mathrm{Cl}$ are complete after one hour, whereas reactions with $\mathrm{P}(o-\text { tolyl })_{2} \mathrm{Cl}$ require 24 hours. ${ }^{51}$ The method of Bolliger and colleagues (Scheme 1.3, bottom scheme) is reliant on the tertiary phosphine groups possessing substituents that can be readily displaced; 52 reactions with alkyl or aryl phosphines would not be expected to proceed due to the strength of the $\mathrm{P}-\mathrm{C}$ bond. Moreover, both of these methods can only be used to produce ligands with $\mathrm{O}$ or NH linkages between the donor groups and ligand backbone.

In the work reported herein, PCPH ligands possessing a proton (rather than a halide) at the C-2 position were used. As they are more commonly used than their halidesubstituted analogues they offer a greater comparability to the scientific literature, and are synthesised from a readily available starting material (dichloro- $m$-xylene). The requirement for $\mathrm{C}-\mathrm{H}$ activation of the ligand to achieve pincer coordination also offers an insight into how manipulation of the ligand's electronic character affects the prevalence of bridging coordination modes, as well as the ease of backbone 
metallation.

\subsection{Palladium-Catalysed Cross-Coupling}

As mentioned previously, phosphine-based palladium pincer complexes have been successfully utilised in the Heck and Suzuki cross-coupling reactions. The Heck reaction was first reported in $1972,{ }^{53}$ and the Suzuki reaction in $1979 ;{ }^{54}$ these palladium-catalysed reactions immediately proved useful, and are now vital tools for carbon-carbon bond formation in synthetic organic chemistry. Richard Heck, Akira Suzuki, and Ei-ichi Negishi were awarded the 2010 Nobel Prize in Chemistry for their discovery of these cross-coupling reactions.

The basis of both the Heck and Suzuki reactions is the reaction between a carbon electrophile (usually an organohalide) and a carbon nucleophile (an alkene or boronic acid/ester), mediated by a palladium catalyst and an external base (Scheme 1.4). The Heck reaction is often performed in basic or highly polar solvents at temperatures above $100{ }^{\circ} \mathrm{C}$, while Suzuki reactions can be carried out at temperatures below $100{ }^{\circ} \mathrm{C}$ in non-polar solvents. This allows the performance and durability of potential catalyst species to be assessed under a wide range of conditions.

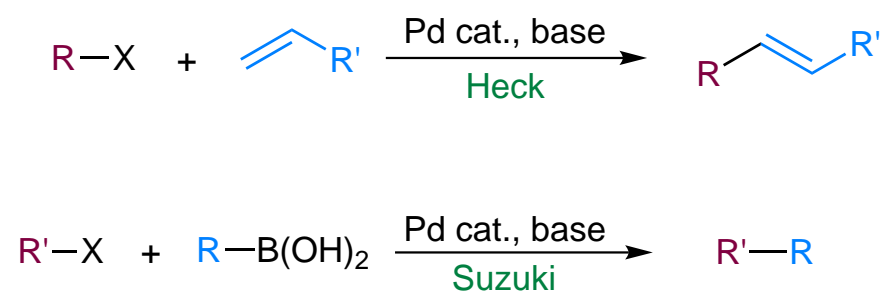

where $\mathrm{R}$ must be unsaturated

Scheme 1.4 Outline of the Heck and Suzuki cross-coupling reactions. The red highlights electrophilic groups, while the blue highlights nucleophilic groups, in order to demonstrate the similarity between the reactions.

The Heck and Suzuki reactions provide a means to quantify how the incorporation of electron-withdrawing groups into PCP pincer ligands affects the catalytic performance of their palladium complexes. The use of palladium pincer species in cross-coupling reactions dates back to $1997 ;^{55}$ this 15 year history, together with the ubiquity of the Heck and Suzuki reactions in the scientific literature ensures an abundance of data for comparison, and makes these reactions suitable yardsticks for 
measuring the catalytic performance of new electron-poor palladium pincer complexes.

\subsection{Research Objectives}

The aims of this project are to synthesise new, electron-poor PCPH and PNP pincer ligands using fluoroaryl electron-withdrawing substituents on the phosphorus donors, and to evaluate their coordination chemistry with platinum, palladium, and rhodium precursors. It is also to investigate how the electronic character of the pincer ligand affects the ease of ligand metallation and pincer complex formation.

To quantify the electronic effects of the electron-withdrawing groups on the metal centre, carbonyl derivatives of these electron-poor pincer complexes will be synthesised and analysed by infrared spectroscopy. The performance of the new palladium compounds will also be assessed in Heck and Suzuki cross-coupling reactions, to determine if any relationship can be established between catalytic activity for these reactions and the electronic nature of the metal centre.

\subsection{Concluding Remarks}

The description "pincer ligand" encompasses a large range of structures, all tridentate, with a preference for forming rigid, well-defined, thermally stable coordination complexes with transition metals. The most renowned of these species possess simple aromatic backbones with one or more phosphorus donor groups. Not only do these phosphorus-containing pincer complexes perform well in reactions that aid organic synthesis, but are also prominent in cutting edge areas of research, such as alkane metathesis and water splitting.

However, to be able to improve on current technologies, an understanding of how the steric and electronic properties of the ligands are manifested in the properties of the metal complexes themselves is desirable. Complexes of electron-poor pincer ligands are not well represented in the scientific literature, yet these complexes have demonstrated unusual coordination geometries and increased catalytic activity over their more electron-rich analogues. Thus, the incorporation of electron-withdrawing 
groups into PCP and PNP coordination motifs should allow the synthesis of a new range of electron-poor phosphorus-containing pincer complexes.

The electronic character of each metal complex will be established by performing infrared spectroscopy on metal carbonyl derivatives of these new pincer complexes, allowing the relative electron density on each metal centre to be established. To assess the impact of the electron-withdrawing groups on the formation of pincer complexes, the coordination chemistry and metallation of the pincer ligands will be studied. Finally, Heck and Suzuki reactions will be carried out with the electronpoor palladium compounds, to determine the extent that the electronic nature of the metal centre affects catalytic activity in cross-coupling reactions. 


\section{Chapter 2}

\section{Ligand Synthesis}

In order to synthesise electron-poor pincer ligands, the appropriate choice of functional groups is required to inductively withdraw electron density away from the donor atoms and any metal bound to them. As mentioned in the previous chapter, electron-poor pincer ligands can be created by modifying the substituents either on the ligand backbone, the "arms" of the pincer that connect the donor groups to the backbone, or the substituents on the donor groups themselves. Modification of the donor groups was deemed to be the most promising route to the formation of electron-deficient pincer complexes; the donor groups bind directly to the metal centre and so have a large influence on the electronic character of the resultant metal complex.

Bis(pentafluorophenyl)phosphine was regarded as the best choice for the phosphorus donor group of the ligand; pentafluorophenylphosphines have been shown to be similar to aryl phosphinites in their ability to produce electron-poor metal centres, although they are not as effective as halophosphines or trifluoromethyl-substituted phosphines in this regard. ${ }^{37}$ The electronic effect of a substituent on a phosphorus donor atom can be approximated with the Tolman Electronic Parameter (TEP), a measure of how each group affects the CO stretching frequency of a nickel carbonyl complex. Larger TEP values indicate lower electron density on the nickel centre, and a less electron-donating or more electron-withdrawing substituent. Values for $\mathrm{C}_{6} \mathrm{~F}_{5}$ are compared to other common substituents in Table 2.1, confirming the electronpoor nature of pentafluorophenylphosphine complexes.

Whereas the phosphinite, trifluoromethyl, and halide substituents all increase the $\pi$-acceptor ability ( $\pi$-acidity) of the phosphorus donor, it is thought that pentafluorophenyl substituents instead drastically reduce the $\sigma$-donor ability of the phosphorus 
Table 2.1 Effect of phosphine substituents on the Tolman Electronic Parameter. ${ }^{37}$ A larger value indicates a more electron-poor metal centre.

\begin{tabular}{lccccccc}
\hline \hline Substituent & ${ }^{t} \mathrm{Bu}$ & $\mathrm{Me}$ & $\mathrm{Ph}$ & $\mathrm{OPh}$ & $\mathrm{C}_{6} \mathrm{~F}_{5}$ & $\mathrm{Cl}$ & $\mathrm{CF}_{3}$ \\
\hline Effect on TEP $\left(\mathrm{cm}^{-1}\right)$ & 0.0 & 2.6 & 4.3 & 9.7 & 11.2 & 14.8 & 19.6 \\
\hline \hline
\end{tabular}

atom, ${ }^{38}$ offering a point of contrast to these groups. Regardless of the exact nature of the electronic influence of pentafluorophosphines, they represent a class of compounds that are effective in producing electron-poor metal centres, and have been sparsely utilised in pincer ligands (with only complexes of ruthenium ${ }^{56}$ and palladium $^{57}$ of one such ligand reported to date). Moreover, work has been reported on the synthesis and coordination chemistry of a bis(trifluoromethyl)phosphine PCP pincer ligand, ${ }^{58}$ which enables direct comparisons to be made between the effect of the perfluorinated ligand substituents $\mathrm{C}_{6} \mathrm{~F}_{5}$ and $\mathrm{CF}_{3}$ on the chemistry of the resultant complexes.

As PCP pincer ligands with 1,3-substituted phenylene backbones are the most ubiquitous of pincer ligands, ${ }^{17}$ these provide an ideal starting point for the incorporation of the bis(pentafluorophenyl)phosphine moieties, as they allow for a high degree of comparability with the scientific literature. Three PCP pincer ligands were established as synthetic targets, with each possessing a different electronic character. The phosphinite ligand 1,3- $\left[\left(\mathrm{C}_{6} \mathrm{~F}_{5}\right)_{2} \mathrm{PO}_{2} \mathrm{C}_{6} \mathrm{H}_{4}\right.$ (referred to as POCOPH, 1) was desirable as it should be the most electron-withdrawing of all the ligands, combining the low $\sigma$-donor ability of the bis(pentafluorophenyl)phosphine with the high $\pi$-acceptor ability of the phosphinite functionality. The phosphine ligand 1,3$\left[\left(\mathrm{C}_{6} \mathrm{~F}_{5}\right)_{2} \mathrm{PCH}_{2}\right]_{2} \mathrm{C}_{6} \mathrm{H}_{4}(\mathrm{PCCCPH}, 2)$ was targeted as it was already known ${ }^{57}$ but had scarcely been investigated in terms of coordination chemistry, and also possessed the more robust and widely used methylene bridges between the backbone and phosphine donors. The final PCP pincer ligand targeted was the asymmetric phosphine-phosphinite 1- $\left[\left(\mathrm{C}_{6} \mathrm{~F}_{5}\right)_{2} \mathrm{PO}\right]-3-\left({ }^{t} \mathrm{Bu}_{2} \mathrm{PCH}_{2}\right)_{2} \mathrm{C}_{6} \mathrm{H}_{4}(\mathrm{POCCPH}, 3)$. This was desirable as it combined the electron-poor bis(pentafluorophenyl)phosphinite group with the electron-rich di-tert-butylphosphine donor group, allowing the mutually trans arrangement of donor and acceptor phosphines in resultant metal complexes to be studied. It was also desirable to investigate whether the introduction of an electron-rich donor would simply reduce the influence of the electron-poor donor, or give rise to new synergic effects. 


\subsection{Synthesis of Pentafluorophenyl-Substituted PCP Pincer Ligands}

The synthesis of bis(pentafluorophenyl)phosphine-substituted pincer ligands first required the synthesis of the appropriate phosphine halide, $\mathrm{Ar}_{2} \mathrm{PX}$, as this comprises the basic building block of the ligand and the means for incorporation of the electronpoor phosphorus donor into the pincer framework. Bis(pentafluorophenyl)phosphine bromide (4), was synthesised according to literature protocols. ${ }^{59}$ This involved a Grignard-type reaction used to attach the pentafluorophenyl substituents to the phosphorus centre; purification by vacuum distillation afforded the product 4 in good yield (Scheme 2.1). The large steric bulk of the pentafluorophenyl group is advantageous in this instance, as it prevents trisubstitution to form the tertiary phosphine. Interestingly, while the reaction proceeds from a phosphine chloride starting material, halide exchange results in the product being a phosphine bromide.
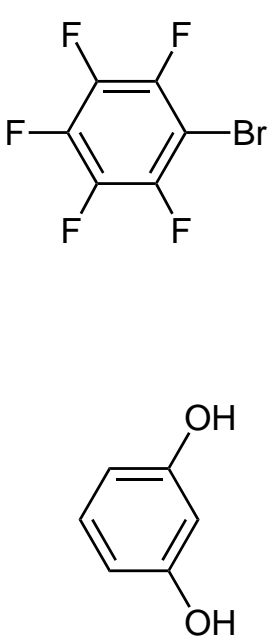<smiles>Fc1c(F)c(F)c(P(Br)c2c(F)c(F)c(F)c(F)c2F)c(F)c1F</smiles>

$475 \%$
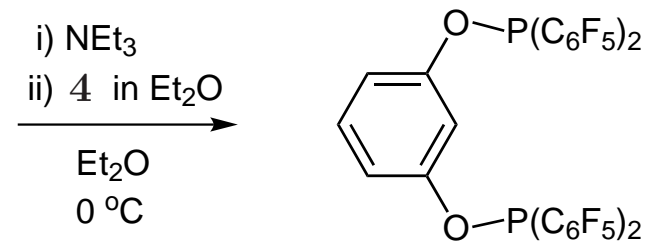

1

$87 \%$

Scheme 2.1 Synthesis of the phosphinite pincer ligand 1.

The synthesis of the POCOPH ligand 1 was then carried out via the reaction of the phosphine halide 4 with resorcinol (benzene-1,3-diol), as is typical for the formation of phosphinite compounds (Scheme 2.1). ${ }^{18}$ The reaction to form ligand 1 was performed according to a published procedure for the synthesis of a pentafluorophenylphosphinite ligand containing a BINOL backbone (where BINOL is 1,1'bi-2-naphthol). ${ }^{60}$ Dropwise addition of a solution of $\mathbf{4}$ in diethyl ether to a cooled mixture of resorcinol and triethylamine in diethyl ether resulted in the formation of a thick white suspension. Isolation of the supernatant liquid by filtration followed by removal of the volatiles in vacuo and recrystallisation from hexane/toluene afforded 
ligand 1 in good yield.

Spectroscopic data for $\mathbf{1}$ was consistent with its formulation as the pentafluorophenyl-substituted POCOPH pincer ligand. The ${ }^{31} \mathrm{P}$ NMR spectrum of 1 displayed a quintet resonance (with ${ }^{3} J_{\mathrm{P}-\mathrm{F}}=35.0 \mathrm{~Hz}$, due to the phosphorus coupling to the four ortho fluorines of the pentafluorophenyl substituents) at $\delta_{\mathrm{P}}=87.1 \mathrm{ppm}$, significantly downfield of the starting material $4\left(\delta_{\mathrm{P}}=12.1\right.$ ppm). This downfield shift is typically observed upon the formation of phosphinite compounds, with representative examples reported by Morales-Morales ${ }^{61}$ and Motoyama. ${ }^{62}$ The ${ }^{1} \mathrm{H}$ and ${ }^{19} \mathrm{~F}$ NMR spectra also displayed the expected number of environments for ligand $\mathbf{1}$ (three proton and three fluorine environments). To date, bis(pentafluorophenyl)phosphinite ligands have not been a significant synthetic target, with only a small number of compounds known, mostly comprising two phosphinite donors linked by a chiral, aliphatic backbone. ${ }^{63,64}$ The POCOPH compound 1 is the first example of the incorporation of the $\mathrm{OP}\left(\mathrm{C}_{6} \mathrm{~F}_{5}\right)_{2}$ functionality into a pincer ligand.

The synthesis of the PCCCPH ligand $\mathbf{2}$ has previously been reported by Chase and colleagues, ${ }^{57}$ preparing the ligand via the reaction of $\mathbf{4}$ with the bis-Grignard reagent 1,3- $\left(\mathrm{ClMgCH}_{2}\right)_{2} \mathrm{C}_{6} \mathrm{H}_{4}$. However, in replicating this procedure, the preparation of the Grignard reagent was found to be difficult and unreliable, typically resulting in low yields of the desired phosphine product (the greatest yield obtained in five attempts being $39 \%$, only slightly lower than the $51 \%$ stated in the literature ${ }^{57}$ ). An improved synthesis of $\mathbf{2}$ was employed, generating the bis-Grignard reagent with the more potent magnesiator, $\left[\mathrm{Mg}(\right.$ anthracene $\left.)(\mathrm{THF})_{3}\right](\mathbf{5})$.

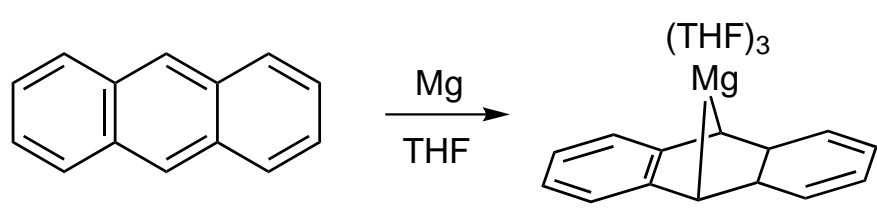

5

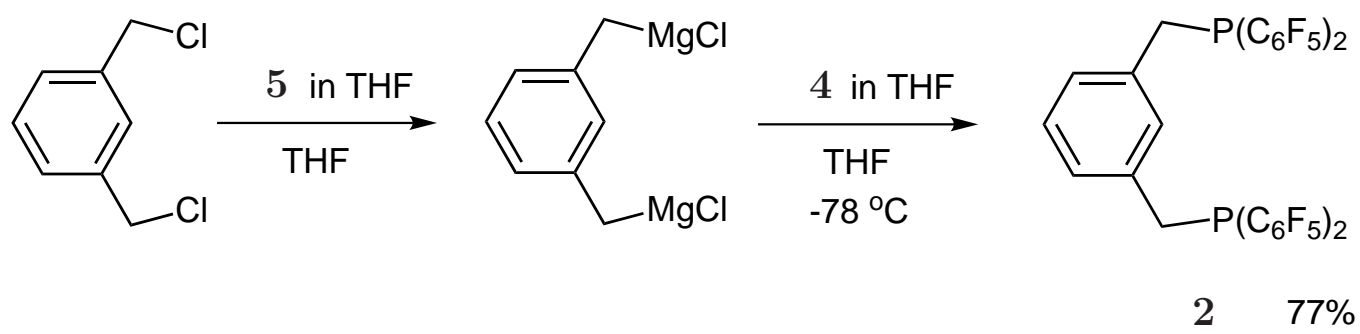

Scheme 2.2 Synthesis of the phosphine pincer ligand 2 . 
The magnesium anthracene complex $\mathbf{5}$ was synthesised by the prolonged reaction of magnesium powder with anthracene in THF, ${ }^{65}$ and isolated as an orange solid after stirring for four days (Scheme 2.2). This magnesium complex 5 was then used to generate the desired bis-Grignard reagent by reaction with $\alpha, \alpha^{\prime}$-dichloro- $m$ xylene in THF. Subsequent reaction of the bis-Grignard reagent with the pentafluorophenylphosphine bromide 4 afforded the desired PCCCPH ligand $\mathbf{2}$. The NMR data were consistent with the synthesis of $\mathbf{2}$, displaying the expected four environments in the ${ }^{1} \mathrm{H}$ NMR spectrum, of which the methylene signal appeared as a doublet, due to coupling to the phosphorus atom. The phosphine signal itself appeared as a quintet in the ${ }^{31} \mathrm{P} \mathrm{NMR}$ spectrum at $-46.3 \mathrm{ppm}$, consistent with literature data for this compound. ${ }^{57}$

Purification of $\mathbf{2}$ was achieved via column chromatography in air, as per the method of Chase, ${ }^{57}$ giving 2 as a white solid in a yield of $67 \%$, substantially greater than those obtained without the magnesium anthracene complex 5. Due to the high air sensitivity and susceptibility to oxidation of most phosphine ligands, column chromatography is not usually employed during their purification. However, the pentafluorophenyl substituents of $\mathbf{2}$ serve to reduce the availability of the lone-pair of electrons on the phosphorus for bonding, making $\mathbf{2}$ sufficiently air-stable as to allow purification by column chromatography in air. Nonetheless, small amounts of the phosphine oxide were detected in samples of $\mathbf{2}$ left standing under ambient conditions overnight, and thus samples of the ligand (as well as all ligands reported herein) were stored under an inert atmosphere.
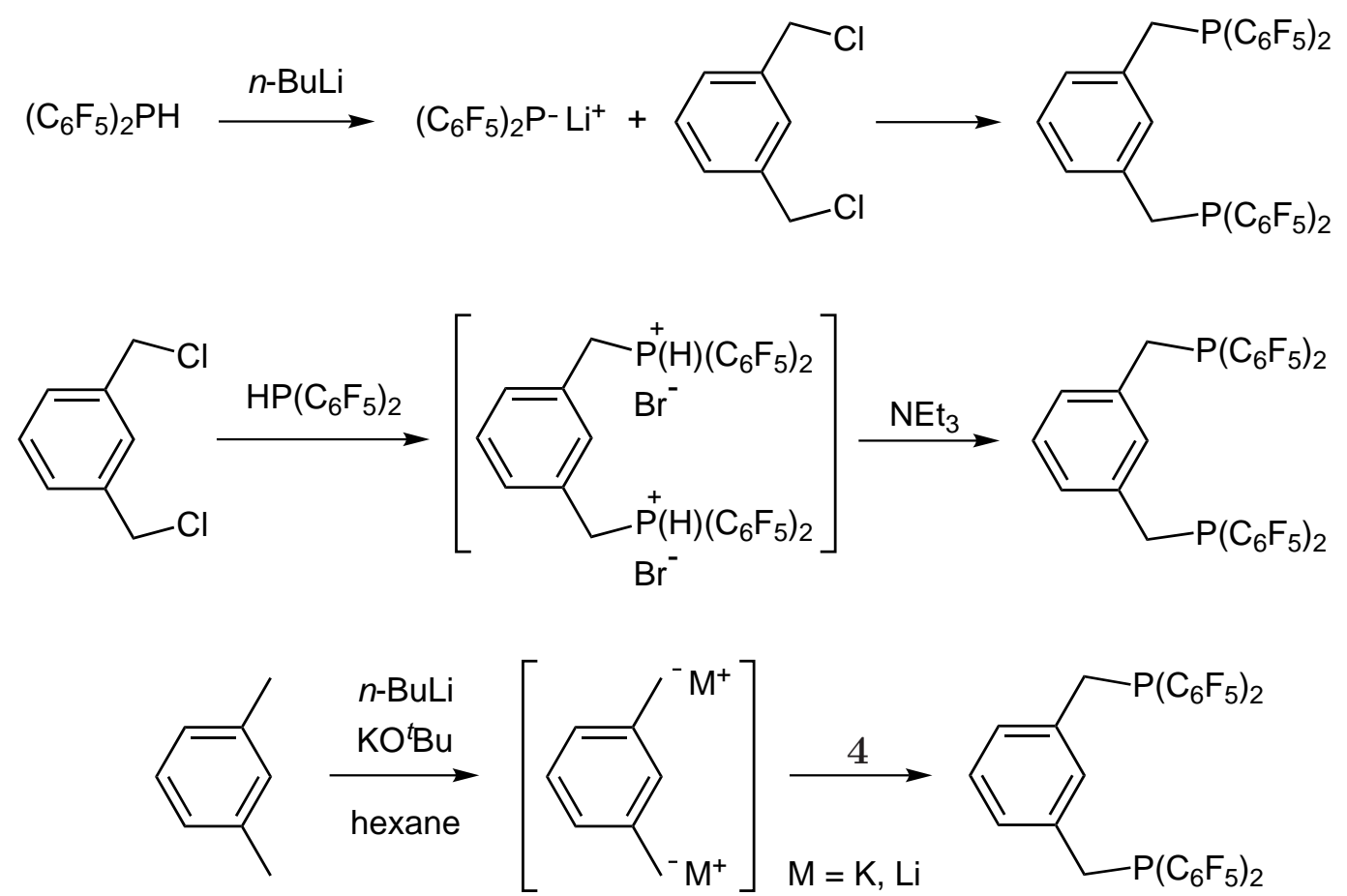

Scheme 2.3 Conventional phosphine syntheses unable to produce 2 . 
The synthesis of the PCCCPH ligand $\mathbf{2}$ via a Grignard-type reaction scheme was required as reports indicated that $\mathbf{2}$ was unable to be synthesised using the two most common synthetic methodologies: by reaction of the benzylic halide either with the lithiated phosphine $\operatorname{LiP}\left(\mathrm{C}_{6} \mathrm{~F}_{5}\right)_{2}$, or with the secondary phosphine $\mathrm{HP}\left(\mathrm{C}_{6} \mathrm{~F}_{5}\right)_{2}$ followed by deprotonation of the intermediate halide salts using an amine base (Scheme 2.3). ${ }^{57}$ This was attributed primarily to the bis(pentafluorophenyl)phosphine being too poor a nucleophile to displace the halides from the benzylic starting material, and also the poor stability of the $\left(\mathrm{C}_{6} \mathrm{~F}_{5}\right)_{2} \mathrm{P}^{-}$anion, decomposing in solution even at $-78^{\circ} \mathrm{C} .{ }^{66 *}$ Similar problems have been encountered in the synthesis of other fluoroarylphosphine phosphine ligands, as described in the review by Pollock and colleagues. ${ }^{39}$ An alternate synthesis of $\mathbf{2}$ via reaction of the phosphine bromide 4 with the dimetallated intermediate 1,3- $\left(\mathrm{MCH}_{2}\right)_{2} \mathrm{C}_{6} \mathrm{H}_{4}$ (where $\mathrm{M}$ is potassium or lithium) was also attempted and found to be unsuccessful, with 2 comprising only a minor part of the reaction mixture. This is likely due to the low selectivity of metallation at the benzylic positions. ${ }^{68}$

The unsymmetrical POCCPH pincer ligand $\mathbf{3}$ was prepared in a similar manner to that of the two previously published examples of phosphine-phosphinite pincer ligands. ${ }^{13,69}$ The synthesis (Scheme 2.4) followed literature procedures for the reduction of 3-hydroxybenzaldehyde to 3-hydroxybenzyl alcohol (6), ${ }^{70}$ and then bromination of the benzylic hydroxyl with phosphorus tribromide to give 3-hydroxybenzyl bromide (7). ${ }^{71}$ Intermediate 7 was reported to degrade over time, so was used in the crude form for the subsequent reaction. The $\mathrm{P}^{t} \mathrm{Bu}_{2}$ moiety was then installed by nucleophilic attack of di-tert-butylphosphine at the benzylic carbon of $\mathbf{7}$, followed by deprotonation of the phosphorus with triethylamine. While such a reaction does not proceed with electron-poor phosphines (and hence could not be used in the synthesis of $\mathbf{2}$ ), the higher nucleophilicity of the tert-butyl-substituted phosphine allowed the reaction to proceed smoothly, giving the benzylic phosphine 8 in a $58 \%$ yield across the two steps from 6 . The synthesis was completed by formation of the phosphinite $\mathrm{P}-\mathrm{O}$ linkage by reaction of the phenol functionality of 8 with the bis(pentafluorophenyl)phosphine bromide 4 , in an almost identical manner to the synthesis of the POCOPH ligand $\mathbf{1}$. The new phosphine-phosphinite ligand $\mathbf{3}$ was isolated as a pale yellow oil, which solidified into a cream-coloured solid on cooling to $-15{ }^{\circ} \mathrm{C}$, in an $86 \%$ yield. This corresponded to an overall yield of $46 \%$ for the four steps from 3-hydroxybenzaldehyde.

The NMR data of ligand 3 showed that the replacement of one of the $\mathrm{OP}\left(\mathrm{C}_{6} \mathrm{~F}_{5}\right)_{2}$ groups of 1 with a $\mathrm{CH}_{2} \mathrm{P}^{t} \mathrm{Bu}_{2}$ group had only a small effect on the chemical shift of

${ }^{*}$ The use of $\left(\mathrm{C}_{6} \mathrm{~F}_{5}\right)_{2} \mathrm{P}^{-}$as a nucleophile has previously been reported in the literature, ${ }^{67}$ but the phosphide itself was unable to be isolated, or trapped by coordination to transition metal ions. Subsequent work has been unable to isolate or reliably use bis(pentafluorophenyl)phosphide. 


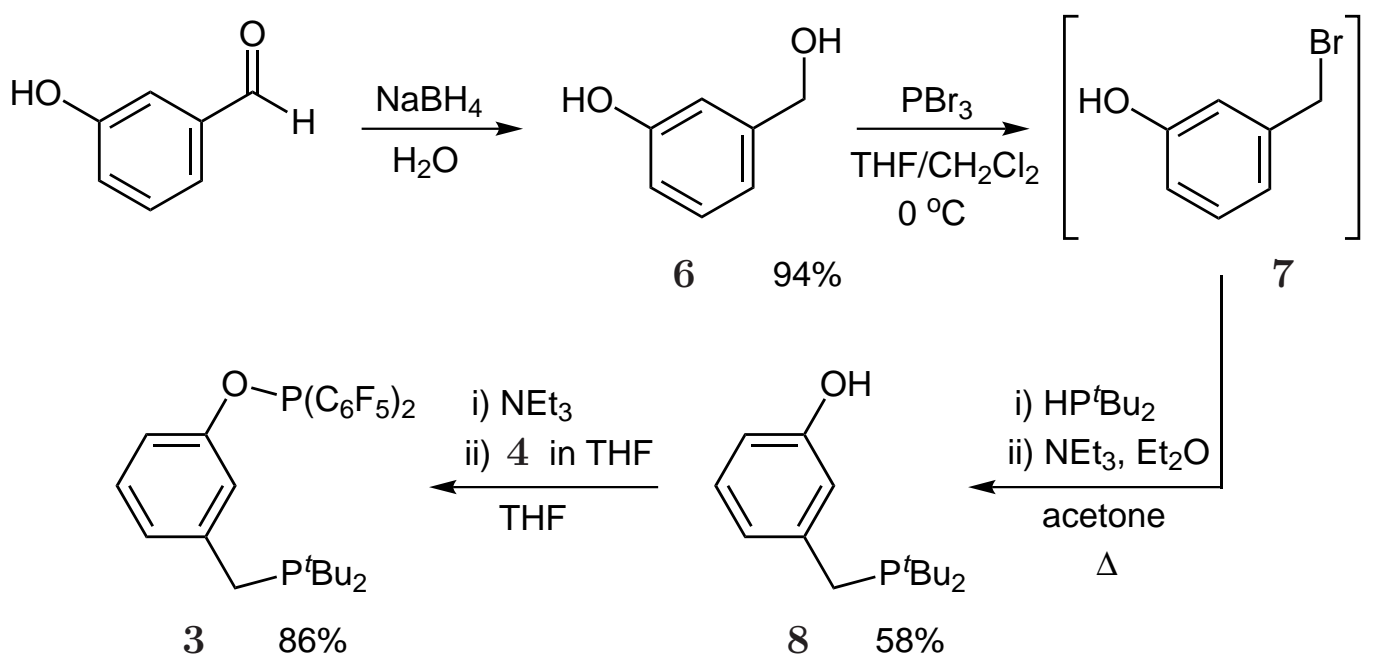

Scheme 2.4 Synthesis of the phosphine-phosphinite pincer ligand 3 .

the remaining phosphinite, which appeared as a quintet resonance in the ${ }^{31} \mathrm{P} \mathrm{NMR}$ spectrum at $82.9 \mathrm{ppm}$, close to the phosphinite chemical shift of $\mathbf{1}\left(\delta_{\mathrm{P}}=87.1 \mathrm{ppm}\right)$. As expected, the phosphorus atom of the $\mathrm{CH}_{2} \mathrm{P}^{t} \mathrm{Bu}_{2}$ group is significantly more shielded, appearing as a singlet in the ${ }^{31} \mathrm{P}$ NMR spectrum at $34.3 \mathrm{ppm}$. The proton spectrum is unremarkable, with four aromatic proton environments observed due to the asymmetry of the ligand backbone, and small P-H couplings displayed by the tert-butyl methyl groups $\left({ }^{3} J_{\mathrm{P}-\mathrm{H}}=10.8 \mathrm{~Hz}\right)$ as well as the benzylic methylene $\left({ }^{2} J_{\mathrm{P}-\mathrm{H}}\right.$ $=2.6 \mathrm{~Hz})$.

The POCCPH ligand $\mathbf{3}$ is the first pincer ligand reported to possess both strongly electron-rich and electron-poor donor groups; the previous two reports of POCCPH ligands both possess the comparatively less electron-poor $\mathrm{OP}^{i} \mathrm{Pr}_{2}$ group. Moreover, both the $\mathrm{P}\left(\mathrm{C}_{6} \mathrm{~F}_{5}\right)_{2}$ and $\mathrm{P}^{t} \mathrm{Bu}_{2}$ moities have been calculated to possess similar cone angles of $171^{\circ}$ for $\mathrm{PPh}\left(\mathrm{C}_{6} \mathrm{~F}_{5}\right)_{2}$, and $170^{\circ}$ for $\mathrm{PPh}^{t} \mathrm{Bu}_{2} \cdot{ }^{37,38}$ Whilst cone angles only provide a rough guide as to the steric influence exerted by the ligands (not taking into account for example, the ability of the planar pentafluorophenyl substituents to interleave or stack, whereas tert-butyl groups cannot), these values indicate that bis(pentafluorophenyl)phosphines and di-tert-butylphosphines are at least comparable in terms of size. Therefore, there should be minimal steric differences between ligands $\mathbf{1}, \mathbf{2}$, and $\mathbf{3}$, allowing any differences in reactivity between resultant complexes to be attributed primarily to electronic effects.

Ligand 3 was found to have undergone significant decomposition over the course of eight months stored at $-15{ }^{\circ} \mathrm{C}$. Analysis of samples of 3 by NMR spectroscopy revealed the presence of a new product $\mathbf{9}$, as well as several unidentified minor products. Spectroscopic data of $\mathbf{9}$ was highly indicative of addition of two fluorine atoms to the phosphorus atoms of the $\mathrm{P}^{t} \mathrm{Bu}_{2}$ group, to form a new phosphorus(V) 
$\mathrm{PF}_{2}{ }^{t} \mathrm{Bu}_{2}$ group. The ${ }^{1} \mathrm{H}$ NMR spectrum of 9 revealed both the methyl protons of the tert-butyl groups and the methylene protons of the benzylic ligand backbone were coupling to two fluorine atoms, with each resonance appearing as a doublet of triplets instead of the doublets observed for ligand 3 . The ${ }^{31} \mathrm{P}$ and ${ }^{19} \mathrm{~F}$ spectra of 9 were also consistent with the formation of a $\mathrm{PF}_{2}{ }^{t} \mathrm{Bu}_{2}$ degradation product; the phosphorus signal appeared as a triplet, and a doublet resonance was observed for the fluorine signal, with each displaying a mutual phosphorus-fluorine coupling constant of $723 \mathrm{~Hz}$.

Table 2.2 Comparison of NMR data of degradation product 9 in $\mathrm{CDCl}_{3}$ with that of $\left(4-\mathrm{NO}_{2}-\mathrm{C}_{6} \mathrm{H}_{4}\right) \mathrm{PF}_{2}{ }^{t} \mathrm{Bu}_{2}$. Data is for the $\mathrm{PF}_{2}{ }^{t} \mathrm{Bu}_{2}$ moiety of each compound.

\begin{tabular}{ll}
\hline \hline \\
Compound $\mathbf{9}$
\end{tabular}

${ }^{a}$ Data from Yandulov and Tran. ${ }^{72}$

The spectroscopic data of $\mathbf{9}$ were very similar to the spectroscopic data reported for $\left(4-\mathrm{NO}_{2}-\mathrm{C}_{6} \mathrm{H}_{4}\right) \mathrm{PF}_{2}{ }^{t} \mathrm{Bu}_{2}$ (Table 2.2). As no resonance in the ${ }^{31} \mathrm{P} \mathrm{NMR}$ spectrum was observed for a $\mathrm{OP}\left(\mathrm{C}_{6} \mathrm{~F}_{5}\right)_{2}$ moiety associated with the degradation product $\mathbf{9}$, the $\mathrm{P}-\mathrm{O}$ bond was presumed to have been cleaved, giving 9 the formula $(3-\mathrm{OH}-$ $\left.\mathrm{C}_{6} \mathrm{H}_{4}\right) \mathrm{PF}_{2}{ }^{t} \mathrm{Bu}_{2}$. The fluorine atoms directly bound to the phosphorus are likely to have originated from the ortho or para positions of the pentafluorophenyl substituents on the phosphinite group, as these aromatic fluorines have been reported to be particularly susceptible to nucleophilic attack, ${ }^{73-75}$ even from phosphorus nucleophiles. ${ }^{76}$ However, the mechanism for the fluorination of the di-tert-butyl phosphine group is not known. Attempts to purify the mixture by column chromatography were unsuccessful, with neither the POCCPH ligand $\mathbf{3}$ or the degradation product $\mathbf{9}$ able to be isolated. As such, the exact nature of $\mathbf{9}$ could not be unambiguously confirmed. 


\subsection{Synthesis of Pentafluorophenyl-Substituted PNP Pincer Ligands}

With the synthesis of the pentafluorophenyl-substituted PCPH pincer ligands $\mathbf{1 ,} \mathbf{2}$, and 3 achieved, the synthesis of similar PNP pincer ligands was explored. The PNP pincer ligands made attractive synthetic targets as they could be prepared with a similar synthetic methodology to that of the previously synthesised PCP pincer ligands, starting from 2,6-substituted pyridine derivatives instead of 1,3-substituted phenylenes. An advantage of the PNP coordination motif is that the ligands contain only neutral donor atoms with available lone pairs of electrons, meaning that no ligand activation step is required to achieve tridentate "pincer" coordination to a metal centre (contrasting with the $\mathrm{C}-2$ deprotonation required for PCP pincer coordination).

From the comparatively straightforward synthesis of the POCOPH ligand $\mathbf{1}$, it was clear that the easiest way to incorporate bis(pentafluorophenyl)phosphine groups into the pincer motif was by direct reaction of the phosphine bromide 4 with an aromatic hydroxyl, to form a new $\mathrm{P}-\mathrm{O}$ bond. As $\mathrm{P}-\mathrm{N}$ bonds could be formed by the analogous reaction with aromatic amines, the new electron-poor PNNNP (10) and PONOP (11) ligands were synthesised via the reaction of 4 with 2,6diaminopyridine and 2,6-dihydroxypyridine respectively (Scheme 2.5). The NMR data were as expected for the new compounds 10 and 11, with each displaying two aromatic proton environments in their ${ }^{1} \mathrm{H}$ NMR spectra, and the expected quintet resonances in the ${ }^{31} \mathrm{P}$ NMR spectra (at $\delta_{\mathrm{P}}=-10.8$ and $70.2 \mathrm{ppm}$ respectively). It was interesting to note that in moving from a phenyl backbone in the POCOPH ligand 1 to a pyridyl backbone in the PONOP ligand 11, the extra electron density furnished to the aromatic ring by the nitrogen served to increase the shielding of the phosphorus donor atoms, shifting the ${ }^{31} \mathrm{P}$ NMR resonance from $87.1 \mathrm{ppm}$ in $\mathbf{1}$ to $70.2 \mathrm{ppm}$ in $\mathbf{1 1 .}$
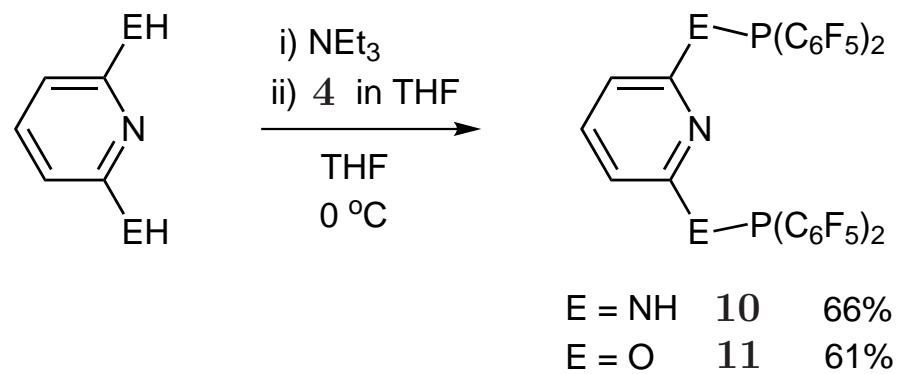

Scheme 2.5 Synthesis of the PNP pincer ligands 10 and 11. 
These reactions were performed under similar conditions to the synthesis of the POCOPH ligand $\mathbf{1}$ (using triethylamine as a base, stirring the reaction mixture at $0{ }^{\circ} \mathrm{C}$ for one hour then warming to room temperature overnight), affording the PNP pincer complexes $\mathbf{1 0}$ and $\mathbf{1 1}$ in modest yields of $66 \%$ and $61 \%$ respectively. Notably, the previously reported syntheses of PONOP and PNNNP ligands required much harsher conditions, with elevated temperatures of $65-80{ }^{\circ} \mathrm{C}$ and either prolonged reaction times (about one week) ${ }^{77}$ or the addition of the strong base nbutyllithium. ${ }^{78,79}$ As these reported syntheses were for ligands that possessed tertbutyl and isopropyl substituents, they were substantially more electron-rich than ligands $\mathbf{1 0}$ and 11. It appears that the significant electron-withdrawing character of the pentafluorophenyl substituents serves to enchance the reactivity of the phosphine bromide 4 with aryl amines and alcohols, facilitating the synthesis of the PNP ligands 10 and 11.

\subsection{Attempted Synthesis of Trifluoromethylaryl- Substituted Ligands}

To offer a point of comparison with the pentafluorophenyl-containing ligands, the synthesis of PCP pincer ligands containing 3,5-bis(trifluoromethyl)phenyl substituents was pursued. Phosphine ligands possessing these trifluoromethylaryl substituents have been shown to possess a slightly more electron-donating character and a slightly smaller steric influence than the analogous pentafluorophenyl phosphines. ${ }^{80}$ However, the smaller size of the $3,5-\left(\mathrm{CF}_{3}\right)_{2} \mathrm{C}_{6} \mathrm{H}_{3}$ group introduces the possibility of trisubstitution to form the tertiary phosphine $\mathrm{P}\left[3,5-\mathrm{C}_{6} \mathrm{H}_{3}\left(\mathrm{CF}_{3}\right)_{2}\right]_{3}$ during the synthesis of the halophosphine ligand precursor $\left[3,5-\left(\mathrm{CF}_{3}\right)_{2} \mathrm{C}_{6} \mathrm{H}_{3}\right]_{2} \mathrm{PCl}(\mathbf{1 2})$ when trichlorophosphine is used as a starting material (as in the synthesis of the pentafluorophenylphosphine bromide 4). To avoid this, the halophosphine $\mathbf{1 2}$ was prepared from the diethylamine-protected phosphine chloride $\mathrm{Et}_{2} \mathrm{NPCl}_{2}$, as the diethylamine group is not displaced by the aryl Grignard reagent, preventing tertiary phosphine formation (Scheme 2.6). ${ }^{81}$ Removal of the diethylamine protecting group by reaction with hydrogen chloride gas gave the desired phosphine chloride species $\mathbf{1 2}$.

With 12 in hand, synthesis of the desired 3,5-bis(trifluoromethyl)phenyl-substituted phosphinite pincer ligand (13) was then attempted from resorcinol (Scheme 2.6), as for the synthesis of the pentafluorophenyl-substituted POCOPH ligand 1. However, reaction between 12 and resorcinol did not proceed selectively at room temperature, affording the desired ligand $\mathbf{1 3}$ (in about $65 \%$ yield, identified by NMR spectroscopy, 


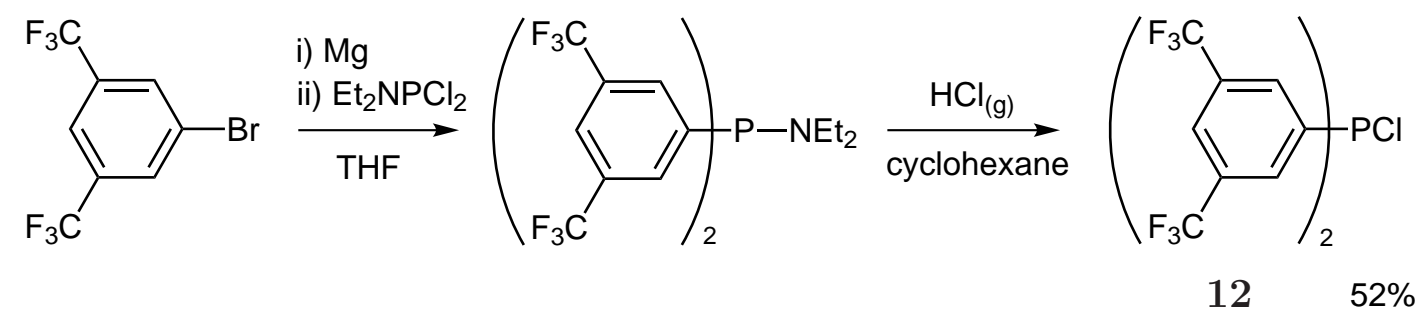

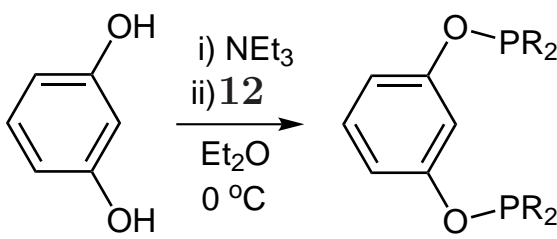

13

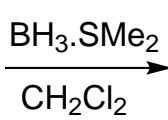

$\mathrm{CH}_{2}$

$13.2 \mathrm{BH}_{3}$<smiles></smiles>

where $\mathrm{R}=3,5-\left(\mathrm{CF}_{3}\right) \mathrm{C}_{6} \mathrm{H}_{3}$

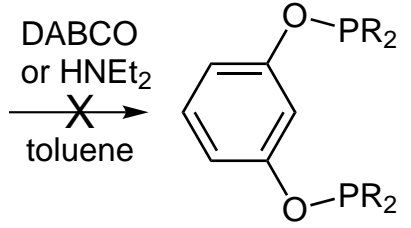

13

+ unidentified

byproducts

Scheme 2.6 Attempted synthesis of PCP pincer ligand $\mathbf{1 3 .}$

$\delta_{\mathrm{P}}=102.0 \mathrm{ppm}$ ) along with four unidentified minor products. Ligand $\mathbf{1 3}$ was not able to be purified by recrystallisation, and proved to be too sensitive to purify by column chromatography in air. To overcome this, $\mathbf{1 3}$ was borane-protected by stirring with a stoichiometric amount of $\mathrm{BH}_{3} \cdot \mathrm{SMe}_{2}$, affording the air-stable adduct $13.2 \mathrm{BH}_{3}$. The reaction mixture was successfully purified by column chromatography on silica. However, attempts at deprotecting the pure $13.2 \mathrm{BH}_{3}$ with either DABCO (1,4-diazabicyclo[2.2.2] octane) ${ }^{82}$ or the less basic diethylamine ${ }^{83}$ were unsuccessful. In both instances deprotection led to the formation of decomposition products which NMR spectroscopy indicated were likely to be $\mathrm{HP}\left[\mathrm{C}_{6} \mathrm{H}_{3}\left(\mathrm{CF}_{3}\right)_{2}\right]_{2}$ and $\mathrm{HP}(\mathrm{O})\left[\mathrm{C}_{6} \mathrm{H}_{3}\left(\mathrm{CF}_{3}\right)_{2}\right]_{2}$ or similar species (at $\delta_{\mathrm{P}}=-42.6$ and $10.6 \mathrm{ppm}$ respectively). This is likely to be promoted through $\mathrm{P}-\mathrm{O}$ cleavage by nucleophilic attack of the amine base on the electron-poor, electrophilic phosphorus centre. Thus ligand 13 could not be isolated and the synthesis of further 3,5-bis(trifluoromethyl)phenylsubstituted ligands was not pursued, owing to the sufficient diversity of the POCOP (1), PCCCP (2), POCCP (3), PNNNP (10), and PONOP (11) ligands already in hand.

\subsection{Concluding Remarks}

A range of bis(pentafluorophenyl)phosphine-substituted PCPH and PNP pincer ligands were prepared using bis(pentafluorophenyl)phosphine bromide (4). Reactions 
between 4 and aromatic alcohols or amines proceeded selectively at room temperature, giving the desired phosphinite or phosphoramine ligand in yields of typically 60-70\%. This method provided a simple yet effective strategy for the synthesis of electron-poor pincer ligands $\mathbf{1}, \mathbf{1 0}$, and $\mathbf{1 1}$, as well as for the mixed phosphinephosphinite ligand $\mathbf{3}$.

The synthesis of the PCCCPH pincer ligand $\mathbf{2}$ proved to be more difficult than for the aforementioned ligands, as common literature protocols for the synthesis of phosphine pincer ligands could not be employed due to the low nucleophilicity of $\mathbf{4}$, along with the low stability of its anionic form, $\left(\mathrm{C}_{6} \mathrm{~F}_{5}\right)_{2} \mathrm{P}^{-}$. Ligand $\mathbf{2}$ was generated via the reaction of $\mathbf{4}$ with the bis-Grignard reagent derived from $\alpha, \alpha^{\prime}$-dichloro- $m$ xylene. Formation of the bis-Grignard reagent from the magnesium anthracene complex $\mathbf{5}$ afforded a substantial increase in the isolated yield of $\mathbf{2}$ when compared to the formation of the Grignard reagent directly from magnesium turnings.

The formation of further electron-poor PCPH pincer ligands with trifluoromethylated aromatic substituents was attempted, but the resultant phosphinite ligand $\mathbf{1 3}$ was unable to be purified by recrystallisation or column chromatography in air. Formation of the borane-protected adduct $13.2 \mathrm{BH}_{3}$ followed by column chromatography resulted in sufficient purification of the protected ligand. However, deprotection could not be achieved by using established literature protocols, and the synthesis of pincer ligands containing 3,5-bis(trifluoromethyl)phenyl substituents was not pursued further. 


\section{Chapter 3}

\section{Coordination Chemistry of PCP Pincer Ligands}

\subsection{Historical Overview}

The first $\kappa^{3}$-PCP complexes were reported by Moulton and Shaw in $1976 .{ }^{84}$ Having noted that cyclometallation reactions were prevalent in complexes with bulky phosphine ligands, they designed a ligand in which an aromatic carbon flanked by two $\mathrm{CH}_{2} \mathrm{P}^{t} \mathrm{Bu}_{2}$ groups would readily undergo ortho-metallation to give a tridentate PCP coordination motif. While the nickel and palladium pincer complexes $[(\mathrm{PCP}) \mathrm{MCl}]$ were readily synthesised from the appropriate metal chlorides, it was noted that the analogous platinum pincer complex was formed in poor yields, with insoluble polymers of the type $\left[(\mathrm{PCPH}) \mathrm{PtCl}_{2}\right]_{\mathrm{x}}$ being formed (Scheme 3.1).

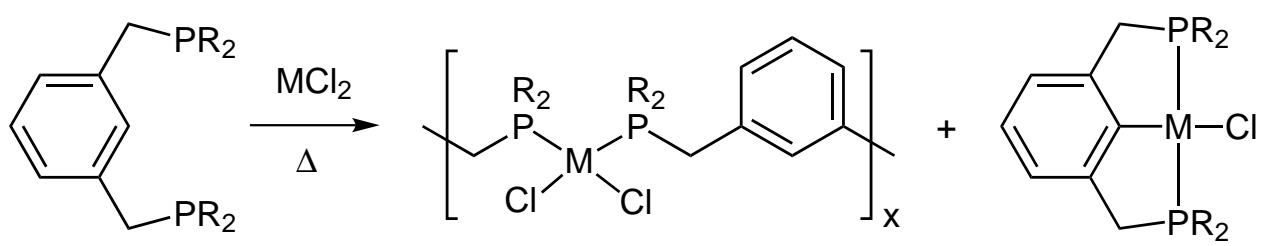

Scheme 3.1 Formation of a cyclometallated $\kappa^{3}$-PCP pincer complex, along with a bridged $\kappa^{2}$-PP oligomer (where $\mathrm{M}$ is typically $\mathrm{Pt}$ or $\mathrm{Pd}$ ). The oligomer may contain metal centres in either cis or trans coordination geometries.

By replacing the aromatic ligand backbone with an alkyl chain, the energy requirement for metallation can be increased, allowing these unmetallated, multimetallic compounds to be isolated and characterised. Reactions between the aliphatic 
phosphines ${ }^{t} \mathrm{Bu}_{2} \mathrm{P}\left(\mathrm{CH}_{2}\right)_{\mathrm{n}} \mathrm{P}^{t} \mathrm{Bu}_{2}$ (where $\mathrm{n}=5-8$ ) and platinum and palladium salts showed that the formation of $\kappa^{2}$-PP bridged dimeric and oligomeric species of the type $\left[\mathrm{MCl}_{2}\left\{{ }^{t} \mathrm{Bu}_{2} \mathrm{P}\left(\mathrm{CH}_{2}\right)_{\mathrm{n}} \mathrm{P}^{t} \mathrm{Bu}_{2}\right\}\right]_{\mathrm{x}}$ was very facile. ${ }^{85}$ Upon heating these compounds, the pentane-bridged diphosphine $(\mathrm{n}=5)$ underwent cyclometallation to produce the tridentate pincer species $\left[\mathrm{PtCl}\left({ }^{t} \mathrm{Bu}_{2} \mathrm{P}\left(\mathrm{CH}_{2}\right)_{2} \mathrm{CH}\left(\mathrm{CH}_{2}\right)_{2} \mathrm{P}^{t} \mathrm{Bu}_{2}\right)\right]$. The metallation of these dimers and oligomers revealed that they were not unwanted byproducts produced during pincer synthesis, but rather were intermediates that in many cases could be further reacted to form the metallated pincer compounds.

Further insight into the importance of these bridged $\kappa^{2}$-PP coordination compounds in the formation of pincer complexes was provided by Rimml and Venanzi in 1983. Reactions using the phenyl-substituted pincer ligand 1,3-bis(diphenylphosphino)- $m$ xylene was seen to produce oligomeric species of the type cis- $\left[(\mathrm{PCPH}) \mathrm{PtX}{ }_{2}\right]_{\mathrm{n}}$ along with the desired PCP pincer species. ${ }^{86}$ None of the corresponding trans-oligomer was observed, leading the authors to speculate that trans-oligomer formation was conducive to pincer complex formation, as it brought the $\mathrm{C}-\mathrm{H}$ bond which is to be activated closer to the metal centre. This was able to explain why tert-butyl phosphine groups assisted the cyclometallation, as their large steric bulk favoured the formation of trans-bridged coordination complexes.

Because bis-phosphines have a known predilection for $\kappa^{2}$-coordination modes, and in many instances ligand cyclometallation is not facile, bridged dimeric and oligomeric structures represent important precursors in the synthesis of $\kappa^{3}$-PCP pincer complexes. However, despite the prevalence of these bridged structures in pincer synthesis, comparatively little work has been reported on their formation during the metallation reaction, and how their presence affects the ease of pincer formation.

Therefore, the coordination chemistry of the electron-deficient PCPH pincer ligands $\mathbf{1}, \mathbf{2}$, and $\mathbf{3}$ with platinum and palladium was investigated. An advantage of utilising electron-poor phosphine ligands in these metallation reactions is that they have the potential to favour coordination modes and geometries that differ from those of their more electron-rich counterparts, an effect that has been previously observed in 5- and 6-coordinate iridium and rhodium complexes. ${ }^{43,45}$ This preference for less conventional coordination geometries was anticipated to stabilise coordination complexes and metallation reaction intermediates not often observed in the literature, in the hope that examination of these species would reveal more about the nature of pincer complex formation. 


\subsection{Coordination to $\operatorname{Pt}(0)$}

Coordination chemistry of the most electron-poor ligand, POCOPH, 1, was initially explored with platinum(0). While the coordination chemistry of ortho-xylyl-type diphosphines with Group 10 metals in the zero oxidation state has long been established, ${ }^{87}$ minimal work has been reported with the analogous meta-substituted ligands. It was hoped that the highly electron-poor nature of $\mathbf{1}$ would facilitate electrophilic metallation of the backbone to produce the tridentate oxidative addition product, [(POCOP)PtR], where $\mathrm{R}$ is an alkyl group arising from a neutral alkene ligand on the platinum precursor accepting the metallated proton.

Initial reactions with $\left[\mathrm{Pt}(\mathrm{nb})_{3}\right]$ and $\left[\mathrm{Pt}(\mathrm{COD})_{2}\right]($ where $\mathrm{nb}=$ norbornene and $\mathrm{COD}=$ 1,5-cyclooctadiene) were carried out in NMR tubes, allowing the reaction progress to be continually monitored by ${ }^{1} \mathrm{H},{ }^{19} \mathrm{~F}$, and ${ }^{31} \mathrm{P}$ NMR spectroscopy. Reactions involving $\left[\mathrm{Pt}(\mathrm{COD})_{2}\right]$ were seen to produce a single product; however, in both toluene- $d_{8}$ and dichloromethane- $d_{2}$ large amounts of poorly-soluble material were present, and spectra possessed broad resonances with a poor signal-to-noise ratio. The product displayed a ${ }^{31} \mathrm{P}$ NMR shift of $108.9 \mathrm{ppm}$ and a ${ }^{1} J_{\mathrm{Pt}-\mathrm{P}}$ coupling of $5448 \mathrm{~Hz}$; the downfield shift of the phosphorus signal and very large platinum-phosphorus coupling indicated coordination of a phosphorus donor trans to the weakly-coordinated $\eta^{2}-1,5$-cyclooctadiene ligand.* Unfortunately, the ${ }^{1} \mathrm{H}$ NMR data could not unambiguously confirm the presence of coordinated 1,5-cyclooctadiene due to the very broad, weak nature of the signals. This data, together with the poorly soluble nature of the product and its similarity to the analogous norbornene species discussed below, allowed the product to be tentatively assigned as an oligomer of the type $\left[(\mathrm{POCOPH}) \operatorname{Pt}\left(\eta^{2}-\mathrm{COD}\right)\right]_{\mathrm{x}}$, where $\mathrm{x}$ may be $>2$, (due to the very low solubility of the observed product, Scheme 3.2).

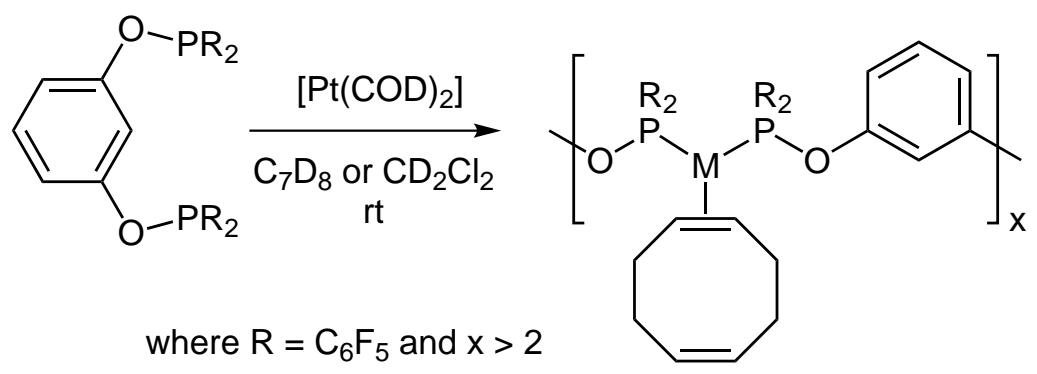

Scheme 3.2 Oligmer formation from the reaction of 1 with $\left[\mathrm{Pt}(\mathrm{COD})_{2}\right]$.

\footnotetext{
${ }^{*}$ In all coordination chemistry with 1 reported herein, ${ }^{1} J_{\mathrm{Pt}-\mathrm{P}}$ values of $>4200 \mathrm{~Hz}$ were diagnostic for coordination of the phosphorus atoms trans to a ligand of low trans-influence, typically a chloride or an $\eta^{2}$-alkene.
} 
Compared to reactions with the COD starting material, NMR-scale reactions between $\left[\mathrm{Pt}(\mathrm{nb})_{3}\right]$ and $\mathbf{1}$ produced substantially smaller quantities of insoluble material and much sharper signals in the NMR spectra. The phosphorus NMR data of the major product $\left(\delta_{\mathrm{P}}=100.5 \mathrm{ppm},{ }^{1} J_{\mathrm{Pt}-\mathrm{P}}=4623 \mathrm{~Hz}\right)$, with a single phosphorus environment and large platinum-phosphorus coupling, along with the observation of coordinated norbornene in the ${ }^{1} \mathrm{H}$ NMR spectrum $\left(\delta_{\mathrm{H}}=2.75 \mathrm{ppm},{ }^{2} J_{\mathrm{Pt}-\mathrm{H}}=70.8 \mathrm{~Hz}\right.$, $\mathrm{HC}=\mathrm{CH})$ was again indicative of the formation of a $[(\mathrm{POCOPH}) \mathrm{Pt}(\mathrm{nb})]_{\mathrm{x}}$ species. The product was isolated by repeating the reaction on a larger scale in toluene, separating the desired product from less soluble polymers and higher oligomers by extraction into dichloromethane (Scheme 3.3). High Resolution Mass Spectrometry (HRMS) confirmed that the product of this reaction was the dimeric species $[(\mathrm{POCOPH}) \mathrm{Pt}(\mathrm{nb})]_{2}(\mathbf{1 4})$, observed as the sodium ion adduct without coordinated norbornene at $m / z=2087 \mathrm{amu}$.

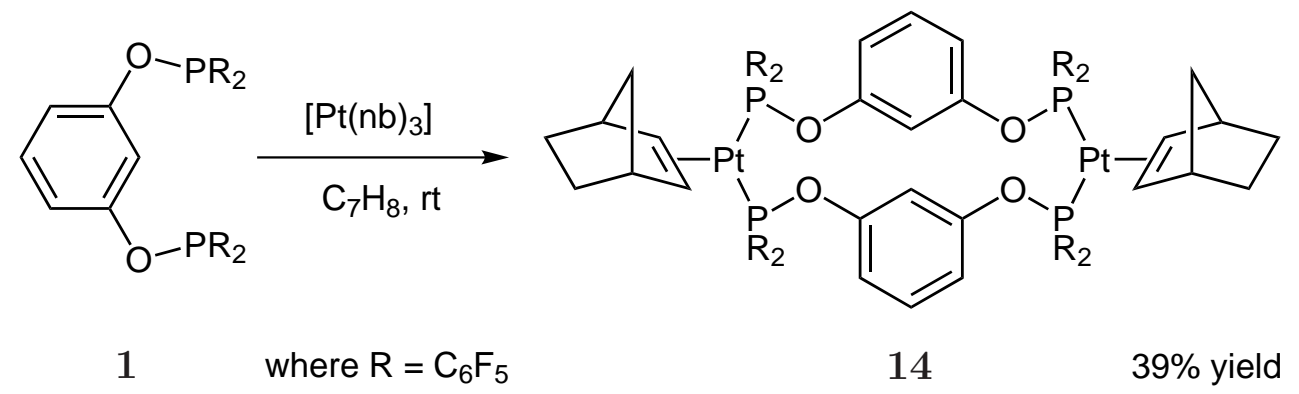

Scheme 3.3 Formation of $[(\mathrm{POCOPH}) \mathrm{Pt}(\mathrm{nb})]_{2}$ (14) from the reaction of $\mathbf{1}$ with $\left[\mathrm{Pt}(\mathrm{nb})_{3}\right]$.

An additional $[(\mathrm{POCOPH}) \mathrm{Pt}(\mathrm{nb})]_{\mathrm{x}}$ species with similar spectroscopic data to 14 was observed as a minor product in many of these reactions. The ${ }^{31} \mathrm{P}$ NMR data $\left(\delta_{\mathrm{P}}=\right.$ $102.1 \mathrm{ppm},{ }^{1} J_{\mathrm{Pt}-\mathrm{P}}=4700 \mathrm{~Hz}$ ) again suggested the coordination of the phosphorus trans to a norbornene ligand, which was observed in the ${ }^{1} \mathrm{H}$ NMR overlapping with the norbornene resonances of $\mathbf{1 4}$. Over time the NMR signals of this oligomeric species disappeared as it was either converted to 14 or precipitated out of solution as polymeric material. The disappearance of this minor product was observed to occur faster in toluene and benzene than in dichloromethane. Where small quantities of this byproduct were found to still be present in reactions of $\mathbf{1}$ and $\left[\mathrm{Pt}(\mathrm{nb})_{3}\right]$ in dichloromethane even after 48 hours at reflux, resuspension of the material in toluene followed by heating to $50{ }^{\circ} \mathrm{C}$ for 12 hours resulted in complete disappearance of this minor product. The reason for these reactions proceeding faster in toluene than dichloromethane may be due to the aromatic solvent providing the ligand with stabilising $\pi-\pi$ interactions during the rearrangement process from oligomer to dimer, thereby minimising the energetic barrier to this transformation. 
Crystals of 14 suitable for single crystal X-ray diffraction were grown from a dichloromethane solution layered with methanol. The X-ray crystal structure (Figure 3.1) supports the NMR and HRMS assignment of $\mathbf{1 4}$ as a dimer, crystallising as the dichloromethane solvate $[(\mathrm{POCOPH}) \mathrm{Pt}(\mathrm{nb})]_{2} \cdot 2 \mathrm{CH}_{2} \mathrm{Cl}_{2}$ in the monoclinic space group $P 2_{1} / n$. As this is a centrosymmetric space group, the asymmetric unit can be proscribed about one half of the molecule, with the other half generated through the centre of inversion. Important bond lengths and angles are summarised in Table 3.1, and crystallographic data collection and refinement parameters are stated in Table 3.2 .

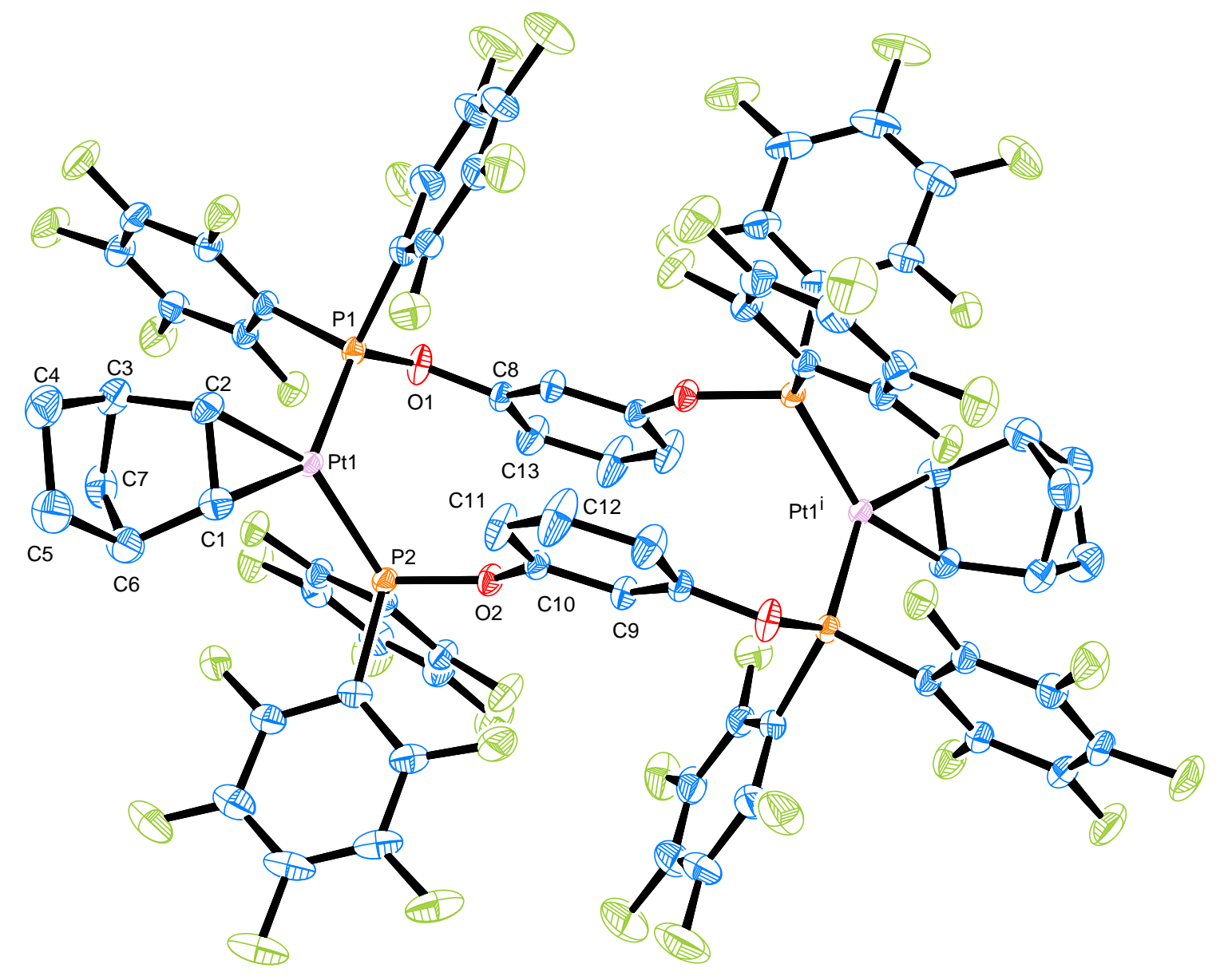

Figure 3.1 ORTEP diagram of $[(\mathrm{POCOPH}) \mathrm{Pt}(\mathrm{nb})]_{2} \cdot 2 \mathrm{CH}_{2} \mathrm{Cl}_{2}$ (14) (50\% probability thermal elipsoids). Dichloromethane solvate and hydrogen atoms omitted for clarity. Atoms denoted by ${ }^{\mathrm{i}}$ are generated from the asymmetric unit by inversion. 
Table 3.1 Selected bond distances $(\AA)$ and angles $\left(^{\circ}\right)$ of $[(\mathrm{POCOPH}) \mathrm{Pt}(\mathrm{nb})]_{2} \cdot 2 \mathrm{CH}_{2} \mathrm{Cl}_{2}(\mathbf{1 4})$.

\begin{tabular}{|c|c|c|c|}
\hline \multicolumn{2}{|c|}{ Bond distances $(\AA)$} & \multicolumn{2}{|l|}{ Bond angles $\left({ }^{\circ}\right)$} \\
\hline Pt1-C1 & $2.153(2)$ & $\mathrm{P} 1-\mathrm{Pt} 1-\mathrm{P} 2$ & $108.04(2)$ \\
\hline $\mathrm{Pt} 1-\mathrm{C} 2$ & $2.139(2)$ & $\mathrm{P} 1-\mathrm{Pt} 1-\mathrm{C} 2$ & $103.80(7)$ \\
\hline Pt1-P1 & $2.2110(6)$ & $\mathrm{P} 2-\mathrm{Pt} 1-\mathrm{C} 1$ & $109.12(6)$ \\
\hline Pt1-P2 & $2.2224(5)$ & $\mathrm{Pt} 1-\mathrm{P} 1-\mathrm{O} 1$ & $121.37(7)$ \\
\hline $\mathrm{C} 1-\mathrm{C} 2$ & $1.434(3)$ & $\mathrm{Pt} 1-\mathrm{P} 2-\mathrm{O} 2$ & $117.14(6)$ \\
\hline Pt1 $\cdots$ Pt1 ${ }^{\mathrm{i}}$ & $8.4535(2)$ & $\mathrm{P} 1-\mathrm{P} 2 \cdots \mathrm{C} 1-\mathrm{C} 2$ & $179.6(2)$ \\
\hline
\end{tabular}

Table 3.2 Crystallographic data of $[(\mathrm{POCOP}) \mathrm{Pt}(\mathrm{nb})]_{2} \cdot 2 \mathrm{CH}_{2} \mathrm{Cl}_{2}$ (14).

\begin{tabular}{|c|c|}
\hline Empirical formula & $\mathrm{C}_{74} \mathrm{H}_{28} \mathrm{~F}_{40} \mathrm{O}_{4} \mathrm{P}_{4} \mathrm{Pt}_{2} \cdot 2 \mathrm{CH}_{2} \mathrm{Cl}_{2}$ \\
\hline Formula weight & 2424.88 \\
\hline Crystal system & Monoclinic \\
\hline Space group & $P 2_{1} / n$ \\
\hline $\mathrm{a} / \AA$ & $14.0338(4)$ \\
\hline $\mathrm{b} / \AA$ & $18.4499(5)$ \\
\hline $\mathrm{c} / \AA$ & $15.2852(4)$ \\
\hline$\alpha /{ }^{\circ}$ & 90.00 \\
\hline$\beta /{ }^{\circ}$ & $91.299(2)$ \\
\hline$\gamma /{ }^{\circ}$ & 90.00 \\
\hline $\mathrm{V} / \AA^{3}$ & $3956.66(19)$ \\
\hline $\mathrm{Z}$ & 2 \\
\hline Cell determination reflections & 9272 \\
\hline Cell determination range, $\theta_{\min } \longrightarrow \theta_{\max } /{ }^{\circ}$ & $2.24 \longrightarrow 32.53$ \\
\hline Temperature/K & $113(2)$ \\
\hline Radiation type & Mo K $\alpha$ \\
\hline Radiation $(\lambda) / \AA$ & 0.71073 \\
\hline Crystal size/ mm & $0.37 \times 0.36 \times 0.31$ \\
\hline $\mathrm{D}_{\text {calc }} / \mathrm{g} \mathrm{m}^{-3}$ & 2.035 \\
\hline $\mathrm{F}(000)$ & 2328 \\
\hline$\mu / \mathrm{mm}^{-1}$ & 3.89 \\
\hline Experimental absorption correction type & None \\
\hline Reflections collected & $117634, R_{\text {equiv }}=0.0467$ \\
\hline Index range $h$ & $-21 \longrightarrow 21$ \\
\hline Index range $k$ & $-26 \longrightarrow 28$ \\
\hline Index range $l$ & $-23 \longrightarrow 22$ \\
\hline$\theta$ range ${ }^{\circ}$ & $2.58 \longrightarrow 33.23$ \\
\hline Independent reflections & 14920 \\
\hline Reflections $[I>2 \sigma(I)]$ & 12169 \\
\hline Restraints/parameters & $0 / 586$ \\
\hline GOF & 1.032 \\
\hline$R_{1}[I>2 \sigma(I)]$ & 0.0279 \\
\hline $\mathrm{w} R 2[I>2 \sigma(I)]$ & 0.0629 \\
\hline$R_{1}$ [all data] & 0.0415 \\
\hline $\mathrm{w} R 2$ [all data] & 0.0678 \\
\hline Residual density/e $\AA^{-3}$ & $-1.221<2.599$ \\
\hline
\end{tabular}


The structure revealed two trigonal planar platinum cores, bridged by two molecules of 1 in a $c i s-\kappa^{2}-\mathrm{PP}$ fashion, with the norbornene bound "in-plane" in a conventional $\eta^{2}$ manner (P1-P2 $\cdots \mathrm{C} 1-\mathrm{C} 2$ torsion angle of $\left.179.6^{\circ}\right)$. While NMR data demonstrated that the norbornene is in a symmetrical coordination environment in solution, crystal packing constraints cause it to be pushed $2.7^{\circ}$ from the normal, closer to P1. Compound $\mathbf{1 4}$ is only the third reported crystal structure ${ }^{\dagger}$ containing the $\left[\mathrm{P}_{2} \mathrm{M}^{0}(\mathrm{nb})\right]$ coordination environment (where $\mathrm{M}$ is metal in the zero oxidation state). In comparison to the previously reported structures, ${ }^{88,89} \mathbf{1 4}$ contains a relatively short norbornene $\mathrm{C}=\mathrm{C}$ bond (1.434(3) $\AA$ compared to $1.460(11)$ and $1.469(8)$ $\AA$ for reported structures) and a longer Pt-C bond (average of 2.146(4) $\AA$ compared to $2.109(15)$ and $2.113(10) \AA$ for reported structures), possibly indicating a weakly bound norbornene. It may be anticipated that the electrophilic platinum centre of 14 generated by the electron-poor phosphinite $\mathbf{1}$ should foster a strong metal-alkene interaction by acting as a better electron acceptor. However, the reduced electron density on the platinum clearly limits the extent to which $\pi$-backbonding occurs in this system, explaining the short norbornene $\mathrm{C}=\mathrm{C}$ bond (through less donation into the $\pi^{*}$ orbital) and the long $\mathrm{Pt}-\mathrm{C}$ distance.

Complex 14 displayed a platinum-carbon coupling of $259 \mathrm{~Hz}$ between the platinum and the coordinated alkene, significantly lower than the corresponding value of $344 \mathrm{~Hz}$ reported for the tert-butyl-substituted compound [(dtbpe)Pt(nb)] (where dtbpe is 1,2-bis(di-tert-butylphosphino)ethane). ${ }^{89}$ This data also suggests a weaker metal-norbornene interaction is present in $\mathbf{1 4}$ than in its more electron-rich analogue, consistent with decreased $\pi$-backbonding to the alkene for the electron-poor complex 14. Comparison of NMR data with similar pentafluorophenyl-substituted compounds was not possible, as compound 14 represents only the second reported instance of a platinum-alkene complex containing a pentafluorophenyl moiety, and the first such compound to be crystallographically characterised. The compound $\left[\left\{\left(\mathrm{C}_{6} \mathrm{~F}_{5}\right)_{3} \mathrm{P}\right\}_{2} \mathrm{Pt}(\mathrm{nb})\right]$ has been reported by the Pringle research group, ${ }^{80}$ but no ${ }^{1} \mathrm{H}$ or ${ }^{13} \mathrm{C}$ NMR data were provided to indicate the strength of the Pt-alkene interaction.

The $\mathrm{P}-\mathrm{Pt}-\mathrm{P}$ angle in 14 of $108.04(2)^{\circ}$ is smaller than the idealised trigonal planar bond angles of $120^{\circ}$, but larger than the significantly constrained $\mathrm{P}-\mathrm{Pt}-\mathrm{P}$ bite angles of approximately $89^{\circ}$ seen in norbornene complexes with chelating rather than bridged phosphines. ${ }^{88,89}$ The observed geometry is in good agreement with that expected for a platinum-alkene complex containing bulky phosphorus-donor ligands, with complexes of the type $\left[\mathrm{Pt}(\mathrm{nb})\left\{\mathrm{P}(\mathrm{OAr})_{3}\right\}_{2}\right]$ displaying $\mathrm{P}-\mathrm{Pt}-\mathrm{P}$ bond angles of around $105^{\circ} .{ }^{90} \mathrm{~A}$ similar coordination geometry is also adopted by cisbridged platinum(II) dimers when a group with a small steric bulk (such as a methyl)

\footnotetext{
${ }^{\dagger}$ According to a search of the Cambridge Structural Database, v5.33, February 2012 update.
} 
is trans to the phosphorus, giving $\mathrm{P}-\mathrm{Pt}-\mathrm{P}$ angles of around $103^{\circ} .{ }^{58,91}$
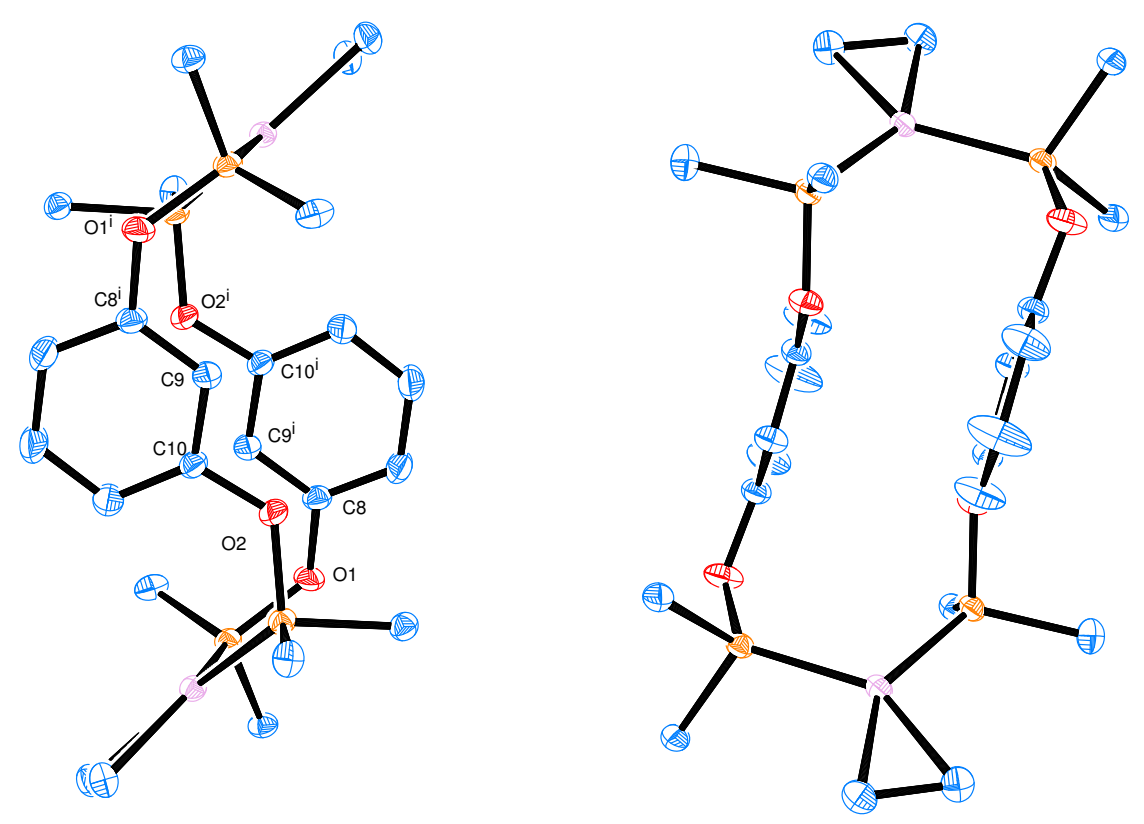

Figure 3.2 ORTEP diagram of $[(\mathrm{POCOPH}) \mathrm{Pt}(\mathrm{nb})]_{2} \cdot 2 \mathrm{CH}_{2} \mathrm{Cl}_{2}$ (14) (50\% probability thermal elipsoids). Dichloromethane solvate, hydrogen atoms, norbornene and pentafluorophenyl substituents (except for carbons bound directly to platinum or phosphorus) omitted for clarity. Atoms denoted by ${ }^{\mathrm{i}}$ are generated from the asymmetric unit by inversion.

Another distinctive feature of the structure of $\mathbf{1 4}$ is the folding of the aryl backbones to lie parallel to each other (Figure 3.2). This is somewhat unusual, as all previously reported cis-dimers have rings canted towards each other by at least $10^{\circ} ;{ }^{58,91,92}$ in 14 they lie within $2^{\circ}$ of parallel. This favourable "parallel displaced" configuration of the aromatic backbones, as well as the short interatomic distances $\left(\mathrm{O} 2 \cdots \mathrm{C} 9^{\mathrm{i}}\right.$ $\left.=3.201(3) \AA, \mathrm{C} 10 \cdots \mathrm{C} 9^{\mathrm{i}}=3.321(3) \AA, \mathrm{C} 10 \cdots \mathrm{C} 10^{\mathrm{i}}=3.387(3) \AA\right)$ indicate that $\pi-\pi$ interactions should occur between the aromatic rings, ${ }^{93}$ conferring additional stability to the dimeric structure. As these favourable intramolecular interactions would not be available to an oligomeric species, this may explain why the oligomeric product initially observed appears to further rearrange to form the dimeric species 14 over time.

With 14 in hand and unambiguously characterised as the platinum-norbornene cisdimer $[(\mathrm{POCOPH}) \mathrm{Pt}(\mathrm{nb})]_{2}$, attempts were made to promote metallation of the aromatic backbone at the 2 -C position. Initially, thermolysis of toluene- $d_{8}$ solutions of 14 at $75^{\circ} \mathrm{C}$ over a period of days was seen to have no significant effect; the only notable change in these solutions being the gradual appearance of decomposition products between 6.2-5.8 ppm in the ${ }^{1} \mathrm{H}$ NMR spectra, accompanied by a slight decrease in signal-to-noise ratio. Increasing the temperature to $100{ }^{\circ} \mathrm{C}$ did not produce 
any observable metallation, instead serving to hasten decomposition.

The strong bases DMAP and DBU were employed in an attempt to promote the metallation under milder conditions, as they have been seen to facilitate metallation of pincer ligands in $\mathrm{Ni}$ (II) complexes. ${ }^{94}$ The addition of DMAP to chloroform- $d$ solutions of $\mathbf{1 4}$ at room temperature resulted in quantitative formation of a new complex, tentatively formulated as trans-[(POCOPH) Pt $(\mathrm{L})]_{2}$ (where $\mathrm{L}$ is a neutral ligand, either norbornene or the amine base) on the basis of NMR data. Instead of the downfield shift of the signal in the ${ }^{31} \mathrm{P}$ NMR spectrum that usually accompanies pincer metallation, this compound displayed an upfield shift and decrease in platinum-phosphorus coupling constant consistent with a trans-dimer $\left(\delta_{\mathrm{P}}\right.$ $=49.5 \mathrm{ppm},{ }^{1} J_{\mathrm{Pt}-\mathrm{P}}=3250 \mathrm{~Hz}$ ), as well as diagnostic signals for the unmetallated ligand 1 in the ${ }^{1} \mathrm{H}$ NMR spectrum. Attempts to isolate this compound were unsuccessful, and attempts to promote metallation of the new trans species led to degradation and a loss of signals in the ${ }^{31} \mathrm{P}$ NMR spectrum. Performing one-pot reactions with $\left[\mathrm{Pt}(\mathrm{nb})_{3}\right]$, ligand $\mathbf{1}$, and $\mathrm{DMAP}$ or DBU were seen to solely produce degradation products, resulting in intractable mixtures that displayed no soluble, phosphorus-containing species.

In an attempt to avoid this decomposition, metallations were attempted using the strong non-nucleophilic base sodium hydride. Reactions were performed on an NMR scale, with an excess of sodium hydride (approximately ten equivalents) added to a dichloromethane- $d_{2}$ solution of $\mathbf{1 4}$. No reaction was observed after heating for 18 hours at $40^{\circ} \mathrm{C}$, indicating that even in the presence of a strong base such as sodium hydride present the metallation reaction was not facile.

Acid was also employed to assist metallation of the ligand backbone to form a $\kappa^{3}$ PCP pincer complex. The addition of acid to $\mathrm{Pt}(0) \eta^{2}$-norbornene complexes has been seen in some cases to result in the formation of the Pt(II) norbornyl species. ${ }^{89}$ In employing the acid as an oxidant it was hoped that this would allow the norbornyl group generated to act as a proton acceptor, leaving as norbornane and forming the cationic, 14-electron pincer complex $[(\mathrm{PCP}) \mathrm{Pt}]^{+}$. Treatment of dichoromethane- $d_{2}$ solutions of 14 with $\mathrm{HBF}_{4} \cdot \mathrm{Et}_{2} \mathrm{O}$ at $-78{ }^{\circ} \mathrm{C}$ was seen to immediately produce a large number of broad, unidentified signals in the ${ }^{1} \mathrm{H},{ }^{19} \mathrm{~F}$ and ${ }^{31} \mathrm{P}$ NMR spectra, with an intractable brown precipitate gradually forming in solution, accompanied by a decrease in signal-to-noise ratio in the NMR spectra of all nuclei studied. Protonation under milder conditions was also attempted using the carbacid $\mathrm{HPhC}\left(\mathrm{SO}_{2} \mathrm{CF}_{3}\right)_{2}$. Solutions of 14 were treated with 1.4 equivalents of $\mathrm{HPhC}\left(\mathrm{SO}_{2} \mathrm{CF}_{3}\right)_{2}$ and subjected to thermolysis in a range of solvents. No reaction was observed after prolonged heating at $40{ }^{\circ} \mathrm{C}$ in dichloromethane- $d_{2}, 60{ }^{\circ} \mathrm{C}$ in benzene- $d_{6}$, and $50{ }^{\circ} \mathrm{C}$ in acetone- $d_{6}$. 
Therefore, while the strong acid $\mathrm{HBF}_{4} \cdot \mathrm{Et}_{2} \mathrm{O}$ led to degradation of the platinum complex, the milder carbacid was not a strong enough oxidant to facilitate metallation to produce a platinum(II) complex, indicating that acid-promoted oxidation is not likely to provide viable route to pincer complexes from these platinum(0) dimers.

\subsection{Coordination to $\mathrm{Pt}(\mathrm{II})$}

\subsubsection{Reactions with $\left[\operatorname{PtMe}_{2}(1,5\right.$-hexadiene $\left.)\right]$}

As attempts at generating a platinum-alkyl pincer complex $[(\mathrm{PCP}) \mathrm{PtR}]$ from the $\mathrm{Pt}(0)$ norbornene dimer 14 were unsuccessful, the $\mathrm{Pt}(\mathrm{II})$ compound $\left[\mathrm{PtMe}_{2}(\mathrm{hex})\right]$ (where hex $=1,5$-hexadiene) was investigated for its utility as a precursor toward the synthesis of a platinum-methyl pincer complex [(PCP)PtMe]. It has been demonstrated in the literature that $[(\mathrm{PCP}) \mathrm{PtMe}]$ complexes can be obtained in moderate yields directly from platinum dimethyl precursors. ${ }^{58}$

As for the corresponding platinum(0) reactions, the coordination chemistry of $\mathbf{1}$ to $\left[\mathrm{PtMe}_{2}(\mathrm{hex})\right]$ was initially performed on a small scale in NMR tubes, allowing real-time monitoring of reaction progress by NMR spectroscopy. Reactions in both toluene- $d_{8}$ and dichloromethane- $d_{2}$ showed the formation of two major species $\mathbf{1 5}$ and 16 at room temperature. Each compound displayed ${ }^{1} \mathrm{H}$ NMR signals consistent with symmetrical, unmetallated aryl ligand backbones, and relatively small platinum-phosphorus couplings $(\approx 2200 \mathrm{~Hz})$ in the ${ }^{31} \mathrm{P}$ NMR spectrum, indicative of phosphorus coordination trans to a methyl group (which possess a large trans influence). This allowed for the formulation of both 15 and $\mathbf{1 6}$ as cis[(POCOPH $\left.) \mathrm{PtMe}_{2}\right]_{\mathrm{x}}$ species. Compounds $\mathbf{1 5}$ and $\mathbf{1 6}$ displayed markedly different solubilities, and as such they were able to be separated and isolated by precipitation of the less soluble $\mathbf{1 6}$ by the addition of hexane to reaction mixtures in toluene.

Analysis by HRMS indicated that the more soluble species $\mathbf{1 5}$ was the dimeric compound cis- $\left[(\mathrm{POCOPH}) \mathrm{PtMe}_{2}\right]_{2}$, with the $[\mathrm{M}+\mathrm{Na}]^{+}$ion detected at $m / z=2147 \mathrm{amu}$. No adequate mass spectrometry results were obtained for 16; in some cases traces of 15 were observed and in others no peaks attributable to a $\left[(\mathrm{POCOPH}) \mathrm{PtMe}_{2}\right]_{\mathrm{x}}$ species were observed. Liquid Chromatography Mass Spectrometry (LCMS) was performed in order to overcome contamination of each species by minor amounts of the other; however, LCMS was unsuccessful in this respect, as both compounds either degraded or were retained on the column. As such, while the HRMS 
data were consistent with the formulation of $\mathbf{1 5}$ as the dimeric compound cis$\left[(\mathrm{POCOPH}) \mathrm{PtMe}_{2}\right]_{2}$, it was not able to be definitively established.

Compound 16 is likely to be a cyclic [(POCOPH $\left.) \mathrm{PtMe}_{2}\right]_{\mathrm{x}}$ oligomer of relatively low molecular weight ( $\mathrm{x}=3$ or 4 ); it is significantly less soluble than the dimeric $\mathbf{1 5}$ in common organic solvents, yet soluble enough that sufficient NMR data could be acquired for characterisation. It is highly unlikely to be a cis-monomer. Such species are well established for complexes of PCPH ligands with alkyl backbones (as the backbone can readily contort out of the coordination plane of the metal to minimise steric strain), ${ }^{95-97}$ but are seldom formed at room temperature in complexes with aromatic ligand backbones, due to the demands of forming a rigid, eight-membered chelate ring. ${ }^{91}$

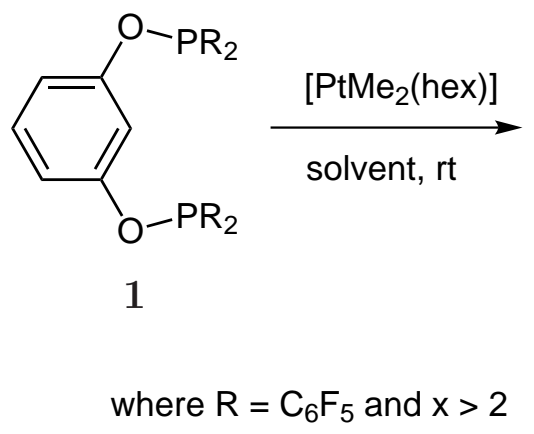

where $\mathrm{R}=\mathrm{C}_{6} \mathrm{~F}_{5}$ and $\mathrm{x}>2$<smiles>C[P+]([PH])(P[PH])POc1cccc(OP[PH](C)([PH])[PH])c1</smiles>

15

toluene, $72 \mathrm{~h}, 78 \%$

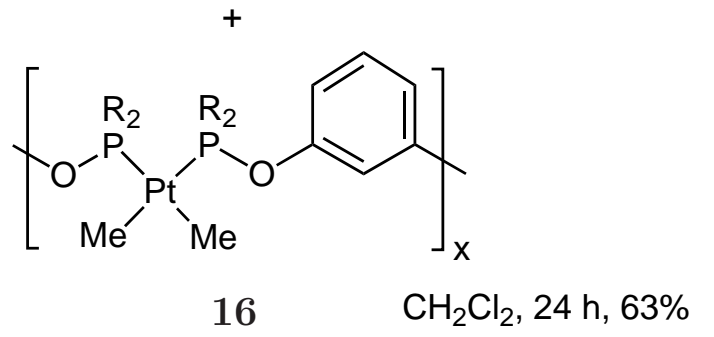

Scheme 3.4 Formation of $\left[(\mathrm{POCOPH}) \mathrm{PtMe}_{2}\right]_{\mathrm{x}}$ species (15 and 16) from the reaction of 1 with $\left[\mathrm{PtMe}_{2}(\right.$ hex $\left.)\right]$.

In the synthesis of 15 (Scheme 3.4), it was observed that longer reaction times and the use of toluene instead of dichloromethane increased the yield of the dimeric complex. This parallels observations in the synthesis of the analogous platinum $(0)$ dimer 14. While the corresponding platinum(0) oligomer was not soluble enough to characterise, it was noted that the dimer synthesis proceeded more rapidly and with the formation of less insoluble white material in benzene and toluene than in dichloromethane. As mentioned above, this may be due to stabilising interactions from the aromatic solvent increasing the rate of reaction.

The platinum $\left[(\mathrm{POCOPH}) \mathrm{PtMe}_{2}\right]_{\mathrm{x}}$ compounds $\mathbf{1 5}$ and $\mathbf{1 6}$ possessed very similar NMR spectroscopic data. However, in ${ }^{1} \mathrm{H}$ NMR spectra of the two species, there was a distinct chemical shift difference between protons on C-2 of each aromatic 
backbone. For the oligomer 16 this proton was observed between the other two aromatic proton environments of the backbone at $\delta_{\mathrm{H}}=6.94 \mathrm{ppm}$, whereas for the dimer 15 this proton was the most upfield of all the aromatic proton signals, appearing at $\delta_{\mathrm{H}}=6.42 \mathrm{ppm}$. This observation of an upfield shift in H-2 again displayed parallels to the norbornene dimer 14 , where the $\mathrm{H}-2$ signal was observed at $\delta_{\mathrm{H}}=6.35 \mathrm{ppm}$. As the solid state structure of $\mathbf{1 4}$ displayed a distinct $\pi-\pi$ interaction between the two ligand backbones, centred about H-2 (Figure 3.2), it is likely that this proton environment is shielded by the shared $\pi$-electrons, accounting for the observation of a upfield shift of H-2 in the two dimeric structures (Figure 3.3). Similar shielding of aromatic protons by $\pi$-stacking has been established in ${ }^{1} \mathrm{H}$ NMR spectroscopy of organic molecules. ${ }^{98}$ It would be very difficult for the oligomeric structure $\mathbf{1 6}$ to adopt a conformation in solution possessing these intramolecular $\pi-\pi$ interactions, and consequently 16 displayed an H-2 resonance at a similar chemical shift to the other aromatic protons of the backbone.

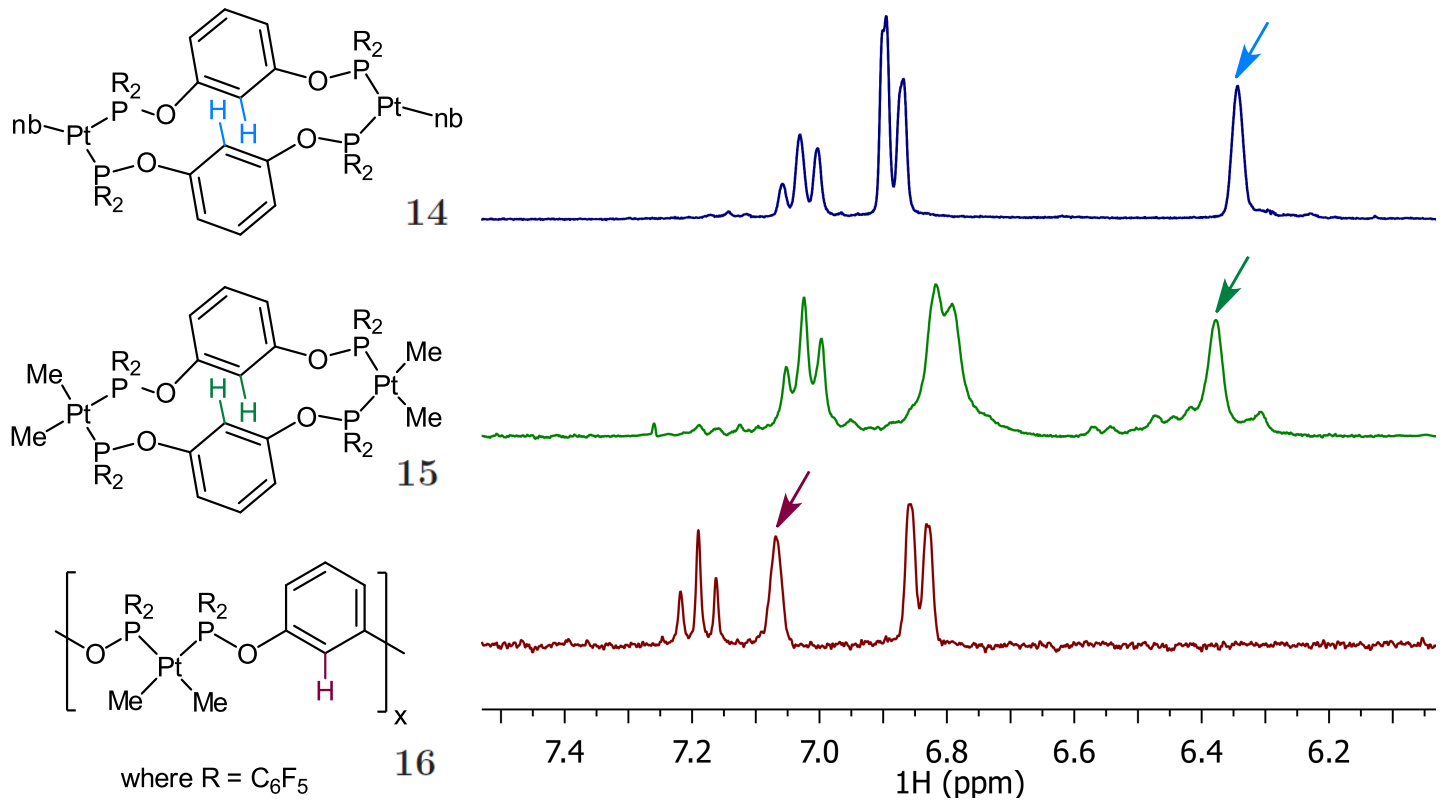

Figure 3.3 Effect of $\pi-\pi$ stacking on the ${ }^{1} \mathrm{H}$ NMR spectra of compounds $14, \mathbf{1 5}$, and $\mathbf{1 6}$ in acetone- $d_{6}$.

Reaction mixtures containing the $\left[(\mathrm{POCOPH}) \mathrm{PtMe}_{2}\right]_{\mathrm{x}}$ species $\mathbf{1 5}$ and $\mathbf{1 6}$ were then heated to promote metallation and formation of the pincer [(POCOP)PtMe] species. Thermolysis of toluene solutions at $90{ }^{\circ} \mathrm{C}$ was seen to produce predominantly one new species, 17. NMR signals of the species were extremely broad, rendering ${ }^{1} \mathrm{H}$ NMR data of little use in structural assignment. However, the ${ }^{31} \mathrm{P}$ NMR spectrum displayed two broad singlets in a 1:1 ratio at 113.1 and $96.3 \mathrm{ppm}$, with platinumphosphorus coupling constants of 2032 and $2722 \mathrm{~Hz}$ respectively. This indicated the presence of phosphorus donors both cis and trans to a methyl group; the high solubility of the species in toluene and the propensity of ligand $\mathbf{1}$ to form bridged 
$\kappa^{2}$-PP dimers (see below) suggested that the complex was likely to be dimeric. This allowed for the tentative assignment of $\mathbf{1 7}$ as cis,trans-[(POCOPH)PtMe $]_{2}$. Similar cis,trans-dimers were isolated from the reaction of ligand $\mathbf{1}$ with platinum dichloride and platinum chloromethyl precursors, and are subsequently discussed in detail.

Metallation of 17 was not observed to any appreciable extent after 20 hours at $90{ }^{\circ} \mathrm{C}$. Upon increasing the temperature to $100{ }^{\circ} \mathrm{C}$, the formation of the desired pincer species [(POCOP $) \mathrm{PtMe}]$ was detected $\left(\delta_{\mathrm{P}}=108.5 \mathrm{ppm},{ }^{1} J_{\mathrm{Pt}-\mathrm{P}}=3760 \mathrm{~Hz}\right.$; verified by comparison with an independently synthesised sample, as is described in the Chapter 4). However, the appearance of this species was also accompanied by the formation of other unknown metallated pincer species, as well as the formation of perfluorinated biphenyl $\left(\mathrm{C}_{12} \mathrm{~F}_{10}\right)$, indicating the degradation of the ligand at these elevated temperatures.

In an attempt to reduce the temperatures required for this metallation, identical reactions were carried out in acetone. It was hoped that a more polar solvent would assist with the proton transfer during metallation and stabilise any charged transition states, minimising the energetic barrier to pincer complex formation. Reactions performed in acetone- $d_{6}$ in sealed NMR tubes heated to $75{ }^{\circ} \mathrm{C}$ were observed again to produce the broad resonances of $\mathbf{1 7}$, without the observation of any products resulting from metallation. The addition of the amine bases DMAP and DBU to toluene solutions of $\left[(\mathrm{POCOPH}) \mathrm{PtMe}_{2}\right]_{\mathrm{x}}$ was also attempted; however, as in the analogous reactions with the platinum-norbornene dimer 14, only decomposition and a loss of signal in ${ }^{31} \mathrm{P}$ NMR spectra was observed.

To investigate whether the difficulty in synthesising the metallated platinum methyl complex [(POCOP)PtMe] was due to the highly electron-deficient nature of the metal centre, or due to the inherent instability of $\mathbf{1}$ possessing highly polarised $\mathrm{P}-\mathrm{O}$ bonds, reactions with the more electron-donating tert-butyl-substituted ligand $\mathbf{3}$ were performed. The reaction between 3 and $\left[\mathrm{PtMe}_{2}(\right.$ hex $\left.)\right]$ was carried out in benzene- $d_{6}$ on an NMR scale. On standing at room temperature a number of products were initially observed by NMR spectroscopy. The major (and only identified) species in solution possessed two broad phosphorus signals in a 1:1 ratio at 88.7 and $48.3 \mathrm{ppm}$, with platinum-phosphorus coupling constants of 2268 and $1825 \mathrm{~Hz}$ respectively. The small downfield shift of the ${ }^{31} \mathrm{P}$ NMR signal of each of the phosphorus donors from those of the free ligand (where $\delta_{\mathrm{P}}=82.9$ and $34.3 \mathrm{ppm}$ ), combined with relatively small one bond platinum-phosphorus couplings (around $2000 \mathrm{~Hz}$ ) was consistent with the formation of a $\kappa^{2}-\mathrm{PP}$ bridged species $c i s-\left[(\mathrm{POCCPH}) \mathrm{PtMe}_{2}\right]_{\mathrm{x}}$.

It is interesting to note that the di-tert-butyl phosphine moiety experienced a greater 

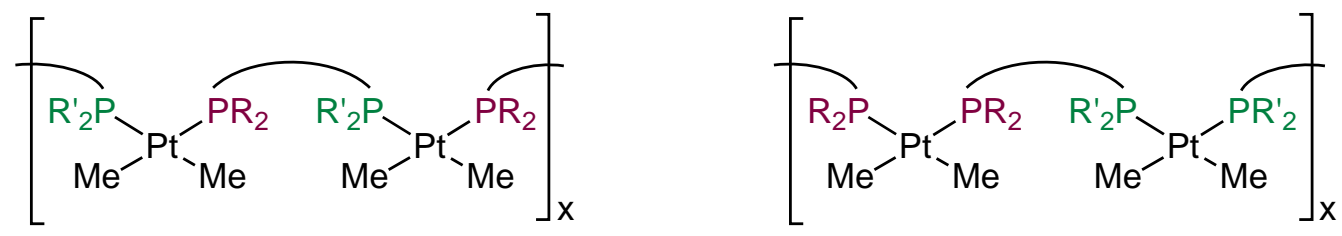

Figure 3.4 The two possible structural isomers that $\left[(\mathrm{POCCPH}) \mathrm{PtMe}_{2}\right]_{\mathrm{x}}$ may adopt in solution.

degree of deshielding than the bis(pentafluorophenyl)phosphinite on coordination to the platinum (with the $\delta_{\mathrm{P}}$ moving downfield by 14.0 for the phosphine and $5.8 \mathrm{ppm}$ for the phosphinite), as the more electron-donating $\mathrm{CH}_{2} \mathrm{P}^{t} \mathrm{Bu}_{2}$ group contributed more electron density to the platinum than the more electron-withdrawing $\mathrm{OP}\left(\mathrm{C}_{6} \mathrm{~F}_{5}\right)_{2}$ group did. Due to this difference in $\sigma$-basicity, it may be expected that initial coordination of the tert-butyl phosphine would be favoured over coordination of the pentafluorophenyl phosphinite, yielding a complex containing two distinct platinum centres (Figure 3.4, right). However, due to the broad nature of the phosphorus resonances (full width at half height maximum of 75 and $98 \mathrm{~Hz}$ respectively) it is difficult to determine the exact coordination environment of the metal; the cis phosphorus-phosphorus coupling expected where different P-donors are coordinated to the same metal centre is on the order of tens of Hertz in similar platinum dimethyl complexes, ${ }^{99}$ meaning that such a coupling may be present but not resolved in this instance.

On standing at room temperature, the NMR resonances of the $\left[(\mathrm{POCCPH}) \mathrm{PtMe}_{2}\right]_{\mathrm{x}}$ species were observed to broaden even further, producing a large number of unidentified signals in the ${ }^{31} \mathrm{P}$ NMR spectrum between 90 and $60 \mathrm{ppm}$. Thermolysis of the reaction mixture at $60{ }^{\circ} \mathrm{C}$ for 48 hours produced only small amounts of the desired [(POCCP)PtMe] pincer species (assigned on the agreement of ${ }^{31} \mathrm{P}$ NMR data with independently synthesised [(POCCP)PtMe], described in the Chapter 4). However, upon increasing the temperature to $85{ }^{\circ} \mathrm{C}$ for 24 hours, degradation of the platinum methyl complex along with formation of perfluorinated biphenyl was observed. As such, the thermolysis of platinum dimethyl dimers did not proceed with sufficient selectivity to provide a viable route to metallated pincer complexes.

\subsubsection{Reactions with $\left[\mathrm{PtCl}_{2}(1,5\right.$-hexadiene $\left.)\right]$}

Due to the difficulty in obtaining the metallated PCP pincer complexes of $\mathbf{1}$ from platinum(0) and platinum(II) dimethyl starting materials, reactions of ligand 1 with 
platinum(II) dichloride starting materials were investigated. Platinum chlorides represent the traditional precursors for the synthesis of pincer complexes. However, the readily available platinum chloride (itself polymeric) and $\left[\mathrm{PtCl}_{2}(\mathrm{NCR})_{2}\right]$ complexes are not ideal for pincer formation, due to a high propensity to form insoluble $\left[(\mathrm{PCPH}) \mathrm{PtCl}_{2}\right]_{\mathrm{x}}$ polymers. ${ }^{84,86}$ The use of alkene-stabilised transition metal starting materials has been observed to minimise this polymer formation. ${ }^{100}$ Platinum dichloro(1,5-hexadiene) was chosen as a starting material, as it is similar to the frequently used $\left[\mathrm{PtCl}_{2}(\mathrm{COD})\right]$, and enabled a direct comparison with the chemistry of the corresponding platinum dimethyl starting material already investigated.

Despite the previous observations that reactions between 1 and platinum precursors proceeded fastest in benzene and toluene, initial investigations into the coordination chemistry of $\mathbf{1}$ with $\left[\mathrm{PtCl}_{2}\right.$ (hex)] were undertaken in dichloromethane, as $\left[\mathrm{PtCl}_{2}\right.$ (hex)] is only sparingly soluble in non-polar solvents. Monitoring of these reactions in situ by NMR spectroscopy revealed the immediate formation of two very similar products with resonances in the ${ }^{31} \mathrm{P}$ NMR spectrum at 53.0 and $51.8 \mathrm{ppm}$, and platinum-phosphorus coupling constants of 4614 and $4503 \mathrm{~Hz}$ respectively. The observation of large ${ }^{1} J_{\mathrm{Pt}-\mathrm{P}}$ values and a ${ }^{31} \mathrm{P}$ chemical shift upfield from that of the free ligand $\left(\delta_{\mathrm{P}}=87.1 \mathrm{ppm}\right)$ indicated that the phosphorus donors were coordinated trans to the chloride (which possesses a low trans-influence). This indicated the formation of cis-[(POCOPH) $\left.\mathrm{PtCl}_{2}\right]_{\mathrm{x}}$ species (likely a dimer and a higher oligomer, as in reactions with norbornene and dimethyl precursors, Scheme 3.5). Due to the presence of broad signals in the ${ }^{1} \mathrm{H}$ NMR spectrum, further spectroscopic characterisation of these compounds was not able to be performed.

Solutions of the cis-[(POCOPH $\left.) \mathrm{PtCl}_{2}\right]_{\mathrm{x}}$ species in dichloromethane were heated at reflux (in a $60{ }^{\circ} \mathrm{C}$ oil bath) for 96 hours to investigate the formation of other stable coordination compounds in solution, and to determine whether metallation was facile at this temperature. Phosphorus-31 NMR spectroscopy revealed the very slow formation of the metallated product [(POCOP $) \mathrm{PtCl}]\left(\delta_{\mathrm{P}}=107.6 \mathrm{ppm}\right)$ over this time, albeit in only trace amounts, with cis-[(POCOPH $\left.) \mathrm{PtCl}_{2}\right]_{\mathrm{x}}$ species still comprising the major part of the reaction mixtures.

The formation of solely cis-[(POCOPH $\left.) \mathrm{PtCl}_{2}\right]_{\mathrm{x}}$ compounds was notable, as platinum dimers with bridging phosphorus donor ligands have been reported with both cis and trans coordination modes. ${ }^{101}$ This is due to a balance between antisymbiotic effects and electrostatic effects during the formation of these compounds. Antisymbiotic effects concern the trans-influence of the donor atoms, with high trans-influence donor ligands preferentially bonding trans to low trans-influence ligands, to prevent mutual destabilisation. ${ }^{102}$ While this is usually applied to $\sigma$-donors, a similar principle 

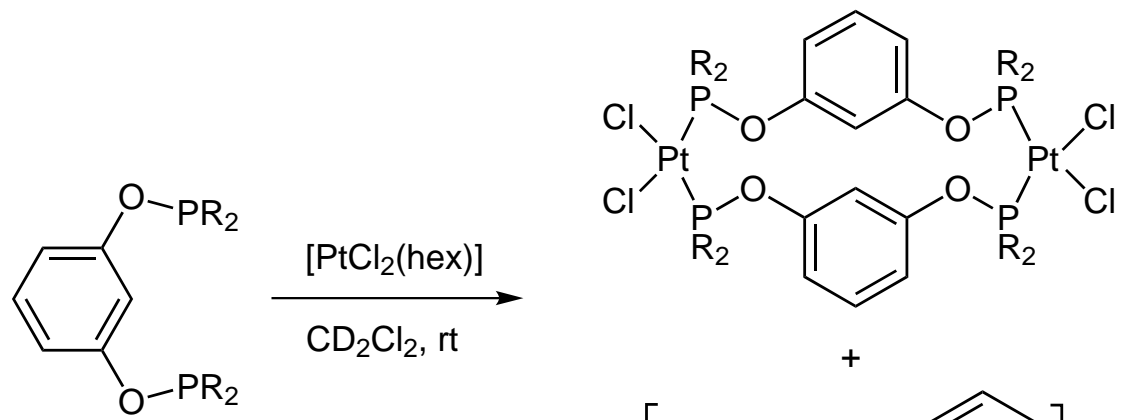

where $\mathrm{R}=\mathrm{C}_{6} \mathrm{~F}_{5}$ and $\mathrm{x}>2$

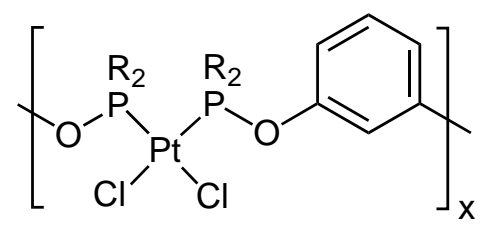

Scheme 3.5 Formation of $\left[(\mathrm{POCOPH}) \mathrm{PtCl}_{2}\right]_{\mathrm{x}}$ species from the reaction of 1 with $\left[\mathrm{PtCl}_{2}(\right.$ hex $\left.)\right]$ in dichloromethane.

applies to $\pi$-acceptors - mutually trans-coordinated $\pi$-acceptors will compete for back-donation from the same d orbital on the metal, leading to destabilisation compared to a mutually cis arrangement. Conversely, electrostatic effects dictate that an alternating arrangement of the negatively charged halide ligands and neutral phosphorus donors around the metal centre will minimise through-space electrostatic repulsion. ${ }^{103}$ Thus, a strong contribution from antisymbiotic effects usually leads to compounds with a cis geometry, whereas electrostatic effects favour the formation of trans complexes. Moreover, it is likely that the polar nature of the dichloromethane solvent favours the formation of the cis complex over the trans, as cis- $\mathrm{PtR}_{2} \mathrm{X}_{2}$ compounds will possess a net dipole, which is cancelled out in a trans arrangement of substituents. While solvent effects may play a part in determining which dimer is favoured, in other reported dimers of the type $\left[(\mathrm{PCPH}) \mathrm{PtCl}{ }_{2}\right]_{2}$, compounds containing phosphine ligands adopted trans coordination geometries, ${ }^{91,97}$ whilst compounds containing phosphinite or phosphite ligands adopted cis coordination geometries. ${ }^{92,104}$ This indicated that the $\pi$-acceptor nature of the ligand in these complexes plays a large part in determining the stereochemistry at the platinum centre, with the $\pi$-acid nature of $\mathbf{1}$ likely to account for the cis geometry of the species observed in solution.

Owing to the failure of reactions in dichloromethane to generate the metallated pincer complex in substantial quantities, the coordination chemistry of $\mathbf{1}$ with $\left[\mathrm{PtCl}_{2}(\right.$ hex $\left.)\right]$ in benzene and toluene was also investigated. The platinum starting material was only very slightly soluble in benzene- $d_{6}$, yet monitoring of reaction mixtures by NMR spectroscopy revealed quantitative formation of a new platinum complex 18 at room temperature.

The NMR spectra of compound 18 showed ${ }^{31} \mathrm{P}$ resonances at $\delta_{\mathrm{P}}=85.4$ and $51.4 \mathrm{ppm}$ 
in an approximately 1:1 ratio. The downfield signal appeared as a quintet $\left({ }^{3} J_{\mathrm{P}-\mathrm{F}}\right.$ $=36.8 \mathrm{~Hz}$ ), while the upfield signal appeared as a singlet, displaying platinumphosphorus coupling of $4531 \mathrm{~Hz}$. The observation of fluorine-phosphorus coupling in the upfield signal was indicative of $\mathbf{1 8}$ possessing an uncoordinated $\mathrm{P}\left(\mathrm{C}_{6} \mathrm{~F}_{5}\right)_{2}$ moiety: phosphorus-fluorine coupling was observed in the ${ }^{31} \mathrm{P}$ NMR spectra of all the free ligands, but was not observed upon coordination to a metal centre. The chemical shift and platinum-phosphorus coupling constant observed for the upfield ${ }^{31} \mathrm{P}$ NMR signal was very similar to that of the $\operatorname{cis}$ - $\left[(\mathrm{POCOPH}) \mathrm{PtCl}_{2}\right]_{\mathrm{x}}$ species previously observed, indicating that 18 contained both an uncoordinated phosphorus and a phosphorus coordinated trans to a chloride. Examination of the ${ }^{1} \mathrm{H}$ NMR spectrum of 18 revealed that it was the 2:1 ligand to metal complex cis- $\left[\left(\kappa^{1}-\mathrm{POCOPH}\right)_{2} \mathrm{PtCl}_{2}\right]$, as no coordinated 1,5-hexadiene was observed, excluding the possibility of the 1:1 complex $\left[\left(\kappa^{1}-\mathrm{POCOPH}\right) \mathrm{PtCl}_{2}\left(\eta^{2}-\mathrm{C}_{6} \mathrm{H}_{10}\right)\right]$. The ${ }^{1} \mathrm{H}$ NMR signals of the aromatic backbone of $\mathbf{1 8}$ were consistent with its formulation as the 2:1 complex, with the four proton environments all having discrete signals in a 1:1:1:1 ratio, as a result of the asymmetry present in the ligand. Unlike for the cis-dimers 14 and 15, the signal of H-2 for this cis-2:1 complex was not shifted upfield due to $\pi-\pi$ interactions between the ligand backbones, with the H-2 environment observed downfield of the other proton environments of the ligand. This indicated that the $\pi$-stacking of ligand backbones in the cis-dimers is only favourable when the backbones are already held in close proximity to each other, as the interactions between backbones were not maintained in absence of a second metal centre.

Upon heating reaction mixtures containing the $2: 1$ complex 18 and $\left[\mathrm{PtCl}_{2}(\right.$ hex $\left.)\right]$ to $80{ }^{\circ} \mathrm{C}$, further reaction to produce a new coordination complex, $\mathbf{1 9}$, was observed. This new species again possessed two ${ }^{31} \mathrm{P}$ NMR signals in a 1:1 ratio, with both signals displaying platinum-phosphorus coupling. The chemical shift and coupling constants of these signals $\left(\delta_{\mathrm{P}}=80.6\right.$ and $55.5 \mathrm{ppm},{ }^{1} J_{\mathrm{Pt}-\mathrm{P}}=3357$ and $4582 \mathrm{~Hz}$ respectively) showed that 19 possessed phosphorus donors coordinated both cis and trans to the chloride ligands, indicating that the free phosphinite groups of $\mathbf{1 8}$ had coordinated in a mutually trans fashion to a $\mathrm{PtCl}_{2}$ moiety, to form cis,trans$\left[(\mathrm{POCOPH}) \mathrm{PtCl}_{2}\right]_{2}$ (19). Again the ${ }^{1} \mathrm{H}$ NMR spectrum of 19 displayed four distinct aromatic proton environments in a 1:1:1:1 ratio, consistent with the formulation of 19 as a cis,trans-dimer. Notably, the ${ }^{1} \mathrm{H}$ NMR chemical shift of the H-2 proton environment in the cis,trans-dimer was downfield of the other aromatic proton environments. Similar to the $2: 1$ complex $\mathbf{1 8}$, this revealed that the $\pi-\pi$ interactions present in the cis-dimers were not present in this cis,trans-dimer 19. This demonstrated that the $\pi$-stacking of ligand backbones was dependent on both metal centres possessing a cis coordination environment; the presence of just one metal centre with trans geometry is prohibitive to the generation of $\pi-\pi$ interactions, likely due to the 
extra physical separation between ligand backbones provided by the trans metal centre.

The cis,trans-dimer 19 was more effectively synthesised by heating the reaction mixture to $80{ }^{\circ} \mathrm{C}$ for approximately two minutes shortly after suspending the reactants in solution, to ensure dissolution of the platinum starting material. Complete formation of 19 was observed by NMR spectroscopy upon standing for 12 hours at room temperature. Removal of the solvent from the NMR sample in vacuo and washing with hexane furnished a sample of $\mathbf{1 9}$ for analysis by HRMS, which confirmed the dimeric nature of 19. It was interesting to observe that during the formation of $\mathbf{1 9}$ - and even during subsequent heating of solutions of $\mathbf{1 9}$ - in no instances was the isomerisation of the cis,trans-dimer to the cis-dimer observed. This is somewhat unexpected, as the cis-dimer was observed to be the thermodynamic product in reactions performed in dichloromethane. Isomerisation from trans- $\left[(\mathrm{PP}) \mathrm{PtCl}_{2}\right]_{2}$ to cis- $\left[(\mathrm{PP}) \mathrm{PtCl}_{2}\right]_{2}$ complexes has previously been observed at temperatures as low as $-50{ }^{\circ} \mathrm{C} ;{ }^{105}$ the absence of a similar isomerisation from 19 to $\mathrm{cis}$ - $\left[(\mathrm{POCOPH}) \mathrm{PtCl} \mathrm{C}_{2}\right]_{2}$ indicated that the cis, trans configuration must be conferred a particular stability in benzene and toluene.

Solutions of 19 were subjected to thermolysis to promote metallation and the formation of the pincer complex [(POCOP)PtCl]. Heating at temperatures of $60{ }^{\circ} \mathrm{C}$ and above resulted in the very slow reaction of $\mathbf{1 9}$ to form a new platinum compound 20, which subsequently reacted to produce the desired pincer species with very good selectivity (Figure 3.5). Intermediate $\mathbf{2 0}$ displayed a single, extremely shielded resonance in the ${ }^{31} \mathrm{P}$ NMR spectrum at $\delta_{\mathrm{P}}=14.0 \mathrm{ppm}$, with a large platinum-phosphorus coupling constant of $4408 \mathrm{~Hz}$. The ${ }^{31} \mathrm{P}$ NMR data indicated the symmetric coordination of the phosphorus donors, each coordinated trans to a chloride. However, while the observed coupling was similar to the earlier reported cis- $\left[(\mathrm{POCOPH}) \mathrm{PtCl}_{2}\right]_{\mathrm{x}}$ species $\left(\delta_{\mathrm{P}} \approx 52 \mathrm{ppm},{ }^{1} J_{\mathrm{Pt}-\mathrm{P}} \approx 4500 \mathrm{~Hz}\right)$, the ${ }^{31} \mathrm{P}$ NMR resonance of 20 appeared almost $40 \mathrm{ppm}$ upfield, confirming that $\mathbf{2 0}$ could not be a previously observed cisdimer or oligomer. Unfortunately, as $\mathbf{2 0}$ was observed as a labile species during the course of a reaction (and was formed and subsequently reacted at elevated temperatures), no ${ }^{1} \mathrm{H}$ NMR signals could be definitively assigned to it.

Potential intermediates in the metallation reaction to form [(POCOP $) \mathrm{PtCl}]$ included an A-frame dimer, ${ }^{106}$ a dimer distorted by intramolecular $\mathrm{H}-\mathrm{Cl}$ interactions, ${ }^{107}$ an arenium complex, ${ }^{108}$ a cis-monomer complex, ${ }^{91}$ and a platinum(IV) complex with a facially-coordinated pincer ligand (Figure 3.6). ${ }^{43}$ However, due to the fact that 20 possessed a large phosphorus-platinum coupling of $4408 \mathrm{~Hz}$, diagnostic of a P-trans-Cl configuration, all but the cis-monomer and fac-platinum(IV) complexes 

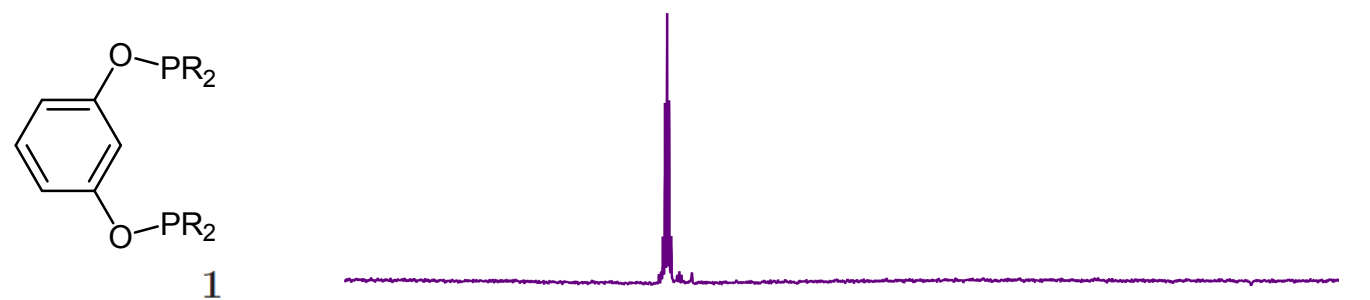<smiles>[R20]Oc1cccc(OP([R2])P([R2])(Cl)(Cl)[PH]([R2])Cl)c1</smiles>

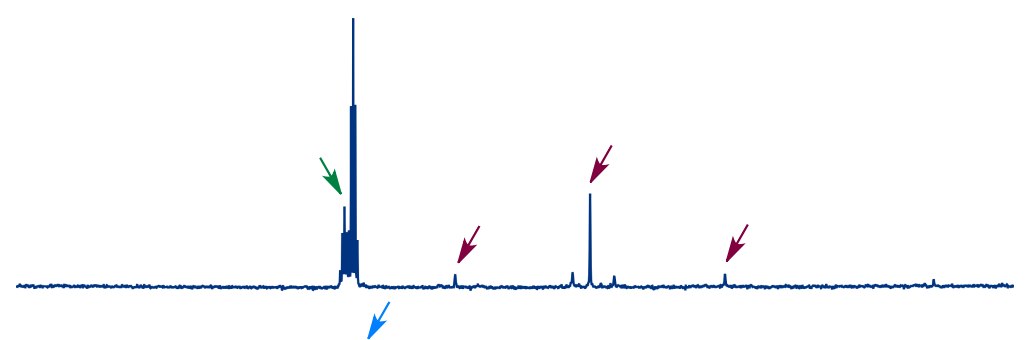<smiles>[R2]P(Cl)P([R2])(Cl)(Cl)Oc1cccc(OP([R20])P([R2])(Cl)(Cl)[PH+]([R2])Cl)c1</smiles>
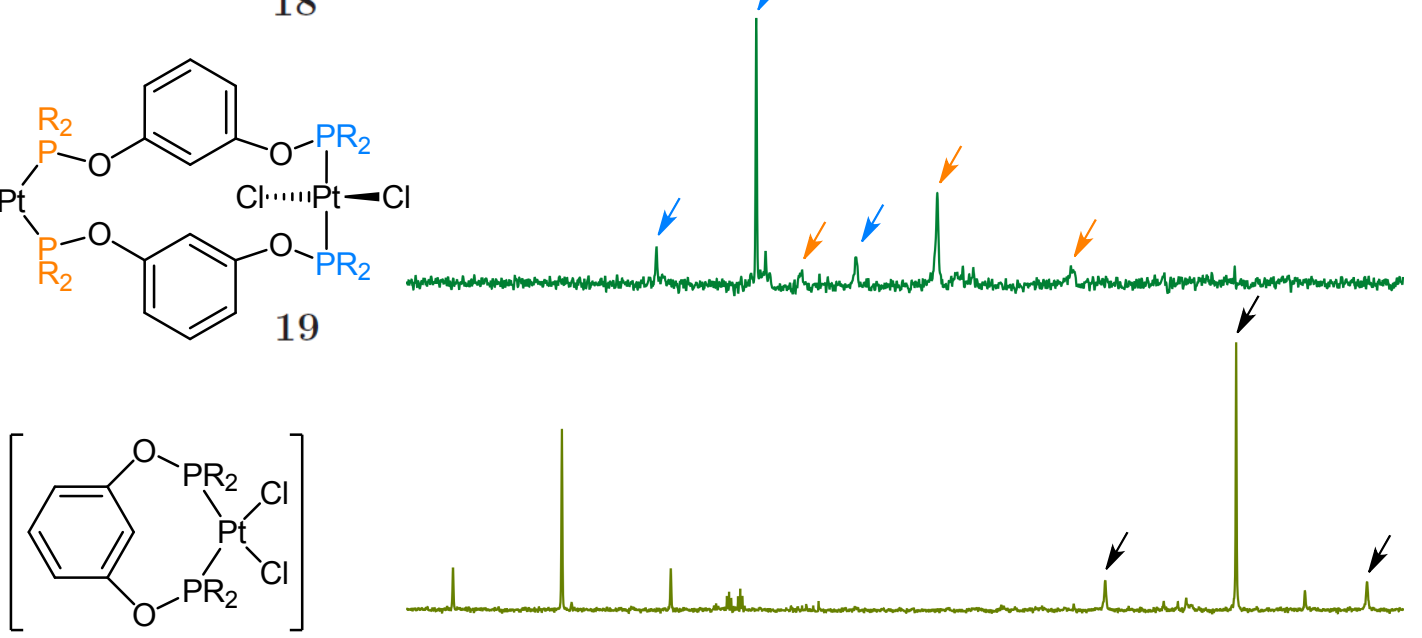

proposed intermediate 20
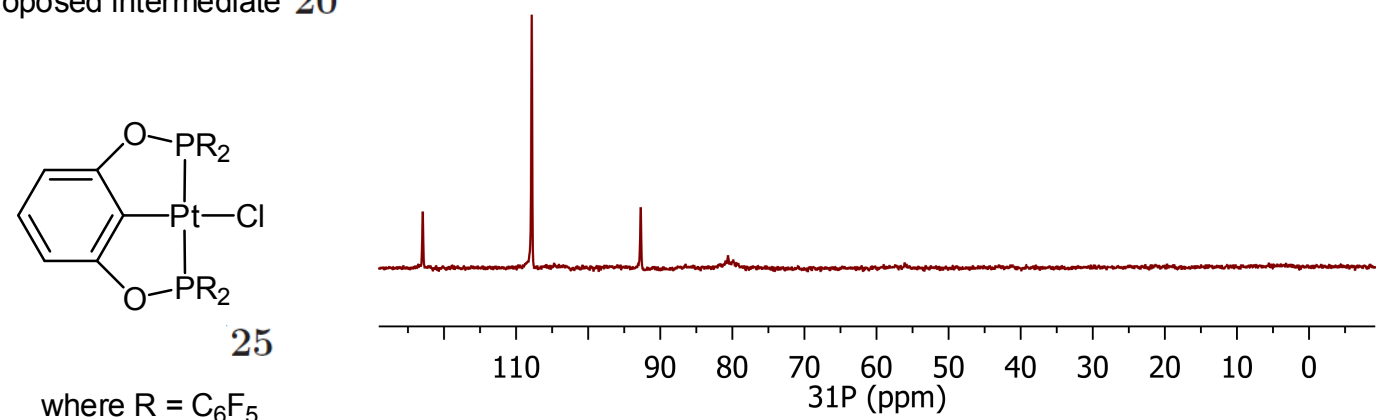

where $\mathrm{R}=\mathrm{C}_{6} \mathrm{~F}_{5}$

Figure 3.5 Stacked plot of ${ }^{31} \mathrm{P}$ NMR spectra showing the course of the metallation reaction to form $[(\mathrm{POCOP}) \mathrm{PtCl}]$ from $\left[\mathrm{PtCl}_{2}(\right.$ hex $\left.)\right]$ in toluene- $d_{8}$. 
had to be discounted as potential intermediates, as each required a mutually trans coordination of phosphorus donors. The fac-platinum(IV) species was the least likely candidate of the two remaining possibilities; no hydride signals were detected in the ${ }^{1} \mathrm{H}$ NMR spectrum of these reaction mixtures, oxidative addition of the aromatic $\mathrm{C}-\mathrm{H}$ bond should produce an intermediate with the metallated ring and hydride occupying mutually cis coordination sites (producing two inequivalent phosphorus environments), and a platinum(IV) species would not be expected to be stable over the course of hours at $60{ }^{\circ} \mathrm{C}$. This left the cis-monomer as the most likely structure for intermediate 20. This structure was consistent with the spectroscopic data. The upfield shift in the ${ }^{31} \mathrm{P}$ NMR resonance (from 80.6 and $55.5 \mathrm{ppm}$ in the cis,transdimer 19, to $14.0 \mathrm{ppm}$ in 20) was attributed to ring strain associated with the formation of a rigid, 8-membered chelate. Analogous shielding of phosphorus atoms due to ring strain has been observed for 4-membered chelate rings with cis-bidentate phosphines. ${ }^{109}$

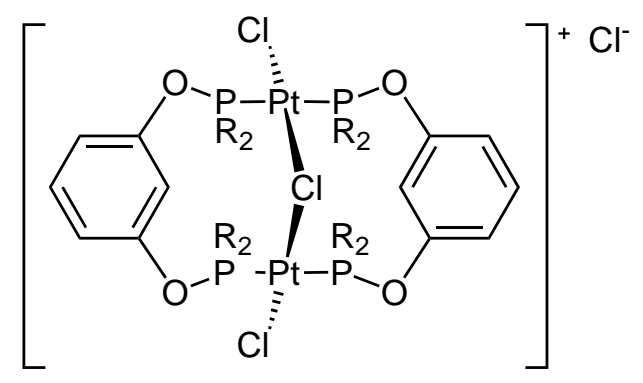

A-frame dimer

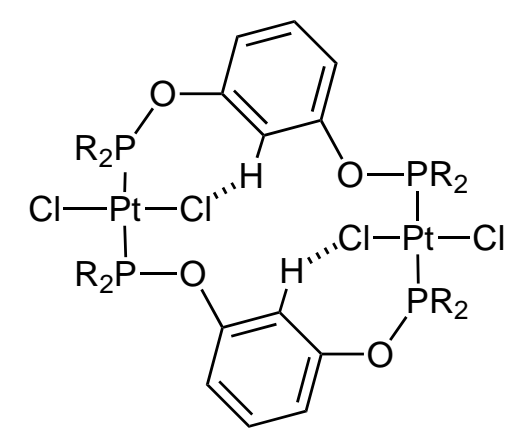

distorted dimer

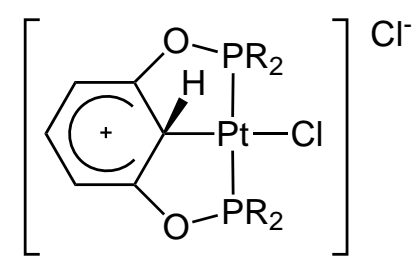

arenium complex

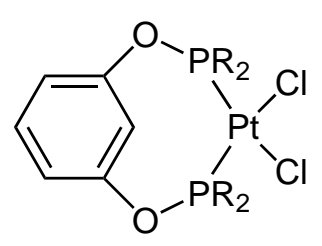

cis-monomer<smiles>[R6]Oc1cccc(OP)c1[P+]([R7])(Cl)Cl</smiles>

fac-platinum(IV)

Figure 3.6 Potential structures for the metallation intermediate 20.

Tentative assignment of a cis-monomeric structure for the metallation intermediate 20 fit with observations of pincer cyclometallation in the literature. Cis-monomeric structures have been proposed as unisolated intermediates for reactions with other PCPH ligands, ${ }^{97,110,111}$ and have been isolated in reactions of metal precursors with PCCCPMe ligands (where cyclometallation is hindered by the presence of a methyl group on C-2). ${ }^{91}$ The formulation of $\mathbf{2 0}$ as a cis-monomer was also in accordance with the behaviour of $\mathbf{2 0}$ in reaction mixtures. A cis-monomeric structure is expected to be high in energy, requiring the redistribution of the bridging ligands onto 
a single metal centre, as well as the formation of a rigid, eight-membered cis-chelate ring. Accordingly, 20 was formed slowly, and only at elevated temperatures. A cismonomeric structure is also predisposed to metallation, with $\mathrm{H}-2$ in close proximity to the metal centre, which can account for the observation that $\mathbf{2 0}$ reacted directly to produce the metallated pincer complex [(POCOP $) \mathrm{PtCl}]$, and was only observed in trace amounts at temperatures above which metallation is facile $\left(>110^{\circ} \mathrm{C}\right)$. As such, the most likely structure for $\mathbf{2 0}$ is that of a cis-monomer, making $\mathbf{2 0}$ an important intermediate observed in the formation of the $[(\mathrm{POCOP}) \mathrm{PtCl}]$ pincer species.

\subsubsection{Reactions with [PtClMe(1,5-hexadiene)]}

The coordination chemistry of $\mathbf{1}$ was also explored with $[\mathrm{PtClMe}(\mathrm{hex})]$, to examine how the presence of both a strong trans-influence (methyl) and weak transinfluence (chloride) ligand would affect the nature of the complexes formed. The replacement of a chloride ligand with a methyl group renders the $[\mathrm{PtClMe}(\mathrm{hex})]$ complex much more soluble in non-polar solvents than the corresponding dichloride complex. This increased solubility was advantageous as, for most starting materials studied, reactions in benzene occurred faster and with less oligomer formation than the corresponding reactions in dichloromethane.

The synthesis of [PtClMe(hex)] has not previously been reported, with the compound observed as a byproduct in the synthesis of $\left[\mathrm{PtMe}_{2}(\mathrm{hex})\right]$, and obtained during the purification of $\left[\mathrm{PtMe}_{2}(\mathrm{hex})\right]$ by sublimation, in yields of typically $<1 \%$. The synthesis of $[\mathrm{PtClMe}($ hex $)]$ was attempted by the addition of one equivalent of dimethylzinc to $\left[\mathrm{PtCl}_{2}(\mathrm{hex})\right]$; however, this was seen to produce the dimethyl complex $\left[\mathrm{PtMe}_{2}(\mathrm{hex})\right]$ as the major product, along with unreacted starting material. The synthesis of $[\mathrm{PtClMe}(\mathrm{hex})]$ was achieved by methylation of the dichloride precursor with an excess of dimethylzinc, then chlorination of the resultant dimethyl complex with one equivalent of acetyl chloride (in a manner similar to the synthesis of the related [PtClMe(COD)] species), ${ }^{112}$ affording the new platinum chloromethyl starting material in a modest overall yield of $46 \%$ for the two-step reaction.

Reactions between the phosphinite ligand 1 and $[\mathrm{PtClMe}($ hex $)]$ in toluene- $d_{8}$ at room temperature were seen to immediately produce a number of spectroscopically similar coordination compounds. The major species in solution displayed a resonance in the ${ }^{31} \mathrm{P}$ NMR spectrum at $\delta_{\mathrm{P}}=89.2 \mathrm{ppm}$ and displayed platinum-phosphorus coupling of $4071 \mathrm{~Hz}$. As there were no other phosphorus signals of significant intensity to be related to this signal, the observation of a single ${ }^{31} \mathrm{P}$ NMR signal shifted down- 
field from that of the free ligand $\mathbf{1}$ was indicative of the formation of a symmetric species containing mutually trans phosphorus donors; trans-[(POCOPH $) \mathrm{PtClMe}]_{2}$. Also present in some reaction mixtures of 1 and $[\mathrm{PtClMe}($ hex $)]$ was the analogous 2:1 complex, trans-[(POCOPH $\left.)_{2} \mathrm{PtClMe}\right]$, displaying ${ }^{31} \mathrm{P}$ NMR resonances for the uncoordinated phosphorus atom at $\delta_{\mathrm{P}}=87.9 \mathrm{ppm}$, possessing the characteristic quintet coupling pattern of the uncoordinated phosphinite. The ligated phosphorus atom appeared at $\delta_{\mathrm{P}}=84.8 \mathrm{ppm}$ with ${ }^{1} J_{\mathrm{Pt}-\mathrm{P}}$ of $4032 \mathrm{~Hz}$; again the ${ }^{31} \mathrm{P}$ NMR data were diagnostic of mutually trans-coordinated phosphorus donors. ${ }^{\ddagger}$ Sufficient proton NMR data for these complexes were not obtained, due to the complex nature of the reaction mixtures.

This initial coordination chemistry of ligand $\mathbf{1}$ with $[\mathrm{PtClMe}(\mathrm{hex})]$ offered some distinct contrasts to that of the same ligand with the analogous $\left[\mathrm{PtCl}_{2}(\right.$ hex $\left.)\right]$ starting material; the 2:1 dichloride complex possessed a cis geometry, while the 2:1 chloromethyl complex adopted a trans geometry. This difference in coordination geometry can be attributed to antisymbiotic effects; the strong trans-influence of the methyl group dictates that it preferentially sits trans to the ligand with the lowest trans-influence, chloride. The stabilisation imparted by this Me-trans-Cl coordination must be sufficient that it can overcome the unfavourable interactions generated upon P-trans-P coordination (which was not observed for the platinum dichloride complexes), whereby the phosphinite ligands compete for backdonation of electron density from the same metal d-orbital.

The energetics of this system must be very finely balanced; on standing at room temperature trans-[(POCOPH $\left.)_{2} \mathrm{PtClMe}\right]$ was seen to slowly isomerise to form the new cis,trans-dimer, cis,trans- $[(\mathrm{POCOPH}) \mathrm{PtClMe}]_{2}(\mathbf{2 1})$ (Scheme 3.6). This isomerisation could be facilitated by gentle heating, with compound $\mathbf{2 1}$ constituting greater than $80 \%$ of the reaction mixture (by ${ }^{31} \mathrm{P}$ NMR spectroscopy) after heating solutions of 1 and [PtClMe(hex)] at $40{ }^{\circ} \mathrm{C}$ overnight. This cis,trans-isomer could then be isolated in spectroscopically pure state by passage of the reaction mixture through a short column of neutral alumina in air (allowing any of the trans-2:1 compound to be oxidised and removed from solution), followed by removal of the solvent in vacuo and washing with cold pentane. High Resolution Mass Spectrometry confirmed the dimeric nature of $\mathbf{2 1}$, with species from the loss of a chloride ligand with and without coordinated acetonitrile (at $m / z=2171$ and 2130 amu respectively) being the only ions detected.

\footnotetext{
${ }^{\ddagger}$ For comparison, the trans-coordinated phosphorus donor in the platinum dichloride cis,transdimer 19 appeared at $\delta_{\mathrm{P}}=80.6 \mathrm{ppm}$, with a ${ }^{1} J_{\mathrm{Pt}-\mathrm{P}}=3357 \mathrm{~Hz}$. It was observed throughout this work that platinum chloromethyl complexes displayed platinum-phosphorus coupling constants about $20 \%$ larger than the analogous platinum dichloride complexes.
} 


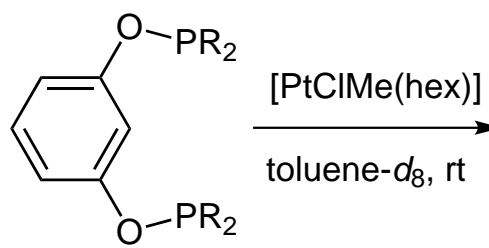

1

where $\mathrm{R}=\mathrm{C}_{6} \mathrm{~F}_{5}$ and
$\mathrm{R}^{\prime}=\mathrm{C}_{6} \mathrm{H}_{4} \mathrm{OP}\left(\mathrm{C}_{6} \mathrm{~F}_{5}\right)_{2}$

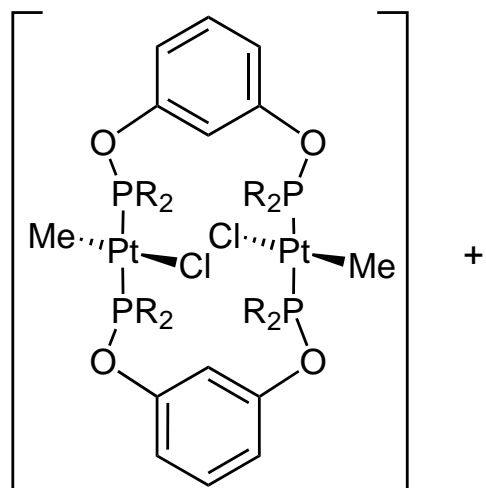<smiles>[R]OP(C)(Cl)([R6]P)O[2H]</smiles>

Scheme 3.6 Formation of cis,trans-[(POCOPH $)$ PtClMe $]_{2}$ species (21) with the trans- $\left[(\mathrm{POCOPH})_{2} \mathrm{PtClMe}\right]$ minor product.

Characterisation of $\mathbf{2 1}$ by ${ }^{31} \mathrm{P}$ NMR spectroscopy revealed three phosphorus environments in a 1:2:1 ratio. The most upfield and most downfield of signals corresponded to one phosphorus donor group each, and displayed chemical shifts and platinumphosphorus coupling constants consistent with phosphorus coordinated trans to a methyl $\left(\delta_{\mathrm{P}}=94.4 \mathrm{ppm},{ }^{1} J_{\mathrm{Pt}-\mathrm{P}}=2066 \mathrm{~Hz}\right)$ and phosphorus coordinated trans to a chloride $\left(\delta_{\mathrm{P}}=62.1 \mathrm{ppm},{ }^{1} J_{\mathrm{Pt}-\mathrm{P}}=5494 \mathrm{~Hz}\right)$. Each of these peaks was observed to be split slightly, due to the weak cis coupling between the two phosphorus nuclei $\left({ }^{2} J_{\mathrm{P}-\mathrm{P}}=15.4 \mathrm{~Hz}\right)$. The most intense phosphorus resonance corresponded to two phosphorus donors, and possessed resonances in the ${ }^{31} \mathrm{P}$ NMR spectrum very similar to that of the trans $2: 1$ complex $\left(\delta_{\mathrm{P}}=90.3 \mathrm{ppm},{ }^{1} J_{\mathrm{Pt}-\mathrm{P}}=4028 \mathrm{~Hz}\right)$, marking this signal as having arisen from the mutually trans-coordinated phosphines. A notable feature of the ${ }^{31} \mathrm{P}$ NMR data of $\mathbf{2 1}$ is that the platinum-phosphorus coupling constants for both the P-trans-P and P-trans-Cl environments were exactly $20 \%$ larger than the analogous coupling constants for the dichloride cis,trans-dimer, indicating that the replacement of a chloride for a methyl at each platinum centre produced a stronger platinum-phosphorus interaction. Proton and phosphorus-31 NMR spectra were also obtained at $50{ }^{\circ} \mathrm{C}$ to confirm that the observed signals were arising from a single, asymmetric dimer rather than a number of dimers in equilibrium. The ratio of ${ }^{1} \mathrm{H}$ and ${ }^{31} \mathrm{P}$ NMR signals did not change (or even broaden substantially) from $20{ }^{\circ} \mathrm{C}$ to $50{ }^{\circ} \mathrm{C}$, confirming that $\mathbf{2 1}$ is a cis,trans-dimer. 


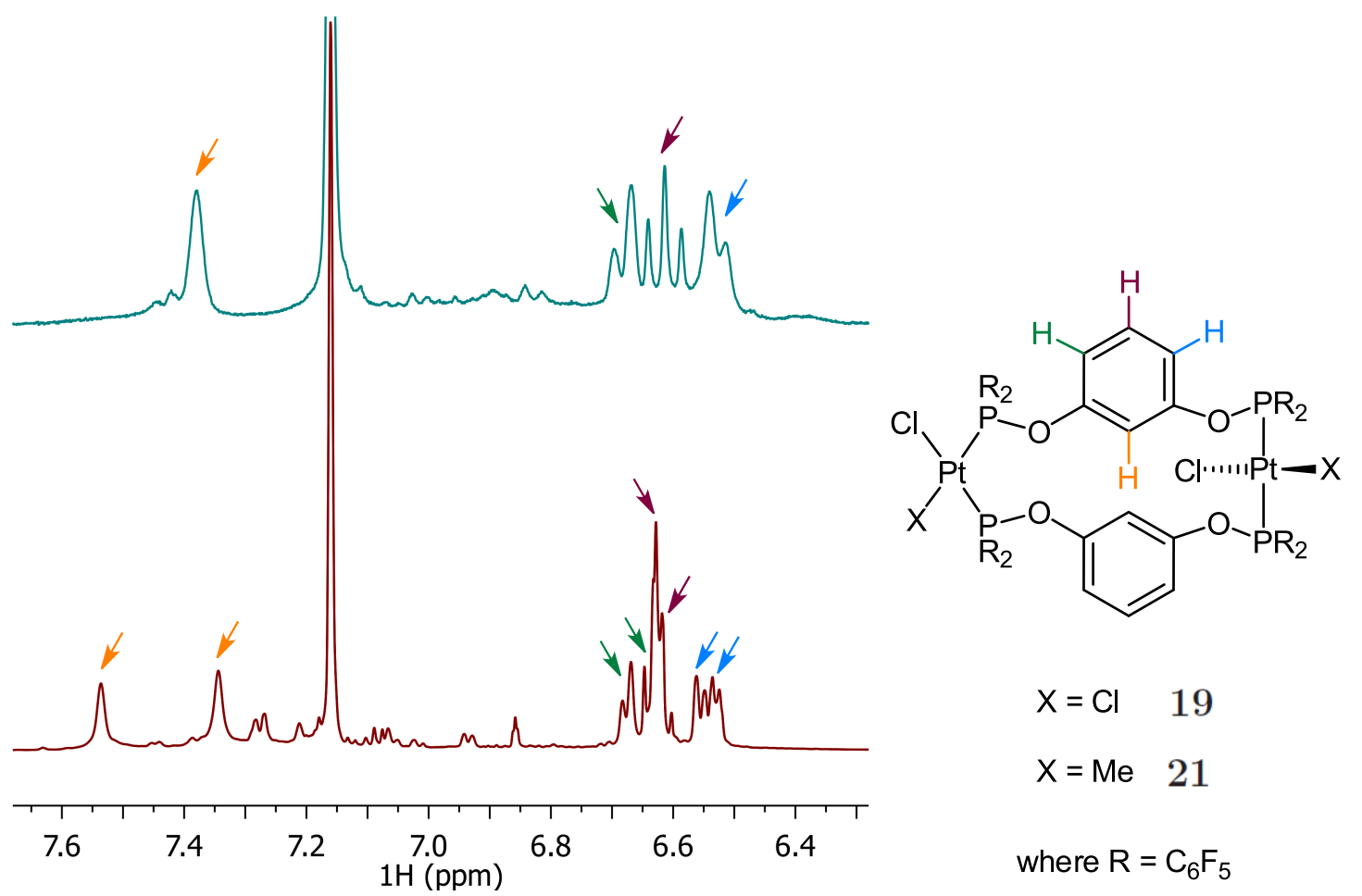

Figure 3.7 Stacked plot of ${ }^{1} \mathrm{H}$ NMR spectra of cis, trans- $\left[(\mathrm{POCOPH}) \mathrm{PtCl}_{2}\right]_{2}(\mathbf{1 9}$, top) and cis,trans-[(POCOPH $) \mathrm{PtClMe}]_{2}(\mathbf{2 1}$, bottom $)$ in benzene- $d_{6}$, demonstrating the spectroscopic similarities between the dimers.

Despite the large chemical shift difference between the two cis-phosphorus atoms in the ${ }^{31} \mathrm{P}$ NMR spectrum of $\mathbf{2 1}$ (approximately $32 \mathrm{ppm}$ ), the aromatic ligand backbones only experienced very small changes in ${ }^{13} \mathrm{C}$ NMR chemical shift (on average, equivalent carbon environments on each ring differed by $0.3 \mathrm{ppm}$ ), which indicated that this asymmetric coordination mode did not result in the polarisation of one ring over the other. The ${ }^{1} \mathrm{H}$ NMR spectrum of $\mathbf{2 1}$ bore a striking similarity to the characteristic ${ }^{1} \mathrm{H}$ NMR spectrum of the corresponding cis,trans-dichloride complex 19, in which the broader H-4,6 signals flanked a sharp H-5 signal, with H-2 shifted slightly downfield (Figure 3.7). This served to demonstrate the spectroscopic similarities between 19 and 21. The use of ${ }^{1} \mathrm{H}-{ }^{31} \mathrm{P}$ Heteronuclear Multiple Bond Correlation (HMBC) NMR experiments proved to be a very useful tool for the full assignment of the spectroscopic data for $\mathbf{2 1}$. As the bis(pentafluorophenyl)phosphine donor groups did not possess any protons and did not display large chemical shift variations of the pentafluorophenyl carbon environments, conventional ${ }^{1} \mathrm{H}-{ }^{13} \mathrm{C}$ HMBC experiments were of little use in establishing the connectivity of both the aromatic ligand backbones and the methyl groups to either the cis- or the trans-coordinated metal centre. Moreover, no ${ }^{1} \mathrm{H}-{ }^{13} \mathrm{C}$ HMBC correlations between the ligand backbone and methyl groups were observed, due to the prohibitively long distance over which the coupling was to occur. However, through the use of ${ }^{1} \mathrm{H}-{ }^{1} \mathrm{H}$ and ${ }^{1} \mathrm{H}^{-13} \mathrm{C} 2 \mathrm{D}$ NMR experiments to fully assign each aromatic ligand backbone, the connectivity between each backbone and the four phosphorus donors was established with ${ }^{1} \mathrm{H}^{-31} \mathrm{P}$ HMBC 
spectroscopy. Similarly, the observation of strong coupling between the protons of each platinum-methyl group and either the mutually cis or the mutually trans phosphorus atoms via ${ }^{1} \mathrm{H}-{ }^{31} \mathrm{P}$ HMBC spectroscopy readily enabled the spectroscopic assignment of each methyl group to a metal centre. Although ${ }^{1} \mathrm{H}-{ }^{31} \mathrm{P}$ HMBC spectroscopy is not a widely used tool in organometallic chemistry, it proved invaluable in the assignment of spectroscopic data for the asymmetric cis,trans-dimer 21.

As for the previous dimeric complexes investigated, 21 was subjected to thermolysis in toluene- $d_{8}$ to examine how facile the metallation reaction was, and also to probe the nature of any intermediates observed. The reaction to form the metallated $[(\mathrm{POCOP}) \mathrm{PtCl}]$ pincer species proceeded faster from the chloromethyl dimer 21 than from the platinum dichloride dimer 19, and without detectable amounts of any intermediate species (Figure 3.8).

The absence of an intermediate observed in the metallation of dimer $\mathbf{2 1}$ is consistent with the metallation reaction requiring rearrangement to a cis-monomeric species before $\mathrm{C}-\mathrm{H}$ activation and pincer complex formation can occur, as was previously indicated during the thermolysis of 19. Due to the platinum-phosphorus interactions being stronger in $\mathbf{2 1}$ than in $\mathbf{1 9}$ (as evidenced by the magnitude of platinum-phosphorus coupling constants), it may be expected that the energy barrier for the rearrangement from the dimer to the monomer will greater for the chloromethyl dimer 21 than for the dichloride dimer 19. However, $\mathrm{C}-\mathrm{H}$ activation of a chloromethyl cis-monomer will be more facile than for a dichloride cis-monomer, as the elimination of methane is more favourable than the elimination of $\mathrm{HCl}$ (discussed in more detail in Chapter 4). Compared to 19, this combination of a higher energy barrier for intermediate formation and a lower energy barrier for metallation of $\mathbf{2 1}$ predictably results in a much shorter-lived intermediate for the metallation of the chloromethyl dimer $\mathbf{2 1}$, and a cis-monomeric intermediate is not detected by NMR spectroscopy.

\subsubsection{Reactions with $\left[\mathrm{PdCl}_{2}(\mathrm{NCMe})_{2}\right]$}

The investigation of reactions between the POCOPH ligand 1 and platinum starting materials revealed the propensity of this phosphinite ligand to form a large number of dimeric and oligomeric structures, which possessed both cis and trans coordination geometries. The advantage of investigating this chemistry with platinum complexes was that the magnitude of the platinum-phosphorus coupling revealed information about the nature of the ligand coordinated trans to the phosphorus-donor, and so 

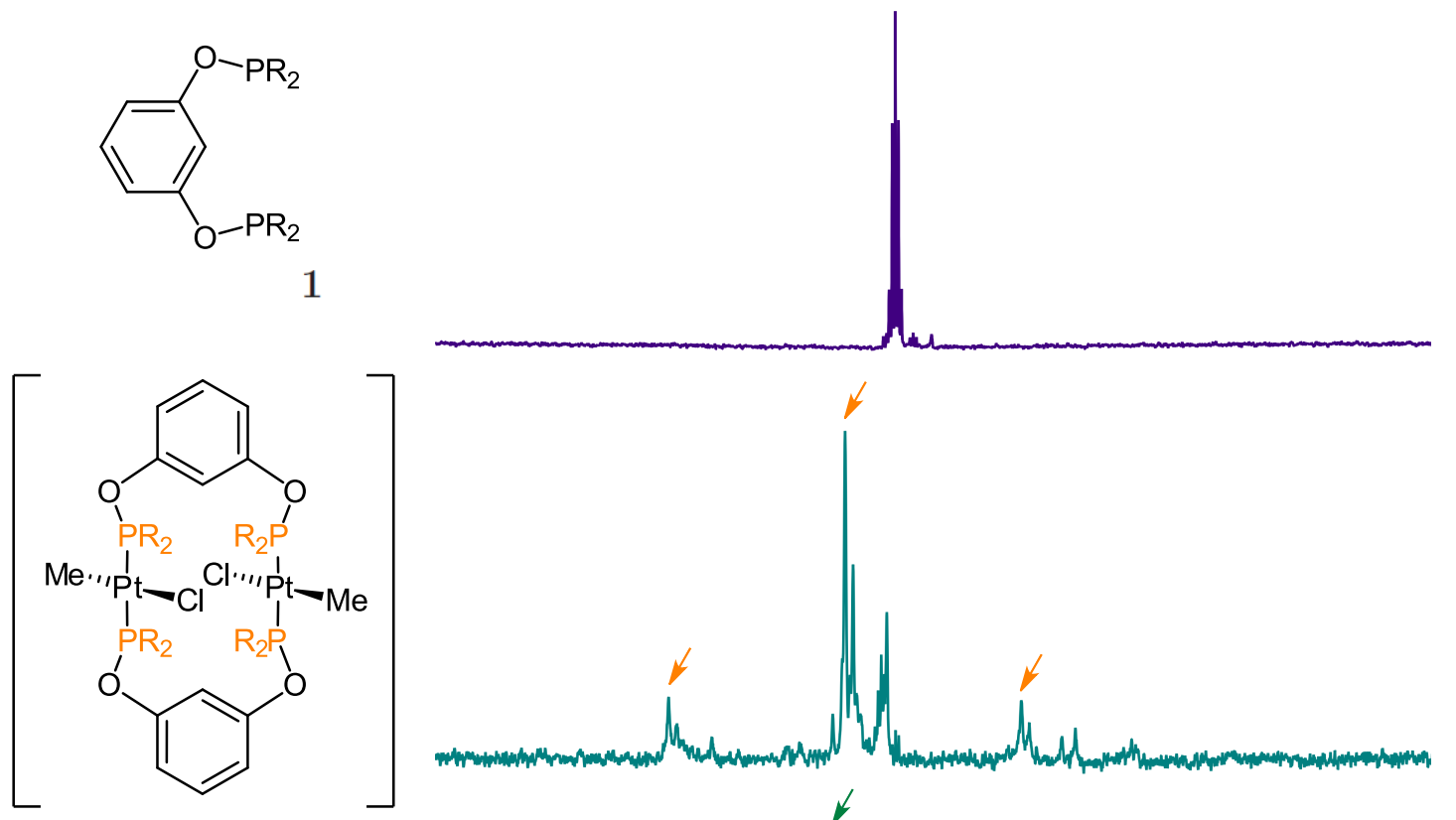

transient intermediate

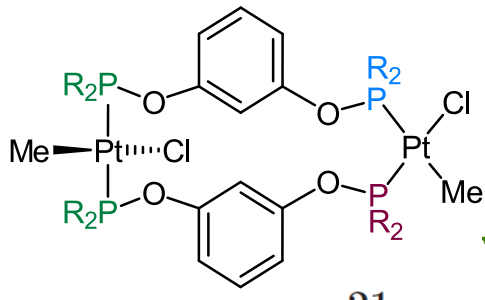

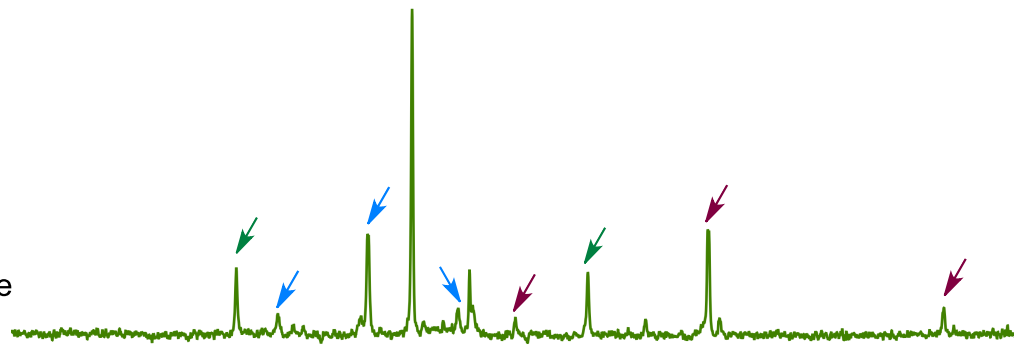

21
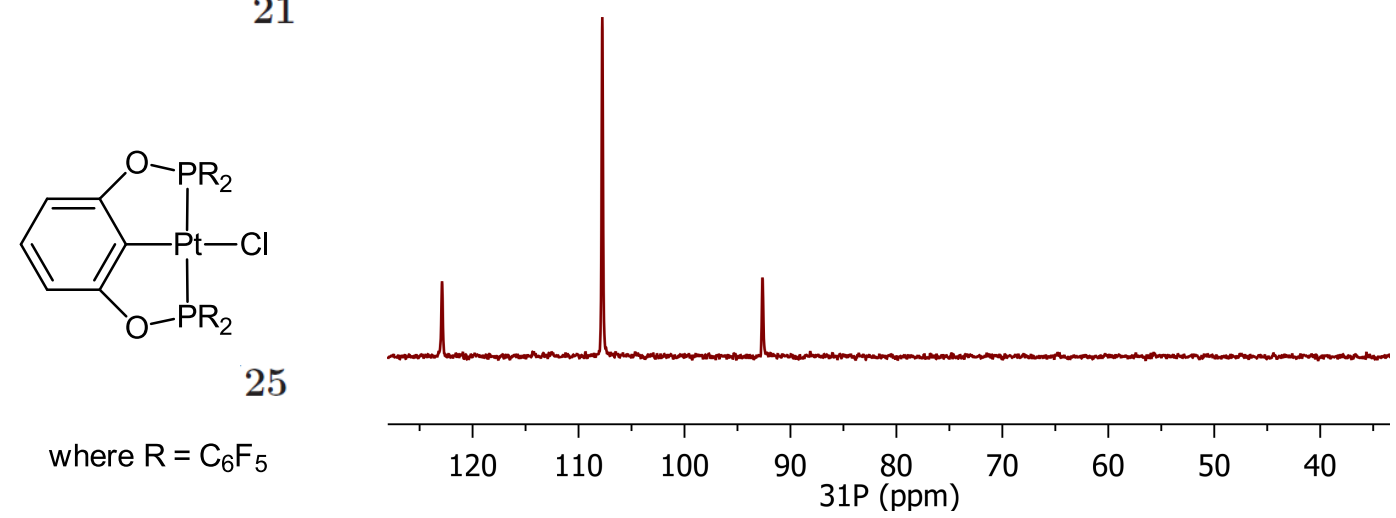

Figure 3.8 Stacked plot of ${ }^{31} \mathrm{P}$ NMR spectra showing the course of the metallation reaction to form $[(\mathrm{POCOP}) \mathrm{PtCl}]$ from $[\mathrm{PtClMe}($ hex $)]$ in toluene- $d_{8}$. 
provided a crucial tool in distinguishing between cis and trans configurations. With the spectroscopic data for the cis-, trans-, and cis,trans-dimers established using platinum chemistry, this spectroscopic data could be employed to help elucidate the structure of products formed in analogous reactions with palladium.

Reactions between 1 and $\left[\mathrm{PdCl}_{2}(\mathrm{NCMe})_{2}\right]$ were initially performed in benzene- $d_{6}$, as this solvent had produced the most interesting results with platinum precursors. Analysis of the reaction mixtures by ${ }^{31} \mathrm{P}$ NMR spectroscopy indicated the initial formation of the symmetric complex 22, which possessed one phosphorus environment in the ${ }^{31} \mathrm{P}$ NMR spectrum, at $\delta_{\mathrm{P}}=88.3 \mathrm{ppm}$. This was likely to be trans- $\left[(\mathrm{POCOPH}) \mathrm{PdCl}_{2}\right]_{2}$ owing to the phosphorus chemical shift slightly downfield of the free ligand (mutually trans phosphorus coordination was observed to appear at chemical shifts close to that of the free ligand in the ${ }^{31} \mathrm{P}$ NMR spectra of platinum complexes, whereas phosphorus coordination trans to a chloride produced a significant upfield shift of this phosphorus resonance). However, this complex possessed broad signals in the ${ }^{1} \mathrm{H}$ NMR spectrum and was seen to react further to give a poorly soluble yellow precipitate, hindering the characterisation of species in these reaction mixtures.

The reactions were repeated in dichloromethane- $d_{2}$ to increase the solubility of the products in solution. NMR spectroscopy revealed the immediate formation of the previously observed trans-dimer $\mathbf{2 2}$, which was observed to isomerise into the corresponding cis,trans-dimer (23) over the course of about an hour at room temperature (Scheme 3.7). This product displayed signals in the ${ }^{31} \mathrm{P}$ NMR spectrum in a 1:1 ratio at 88.7 and $78.5 \mathrm{ppm}$ (for the $\mathrm{P}$-trans-P and $\mathrm{P}$-trans-Cl environments respectively), shifted downfield from similar environments in the platinum analogue 19, as the less electron-rich palladium did not shield the phosphinite ligands to the extent that platinum did. The ${ }^{31} \mathrm{P}$ NMR resonance of the phosphorus nuclei attached to the cis-coordinated palladium centre also appeared slightly broader than those on the trans-coordinated palladium, an effect also seen in the spectra of the analogous platinum complex 19. The proton NMR spectrum of 23 also resembles that of the platinum cis,trans-dimer 19: the chemical shifts and lineshapes for the resonances of protons H-2, H-4, and H-6 are similar, but the H-5 signal for the palladium complex appears approximately $0.5 \mathrm{ppm}$ upfield of that in the platinum complex. It is not immediately clear why this may be; however, it may indicate that these similar species adopt slightly different conformations in solution.

It was found that $\mathbf{2 3}$ could be readily obtained from dichloromethane solutions of $\mathbf{1}$ and $\left[\mathrm{PdCl}_{2}(\mathrm{NCMe})_{2}\right]$ heated at reflux overnight. The dimeric nature of this cis, trans species was confirmed by HRMS analysis, detected as the $[\mathrm{M}-\mathrm{Cl}]^{+}$ion, as $m / z=$ 


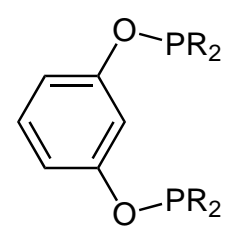

$\left[\mathrm{PdCl}_{2}(\mathrm{NCMe})_{2}\right]$

$\mathrm{CH}_{2} \mathrm{Cl}_{2}, \mathrm{rt}$

where $\mathrm{R}=\mathrm{C}_{6} \mathrm{~F}_{5}$

1

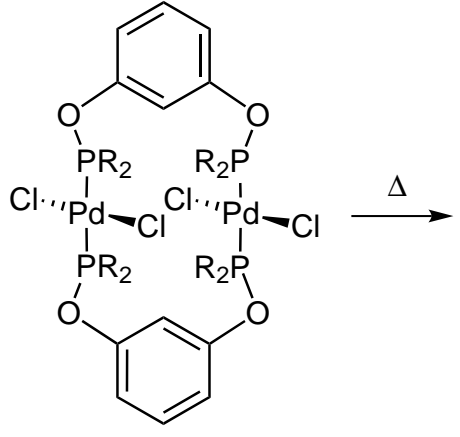

22

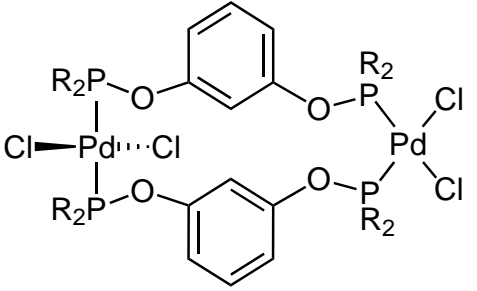

23
$83 \%$

Scheme 3.7 Formation of cis,trans- $\left[(\mathrm{POCOPH}) \mathrm{PdCl}_{2}\right]_{2}$ species (23) via the trans-[(POCOPH $\left.) \mathrm{PdCl}_{2}\right]_{2}$ intermediate $(\mathbf{2 2})$.

1995 amu. This also confirmed that the initial reaction product 22 was likely to be a dimer (rather than a higher oligomer), due to its ready isomerisation to $\mathbf{2 3}$ and its solubility in benzene. On continued reflux in dichloromethane, 23 was not seen to react any further; however, upon heating above $100{ }^{\circ} \mathrm{C}$ in toluene quantitative conversion to the metallated $[(\mathrm{POCOP}) \mathrm{PdCl}]$ pincer species was observed. During this metallation reaction a transient intermediate was observed by ${ }^{31} \mathrm{P}$ NMR spectroscopy as a single resonance at $\delta_{\mathrm{P}}=45.6 \mathrm{ppm}$; while this chemical shift was suitably downfield of the signals corresponding to 23 , insufficient data were gathered on this species to speculate as to whether this may indicate the presence of a cis-monomeric complex as a metallation intermediate.

The initial formation of the palladium trans-dimer 22 in dichloromethane, whereby under identical conditions for platinum a cis-dimer is favoured, is likely to be due to electrostatic effects. The greater ionic character of metal-ligand bonding on palladium serves to enhance the electrostatic repulsion between mutually cis chloride ligands, destabilising the cis geometry favoured by antisymbiotic and solvent effects. This also serves to account for the formation of a trans-dimer in the absence of strongly trans directing ligands. The preference of platinum and palladium dimers of the type $\left[(\mathrm{POCOPH}) \mathrm{MCl}_{2}\right]_{2}$ to adopt a stable cis, trans configuration is interesting, especially considering that the three examples of cis,trans dimers $(\mathbf{1 9}, \mathbf{2 1}$, and 23) represent complexes both with and without strongly trans directing ligands, and rearrange to form these asymmetric dimers from the initial formation of both cis and trans complexes. For coordination complexes of the phosphinite ligand $\mathbf{1}$, the cis,trans-dimeric structure must offer a balance between antisymbiotic and electrostatic effects that is energetically favourable for all compounds, despite their differences. 


\subsubsection{Literature Precedent for cis,trans-Dimers}

Dimeric compounds containing two metal centres with identical donor groups but different coordination geometry are extremely rare in the literature. To date, only six examples of such compounds have been published, ${ }^{113-116}$ falling into three different classes (Figure 3.9). The most remarkable feature of these structures is that they do not bear any striking similarities to one another. Nitrogen, phosphorus, arsenic, and antimony donor ligands are all represented, linked by backbones of one, three, or eleven atoms in length.

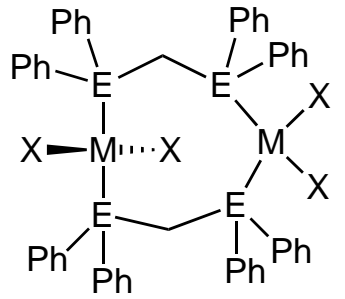

$$
\begin{aligned}
& E=S b, A s \\
& M=P t, P d \\
& X=B r, C l
\end{aligned}
$$

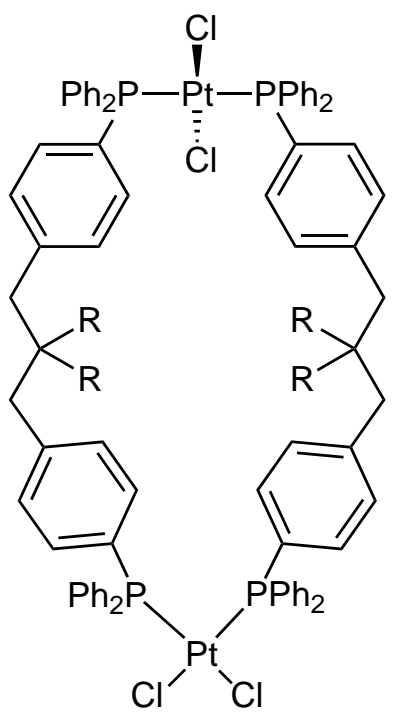

$\mathrm{R}=\mathrm{P}(\mathrm{O})(\mathrm{OEt})_{2}$

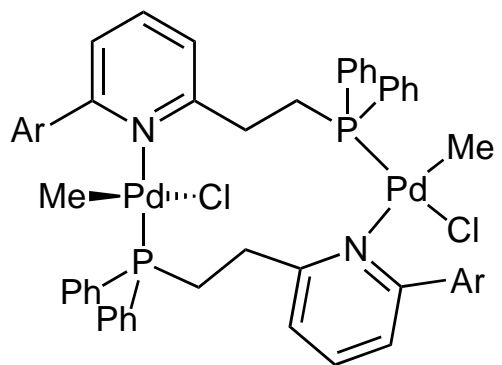

$\mathrm{Ar}=\mathrm{Ph}$, Naphth

Figure 3.9 Previously reported cis,trans-dimers.

All of the reported cis,trans-dimers contain aromatic substituents on donor atoms, and all contain at least one halide on each metal centre. As aryl substitution is seen to decrease the $\sigma$-donor ability of pnictogens, ${ }^{38}$ this may indicate that the formation of cis,trans-dimers is favoured by, or at least accessible to, poorly $\sigma$-donating ligands. This is likely due to the rearrangement processes required for the formation of these complexes; while the in situ formation of previously reported cis,trans-dimers has not been investigated, this work indicates that they are formed through the rearrangement of symmetric isomers in solution (as observed in the formation of the platinum chloromethyl cis,trans-dimer 21 and the palladium dichloro cis,transdimer 23). During such a rearrangement a pnictogen ligand will have to migrate between orbitals on the platinum, with the energy barrier to this migration being smaller for poorer $\sigma$-donor ligands, due to their weaker interactions with the metal centre. 
The steric influence exerted by the ligands will also play a role in enabling the formation of cis,trans-dimers. The effect of sterics was observed in the formation of dimeric species with bridging pyridine-phosphine ligands: a cis,trans coordination geometry was favoured for phenyl and 1-naphthyl substituents on the pyridyl ring, while a trans,trans coordination geometry was favoured for ligands with the bulkier 9-anthracyl substituent. ${ }^{115}$ Therefore it is plausible that for a cis, trans dimeric structure to be favoured, there needs to be a fine balance of steric and electronic factors at work, that serve to destabilise the cis-dimer to the point where isomerisation to the trans-dimer is almost favoured, with cis,trans coordination providing a thermodynamically stable trade-off between the two conventional dimeric forms. It is possible that such an effect is observed in this work; the compound $\left[(\mathrm{POCOPH}) \mathrm{PtCl}_{2}\right]_{2}$ adopted a thermodynamically stable cis-dimeric structure in dichloromethane, but formed an equally stable cis,trans-dimer in toluene, due to the absence of the polar solvent's stabilisation of cis coordination modes. While cis,trans-dimer formation appears to require a fine balance between steric and electronic effects, along with the ability to readily isomerise between coordination geometries, it is not clear how this unusual coordination geometry lowers the total energy of the system to an extent where the cis,trans-dimer is favoured as the thermodynamic product.

\subsection{Concluding Remarks}

The coordination chemistry of group 10 metals with the phosphinite donor ligand 1 was typified by the formation of a range of dimeric species, displaying cis, trans, and the unusual cis,trans coordination geometries (Table 3.3). This was primarily due to the electron-poor nature of $\mathbf{1}$ hindering metallation (discussed further in the Chapter 4), and allowing for coordination compounds and metallation intermediates to be more readily studied than with similar electron-rich ligands.

The coordination chemistry of $\mathrm{Pt}(0)$-alkene species with 1 was typified by the formation of oligomeric compounds of the type cis-[(POCOPH)Pt $\left(\eta^{2}\right.$-alkene $\left.)\right]_{\mathrm{x}}$, with poor solubilities and unknown molecular weights. However, reaction between $\left[\mathrm{Pt}(\mathrm{nb})_{3}\right]$ and 1 afforded the dimeric species 14, the first crystallographically characterised example of a platinum-alkene complex containing a pentafluorophenyl phosphine group. The solid state structure of $\mathbf{1 4}$ revealed a weakly bound norbornene group - due to the electron-poor metal centre being unable to participate adequately in $\pi$-backbonding — and also a stabilising "parallel displaced" $\pi$ - $\pi$ interaction between the aromatic ligand backbones. Attempts at generating platinum(II) pincer species 
Table $3.3 \quad{ }^{31} \mathrm{P}$ NMR data of dimeric compounds containing ligand $\mathbf{1}$.

\begin{tabular}{|c|c|c|c|c|}
\hline Formula & Compound & Coordination & $\delta_{\mathrm{P}}(\mathrm{ppm})$ & ${ }^{1} J_{\mathrm{Pt}-\mathrm{P}}(\mathrm{Hz})$ \\
\hline cis-[(POCOPH $\left.) \mathrm{Pt}(\mathrm{nb})_{2}\right]_{2}$ & 14 & cis & 100.5 & 4623 \\
\hline cis- $\left[(\mathrm{POCOPH}) \mathrm{PtMe}_{2}\right]_{2}$ & 15 & cis & 89.9 & 2202 \\
\hline \multirow[t]{2}{*}{ cis- $\left[\left(\kappa^{1}-\mathrm{POCOPH}\right)_{2} \mathrm{PtCl}_{2}\right]$} & 18 & uncoordinated & 85.4 & - \\
\hline & & cis & 51.4 & 4531 \\
\hline \multirow[t]{2}{*}{ cis,trans $-\left[(\mathrm{POCOPH}) \mathrm{PtCl}_{2}\right]_{2}$} & 19 & trans & 80.6 & 3357 \\
\hline & & cis & 55.5 & 4582 \\
\hline \multirow[t]{3}{*}{ cis,trans- $[(\mathrm{POCOPH}) \mathrm{PtClMe}]_{2}$} & 21 & cis $($ trans-Me) & 94.4 & 2066 \\
\hline & & trans & 90.3 & 4028 \\
\hline & & cis $($ trans $-\mathrm{Cl})$ & 62.1 & 5494 \\
\hline \multirow{3}{*}{ cis,trans $-\left[(\mathrm{POCOPH}) \mathrm{PdCl}_{2}\right]_{2}$} & 22 & trans & 88.3 & - \\
\hline & 23 & trans & 88.7 & - \\
\hline & & cis & 78.5 & - \\
\hline
\end{tabular}

through metallation of the ligand backbone were unsuccessful, regardless of whether the metallation was attempted under acidic, basic, or thermal conditions.

The coordination chemistry of $\mathbf{1}$ with $\left[\mathrm{PtMe}_{2}(\mathrm{hex})\right]$ was found be similar to the coordination chemistry of $\mathbf{1}$ with platinum(0), forming the $\kappa^{2}$-PP bridged dimer cis-[(POCOPH $\left.) \mathrm{PtMe}_{2}\right]_{2}$ at room temperature, along with small amounts of a higher oligomer, cis-[(POCOPH $\left.) \mathrm{PtMe}_{2}\right]_{\mathrm{x}}$. Traces of the metallated pincer species [(POCOP)PtMe] were detected during thermolysis of solutions of $\mathbf{1 5}$ and 16, but these reactions lacked selectivity and were prone to decomposition. Monitoring of the reactions by NMR spectroscopy suggested that a new dimer, cis,trans- $\left[(\mathrm{POCOPH}) \mathrm{PtMe}_{2}\right]_{2}$ (17) may be formed as an intermediate in the metallation reaction. Analogous reactions with the more electron-donating POCCPH ligand $\mathbf{3}$ allowed the metallated pincer complex to be formed at a lower reaction temperature, although decomposition and a lack of selectivity was still observed. Therefore, for pincer ligands containing the electron-withdrawing bis(pentafluorophenyl)phosphinite functionality, $\left[\mathrm{PtMe}_{2}(\mathrm{hex})\right]$ did not represent a viable starting material for the formation of the metallated [(PCP)PtMe] pincer complex.

Reactions between 1 and platinum chloride starting materials proved to be better suited for the synthesis of pincer complexes, with the metallated products formed selectively upon prolonged heating of reaction mixtures. The coordination chemistry between platinum chloride starting materials and ligand $\mathbf{1}$ at room temperature proved to be especially interesting, with the unexpected formation of cis,transdimers 19 and $\mathbf{2 1}$ for both the dichloride and chloromethyl platinum complexes. Spectroscopic investigation into the formation of these compounds revealed that each had an initial preference for opposing coordination geometries before rearrangement into the cis,trans-dimer; the dichloride starting material formed the cis-monomeric 
compound 18, while the chloromethyl starting material produced a trans-dimer.

Spectroscopic data obtained from the coordination of $\mathbf{1}$ to platinum was used to assist in identification of coordination compounds formed in reactions with the palladium precursor $\left[\mathrm{PdCl}_{2}(\mathrm{NCMe})_{2}\right]$. Data indicated an initial preference for transdimer formation, even in polar solvents, followed by a rapid rearrangement to form another cis,trans-dimeric species, 23. All three cis,trans-dimers (19, 21, and 24) were found to be stable in aromatic solvents at temperatures up to $60{ }^{\circ} \mathrm{C}$, with extended thermolysis of these solutions promoting $\mathrm{C}-\mathrm{H}$ activation to form metallated pincer complexes.

These cis,trans-dimers represent a very rare coordination geometry, with only six other compounds reported to date. Comparison of previously published species with the compounds described in this work show that a surprisingly diverse range of donor atoms and backbone lengths are present in cis,trans-dimers. However, all compounds appear to have pnictogen donor atoms capable of ready migration between metal orbitals, allowing for facile isomerisation from any kinetic products formed to the thermodynamically favoured cis,trans-dimer. It also appears that the balance between steric and electronic effects play a crucial role in disfavouring the cis- and trans-dimers, producing the cis,trans configuration. As such, these unusual dimers present an unanticipated class of compounds formed during the synthesis of PCP pincer complexes. 


\section{Chapter 4}

\section{Synthesis and Reactivity of PCP Pincer Complexes}

The initial coordination chemistry of the electron-poor POCOPH pincer ligand $\mathbf{1}$ with platinum and palladium precursors (outlined in Chapter 3) revealed that incorporation of electron-withdrawing groups onto the ligand backbone served to make ligand metallation less facile, instead favouring the formation of bridged, oligomeric structures. To probe the barrier to ligand metallation and pincer complex formation, the effect of the transition metal starting material was investigated.

\subsection{Synthesis of PCP Pincer Complexes}

From the exploration of the coordination chemistry of the phosphinite ligand 1 with platinum and palladium starting materials, it was clear that metal chloride starting materials offered the most successful route to the metallated pincer complex. However, the elevated reaction temperatures and long reaction times required to facilitate metallation in some instances were observed to lead to the partial decomposition of the desired species in solution. As it became clear that there was a reasonable degree of difficulty in the convenient synthesis and isolation of these compounds, a range of starting materials were assessed for their ability to readily produce the metallated pincer complexes. There have been no direct comparisons of starting materials for the synthesis of PCP pincer compounds reported in the literature to date. 


\subsubsection{Effect of Metal Precursor on PCP Pincer Synthesis}

Owing to the difficulty in generating the pincer complex [(POCOP)PtCl] (25) from the $\left[\mathrm{PtCl}_{2}(\right.$ hex $\left.)\right]$ starting material, reactions with the more commonly used $\left[\mathrm{PtCl}_{2}(\mathrm{COD})\right]$ starting material were performed. By undertaking reactions with the hexadiene and cyclooctadiene starting materials simultaneously under identical conditions, it was observed that reactions with $\left[\mathrm{PtCl}_{2}(\mathrm{COD})\right]$ produced 25 at a faster rate than those with $\left[\mathrm{PtCl}_{2}(\right.$ hex $\left.)\right]$. However, both reactions were observed to proceed very slowly; after 120 hours at reflux in toluene reactions performed with the cyclooctadiene starting material had reached approximately $75 \%$ completion, while the hexadiene starting material had only achieved about a $50 \%$ conversion (as assessed by integrations of signals in the ${ }^{1} \mathrm{H}$ and ${ }^{19} \mathrm{~F}$ NMR spectra). As for reactions with $\left[\mathrm{PtCl}_{2}(\right.$ hex $\left.)\right]$, reactions with $\left[\mathrm{PtCl}_{2}(\mathrm{COD})\right]$ were also observed to proceed via the cis,trans-dimer 19. However while reactions between ligand $\mathbf{1}$ and $\left[\mathrm{PtCl}_{2}(\right.$ hex $\left.)\right]$ were observed to produce dimeric species such as $\mathbf{1 9}$ at room temperature, no reaction occurred between 1 and $\left[\mathrm{PtCl}_{2}(\mathrm{COD})\right]$ at room temperature, even after 72 hours.

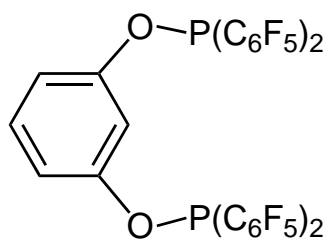

1
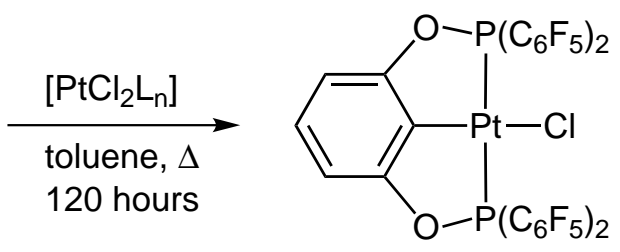

25

Scheme 4.1 Effect of starting material on the progress of the metallation of ligand 1 to form 25. Percentage values represent the approximate conversion of ligand to the metallated product, as observed by ${ }^{31} \mathrm{P}$ NMR spectroscopy.

Another starting material that has been used successfully in the synthesis of platinum pincer compounds is $\left[\mathrm{PtCl}_{2}\left(\mathrm{SEt}_{2}\right)_{2}\right] .{ }^{117}$ Reactions between $\left[\mathrm{PtCl}_{2}\left(\mathrm{SEt}_{2}\right)_{2}\right]$ and 1 were observed to proceed much faster than those performed with the platinumdiene precursors; formation of the pincer complex $\mathbf{2 5}$ was observed to be about $75 \%$ complete after 64 hours, reaching completion after 120 hours at reflux in toluene (Scheme 4.1). Compared to the reactions performed with $\left[\mathrm{PtCl}_{2}\right.$ (diene)] precursors, considerably smaller amounts of the cis,trans-dimer intermediate were observed throughout the course of the reaction. In reactions between $\left[\mathrm{PtCl}_{2}\left(\mathrm{SEt}_{2}\right)_{2}\right]$ and $\mathbf{1}$ at room temperature, the free ligand $\mathbf{1}$, along with a species that has tentatively been assigned as the 1:1 complex $\left[\left(\kappa^{1}-\mathrm{POCOPH}\right) \mathrm{PtCl}_{2}\left(\mathrm{SEt}_{2}\right)\right]$ are the major components of the reaction mixture. This new platinum complex possessed the characteristic quintet signal of the uncoordinated phosphinite at $85.4 \mathrm{ppm}$ in the 
${ }^{31} \mathrm{P}$ NMR spectrum, with a corresponding singlet at $68.9 \mathrm{ppm}$ that displayed a platinum-phosphorus coupling constant of $4240 \mathrm{~Hz}$, likely to represent phosphorus coordination trans to a chloride. The alternate isomer with the diethyl sulfide coordinated trans to the phosphorus could not be ruled out, but was unlikely, as phosphorus coordination trans to sulfur should result in platinum-phosphorus coupling constants about 500-1000 Hz smaller than for phosphorus coordination trans to chloride, ${ }^{118,119}$ which typically had values between $4600-4200 \mathrm{~Hz}$ for platinum dichloride complexes of $\mathbf{1}$.

The spectroscopic data of this 1:1 complex was very similar to that of the 2:1 complex 18, the major difference being the appearance of the coordinated phosphorus atom downfield from that in $\mathbf{1 8}\left(\delta_{\mathrm{P}}=51.4 \mathrm{ppm}\right)$. The broad signals of coordinated diethyl sulfide were observed in the ${ }^{1} \mathrm{H}$ NMR spectrum, but due to overlap with free diethyl sulfide and resonances of the starting material, the presence of diethyl sulfide in the new platinum complex could not be definitively established. However, on the basis that this species contains a $\kappa^{1}$-bound phosphinite ligand and is not the 2:1 complex previously observed, a 1:1 complex with bound diethyl sulfide is the most likely structure. Despite some uncertainty as to the nature of this species, it is clear that at room temperature, the reaction between ligand $\mathbf{1}$ and $\left[\mathrm{PtCl}_{2}\left(\mathrm{SEt}_{2}\right)_{2}\right]$ produced none of the previously observed dimeric or oligomeric $\left[(\mathrm{POCOPH}) \mathrm{PtCl}_{2}\right]_{\mathrm{x}}$ species.

From these results, it is clear that for $\left[\mathrm{PtCl}_{2} \mathrm{~L}_{\mathrm{n}}\right]$ starting materials, the ease of formation of the pincer complex $\mathbf{2 5}$ depends on the ancillary ligand $\mathrm{L}_{\mathrm{n}}$, with diethyl sulfide $>$ cyclooctadiene $>$ hexadiene. This order of reactivity correlated roughly with the ability of these neutral ligands to bind to platinum: cyclooctadiene has been reported to displace hexadiene from platinum, ${ }^{120}$ but dimethyl sulfide and cyclooctadiene have been reported to displace each other from platinum, indicating that they bind with a similar strength to the metal centre. ${ }^{121,122}$ However, upon $\kappa^{1}$-coordination of ligand 1 to the platinum, diethyl sulfide would be expected to be a significantly better ligand than the $\eta^{2}$-COD; the COD is no longer stabilised by the chelate effect, and the electron-withdrawing nature of the phosphinite does not provide the metal centre with significant electron density to stabilise the COD by $\pi$-backbonding.

This stability series (diethyl sulfide $>$ cyclooctadiene $>$ hexadiene) also correlated well with qualitative observations of the amount of cis,trans-dimer 19 present in reaction mixtures; the more readily displaced the ancillary ligand, the more facile the formation of dimer 19, the longer 19 persisted in solution, and the slower the formation of the metallated pincer species. It can be surmised that platinum precursors with more difficult to displace neutral ligands are less prone to dimer formation; 
once a $\kappa^{1}$-[(PCPH) $\left.\mathrm{PtCl}_{2} \mathrm{~L}\right]$ intermediate is formed, not only will the pendant phosphorus donor have to displace a strongly-bound ligand from the metal to form a dimer, it will also be in competition with the already displaced, strongly binding ancillary ligand to do so. Therefore, neutral ligands that bind more strongly to this $\kappa^{1}$-[(PCPH) $\left.\mathrm{PtCl}_{2} \mathrm{~L}\right]$ intermediate will be displaced from the platinum centre by phosphorus at temperatures closer to those required for metallation, minimising the build up of dimer species in solution (Figure 4.1). The observation of increased levels of dimer in the less facile metallation reactions is in agreement with the earlier postulation that the presence of 19 results in an increased barrier to metallation, due to the energy costs of rearrangement from this stable configuration into one from which metallation can readily occur (such as a cis-monomeric configuration). Empirically, palladium starting materials with $\mathrm{PPh}_{3}$ and $\mathrm{COD}$ ligands have been observed to reduce the formation of $\left[(\mathrm{PCP}) \mathrm{PdCl}_{2}\right]_{\mathrm{x}}$ species and increase yields of the metallated product when compared to starting materials with the more readily displaced nitrile ancillary ligands. ${ }^{123}$

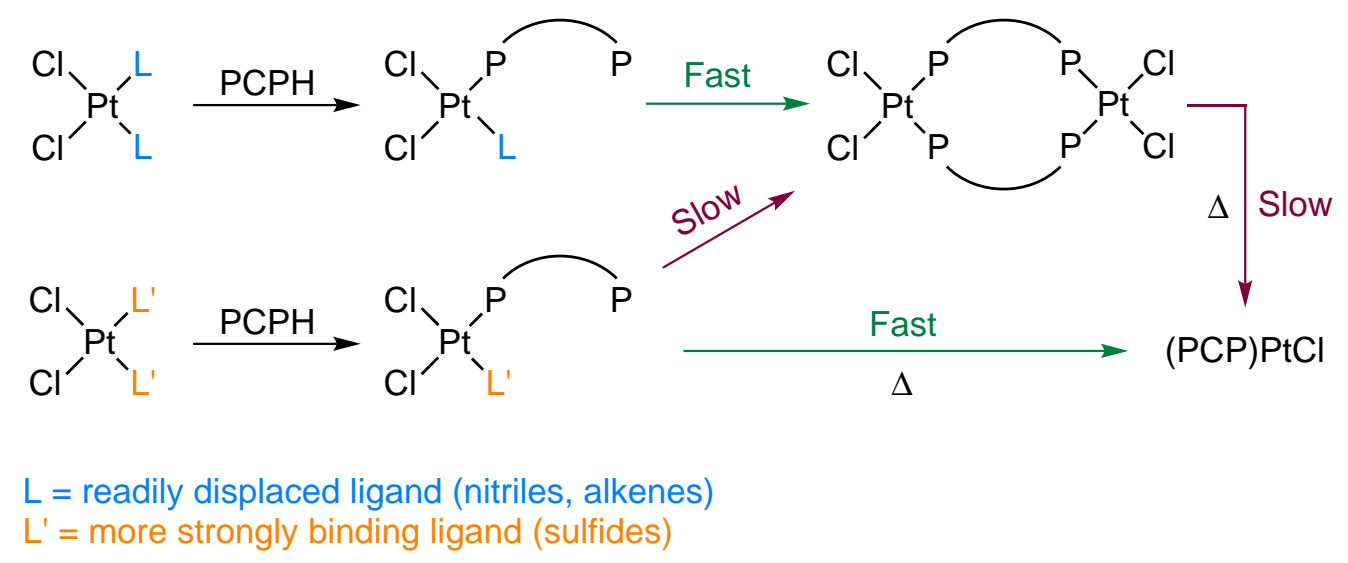

Figure 4.1 Effect of ancillary ligand binding strength on dimer formation. Ligands that are more readily displaced from the $\kappa^{1}$ - $\left.(\mathrm{PCPH}) \mathrm{PtCl}_{2} \mathrm{~L}\right]$ intermediate will favour dimer formation (top), while less readily displaced ligands will favour monomer formation (bottom). Ligand metallation to form the pincer complex is more facile from the monomer than the dimer.

As altering the ancillary ligand(s) on the metal precursor was observed to have a small but significant effect on the ease of formation of $\mathbf{2 5}$, the effect of altering the anionic ligand on the metal centre was examined. Replacement of a chloride ligand on $\left[\mathrm{PtCl}_{2}(\mathrm{COD})\right]$ with a hexamethyldisilazide group provides the resulting platinum complex with an internal base functionality, which has been observed to assist in the synthesis of cyclometallated pincer species at lower temperatures than can be achieved using platinum dichloride starting materials. ${ }^{124}$ Reactions performed in benzene at reflux were reported to achieve metallation of even the difficult cyclohexyl backbones within six hours. However, no significant reaction 
between $\left[\mathrm{PtCl}\left\{\mathrm{N}\left(\mathrm{SiMe}_{3}\right)_{2}\right\}(\mathrm{COD})\right]$ and ligand 1 was observed in benzene- $d_{6}$ after 33 hours at $80^{\circ} \mathrm{C}$; only after a further 20 hours at reflux in toluene were significant amounts of $\mathbf{2 5}$ detected. While the internal base functionality served to reduce the time required for metallation to occur, it did not significantly reduce the temperature at which metallation was facile. Attempts to promote more facile metallation from the dichloride starting materials $\left[\mathrm{PtCl}_{2}(\mathrm{hex})\right]$ and $\left[\mathrm{PtCl}_{2}\left(\mathrm{SEt}_{2}\right)_{2}\right]$ via the addition of triethylamine as external base were unsuccessful, as the formation of $\mathbf{2 5}$ was observed to proceed at approximately the same rate regardless of whether the external base was present or absent.

The effect of the anionic ligand on the metallation reaction was also investigated through the use of the platinum chloromethyl starting material [PtClMe(hex)], as the similar $[\mathrm{PtClMe}(\mathrm{COD})]$ starting material has been used to good effect in the synthesis of pincer complexes in the literature. ${ }^{58,100}$ Reactions between ligand 1 and $[\mathrm{PtClMe}($ hex $)]$ showed that complete formation of [(POCOP $) \mathrm{PtCl}]$ had occurred after 48 hours at reflux in toluene, substantially quicker than reactions performed with the dichloride starting material $\left[\mathrm{PtCl}_{2}(\mathrm{hex})\right]$. This result is interesting when considering that reactions between the platinum dimethyl derivative $\left[\mathrm{PtMe}_{2}(\mathrm{hex})\right]$ and ligand 1 produced only small amounts of the pincer complex [(POCOP)PtMe].

The observation that the platinum chloro(methyl) starting material offers a more efficient route to metallated pincer complexes than the analogous platinum dichloride starting material is likely due to the methyl group acting as a better proton acceptor than the chloride, with the methane generated also likely to be a better leaving group than hydrogen chloride. No traces of [(POCOP)PtMe] are detected in reactions starting from $[\mathrm{PtClMe}(\mathrm{hex})]$, demonstrating methyl group has a much larger affinity for the metallated proton than the chloride group. However, as only small amounts of the metallated product were observed in reactions between $\mathbf{1}$ and the dimethyl starting material $\left[\mathrm{PtMe}_{2}(\mathrm{hex})\right]$, the chloride ligand must also assist the reaction. One such way this may occur is for the chloride to generate favourable $\mathrm{H} \cdots \mathrm{Cl}$ interactions with the $\mathrm{C}-\mathrm{H}$ bond being broken, weakening the bond and lowering the energy required for $\mathrm{C}-\mathrm{H}$ activation. In such a $\mathrm{H} \cdots \mathrm{Cl}$ interaction, the chloride may also act as a shuttle, assisting the proton transfer to the methyl leaving group. Theoretical calculations have shown that this chloride-assisted proton transfer is favoured during the oxidative addition of methane in the Shilov reaction. ${ }^{125}$ In the synthesis of pincer compounds, a dimeric intermediate of the type $[(\mathrm{PCPH}) \mathrm{PtClX}]_{2}$ has been isolated and crystallographically characterised, in which the chloride ligands interact strongly with the proton on C-2 of the ligand backbone, resulting in a weakened $\mathrm{C}-\mathrm{H}$ bond (as seen in the distorted dimer structure mentioned in Chapter 3, Figure 3.6). ${ }^{107}$ It is thought that the chloride ligand helps 
to facilitate metallation through this interaction .
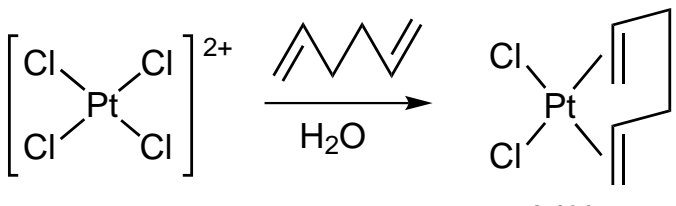

i) $\mathrm{ZnMe}_{2}$ in $\mathrm{Et}_{2} \mathrm{O}$

ii) $\mathrm{CH}_{3} \mathrm{COCl}$

$94 \%$<smiles></smiles>

$46 \%$

$\mathrm{POCOPH}$

toluene, $\Delta$

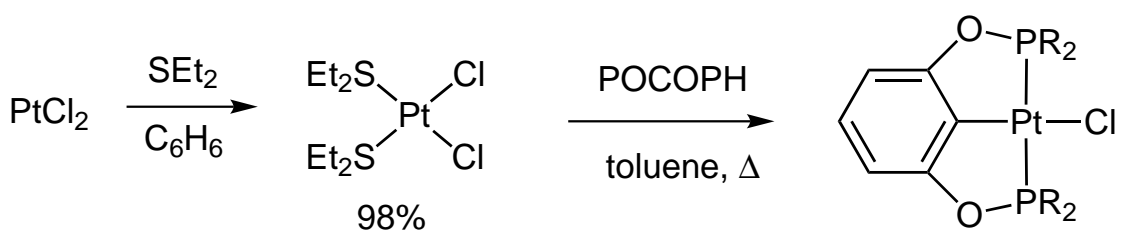

47\% from [PtCIMe(hex)]

( $20 \%$ overall)

$33 \%$ from $\left[\mathrm{PtCl}_{2}\left(\mathrm{SEt}_{2}\right)_{2}\right]$

(32\% overall)

Scheme 4.2 Synthesis of the pincer complex 1 via platinum dichloride and chloromethyl starting materials.

While the experimental data indicated that for the synthesis of $\mathbf{2 5}$, the reactivity of starting materials was in the order of $\left[\mathrm{PtCl}\left\{\mathrm{N}\left(\mathrm{SiMe}_{3}\right)_{2}\right\}(\mathrm{COD})\right]>[\mathrm{PtClMe}($ hex $)]$ $>\left[\mathrm{PtCl}_{2}\left(\mathrm{SEt}_{2}\right)_{2}\right]>\left[\mathrm{PtCl}_{2}(\mathrm{COD})\right]>\left[\mathrm{PtCl}_{2}(\right.$ hex $\left.)\right]$, the ease of starting material synthesis should also be considered. The syntheses of the most reactive compounds $\left[\mathrm{PtCl}\left\{\mathrm{N}\left(\mathrm{SiMe}_{3}\right)_{2}\right\}(\mathrm{COD})\right]^{124}$ and $[\mathrm{PtClMe}($ hex $)]$ (see experimental section) both required organometallic starting materials of the type $\left[\mathrm{PtCl}_{2}\right.$ (diene)], which were then treated with pyrophoric reagents and gave only modest yields of the desired species (approximately $45-70 \%$ ). However, the less reactive $\left[\mathrm{PtCl}_{2}\left(\mathrm{SEt}_{2}\right)_{2}\right]$ could be synthesised from the treatment of platinum(II) chloride with diethyl sulfide, and obtained in yields of typically $>95 \%$. So while the monochloride starting materials were observed to require shorter reaction times and gave greater yields of the metallated product 25, it must be taken into account whether this value represents a greater overall yield from the original platinum starting material (Scheme 4.2).

\subsubsection{Effect of the Ligand on PCP Pincer Synthesis}

The cyclometallation reaction to form pincer complexes has been known to be dependent upon the steric bulk on the donor atoms of the ligand, with bulky groups such as tert-butyls favouring pincer formation. However, the electronic aspects of the cyclometallation reaction have been reported to vary depending on the nature of 
the metal and ligand; ${ }^{126}$ while numerous studies have been devoted to cyclometallation reactions in the literature, the electronic aspects of metallation to form PCP pincer complexes have scarcely been reported, save for reports that ligands containing $\pi$-accepting phosphite functionalities are unusually difficult to metallate. ${ }^{92,104}$ From the reactions between the POCOPH ligand 1 and platinum starting materials, it was clear that metallation in this instance was substantially less facile than reported reactions with other PCP pincer ligands. While this proved to be valuable in investigating the coordination chemistry of $\mathbf{1}$, it was clearly detrimental to the convenient synthesis of the pincer complex 25. To provide an indication of how the electronic nature of the ligand affected the ease of metallation, the pincer ligands $\mathrm{POCOPH}(\mathbf{1}), \mathrm{PCCCPH}(\mathbf{2})$, and $\mathrm{POCCPH}(\mathbf{3})$ were reacted with $\left[\mathrm{PtCl}_{2}\left(\mathrm{SEt}_{2}\right)_{2}\right]$ in NMR tubes. This allowed frequent in situ monitoring of the reaction progress by NMR spectroscopy, enabling the time each reaction required to reach completion to be evaluated.

For these small-scale reactions, it was observed that the reaction with the most electron-donating POCCPH ligand 3 reached completion first, after 17 hours at reflux in toluene- $d_{8}$. The reaction with the PCCCPH ligand 2 reached completion after 33 hours, almost double the time of ligand $\mathbf{3}$, while the reaction with electronpoor POCOPH ligand $\mathbf{1}$ occurred very slowly and had only reached about $50 \%$ completion after 33 hours at reflux in toluene. As ligands 1, 2, and $\mathbf{3}$ all have a similar steric bulk this is a clear indication that more electron-donating ligands increase the ease of the metallation reaction.

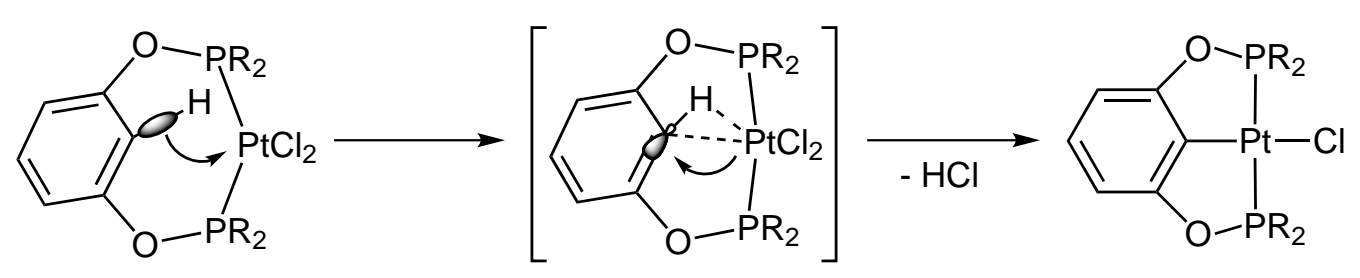

Scheme 4.3 Metallation of the pincer backbone to form 25. Donation of electron density from the $\mathrm{C}-\mathrm{H} \sigma$-orbital to the metal forms an agostic transition state. Backdonation from the metal into the $\mathrm{C}-\mathrm{H} \sigma^{*}$-orbital completes the bond scission.

As these cyclometallation reactions have been observed to proceed via agostic intermediates/transition states, ${ }^{127}$ it may seem intuitive for an electron deficient metal centre to enhance this $\mathrm{C}-\mathrm{H}$ activation step by withdrawing electron density from the $\mathrm{C}-\mathrm{H} \sigma$-bonding orbital in an electrophilic manner. However once the $\mathrm{C}-\mathrm{H}$ agostic interaction with the metal is established, backdonation from the metal into the $\mathrm{C}-\mathrm{H} \sigma^{*}$-antibonding orbital is required to complete the scission of this bond (Scheme 4.3). ${ }^{128}$ This backdonation is difficult for electron-poor metal complexes 
to achieve, and hence cyclometallation reactions with $\mathbf{1}, \mathbf{2}$, and $\mathbf{3}$ are seen to be less facile than for reported reactions with more basic phosphine ligands. ${ }^{129}$ Furthermore, pincer ligands with electron-withdrawing groups may generate less stable agostic intermediates. As the aromatic backbone is attached at C-1 and C-3 to the electron-poor phosphorus donors, any inductive withdrawal of electron density from the $\pi$-system of the ligand backbone should cause a contraction of the orbitals on C2 , destabilising any agostic metal-ligand interactions. This effect has been observed for reactions with palladium, whereby the incorporation of electron-withdrawing groups ortho to aromatic $\mathrm{C}-\mathrm{H}$ bonds decreases the rate of the metallation reaction. ${ }^{130,131}$ This difficulty in promoting metallation with electron-poor ligands was also observed in the initial reports of the coordination chemistry of the PCCCPH ligand 2 with ruthenium, whereby attempts to produce the pincer complex by direct cyclometallation were unsuccessful, and a transcyclometallation reaction scheme had to be employed. ${ }^{56}$

Upon scaling up these reactions to synthesise the $[(\mathrm{PCP}) \mathrm{PtCl}]$ pincer complexes of ligands $\mathbf{1}, \mathbf{2}$, and $\mathbf{3}$, the yields obtained did not directly correlate to the speed of the metallation reaction; a more facile metallation reaction did not necessarily result in an increased yield. Compound [(POCOP $) \mathrm{PtCl}](\mathbf{2 5})$ was isolated in a $33 \%$ yield, [(PCCCP)PtCl] (26) in a $68 \%$ yield, and [(POCCP $) \mathrm{PtCl}](\mathbf{2 7})$ in a $50 \%$ yield. It therefore appeared that as the number of $\mathrm{P}-\mathrm{O}$ bonds in the ligand increased, so the yield of the metallated pincer complex decreased. This was supported by the observation of traces of resorcinol in ${ }^{1} \mathrm{H}$ NMR spectra of reactions between 1 and platinum precursors that had been subjected to prolonged thermolysis (greater than 100 hours at reflux in toluene), highlighting the lower stability of $\mathrm{P}-\mathrm{O}$ bonds when compared to $\mathrm{P}-\mathrm{C}$ bonds.

\subsection{Reactions of $\mathrm{Pt} / \mathrm{Pd}$ Pincer Compounds}

\subsubsection{Synthesis of Pincer Metal-Carbonyl Compounds}

With platinum PCP pincer complexes 25, 26, and 27 in hand, the corresponding palladium pincer complexes were synthesised from $\left[\mathrm{PdCl}_{2}\left(\mathrm{NCMe}_{2}\right]\right.$. Palladium has demonstrated a higher reactivity than platinum for ligand cyclometallation reactions, which has been attributed to its greater electrophilicity assisting with the initial agostic interaction between the metal centre and the $\mathrm{C}-\mathrm{H}$ bond being cleaved (the first step in Scheme 4.3). ${ }^{132,133}$ Therefore, shorter reaction times were required 
to achieve ligand metallation on palladium, generally affording slightly higher yields of complexes [(POCOP $) \mathrm{PdCl}](\mathbf{2 8}),[(\mathrm{PCCCP}) \mathrm{PdCl}](\mathbf{2 9})$, and $[(\mathrm{POCCP}) \mathrm{PdCl}]$ (30) than for the analogous platinum complexes.

The NMR spectra of the platinum and palladium complexes were all consistent with the formation of tridentate pincer complexes. The ${ }^{31} \mathrm{P}$ NMR spectra all displayed signals downfield from those of the free ligand and unmetallated intermediates, consistent with deshielding of the phosphorus atoms upon coordination to the metal centre, followed by the loss of an electron-rich chloride ligand upon metallation. The platinum-phosphorus coupling constants of $\mathbf{2 5 , 2 6}$, and $\mathbf{2 7}$ ranged from $3088 \mathrm{~Hz}$ to $3663 \mathrm{~Hz}$, indicative of mutual trans-coordination of the phosphorus donors. Complexes of the asymmetric POCCP ligand $\mathbf{3}$ also displayed large trans phosphorus coupling of 460-470 Hz, significantly larger than that observed for the only other Group 10 metal phosphine-phosphinite pincer complex reported $(388 \mathrm{~Hz}) .{ }^{13}$ This unusual coordination of mutually trans donating and accepting phosphine ligands may provide a synergy that strengthens this phosphorus-phosphorus coupling interaction.

The loss of a signal for the $\mathrm{H}-2$ proton environment was evident in the ${ }^{1} \mathrm{H}$ NMR spectra of all compounds, with the remaining proton resonances shifted slightly upon metallation. The methylene proton signals of the PCCCP complexes $[(\mathrm{PCCCP}) \mathrm{PtCl}](\mathbf{2 6})$ and $[(\mathrm{PCCCP}) \mathrm{PdCl}](\mathbf{2 9})$ had changed in appearance, from a doublet in the free ligand $\mathbf{2}$ to virtual triplets in the metal complexes due to strong coupling through the metal atom to the second phosphorus donor. All spectroscopic data for the previously synthesised complex $\mathbf{2 9}$ were in agreement with that previously reported; ${ }^{57}$ however, the isolated yield of $64 \%$ was significantly lower than the literature yield of $82 \%$. As the previously reported synthesis proceeded via a two step reaction from the less readily synthesised $\left[\mathrm{Pd}(\mathrm{NCMe})_{4}\right]\left[\mathrm{BF}_{4}\right]_{2}$ starting material, the synthesis reported herein can be seen as an alternate preparation that offers a more convenient but slightly lower yielding route to 29. In all $[(\mathrm{PCP}) \mathrm{MCl}]$ complexes synthesised the ${ }^{19} \mathrm{~F}$ NMR spectra are unremarkable, each showing three signals in a 2:1:2 (ortho:para:meta) ratio, consistent with free rotation of the pentafluorophenyl groups.

To assess the electronic characteristics of each metal centre, the metal-carbonyl derivatives $[(\mathrm{PCP}) \mathrm{M}(\mathrm{CO})]^{+}$were prepared by chloride abstraction with silver hexafluoroantimonate followed by exposure to carbon monoxide gas. The $\mathrm{C}-\mathrm{O}$ stretches were measured by infrared (IR) spectroscopy, and are listed in Table 4.1. The $\mathrm{C}-\mathrm{O}$ stretching frequency of metal carbonyls is very sensitive to the electron density on the metal centre, with more electron-rich metal centres displaying a greater de- 
Table 4.1 Infrared $\mathrm{C}-\mathrm{O}$ stretches of pincer carbonyl complexes.

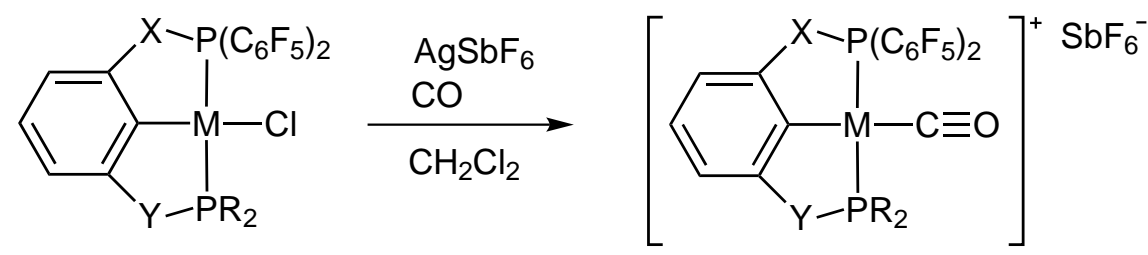

\begin{tabular}{lcccccc}
\hline \hline Formula & Compound & $\mathrm{X}$ & $\mathrm{Y}$ & $\mathrm{R}$ & $\nu_{\mathrm{C}-\mathrm{O}}\left(\mathrm{cm}^{-1}\right)$ & $\Delta_{\delta \mathrm{m}, \mathrm{p}}{ }^{a}(\mathrm{ppm})$ \\
\hline$[(\mathrm{POCOP}) \mathrm{Pt}(\mathrm{CO})]\left[\mathrm{SbF}_{6}\right]$ & $\mathbf{3 1}$ & $\mathrm{O}$ & $\mathrm{O}$ & $\mathrm{C}_{6} \mathrm{~F}_{5}$ & 2145 & 15.8 \\
{$[(\mathrm{PCCCP}) \mathrm{Pt}(\mathrm{CO})]\left[\mathrm{SbF}_{6}\right]$} & $\mathbf{3 2}$ & $\mathrm{CH}_{2}$ & $\mathrm{CH}_{2}$ & $\mathrm{C}_{6} \mathrm{~F}_{5}$ & 2127 & 13.3 \\
{$[(\mathrm{POCCP}) \mathrm{Pt}(\mathrm{CO})]\left[\mathrm{SbF}_{6}\right]$} & $\mathbf{3 3}$ & $\mathrm{O}$ & $\mathrm{CH}_{2}$ & ${ }^{t} \mathrm{Bu}$ & 2111 & 13.8 \\
{$[(\mathrm{POCOP}) \mathrm{Pd}(\mathrm{CO})]\left[\mathrm{SbF}_{6}\right]$} & $\mathbf{3 4}$ & $\mathrm{O}$ & $\mathrm{O}$ & $\mathrm{C}_{6} \mathrm{~F}_{5}$ & 2170 & 15.1 \\
{$[(\mathrm{PCCCP}) \mathrm{Pd}(\mathrm{CO})]\left[\mathrm{SbF}_{6}\right]$} & $\mathbf{3 5}$ & $\mathrm{CH}_{2}$ & $\mathrm{CH}_{2}$ & $\mathrm{C}_{6} \mathrm{~F}_{5}$ & 2148 & 13.4 \\
{$[(\mathrm{POCCP}) \mathrm{Pd}(\mathrm{CO})]\left[\mathrm{SbF}_{6}\right]$} & $\mathbf{3 6}$ & $\mathrm{O}$ & $\mathrm{CH}_{2}$ & ${ }^{t} \mathrm{Bu}$ & 2140 & 13.9 \\
\hline \hline
\end{tabular}

${ }^{a}$ Value of $\Delta_{\delta \mathrm{m}, \mathrm{p}}$ is for the $[(\mathrm{PCP}) \mathrm{MCl}]$ species, to reflect that this parameter may be used as an alternate evaluation of electron density within the pincer complex.

gree of $\pi$-backbonding to the carbonyl ligand, meaning that the carbonyl stretch is shifted to lower wavenumbers with increasing electron density on the metal. The observed magnitude of the $\mathrm{C}-\mathrm{O}$ stretching frequency followed the pattern POCOP $>$ PCCCP > POCCP; the enhanced $\pi$-acceptor ability of the phosphinite POCOP generated the most electron-poor metal centres, while the strong $\sigma$-donor ability of the tert-butyl phosphine in POCCP resulted in the most electron-rich metal centres.

Of note is the observation that the $\mathrm{C}-\mathrm{O}$ stretches of compounds $\mathbf{3 1}, \mathbf{3 4}$, and 35 appeared at wavenumbers greater than that of free carbon monoxide $\left(2143 \mathrm{~cm}^{-1}\right)$. While it appears counterintuitive that bonding to a metal centre may increase the strength of this carbon-oxygen bond, examination of the molecular orbital diagram of carbon monoxide (Figure 4.2) reveals that the HOMO is a $\sigma^{*}$ antibonding orbital. Therefore, loss of electron density from this orbital upon coordination to the metal centre will increase the $\mathrm{C}-\mathrm{O}$ bond order, resulting in a shorter, stronger bond. Similarly, the LUMO is a $\pi^{*}$ antibonding orbital; during $\pi$-backbonding the metal centre donates electron density into this antibonding orbital, reducing the $\mathrm{C}-\mathrm{O}$ bond order and weakening of the $\mathrm{C}-\mathrm{O}$ bond as the amount of backdonation increases. Additionally, electrostatic factors may further serve to increase the $\mathrm{C}-\mathrm{O}$ stretching frequency of metal-carbonyl complexes in complexes where minimal $\pi$-backbonding occurs. ${ }^{134}$

Based on reported $\mathrm{C}-\mathrm{O}$ stretching frequencies, the palladium complexes $\mathbf{3 4}, \mathbf{3 5}$, 36, and the platinum complex 31 represent some of the most electron-poor PCP pincer compounds reported to date. The highest $\mathrm{C}-\mathrm{O}$ stretching frequency reported for a palladium $\mathrm{PCP}$ pincer compound is $2141 \mathrm{~cm}^{-1},{ }^{135}$ significantly lower than the $2170 \mathrm{~cm}^{-1}$ displayed by the POCOP complex 34 . A platinum 


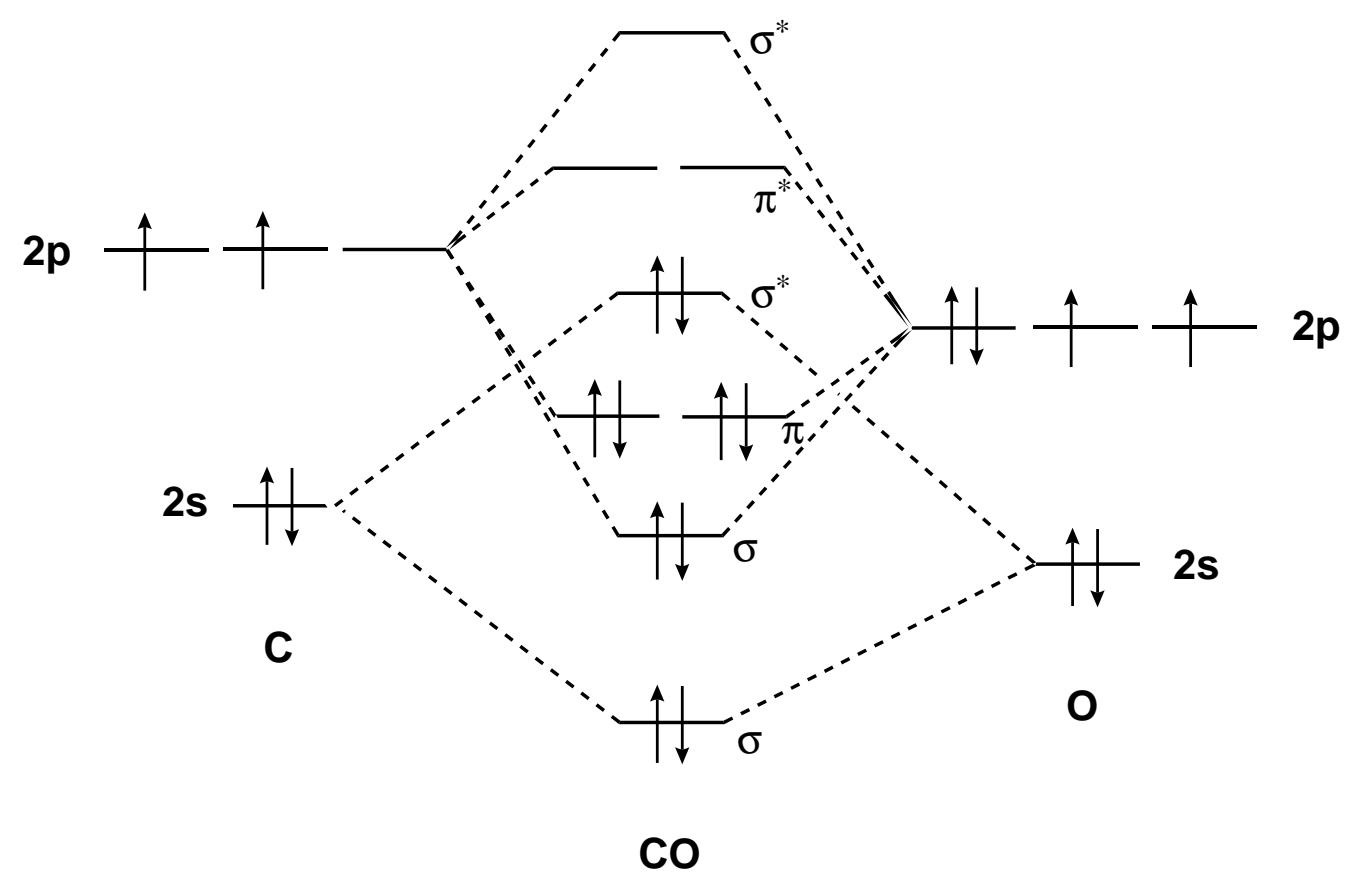

Figure 4.2 Molecular orbital diagram of carbon monoxide.

bis(trifluoromethyl)phosphine pincer complex has been reported to display a $\mathrm{C}-\mathrm{O}$ stretch at $2143 \mathrm{~cm}^{-1},{ }^{58}$ higher than the analogous bis(pentafluorophenyl)phosphine 32, but slightly lower than the phosphinite $\mathbf{3 1}$, demonstrating the enhanced $\pi$ acceptor capability of trifluoromethyl phosphines over pentafluorophenyl phosphines. Most reported alkyl phosphine PCP pincer complexes have displayed $\mathrm{C}-\mathrm{O}$ stretches in the range $2070-2080 \mathrm{~cm}^{-1} ;^{136,137}$ as the complexes reported herein possess values from $2111-2170 \mathrm{~cm}^{-1}$ they represent unusual examples of pincer complexes with very electron-poor metal centres.

Another parameter that provides information on the electronic character of these complexes is $\Delta_{\delta \mathrm{m}, \mathrm{p}}$, the difference in chemical shift between the meta and para fluorines of the $\mathrm{P}\left(\mathrm{C}_{6} \mathrm{~F}_{5}\right)_{2}$ group. This has commonly been used to determine the degree of coordination of pentafluorophenyl borate anions, ${ }^{138}$ but can also provide an indication of the electron density on the phosphorus atom in pentafluorophenyl phosphine ligands. ${ }^{57}$ Values for the ligands POCOPH (1), PCCCPH (2), and POC$\mathrm{CPH}(\mathbf{3})$ are 12.0, 11.1, and 11.8 ppm respectively, and reflected the slightly more electron poor nature of the pentafluorophenyl phosphinite group compared to the pentafluorophenyl phosphine group. The $\Delta_{\delta \mathrm{m}, \mathrm{p}}$ values for the metallated complexes (Table 4.1) are predictably higher than for the free ligands, representing the loss of electron density from the phosphorus donors upon metal coordination. Of note is that for $\mathbf{3}$, the $\Delta_{\delta \mathrm{m}, \mathrm{p}}$ value is very similar to $\mathbf{1}$ for the free ligand ( $0.2 \mathrm{ppm}$ lower), indicating that the electron rich tert-butyl phosphine is too far removed from the pentaflurophenyl phosphinite to affect it electronically. However, upon metal coordination, there is a $2.0 \mathrm{ppm}$ difference (on platinum) in $\Delta_{\delta \mathrm{m}, \mathrm{p}}$ values, indicating the 
increased donor ability of the tert-butyl phosphine has reduced the demand of the metal for electron density from the phosphinite. While these values are an interesting measure of the electronics of the pentafluorophenyl ring (and the phosphorus donor by association), as seen in Table 4.1 they do not correlate well with the $\mathrm{C}-\mathrm{O}$ stretching frequencies between complexes of different metals, or between complexes with different numbers of pentafluorophenyl-substituted donors.

The NMR data obtained for the carbonyl compounds $31,32,33,34,35$, and 36 was consistent with the synthesis of a $[(\mathrm{PCP}) \mathrm{M}(\mathrm{CO})]^{+}$complex in each instance. Spectroscopically there were minor differences between each carbonyl complex and its parent chloride. The ${ }^{31} \mathrm{P}$ NMR signal of the $\mathrm{P}\left(\mathrm{C}_{6} \mathrm{~F}_{5}\right)_{2}$ groups experienced small chemical shift differences of 2-6 ppm, while the $\mathrm{P}^{t} \mathrm{Bu}_{2}$ groups experienced substantial deshielding of between 19-26 ppm, a consequence of the cationic metal centre being able to extract further electron density from the more donating tert-butyl phosphines, but less so from the pentafluorophenyl phosphines or phosphinites. The pentafluorophenyl groups do experience this increased electron demand to some extent, as each carbonyl complex possessed $\Delta_{\delta \mathrm{m}, \mathrm{p}}$ values about 1-3 ppm higher than for the analogous chloride complex. Similarly, ${ }^{1} \mathrm{H}$ NMR spectra showed that the proton environments of each ligand backbone were shifted downfield by an average of about $0.4 \mathrm{ppm}$, reflecting the loss of electron density from the metal centre upon the formation of these cationic carbonyl complexes.

The presence of the carbonyl ligand was confirmed in each instance by ${ }^{13} \mathrm{C}$ NMR spectroscopy, with all of the CO ligands appearing between $\delta_{\mathrm{C}}=177$ and $181 \mathrm{ppm}$. Platinum-phosphorus coupling was able to be resolved for the PCCCP and POCCP complexes 32 and 33, having values of 1068 and $1064 \mathrm{~Hz}$ respectively, consistent with the presence of a platinum-bound carbonyl.

Single crystals of the platinum carbonyl [(POCOP)Pt(CO)] $\left[\mathrm{SbF}_{6}\right]$ (31) suitable for single crystal X-ray diffraction were grown by slow evaporation from a dichloromethane solution of $\mathbf{3 7}$ under a nitrogen atmosphere at room temperature. While there are many reported crystal structures of the phosphine PCCCP pincer complexes, there are significantly fewer examples of the corresponding phosphinite POCOP species. Compound 31 represents the first example of a crystallographically characterised Group $10[(\mathrm{POCOP}) \mathrm{M}(\mathrm{CO})]^{+}$complex, and only the third X-ray crystal structure of a POCOP platinum pincer species.

The X-ray crystal structure (Figure 4.3) showed the expected tridentate $\kappa^{3}$-PCP coordination of the pincer ligand, with the carbon monoxide ligand coordinated to the platinum trans to the metallated carbon of the pincer backbone. Impor- 
tant structural data are given in Table 4.2. The solid state structure revealed a considerable distortion about the platinum centre in 31 ; while the $\mathrm{C}-\mathrm{Pt}-\mathrm{CO}$ angle is close to the expected $180^{\circ}\left(178.72(9)^{\circ}\right)$, the $\mathrm{P}-\mathrm{Pt}-\mathrm{P}$ angle of $157.73(2)^{\circ}$ is substantially smaller than for idealised square planar geometry. This distortion is common for similar pincer complexes, with platinum PCCCP pincer complexes typically possessing small $\mathrm{P}-\mathrm{Pt}-\mathrm{P}$ angles in the range $161-168^{\circ},{ }^{58}$ while the only other example of a $[(\mathrm{POCOP}) \mathrm{PtX}]$ species demonstrated a relatively small $\mathrm{P}-\mathrm{Pt}-\mathrm{P}$ angle of $161.20(4)^{\circ} .{ }^{129}$ The small $\mathrm{P}-\mathrm{Pt}-\mathrm{P}$ angle in $\mathbf{3 1}$ was likely to be due to the electron-poor phosphorus atoms inducing polarisation and shortening of the $\mathrm{P}-\mathrm{O}$ bonds (average bond length of 1.607(4) $\AA$, compared to 1.644(10) $\AA$ in a more electron-rich platinum phosphinite ${ }^{129}$ ), resulting in more strained five-membered chelate rings. This influence of short $\mathrm{P}-\mathrm{O}$ bonds in decreasing the $\mathrm{P}-\mathrm{Pt}-\mathrm{P}$ angle is also observed in an isoelectronic iridium carbonyl complex possessing similarly electron-poor tris(trifluoromethyl)phenyl phosphinite donors. ${ }^{46}$

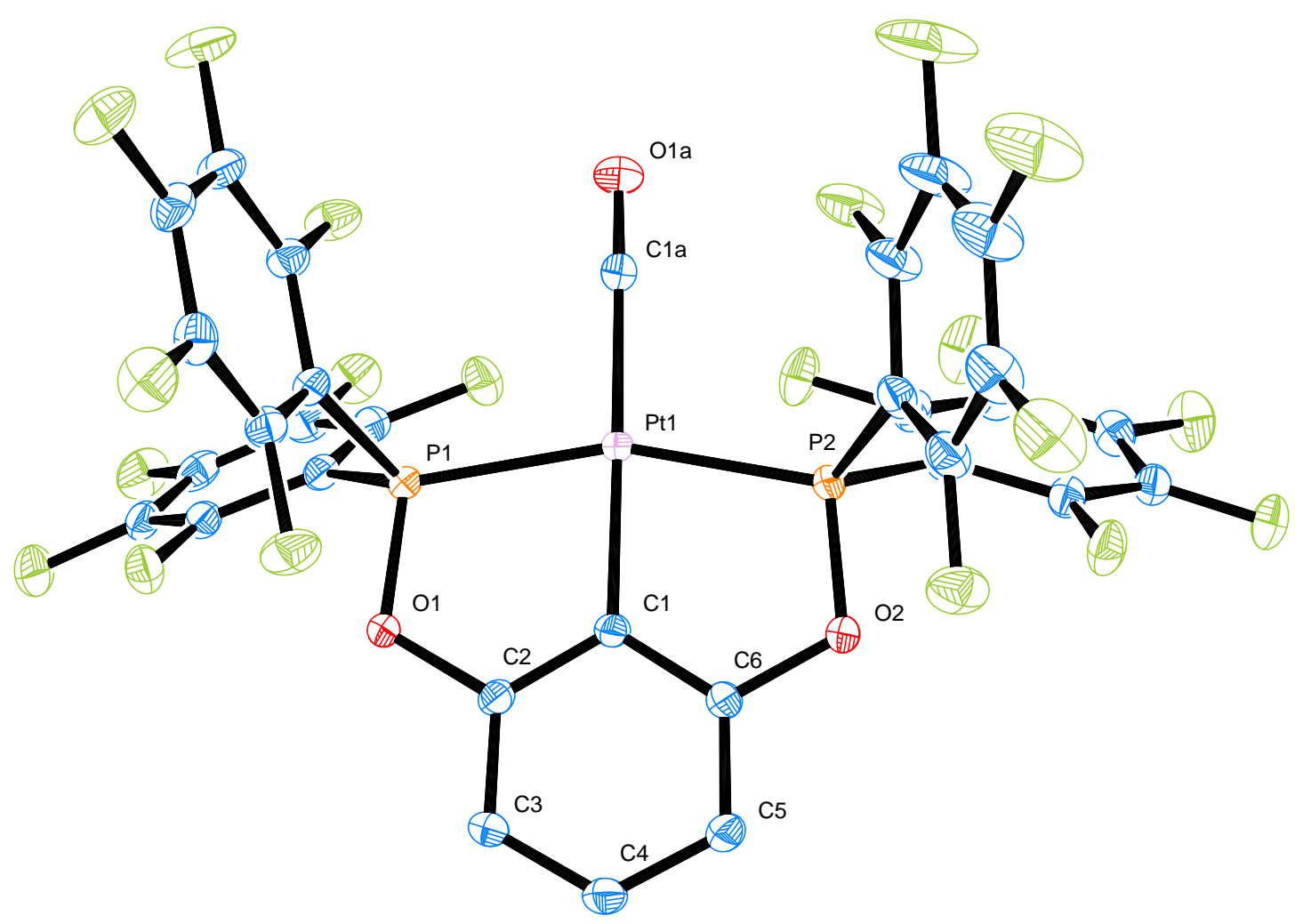

Figure 4.3 ORTEP diagram of [(POCOP)Pt(CO)] $\left[\mathrm{SbF}_{6}\right](\mathbf{3 1})$ (50\% probability thermal elipsoids). Hexafluoroantimonate counterion and hydrogen atoms omitted for clarity.

The $\mathrm{C}-\mathrm{O}$ and $\mathrm{Pd}-\mathrm{C}$ bond lengths for the carbonyl ligand of $1.117(3) \AA$ and $1.962(2) \AA$ were broadly consistent with the IR data $\left(\nu(\mathrm{C}-\mathrm{O})=2145 \mathrm{~cm}^{-1}\right)$, and 
Table 4.2 Selected bond distances $(\AA)$ and angles $\left(^{\circ}\right)$ for complex $[(\mathrm{POCOP}) \mathrm{Pt}(\mathrm{CO})]\left[\mathrm{SbF}_{6}\right](\mathbf{3 1})$.

\begin{tabular}{|c|c|c|c|}
\hline \multicolumn{2}{|c|}{ Bond distances $(\AA)$} & \multicolumn{2}{|l|}{ Bond angles $\left({ }^{\circ}\right)$} \\
\hline Pt1-P1 & $2.2748(6)$ & $\mathrm{P} 1-\mathrm{Pt} 1-\mathrm{C} 1 \mathrm{a}$ & $99.59(7))$ \\
\hline $\mathrm{Pt} 1-\mathrm{P} 2$ & $2.2829(6)$ & $\mathrm{P} 2-\mathrm{Pt} 1-\mathrm{C} 1 \mathrm{a}$ & $102.33(7)$ \\
\hline Pt1-C1 & $2.008(2)$ & $\mathrm{P} 1-\mathrm{Pt} 1-\mathrm{C} 1$ & $79.35(6)$ \\
\hline Pt1-C1a & $1.962(2)$ & $\mathrm{P} 2-\mathrm{Pt} 1-\mathrm{C} 1$ & $78.78(6)$ \\
\hline $\mathrm{C} 1 \mathrm{a}-\mathrm{O} 1 \mathrm{a}$ & $1.117(3)$ & $\mathrm{C} 1-\mathrm{Pt} 1-\mathrm{C} 1 \mathrm{a}$ & $178.72(9)$ \\
\hline $\mathrm{Pt} 1 \cdots \mathrm{F}-\mathrm{SbF}_{5}$ & $3.529(6)$ & Pt1-C1a-O1a & $179.2(2)$ \\
\hline
\end{tabular}

Table 4.3 Crystallographic data for complex [(POCOP)Pt(CO)][ $\left.\mathrm{SbF}_{6}\right]$ (31).

\begin{tabular}{|c|c|}
\hline Empirical formula & $\mathrm{C}_{31} \mathrm{H}_{3} \mathrm{~F}_{20} \mathrm{O}_{3} \mathrm{P}_{2} \mathrm{Pt} \cdot \mathrm{SbF}_{6}$ \\
\hline Formula weight & 1296.11 \\
\hline Crystal system & Monoclinic \\
\hline Space group & $P 2_{1} / n$ \\
\hline $\mathrm{a} / \AA ̊ \AA$ & $10.0907(3)$ \\
\hline $\mathrm{b} / \AA$ & $19.0384(5)$ \\
\hline$c / \AA$ & $18.9040(5)$ \\
\hline$\alpha /{ }^{\circ}$ & 90.00 \\
\hline$\beta /{ }^{\circ}$ & $103.6740(10)$ \\
\hline$\gamma /{ }^{\circ}$ & 90.00 \\
\hline $\mathrm{V} / \AA^{3}$ & $3528.73(17)$ \\
\hline $\mathrm{Z}$ & 4 \\
\hline Cell determination reflections & 9004 \\
\hline Cell determination range, $\theta_{\min } \longrightarrow \theta_{\max } /^{\circ}$ & $2.111 \longrightarrow 31.921$ \\
\hline Temperature/K & $113(2)$ \\
\hline Radiation type & Mo K $\alpha$ \\
\hline Radiation $(\lambda) / \AA$ & 0.71073 \\
\hline Crystal size/ mm & $0.5 \times 0.14 \times 0.14$ \\
\hline $\mathrm{D}_{\text {calc }} / \mathrm{g} \mathrm{m}^{-3}$ & 2.440 \\
\hline $\mathrm{F}(000)$ & 2424 \\
\hline$\mu / \mathrm{mm}^{-1}$ & 4.99 \\
\hline Experimental absorption correction type & Multi-scan (SADABS) \\
\hline $\mathrm{T}_{\max }, \mathrm{T}_{\min }$ & $0.7463,0.5142$ \\
\hline Reflections collected & $101603, R_{\text {equiv }}=0.0390$ \\
\hline Index range $h$ & $-14 \longrightarrow 15$ \\
\hline Index range $k$ & $-28 \longrightarrow 28$ \\
\hline Index range $l$ & $-28 \longrightarrow 28$ \\
\hline$\theta$ range $/^{\circ}$ & $2.37 \longrightarrow 32.07$ \\
\hline Independent reflections & 11960 \\
\hline Reflections $[I>2 \sigma(I)]$ & 10251 \\
\hline Restraints/parameters & $0 / 653$ \\
\hline GOF & 1.053 \\
\hline$R_{1}[I>2 \sigma(I)]$ & 0.0233 \\
\hline $\mathrm{w} R 2[I>2 \sigma(I)]$ & 0.0558 \\
\hline$R_{1}$ [all data] & 0.0415 \\
\hline $\mathrm{w} R 2$ [all data] & 0.0595 \\
\hline Residual density/e $\AA^{-3}$ & $-0.613<3.016$ \\
\hline
\end{tabular}


indicated carbon monoxide coordination to an electron-poor metal centre. These values were very similar to the corresponding values reported for an electron-poor trifluoromethyl phosphine pincer compound (1.114(6) $\AA$, 1.964(4) $\AA$, and $2143 \mathrm{~cm}^{-1}$ respectively), ${ }^{58}$ with a sufficiently shorter $\mathrm{C}-\mathrm{O}$ bond and longer $\mathrm{Pd}-\mathrm{C}$ bond than in the more strongly $\pi$-backbonding isopropyl phosphine pincer complex (1.131(4) $\AA$, 1.919(3) $\AA$, and $\left.2080 \mathrm{~cm}^{-1}\right) .{ }^{137}$ In this case, these results confirmed the strong $\mathrm{C}-\mathrm{O}$ bond suggested by IR spectroscopy. However, care must be taken when comparing small changes in X-ray crystal data, as the solid state measurement reflects the average distribution of electron density within the crystal lattice, and hence is susceptible to disorder and defects.
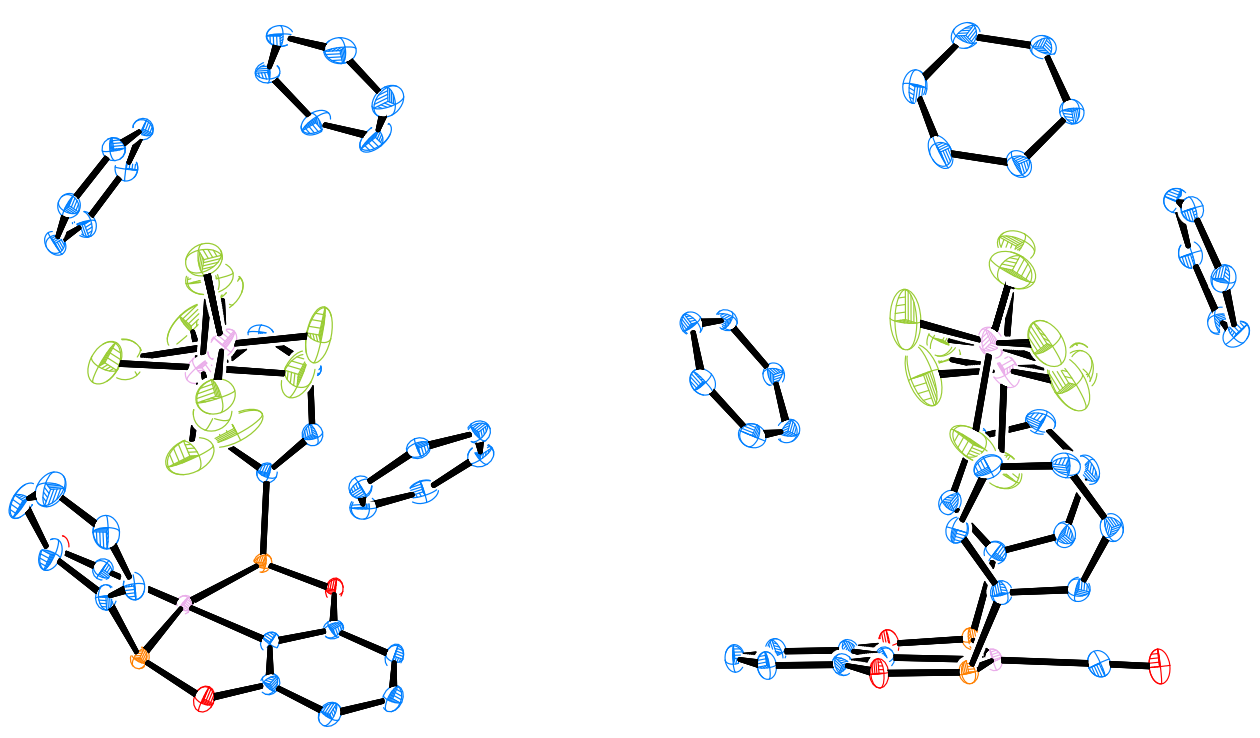

Figure 4.4 ORTEP diagram of $[(\mathrm{POCOP}) \mathrm{Pt}(\mathrm{CO})]\left[\mathrm{SbF}_{6}\right](\mathbf{3 1})$ showing two view of the packing of the hexafluoroantimonate anion (50\% probability thermal elipsoids). Hydrogen atoms, aromatic fluorines, and downward-facing pentafluorophenyl substituents on the phosphorus atoms omitted for clarity. Aromatic rings surrounding the anion represent pentafluorophenyl substituents of adjacent molecules of $\mathbf{3 1}$.

The hexafluoroantimonate counterion in $\mathbf{3 1}$ displayed some disorder, which could be modelled with two superimposed and slightly offset $\mathrm{SbF}_{6}$ octahedra, that refined to adopt an approximately 40:60 occupancy ratio. In the solid state structure the anion occupies a void in which it sits almost directly above the coordination plane of the platinum, enclosed by two pentafluorophenyl rings of the same molecule, as well as three pentafluorophenyl rings from adjacent molecules of $\mathbf{3 1}$ (Figure 4.4). The Pt ... F distance is 3.529(6) A, slightly larger than the sum of the van der Waals radii for platinum and fluorine $(3.32 \AA)$, indicating that there is not likely to be any significant interaction between the electron-poor platinum centre and electron rich counterion. A similar, slightly shorter Pt . . F distance of 3.333(7) A was observed in the crystal structure of a similar, trifluoromethyl-substituted platinum pincer 
complex, ${ }^{58}$ which suggested that such a packing arrangement may be favoured in the solid state for such structures.

All of the palladium carbonyl compounds underwent gradual loss of bound carbon monoxide, as has been noted in the literature for similar palladium species. ${ }^{52,135,136}$ The completely decarbonylated adduct of 35, [(PCCCP)Pd] $\left[\mathrm{SbF}_{6}\right](\mathbf{3 9})$ was isolated in a spectroscopically pure state, having gradually formed over the course of months under ambient conditions. Infrared spectroscopy of $\mathbf{3 9}$ revealed a spectrum almost identical to that of the carbonyl complex 35, with the absence of the $\mathrm{C}-\mathrm{O}$ stretch at $2148 \mathrm{~cm}^{-1}$. The $\mathrm{Sb}-\mathrm{F}$ stretch of the counterion was observed at $665 \mathrm{~cm}^{-1}$, which confirmed that the product remained ionic (Figure 4.5).

Solid samples of $\mathbf{3 4}$ and $\mathbf{3 6}$ had also undergone loss of carbon monoxide under ambient conditions, with the decarbonylated species 38 and 40 comprising significant amounts of the material. Characterisation of the new species 38, 39, and 40 by NMR spectroscopy revealed that all species displayed ${ }^{1} \mathrm{H},{ }^{31} \mathrm{P}$ and ${ }^{19} \mathrm{~F} \mathrm{NMR}$ signals shifted upfield from those of the parent palladium carbonyl species. The ${ }^{1} \mathrm{H}$ and ${ }^{19} \mathrm{~F}$ signals of both species, along with the $\mathrm{P}^{t} \mathrm{Bu}_{2}{ }^{31} \mathrm{P} \mathrm{NMR}$ signal of 40 , also appeared downfield of the corresponding palladium chloride species, giving the impression that these compounds represented almost a spectroscopic 'average' between the $[(\mathrm{PCP}) \mathrm{PdCl}]$ and $[(\mathrm{PCP}) \mathrm{Pd}(\mathrm{CO})]^{+}$species. This effect has previously been observed in the spectroscopic data of PCP pincer chlorides subjected to halide abstraction. ${ }^{139}$

Carbon-13 NMR data of $\mathbf{3 9}$ indicated that no carbon-containing species were bound in the coordination site vacated by the carbon monoxide, while the ${ }^{19} \mathrm{~F}$ NMR spectrum displayed only the three sharp peaks in a 2:1:2 ratio expected for the freely rotating $\mathrm{C}_{6} \mathrm{~F}_{5}$ groups, ruling out stabilisation of the decarbonylated species by longlived $\mathrm{C}-\mathrm{F} \cdots \mathrm{Pd}$ interactions. The similarity in chemical shift of the ortho-fluorine environments between 35 and the decarbonylated complex $39\left(\delta_{\mathrm{F}}=-128.7 \mathrm{ppm}\right.$ and -129.7 ppm respectively) also indicated that there was no rapid, fluxional interaction between the metal and the ortho-fluorine atoms. No signals attributed to the hexafluoroantimonate counterion were observed in the ${ }^{19} \mathrm{~F}$ NMR spectrum; however, as antimony possesses two quadrupolar NMR active isotopes $\left({ }^{121} \mathrm{Sb}\right.$ and ${ }^{123} \mathrm{Sb}$ ), hexafluoroantimonate anions often appear as extremely broad signals and may be hard to detect by ${ }^{19} \mathrm{~F}$ NMR spectroscopy. Thus it is difficult to rule out stabilisation of these decarbonylated species by hexafluoroantimonate coordination. Infrared spectroscopy did not provide evidence either for or against anion coordination - while the single band for the $\mathrm{Sb}-\mathrm{F}$ stretch observed at $665 \mathrm{~cm}^{-1}$ may indicate anion coordination is unlikely (Figure 4.5), a single "broad asymmetric ab- 


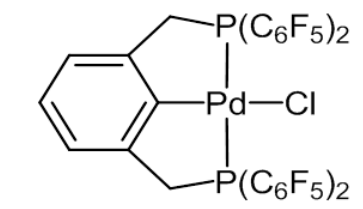

$\downarrow \begin{aligned} & \mathrm{AgSbF} \\ & \mathrm{CO}\end{aligned}$

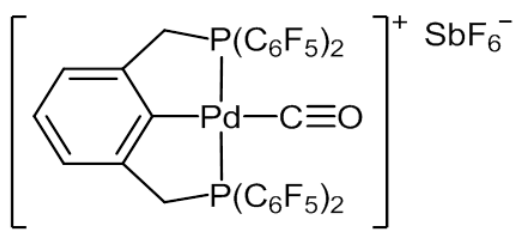

co $\|_{2}$

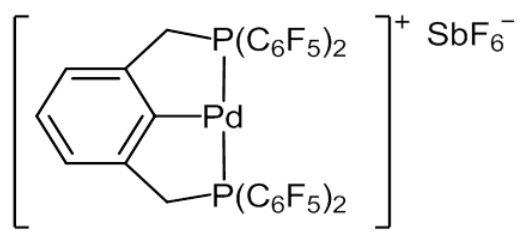

39

35
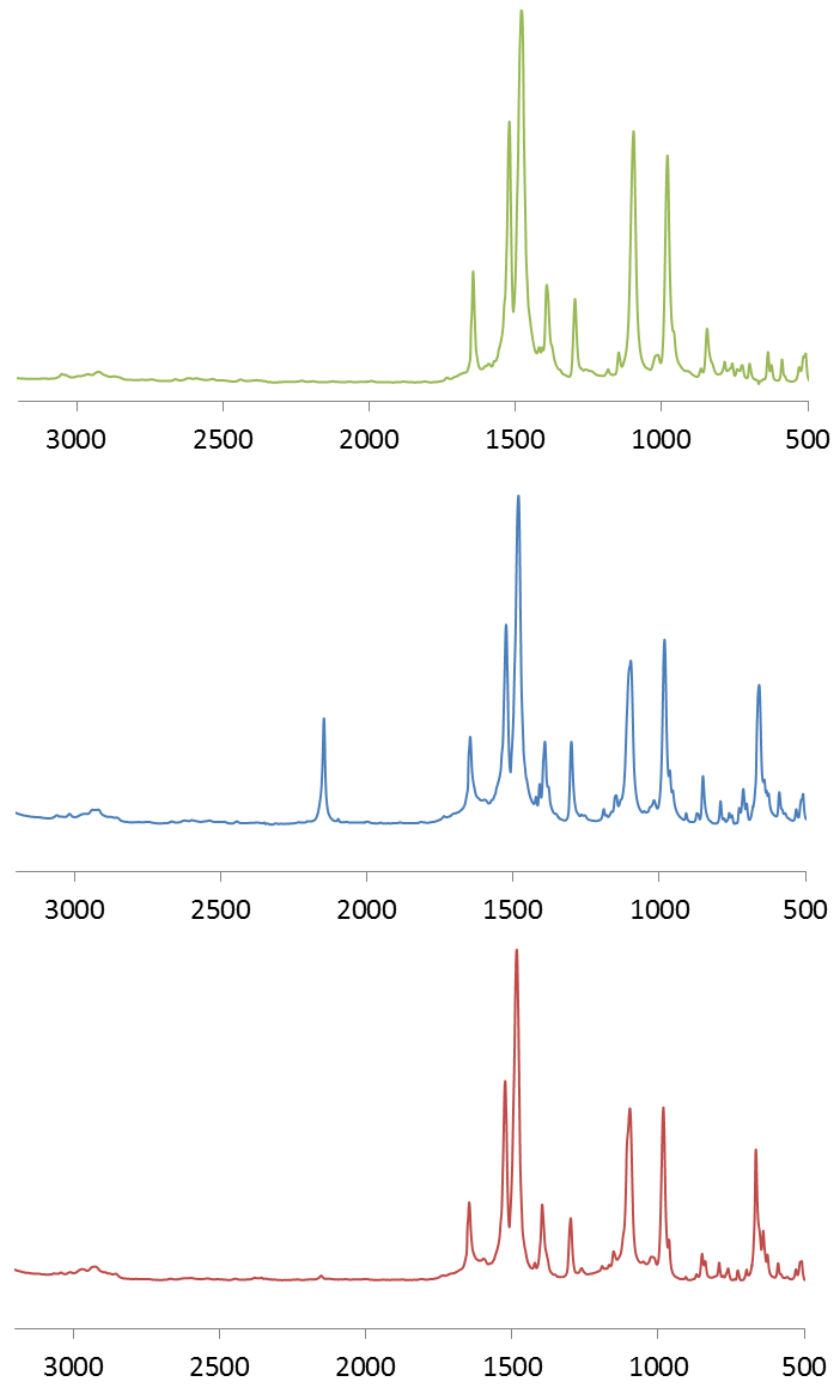

Figure 4.5 IR spectra showing carbonylation of 29 and subsequent decarbonylation of 35. The $\mathrm{C}-\mathrm{O}$ stretch of the carbonyl complex appears at $2148 \mathrm{~cm}^{-1}$, and the $\mathrm{SbF}$ stretch of the hexafluoroantimonate counterion is observed around $650 \mathrm{~cm}^{-1}$ for both of the cationic species, but not the starting material. 
sorption" at $660 \mathrm{~cm}^{-1}$ has been observed in an instance where hexafluoroantimonate coordination has been proven by X-ray crystallography. ${ }^{140}$

To further investigate the possibility of anion coordination to the decarbonylated palladium centre, the tetrafluoroborate salt of the carbonyl $[(\mathrm{PCCCP}) \mathrm{Pd}(\mathrm{CO})]\left[\mathrm{BF}_{4}\right]$ and decarbonylated species $[(\mathrm{PCCCP}) \mathrm{Pd}]\left[\mathrm{BF}_{4}\right]$ were synthesised, to enable the ${ }^{19} \mathrm{~F}$ NMR chemical shifts of the anion to be established. Fluorine NMR spectroscopy did not reveal any significant differences between the anion in the carbonyl complex $\mathbf{3 5}$ and the decarbonylated complex 39, as the anion appeared as either a broad singlet or two sharp singlets in a $1: 4$ ratio (due to ${ }^{10} \mathrm{~B}$ and ${ }^{11} \mathrm{~B}$ ) around $\delta_{\mathrm{F}}=-153 \mathrm{ppm}$ for both species. Free $\mathrm{BF}_{4}{ }^{-}$has been observed at $\delta_{\mathrm{F}}=-151 \mathrm{ppm}$ and coordinated $\mathrm{BF}_{4}{ }^{-}$at $-163 \mathrm{ppm},{ }^{141}$ therefore this data indicated that the decarbonylated species may possess a slight degree of metal-anion interaction in solution at room temperature, but the tetrafluoroborate anion in both 35 and 39 can be regarded as non-coordinating.

In order to determine whether small amounts of water were coordinating to the palladium centre, a solution of the decarbonylated species 39 in dichloromethane- $d_{2}$ was treated with $2 \mu \mathrm{L} \mathrm{D} \mathrm{D}_{2} \mathrm{O}$ (approximately ten equivalents) and warmed gently to promote $\mathrm{H}_{2} \mathrm{O} / \mathrm{D}_{2} \mathrm{O}$ exchange. Analysis of the sample by NMR spectroscopy did not reveal any discernible changes resulting from treatment with $\mathrm{D}_{2} \mathrm{O}$, and IR spectroscopy did not show the expected O-D stretch around $2400-2700 \mathrm{~cm}^{-1}$. Thus it is unlikely that $\mathrm{H}_{2} \mathrm{O}$ occupies the vacant coordination site generated upon loss of carbon monoxide in solution. However, IR spectroscopy performed on solid samples of $\mathbf{3 5}$ that had stood for weeks under ambient conditions revealed a drastic decrease in the intensity of the $\mathrm{C}-\mathrm{O}$ stretch, along with the appearance of a sharp peak at $3672 \mathrm{~cm}^{-1}$. This was attributed to the presence of coordinated $\mathrm{H}_{2} \mathrm{O}$, indicating that adventitious water took the place of carbon monoxide when this decarbonylation occured in the solid state. As there was evidence for the decarbonylated species 39 binding water in the solid state but not in solution, it is likely that in solution the "vacant" coordination site is occupied by solvent molecules. However, this was unable to be conclusively established by NMR or infrared spectroscopy.

Quantitative regeneration of each of the palladium carbonyl complexes 34, 35, and 36 was achieved by passage of carbon monoxide through dichloromethane- $d_{2}$ solutions of the decarbonylated species for 15 minutes at room temperature. The carbonylation was found to be reversible, with displacement of carbon monoxide from the metal centre observed upon passage of inert gas (argon, nitrogen, or methane)

*As the $\mathrm{O}-\mathrm{H}$ stretch for $\mathrm{H}_{2} \mathrm{O}$ would be expected around $3500-3700 \mathrm{~cm}^{-1}$, Hooke's Law predicts that on doubling the mass of the hydrogen atom (upon deuterium substitution) the stretching frequency will decrease by a factor of $\sqrt{2}$, therefore appearing in the vicinity of $2400-2700 \mathrm{~cm}^{-1}$. 

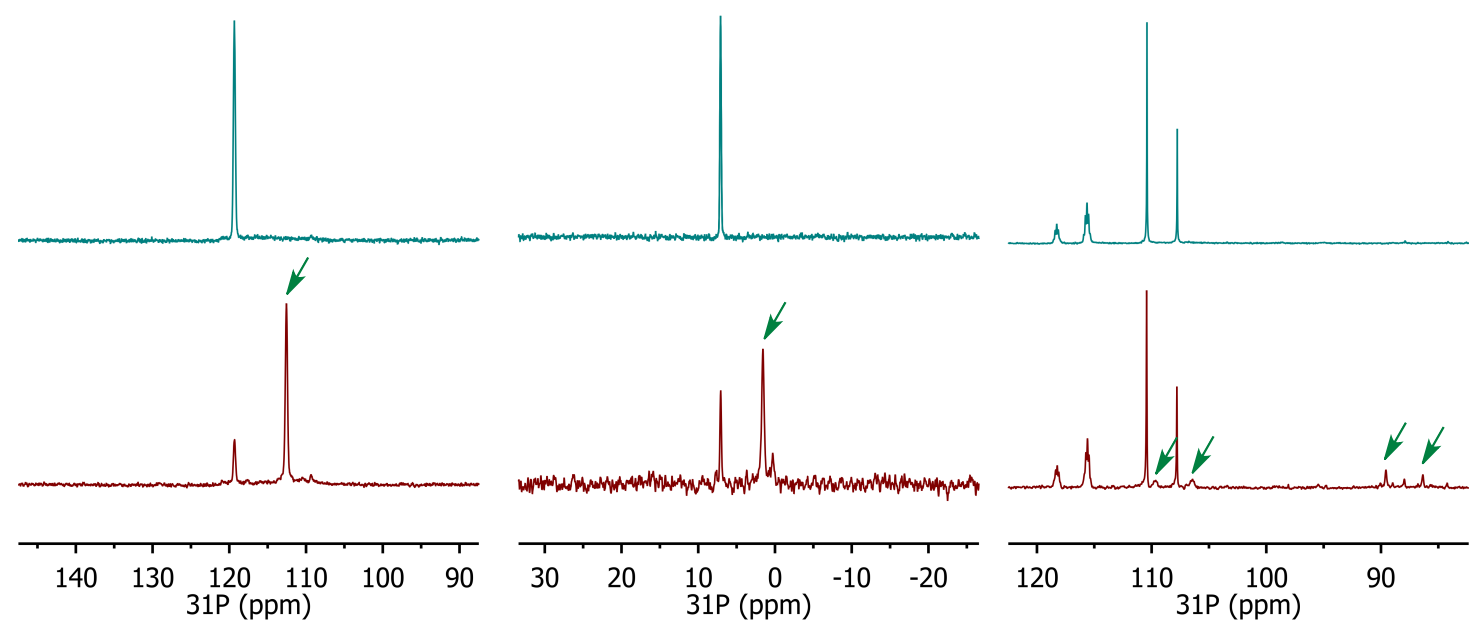

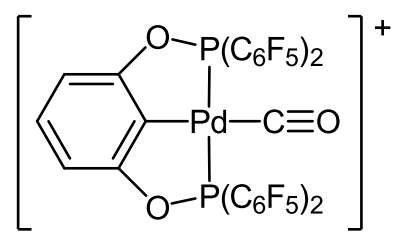

34

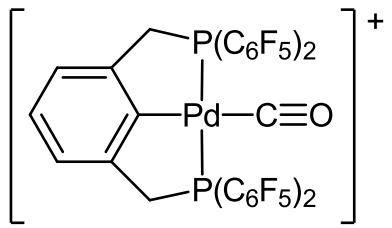

35

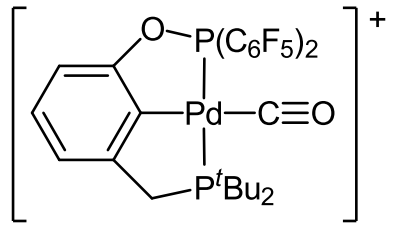

36

$$
v(\mathrm{C}-\mathrm{O})=2170 \mathrm{~cm}^{-1}
$$

$$
v(\mathrm{C}-\mathrm{O})=2148 \mathrm{~cm}^{-1}
$$$$
v(\mathrm{C}-\mathrm{O})=2140 \mathrm{~cm}^{-1}
$$

Figure 4.6 Phosphorus-31 NMR spectra showing the decarbonylation of compounds 34,35 , and 36 in dichloromethane- $d_{2}$. Nitrogen was passed through solution for 30 minutes between obtaining top and bottom spectra. Resonances attributed to decarbonylated products are indicated by an arrow.

through these same solutions. The decarbonylated species were seen to slowly decompose over time in solution even under an inert atmosphere, as broad resonances appeared in the ${ }^{1} \mathrm{H}$ NMR spectra, accompanied by a decrease in the signal to noise ratio and the appearance of dark precipitate in solution. As previously mentioned, palladium pincer carbonyl complexes have been observed to undergo decarbonylation, however this is the first report of reversible carbon monoxide uptake for these species. The Brookhart research group has reported an electron-poor iridium PCP pincer complex capable of undergoing exchangeable binding of small gaseous molecules in the solid state, ${ }^{46}$ but in this species the carbon monoxide is unable to be displaced.

The ease of decarbonylation of each of the carbonyl complexes 34,35 , and 36 was investigated by bubbling nitrogen through a dichloromethane- $d_{2}$ solution of the carbonyl compound for 30 minutes, then assessing the proportion of decarbonylated species present by NMR spectroscopy. The ease of carbonyl displacement for each species was in broad agreement with the IR stretching frequency of the $\mathrm{C}-\mathrm{O}$ bond; the larger the $\mathrm{C}-\mathrm{O}$ stretching frequency, the more readily the decarbonyla- 
tion occurred (Figure 4.6). While there existed a moderate difference between $\mathrm{C}-\mathrm{O}$ stretching frequencies of the POCOP (34) and PCCCP (35) carbonyl complexes $\left(\nu(\mathrm{C}-\mathrm{O})=2170\right.$ and $2148 \mathrm{~cm}^{-1}$ respectively $)$, both decarbonylation reactions were observed to proceed swiftly (progressing approximately $90 \%$ and $75 \%$ respectively after 30 minutes at room temperature). However, while the more electron-rich POCCP complex 36 possessed a $\mathrm{C}-\mathrm{O}$ stretching frequency close to that of $\mathbf{3 5}$ $\left(\nu(\mathrm{C}-\mathrm{O})=2140 \mathrm{~cm}^{-1}\right)$, its decarbonylation was much less facile, having progressed only about $10 \%$ under identical conditions to those above. Thus it appears that $\mathrm{Pd}-\mathrm{CO} \pi$-backbonding effects play a large part in the stabilisation of these carbonyl complexes; decarbonylation was facile for compounds expected to possess negligible backbonding (34 and $\mathbf{3 5}$ ), but occurred significantly more slowly for the most electron-rich compound, which is most capable of $\pi$-backbonding $\mathbf{3 6}$.

\subsubsection{Methylation of Pincer Chloride Complexes}

As the platinum methyl complexes [(PCP)PtMe] could not be synthesised directly from $\left[\mathrm{PtMe}_{2}(\mathrm{hex})\right]$, methylation of the pincer chloride complexes was examined. Methylation of [(PCCCP)PtCl] pincer complexes has previously been reported using methyllithium for electron-rich complexes, ${ }^{142}$ and methylmagnesium bromide for electron-poor complexes. ${ }^{58}$

Methylation reactions were first trialled on a small scale with the most electronpoor compound [(POCOP)PtCl] (25). Reaction of 25 with methylmagnesium iodide ( $0.5 \mathrm{M}$ solution in ether) was seen to produce a new species $4 \mathbf{1 1}$, which had spectroscopic data similar to the starting material and the anticipated methyl complex $\left(\delta_{\mathrm{P}}=109.2 \mathrm{ppm},{ }^{1} J_{\mathrm{Pt}-\mathrm{P}}=3541 \mathrm{~Hz}\right)$, but lacked the expected platinum-methyl resonance in the ${ }^{1} \mathrm{H}$ NMR spectrum. Analysis of 41 by HRMS was unsuccessful, as only peaks due to degradation of the ligand were observed, making it difficult to confirm a whether a methyl or an iodide was coordinated to the metal centre. However, the analogous POCCP complex [(POCCP)PtI $(42)$ was synthesised with methylmagnesium iodide in an identical manner, and confirmed as the platinum iodide species by HRMS, with the $[\mathrm{M}+\mathrm{Na}]^{+}$ion detected at $959 \mathrm{amu}$. Comparison of spectroscopic data between $\mathbf{4 1}$ and $\mathbf{4 2}$ indicated that the POCOP complex $\mathbf{4 1}$ was also the platinum iodide complex [(POCOP $) \mathrm{PtI}]$. As no traces of the desired methyl complex were detected in either of the reaction mixtures with methylmagnesium iodide, this indicated that the Grignard reagent was not a strong enough methylating agent to facilitate the formation of these platinum-methyl complexes. 
Reactions between 25 and the stronger methylating agent methyllithium (1.2 $\mathrm{M}$ in ether) were observed to produce the desired methyl compound [(POCOP)PtMe] (43; $\left.\delta_{\mathrm{P}}=108.5 \mathrm{ppm},{ }^{1} J_{\mathrm{Pt}-\mathrm{P}}=3752 \mathrm{~Hz}\right)$ in only small amounts, along with considerable quantities of byproduct. One byproduct that could be identified was $\mathrm{PMe}\left(\mathrm{C}_{6} \mathrm{~F}_{5}\right)_{2}$. This displayed a quintet resonance in the ${ }^{31} \mathrm{P}$ NMR spectrum, which marked it as an uncoordinated ligand containing the $\mathrm{P}\left(\mathrm{C}_{6} \mathrm{~F}_{5}\right)_{2}$ functionality. The ${ }^{31} \mathrm{P}$ NMR chemical shift at $\delta_{\mathrm{P}}=-53.1 \mathrm{ppm}$, significantly downfield of the phosphinite ligand $\mathbf{1}$ (87.1 ppm), and close to that of the phosphine ligand $2(-46.3 \mathrm{ppm})$, indicated that this product was a phosphine rather than phosphinite. Unfortunately the reaction mixture could not be purified to an extent where the nature of $\mathrm{PMe}\left(\mathrm{C}_{6} \mathrm{~F}_{5}\right)_{2}$ could be unambiguously confirmed by ${ }^{1} \mathrm{H}$ NMR spectroscopy. However, the ${ }^{31} \mathrm{P}$ NMR data is in good agreement with that previously reported for $\mathrm{PMe}\left(\mathrm{C}_{6} \mathrm{~F}_{5}\right)_{2},{ }^{143}$ indicating that this phosphine had likely been formed by nucleophilic attack by the methyl anion on the phosphorus atom of the phosphinite ligand, cleaving the $\mathrm{P}-\mathrm{O}$ bond. The nucleophilic substitution of -OR groups on phosphorus by organolithium reagents has been established in the literature, being employed in the synthesis of phosphine ligands from phosphite, phosphonite, or phosphinite precursors. ${ }^{144,145}$ In this instance, despite coordination of the phosphinite to platinum, the pentafluorophenyl substituents render the phosphorus donor sufficiently nucleophilic that methylation at the phosphorus is competitive with methylation at the metal centre.

As methylmagnesium iodide had proven too weak and methyllithium had proven too strong to effectively methylate $\mathbf{2 5}$, dimethyl zinc was investigated as an alternate methylating agent. A solution of dimethyl zinc in ether was synthesised by the addition of methyl iodide to a suspension of magnesium and zinc powders in diethyl ether, isolating the dimethyl zinc solution by co-distillation with the diethyl ether solvent. Reactions between 25 and dimethyl zinc were observed to produce exclusively the desired platinum methyl complex 43. It appears that for this very electron-poor platinum compound, dimethyl zinc possesses an effective combination of high activity without sufficient nucleophilicity to facilitate $\mathrm{P}-\mathrm{O}$ cleavage, making it an effective methylating agent (Scheme 4.4).

The remaining platinum chloride compounds $\mathbf{2 6}$ and $\mathbf{2 7}$ were subsequently treated with dimethylzinc solutions, in order to establish whether methylation occurred as selectively for these more electron-rich compounds as for $\mathbf{2 5}$. In the case of the PCCCP complex 26, methylation with dimethyl zinc was observed to proceed smoothly, giving quantitative formation of the desired product [(PCCCP)PtMe] (44) after just three hours at room temperature. On standing for two months at room temperature in a stoppered NMR tube, significant conversion (>90\%) from the methyl complex 44 back to the chloride starting material 26 was observed. This unexpected result 


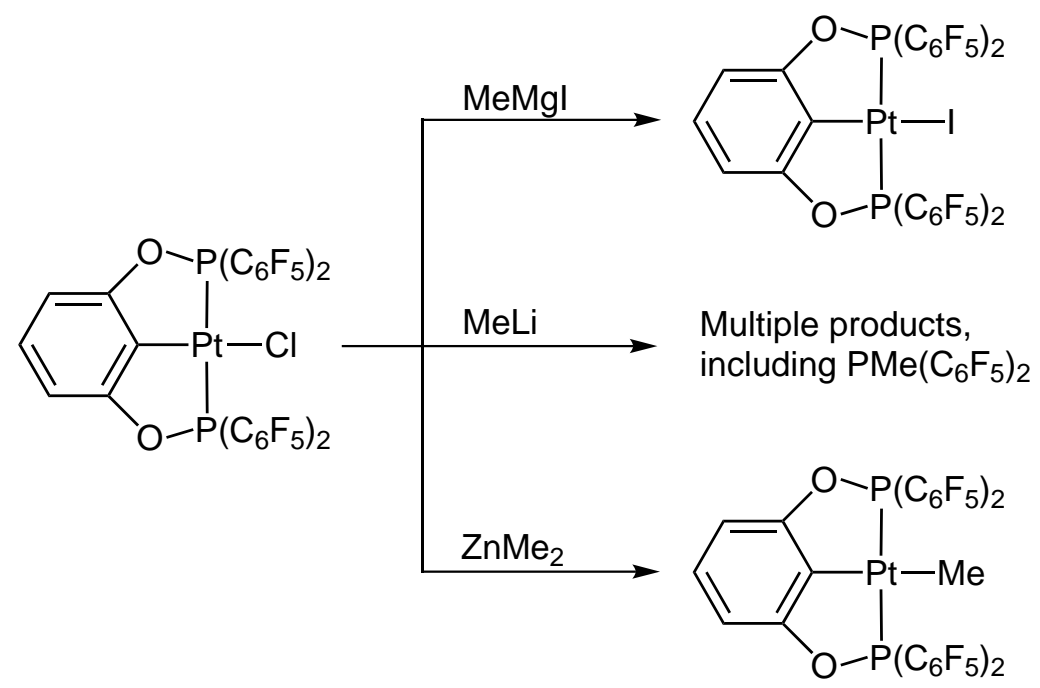

Scheme 4.4 The effect of different methylating agents on compound $\mathbf{2 5}$.

was likely due to diffusion of water through the rubber septum; the action of this water on the zinc chloride would have produced small amounts of $\mathrm{HCl}$ in solution, which protonated the methyl complex, irreversibly eliminating methane and reforming the parent platinum chloride (Scheme 4.5).

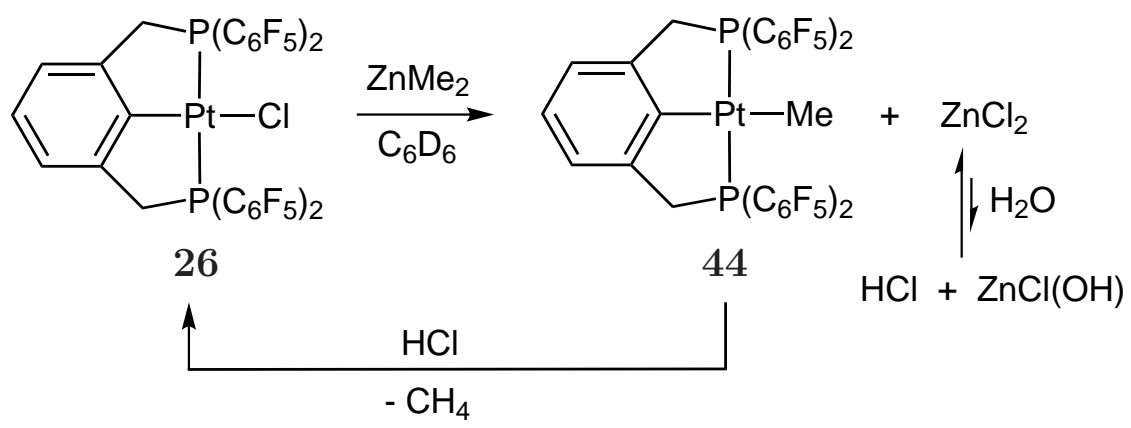

Scheme 4.5 Synthesis of 44 and conversion back to 26 by action of ambient $\mathrm{H}_{2} \mathrm{O}$.

Methylation of the POCCP complex 27 proceeded slowly, attaining only a $50 \%$ conversion to the methyllated product [(POCCP)PtMe] (45) after 44 hours at room temperature. Moderate amounts of a byproduct were also formed (present in about a 1:4 ratio with 45 ), which was revealed to be the platinum iodide complex [(POCCP)PtI $(42)$ on comparison with an authentic sample of $\mathbf{4 2}$. It transpired that the source of iodide in the reaction mixture was methyl iodide, which was present as unreacted starting material from the synthesis of the dimethyl zinc solution, as the methyl iodide possesses a similar boiling point to dimethylzinc $\left(44^{\circ} \mathrm{C}\right.$ and $46{ }^{\circ} \mathrm{C}$ respectively). The iodide complex 42 was observed to be stable in the presence of large (greater than 10-fold) excesses of dimethyl zinc, and did not un- 
dergo methylation even upon heating to $70{ }^{\circ} \mathrm{C}$ for 40 hours.

As reaction of $\mathbf{2 7}$ with methylmagnesium iodide had been observed to result in halide exchange, further methylation of $\mathbf{2 7}$ was attempted with methyllithium. Reaction between $\mathbf{2 7}$ and methyllithium was observed to produce predominantly the methylated product ( $>95 \%$ by NMR spectroscopy) 45 within 30 minutes at room temperature. This was in contrast to reactions between methyllithium and the POCOP complex 25, which yielded a large number of degradation products.

In order to eliminate the possibility of halide exchange in these methylation reactions, a methyl iodide-free dimethyl zinc solution was prepared by repeating the aforementioned synthesis, using dibutyl ether rather than diethyl ether as the solvent. The high boiling point of dibutyl ether $\left(142{ }^{\circ} \mathrm{C}\right)$ enabled neat dimethyl zinc to be isolated by fractional distillation of the reaction mixture, whereby it was diluted with toluene to concentration of approximately $1.5 \mathrm{M}$.

Upscaling the test methylations using the methyl iodide-free dimethyl zinc solution in toluene afforded the POCOP and PCCCP platinum methyl compounds $\mathbf{4 3}$ and 44 in yields of $76 \%$ and $87 \%$ respectively. However, when the reaction of [(POCCP) PtCl] 27 with methyllithium was repeated on a preparative scale (rather than on an NMR scale), NMR spectroscopy indicated that the reaction had produced a complex mixture containing a new product, $\mathbf{4 6}$, as well as smaller amounts of the desired POCCP platinum methyl complex 45, other unidentified minor products, and unreacted starting material 27. It is not clear why this discrepancy in reaction selectivity should occur. Even when carried out with substoichiometric amounts of methyllithium, reaction mixtures contained predominantly the byproduct 46 and starting material. Subsequent treatment of these reaction mixtures with methyllithium did not promote further formation of the byproduct $\mathbf{4 6}$, but led to the formation of a number of unidentified species. Further attempts to isolate 46 from these mixtures were ultimately unsuccessful.

While the byproduct 46 was not isolated, NMR data obtained of the reaction mixtures indicated it was likely to be a $\left[\left(\mathrm{P}^{\mathrm{Me}} \mathrm{OCCP}\right) \mathrm{Pt}\left(\mathrm{C}_{6} \mathrm{~F}_{5}\right)\right]$ complex, in which the methyl nucleophile had attacked the phosphinite group, causing a pentafluorophenyl group to migrate from the phosphorus to the platinum (Figure 4.7). Phosphorus31 NMR spectroscopy revealed 46 possessed the distinctive two doublet signals of metallated complexes of the POCCP ligand 3; however, while the $\mathrm{P}^{t} \mathrm{Bu}_{2}$ group appeared close to that of the starting material $\left(\delta_{\mathrm{P}}=73.8 \mathrm{ppm}\right.$, compared to $70.2 \mathrm{ppm}$ for starting material), the signal for the phosphinite group had been shifted significantly downfield (132.0 ppm, compared to $108.7 \mathrm{ppm}$ for the starting material). 
This large downfield shift was consistent with the $\mathrm{P}-\mathrm{O}$ bond of the phosphinite remaining unbroken, and substitution of the methyl group onto the phosphorus, as alkylphosphinites appear downfield of arylphosphinites. ${ }^{\dagger}$

Incorporation of the methyl nucleophile onto the phosphinite was also indicated by a ${ }^{1} \mathrm{H}-{ }^{31} \mathrm{P}$ HMBC experiment, with a signal at $\delta_{\mathrm{H}}=1.68$ observed to couple solely to the phosphinite group at $\delta_{\mathrm{P}}=132.0 \mathrm{ppm}$. This signal also displayed the expected phosphorus and platinum coupling $\left({ }^{2} J_{\mathrm{P}-\mathrm{H}}=9.5 \mathrm{~Hz},{ }^{3} J_{\mathrm{Pt}-\mathrm{H}}=26.6 \mathrm{~Hz}\right)$ in the ${ }^{1} \mathrm{H}$ NMR spectrum.

The fate of the displaced pentafluorophenyl group was revealed by ${ }^{19} \mathrm{~F}$ NMR spectroscopy, with the appearance of distinctive signals with platinum coupling at $\delta_{\mathrm{F}}=$ -114.3 and $-115.5 \mathrm{ppm}\left({ }^{3} J_{\mathrm{Pt}-\mathrm{F}}=267\right.$ and $230 \mathrm{~Hz}$ respectively $)$. This was consistent with data for a previously reported $\left[(\mathrm{PCP}) \mathrm{Pt}\left(\mathrm{C}_{6} \mathrm{~F}_{5}\right)\right]$ species $\left(\delta_{\mathrm{F}}=-107.2 \mathrm{ppm}\right.$, $\left.{ }^{3} J_{\mathrm{Pt}-\mathrm{P}}=235 \mathrm{~Hz}\right) .{ }^{146}$ Integration of platinum-pentafluorophenyl and phosphoruspentafluorophenyl ${ }^{19} \mathrm{~F}$ NMR resonances confirmed that the two pentafluorophenyl groups were present in a 1:1 ratio. As the new methyl pentafluorophenyl phosphinite was chiral, the tert-butyl groups appeared diastereotopic in the ${ }^{1} \mathrm{H}$ NMR spectrum, as did the ortho and meta fluorines of the $\mathrm{Pt}-\mathrm{C}_{6} \mathrm{~F}_{5}$ group (which experienced restricted rotation). So while the byproduct $\mathbf{4 6}$ could not be unambiguously identified, there was sufficient evidence to indicate that reaction of $\mathbf{2 7}$ with methyllithium results in methylation at the phosphinite, along with pentafluorophenyl migration from the phosphinite to the platinum centre.

A similar reaction, involving methylation at phosphorus followed the migration of an aryl substituent onto a metal centre has been observed by Nakajima and colleagues upon treatment of an iron PNP pincer complex with dimethylmagnesium. ${ }^{147}$ Furthermore, pentafluorophenyl migration from a bis(pentafluorophenyl)phosphine group to a metal centre has been reported to occur on rhodium carbonyl clusters containing $\mathrm{P}\left(\mathrm{C}_{6} \mathrm{~F}_{5}\right)_{2}$ groups. ${ }^{148}$ Mass spectrometry data for the POCCP compounds reported herein also indicated that the $\mathrm{P}-\mathrm{C}$ bond of the bis(pentafluorophenyl)phosphinite group was prone to cleavage. Data obtained for the POCCP methyl complex 45 subsequently synthesised (vide infra) revealed large peaks corresponding to $\left[\mathrm{M}-\mathrm{C}_{6} \mathrm{~F}_{5}+\mathrm{MeOH}\right]^{+}(690 \mathrm{amu})$ and $\left[\mathrm{M}-\mathrm{C}_{6} \mathrm{~F}_{5}+\mathrm{NCMe}\right]^{+}$ (699 amu), as well as the expected peaks due to loss of a methyl group. Mass spectroscopy also indicated that the POCCP chloride complexes [(POCCP $) \mathrm{PtCl}]$, 27, and [(POCCP $) \mathrm{PdCl}], \mathbf{3 0}$, also underwent facile loss of a pentafluorophenyl substituent. For both 27 and 30, significant peaks were observed in the mass spectra

\footnotetext{
${ }^{\dagger}$ An example of this can be observed in the ${ }^{31} \mathrm{P}$ NMR data for [(POCOP)PdI] complexes of Kimura, ${ }^{51}$ where the diphenylphosphinite complex has a $\delta_{\mathrm{P}}=144.0 \mathrm{ppm}$, whereas the diethylphosphinite complex appears downfield at $\delta_{\mathrm{P}}=176.9 \mathrm{ppm}$.
} 

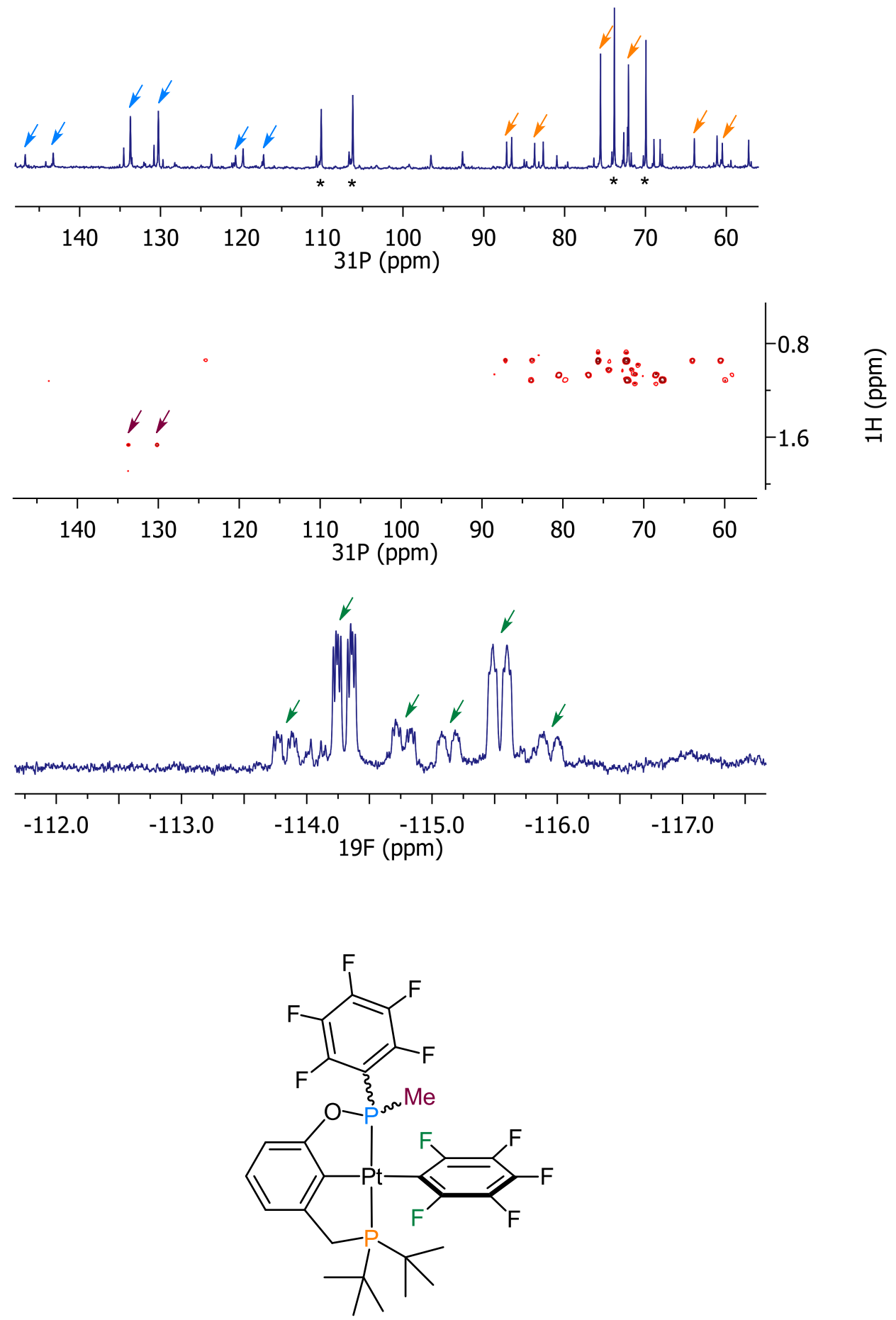

Figure 4.7 Spectroscopic data for byproduct 46, showing, from top to bottom, the ${ }^{31} \mathrm{P}$ NMR spectrum, the ${ }^{1} \mathrm{H}-{ }^{31} \mathrm{P}$ HMBC spectrum, and the ${ }^{19} \mathrm{~F}$ NMR spectrum. The highlighted correlation in the ${ }^{1} \mathrm{H}-{ }^{31} \mathrm{P}$ HMBC spectrum revealed the presence of a methyl group (red) attached to the phosphorus atom of the phosphinite group (blue). Distinctive ${ }^{195} \mathrm{Pt}$ coupling on the ortho-fluorine resonances (green) of a $\mathrm{C}_{6} \mathrm{~F}_{5}$ group in the ${ }^{19} \mathrm{~F}$ NMR indicated it was directly bound to the metal centre. Signals marked with an asterisk denote starting material 27 (peaks due to ${ }^{195} \mathrm{Pt}$ coupling in 27 not indicated). 
at $\mathrm{M}-144 \mathrm{amu}$, corresponding to $\left[\mathrm{M}-\mathrm{C}_{6} \mathrm{~F}_{5}-\mathrm{Cl}+\mathrm{OH}+\mathrm{NCMe}\right]^{+}$. Whilst the formation of these ions occurred under high energy conditions, the HRMS data revealed a predilection for the metallated POCCP ligand 3 to undergo the $\mathrm{P}-\mathrm{C}$ cleavage observed in the formation of the pentafluorophenyl-migration product 46 .

The synthesis of the desired POCCP platinum methyl complex 45 was subsequently achieved by treatment of the parent platinum chloride $\mathbf{2 7}$ with the methyl iodide-free dimethyl zinc solution, as for the syntheses of the other platinum methyl compounds 43 and 44. However, due to the more electron-rich nature of the starting material $\mathbf{2 7}$, the methylation required a large excess of dimethyl zinc and prolonged reaction times (54 hours, compared to five hours for the formation of 43 and 44 ), and afforded the desired compound $\mathbf{4 5}$ in a $63 \%$ yield.

Characterisation of platinum methyl complexes 43,44 , and 45 by ${ }^{13} \mathrm{C}$ NMR spectroscopy revealed the presence of platinum-methyl groups between -12 and -17 ppm, with platinum-carbon coupling constants of around $500 \mathrm{~Hz}$. For the PCCCP and POCCP compounds 44 and 45, carbon C-2 displayed a massive downfield shift of about $25 \mathrm{ppm}$ on methylation. This was consistent with the replacement of an electron-rich chloride for the more poorly donating methyl group. The chemical shift of the C-2 environment of the POCOP complex 43 could not be conclusively determined due to overlap with other signals.

Analogous methylation of the POCOP and PCCCP palladium compounds $\mathbf{2 8}$ and 29 was attempted. Reactions performed with solutions of dimethylzinc in ether in both cases led to the formation of black solutions, while ${ }^{31} \mathrm{P}$ and ${ }^{19} \mathrm{~F}$ NMR spectroscopy indicated the formation of a multitude of unidentified products in each reaction. Reaction between $\mathbf{2 9}$ and methylmagnesium iodide was also attempted, in the hope that the milder Grignard reagent would promote selective methylation without degradation. However, in situ monitoring of methylation reactions attempted with methylmagnesium iodide did not reveal the presence of any palladium-methyl resonances in the ${ }^{1} \mathrm{H}$ NMR; only the appearance of broad, unidentified signals attributed to decomposition products were observed in the NMR spectra. Methylation of these palladium chlorides was not achieved with organometallic reagents; milder routes to methylation may be required for these electron-poor palladium compounds, as they are less robust than their platinum analogues. 


\subsubsection{Protonation of Platinum Methyl Complexes}

As mentioned in Chapter 1, the low temperature protonation of a rhodium PNP pincer complex by the Brookhart research group yielded the first stable metalmethane $\sigma$-complex able to be fully characterised by NMR spectroscopy. ${ }^{25}$ As the [(PCP)PtMe] complexes synthesised were isoelectronic with [(PNP)RhMe] complexes, the protonation reactions of these platinum methyl pincer complexes were examined in an attempt to determine the extent to which the electronic character of the ligand affected the reactivity of the Pt-Me group.
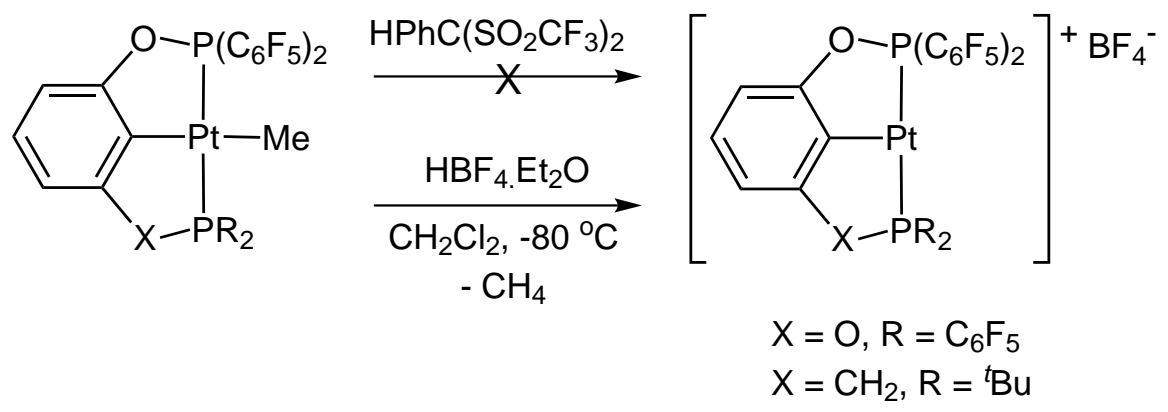

Figure 4.8 Protonation of $\mathbf{4 3}$ and $\mathbf{4 5}$ at low temperature.

The most electron-poor and least electron-poor of the platinum methyl complexes, [(POCOP)PtMe] (43) and [(POCCP)PtMe] (45) respectively, were dissolved in dichloromethane- $d_{2}$, cooled to $-196{ }^{\circ} \mathrm{C}$, and treated with a slight excess of the carbacid $\mathrm{HPhC}\left(\mathrm{SO}_{2} \mathrm{CF}_{3}\right)_{2}$ (Figure 4.8). Each sample was placed in the NMR instrument and allowed to warm to $-80{ }^{\circ} \mathrm{C}$, with the sample monitored continuously by ${ }^{1} \mathrm{H}$ and ${ }^{19} \mathrm{~F}$ NMR spectroscopy. After twenty minutes in the instrument, neither reaction had progressed at all, and samples were withdrawn and maintained at $-78^{\circ} \mathrm{C}$. Each sample in turn was treated with an equimolar amount of tetrafluoroboric acid and immediately placed in the instrument at $-80^{\circ} \mathrm{C}$ for further analysis. NMR spectroscopy of these reaction mixtures showed that both samples had reacted immediately and completely with the acid, with only methane and the cationic $[(\mathrm{PCP}) \mathrm{Pt}]^{+}$detected in each instance. Non-coordinated $\mathrm{BF}_{4}{ }^{-}$was detected in the ${ }^{19} \mathrm{~F}$ NMR spectrum at $\delta_{\mathrm{F}}=-152 \mathrm{ppm}$ for each sample (coordinated $\mathrm{BF}_{4}{ }^{-}$has been observed at $\delta_{\mathrm{F}}=-169 \mathrm{ppm}$ for a similar cationic PCP pincer species ${ }^{139}$ ), ruling out $\mathrm{CH}_{4}$ displacement from the coordination sphere of the metal by $\mathrm{BF}_{4}{ }^{-}$, and indicating that the parent methyl complexes $\mathbf{4 3}$ and $\mathbf{4 5}$ do not interact with any liberated methane at this temperature. Similar to the decarbonylated palladium complexes 38, 39, and 40, the "vacant" coordination site on the metal centre is likely to be occupied by solvent in solution, owing to the instability and high reactivity of 14electron species. 


\subsection{Concluding Remarks}

A major drawback to the synthesis of electron-deficient PCP pincer complexes is the increased energy barrier to ligand metallation for electron-poor ligands. The choice of starting material has a pronounced effect on the ease of synthesis of metallated pincer complexes. Altering the ancillary ligands on $\left[\mathrm{PtCl}_{2} \mathrm{~L}_{\mathrm{n}}\right]$ species was observed to increase the rate of metallation with increasing binding strength of donor ligand. Although this result appeared somewhat counterintuitive, readily displaced ancillary ligands favoured the formation of dimeric intermediates, which increased the barrier to metallation, as they underwent an energy-consuming rearrangement process to allow metallation to occur. With the use of more strongly donating diethyl sulfide ligands in place of 1,5-hexadiene, dimer formation was noticeably diminished and metallation of ligand $\mathbf{1}$ proceeded approximately twice as fast.

A significant increase in the reactivity of the starting material could be achieved by the substitution of an anionic chloride ligand for either a hexamethyldisilazane group or a methyl group. These substituents provided good proton-accepting leaving groups, reducing the reaction times and temperatures required for metallation. However, as these starting materials required more complicated syntheses than the simple platinum dichlorides, the increase in reactivity they provided may not offer a practical advantage in terms of time saved or overall atom economy.

The electronic nature of the ligand also played a large part in the ease of forming PCP pincer complexes. Metallation was seen to be significantly more facile for less electron-poor ligands, due to their increased ability to donate electron density into the $\sigma^{*}$-antibonding orbital of the $\mathrm{C}-\mathrm{H}$ bond being cleaved. However, the rate of metallation was not proportional to the yields of each complex obtained; it was observed that $\mathrm{P}-\mathrm{O}$ bonds were unstable for prolonged periods under metallation conditions, and the fewer $\mathrm{P}-\mathrm{O}$ bonds a ligand possessed the greater the yield of the metallated complex.

With the successful synthesis of the platinum and palladium $[(\mathrm{PCP}) \mathrm{MCl}]$ pincer compounds, these were subjected to halide abstraction and treatment with carbon monoxide to produce the metal carbonyl species $[(\mathrm{PCP}) \mathrm{M}(\mathrm{CO})]^{+}$. Examination of the carbonyl stretching frequency by infrared spectroscopy confirmed that POCOP complexes were the most electron-poor, and POCCP complexes the least electronpoor. It also revealed that a number of these compounds possessed $\mathrm{C}-\mathrm{O}$ stretching frequencies greater than that of free carbon monoxide, and were among the most electron-deficient pincer compounds known to date. Crystallographic characterisation of $[(\mathrm{POCOP}) \mathrm{Pt}(\mathrm{CO})]\left[\mathrm{SbF}_{6}\right]$ revealed the expected tridentate, planar coordi- 
nation of the ligand, as well as a short carbonyl $\mathrm{C}-\mathrm{O}$ distance arising from the electron-deficient metal centre.

Whilst the platinum carbonyl complexes proved to be indefinitely stable, the palladium carbonyl species underwent gradual loss of carbon monoxide over time in the solid state. This CO loss could be encouraged by the passage of inert gas though solutions of the palladium carbonyl, with the carbonyl complex regenerated upon the introduction of carbon monoxide into solution. This reversible carbonyl binding was observed be more facile with decreasing electron density on the metal centre.

Methylation of the $[(\mathrm{PCP}) \mathrm{PtCl}]$ species was achieved via the use of dimethyl zinc; reactions with methyl magnesium iodide led to halide exchange and the formation of platinum iodide species, while methyllithium led to decomposition of the electron-poor complexes. The methylation of $[(\mathrm{POCCP}) \mathrm{PtCl}]$ with methyllithium was observed to be facile and selective on an NMR scale, but upon scaling up led to nucleophilic attack on the phosphinite donor, displacing a pentafluorophenyl group which migrated to the metal to form the unusual byproduct $\left[\left(\mathrm{P}^{\mathrm{Me}} \mathrm{OCCP}\right) \mathrm{Pt}\left(\mathrm{C}_{6} \mathrm{~F}_{5}\right)\right]$. In all cases treatment of the corresponding palladium pincer complexes with methylating agents led to decomposition.

Ligand electronic effects play a large part in dictating the synthesis and reactivity of platinum and palladium PCP pincer complexes. Species with highly electronwithdrawing ligands are significantly harder to synthesise than their more electronrich analogues, and are more susceptible to decomposition or unwanted side reactions during reactions with nucleophiles. However, possessing a highly electron-deficient metal centre was seen to be a boon for the palladium pincer carbonyl species, as it favoured the reversible binding of carbon monoxide, and also allowed the electrondeficient platinum chlorides to react rapidly and selectively with dimethyl zinc. 


\section{Chapter 5}

\section{Synthesis and Reactivity of PNP Pincer Complexes}

In Chapters 3 and 4, it was observed that the incorporation of electron-withdrawing bis(pentafluorophenyl)phosphine substituents into the PCP pincer framework resulted in significant energetic barriers to ligand metallation and pincer complex formation. Metallation reactions typically required prolonged thermolysis, and proceeded through a number of stable oligomeric intermediates. To circumvent the need for ligand $\mathrm{C}-\mathrm{H}$ activation, the synthesis of PNP pincer complexes was investigated. The pyridyl backbone of PNP pincer ligands possesses a nitrogen donor with a lone pair of electrons, meaning the only bond broken in the metallation reaction is between the metal centre and the ligand being displaced by the pyridyl group. Without the requirement for scission of a strong $\mathrm{C}-\mathrm{H}$ bond, tridentate pincer coordination should be achieved under significantly milder conditions with electron-poor PNP ligands than for the analogous PCPH ligands.

While it may assist in pincer complex formation, moving from a phenyl to a pyridyl ligand backbone will have a pronounced effect on the character of the metal complex produced. When metallated, PCP pincer ligands are nominally monoanionic, due to the deprotonated aromatic carbon, while PNP ligands carry no formal charge. This means that when identical metal complexes are prepared with PCP and PNP ligands, the complex with the PNP ligand will carry an extra +1 charge. Moreover, because the pincer coordination motif places the donor atom of the ligand backbone trans to an available coordination site on the metal centre, PCP and PNP complexes will possess different trans effects. Computational studies on platinum complexes have shown that the donor atom trans to the active site has a large influence on the energy barrier for $\mathrm{C}-\mathrm{H}$ activation reactions, with more electronegative donor atoms 
producing a lower energy barrier. ${ }^{149,150}$ Therefore, PNP pincer complexes may be better suited for small small molecule activation reactions than PCP pincer complexes.

\subsection{Synthesis of Platinum PNP Complexes}

As the ease of PCP pincer complex formation was rigorously investigated with a number of platinum chloride starting materials, the coordination chemistry of the PNNNP and PONOP pincer ligands $\mathbf{1 0}$ and $\mathbf{1 1}$ was initially investigated with the same platinum precursors. This would enable a direct comparison of the ease of pincer complex formation between the electron-poor PCPH and PNP pincer ligands, which would also help to explore whether significant barriers to electron-poor pincer complex formation existed, other than $\mathrm{C}-\mathrm{H}$ activation.

Previous reports indicated that the formation of electron-rich $[(\mathrm{PNP}) \mathrm{PtX}]^{+}$species occured in a matter of hours from $\left[\mathrm{PtX}_{2}\right.$ (diene)] precursors at room temperature in dichloromethane. ${ }^{78}$ Ligands $\mathbf{1 0}$ and $\mathbf{1 1}$ were reacted with $\left[\mathrm{PtCl}_{2}(\mathrm{COD})\right]$ at room temperature in dichloromethane- $d_{2}$, with reaction mixtures monitored in situ by NMR spectroscopy. Results showed that reactions with the PNNNP pincer ligand 10 proceeded smoothly but slowly, with the quantitative formation of the pincer complex $[(\mathrm{PNNNP}) \mathrm{PtCl}]^{+}(\mathbf{4 7})$ observed after 22 hours at room temperature. Reactions with the PONOP ligand $\mathbf{1 1}$ proceeded extremely slowly - after 48 hours at room temperature, starting materials still comprised the greater part of the reaction mixture.

The $[(\mathrm{PNNNP}) \mathrm{PtCl}]^{+}$complex 47 was isolated as the chloride salt upon upscaling of the initial exploratory reaction (Scheme 5.1). Mass spectrometry data confirmed the formulation of $\mathbf{4 7}$ as the cationic PNP pincer complex [(PNNNP)PtCl $]^{+}$, with the $[\mathrm{M}]^{+}$ion detected at $1066 \mathrm{amu}$. The NMR spectra of this compound displayed the expected three environments in the ${ }^{1} \mathrm{H}$ and ${ }^{19} \mathrm{~F}$ NMR spectra. The ${ }^{31} \mathrm{P}$ NMR spectrum displayed a singlet resonance at $\delta_{\mathrm{P}}=30.6 \mathrm{ppm}$ (free ligand $\mathbf{1 0}$ appeared at $\delta_{\mathrm{P}}=-10.8 \mathrm{ppm}$ ), with platinum-phosphorus coupling consistent with a mutually trans arrangement of phosphorus donors $\left({ }^{1} J_{\mathrm{Pt}-\mathrm{P}}=3153 \mathrm{~Hz}\right)$, as expected upon pincer coordination.

The unexpectedly low reactivity of the PONOP ligand $\mathbf{1 1}$ could be attributed in part to it being significantly more electron-poor than the PNNNP ligand 10. This differ- 


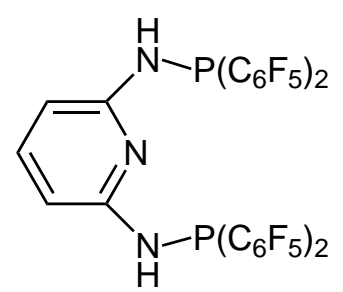

10

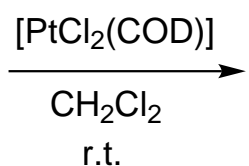

r.t.

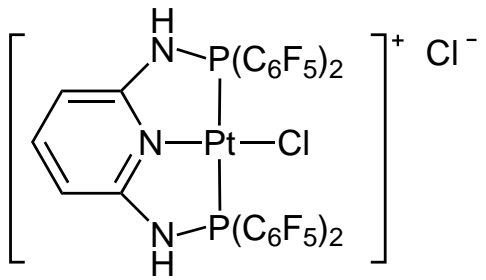

47
$37 \%$ yield

Scheme 5.1 Synthesis of the platinum PNNNP pincer compound 47.

ence in electronic character has previously been observed for PCP pincer complexes - carbonyl stretching frequencies reported for POCOP and PNCNP complexes with identical substituents have indicated that complexes of phosphoramines are significantly more electron-rich than those of analogous phosphinites. ${ }^{52}$ This observation was also supported by the ${ }^{19} \mathrm{~F}$ NMR $\Delta_{\delta \mathrm{m}, \mathrm{p}}$ values for these PNP ligands $(10.6 \mathrm{ppm}$ for 10, $12.2 \mathrm{ppm}$ for 11), which demonstrated that the phosphorus donors of the PNNNP ligand 10 were more electron-rich than those of the PONOP ligand 11.

In order to promote the pincer coordination of the more electron-poor ligand $\mathbf{1 1}$, the synthesis of $[(\mathrm{PONOP}) \mathrm{PtCl}]^{+}$was subsequently attempted in acetone, as the more polar solvent should better stabilise the charge developed during the formation of the desired cationic PNP pincer complex (according to the Hughes-Ingold Rules). ${ }^{151}$ Reactions were performed on an NMR scale between ligand $\mathbf{1 1}$ and platinum dichlorides $\left[\mathrm{PtCl}_{2}(\right.$ hex $\left.)\right]$ and $\left[\mathrm{PtCl}_{2}\left(\mathrm{SEt}_{2}\right)_{2}\right]$ in acetone- $d_{6}$. Analysis of the reaction mixtures by NMR spectroscopy revealed that both reactions produced the same product (48). However, reactions performed with $\left[\mathrm{PtCl}_{2}\left(\mathrm{SEt}_{2}\right)_{2}\right]$ proceeded more selectively than reactions with $\left[\mathrm{PtCl}_{2}(\mathrm{hex})\right]$, resulting in quantitative formation of the new species, 48, in solution after 15 hours at room temperature.

Unfortunately, the NMR data obtained for compound 48 indicated that it was not the desired PNP pincer complex. For all of the pincer complexes reported herein, the ${ }^{31} \mathrm{P}$ NMR chemical shift of the phosphorus donors was observed downfield from that of the free ligand, due to the displacement of an electronegative chloride ligand upon backbone coordination. In 48 , the ${ }^{31} \mathrm{P}$ resonance was shifted significantly upfield from that of the free ligand (at $\delta_{\mathrm{P}}=29.0 \mathrm{ppm}$, compared to $70.2 \mathrm{ppm}$ for ligand 11). Together with the observation of a large platinum-phosphorus coupling constant $\left({ }^{1} J_{\mathrm{Pt}-\mathrm{P}}=4149 \mathrm{~Hz}\right)$, this was consistent with compound 48 being the cis-bridged oligomeric species cis-[(PONOP) $\left.\mathrm{PtCl}_{2}\right]_{\mathrm{x}}$ (Scheme 5.2). For the analogous platinum POCOPH coordination chemistry discussed in Chapter 3 , the ${ }^{31} \mathrm{P}$ NMR resonances of phosphorus donors of unmetallated ligands coordinated trans to a chloride ligand (in cis- $\left[(\mathrm{POCOPH})_{2} \mathrm{PtCl}_{2}\right], \mathbf{1 8}$, and cis,trans-[(POCOPH$\left.\left.) \mathrm{PtCl}_{2}\right]_{2}, \mathbf{1 9}\right)$ were shifted upfield from the free ligand by about $34 \mathrm{ppm}$, and displayed platinum-phosphorus 
coupling of about $4500 \mathrm{~Hz}$. This was consistent with the upfield shift from starting material of $41.2 \mathrm{ppm}$ and platinum-phosphorus coupling of $4149 \mathrm{~Hz}$ observed for 48 , especially as PNP complexes have been observed to display platinum-phosphorus couplings around 400-500 $\mathrm{Hz}$ less than their PCP analogues.* Analogy with the coordination chemistry of the POCOPH ligand also suggested that the oligomer 48 is a dimer. Analysis of this oligomer by mass spectrometry gave only signals attributed to degradation of $\mathbf{4 8}$ inside the instrument, and so the nuclearity of $\mathbf{4 8}$ was not established. Reaction mixtures containing the oligomer 48 in acetone were heated to reflux for 48 hours in an attempt to promote the formation of the tridentate PONOP pincer complex. However, compound 48 appeared stable in acetone solutions up to $60{ }^{\circ} \mathrm{C}$, and no further reaction was observed.



Scheme 5.2 Formation of the oligomeric platinum PONOP compound 48. Synthesis of the corresponding PONOP pincer complex was not observed even upon prolonged thermolysis.

To facilitate the formation of the PONOP pincer complex, reactions were also undertaken between the PONOP ligand 11 and $\left[\mathrm{PtCl}_{2}(\mathrm{NCMe})_{2}\right]$ in acetonitrile- $d_{3}$, as well as between 11, $\left[\mathrm{PtCl}_{2}\left(\mathrm{SEt}_{2}\right)_{2}\right]$ and $\mathrm{NaSbF}_{6}$ in acetone- $d_{6}$. Similar reaction conditions have been successfully employed in the synthesis of tert-butyl-substituted PONOP pincer complexes of platinum and palladium. ${ }^{153}$ However, with the electronpoor ligand 11, neither of these reaction mixtures showed any signs of the desired pincer complex $[(\mathrm{PONOP}) \mathrm{PtCl}]^{+}$. Analysis of both reaction mixtures by NMR spectroscopy revealed quantities of the oligomeric bridged species 48 . The observation of $\mathbf{4 8}$ having formed from starting materials with differing ancillary ligands helped to confirm its formulation as $\left[(\mathrm{PONOP}) \mathrm{PtCl}_{2}\right]_{\mathrm{x}}$, as it ruled out 48 possessing any neutral ligands other than the PONOP ligand $\mathbf{1 1}$ (such as diethyl sulfide).

Reactions under more harsh conditions were not attempted, as the reason for the investigation of PNP pincer ligands was that they generally allowed for pincer complex formation under more mild conditions than for analogous PCPH pincer ligands. The coordination chemistry of ligands $\mathbf{1 0}$ and $\mathbf{1 1}$ with platinum dichloride starting

${ }^{*}$ For example, where the phosphine donor is $\mathrm{CH}_{2} \mathrm{P}^{t} \mathrm{Bu}_{2}$, the ${ }^{31} \mathrm{P}$ NMR data for the PCNCP pincer complex is $\delta_{\mathrm{P}}=53.7 \mathrm{ppm},{ }^{1} J_{\mathrm{Pt}-\mathrm{P}}=2403 \mathrm{~Hz} ;{ }^{152}$ for the PCCCP pincer complex it is $\delta_{\mathrm{P}}=$ $64.9,{ }^{1} J_{\mathrm{Pt}-\mathrm{P}}=2881 \mathrm{~Hz} \cdot{ }^{84}$ 
materials had clearly demonstrated that pincer complex formation was significantly more facile for the more electron-rich PNNNP ligand $\mathbf{1 0}$ than for the electron-poor PONOP ligand 11. This low reactivity of ligand 11 was likely to be due to its inability to displace a chloride ligand from the metal centre. As the synthesis of PNP pincer complexes requires halide displacement from the metal by the nitrogen donor of the pyridyl backbone, the decreased electron density on metal centres with electron-poor ligands should lead to an increased electrostatic interaction between the metal and halide ligand, decreasing the ease of halide displacement. It may also be anticipated that cation formation for complexes of the more electron-poor PONOP ligand 11 would be less favourable than for complexes of the PNNNP ligand 10, as there would be less electron density available for the PONOP ligand to stabilise the positive charge developed on the metal centre.

\subsection{Synthesis of Rhodium PNP Species}

While reactions of the electron-poor PNP ligands $\mathbf{1 0}$ and $\mathbf{1 1}$ with platinum dichloride starting materials provided an effective comparison of the ease of pincer complex formation between PCPH and PNP ligands, the reactivity of PNP pincer complexes was investigated using rhodium(I) chloride compounds, as they were neutral and isoelectronic with the Group $10[(\mathrm{PCP}) \mathrm{MCl}]$ complexes reported in the Chapter 4. The preparation of iridium PNP pincer complexes may have offered more direct comparisons with the chemistry of the platinum PCP pincer compounds that are the predominant focus of this work, but it is the analogous rhodium compounds that have displayed the more interesting chemistry. Recent examples of methane $\sigma$-complexation ${ }^{25}$ and amine $\mathrm{N}-\mathrm{H}$ activation ${ }^{154}$ facilitated by rhodium PNP pincer complexes serve to highlight the utility of and interest in these rhodium species. An added advantage of rhodium is that it is NMR active $\left({ }^{103} \mathrm{Rh}\right.$ has a spin of $\frac{1}{2}$ and a natural abundance of $100 \%$ ), and therefore is able to provide additional spectroscopic data on the coordination environment of the rhodium centre.

Initial reactions between PNNNP and PONOP ligands 10 and 11 with the rhodium and iridium precursors $[\mathrm{RhCl}(\mathrm{COD})]_{2}$ and $\left[\mathrm{IrCl}(\mathrm{COE})_{2}\right]_{2}$ indicated that the respective $[(\mathrm{PNP}) \mathrm{MCl}]$ pincer complexes were readily formed in solution. ${ }^{\dagger}$ The PNNNP complexes offered an interesting point of difference from those of the PONOP ligand, as the presence of $\mathrm{N}-\mathrm{H}$ groups linking the phosphorus donors to the aromatic backbone provided a site for deprotonation on the ligand, which may lead

\footnotetext{
${ }^{\dagger}$ This work was carried out in conjunction with an undergraduate research project performed by Emma Aitken.
} 
to "non-innocent" behaviour. Complexes of the analogous PCNCP ligands, possessing $\mathrm{CH}_{2}$ rather than $\mathrm{NH}$ linker groups, have demonstrated a wide range of reactions based upon reversible deprotonation and reprotonation of the benzylic positions (Scheme 5.3). ${ }^{155}$ Such behaviour exists predominantly due to the ability of the pyridyl backbone to undergo dearomatisation, with the nitrogen donor atom becoming formally anionic and bonding in a covalent (rather than dative) fashion to the metal centre.

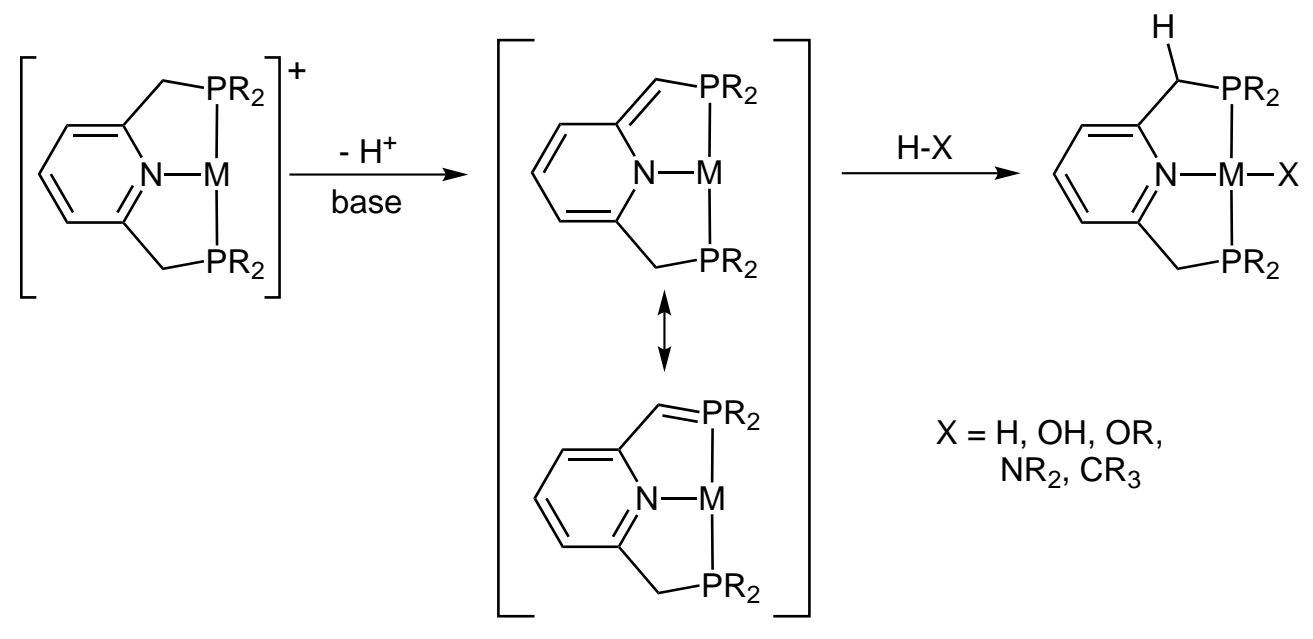

Scheme 5.3 Non-innocent behaviour of PCNCP pincer complexes. Figure adapted from Gunanathan et al., ${ }^{155}$ ancillary ligands on the metal centre omitted for clarity.

Non-innocent ligand behaviour offers advantages in catalytic transformations; by possessing a remote proton acceptor site it aids $\mathrm{H}-\mathrm{X}$ bond activation by allowing the cleavage to occur without a change in metal oxidation state, while the physical separation between the $\mathrm{H}$ and $\mathrm{X}$ groups can minimise the reverse $\mathrm{H}-\mathrm{X}$ elimination reaction. This has enabled rhodium PNP and PNN complexes to display high activities for a variety of $\mathrm{N}-\mathrm{H}, \mathrm{C}-\mathrm{H}$, and $\mathrm{H}-\mathrm{H}$ bond activations. ${ }^{154,156}$ Analogous reports of non-innocent behaviour of PNNNP ligands are substantially rarer; the reversible deprotonation of a $\mathrm{N}-\mathrm{H}$ linker group of a PNNNP pincer ligand has only been reported on one occasion. ${ }^{157}$

The rhodium PNNNP complex [(PNNNP)RhCl] (49) was synthesised according to a reported method for the synthesis of $[(\mathrm{NNN}) \mathrm{RhCl}]$ pincer complexes. ${ }^{158}$ The PNNNP ligand 10 and the rhodium starting material $[\mathrm{RhCl}(\mathrm{COD})]_{2}$ were dissolved in hot toluene, and within minutes the desired product began to crystallise out of the reaction mixture as the toluene solvate, $[(\mathrm{PNNNP}) \mathrm{RhCl}] \cdot \mathrm{C}_{7} \mathrm{H}_{8}$. The toluene of crystallisation was removed by washing with dichloromethane, giving 49 in good yields. The formulation of 49 as [(PNNNP)RhCl] was confirmed by mass spectrometry, with the observation of the $[\mathrm{M}-\mathrm{Cl}]^{+}$ion at $m / z=940 \mathrm{amu}$, and absence of 
any ion corresponding to a dimer confirming that the chloride bridges of the starting material had been cleaved during the synthesis of 49 . While analysis of 49 by NMR spectroscopy in acetone- $d_{6}$ gave data consistent with [(PNNNP)RhCl], it revealed that the $\mathrm{N}-\mathrm{H}$ groups readily underwent $\mathrm{H} / \mathrm{D}$ exchange with the acetone- $d_{6}$ solvent. This was most evident in the ${ }^{31} \mathrm{P}$ NMR spectrum: the phosphorus signal appeared as three overlapping doublets, due to the isotopomers 49, 49- $d$, and 49- $d_{2}$ (Figure 5.1). Complete deuteration of the $\mathrm{N}-\mathrm{H}$ groups could be achieved by the addition of one drop of $\mathrm{D}_{2} \mathrm{O}$ to acetone- $d_{6}$ solutions of $\mathbf{4 9}$.

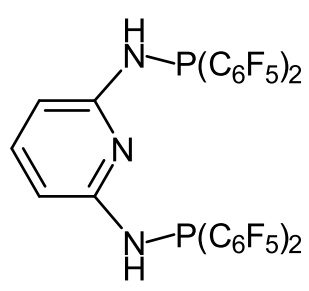

$\stackrel{[\mathrm{RhCl}(\mathrm{COD})]_{2}}{\longrightarrow}$ toluene $100^{\circ} \mathrm{C}$ $[(\mathrm{PNNNP}) \mathrm{RhCl}]$
$[(\mathrm{PNNNP}) \mathrm{RhCl}]-d_{1}$
$[(\mathrm{PNNNP}) \mathrm{RhCl}]-d_{2}$

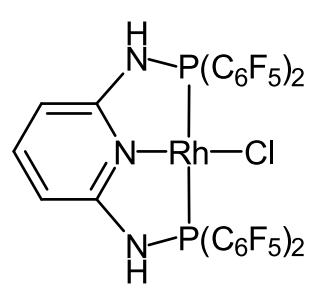

49

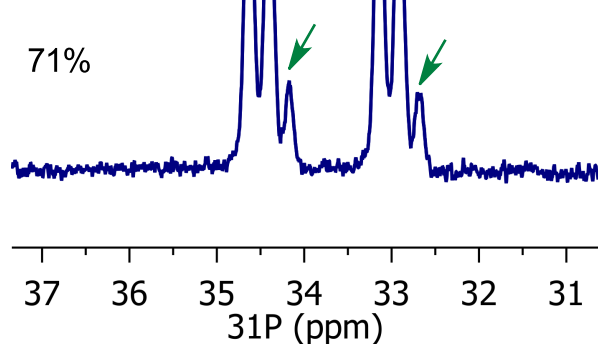

Figure 5.1 Synthesis of the PNNNP rhodium complex 49, and ${ }^{31} \mathrm{P}$ NMR spectrum of 49 in acetone- $d_{6}$ showing isotopomers resulting from facile $\mathrm{H} / \mathrm{D}$ exchange of $\mathrm{N}-\mathrm{H}$ protons.

The methylation of the PNNNP pincer chloride complex 49 was subsequently investigated. Methylation of the [(PCP)PtCl] complexes had indicated that dimethylzinc possessed a sufficiently low nucleophilicity and high activity to be an appropriate choice of methylating agent for these bis(pentafluorophenyl)phosphine-substituted complexes (as described in Chapter 4). Dimethylzinc has also been used to good effect in the literature for methylation of [(PCNCP $) \mathrm{RhCl}]$ species, as the more basic methyllithium has been observed to result in benzylic deprotonation of the ligand backbone rather than methylation. ${ }^{159,160}$

Reactions between complex 49 and dimethylzinc solutions in toluene were first investigated on an NMR scale in benzene- $d_{6}$ and toluene- $d_{8}$ (as toluene allowed for reaction mixtures to be cooled below $4^{\circ} \mathrm{C}$ ). The low solubility of the rhodium chloride starting material 49 in benzene and toluene allowed the appearance of new, soluble intermediates and products in the reaction mixture to be easily studied by NMR spectroscopy. Analysis of reaction mixtures by NMR spectroscopy revealed 
that the reaction proceeded rapidly, forming a new product within 30 minutes at room temperature. This new product possessed NMR spectroscopic data consistent with it being the desired rhodium(I) methyl complex, [(PNNNP)RhMe] (50); the resonance for the rhodium methyl group was observed in the ${ }^{1} \mathrm{H}$ NMR spectrum as a broad triplet (at $\delta_{\mathrm{H}}=1.09 \mathrm{ppm}$ ), and the increase in the magnitude of the rhodiumphosphorus coupling constant (from 180 to $218 \mathrm{~Hz}$ ) was consistent with moving from a $[(\mathrm{PNP}) \mathrm{RhCl}]$ to a $[(\mathrm{PNP}) \mathrm{RhMe}]$ complex. The observation of three sharp, discrete resonances for the ortho, meta, and para aromatic fluorine resonances in the ${ }^{19} \mathrm{~F}$ NMR spectrum was also consistent with 50 being a rhodium(I) compound, as the increased steric congestion at rhodium(III) centres in complexes of ligand 10 resulted in restricted rotation of the pentafluorophenyl substituents and inequivalence of fluorine environments in ${ }^{19} \mathrm{~F}$ NMR spectra.

Unfortunately, the desired rhodium methyl complex 50 proved unstable in the reaction mixture at room temperature; the orange solution gave way to a dark solution with a brown precipitate within two hours at room temperature, accompanied by a decrease in signal-to-noise in the NMR spectra consistent with degradation or precipitation of PNP rhodium species out of solution. Prior to precipitate formation, the appearance of a new compound (51) in solution was observed. This species appeared to be a degradation product from the reaction between the rhodium methyl complex 50 and dimethylzinc: 51 was only detected in solution following the formation of 50, and was present in greater amounts in reaction mixtures when greater amounts of dimethylzinc were used.

Spectroscopic data revealed that the degradation product 51 was an asymmetric species. The ${ }^{1} \mathrm{H}$ NMR spectrum showed that the symmetry of the ligand backbone had been destroyed; the signals for protons meta to the pyridyl nitrogen ( $\mathrm{H}-4$ and $\mathrm{H}-$ 6 , following the numbering scheme used for the PCP pincers) appeared as separate doublet resonances. The signal for H-5 was obscured by resonances from the toluene in the dimethylzinc solution, but COSY NMR experiments revealed that both $\mathrm{H}-4$ and H-6 coupled to a resonance at approximately $7.1 \mathrm{ppm}$, proton H-5. Only one of the $\mathrm{N}-\mathrm{H}$ protons was observed, shifted slightly upfield from the $\mathrm{N}-\mathrm{H}$ protons of the rhodium(I) chloride and methyl complexes. The second $\mathrm{N}-\mathrm{H}$ proton could not be detected by COSY or ${ }^{1} \mathrm{H}-31 \mathrm{P}$ NMBC experiments, therefore it was likely that the deprotonation of an $\mathrm{N}-\mathrm{H}$ group by dimethylzinc had given rise to the asymmetry of 51. This was also supported by the observation of methane in the ${ }^{1} \mathrm{H}$ NMR spectrum (at $\delta_{\mathrm{H}}=0.17 \mathrm{ppm}$ ), and was consistent with the observation that the presence of more dimethylzinc led to an increased the amount of $\mathbf{5 1}$ in solution.

This $\mathrm{N}-\mathrm{H}$ deprotonation was also supported by ${ }^{31} \mathrm{P}$ NMR spectroscopy. The ${ }^{31} \mathrm{P}$ 
NMR data of 51 showed the presence of two different phosphorus environments, which appeared as doublets of doublets at $\delta_{\mathrm{P}}=74.4$ and $44.7 \mathrm{ppm}$, with each phosphorus donor coupling to the other strongly $\left({ }^{2} J_{\mathrm{P}-\mathrm{P}}=522 \mathrm{~Hz}\right)$. This spectroscopic data were consistent with that observed for the benzylic deprotonation of PCNCP ligands. ${ }^{156}$ However, unlike for PCNCP complexes, deprotonation at the "arms" of the ligand did not appear to induce dearomatisation of the ligand backbone. Whereas dearomatisation of the pyridyl backbone has been observed to shift ${ }^{1} \mathrm{H}$ NMR resonances of the pyridyl protons upfield (as they are less aromatic and more alkene-like), ${ }^{152,161}$ in compound $\mathbf{5 1}$ each of the proton environments on the ligand backbone are similar in chemical shift to those of the PNNNP rhodium methyl complex 50 (Figure 5.2). This $\mathrm{N}-\mathrm{H}$ deprotonation without backbone dearomatisation has previously been observed on an PNNNP iron complex. ${ }^{78}$

The asymmetric compound $\mathbf{5 1}$ also possessed two distinctive, high-field resonances in the ${ }^{1} \mathrm{H}$ NMR spectrum. A Rh-Me group was observed at $\delta_{\mathrm{H}}=1.21 \mathrm{ppm}$, similar in chemical shift and lineshape to the methyl group of the rhodium(I) methyl complex $\left(\delta_{\mathrm{H}}=1.16 \mathrm{ppm}\right)$. In both cases the resonances were too broad to discern any rhodium-proton coupling. Interestingly, a further high-field resonance was observed in the ${ }^{1} \mathrm{H}$ NMR spectrum at $-0.17 \mathrm{ppm}$, and was much sharper than any of the rhodium-methyl signals. This indicated that the proton environment did not experience significant rhodium or phosphorus coupling, and so was suggestive of a zinc-methyl group rather than a second rhodium-methyl group. ${ }^{\ddagger}$ This high-field signal also integrated for three protons against the other proton environments of $\mathbf{5 1}$ in all the reaction mixtures studied, which confirmed that the $\mathrm{Zn}-\mathrm{Me}$ moiety was associated with the rhodium complex.

The formation of a methylzinc adduct of a rhodium PNP pincer complex has been reported in a similar reaction with dimethylzinc, ${ }^{159}$ but was not structurally characterised. As such, the structure the rhodium-methylzinc adduct 51 cannot be ascertained with the data on hand. Organozinc fragments have been reported to bind to rhodium centres in both bridging ${ }^{162}$ and terminal ${ }^{163}$ coordination modes, and may also form outer-sphere adducts through interactions with the ligand. ${ }^{164}$ Fluorine-19 NMR data for 52 revealed restricted rotation of the pentafluorophenyl substituents of the ligand, which was observed in spectra of the six-coordinate rhodium complexes $[(\mathrm{PNNNP}) \mathrm{RhClMeI}]$ and $\left[(\mathrm{PNNNP}) \mathrm{RhI}_{3}\right]$ (vide infra) but not in spectra of four-coordinate $[(\mathrm{PNNNP}) \mathrm{RhX}]$ species. This suggests the presence of a degree

\footnotetext{
¥Analysis of full width at half maximum values for the Rh-Me and Zn-Me groups helped to confirm their assignment as such. Averaging data for seven ${ }^{1} \mathrm{H}$ spectra over four different methylation reactions, the Rh-Me resonance $\left(\delta_{\mathrm{H}}=1.21 \mathrm{ppm}\right)$ possessed an average FWHM of $24.5 \mathrm{~Hz}$, while the Zn-Me resonance $\left(\delta_{\mathrm{H}}=-0.17\right)$ possessed an average FWHM of $6.3 \mathrm{~Hz}$. This demonstrated that the signal at $-0.17 \mathrm{ppm}$ did not display the requisite coupling to rhodium and phosphorus to correspond to a second Rh-Me group.
} 

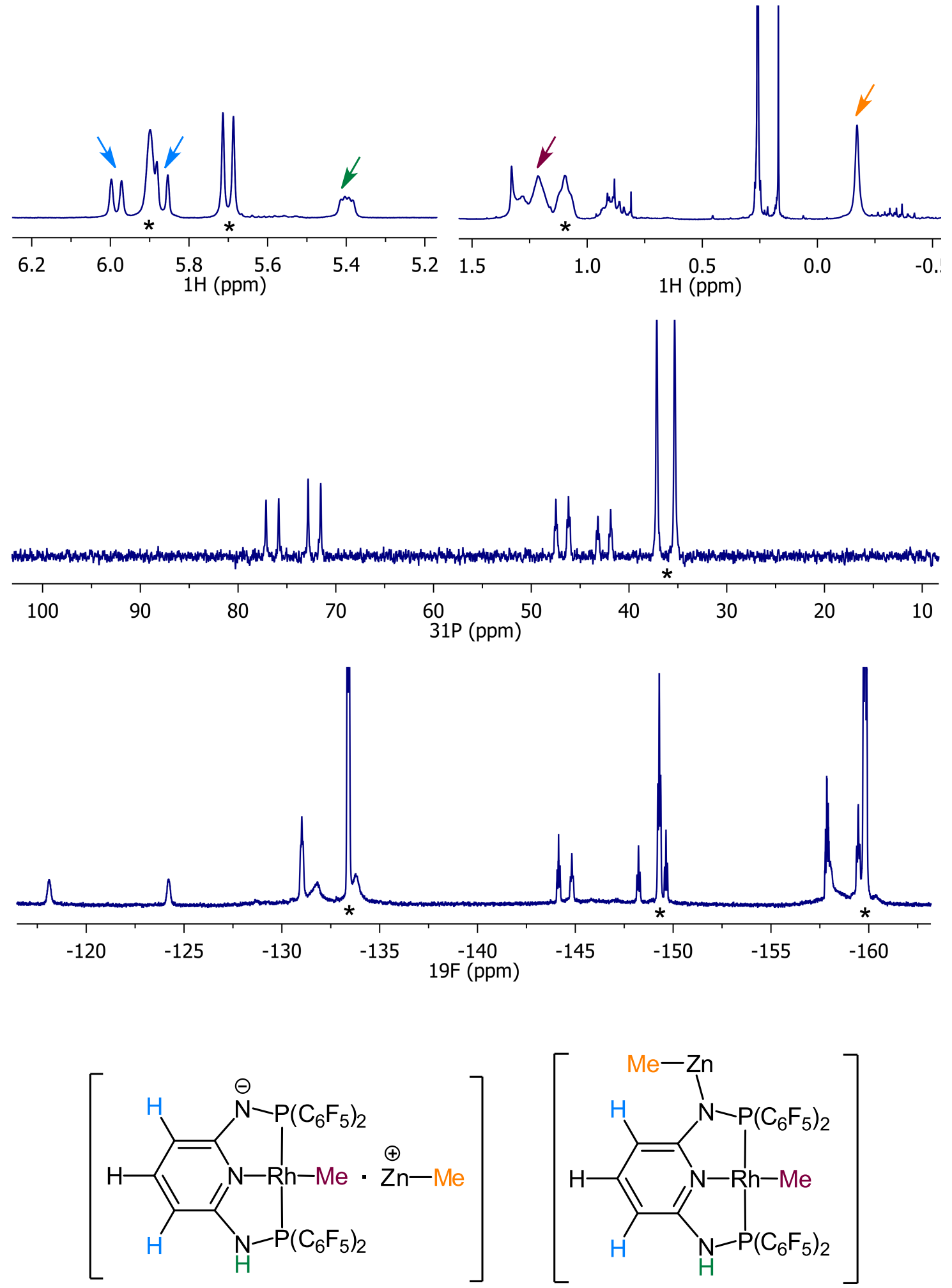

Figure 5.2 Multinuclear NMR spectra for the rhodium-methylzinc adduct 51 in toluene- $d_{8}$. Structure comprising the functional groups suggested by the data at bottom left, with a possible structural configuration for 51 at bottom right. Asterisks denote resonances arising from the rhodium(I) methyl complex $\mathbf{5 0 .}$ 
of steric crowding about the rhodium centre of $\mathbf{5 1}$, but it is not possible to conclude what moiety is responsible for this hindrance. Thus, while the NMR data (Figure 5.2) is consistent with the formulation of $\mathbf{5 1}$ as a deprotonated methylzinc adduct $\left[\left(\mathrm{PN}^{*} \mathrm{NNP}\right) \mathrm{RhMe} \cdot \mathrm{ZnMe}\right]$, it is difficult to speculate further on its nature.

In an attempt to isolate the rhodium methyl complex 50 without the formation of 51 and subsequent degradation, the PNNNP rhodium chloride complex 49 was treated with substoichiometric amounts of dimethylzinc in toluene- $d_{8}$ at $-15{ }^{\circ} \mathrm{C}$. As the starting PNNNP rhodium chloride complex 49 was only sparingly soluble in toluene, it was hoped that decantation of the supernatant from the reaction mixture would allow the isolation of $\mathbf{5 0}$, without excess dimethyzinc leading to degradation. However, in all reactions involving the treatment of 49 with dimethylzinc, small but significant amounts (c.a. 10\%) of the methylzinc adduct 51 were detected. Methylation reactions were also attempted in THF, where the Lewis acid-base interactions between the solvent and the zinc were seen to reduce the Brønstead basicity of the dimethyzinc, and produce significantly smaller quantities of the methylzinc adduct 51. However, reactions in THF demonstrated a lower selectivity than those in toluene, producing significant quantities of unidentified byproducts.

Attempts at the methylation of a rhodium PNP pincer complex with dimethylzinc have previously been reported to result in dark brown solutions of a rhodium zinc adduct, $\left[(\mathrm{PNP}) \mathrm{Rh}\left(\mathrm{CH}_{3}\right) \mathrm{Zn}\left(\mathrm{CH}_{3}\right) \mathrm{X}\right]$, from which the zinc could be abstracted with bipy to yield the rhodium(I) methyl complex. ${ }^{159}$ It appeared that in reactions of $\mathbf{4 9}$ with dimethylzinc that the initially formed rhodium methyl complex, 50, further reacted with dimethylzinc to form a soluble methylzinc adduct, 51, which decomposed to form an insoluble residue, from which the zinc might be removed to yield a rhodium pincer complex. However, attempts at zinc abstraction by treatment of these residues with bipy were unsuccessful, as they did not yield any soluble species with identifiable resonances in the ${ }^{31} \mathrm{P}$ or ${ }^{19} \mathrm{~F}$ NMR spectra. Ultimately, the dark suspensions formed by the decomposition (or further reaction) of the rhodium methyl complex $\mathbf{5 0}$ proved to be intractable.

In attempting to synthesise the rhodium methyl complex 50 using solutions of dimethylzinc in diethyl ether that contained methyl iodide (as described in Chapter 4 ), further evidence that dimethylzinc facilitates $\mathrm{N}-\mathrm{H}$ deprotonation in PNNNP rhodium complexes was obtained. Reactions with dimethylzinc in ether proceeded with a low selectivity, and formed a number of products with broad doublets between 2.8-2.2 ppm in the ${ }^{1} \mathrm{H}$ NMR spectra. Extraction of these reaction mixtures with pentane/diethyl ether yielded small amounts of material sufficiently pure to allow tentative characterisation of one of the major components of the reaction 
mixture (53) by NMR spectroscopy. The ${ }^{31} \mathrm{P}$ NMR spectrum of $\mathbf{5 3}$ was similar to that of the asymmetric methylzinc adduct 51, with two inequivalent phosphorus environments appearing as doublets of doublets at $\delta_{\mathrm{P}}=85.9$ and $50.9 \mathrm{ppm}$, with rhodium-phosphorus coupling constants $\left({ }^{1} J_{\mathrm{Rh}-\mathrm{P}}=150\right.$ and $135 \mathrm{~Hz}$ respectively) suggestive of a rhodium(III) complex. The ${ }^{1} \mathrm{H}$ NMR spectrum of $\mathbf{5 3}$ was also similar to that of the methylzinc adduct 51, with three separate aromatic protons and one $\mathrm{N}-\mathrm{H}$ proton observed from $7.0-5.5 \mathrm{ppm}$, as well as two distinctive broad triplet resonances of rhodium-methyl groups, which appeared at 1.43 and $0.14 \mathrm{ppm}$ respectively. Where the new compound $\mathbf{5 3}$ differed significantly from that of the methylzinc adduct was that the sharp resonance of the $\mathrm{Zn}-$ Me group at $-0.17 \mathrm{ppm}$ was absent, while a broad doublet appeared at $2.62 \mathrm{ppm}\left({ }^{3} J_{\mathrm{P}-\mathrm{H}}=9.0 \mathrm{~Hz}\right)$ and integrated for three protons. This ${ }^{1} \mathrm{H}$ NMR data were consistent with the presence of an N-methyl group on the ligand backbone, ${ }^{165}$ indicating that the presence of methyl iodide in the dimethylzinc solutions had resulted in methylation at the $\mathrm{N}-\mathrm{H}$ position (Scheme 5.4). Therefore, this electrophilic N-methylation required to form 53 in reaction mixtures was seen as further evidence of $\mathrm{N}-\mathrm{H}$ deprotonation by the dimethylzinc.

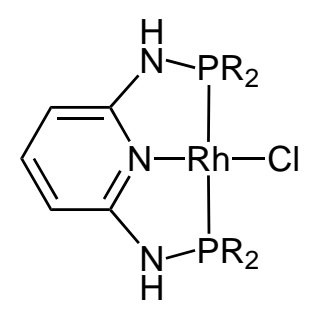

49

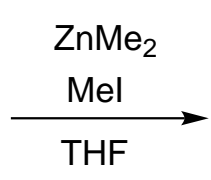

53

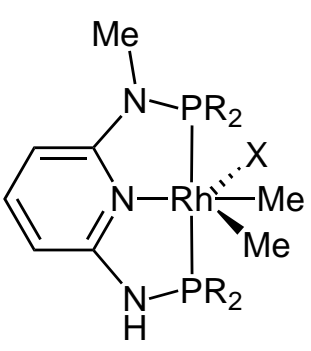

$\mathrm{X}=\mathrm{Cl}$ or I

$\mathrm{R}=\mathrm{C}_{6} \mathrm{~F}_{5}$

Scheme 5.4 Electrophilic methylation at an N-H position of the PNNNP ligand by methyl iodide in the presence of dimethylzinc.

To ensure that $\mathrm{H} / \mathrm{Me}$ exchange between methyl iodide and 49 was not occurring in a manner similar to H/D exchange, 49 was treated with a large excess of methyl iodide in acetone- $d_{6}$. Analysis of the reaction mixture by NMR spectroscopy revealed the immediate and quantitative formation of a new species 54 that possessed only one phosphine environment, and which ${ }^{1} \mathrm{H}$ NMR spectroscopy indicated had a $\mathrm{Rh}-\mathrm{Me}$ group (which appeared as a broad triplet of doublets at $\delta_{\mathrm{H}}=1.13 \mathrm{ppm}$ ), but not an N-methyl group. As for the previously discussed reaction intermediates, the small magnitude of the rhodium-phosphorus coupling constant $\left({ }^{1} J_{\mathrm{Rh}-\mathrm{P}}=133 \mathrm{~Hz}\right)$ and the observation of restricted rotation in the ${ }^{19} \mathrm{~F}$ NMR spectrum indicated that 54 was a rhodium(III) complex, likely to be [(PNNNP)RhClMeI], produced by the oxidative addition of methyl iodide to the starting material 49. Such oxidative additions have been reported as being facile for other other PNP rhodium(I) pincer 
complexes. ${ }^{156,166,167}$ The reaction was repeated on a large scale in THF, providing $\mathbf{5 4}$ as a red-orange solid in $83 \%$ yield. Analysis of $\mathbf{5 4}$ by mass spectrometry confirmed its formulation as the methyl iodide oxidative addition product [(PNNNP)RhClMeI], with the $[\mathrm{M}+\mathrm{Na}]^{+}$ion detected at $m / z=1140 \mathrm{amu}$. Compound $\mathbf{5 4}$ was observed to be stable in acetone- $d_{6}$ in the presence of methyl iodide over the course of 12 hours, with no sign of reaction at the $\mathrm{N}-\mathrm{H}$ groups. This confirmed that to achieve $\mathrm{N}$-methylation, dimethylzinc was required to deprotonate an $\mathrm{N}-\mathrm{H}$ group, which was then able to be methylated in the presence of the electrophilic methyl group of methyl iodide.

As the methylation reactions with dimethylzinc had been shown to promote ligand deprotonation, methylation with methylmagnesium iodide was pursued. Whereas methylmagnesium iodide was seen to be unsuccessful for the methylation of the $[(\mathrm{PCP}) \mathrm{PtCl}]$ compounds, it was envisaged that the increased steric bulk of the rhodium(III) starting material [(PNNNP)RhClMeI], 54, would promote nucleophilic attack by the smaller methyl group rather than by the larger iodide. Similar PNP rhodium(III) iodide complexes have been reported as possessing cationic, fivecoordinate structures with iodide counterions, ${ }^{166}$ which indicated that iodide dissociation from 54 may be facile, which would increase its susceptibility to nucleophilic attack by a methyl group. Treatment of the new rhodium(III) chloromethyl iodide complex $\mathbf{5 4}$ with methylmagnesium iodide in THF was observed to produce a new product 55 with a ${ }^{31} \mathrm{P}$ NMR resonance at $36.6 \mathrm{ppm}$ and a rhodium-phosphorus coupling of $115 \mathrm{~Hz}$. This species did not contain an observable Rh-Me group, and so was postulated to be a rhodium trihalide complex [(PNNNP) $\left.\mathrm{RhX}_{3}\right]$.

Similarly, treatment of the rhodium(I) chloride starting material 49 with methylmagnesium iodide resulted in the formation of the same rhodium(III) complex, $\mathbf{5 5}$. In situ monitoring of this reaction by NMR spectroscopy indicated that it proceeded via a rhodium(I) intermediate 56, with a ${ }^{31} \mathrm{P}$ NMR signal at $\delta_{\mathrm{P}}=40.5 \mathrm{ppm}$ $\left({ }^{1} J_{\mathrm{Rh}-\mathrm{P}}=170 \mathrm{~Hz}\right)$. The lack of any discernible $\mathrm{Rh}-$ Me resonance in the ${ }^{1} \mathrm{H} \mathrm{NMR}$ spectrum, together with a rhodium-phosphorus coupling constant similar to that of the rhodium(I) chloride complex $49(180 \mathrm{~Hz})$, indicated that this intermediate is likely to be the product of halide exchange with the methylmagnesium iodide, [(PNNNP)RhI]. The ready oxidation of the PNNNP rhodium chloride complex 49 to the rhodium(III) complex 55 in the presence methylmagnesium iodide has parallels to chemistry reported by the Albrecht research group. ${ }^{168}$ In that work, the formation of a rhodium(III) N-heterocyclic carbene complex from a rhodium(I) chloride starting material was facilitated by potassium iodide - the unexpected oxidation was attributed to the presence of iodine, which was generated from the aerobic oxidation of the iodide anion. The formation of iodine in solutions of organomagne- 
sium iodides has been reported by Wehmschulte and associates, who also attribute its formation to aerobic oxidation of iodide ions in solution by ambient air. ${ }^{169}$

In order to verify the nature of the product $\mathbf{5 5}$ and intermediate $\mathbf{5 6}$, the rhodium(I) iodide and rhodium(III) triiodide PNNNP pincer compounds were independently synthesised. A solution of the rhodium(I) chloride 49 in acetone- $d_{6}$ was treated with an excess of sodium iodide in a Finkelstein-type reaction, with NMR spectroscopy revealing quantitative formation of $[(\mathrm{PNNNP}) \mathrm{RhI}]$ after ten minutes at room temperature. The rhodium(I) iodide species was isolated, dissolved in benzene- $d_{6}$ and treated with iodine, immediately forming [(PNNNP) $\left.\mathrm{RhI}_{3}\right]$. The formulae of these compounds were confirmed by HRMS, while NMR spectroscopy confirmed the rhodium(I) iodide complex to be identical to the reaction intermediate $\mathbf{5 6}$, while the rhodium(III) iodide complex was found to be the same as the product of reactions involving methylmagnesium iodide. These reactions are summarised in Scheme 5.5.

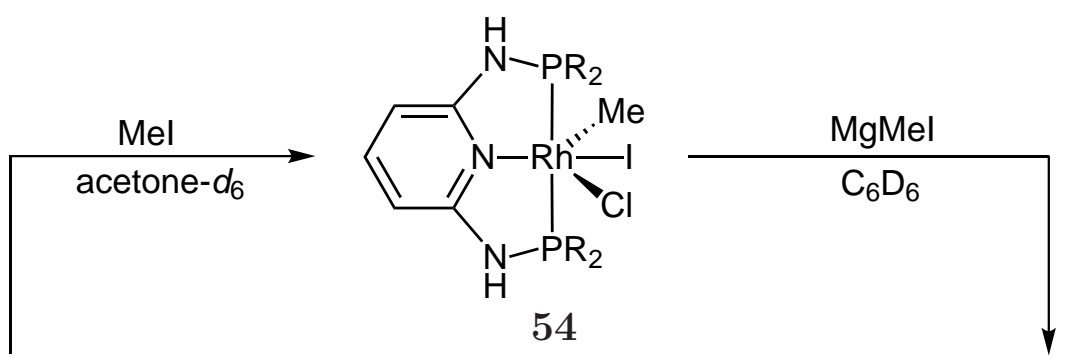

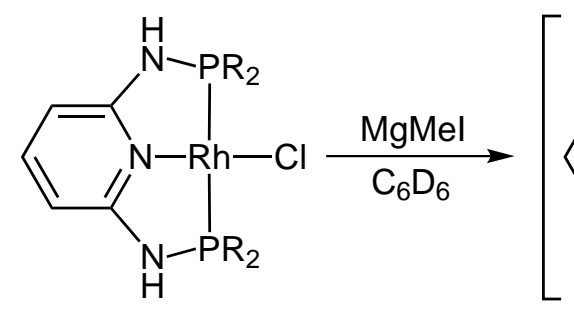

49

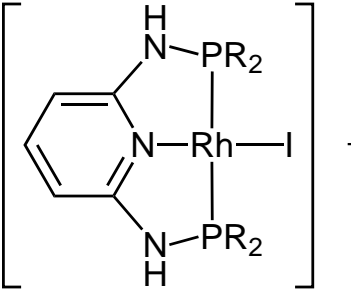

56

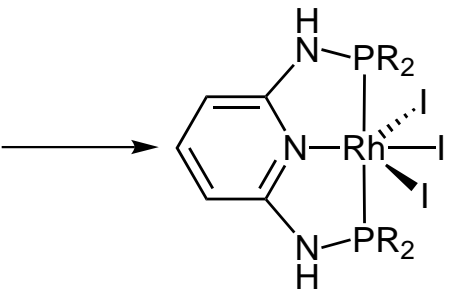

55

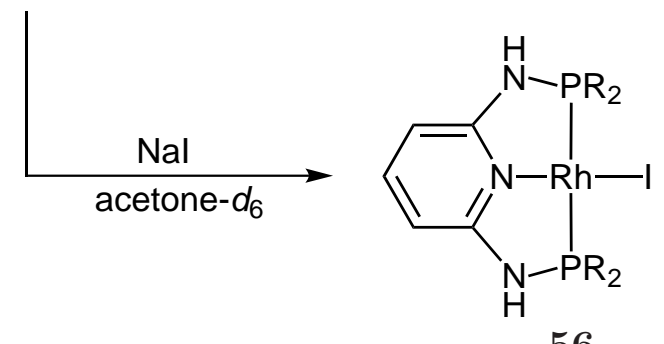

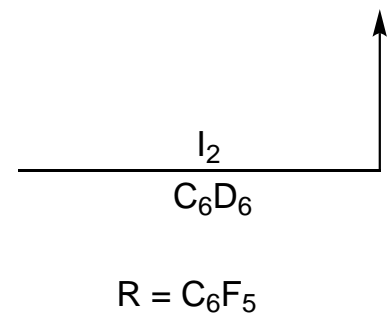

56

Scheme 5.5 Attempts at producing rhodium(I) and rhodium(III) methyl complexes via methylation of $\mathbf{4 9}$ with methylmagnesium iodide. The synthesis of compounds 56 and 55 by treatment of 49 with sodium iodide then iodine was used to verify the nature of these compounds.

It therefore appeared that, much like the platinum chloride PCP pincer complexes, these isoelectronic rhodium PNP species do not undergo methylation with methyl- 
magnesium iodide, rather they undergo halide exchange. However, unlike the platinum compounds, they are very susceptible to oxidation (to rhodium(III) species), and in all cases treatment of these PNP rhodium pincer complexes with methylmagnesium iodide eventually led to the formation of the PNNNP rhodium(III) triiodide complex 55 .

\subsection{Concluding Remarks}

The synthesis of complexes of the electron-poor PNP pincer ligands 10 and 11 was investigated. As the pyridyl ligand backbone can coordinate to metal centres without the $\mathrm{C}-\mathrm{H}$ activation required for complexes of $\mathrm{PCPH}$ pincer ligands it was hoped that pincer formation would be more facile for electron-poor PNP ligands than for the analogous PCP ligands.

Reaction of the PNNNP and PONOP pincer ligands 10 and 11 with platinum dichloride starting materials was carried out. The reaction of the PNNNP ligand occurred slowly compared to those of more electron-rich ligands reported in the literature, forming the desired platinum PNNNP pincer complex $[(\mathrm{PNNNP}) \mathrm{PtCl}]^{+}$, 47, upon prolonged reaction at room temperature. However, reactions of the more electron-poor PONOP ligand did not yield a pincer complex, despite the use of polar solvents and application of heat to reaction mixtures. Instead, these reactions yielded the PONOP-bridged cis-oligomer 48; a reactivity which bore similarities to the bridging coordination modes favoured by the POCOPH ligand $\mathbf{1}$. It was proposed that the pronounced difference in reactivity between ligands $\mathbf{1 0}$ and $\mathbf{1 1}$ was due to an increased energy barrier for halide displacement and cation formation with decreasing electron density on the metal centre.

The synthesis of the rhodium PNP pincer complexes was subsequently investigated, as PNP complexes of Group 9 metals in the +1 oxidation state are isoelectronic with PCP pincer complexes of Group 10 metals in the +2 oxidation state. The rhodium(I) PNNNP pincer complex [(PNNNP)RhCl], 49, was obtained in good yield from $[\mathrm{RhCl}(\mathrm{COD})]_{2}$. The methylation of $\mathbf{4 9}$ with dimethylzinc solutions was investigated, and while it appeared that the rhodium(I) methyl complex [(PNNNP)RhMe], 50, was formed in solution, it was not able to be isolated. Careful in situ analysis of reactions between 49 and dimethylzinc revealed the presence of an asymmetric species as a minor product, which was deemed to be the methylzinc adduct $\mathbf{5 1}$, having arisen from $\mathrm{N}-\mathrm{H}$ deprotonation of the ligand by an organozinc species in 
solution. Further evidence of $\mathrm{N}-\mathrm{H}$ deprotonation was indicated by the formation of an asymmetric, N-methylated product $\mathbf{5 3}$ when methyl iodide was present in the dimethylzinc solution.

The reaction of methyl iodide with the PNNNP rhodium(I) chloride 49 in the absence of dimethylzinc resulted in facile oxidative addition to produce the rhodium(III) complex [(PNNNP)RhClMeI], 54. Attempts at the methylation of both rhodium(I) and rhodium(III) complexes 49 and 54 with the milder methylating agent methylmagnesium iodide resulted in formation of the rhodium(III) triodide complex, [(PNNNP)RhI $\left.\mathrm{Rh}_{3}\right], \mathbf{5 5}$, in both instances. This was confirmed by independent synthesis of the rhodium(I) and rhodium(III) iodide complexes. The facile formation of rhodium iodide complexes revealed the inability of methylmagnesium iodide to methylate these electron-poor rhodium PNNNP pincer complexes, as well as reinforcing the prevalence of oxidative addition reactions in the chemistry of these rhodium(I) species. 


\section{Chapter 6}

\section{Catalytic Activity of Palladium Pincer Complexes}

\subsection{Palladium Pincer Complexes in Catalysis}

Since their inception, pincer complexes of palladium have demonstrated high activities and selectivities in a number of synthetically useful catalytic reactions. ${ }^{16,17}$ Their use in catalysis was first reported in 1993 by Seligson and Trogler, who employed a PCP palladium complex with an alkyl backbone to facilitate hydroamination of activated alkenes. ${ }^{170}$ It was found that the rigid, tridentate coordination of the pincer ligand helped reduce catalyst decomposition, and placed the strongly trans-directing alkyl group trans to the active site. This resulted in a higher reaction rate, and a greater catalyst stability and selectivity when compared to bis-monodentate and bidentate chelate phosphine analogues.

Pincer complexes began to garner considerable interest in the field of homogeneous catalysis, and were first employed in palladium-catalysed cross-coupling reactions by the Milstein research group in 1997. ${ }^{55}$ Their use of PCP palladium complexes in the Heck reaction revealed these species to be amongst the most active catalysts known at the time. This was attributed to the remarkable stability of these species, with no catalyst degradation observed even after 300 hours at the $140{ }^{\circ} \mathrm{C}$ employed in the reactions.

The analogous investigation of palladium pincer complexes in the Suzuki crosscoupling reaction was not reported until three years later. It was demonstrated by Bedford and co-workers that POCOP palladium triflates were very active for 
the coupling of electronically deactivated and sterically hindered aryl bromides with phenylboronic acid. ${ }^{171}$ These pincer complexes were inexpensive, easily synthesised, and could carry out reactions at loadings as low as $0.0001 \mathrm{~mol} \%$, giving turnover numbers (TONs) amongst the highest then reported for the substrates examined.

As well as being highly active in palladium-mediated cross-coupling reactions, ECE pincer complexes have proven to be extremely effective catalysts for the allylation of electrophiles. The Szabó research group have demonstrated that SeCSe palladium pincer complexes are particularly effective in the synthesis of allylboranes and allylbenzene derivatives. ${ }^{172,173}$ These catalysts are tolerant to a variety of functional groups, with reactions proceeding under very mild conditions, generating products with a greater selectivity than the more commonly employed $\left[\mathrm{Pd}_{2}(\mathrm{dba})_{3}\right]$ and $\left[\mathrm{Pd}\left(\mathrm{PPh}_{3}\right)_{4}\right]$ species. The efficacy of pincer complexes in these reactions is attributed to the chelate effect reducing the possibility of ligand exchange, whilst the tridentate ligand leaves only one vacant coordination site on the metal, minimising unintended side reactions.

Since these initial reports, catalysis performed by pincer complexes of palladium has generated a good deal of excitement and controversy. The well defined, robust nature of pincer complexes, combined with their demonstration of considerable catalytic activity has made them attractive targets for research into the Heck and Suzuki reactions. It was hoped that their high thermal stability and ability to incorporate donor groups of different electronic and steric character would allow for the generation of highly active and selective catalysts that would resist leaching of palladium into the products synthesised.

\subsection{Mechanistic Implications of Catalysis with PCP Pincer Complexes}

The fixed, tridentate nature of ECE pincer ligands has raised questions over the reaction mechanisms pincer complexes employ during catalysis. It has long been known that the Heck reaction operates through a catalytic cycle dependent on a $\operatorname{Pd}(0)$ active catalyst (Figure 6.1). ${ }^{174}$ The $\operatorname{Pd}(0)$ active species undergoes oxidative addition of the aryl halide to yield a $\mathrm{Pd}(\mathrm{II})$-aryl halide complex, which is then capable of inserting the alkene substrate into the Pd-aryl bond. The subsequent alkyl-palladium species generated undergoes $\beta$-hydride elimination, giving the coupled styrene derivative and a palladium-hydrido species, which is reduced back to 


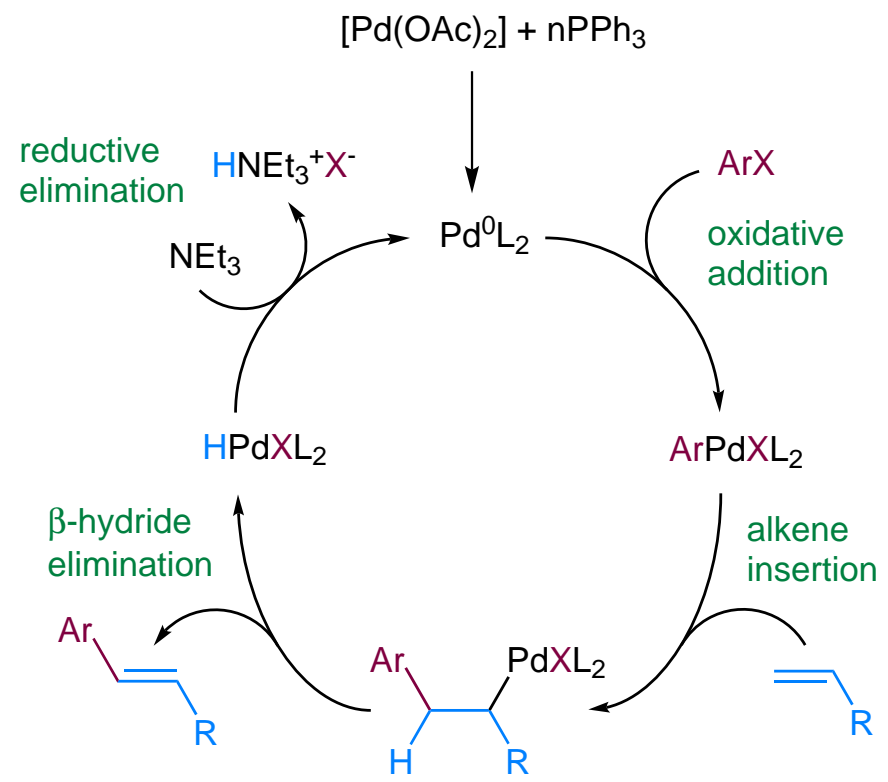

Figure 6.1 Outline of the classic "textbook" Heck catalytic cycle.

the active catalyst species through the action of the base. Conceptually the Suzuki reaction is very similar, except that the intermediate $\mathrm{Pd}(\mathrm{II})$-aryl species undergoes transmetallation with an arylboronic acid, rather than undergoing alkene insertion.

Recent investigations have shown that this classic "textbook" catalytic cycle for the Heck reaction may not be an entirely accurate representation of the course of the reaction. Research by Amatore and Jutand has demonstrated that anionic, five-coordinate $\mathrm{Pd}(\mathrm{II})$ species play a role as vital intermediates in the catalytic cycle. ${ }^{175}$ However, the essence of the mechanism remains the same; the $\operatorname{Pd}(0) / \operatorname{Pd}(\mathrm{II})$ oxidative addition and reductive elimination cycle forms the foundation of these cross-coupling reactions.

This creates a conundrum when considering catalysis by ECE palladium pincer complexes. How can the $\operatorname{Pd}(0) / \operatorname{Pd}(\mathrm{II})$ catalytic cycle be in operation when the catalyst is effectively "locked" in a $\mathrm{Pd}(\mathrm{II})$ resting state by the strong $\mathrm{Pd}-\mathrm{C}$ bond? This has been a matter of much conjecture in the literature, ${ }^{176}$ and there are two main schools of thought as to how this catalysis is carried out. It was originally proposed that the cyclometallated $\mathrm{P}-\mathrm{C}$ chelates of the type $\mathbf{5 7}$ (Figure 6.2) formed in reaction mixtures of $\mathrm{Pd}(\mathrm{OAc})_{2}$ and $\mathrm{P}(\text { o-tolyl })_{3}$ served as active Heck and Suzuki catalysts, operating through a $\mathrm{Pd}(\mathrm{II}) / \mathrm{Pd}(\mathrm{IV})$ mechanism. ${ }^{177,178} \mathrm{~A}$ mechanism for this transformation was developed by Bernard Shaw, ${ }^{179}$ and upon the discovery of PCP palladium Heck catalysts, their reaction mechanism was deemed to proceed via this $\mathrm{Pd}(\mathrm{II}) / \mathrm{Pd}(\mathrm{IV})$ pathway. ${ }^{55,180}$

The idea that cyclometallated palladium compounds accessed the $\operatorname{Pd}(\mathrm{IV})$ oxidation state during catalysis was met with less than unanimous agreement. It had 


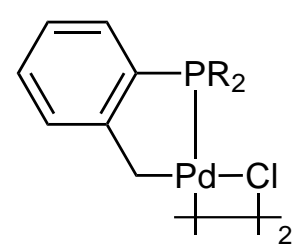

57

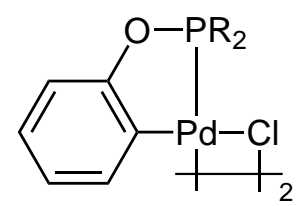

58

Figure 6.2 Representative examples of cyclometallated $\mathrm{P}-\mathrm{C}$ chelates.

been demonstrated by Louie and Hartwig in 1996 that $\mathrm{P}-\mathrm{C}$ chelates of the type 57 could be reduced under catalytic conditions, forming phosphine-stabilised $\operatorname{Pd}(0)$ complexes, which served as the active catalyst species. ${ }^{181}$ A breakthrough in the field came in 2000, when it was discovered that under catalytic conditions, $\mathrm{Pd}(\mathrm{II})$ species could be reduced to palladium nanoparticles, which efficiently catalysed cross-coupling reactions via a conventional $\mathrm{Pd}(0) / \mathrm{Pd}(\mathrm{II})$ cycle. ${ }^{182}$ It was subsequently found that $\mathrm{PCP}^{183}$ and $\mathrm{SCS}^{184}$ palladium pincer complexes were likely to serve as depot forms of $\operatorname{Pd}(0)$ nanoparticles rather than serving as the active catalyst species themselves. In an assessment of the mechanism of the Heck reaction in 2006, de Vries stated "the conclusion seems justified that all palladacycles and pincers decompose during the Heck reaction at high temperatures to form palladium colloids." 185

However, recent developments in the field of $\mathrm{Pd}(\mathrm{IV})$ chemistry have again encouraged debate as to whether high oxidation state pathways exist for these crosscoupling reactions. It has been demonstrated that in the presence of strong oxidants (such as trivalent iodine(III) salts, $\mathrm{IXR}_{2}$ ), $\mathrm{Pd}(\mathrm{IV})$ species play an important role in catalytic cycles. ${ }^{186,187}$ It has also been shown that when these iodonium salts are used in place of aryl halides in pincer-catalysed Heck reactions, the reaction is likely to proceed through a $\mathrm{Pd}(\mathrm{II}) / \mathrm{Pd}(\mathrm{IV})$ cycle (Scheme 6.1). ${ }^{188}$

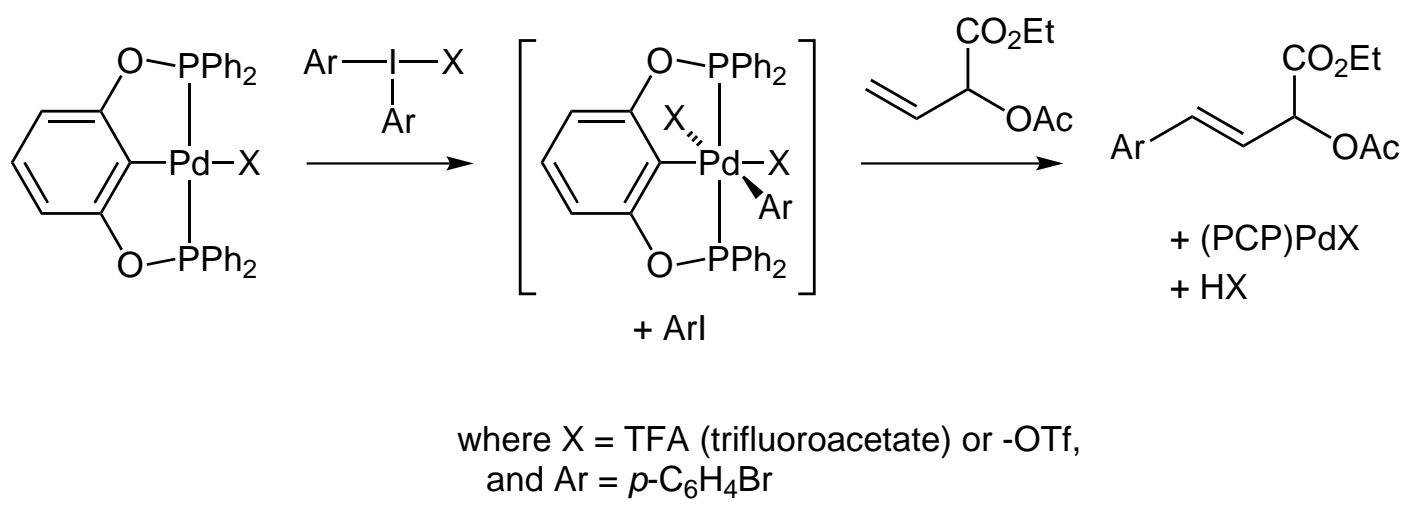

Scheme 6.1 $\mathrm{Pd}(\mathrm{IV})$ intermediates in the Heck reaction promoted by strong oxidants. 
In the absence of such strong oxidants, it has been proposed on the basis of computational calculations that $\mathrm{Pd}(\mathrm{IV})$ species are thermally accessible intermediates in the Heck and Negishi reactions between PCP pincer complexes and aryl halides in polar solvents at elevated temperatures. ${ }^{189,190}$ Supporting these calculations, the first example of $\mathrm{Ar}-\mathrm{X}$ oxidative addition to form a $\mathrm{Pd}(\mathrm{IV})$ species has recently been published in the scientific literature. ${ }^{191}$ In this example, chelate-assisted oxidative addition of an aryl iodide species to an ONC palladium pincer complex yielded the stable, crystallographically characterised $\mathrm{Pd}(\mathrm{IV})$ product at room temperature (Scheme 6.2). This emphasises that despite many ECE palladium pincer complexes acting as sources of a $\mathrm{Pd}(0)$ active catalyst, $\mathrm{Pd}(\mathrm{II}) / \mathrm{Pd}(\mathrm{IV})$ cycles should continue to be assessed for their viability in cross-coupling reactions.

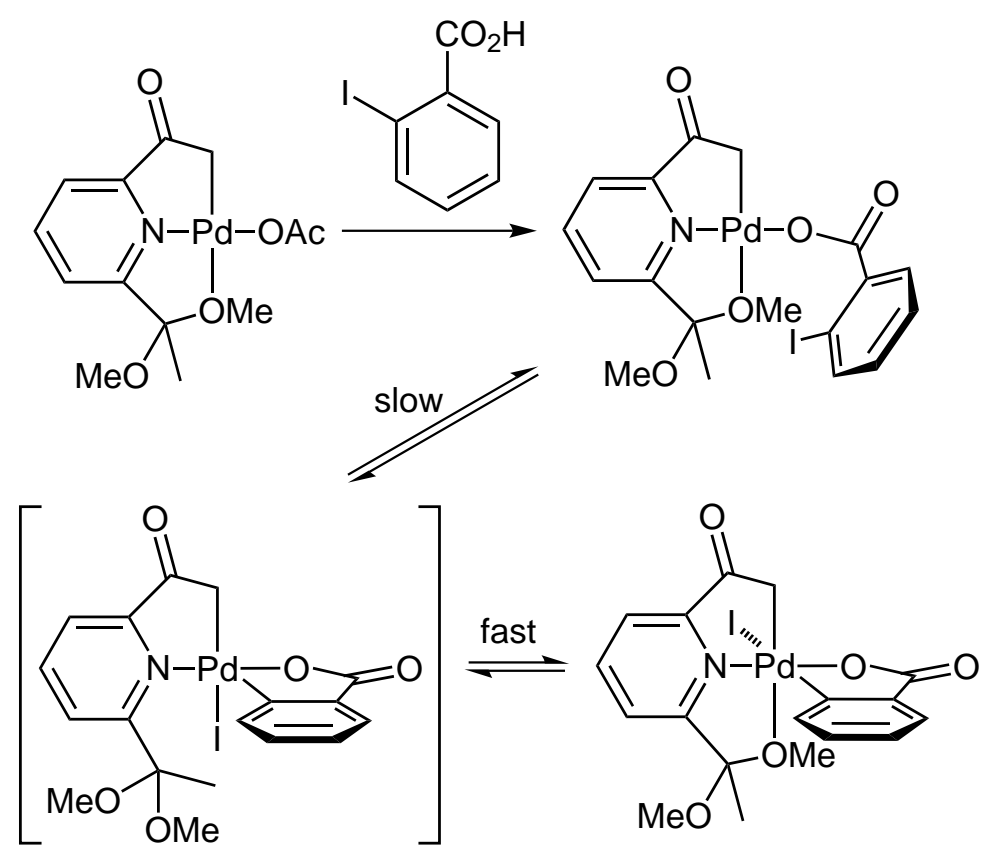

Scheme 6.2 Chelate-assisted oxidative addition to form a $\operatorname{Pd}(\mathrm{IV})$ complex.

\subsection{Performance of $[(\mathrm{PCP}) \mathrm{PdCl}]$ Species in the Heck Reaction}

Owing to the high activity of PCP pincer-based catalysts, the electron-poor pincer complexes [(POCOP $) \mathrm{PdCl}](\mathbf{2 8}),[(\mathrm{PCCCP}) \mathrm{PdCl}](\mathbf{2 9})$, and [(POCCP $) \mathrm{PdCl}]$ (30) had their performance in the Heck reaction evaluated. The basic Heck crosscoupling between bromobenzene and styrene was used to evaluate each catalyst. A survey of the literature revealed that N-methylpyrrolidone (NMP) and dimethylformamide (DMF) were commonly employed solvents, with most reactions also using 
an inorganic base (such as a phosphate or carbonate), typically at temperatures of $140{ }^{\circ} \mathrm{C}$.

Initial reactions focused on briefly optimising the base and solvent systems, and determining average TONs for each catalyst species. Methodology for a typical Heck reaction is outlined in the experimental section. Analysis of all Heck reactions was performed by gas chromatography mass spectrometry (GCMS). To avoid any bias arising from solvent evaporation between sampling and analysis, samples were stored in the dark at $-15{ }^{\circ} \mathrm{C}$ and spiked with 2-methylnaphthalene as an internal standard. Storing the samples in the dark also served to minimise cis/trans isomerisation of stilbene, as samples that had been left in direct sunlight before being subjected to re-analysis showed substantially increased levels of cis-stilbene. All reactions were performed in duplicate at the least, with quoted values reflecting the averages of each reaction performed.

\subsubsection{Results of Heck Reactions}

Data obtained from the Heck reactions (Table 6.1) showed that the optimal reaction conditions were achieved in DMF with potassium carbonate as a base. Whilst using potassium phosphate monohydrate resulted in slightly higher yields than potassium carbonate, more consistent results were obtained with the carbonate. Utilising $\mathrm{DMF}$ as a solvent for these reactions gave much higher conversions than reactions performed with NMP - POCOP and POCCP catalysts 28 and 30, which exhibited modest TONs in DMF, were found to be completely inactive in NMP. Moreover, in all reactions performed in NMP with 29, a small amount of biphenyl (arising from the homocoupling of bromobenzene) was detected. No biphenyl was detected in any of the reactions performed in DMF. The reaction in DMF was also repeated without the addition of a catalyst, and no cross-coupling products were observed. This confirmed that the palladium pincer complexes were responsible for the activity, either as the catalyst or precatalyst; the reaction was not occurring under metal-free conditions or being promoted by persistent palladium residues adsorbed onto the surface of the reaction vessel.

Of the three complexes tested in these Heck reactions, activity was of the order $[(\mathrm{PCCCP}) \mathrm{PdCl}](\mathbf{2 9})>[(\mathrm{POCOP}) \mathrm{PdCl}](\mathbf{2 8}) \gg[(\mathrm{POCCP}) \mathrm{PdCl}](\mathbf{3 0})$. At a catalyst loading of 0.002 mole $\%$ all three compounds gave similar conversions of about $20-25 \%$ in 16 hours. However, on increasing the catalyst loading to 0.004 mole \% and extending the reaction time to 22 hours it became clear that compounds 28 and 
Table 6.1 Results of the Heck reaction with 28, 29, and 30.

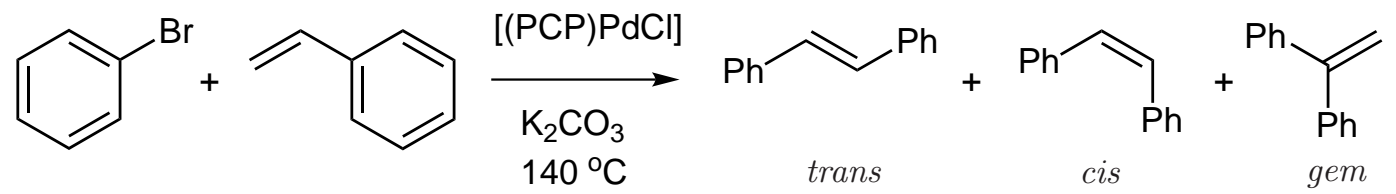

\begin{tabular}{|c|c|c|c|c|c|c|}
\hline Ligand & Time (h) & Solvent & {$[\mathrm{Pd}](\mathrm{mol} \%)$} & Yield $^{a}(\%)$ & TON & gem/cis/trans \\
\hline \multirow{5}{*}{ POCOP } & 4 & DMF & 0.002 & 13 & 6,400 & $1 / 1 / 41$ \\
\hline & 16 & DMF & 0.002 & 18 & 8,900 & $1 / 5 / 14$ \\
\hline & 16 & DMF & 0.005 & 45 & 9,100 & $1 / 0 / 16$ \\
\hline & 22 & DMF & 0.004 & 68 & 17,000 & $1 / 2 / 27$ \\
\hline & 16 & NMP & 0.002 & 0 & 0 & $0 / 0 / 0$ \\
\hline \multirow{6}{*}{ PCCCP } & 4 & $\mathrm{DMF}$ & 0.002 & 4 & 2,200 & $0 / 0 / 1$ \\
\hline & 16 & $\mathrm{DMF}$ & 0.002 & 24 & 12,000 & $1 / 4 / 15$ \\
\hline & $16^{b}$ & DMF & 0.002 & 36 & 18,000 & $1 / 2 / 17$ \\
\hline & 16 & $\mathrm{DMF}$ & 0.005 & 54 & 11,000 & $1 / 0 / 16$ \\
\hline & 22 & DMF & 0.004 & 89 & 22,000 & $1 / 2 / 21$ \\
\hline & 16 & NMP & 0.002 & 24 & 12,000 & $1 / 2 / 17^{c}$ \\
\hline \multirow{5}{*}{ POCCP } & 4 & $\overline{\mathrm{DMF}}$ & 0.002 & 0 & 0 & $0 / 0 / 0$ \\
\hline & 16 & $\mathrm{DMF}$ & 0.002 & 25 & 13,000 & $1 / 4 / 15$ \\
\hline & 16 & DMF & 0.005 & 24 & 5,000 & $1 / 0 / 20$ \\
\hline & 22 & $\mathrm{DMF}$ & 0.004 & 16 & 4,000 & $1 / 14 / 16$ \\
\hline & 16 & NMP & 0.002 & 0 & 0 & $0 / 0 / 0$ \\
\hline
\end{tabular}

${ }^{a}$ Determined by GCMS and based on bromobenzene and product.

${ }^{b}$ Reaction performed with $\mathrm{K}_{3} \mathrm{PO}_{4} \cdot \mathrm{H}_{2} \mathrm{O}$ instead of $\mathrm{K}_{2} \mathrm{CO}_{3}$.

${ }^{c} 3$ equivalents of biphenyl also detected.

29 were more active than 30 . Under these conditions 29 gave the greatest average TON of 21,000. Complex 28 showed a similar activity giving an average TON of 18,000, while 30 displayed TONs of only 4,000.

In the literature, many PCP pincer catalysts have attained TONs of around 100,000 for the Heck reaction, with the greatest reported TON to date being 8,900,000. ${ }^{180}$ Albeit many of these results are obtained using the more reactive aryl iodide substrates; lower activities can be expected (and in most cases are observed) for reactions involving aryl chlorides or bromides. More recently, an aminophosphine PNCNP pincer complex was found to give TONs of 5,000,000 in the cross-coupling of styrene and bromobenzene. ${ }^{135}$ The pincer complexes 28, 29 and $\mathbf{3 0}$ therefore display very modest activities in the Heck reaction; however, this is the first account of Heck catalysis using a complex containing a cyclometallated $\mathrm{CH}_{2} \mathrm{P}\left(\mathrm{C}_{6} \mathrm{~F}_{5}\right)_{2}$ or $\mathrm{OP}\left(\mathrm{C}_{6} \mathrm{~F}_{5}\right)_{2}$ moiety. The pentafluorophenyl-substituted PCCCP complex 29 also displays a much higher activity than its perfluoroalkyl-substituted analogues, which recently demonstrated TONs on the order of hundreds - rather than tens of thousands - of cycles. ${ }^{192}$ Whilst complexes $\mathbf{2 8}, \mathbf{2 9}$, and $\mathbf{3 0}$ appear to be less active than their non-fluorinated phosphine and phosphinite PCP counterparts, they generally display a higher activity than NCN or SCS ligated complexes. ${ }^{193,194}$ 
The reactions of the two most active palladium complexes, 28 and $\mathbf{2 9}$, were monitored at regular intervals by GCMS. The plots of conversion against time for these reactions (Figure 6.3, top) confirmed that $\mathbf{2 9}$ was slightly more active than $\mathbf{2 8}$, and also demonstrated that both reactions still proceeded up to 22 hours at $140{ }^{\circ} \mathrm{C}$. Extrapolation to the point of $50 \%$ conversion gave turnover frequencies (TOFs) of 900 for $\mathbf{2 8}$ and 2,300 for $\mathbf{2 9}$. These values pale in comparison to the TOF of 140,000 reported for the identical coupling of bromobenzene and styrene catalysed by a PNCNP pincer complex, ${ }^{135}$ and are towards the low end of the TOFs generally observed for PCP pincer complexes (of the order of 1,000-10,000). ${ }^{16}$

Importantly, these plots suggested that the reactions involving 29 may display sigmoidal (S-shaped) kinetics (Figure 6.3, bottom graph). Such reactions display an induction period, during which time only a slight amount of product is formed while the catalyst precursor decomposes or reacts to form the active catalyst species. For pincer catalysed cross-coupling reactions the observation of an induction period is extremely indicative of decomposition to a nanoparticulate active catalyst. ${ }^{183}$ An induction period was not detected in reactions performed with $\mathbf{2 8}$, but may be present during the initial stages of the reaction for which data were not collected. To confirm that the active catalyst in these Heck reactions was a $\operatorname{Pd}(0)$ species (rather than the intact $\mathrm{Pd}(\mathrm{II})$ pincer complex), the mercury poisoning test was employed. This entailed the addition of a large excess of elemental mercury to the reaction mixture, to amalgamate any heterogeneous $\operatorname{Pd}(0)$ deposited during the reaction and hence negate any catalytic activity from $\operatorname{Pd}(0)$ species. ${ }^{195,196}$ Whilst elemental mercury has been shown to degrade molecular $\operatorname{Pd}(0)$ and $\operatorname{Pt}(0)$ alkene complexes, ${ }^{197,198}$ it does not react with $\mathrm{Pd}(\mathrm{II})$ pincer complexes, and hence is diagnostic for whether the pincer complex is acting as a catalyst or a source of active $\operatorname{Pd}(0)$ in a particular reaction. ${ }^{176}$

Mercury poisoning tests were carried out by repeating the Heck reactions catalysed by 28 and 29 with the addition of approximately $50 \mathrm{mg}$ (about 6,000 equivalents) of elemental mercury to each reaction mixture. Solutions were thoroughly stirred for two minutes prior to the addition of the catalyst to divide and distribute the mercury evenly throughout. Results are shown on the graphs in Figure 6.3; no trace of product was detected in any of the reaction mixtures in which mercury was present. This confirmed that pincer complexes themselves were not active catalysts for the Heck reaction, and instead acted as sources of catalytically active $\operatorname{Pd}(0)$, as is seen in the literature for almost all cyclometallated palladium compounds. ${ }^{185}$ 

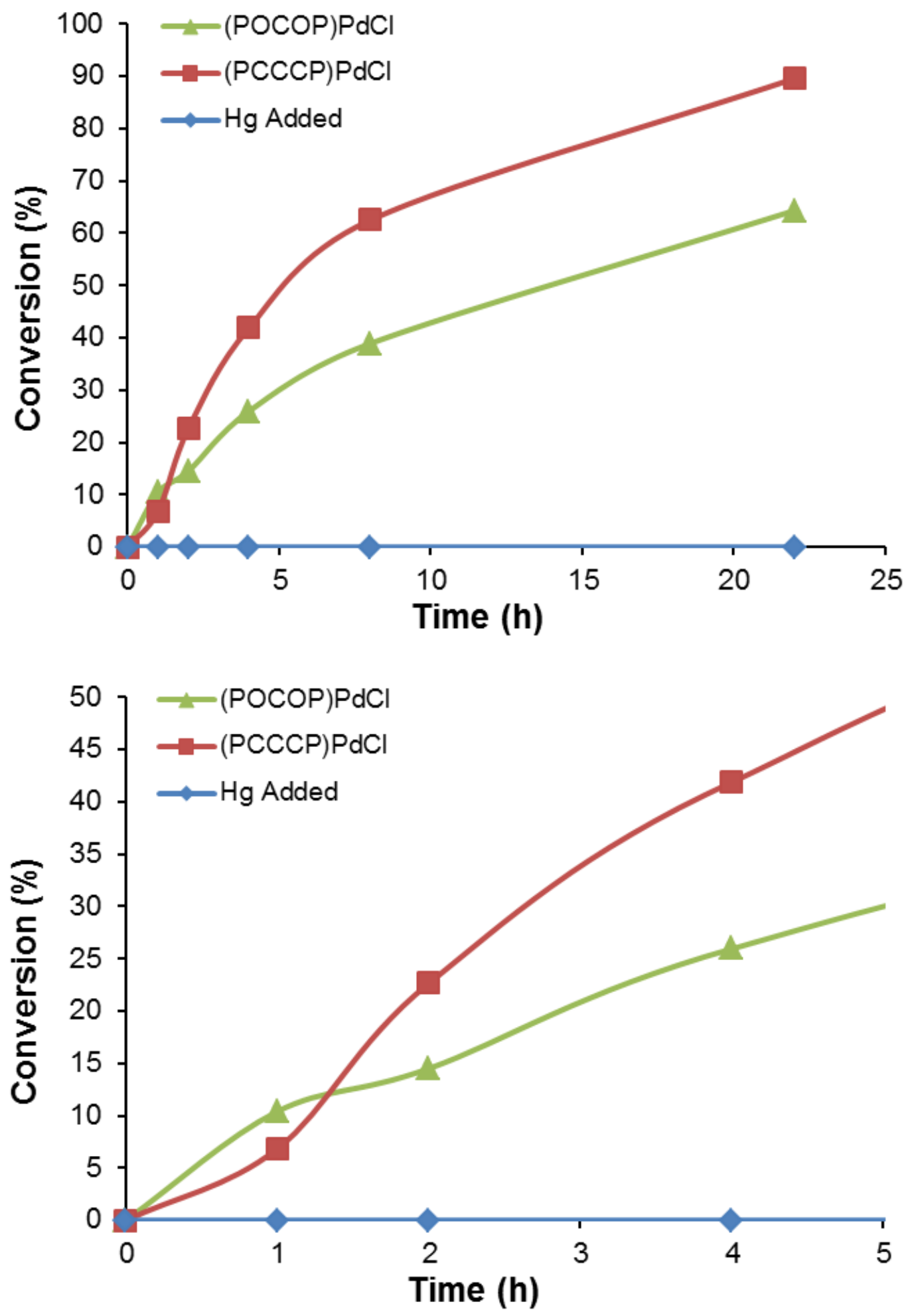

Figure 6.3 Conversion vs time: $\mathrm{PhBr} /$ styrene $/ \mathrm{K}_{2} \mathrm{CO}_{3}$ at $140{ }^{\circ} \mathrm{C}$ with $4 \mathrm{mmol} \%$ of complexes 28 and 29. Full duration of the reaction (top), and first four hours (bottom), showing the presence of an induction period. 


\subsubsection{Active Species Generation and Reaction Mechanism}

As these Heck reactions have been established to proceed through a heterogeneous $\operatorname{Pd}(0)$ active species, two pathways have been proposed in the literature for the reduction of the $\mathrm{Pd}(\mathrm{II})$ precatalyst. In the first, proposed originally by Louie and Hartwig ${ }^{181}$ then revised by Sommer and colleagues (Scheme 6.3), ${ }^{199}$ a phosphorusdonor group of the pincer ligand is displaced by the amine base used, giving a cyclometallated palladium-amine intermediate. This is able to undergo $\beta$-hydride elimination from an alkyl group of the coordinated amine, to form a palladium hydride complex. As the cyclometallated carbon and hydride occupy coordination sites that are mutually cis-coordinated, reductive elimination of the aryl backbone of the pincer should be facile, yielding a $\operatorname{Pd}(0)$ species.

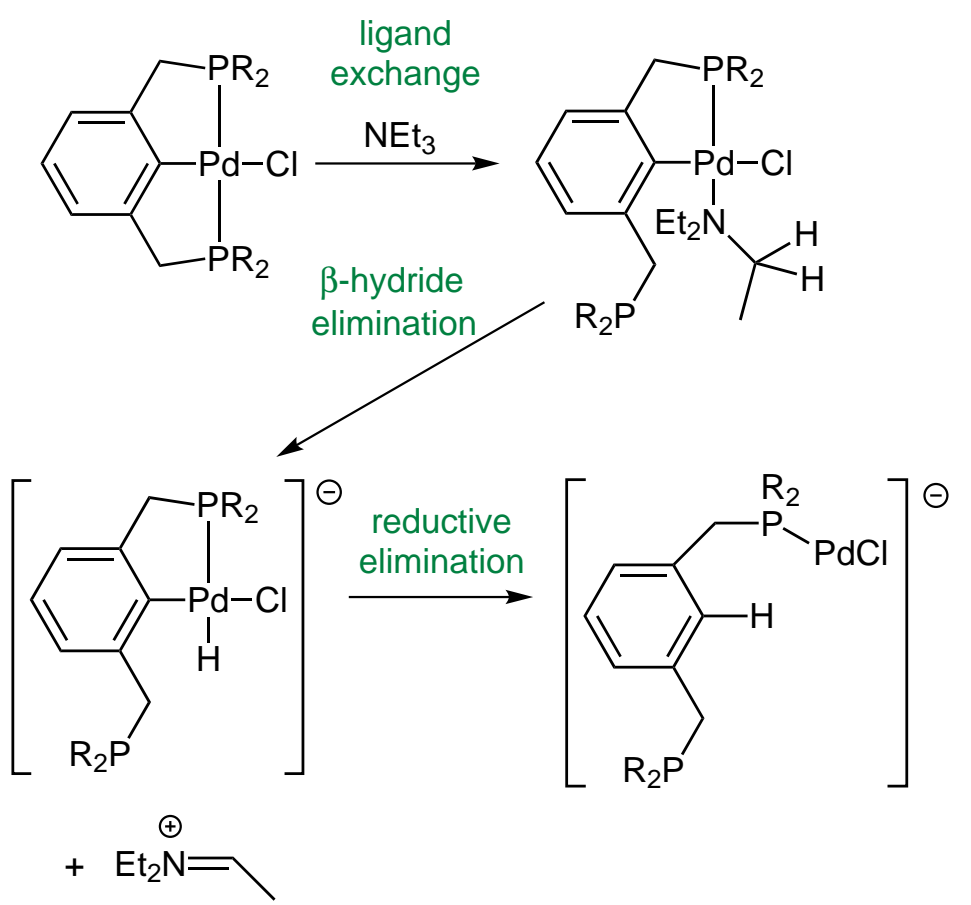

Scheme 6.3 Decomposition pathway for PCP pincer complexes in the presence of an amine base.

A second route to the $\operatorname{Pd}(0)$ species was proposed by Beletskaya ${ }^{200}$ for cyclometallated $\mathrm{N}-\mathrm{C}$ chelates, but may also be applicable for the decomposition of PCP pincer complexes (Scheme 6.4). In this pathway a phosphorus donor group dissociates from the palladium and is replaced with the $\pi$-coordinated alkene. This is able to undergo insertion into the palladium-carbon bond of the pincer ligand, much like in the classical Heck mechanism (Figure 6.1). However, in this configuration $\beta$-hydride elimination is unable to occur, as the seven-membered ring formed in the insertion step prevents the $\beta$-hydrogen from interacting with the palladium. Dissociation of the second phosphorus donor allows free rotation that brings the hydrogen into the proximity of the palladium, rendering the insertion product susceptible to $\beta$-hydride 
elimination and giving a styryl-substituted pincer ligand. The palladium-hydrido species generated is subsequently reduced to $\operatorname{Pd}(0)$ through hydride abstraction by the inorganic base, just as in the final step of the classical Heck reaction mechanism.

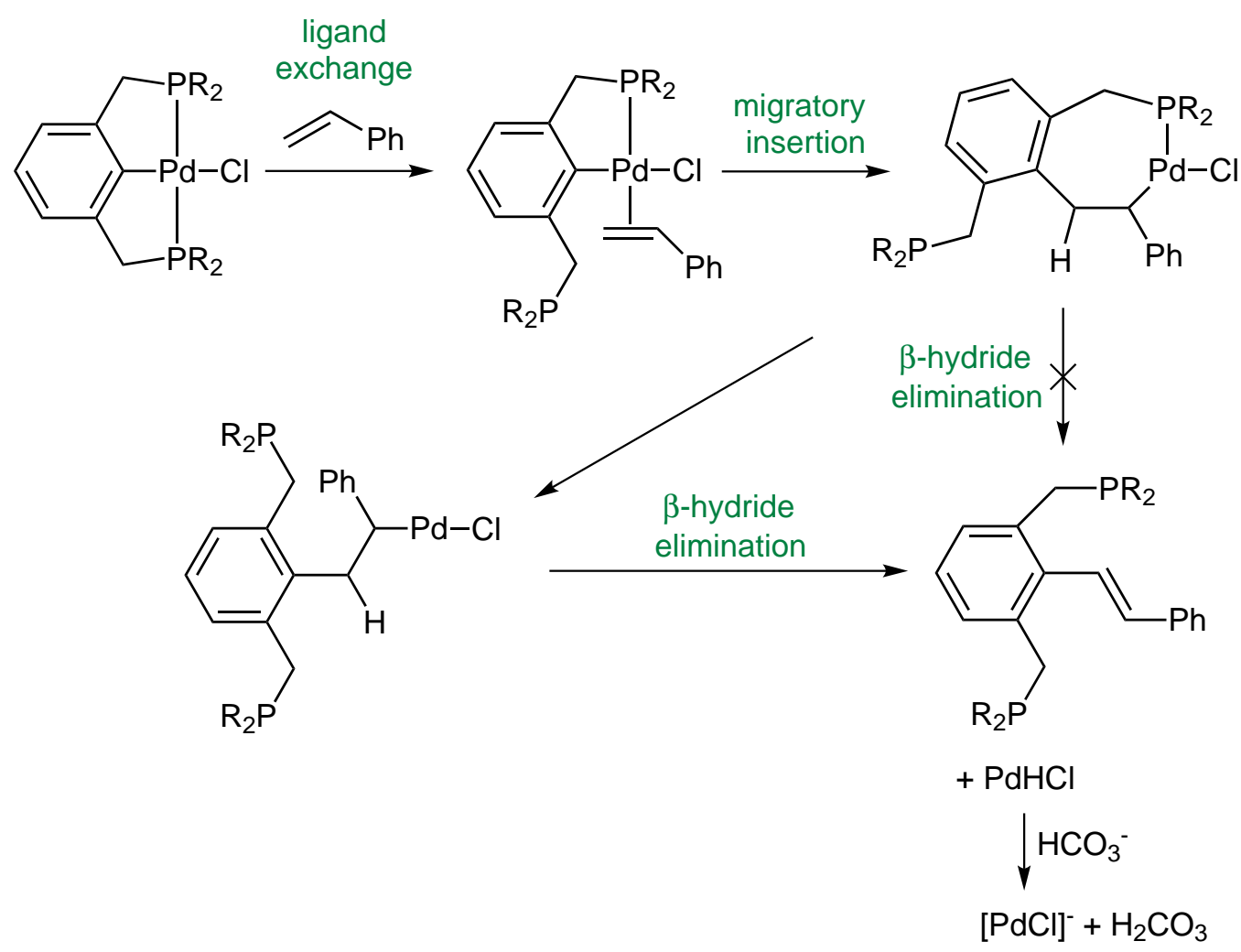

Scheme 6.4 Alternate decomposition pathway for PCP pincer complexes in the Heck reaction.

As these decomposition pathways are able to yield molecular $\operatorname{Pd}(0)$ species, and it has already been mentioned that mercury may poison some molecular $\operatorname{Pd}(0)$ species, further evidence was needed to unambiguously state that the active catalyst is a colloidal (rather than molecular) $\operatorname{Pd}(0)$ species. An observation in agreement with a nanoparticulate active catalyst is the behaviour of $\mathbf{3 0}$ at different catalyst loadings. This complex achieved a 25\% conversion after 16 hours at a 0.002 mole \% loading, but counterintuitively, the conversion decreased to $16 \%$ after 22 hours at double the catalyst loading (0.004 mole \%). If a catalytic cycle involving a molecular catalyst species was operating, a greater yield of product would be expected at a greater catalyst loading. However, when nanoparticles are the active catalyst, the activity is extremely concentration dependent. Low concentrations of catalyst precursor often demonstrate a disproportionately high activity due to the formation of smaller colloidal particles of palldium, which have a greater ratio of palladium at the surface (active) sites than larger particles, and hence are more active. ${ }^{185}$ Moreover, constant reaction of the aryl bromide with the surface of the nanoparticles prevents aggregation and Ostwald ripening; at a low palladium concentration more aryl bromide will be present per palladium atom, so this effect will be more pronounced. ${ }^{185}$ The rate 
of decompostion to form the active $\operatorname{Pd}(0)$ catalyst will depend on the coordination environment of the metal and therefore be specific to each complex, ${ }^{201}$ explaining why $\mathbf{3 0}$ was the only complex used that displayed a decrease in conversion moving from 0.002 to 0.005 mole \% loading.

Since the three complexes evaluated all showed similar product ratios (roughly 1:2:20 gem:cis:trans) the same active catalyst is expected in all reactions. It is worth noting that while 30 displayed an almost 1:1 cis:trans ratio after 22 hours, this is not seen as indicative of a molecular active catalyst. Increasing the steric bulk of the active catalyst is seen to increase the yield of the gem rather than cis product. ${ }^{202}$ In this case, the predominance of the cis isomer was not seen in shorter reactions with $\mathbf{3 0}$, and so the increased amount present in the longest reactions is likely to be due to cis/trans isomerisation in solution pending analysis.

From the decomposition pathways previously outlined (Scheme 6.3 and Scheme 6.4), a key step in the formation of the active catalyst species is the dissociation of one of the phosphorus donor atoms from the palladium. This allows for the formation of a complex with the correct cis-geometry for the reductive elimination or alkene insertion to occur. Dissociation of the remaining phosphorus donor group is then required for the formation of colloidal palladium. This requirement for $\mathrm{Pd}-\mathrm{P}$ dissociation can help explain the different rates of reactivity seen between compounds $\mathbf{2 8}, \mathbf{2 9}$, and 30 in the Heck reaction. $\mathrm{P}-\mathrm{O}$ bonds are notorious for undergoing facile hydrolysis; ${ }^{203,204}$ at millimole percent catalyst loadings it can be assumed that the amount of adventitious water present is sufficient to facilitate this cleavage. Moreover, under these basic reaction conditions (DMF solvent, excess potassium carbonate) this reaction will be more facile, as hydroxide is a better nucleophile than water. Once a $\mathrm{P}-\mathrm{O}$ bond is broken, the chelate effect can no longer provide additional stabilisation to the phosphine hydroxide ligand, allowing it to more readily dissociate from the complex. Once the dissociation has occurred, phosphinous acid/phosphine oxide tautomerism will reduce the availability of the phosphorus lone pair of electrons for ligand-metal bonding, making the coordination site cis to the $\mathrm{P}-\mathrm{C}$ bond more readily available, facilitating precatalyst decomposition (Scheme 6.5). Similar P-O cleavage was observed in reactions between methyllithium the POCOP platinum complex 25 (Chapter 4), where $\mathrm{PMe}\left(\mathrm{C}_{6} \mathrm{~F}_{5}\right)_{2}$ was observed as a minor product.

The ability for $\mathrm{P}-\mathrm{O}$ bond hydrolysis to hasten complex degradation was demonstrated in the relative rates of active catalyst formation between $\mathbf{2 8}$ and $\mathbf{2 9}$; data suggested that the PCCCP-ligated precatalyst displayed a longer induction period than the POCOP-ligated species (Figure 6.3, bottom). However, because 29 underwent a less facile decomposition it proved to be the better catalyst precursor. A 

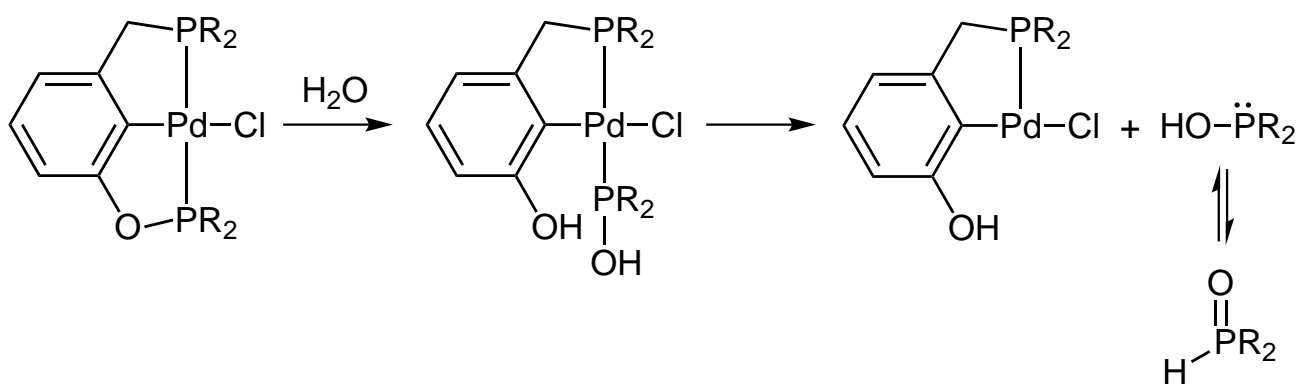

Scheme 6.5 $\mathrm{P}-\mathrm{O}$ bond hydrolysis in a PCP pincer complex and subsequent tautomerism between phosphinous acid and phosphine oxide forms.

gradual deposition of colloidal palladium over time helps to prevent Ostwald ripening and palladium black formation, ensuring a steady stream of active palladium nanoparticles are entering the reaction mixture. This 'slow release' of active palladium has been repeatedly invoked to explain why the tridentate pincer compounds are better precatalysts in the harsh conditions of the Heck reaction than analogous bidentate cyclometallated compounds. ${ }^{205}$

This effect was also observed when comparing compound 28 to its non-fluorinated analogues. While $\mathbf{2 8}$ achieved a TON of 18,000 for the cross-coupling of styrene with bromobenzene, replacing the electron-withdrawing pentafluorophenyl substituents on the phosphorus donor with electron-donating isopropyl groups gave a reported TON of 140,000 for the identical reaction. This dramatic difference in activities can be attributed to the electronic effects these substituents have on the phosphorus centres. Because they are more nucleophilic and better able to stabilise a negative charge, phosphine ligands with electron-withdrawing substituents have been shown to be much more susceptible to hydrolysis under basic conditions than their more electron-rich counterparts. ${ }^{206,207}$ This propensity for hydrolysis and subsequent degradation shown by pentafluorophenyl-substituted PCP pincer complexes renders them less efficient at depositing catalytically active palladium nanoparticles than their non-fluorinated counterparts, making them poorer catalysts for the Heck reaction.

Counterintuitive to the notion that more stable precatalysts result in higher TONs was the observation that the complex with both phosphine and phosphinite functionalities, 30, was a poorer precatalyst for the Heck reaction than the phosphinite complex 28. The electronic effect that the ligands impart on the metal centre is responsible for this. As has been previously mentioned, tert-butyl phosphines are exceedingly good $\sigma$-donors, while pentafluorophenyl phosphines and phosphinites are very poor $\sigma$-donors. ${ }^{37,38}$ This results in increased electron density on the palladium in 30 compared to $\mathbf{2 8}$; in the carbonyl analogues the $\mathrm{C}-\mathrm{O}$ stretch is seen to decrease 
from $2170 \mathrm{~cm}^{-1}$ in $[(\mathrm{POCOP}) \mathrm{Pd}(\mathrm{CO})]^{+}(\mathbf{3 4})$ to $2140 \mathrm{~cm}^{-1}$ in $[(\mathrm{POCCP}) \operatorname{Pd}(\mathrm{CO})]^{+}$ (36). Increasing the electron density on a metal centre has been shown to increase the energy barriers for alkene insertion and also reductive elimination. ${ }^{208,209}$ The effect of replacing a poorly donating pentafluorophenyl group with a strongly donating tert-butyl group therefore should increase the energy requirement for the decomposition pathways shown in Scheme 6.3 and Scheme 6.4, making formation of the initial $\mathrm{Pd}(0)$ species less facile. Furthermore, the strong $\mathrm{Pd}-\mathrm{P}^{t} \mathrm{Bu}_{2}$ bond may hinder colloidal palladium formation, as the $\operatorname{Pd}(0)$ centres will not be able to aggregate and effectively form nanoparticles until the phosphine has dissociated. A similar phenomenon has previously been reported in the literature, whereby the presence of tert-butyl phosphine donors improve the stability of catalyst precursors, but at the cost of a lower activity. ${ }^{183}$

There is clearly a fine balance in the relationship between ligand electronic effects and precatalyst activity. Complexes containing either very strongly donating or very weakly donating ligands display surprisingly modest activities as Heck precatalysts; a feature that is exacerbated rather than moderated when both donor types are combined in the same ligand.

Evidently these are complicated reaction mixtures. A number of potential $\mathrm{Pd}(\mathrm{II})$ to $\operatorname{Pd}(0)$ decomposition pathways are available, and there are surely a number of different factors at work influencing palladium nanoparticle formation. Whilst this section has sought to explain the observed reactivities of compounds 28, 29 and 30 , the factors determining the activity of PCP pincer complexes may not play a part in reactions catalysed by other ECE pincer species. Van Koten notes that for SCS pincer-porphyrin complexes, the more electron-rich the porphyrin, the faster the active catalyst formation and the higher the conversions obtained. ${ }^{201}$ Conversely, the work reported herein, along with that of Eberhard ${ }^{183}$ suggests that for PCP pincer complexes the presence of an electron-rich $\mathrm{P}^{t} \mathrm{Bu}_{2}$ groups slows down the formation of $\operatorname{Pd}(0)$ species and results in poor catalyst activity. Therefore, the factors that influence palladium nanoparticle formation for one group of pincer complexes may not pertain to pincer complexes as a whole, and ligand electronic effects in each case must be balanced such that the desired 'slow-release' of the nanoparticulate active catalyst into solution is obtained. 


\subsection{Performance of $[(\mathrm{PCP}) \mathrm{PdCl}]$ Species in the Suzuki Reaction}

Pincer complexes 28-30 were also assessed for their activity in the Suzuki reaction. While the Suzuki reaction is conceptually similar to the Heck reaction - it is a palladium-catalysed $\mathrm{C}-\mathrm{C}$ bond-forming reaction involving an aryl halide - the use of a boronic acid- or boronic ester-substituted aryl or vinyl group means that these reactions can generally be carried out under milder conditions than Heck reactions. It was hoped that under these conditions there would be less propensity for complex decomposition and palladium nanoparticle formation. The Suzuki reaction was therefore viewed as a greater hope for the observation of a $\mathrm{Pd}(\mathrm{II}) / \mathrm{Pd}(\mathrm{IV})$ catalytic cycle involving an intact palladium pincer complex.

Initial reactions were performed with phenylboronic acid and 4-bromoanisole in toluene at $100{ }^{\circ} \mathrm{C}$ (see Experimental Chapter for details). Reactions in toluene at $100{ }^{\circ} \mathrm{C}$ are common for pincer species in the literature, thereby offering a good degree of comparability with established work. 4-Bromoanisole was chosen as the aryl halide for these reactions - being an electronically-deactivated aryl bromide it should prove sufficiently difficult to couple, therefore providing a clear difference in turnovers between good and bad catalysts. Reactions with aryl iodides generally give high TONs even with poor catalysts, while reactions with aryl chlorides do not proceed readily, so are unsuitable for initial reactivity studies. As in the Heck reactions, both potassium carbonate and potassium phosphate were trialled as external bases. Preliminary investigations to determine optimal catalyst loadings were carried out in toluene- $d_{8}$ and analysed by NMR (as this had a higher throughput than GCMS). NMR proved to be a valid analytical tool, giving similar results to GCMS. However, due to the higher cost of solvents, NMR reactions were necessarily performed on a smaller scale, giving rise to mass transfer problems and a noticeably lower reproducibility than reactions carried out for GCMS analysis. Control reactions were also performed after these initial studies using the same glassware, to ensure that there was no activity due to persistent palladium residues that had survived the cleaning process. As in the Heck analysis, all reactions were performed in at least duplicate; quoted values for conversions reflect the averages of all identical analyses. 


\subsubsection{Results of Suzuki Reactions}

Results of the Suzuki reactions (Table 6.2) show that the activity of the complexes is of the order $[(\mathrm{POCOP}) \mathrm{PdCl}](\mathbf{2 8})>[(\mathrm{POCCP}) \mathrm{PdCl}](\mathbf{3 0})>[(\mathrm{PCCCP}) \mathrm{PdCl}]$ (29). The most active catalyst, 28, was tested under a range of reaction conditions, and was found to be slightly air sensitive, with greater yields obtained in reactions performed under inert atmospheres than those performed under ambient conditions. The addition of water to these reactions somewhat surprisingly increased the turnovers - it was expected that in the presence of water $\mathrm{P}-\mathrm{O}$ bond hydrolysis in 28 would be more facile and the TON would be reduced. The increased activity can be attributed in part to an increased solubility of the inorganic base in the presence of water, but primarily due to the increased concentration of hydroxide ions in the reaction mixture. Whereas in the Heck reaction the base is used to regenerate the active catalyst after $\beta$-hydride elimination, in the Suzuki reaction the base aids in transmetallation of the boron to the palladium. It is not clear whether this transmetallation proceeds via base coordination to the metal ${ }^{210}$ or to the boron, ${ }^{211}$ but in either case the high nucleophilicity of the $\mathrm{OH}^{-}$renders it much more effective at facilitating transmetallation than the inorganic bases alone. This also served to explain why the potassium phosphate was a better base for these reactions than potassium carbonate - the phosphate is used in its monohydrate form, meaning that as well as being a slightly stronger base, the water of crystallisation will provide the reaction mixture with additional hydroxide ions. Hydroxide bases themselves were not considered for this reaction as their increased basicity could potentially lead to more rapid catalyst degradation.

Reactions performed with potassium phosphate in the presence of a small amount of water produced the best results for the coupling of phenylboronic acid and 4bromoanisole catalysed by compound $\mathbf{2 8}$, giving $88 \%$ conversion and an average TON of 176,000 . These results indicate that $\mathbf{2 8}$ is amongst the most active pincer complexes for Suzuki reactions with aryl bromides. The most active pincer complexes have been reported by Bolliger and associates, ${ }^{52}$ whose PNCNP and POCOP complexes perform the coupling extremely rapidly, generating TONs of around 95,000 (with a 95\% conversion) in just 5 minutes for the coupling of 4bromoanisole with phenylboronic acid. Bedford ${ }^{212}$ and Garagorri ${ }^{78}$ have also reported highly active POCOP and PNCNP complexes, attaining TONs of 190,000 and 180,000 respectively for this cross-coupling. However, these numbers represent conversions of just $19 \%$ and $18 \%$; increasing the catalyst loading to obtain conversions in excess of $70 \%$ gives TONs of 7,200 and 77,000 respectively. Additionally, Bedford and Garagorri's reactions were carried out above $100{ }^{\circ} \mathrm{C}$ for at least 16 hours, demonstrating that the achievement of 176,000 turnovers after two hours at 
Table 6.2 Results of the Suzuki reaction with 28, 29, and 30 .<smiles>[X]c1ccc([PH2+])cc1</smiles>

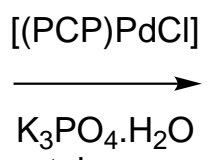
toluene<smiles>[R]c1ccc(-c2ccccc2)cc1</smiles>

\begin{tabular}{|c|c|c|c|c|c|c|}
\hline Ligand & $\overline{\text { Time }(\mathrm{h})}$ & $\overline{\text { Temp }\left({ }^{\circ} \mathrm{C}\right)}$ & $\overline{\mathrm{ArX}}$ & {$[\mathrm{Pd}](\mathrm{mol} \%)$} & Yield $^{a}(\%)$ & $\overline{\mathrm{TON}}$ \\
\hline \multirow{12}{*}{ POCOP } & $14^{b}$ & 100 & 4-bromoanisole & 0.001 & 39 & 39,000 \\
\hline & 20 & 100 & 4-bromoanisole & 0.001 & 89 & 89,000 \\
\hline & 2 & 100 & 4-bromoanisole & 0.001 & 74 & 74,000 \\
\hline & $1^{c}$ & 100 & 4-bromoanisole & 0.001 & 64 & 64,000 \\
\hline & 2 & 80 & 4-bromoanisole & 0.001 & 55 & 55,000 \\
\hline & $2^{c}$ & 80 & 4-bromoanisole & 0.001 & 44 & 44,000 \\
\hline & 2 & 60 & 4-bromoanisole & 0.001 & 13 & 13,000 \\
\hline & 2 & 100 & 4-bromoanisole & 0.0005 & 77 & 144,000 \\
\hline & $2^{d}$ & 100 & 4-bromoanisole & 0.0005 & 35 & 70,000 \\
\hline & $2^{e}$ & 100 & 4-bromoanisole & 0.0005 & 88 & 176,000 \\
\hline & 2 & 100 & 4-chloroanisole & 0.0005 & 0 & 0 \\
\hline & 20 & 100 & 4-chlorobenzaldehyde & 0.05 & 10 & 200 \\
\hline \multirow{3}{*}{ PCCCP } & 20 & 100 & 4-bromoanisole & 0.001 & 56 & 56,000 \\
\hline & 2 & 100 & 4-bromoanisole & 0.001 & 25 & 25,000 \\
\hline & $1^{c}$ & 100 & 4-bromoanisole & 0.001 & 2 & 2,000 \\
\hline \multirow{8}{*}{ POCCP } & $14^{b}$ & 100 & 4-bromoanisole & 0.001 & 17 & 17,000 \\
\hline & 20 & 100 & 4-bromoanisole & 0.001 & 66 & 66,000 \\
\hline & 2 & 100 & 4-bromoanisole & 0.001 & 53 & 53,000 \\
\hline & $1^{c}$ & 100 & 4-bromoanisole & 0.001 & 6 & 6,000 \\
\hline & 2 & 80 & 4-bromoanisole & 0.001 & 14 & 14,000 \\
\hline & 2 & 60 & 4-bromoanisole & 0.001 & 0 & 0 \\
\hline & 2 & 100 & 4-chloroanisole & 0.0005 & 0 & 0 \\
\hline & 20 & 100 & 4-chlorobenzaldehyde & 0.05 & 60 & 1,200 \\
\hline $\mathrm{DPPF}^{f}$ & 2 & 100 & 4-bromoanisole & 0.001 & 77 & 77,000 \\
\hline
\end{tabular}

${ }^{a}$ Determined by GCMS and based on aryl halide and biphenyl.

${ }^{b}$ Reaction performed with $\mathrm{K}_{2} \mathrm{CO}_{3}$ instead of $\mathrm{K}_{3} \mathrm{PO}_{4} \cdot \mathrm{H}_{2} \mathrm{O}$.

${ }^{c}$ Reaction performed with the addition of $\sim 30,000$ equivalents $\mathrm{Hg}$.

${ }^{d}$ Reaction performed in air.

${ }^{e}$ Reaction performed with the addition of $0.1 \mathrm{~mL}$ water.

${ }^{f}$ The formula of the complex used was [(DPPF) $\left.\mathrm{PdCl}_{2}\right]$. 
$100{ }^{\circ} \mathrm{C}$ by $\mathbf{2 8}$ is very impressive for this class of complex.

While compound $\mathbf{2 8}$ displays a very high activity for a pincer complex in the Suzuki reaction, this activity is substantially lower than is displayed by other palladium species for this reaction. Cyclometallated $\mathrm{P}-\mathrm{C}$ chelates such as $\mathbf{5 8}$ (Figure 6.2) have been observed to give TONs approaching 9,000,000 for reactions with electronicallydeactivated aryl bromides. ${ }^{212}$ One of the most effective systems for the Suzuki reaction - Buchwald's 2-dicyclohexylphosphinobiphenyl ligands with $\left[\mathrm{Pd}(\mathrm{OAc})_{2}\right]$ - catalyses reactions with electronically-deactivated aryl chlorides at room temperature. ${ }^{213}$ In comparison, Suzuki reactions using aryl chlorides do not proceed well with these pincer complexes. Reactions with the electronically-activated 4chlorobenzaldehyde only proceeded with very moderate turnovers of 1,200 (complex 30) and 200 (complex 28) after 18 hours at $100{ }^{\circ} \mathrm{C}$. Interestingly, the reactivity displayed here was the reverse of that shown in reactions involving electron-poor aryl bromides - the POCCP complex 30 was more active than the POCOP complex 28.

To assess the activity of these pincer complexes in the Suzuki reaction, they were compared to a commercially available catalyst, $\left[(\mathrm{DPPF}) \mathrm{PdCl}_{2}\right]$. This species was chosen as it is a highly active catalyst for cross-coupling reactions and is commonly used in organic synthesis. Reactions with 28, 29, 30 and $\left[(\mathrm{DPPF}) \mathrm{PdCl}_{2}\right]$ were monitored by GCMS to observe the speed at which the reactions proceeded, and also to probe the nature of induction periods and catalyst lifetime. Results from these reactions (Figure 6.4, top) show that as well as giving the greatest conversions, complex 28 also promotes a very rapid reaction. Extrapolating from the point of $50 \%$ conversion gives TOFs of 330,000 for complex 28, 88,000 for [(DPPF) $\mathrm{PdCl}_{2}$ ] and 29,000 for complex 30. A TOF value for $\mathbf{2 9}$ could not be established as the reaction had not reached $50 \%$ conversion during the time allotted. These data show that over the first hour, the POCOP complex $\mathbf{2 8}$ outperforms the commerically available $\left[(\mathrm{DPPF}) \mathrm{PdCl}_{2}\right]$, reaching the $50 \%$ conversion mark in almost one quarter of the time for the DPPF complex. However, it appears catalyst stability is an issue under these conditions, with both $\mathbf{2 8}$ and $\mathbf{3 0}$ displaying a much reduced activity after 30 minutes, so much so that around the 90 minute mark [(DPPF) $\left.\mathrm{PdCl}_{2}\right]$ surpasses the turnovers achieved by 28 .

This poor catalyst lifetime was also demonstrated by testing the supernatant for catalytic activity. The coupling of 4-bromoanisole with phenylboronic acid in the presence of $1 \mathrm{mmol} \% \mathbf{2 8}$ was performed; after 30 minutes the supernatant containing the catalyst was reused with fresh starting materials, then after an additional 30 minutes the supernatant was used in a third subsequent reaction. Reactions were 

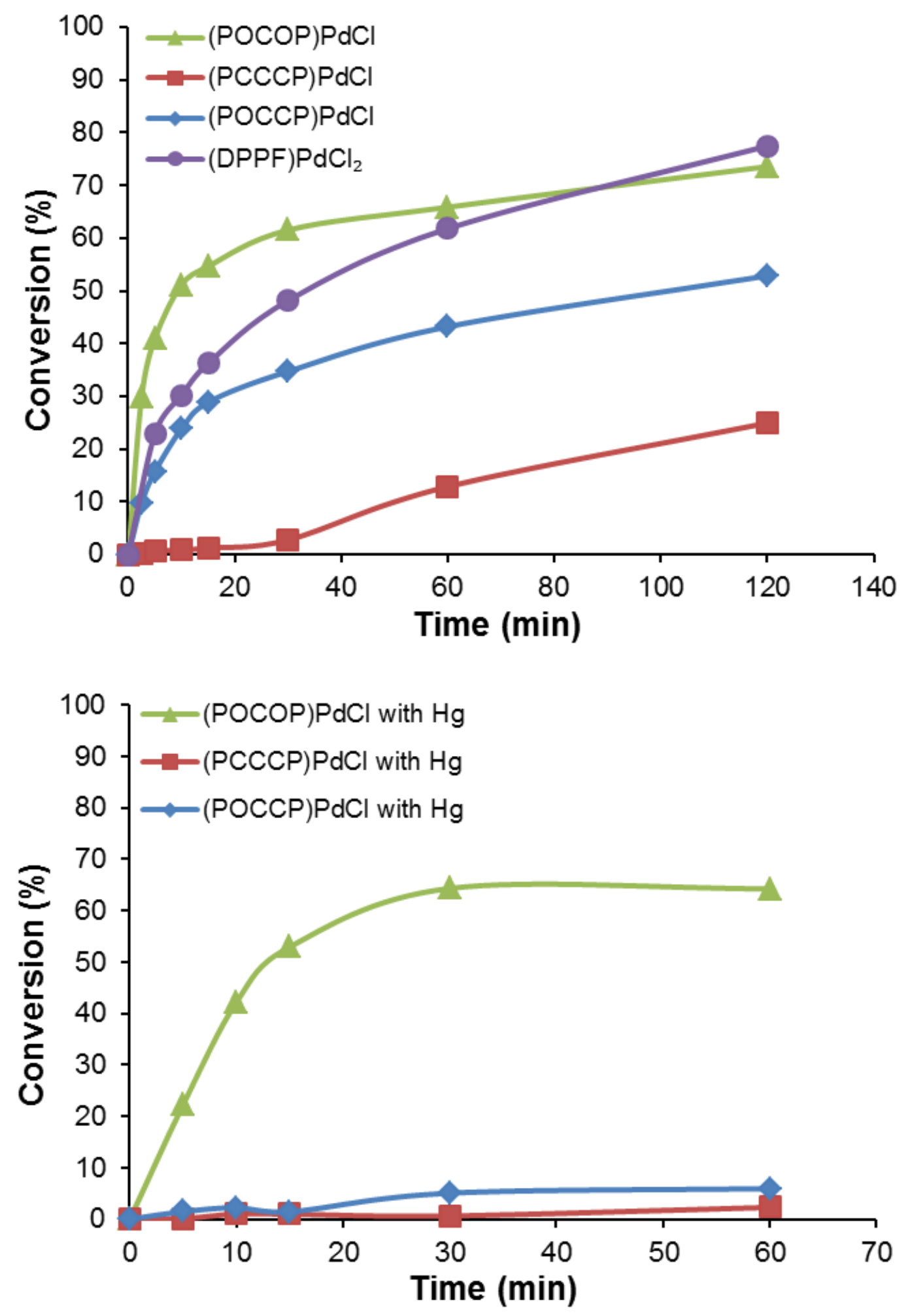

Figure 6.4 Conversion vs time: 4-bromoanisole/phenylboronic acid $/ \mathrm{K}_{3} \mathrm{PO}_{4} \cdot \mathrm{H}_{2} \mathrm{O}$ at $100{ }^{\circ} \mathrm{C}$ with $1 \mathrm{mmol} \%$ of complexes 28, 29, 30 and [(DPPF)PdCl $\mathrm{P}_{2}$. Standard reaction conditions (top), reactions with the addition of approximately 30,000 equivalents mercury (bottom). 
performed in duplicate, with the activity of $\mathbf{2 8}$ seen to decrease upon each subsequent reuse. Initially, a $73 \%$ conversion into the biphenyl product was observed, dropping to $50 \%$ then $21 \%$ on the second and third reuses respectively. The dramatic loss of activity between runs confirmed the decomposition of the active species, and indicated that severe difficulties may be encountered with catalyst recovery and reuse in this system.

As well as illustrating the rapidity at which the POCOP complex $\mathbf{2 8}$ carried out these Suzuki reactions, the plots of conversion against time (Figure 6.4) also demonstrate why the PCCCP complex 29 performed so poorly. The reaction catalysed by 29 clearly displays an induction period of approximately 30 minutes, during which time only traces of product are formed. As in the Heck reactions, the induction period and subsequent sigmoidal kinetics are characteristic of a colloidal $\operatorname{Pd}(0)$ active catalyst. To probe the nature of the active species in these Suzuki reactions, mercury poisoning experiments were again undertaken. The addition of one drop of mercury (about 30,000 equivalents per palladium) to the reaction mixtures was seen to dramatically reduce the activity of compounds $\mathbf{2 9}$ and $\mathbf{3 0}$ (Figure 6.4, bottom). After one hour in the presence of mercury, the TON of $\mathbf{3 0}$ dropped from 43,000 to 6,000, while the TON of $\mathbf{2 9}$ dropped from 13,000 to 2,000. However, somewhat surprisingly, the POCOP compound $\mathbf{2 8}$ was seen to give essentially the same conversions regardless of whether mercury was present or not (TON of 66,000 without mercury and 64,000 with mercury, after one hour). Since the mercury poisoning experiments had been seen to give no traces of product in Heck reactions with $\mathbf{2 8}$, this served to establish that under the milder Suzuki reaction conditions the catalytic activity was due to a homogeneous palladium species in solution, rather than heterogeneous palladium nanoparticles.
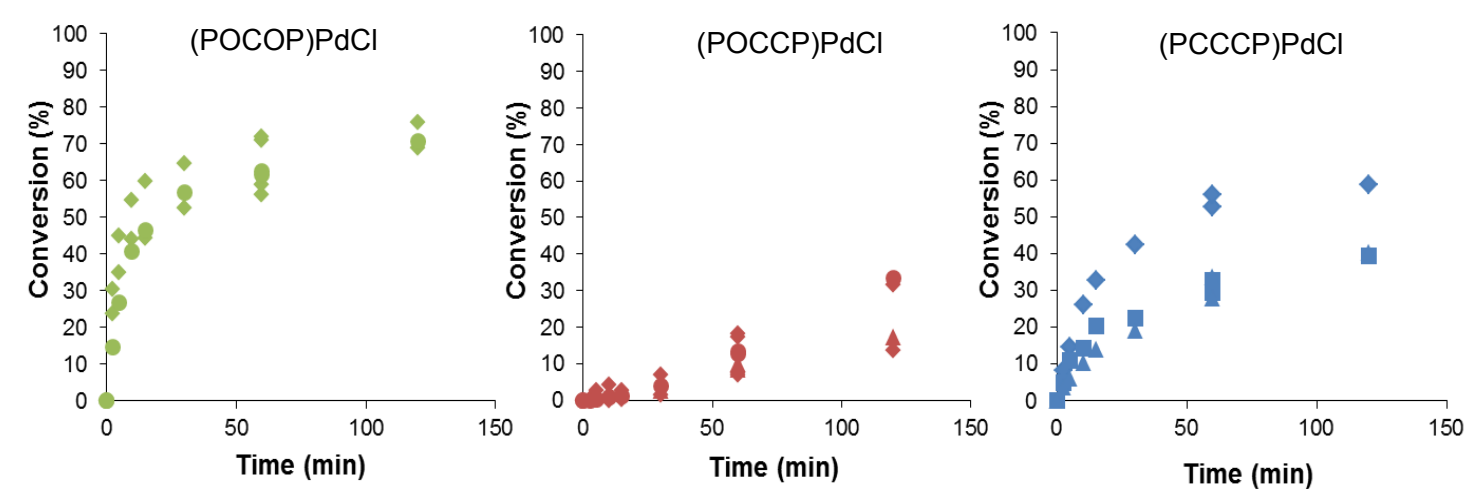

Figure 6.5 Raw data for Suzuki reactions at $100{ }^{\circ} \mathrm{C}$ catalysed by (from left to right) pincer complex 28,29 , and 30 . 
It has been reported that kinetic irreproducibility is also an indicator of complex degradation to produce a heterogeneous active catalyst, as the nanoparticle formation is highly sensitive to reaction conditions. ${ }^{214}$ Examination of the raw data from these Suzuki reactions (Figure 6.5) revealed that reactions performed with compound $\mathbf{2 8}$ were more reproducible than those performed with $\mathbf{2 9}$ and $\mathbf{3 0}$. The percentage of starting material converted to product in reactions catalysed by $\mathbf{2 8}$ was spread over a $6 \%$ range (69-76\% conversion) after 2 hours at $100{ }^{\circ} \mathrm{C}$, compared to $20 \%$ and $19 \%$ (13-33\% and 39-58\% conversion) for 29 and 30 respectively. This provided a further indication that Suzuki reactions catalysed by the POCOP complex $\mathbf{2 8}$ proceed through a homogeneous active species, while the PCCCP complex 29 and the POCCP complex 30 degraded under Suzuki conditions to produce heterogeneous active catalysts.

To probe for the presence of an induction period in reactions involving $\mathbf{3 0}$, and to further investigate the nature of the active species in these Suzuki reactions, reactions with complexes $\mathbf{2 8}$ and $\mathbf{3 0}$ were monitored at the lower temperatures of $80{ }^{\circ} \mathrm{C}$ and $60{ }^{\circ} \mathrm{C}$, with the results shown in Figure 6.6. Performing kinetic studies at lower temperatures has been shown to dramatically reduce the rate of palladium nanoparticle formation, revealing sigmoidal kinetic behaviour in reactions that displayed none at higher temperatures. ${ }^{215}$ The most striking feature of these plots is that even at $80{ }^{\circ} \mathrm{C}$, compound 28 maintained a high activity, producing 55,000 turnovers at a TOF of 50,000. Conversely, the activity of $\mathbf{3 0}$ was reduced dramatically, giving only 14,000 turnovers after the two hour reaction had concluded. Further reduction of the temperature to $60{ }^{\circ} \mathrm{C}$ rendered $\mathbf{2 8}$ much less effective (TON of 13,000), while no traces of product were detected in the reaction of $\mathbf{3 0}$ at this temperature.

Looking at an expanded view of the reactions (Figure 6.6, bottom) the presence of an induction period is clearly visible for the reaction of the POCCP complex 30 at $80{ }^{\circ} \mathrm{C}$, with only traces of product detected up until the 20 minute mark, at which point the reaction commenced. For the reaction of the POCOP complex 28 at $60{ }^{\circ} \mathrm{C}$, the results are not quite as clear-cut, as a slight bump appears in the curve after 10 minutes. However, the 4-methoxybiphenyl product was detected in the first sample withdrawn after 2.5 minutes and appeared in ever-increasing amounts in every sample taken forthwith, rather than being present in trace amounts for the induction period, then appearing in increasing amounts. Thus the data were not conclusive as to whether sigmoidal kinetics are likely to be in effect for Suzuki reactions involving 28. However if degradation of the pincer complex to an active species does occur, it must necessarily be very rapid to consistently produce detectable amounts of product after two minutes at $60{ }^{\circ} \mathrm{C}$. 

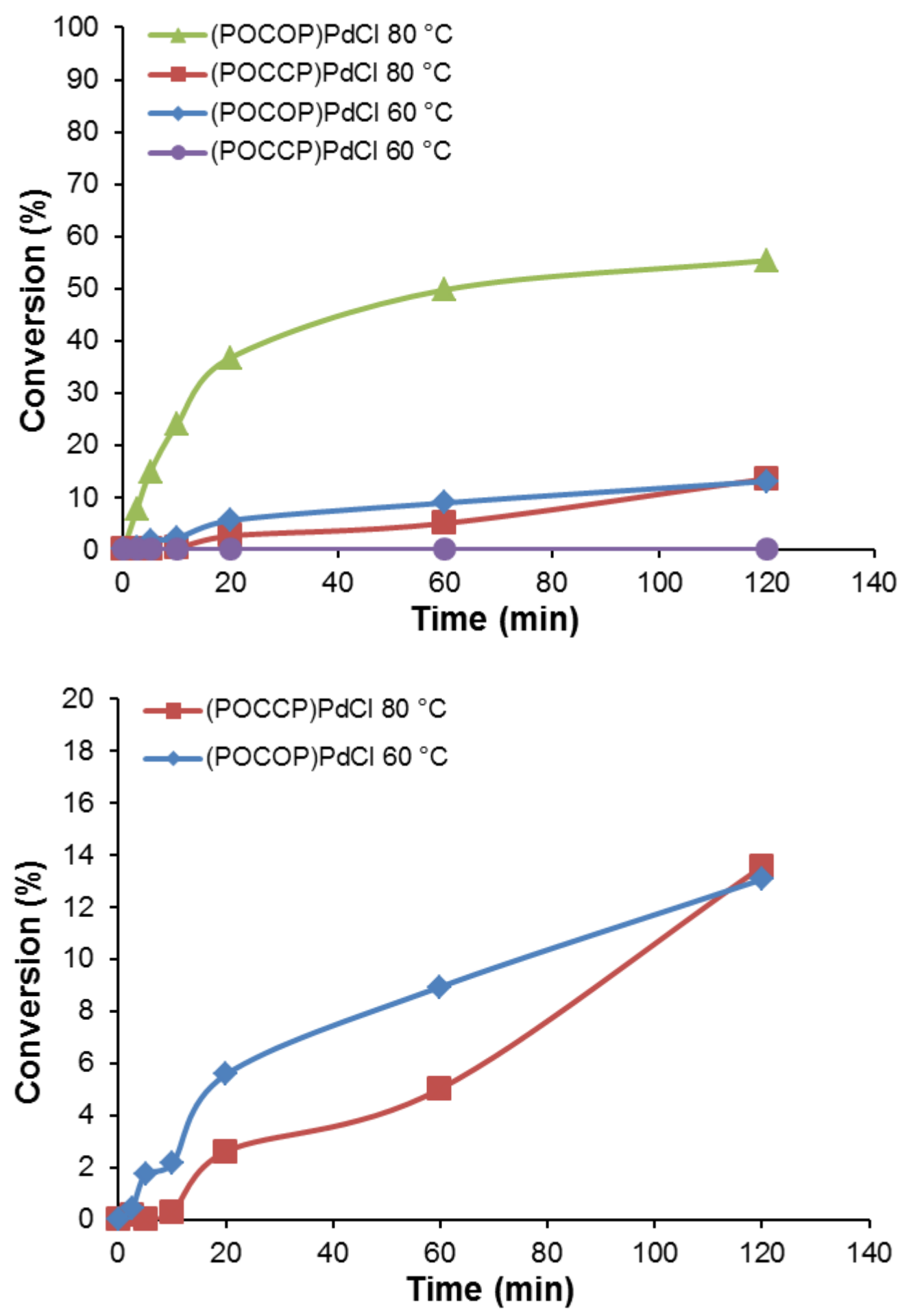

Figure 6.6 Conversion vs time: 4-bromoanisole/phenylboronic acid $/ \mathrm{K}_{3} \mathrm{PO}_{4} \cdot \mathrm{H}_{2} \mathrm{O}$ at $80{ }^{\circ} \mathrm{C}$ and $60^{\circ} \mathrm{C}$ with $1 \mathrm{mmol} \%$ of complexes 28 and 30. All data (top), expanded view of selected reactions (bottom). 
Mercury poisoning reactions were also repeated for the reaction with compound $\mathbf{2 8}$ at $80^{\circ} \mathrm{C}$. This was done to ensure that the negative result in the mercury drop test performed at $100{ }^{\circ} \mathrm{C}$ was not due to the formation of $\operatorname{Pd}(0)$ being so rapid that the cross-coupling was completed before the mercury could amalgamate the palladium, as has been proposed for some rapid Suzuki reactions in the literature. ${ }^{216}$ Upon reducing the reaction temperature from $100{ }^{\circ} \mathrm{C}$ to $80{ }^{\circ} \mathrm{C}$, the observed TOF for 28 droped from 330,000 to approximately 50,000 in the absence of mercury; a value close to that of the POCCP species 30 at $100{ }^{\circ} \mathrm{C}$ (TOF of 29,000). As 30 returned a positive mercury drop test at $100{ }^{\circ} \mathrm{C}$ (a drastic reduction in activity was observed), it can be concluded that at $80{ }^{\circ} \mathrm{C}$ nanoparticle formation from 28 would not be so rapid as to give a spurious result in the mercury drop test.

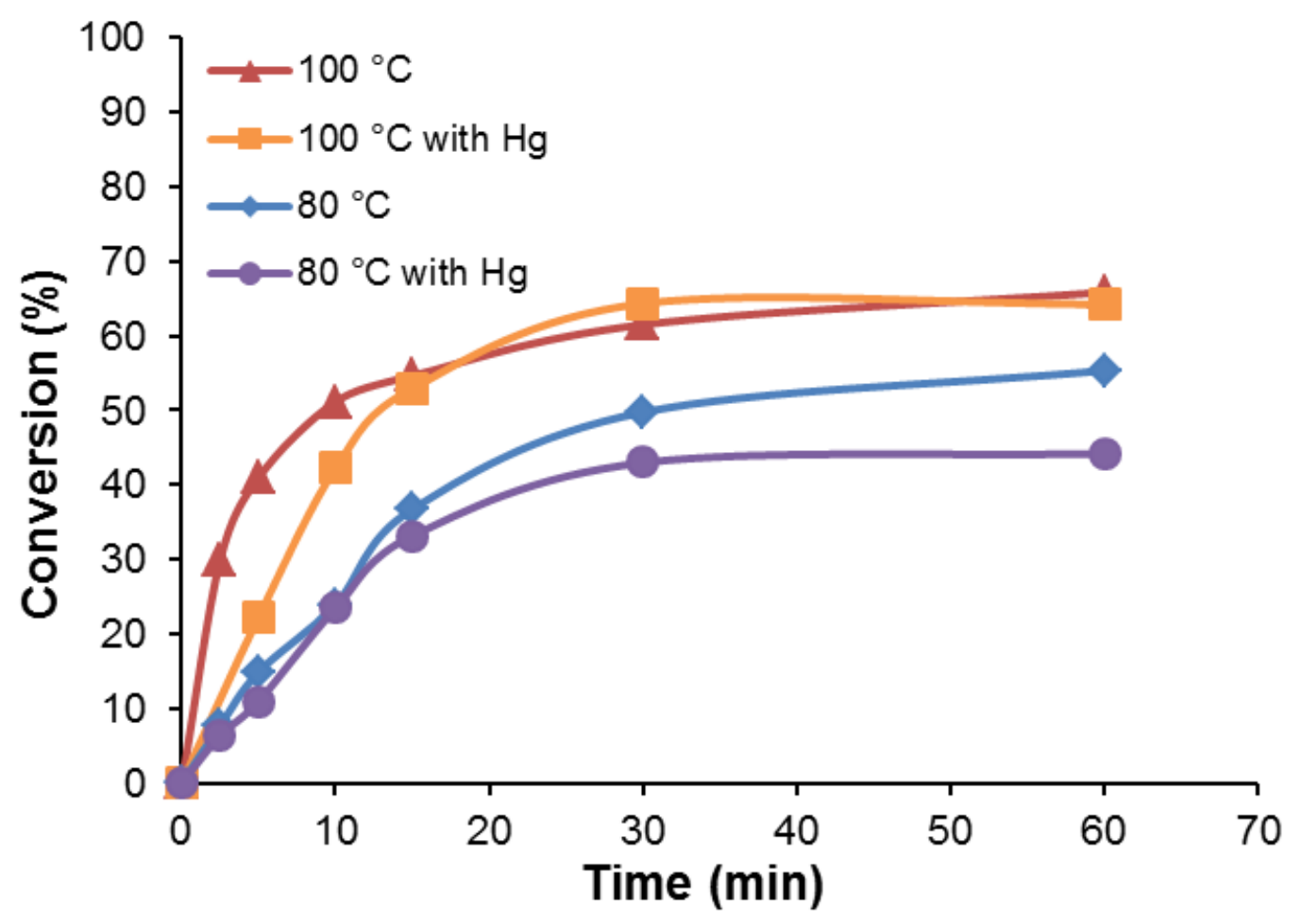

Figure 6.7 Conversion vs time: 4-bromoanisole/phenylboronic acid $/ \mathrm{K}_{3} \mathrm{PO}_{4} \cdot \mathrm{H}_{2} \mathrm{O}$ at $100{ }^{\circ} \mathrm{C}$ and $80{ }^{\circ} \mathrm{C}$ with $1 \mathrm{mmol} \%$ of complex 28 in the presence and absence of 30,000 equivalents of mercury.

Again, the mercury drop test with 28 was negative; reactions at $80{ }^{\circ} \mathrm{C}$ gave essentially the same conversions whether mercury was present or not, just as they had at $100{ }^{\circ} \mathrm{C}$. Looking at a plot of the reaction progress for these mercury poisoning experiments (Figure 6.7), it is evident that in both instances when mercury is present the activity is halted after about 30 minutes, while in the absence of mercury the reaction continues to progress after 60 minutes (albeit slowly). This is indicative that there are contributions to activity from both homogeneous and heterogeneous active species occurring concurrently in these reaction mixtures. In reactions involving 29 and 30 the activity was severely diminished by mercury poisoning, but not 
eliminated altogether. This was in contrast to the Heck reaction, where no traces of product were observed in reactions where mercury was added, and indicated that a small amount of activity in these Suzuki reactions was probably due to homogeneous catalysis by palladium complexes in solution. For 28 the contribution from homogeneous catalysis was much greater, to the point where mercury addition was seen only to reduce activity after about 30 minutes, by which time colloidal degradation products of the homogeneous active catalyst must be responsible for the activity.

The effectiveness of compound $\mathbf{2 8}$ in catalysing the Suzuki reaction on a large scale was also investigated. The reaction between 4-bromoanisole and phenylboronic acid was performed in a round bottom flask at 20 times the scale of the previous reactions, with a catalyst loading of $1 \mathrm{mmol} \%$. After 12 hours at $100{ }^{\circ} \mathrm{C}$ the reaction was stopped and the product isolated by extraction with ethyl acetate, followed by column chromatography on alumina to remove the excess phenylboronic acid. The 4-methoxybiphenyl was isolated in a $98 \%$ yield, giving a turnover number for $\mathbf{2 8}$ of 98,000 and indicating that this catalyst continues to perform well upon upscaling of the Suzuki reaction.

\subsubsection{Active Species Generation and Reaction Mechanism}

The observation that Suzuki reactions catalysed by compound 28 proceeded via a homogeneous active species meant the nature of the reaction mechanism needed to be addressed. Like the Heck reaction, the Suzuki reaction is typically catalysed out by a low-coordinate $\operatorname{Pd}(0)$ active species, with oxidative addition of the aryl halide to form a $\mathrm{Pd}(\mathrm{II})$ intermediate a key step in the mechanism (Figure 6.8). As this is conceptually similar to the Heck reaction, the $\mathrm{Pd}(\mathrm{II}) /(\mathrm{IV})$ catalytic cycle proposed for pincer complexes participating in the Heck reaction has also been invoked to explain their reactivity in the Suzuki reaction. ${ }^{52,217}$

In this work, it is highly unlikely that reactions catalysed by 28 proceed through a $\mathrm{Pd}(\mathrm{II}) /(\mathrm{IV})$ catalytic cycle involving the intact pincer complex. The primary reason for this is electronic; $\mathbf{2 8}$ contains a very electron-poor metal centre. Palladium(IV) species are regarded as being significantly less stable than the corresponding platinum(IV) complexes, ${ }^{218}$ hence for the oxidative addition step to be facile, the $\mathrm{Pd}(\mathrm{IV})$ product must be stabilised through electron donation to the metal from the surrounding ligands. As discussed in Chapter 4, the $\mathrm{C}-\mathrm{O}$ stretching frequencies of the carbonyl derivatives $[(\mathrm{POCOP}) \mathrm{Pd}(\mathrm{CO})]^{+},[(\mathrm{PCCCP}) \mathrm{Pd}(\mathrm{CO})]^{+}$, and 


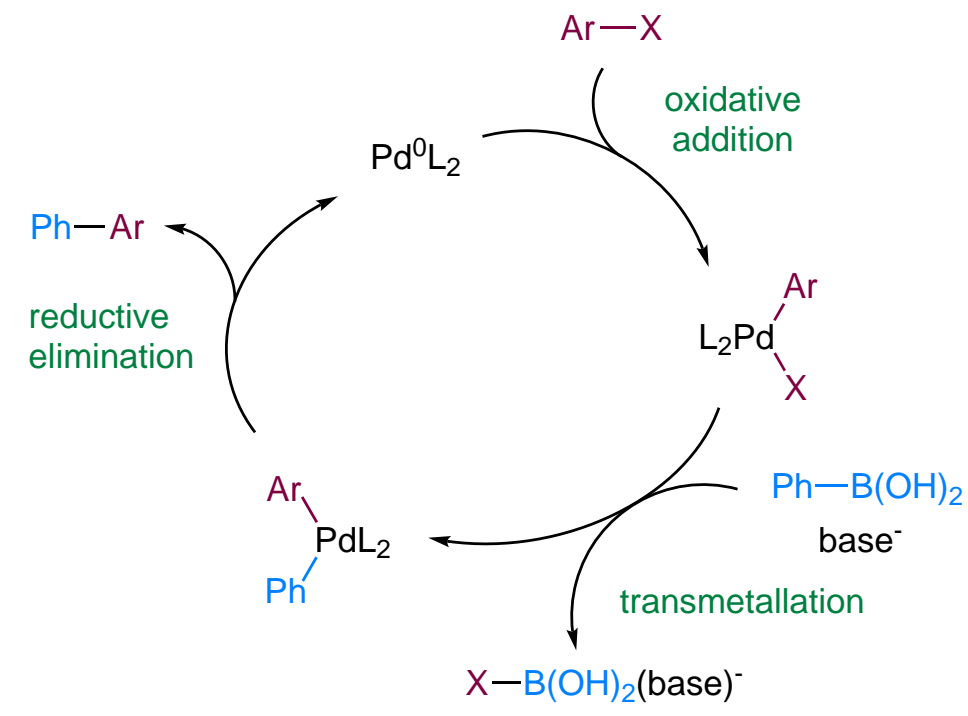

Figure 6.8 Outline of the classic "textbook" Suzuki catalytic cycle.

$[(\mathrm{POCCP}) \mathrm{Pd}(\mathrm{CO})]^{+}$(compounds $\mathbf{3 4}, \mathbf{3 5}$, and 36 respectively) provided an indication of the electron density present on each metal centre. With a carbonyl $\mathrm{C}-\mathrm{O}$ stretching frequency of $2170 \mathrm{~cm}^{-1}$, complex 34 displayed the most electron-poor palladium centre of any pincer compound reported to date, and was significantly more electron-poor than the corresponding PCCCP and POCCP palladium complexes 35 and 36 (carbonyl $\mathrm{C}-\mathrm{O}$ stretches of 2148 and $2140 \mathrm{~cm}^{-1}$ respectively). As any $\mathrm{Pd}(\mathrm{IV})$ intermediates generated from 28 will undergo minimal electronic stabilisation, they will be high in energy and consequently be poor candidates for intermediates in a reaction that was observed to be facile at temperatures as low as $60{ }^{\circ} \mathrm{C}$. This rationale that an electron-poor metal centre will not readily undergo oxidative addition has been bolstered through the use of computational chemistry. Density Functional Theory (DFT) calculations on similar palladium pincer complexes have demonstrated that for this oxidative addition, "the higher the electron density on the metal centre, the lower the ground state energy and energetic barrier." 189 To confirm this and correlate electron density on the metal centre with ease of oxidation, cyclic voltammetry (CV) was performed on complexes $\mathbf{2 8}, \mathbf{2 9}$, and $\mathbf{3 0 .}$ However, meaningful results were unable to be obtained. Fortunately, a 2011 paper by Polukeev and colleagues ${ }^{219}$ confirmed that the carbonyl $\mathrm{C}-\mathrm{O}$ stretch of a pincer carbonyl adduct directly corresponds to the ease of oxidation of its metal centre. From these carbonyl stretching frequencies, this indicates the ease of oxidation of these complexes would be $\mathbf{3 0}>\mathbf{2 9}>\mathbf{2 8}$. Since the compound that should be the most difficult to oxidise is the species that reacts via homogeneous catalysis, from an electronics point of view a $\mathrm{Pd}(\mathrm{II}) /(\mathrm{IV})$ mechanism for this catalysis is highly unlikely.

Furthermore, there is a steric barrier to $\mathrm{Pd}(\mathrm{II}) /(\mathrm{IV})$ catalysis. To achieve reductive elimination of the biphenyl product from a $\mathrm{Pd}(\mathrm{IV})$ species, the aryl groups need to 
occupy mutually cis coordination sites, and be oriented in a "face-on" conformation to facilitate effective orbital overlap. However, it has been demonstrated that in tert-butyl-substituted PCP pincer complexes, the steric demands of the bulky tertbutyl groups above and below the coordination plane of the aryl groups force them to sit in a flat, "side-on" manner, significantly increasing the energy barrier for $\mathrm{C}-\mathrm{C}$ reductive elimination (Figure 6.9). ${ }^{220}$ Bis(pentafluorophenyl)phosphine groups have a similar steric demand to di-tert-butyl phosphine groups, ${ }^{37}$ therefore the coordinated aryl groups would be expected adopt a "side-on" conformation, providing another restriction to biphenyl formation. Hence because of the electron-poor, sterically crowded nature of the POCOP complex 28, a mechanism involving Pd(IV) intermediates is highly unfavourable and can be disregarded as a plausible reaction pathway in this instance.

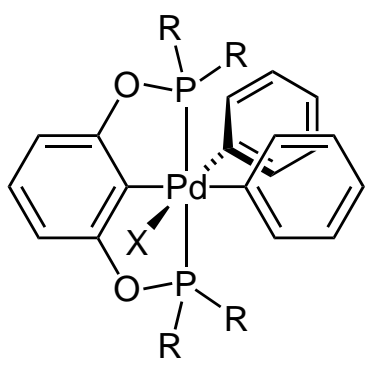

$\mathrm{R}=$ small steric bulk Aryl groups "face-on"

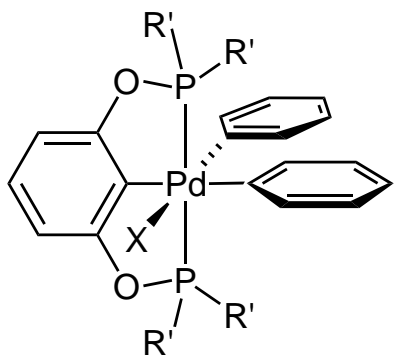

$\mathrm{R}^{\prime}$ = large steric bulk Aryl groups "side-on"

Figure 6.9 Steric effect of phosphorus substituents on aryl coordination. Reductive elimination of aryl groups is favoured by a "face-on" conformation.

Another consideration for the mechanism of the Suzuki reaction catalysed by $\mathbf{2 8}$ is a concerted, redox-free pathway (Figure 6.10). Such a pathway was proposed in 2009 by Olsson and Wendt, ${ }^{221}$ and has been subsequently invoked to explain the reactivity of pincer catalysts in both the Suzuki and Negishi cross-coupling reactions. ${ }^{190,222}$ The key to this mechanism is that the first step - the transmetallation of the phenyl group from the boron to the palladium - can occur via a four-membered transition state, without any change in the oxidation state of the metal. ${ }^{211}$ The palladiumphenyl species generated by transmetallation then reacts with the aryl halide in a metathesis-type reaction. This is almost identical to the transmetallation step, proceeding through a similar four-membered transition state in a concerted fashion, with $\mathrm{Pd}-\mathrm{Br}$ bond formation occurring concurrently with $\mathrm{Pd}-\mathrm{C}$ bond cleavage. This produces the biphenyl product and also regenerates the (PCP)PdX active catalyst, leaving the pincer framework intact.

While this redox-free mechanism is a viable possibility for the Suzuki reaction, an 


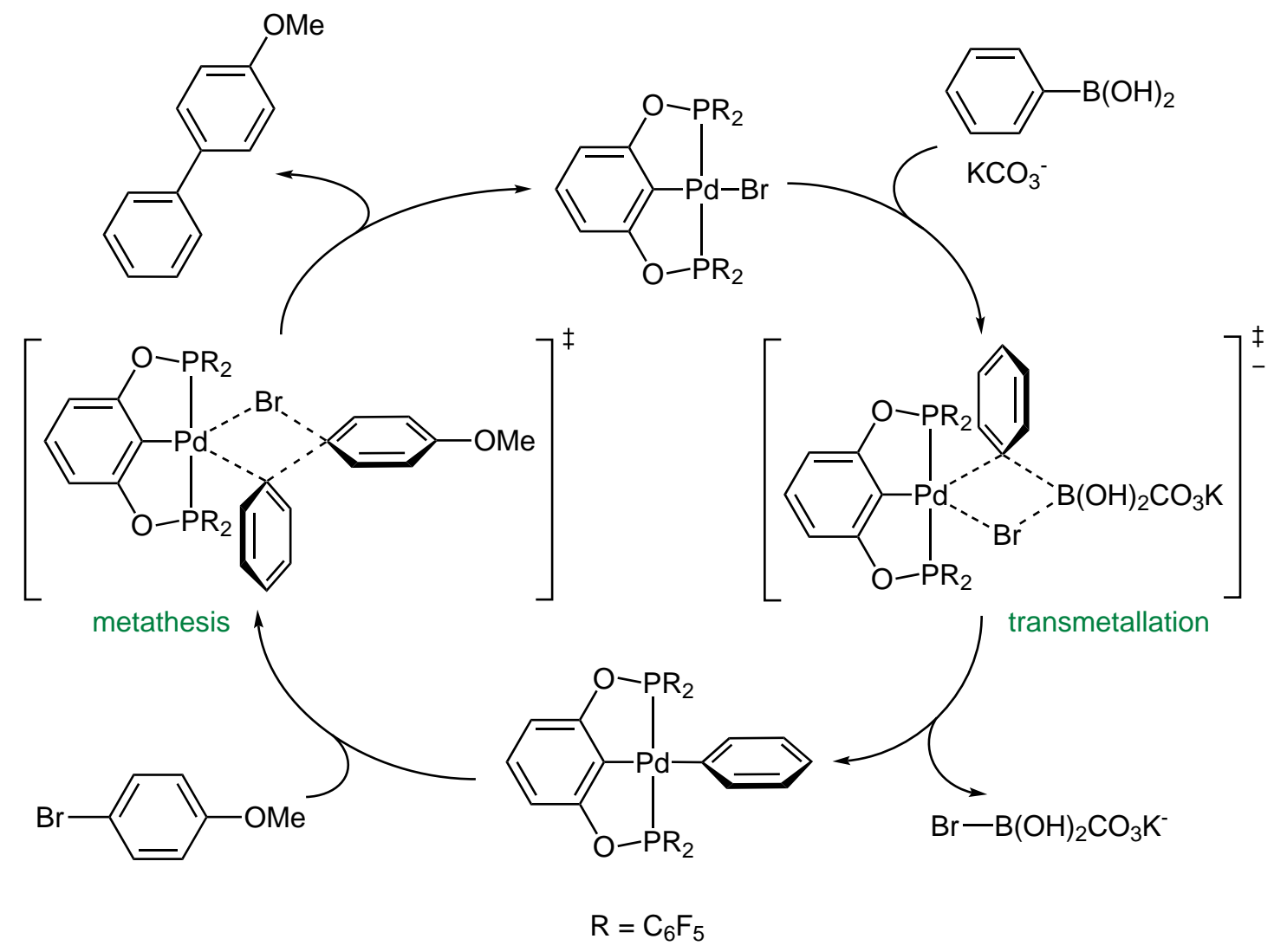

Figure 6.10 Proposed redox-free mechanism for compound 28 in the Suzuki reaction.

analogous mechanism is not possible for the Heck reaction. The Heck reaction requires alkene insertion into a palladium-aryl bond, meaning that oxidative addition of the aryl halide to the palladium has to be the first mechanistic step. In the Suzuki reaction there is no insertion step, meaning either the aryl halide oxidative addition or transmetallation of the arylboronic acid may occur first. As the transmetallation step occurs through a redox-free pathway, when the reaction is initiated by transmetallation it allows for the possibility of a redox-free mechanism, so long as the product-forming metathesis step is facile. Such metathesis steps are not well known for palladium, they are more prevalent in the chemistry of the harder, early transition metals. ${ }^{223}$ However, reports have indicated that these concerted, four-membered transition states are favoured for some palladium pincer complexes as a lower energy alternative to an oxidative addition/reductive elimination pathway. ${ }^{224,225}$ Moreover, in the well-established cross-coupling of vinyl epoxides with organoboronic acids, the nucleophilic attack step is conceptually similar to the metathesis. ${ }^{172,226,227}$ In this reaction, an aryl or vinyl group is transmetallated from a boronic acid to a palladium pincer species, as in the first step of Figure 6.10. The palladium-aryl species then undergoes nucleophilic attack on a vinyl epoxide, which can be seen as analogous to the metallated carbon of the phenyl group attacking the ipso-carbon of the aryl bromide in the second step of Figure $6.10 .{ }^{226}$ The fact that this reaction is catalysed 
by palladium pincer complexes under mild conditions via a redox-free mechanism is a good indication that a similar redox-free mechanism may be in operation in the Suzuki reaction.

This proposed mechanism is consistent with the reactivity differences between compounds 28, 29, and 30. For proton transfer reactions on late transition metals that can proceed via oxidative addition or $\sigma$-bond metathesis pathways, it has been observed that the four-centred metathesis transition state was favoured for less electron-rich metal centres. ${ }^{223}$ Similarly, in the aforementioned vinyl epoxide crosscoupling, it was noted that complexes with electron-poor metal centres catalysed the reaction significantly faster than their electron-rich counterparts. This trend of a higher reactivity for more electron-poor metal centres can explain the results obtained in these Suzuki reactions. For the most electron-poor complex 28, the $\mathrm{C}-\mathrm{Br}$ bond metathesis step is facile and the reaction is catalysed by a palladium pincer complex in solution. For the comparatively more electron-rich complexes 29 and 30 the metathesis step will be less favourable, meaning that the colloidal palladium decomposition products catalyse the reaction faster than the parent pincer complex can, and the reactions proceed predominantly through a heterogeneous active species. As this mechanism is not possible for the Heck reaction, this also explains why small amounts (c.a. 2-5\%) of product appear in mercury-poisoned Suzuki reactions catalysed by $\mathbf{2 9}$ and $\mathbf{3 0}$, but no traces of product are detected in analogous Heck reactions: the catalyst precursors display a limited activity proceeding via this redox-free mechanism, and can therefore can undergo a small number of turnovers before the heterogeneous $\operatorname{Pd}(0)$ is liberated and amalgamated.

Comparing the TONs displayed by compound 28 with literature values also supports the notion of the Suzuki reaction proceeding through this redox-free mechanism. Both pincer species in the literature that are deemed to catalyse the reaction in this manner are considerably more electron-rich than the POCOP complex 28, consisting of aliphatic (rather than aromatic) backbones, and cyclohexyl ${ }^{222}$ or tertbutyl $^{221}$ substituted phosphine donor groups. These species displayed TONs of 9,100 and 1,100 respectively, with a greater activity shown by the $\mathrm{PCy}_{2}$-substituted complex than by the more strongly electron-donating $\mathrm{P}^{t} \mathrm{Bu}_{2}$-substituted complex. As expected, under similar conditions the more electron-poor POCOP complex $\mathbf{2 8}$ significantly outperformed its more electron-rich analogues, with a TON of 74,000. This is consistent with all three complexes catalysing the Suzuki reaction through a redox-free pathway, with the exceptional stability conferred to the literature compounds by their aliphatic backbones and strong $\mathrm{P}$-donor ligands preventing $\operatorname{Pd}(0)$ deposition, and allowing the contributions to catalysis by the intact pincer species to be observed. 


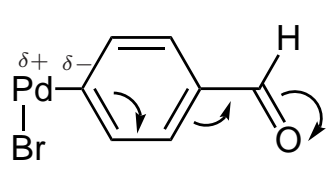

1

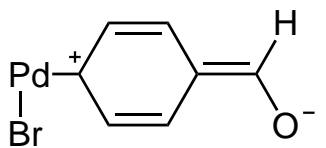

Electron-withdrawing group

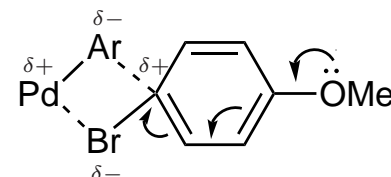

1

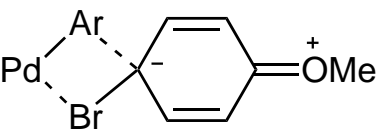

Electron-donating group

Figure 6.11 Comparison of resonance stabilisation in oxidative addition/reductive elimination mechanism (left) and redox-free mechanism (right). Ancillary ligands omitted for clarity.

Furthermore, this redox-free mechanism provides an explanation as to why the more electron-rich POCCP complex 30 outperformed the POCOP complex 28 in the cross-coupling of 4-chlorobenzaldehyde (TONs of 1,200 vs 200 respectively). Due to the differences in the electronic character of oxidative addition compared to $\mathrm{C}-\mathrm{X}$ bond metathesis, what constitutes an 'activated' or 'deactivated' aryl halide will differ between the oxidative addition/reductive elimination mechanism and the redoxfree mechanism. When the aryl halide undergoes oxidative addition to the metal centre, a partial negative charge $(\delta-)$ is developed on the metallated carbon atom. Aryl groups with para-substituted electron-withdrawing groups can stabilise this species through resonance effects, drawing some of the negative charge away and making the oxidative addition reaction more facile (Figure 6.11, left). Conversely, during $\mathrm{C}-\mathrm{X}$ bond metathesis, a partial positive charge $(\delta+)$ is developed on this same carbon of the aryl halide (as it is bound to the electronegative halogen rather than the electropositive metal centre). This charge can be stabilised in a similar fashion through resonance effects from para-positioned electron-donating groups (Figure 6.11, right). Hence, for an oxidative addition/reductive elimination pathway aryl halides possessing para electron-withdrawing groups will be 'activated', whereas for the redox-free pathway aryl halides with para electron-donating groups will be 'activated'. This decreased activity when moving from traditionally 'deactivated' (electron-donating) to 'activated' (electron-withdrawing) aryl halides has been previously observed where a redox-free mechanism was proposed; ${ }^{221}$ however, it was marked as a "surprising" result and not attributed to the innate nature of the redox-free mechanism itself. In this work, the observation that $\mathbf{2 8}$ outperforms 30 with the electron-donating 4-bromoanisole, while 30 outperforms 28 with the electron-withdrawing 4-chlorobenzaldehyde is consistent with $\mathbf{2 8}$ proceeding through 


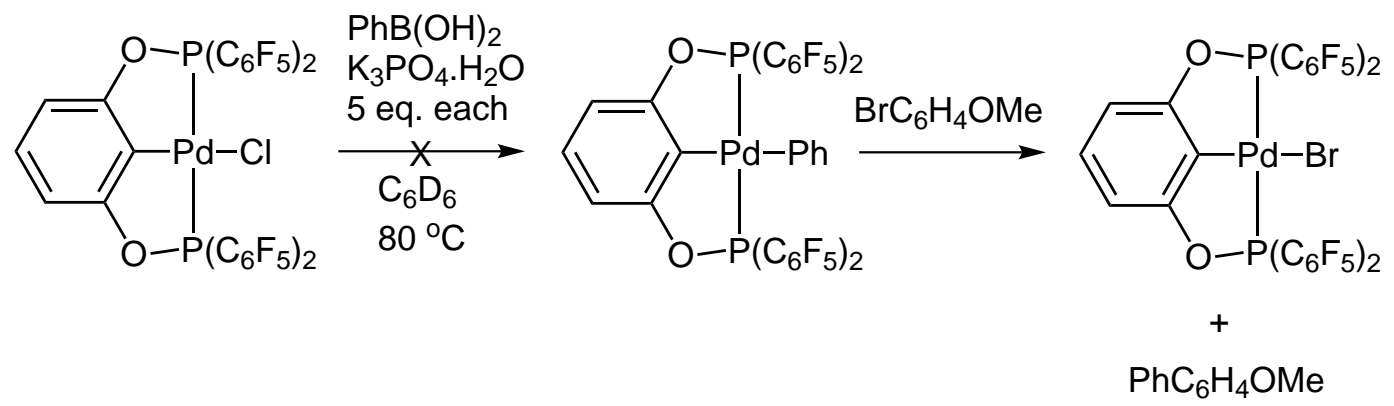

Scheme 6.6 NMR-scale Suzuki reaction attempted with 28.

a redox-free mechanism, while $\mathbf{3 0}$ proceeds via a (heterogeneous) oxidative addition/reductive elimination cycle.

Whilst all the experimental evidence was in good agreement with the Suzuki reaction of $\mathbf{2 8}$ proceeding through a redox-free pathway, confirmation of this was attempted with NMR experiments. The reaction between 28 and excess phenylboronic acid in the presence of potassium phosphate (Scheme 6.6) was attempted on an NMR scale in order to observe the palladium-phenyl intermediate [(POCOP)PdPh], which could then be reacted directly with the 4-bromoanisole to confirm that the Suzuki reaction did proceed through the intact pincer complex. While the previous results had indicated that $\mathbf{2 8}$ was stable for a number of hours when heated in the presence of base, in the presence of a large excess of phenylboronic acid and base at elevated temperatures solutions of $\mathbf{2 8}$ discoloured within two minutes, turning first a bright orange and then a dark yellow/brown colour, indicative of complex degradation. This was confirmed by NMR spectroscopy; after five minutes at $80{ }^{\circ} \mathrm{C}$ only trace amounts of $\mathbf{2 8}$ remained in solution.

This introduced the possibility that Suzuki reactions involving 28 under these conditions were proceeding via a homogeneous $\mathrm{Pd}(0) / \mathrm{Pd}(\mathrm{II})$ mechanism, catalysed by a molecular $\operatorname{Pd}(0)$ degradation product. Such a scenario has not been observed or even postulated for palladium PCP pincer complexes, but the notion of a molecular degradation product serving as the active catalyst species was posited for cyclometallated P-C chelate complexes by Bedford and colleagues in $2003 .{ }^{212}$ In that work, the active catalyst was identified as a biphenyl species arising from the coupling of the phenyl group from the boronic acid with the ligand backbone (Scheme 6.7). These degradation products bear a close resemblence to complexes of the extremely effective Buchwald-type ligands such as SPhos and XPhos: they consist of a singular phosphorus donor atom attached to a bulky biphenyl group. ${ }^{213}$ As such, these cyclometallated $\mathrm{P}-\mathrm{C}$ chelates demonstrated remarkable activity in the Suzuki reaction, catalysing the cross-coupling of 4-bromoanisole and phenylboronic acid with TONs in excess of 4,000,000. ${ }^{212}$ 


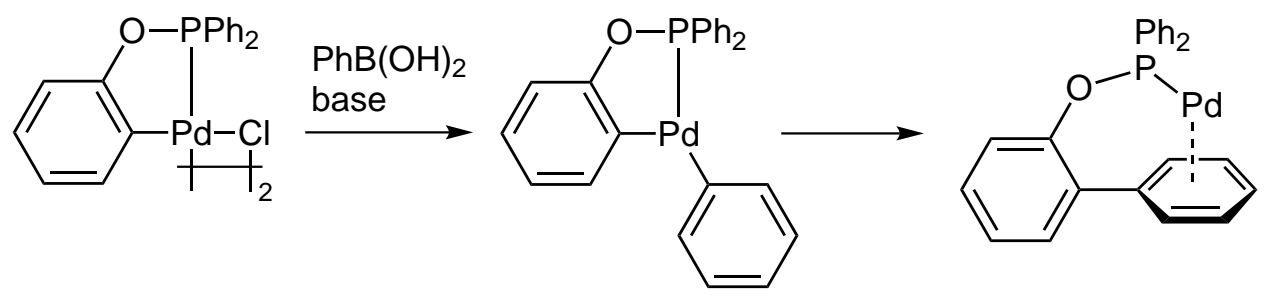

Scheme 6.7 Pathway for $\mathrm{Pd}(0)$ active catalyst formation in $\mathrm{P}-\mathrm{C}$ chelates. ${ }^{212}$

However, for pincer complexes the formation of a similar monodentate, biphenylsubstituted phosphine complex should be substantially less facile. Whereas in $\mathrm{P}-\mathrm{C}$ chelate complexes the coordination site cis to the metallated aryl ring is occupied by a relatively easy to displace bridging halide, in pincer complexes the displacement of a more strongly binding phosphorus donor group is required to achieve phenylation of the aryl backbone of the pincer complex. There is the possibility that $\mathrm{P}-\mathrm{O}$ bond hydrolysis and subsequent phosphinous acid dissociation (as in Scheme 6.5) creates this vacant coordination site cis to the metallated aryl backbone. This hydrolysis and biphenyl active catalyst formation needs to be extremely facile, as no induction periods were definitively observed for Suzuki reactions with 28, even at $60{ }^{\circ} \mathrm{C}$. While such a fast hydrolysis is possible, if this reaction was rapid, it would be expected that a second hydrolysis event would also be rapid, leading to equally fast catalyst degradation and 2,6-dihydroxybiphenyl formation. Moreover, the POCCP complex 30 would not be succeptable to a second hydrolysis, so would be expected to form a more stable biphenylphosphine active catalyst. As Suzuki reactions with 30 proceeded through a heterogeneous rather than homogeneous active catalyst, this pathway for active catalyst formation is unlikely to occur in these reactions.

To determine if the pincer complex decomposition was yielding homogeneous $\operatorname{Pd}(0)$ species capable of catalysing the Suzuki reaction, these degradation products were analysed by GCMS in tandem with NMR spectroscopy. As in the first step of Scheme 6.6, palladium complexes $\mathbf{2 8}, \mathbf{2 9}$ and $\mathbf{3 0}$ were reacted with excess phenylboronic acid and potassium phosphate in benzene- $d_{6}$ at $80{ }^{\circ} \mathrm{C}$. Hexafluorobenzene was used as an internal standard so that ${ }^{19} \mathrm{~F}$ NMR spectroscopy could be used quantitatively to determine the degree of decomposition, as well as the proportions of different degradation products present. Control experiments were also undertaken with $\mathbf{2 8}$ and phenylboronic acid, as well as $\mathbf{2 8}$ and potassium carbonate, to assess the effect of each of the starting materials individually on the pincer complex.

Monitoring the reactions by NMR spectroscopy, it was clear that phenylation of the pincer complex $\mathbf{2 8}$ by the base-activated phenylboronic acid facilitates pincer complex degradation. In the presence of both phenylboronic acid and potassium carbonate, less that $1 \%$ of $\mathbf{2 8}$ remained in solution and was detectable by ${ }^{19} \mathrm{~F}$ NMR 
spectroscopy after 5 minutes at $80{ }^{\circ} \mathrm{C}$. Conversely, in the presence of the base alone the rate of decomposition was decidedly slower (greater than $40 \%$ of $\mathbf{2 8}$ remained after three hours at $80{ }^{\circ} \mathrm{C}$ ), and 28 was seen to be extremely stable in the presence of phenylboronic acid alone (only trace amounts of decomposition products were observed after 24 hours at $80{ }^{\circ} \mathrm{C}$ ).

Compounds 29 and $\mathbf{3 0}$ displayed markedly different reactivities to compound $\mathbf{2 8}$ in reactions with phenylboronic acid and potassium carbonate. The POCOP complex 28 decomposed to give complicated mixtures of phosphorus and fluorine-containing products that were not readily identifiable by ${ }^{31} \mathrm{P}$ NMR spectroscopy. Conversely, the PCCCP and POCCP complexes both reacted to form typically three or four different stable molecular species. However, due to the number of compounds present in these reaction mixtures the decomposition products were not able to be unambiguously characterised. Data from ${ }^{31} \mathrm{P}$ NMR spectra of these reactions suggests that these species were asymmetric $\mathrm{Pd}$ (II) complexes; they typically possessed one phosphorus environment at a similar chemical shift to the starting material and one phosphorus environment shifted 20-40 ppm downfield of the starting material, with $\mathrm{P}-\mathrm{P}$ coupling values of approximately $400-500 \mathrm{~Hz}$, typical for a trans twobond phosphorus-phosphorus coupling. The concentration of these species decreased slowly over time, indicating that these compounds were potential precatalysts for the Suzuki reaction; being formed rapidly then undergoing a gradual decomposition to deposit $\operatorname{Pd}(0)$ nanoparticles into solution.

After monitoring by NMR spectroscopy for seven hours at $80^{\circ} \mathrm{C}$, these reaction mixtures were analysed by GCMS. Samples from each reaction were hydrolysed prior to GCMS analysis, in order to cleave $\mathrm{P}-\mathrm{O}$ bonds and therefore create more volatile species in solution. Results were compared to samples that had not been subjected to hydrolysis, to assist with the identification of species that had arisen from the $\mathrm{P}-\mathrm{O}$ cleavage. Somewhat surprisingly in all reaction mixtures with phenylboronic acid and potassium carbonate, the major volatile products were decafluorobiphenyl, 2,3,4,5,6-pentafluorobiphenyl, and biphenyl. The ${ }^{19} \mathrm{~F}$ NMR spectra of these decomposition reactions indicated that initially pentafluorobiphenyl was formed, then as the decomposition reactions proceeded pentafluorobiphenyl formation ceased and the concentration of decafluorobiphenyl slowly increased (Figure 6.12). The gradual formation of decafluorobiphenyl was also observed in the decomposition of $\mathbf{2 8}$ in the presence of base alone. Biaryl formation in cis-bidentate $\mathrm{P}-\mathrm{P}$ chelate complexes has been reported as occurring through the insertion of palladium-aryl centres into phosphorus-aryl bonds. ${ }^{228}$ Thus, a similar decomposition pathway may be present for these complexes, with the requisite palladium-phenyl species being generated by the boronic acid (as in the first step of Figure 6.10). Alternately, the phenylboronic 
acid could directly phenylate one of the phosphorus donors via nucleophilic attack, in a manner analogous to the previously proposed $\mathrm{P}-\mathrm{O}$ hydrolysis, a reaction that would necessarily be more facile at the $\mathrm{OP}\left(\mathrm{C}_{6} \mathrm{~F}_{5}\right)_{2}$ groups compared to the more electron-rich and less Lewis acidic $\mathrm{CH}_{2} \mathrm{P}\left(\mathrm{C}_{6} \mathrm{~F}_{5}\right)_{2}$ and $\mathrm{CH}_{2} \mathrm{P}^{t} \mathrm{Bu}_{2}$ groups. Either of these pathways would account for the initial appearance of pentafluorobiphenyl, and the observation that the decomposition was much more facile in the presence of both phenylboronic acid and base.
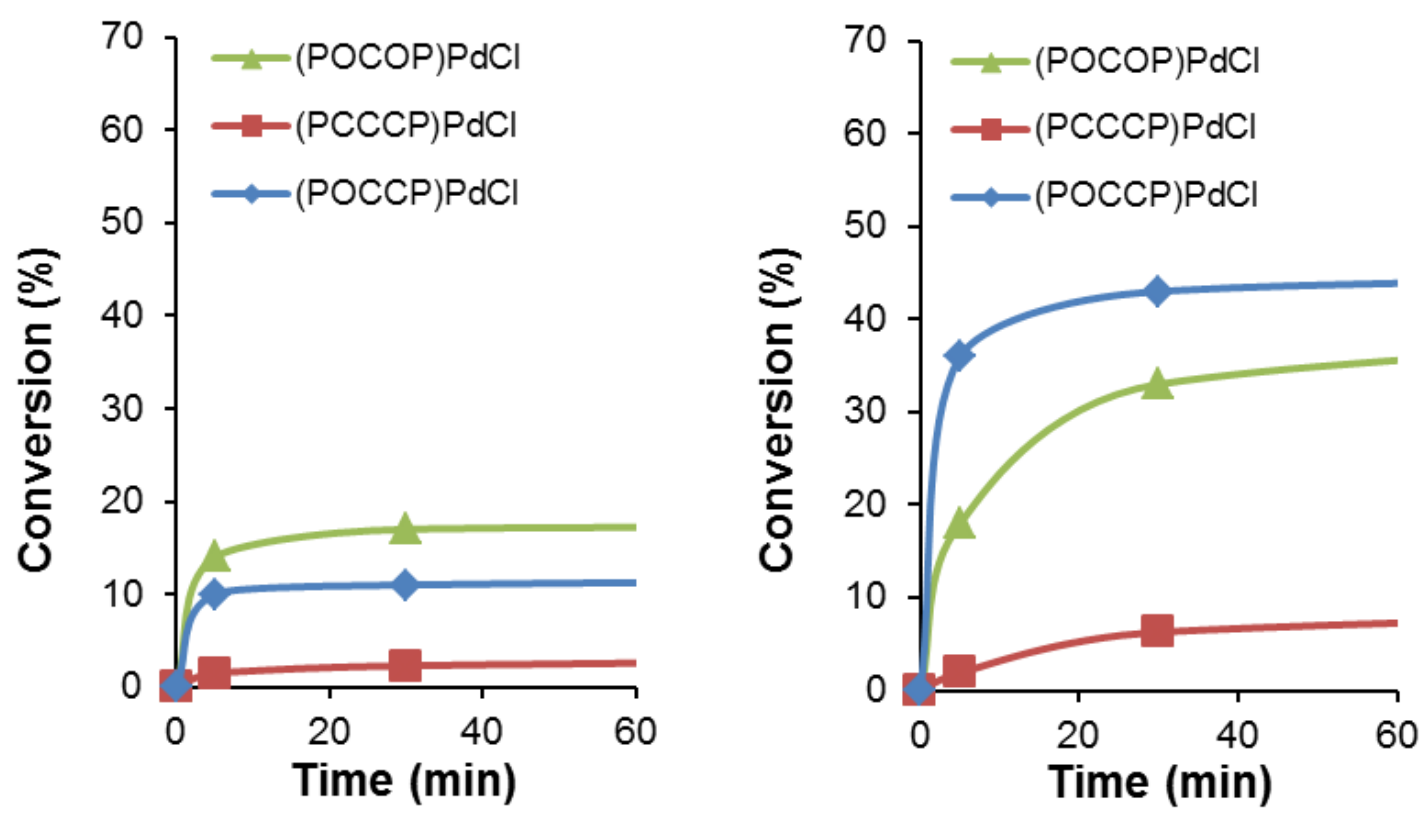

Figure 6.12 Plot of the appearance of pentafluorobiphenyl (left) and decafluorobiphenyl (right) in NMR-scale catalyst degradation experiments. Conversions represent the moles of pentafluorophenyl generated per mole of pentafluorophenyl present in the starting material. Data obtained at $\mathrm{t}=180$ min was plotted but is not displayed.

During this decomposition, it is interesting to note the difference in rates of biphenyl formation between the complexes (Figure 6.12), particularly with respect to the catalytic activity of each species. Biphenyl formation plateaued after 30 minutes at $80{ }^{\circ} \mathrm{C}$ for the POCOP complex 28, and after 10 minutes for the POCCP complex 30, with the formation of the major biphenyl component (decafluorobiphenyl) being much more rapid in the case of $\mathbf{3 0}$. This correlated well with the heterogeneous catalytic activity of each complex; there was an approximately 10 minute induction period at $80{ }^{\circ} \mathrm{C}$ for 30 , after which time heterogeneous catalysis commenced. For 28, mercury poisoning experiments have shown that homogeneous catalysis concluded after about 30 minutes, after which time there was still a contribution to catalysis from a heterogeneous active species. For the PCCCP complex 29, only small amounts of biphenyl product were detected, while minimal catalytic activity was observed in the first hour even at $100{ }^{\circ} \mathrm{C}$. Hence the formation of these fluorinated biphenyls can be seen as a precursor to the formation of colloidal palladium 
and heterogeneous catalysis, and potentially offers an explanation as to the fate of the homogeneous active species in the case of $\mathbf{2 8}$.

Further analysis of reaction mixtures by GCMS did not reveal any traces of 2,6substituted biphenyls, confirming that a biphenylphosphine- or phosphinite-ligated active species (as in Scheme 6.7) is not formed with 28, or indeed any of the palladium complexes. However, in reactions involving the POCOP complex 28, there were small amounts of the phosphines $\mathrm{P}\left(\mathrm{C}_{6} \mathrm{~F}_{5}\right)_{3}$ and $\mathrm{PPh}\left(\mathrm{C}_{6} \mathrm{~F}_{5}\right)_{2}$, as well as the phosphinite $\mathrm{P}(\mathrm{OPh})\left(\mathrm{C}_{6} \mathrm{~F}_{5}\right)_{2}$ present. Amongst the decomposition products from 30, minor traces of $\mathrm{PPh}^{t} \mathrm{Bu}_{2}$ were observed, but no other phosphorus-containing species were identified from the decomposition of complexes 29 and 30. This may indicate that in the Suzuki reaction, compound $\mathbf{2 8}$ is reduced to a low coordinate $\operatorname{Pd}(0)$ complex, stabilised by the presence of phosphorus-donor ligands in solution. Such a species has previously been proposed as the active catalyst in $\mathrm{C}-\mathrm{S}$ crosscoupling reactions catalysed by a $[(\mathrm{POCOP}) \mathrm{NiCl}]$ species. ${ }^{229}$

The possibility of the active species in Suzuki reactions involving $\mathbf{2 8}$ being a $\operatorname{Pd}(0)$ complex bound to monodentate phosphine ligands formed from the degradation of the pincer complex certainly bears consideration. This is able to explain how the reaction proceeds when the parent complex is not stable for more than a few minutes under catalytic conditions, and also follows the established $\operatorname{Pd}(0) /(\mathrm{II})$ reaction mechanism. On the contrary, it does not explain the reactivity difference between 28 and 30 in coupling "activated" aryl chlorides. Nor does it account for lack of an observed induction period, even at low temperatures - both the cyclometallated $\mathrm{P}-\mathrm{C}$ chelates and $[(\mathrm{POCOP}) \mathrm{NiCl}]$ complexes display distinct induction periods during the active catalyst formation. ${ }^{212,229}$ Due to the fact that this reaction proceeds so rapidly and may be catalysed by such small amounts of palladium, it is extremely difficult to conclude whether Suzuki reactions involving 28 proceed through a redoxfree mechanism catalysed by the pincer complex, or whether the pincer complex acts as a precatalyst for a homogeneous, phosphine-ligated $\operatorname{Pd}(0)$ catalyst. One thing is clear though, unlike the complexes 29 and 30, the POCOP complex 28 catalyses the Suzuki reaction through a homogeneous active species, and is one of the most active pincer catalysts for this reaction reported to date. 


\subsection{Concluding remarks}

The palladium pincer compounds [(POCOP $) \mathrm{PdCl}](\mathbf{2 8})$, [( $\mathrm{PCCCP}) \mathrm{PdCl}](\mathbf{2 9})$, and $[(\mathrm{POCCP}) \mathrm{PdCl}]$ (30) were assessed for their activity as catalysts in the Heck and Suzuki cross-coupling reactions. While catalysts containing fluoroaryl phosphines traditionally have not demonstrated high activities, ${ }^{80}$ it was hoped that the electronic and structural variations provided by this series of compounds would provide insight into factors that assist efficient catalyst design.

In the Heck reaction, it was observed that compounds 28, 29, and 30 displayed modest activities, giving TONs of 4,000-22,000 after 22 hours. By undertaking mercury poisoning experiments, it was shown that all the palladium pincer complexes functioned as precatalysts, decomposing under catalytic conditions to give catalytically active palladium nanoparticles. The PCCCP complex $\mathbf{2 9}$ was the most active precatalyst; unlike the POCOP complex 28 it contains no readily-hydrolysed $\mathrm{P}-\mathrm{O}$ bonds and therefore underwent a longer induction period, but proved to be more efficient at providing the reaction mixture with a steady stream of active palladium. Incorporation of an electron-donating di-tert-butyl phosphine group into the pincer framework (30) did not stabilise the precatalyst and assist with the desired 'slow-release' of active palladium into solution. Instead this precatalyst displayed the worst features of the two functionalities; it was still prone to $\mathrm{P}-\mathrm{O}$ hydrolysis while undergoing a less facile decomposition to $\operatorname{Pd}(0)$, making for poor performance in the Heck reaction.

These electron-poor PCP pincer complexes proved to be substantially more active in the Suzuki reaction. Compound 28 in particular was highly active, giving an average TON of 176,000 in just two hours, making it one of the most active pincer complexes reported for the Suzuki reaction to date. This corresponds to just $5 \mathrm{ppm}$ of palladium required for a conversion of $88 \%$. Comparison to [( $\mathrm{DPPF}) \mathrm{PdCl}_{2}$ ] showed that while $\mathbf{2 8}$ gave similar turnovers to the commercially available catalyst after two hours, $\mathbf{2 8}$ performed the reaction at a substantially faster rate, reaching the $50 \%$ conversion mark almost four times faster than [(DPPF) $\left.\mathrm{PdCl}_{2}\right]$. This activity was somewhat unexpected, especially as $\mathbf{2 8}$ outperformed the activity reported for its proteo analogue, ${ }^{171}$ a feat not often reported for palladium-catalysed cross coupling reactions.

Performing low temperature kinetic tests as well as mercury poisoning experiments on these Suzuki reactions indicated that reactions involving 29 and 30 proceeded via a heterogeneous active catalyst — as in the Heck reactions — whereas reactions with 28 were catalysed by a homogeneous species. In considering the nature 
of this homogeneous active species, the sterically-crowded, electron-poor nature of 28 renders catalysis through a $\operatorname{Pd}(\mathrm{II}) /(\mathrm{IV})$ cycle highly unlikely. However, due to aryl halide oxidative addition not being an explicit requirement for the Suzuki reaction, catalysis through an alternate, redox-free mechanistic pathway is plausible. This mechanism offers parallels with established $\mathrm{Pd}(\mathrm{II})$ chemistry, and can explain the observed reactivity differences between 28 and similar pincer complexes in the literature, as well as relatively poor performance of $\mathbf{2 8}$ in coupling "activated" aryl chlorides. However, assessment of the stability of these pincer complexes under pseudo-catalytic conditions revealed that $\mathbf{2 8}$ has a dramatically shorter lifetime than compounds 29 and 30, yielding degradation products that suggested the active species may be a $\operatorname{Pd}(0)$ centre stabilised by tertiary phosphine ligands such as $\mathrm{P}\left(\mathrm{C}_{6} \mathrm{~F}_{5}\right)_{3}$ present in solution. As these reactions proceeded rapidly in the presence of very little palladium, it was very difficult to unambiguously identify the active species present in reactions involving 28.

From these investigations, it could be concluded that the performance of pincer species in palladium-catalysed cross-coupling reactions does not have a simple correlation with the electronic nature of the metal centre. It appears instead that both the stability and the electronic character of the phosphorus donor groups needs to be considered, in conjunction with the nature of active catalyst formation under catalytic conditions. In the Heck reaction, [(PCCCP)PdCl] (29) considerably outperforms [(POCCP $) \mathrm{PdCl}](\mathbf{3 0})$ as a precatalyst, while in the Suzuki reactions the reverse is true, despite all of these reactions proceeding via a heterogeneous active species. The reason for this becomes evident when considering the reaction conditions - the Heck reaction is performed at high temperature in the absence of a good nucleophile, while the Suzuki reaction is performed at considerably lower temperatures in the presence of base-activated phenylboronic acid, which acts as a phenylating agent.

In the Heck reaction, displacement of a phosphorus donor group from the palladium is an essential step in colloidal palladium formation (Scheme 6.3, Scheme 6.4), therefore the replacement of a poorly donating $\mathrm{P}\left(\mathrm{C}_{6} \mathrm{~F}_{5}\right)_{2}$ group with a strongly donating $\mathrm{P}^{t} \mathrm{Bu}_{2}$ group hinders ligand dissociation, and subsequently leads to a lower catalyst activity. Moreover, as the active catalyst formation is slow (half lives of the order of 5-10 hours are common, Figure 6.3), thermal stability of the precatalyst is of benefit, with the more robust PCCCP species $\mathbf{2 9}$ demonstrating a higher activity than the complexes comprising the more labile $\mathrm{P}-\mathrm{O}$ bonds, 28 and $\mathbf{3 0}$. Conversely, in the Suzuki reaction nucleophilic attack on the phosphorus centre appears to play a major part in the active catalyst formation, as evidenced by the observation of fluorinated biphenyls in GCMS and NMR analysis of reaction mixtures. With the 
similarity of this nucleophilic attack to $\mathrm{P}-\mathrm{O}$ hydrolysis (Scheme 6.5) and the correlation of low activities with low amounts of biphenyl formation in the case of the PCCCP complex 29, this indicates that in the presence of a nucleophile, incorporation of the less robust and more Lewis acidic phosphinite functionality into the precatalyst aids heterogeneous active catalyst formation. These results emphasise that whilst the Heck and Suzuki reactions are conceptually similar, they have individual, specific, and even conflicting factors than need to be taken into account when considering efficient precatalyst design.

Drawing further conclusions on how the structural and electronic nature of PCP pincer complexes affect their activities in these reactions is complicated by the observation that the POCOP complex $\mathbf{2 8}$ catalyses the Suzuki reaction via a homogeneous active species. While the exact nature of the catalyst species is uncertain, this does highlight that conceptually small structural modifications may have a large effect on the nature of the catalysis. A further point of emphasis is that pincer complexes may not be as robust under catalytic conditions as many have anticipated, and further, due to the high activity of colloidal and low-ligated homogeneous palladium species, confirmation of the active catalyst species in solution is extremely difficult. This is put best by Phan and colleagues, "indeed, if ppt levels of palladium are capable of easily catalyzing some reactions, probing the nature of the true catalyst by most spectroscopic or other techniques may currently be impossible". ${ }^{176}$ 


\section{Chapter 7}

\section{Conclusions}

Transition metal complexes stabilised by phosphorus-containing pincer ligands have been at the forefront of chemical research in recent years. They have facilitated the selective bond cleavage of some of the most difficult-to-activate substrates, and have shown considerable promise in two catalytic technologies that may help alleviate a potential energy crisis in the years to come: alkane metathesis and water splitting. The rigid, well-defined coordination geometry of pincer complexes generally minimises decomposition reactions, providing pincer complexes with a stability that helps to ensure they remain intact and catalytically active even under demanding reaction conditions.

While much of the research undertaken with pincer complexes involves the use of electron-rich ligands, this study entailed the synthesis of PCP and PNP pincer ligands bearing bulky electron-poor bis(pentafluorophenyl)phosphine substituents. It was envisaged that the incorporation of these electron-poor groups into the pincer coordination motif would provide an insight into the factors that influence the synthesis and reactivity of these PCP and PNP pincer complexes.

The synthesis of the PCP and PNP pincer ligands was performed by attaching the electron-poor phosphines to phenyl or pyridyl backbones with $\mathrm{CH}_{2}, \mathrm{NH}$, or $\mathrm{O}$ linkages. Installation of the phosphines via condensation reactions between bis(pentafluorophenyl)phosphine bromide and aromatic alcohol or amine functionalities was observed to give the desired pincer ligands in moderate to good yields, with the added advantage of being carried out under milder conditions than have been reported for the synthesis of more electron-rich analogues. This synthetic methodology was also tolerant of other functionalities present on the ligand, enabling the synthesis of the mixed phosphine-phosphinite POCCPH ligand 3 . The previously 
reported diphosphine PCCCPH ligand $\mathbf{2}$ was also synthesised; by using magnesiumanthracene methods to produce the required Grignard reagent, ligand $\mathbf{2}$ was obtained in a greater yield than previously reported.

The coordination chemistry of the most electron-poor POCOPH pincer ligand 1 was investigated with a range of platinum and palladium starting materials. Reaction of 1 with $\left[\mathrm{Pt}(\mathrm{nb})_{3}\right]$ yielded the cis-bridged dimer $[(\mathrm{POCOPH}) \mathrm{Pt}(\mathrm{nb})]_{2}(\mathbf{1 4})$, which was characterised by single crystal X-ray diffraction, and was shown to be stabilised by favourable $\pi-\pi$ interactions between the aromatic ligand backbones. A similar preference for the formation of $\kappa^{2}$-PP bridged structures was indicated in reactions between ligand 1 and $\left[\mathrm{PtMe}_{2}(\mathrm{hex})\right]$. None of the species arising from norborneneor methyl-substituted starting materials could be induced to undergo metallation to form platinum PCP pincer complexes.

Reactions of ligand $\mathbf{1}$ with platinum and palladium dichloride or chloromethyl starting materials led to the formation of rare examples of cis,trans-dimers 19, 21, and 23 as precursors to the metallated PCP pincer complexes. The formation of these cis,trans-dimers is likely to be the result of having to balance steric and electronic effects - bulky bridging ligands are seen to favour trans-coordination modes due to steric constraints, while $\pi$-acceptor donor groups favour cis-coordination in order to minimise unfavourable electronic interactions. The extensive coordination chemistry demonstrated by ligand $\mathbf{1}$ was in part attributed to its electron-poor nature increasing the barrier to $\mathrm{C}-\mathrm{H}$ activation and ligand metallation, favouring the formation of bridged coordination complexes over mononuclear pincer species.

As ligand 1 proved difficult to metallate, the influence of both ligand and starting material on pincer complex formation was assessed. It was revealed that the ease of ligand metallation increases with increasing electron-donating ability of the ligand. Substitution of a bis(pentafluorophenyl)phosphinite group on the ligand for a ditert-butyl phosphine group - moving from ligand $\mathbf{1}$ to ligand $\mathbf{3}$ - resulted in the formation of roughly double the amount of metallated product in half the time. It was also determined that the nature of the ancillary ligand on the platinum starting material had a significant influence on the ease of pincer complex formation. Somewhat counterintuitively, strongly binding ancillary ligands promoted faster ligand metallation, as their less facile dissociation from the metal centre disfavoured the formation of thermodynamically stable dimers and oligomers as metallation intermediates.

The electronic character of ligands $\mathbf{1}, \mathbf{2}$, and $\mathbf{3}$ were assessed via the synthesis of the appropriate platinum or palladium carbonyl species, $[(\mathrm{PCP}) \mathrm{M}(\mathrm{CO})]^{+}$. Infrared 
spectroscopy of these carbonyl complexes confirmed the expected trend, from most electron-poor to most electron-rich, POCOP $>$ PCCCP $>$ POCCP. The palladium POCOP carbonyl $\mathbf{3 4}$ possessed the largest value reported to date for the $\mathrm{C}-\mathrm{O}$ stretching frequency of a pincer carbonyl complex in the IR spectrum, while the platinum POCOP complex 31 and the palladium PCCCP complex 35 also displayed $\mathrm{C}-\mathrm{O}$ stretching frequencies greater than that of free carbon monoxide. All three of the palladium carbonyl complexes demonstrated the reversible binding of carbon monoxide, with the displacement of the CO ligand increasing in ease with increasing magnitude of the $\mathrm{C}-\mathrm{O}$ stretching frequency in the infrared spectrum. This observation was attributed to the more electron-poor metal centres being less able to bind the carbon monoxide via $\pi$-backbonding.

The synthesis of platinum methyl pincer complexes [(PCP)PtMe] from the parent platinum chlorides was investigated. Reactions with methylmagnesium iodide were found to result in halide exchange to produce the corresponding platinum iodide species, while the greater nucleophilicity of methyllithium resulted in nucleophilic attack at the electron-deficient $\mathrm{P}\left(\mathrm{C}_{6} \mathrm{~F}_{5}\right)_{2}$ centres. In the case of $[(\mathrm{POCCP}) \mathrm{PtCl}]$ (27), this nucleophilic attack at phosphorus was observed to result in pentafluorophenyl migration to the platinum, producing a platinum pentafluorophenyl complex (46), amongst other byproducts. Successful methylation was achieved with the less nucleophilic dimethylzinc; however, the more electron-rich pincer complex $\mathbf{2 7}$ underwent methylation at a significantly slower rate than its more electron-poor counterparts 25 and 26 did.

Reaction of the PNP ligands 10 and 11 with platinum chloride starting materials revealed a large electronic influence on pincer complex formation. Whereas PNP pincer complex formation proceeded under mild conditions for the more electron-rich PNNNP ligand 10, reactions with the more electron-poor PONOP ligand $\mathbf{1 1}$ did not yield any traces of the pincer species. This was likely due to the chloride dissociation required for PNP pincer formation becoming less facile for more electron-poor metal centres. The chemistry of $[(\mathrm{PNNNP}) \mathrm{RhCl}](49)$ was also investigated, as it was isolectronic with the Group 10 PCP pincer complexes studied. Despite possessing a relatively electron-withdrawing ligand, the chemistry of 49 was dominated by facile oxidation to rhodium(III) species. Furthermore, reactions with dimethylzinc resulted in the deprotonation of an $\mathrm{N}-\mathrm{H}$ group on the ligand, to form a methylzinc adduct (51), which was observed to be stabilised by Lewis acid-base interactions with ethers.

The catalytic activity of the electron-poor [( $\mathrm{PCP}) \mathrm{PdCl}]$ complexes 28, 29, and $\mathbf{3 0}$ were assessed in Heck and Suzuki cross-coupling reactions. All three complexes were 
found to possess only modest activity in the Heck reaction, in all cases functioning as precatalysts, which decomposed to give catalytically-active $\operatorname{Pd}(0)$ colloids. Under the milder reaction conditions required for the Suzuki reactions, the most electronpoor complex, [(POCOP)PdCl], 28, was able to couple electronically-deactivated aryl bromides with a high activity via a homogeneous active species. This reactivity is consistent with that expected for an intact pincer species operating through a redox-free mechanism. However, the susceptibility of these electron-poor pincer species to nucleophilic attack and decomposition means that catalysis by unidentified homogeneous degradation products cannot be ruled out.

While electron-withdrawing PCP and PNP pincer ligands possessing phosphinite or phosphinamide linkers can be more readily synthesised than their more electrondonating analogues, there are significant difficulties associated with the metallation of these electron-deficient ligands. The resultant electron-poor pincer complexes are also rendered increasingly susceptible to nucleophilic attack at the phosphorus and degradation, counteracting the stability conferred to complexes by the pincer coordination motif. However, the increased barrier to ligand metallation makes such species ideally suited to investigating the coordination chemistry and metallation of pincer ligands. Furthermore, it appears that highly electron-deficient POCOP palladium complexes display reversible uptake of carbon monoxide, and enhanced activity in the Suzuki reaction over their more electron-rich counterparts.

An area of this work that may be explored in future investigations is the isolation and characterisation of the deprotonated form of the rhodium PNNNP pincer complex [(PNNNP)RhCl], 49. Research into the behaviour of this deprotonated species in stoichiometric and catalytic reactions may reveal whether 49 is capable of the noninnocent behaviour that is prevalent in the chemistry of related PCNCP complexes. Further work into conclusively determining the nature of the active species arising from [(POCOP $) \mathrm{PdCl}], 28$, in the Suzuki reaction would also be beneficial. One way in which this may be achieved is through the comparison of the activity of $\mathbf{2 8}$ with that of independently synthesised potential degradation products, to determine if a degradation product is responsible for the high homogeneous catalytic activity of 28. Moreover, characterisation of any of the cis,trans dimers by X-ray diffraction would be highly desirable. 


\section{Chapter 8}

\section{Experimental}

\subsection{General Procedures}

All reactions and manipulations of products and reagents were carried out under an inert nitrogen atmosphere using standard Schlenk techniques unless otherwise stated. Dichloromethane, diethyl ether, and tetrahydrofuran were dried by heating to reflux over the appropriate drying agent (calcium hydride for dichloromethane, sodium/benzophenone ketyl for ethers) and distilled prior to use. Other solvents were degassed and purged with nitrogen before use, with most stored over the appropriate size molecular sieve. ${ }^{230}$ Methyl iodide, dibutyl ether, bromobenzene, and styrene were purified by passage through a short neutral alumina column prior to use. The compounds 3-hydroxybenzyl alcohol, ${ }^{70}$ 3-hydroxybenzyl bromide, ${ }^{71}$ (3-hydroxybenzyl)di-tertbutyl phosphine, ${ }^{13}$ and bis(pentafluorophenyl)bromophosphine, ${ }^{231}$ were synthesised using literature procedures. The transition metal precursors $\left[\mathrm{PdCl}_{2}\left(\mathrm{NCMe}_{2}\right]{ }^{232}\right.$ $\left[\mathrm{PtCl}(\mathrm{COD}) \mathrm{N}\left(\mathrm{SiMe}_{3}\right)_{2}\right],{ }^{124}\left[\mathrm{PtCl}_{2}(\right.$ hex $\left.)\right],{ }^{233}\left[\mathrm{PtCl}_{2}\left(\mathrm{SEt}_{2}\right)_{2}\right],{ }^{117}\left[\mathrm{PtMe}_{2}(\right.$ hex $\left.)\right],{ }^{234}$ and $[\mathrm{RhCl}(\mathrm{COD})]_{2},{ }^{235}$ were prepared by literature methods. The platinum compounds $\left[\mathrm{PtCl}_{2}(\mathrm{COD})\right]^{236}$ and $\left[\mathrm{Pt}(\mathrm{nb})_{3}\right]^{234}$ were obtained from colleagues at Victoria University of Wellington, having been prepared by literature procedures.

NMR spectra were obtained using a Varian Unity Inova $300\left(300 \mathrm{MHz}\right.$ for ${ }^{1} \mathrm{H}, 121$ $\mathrm{MHz}$ for ${ }^{31} \mathrm{P}$, and $282 \mathrm{MHz}$ for $\left.{ }^{19} \mathrm{~F}\right)$, a Varian Unity Inova $500\left(500 \mathrm{MHz}\right.$ for ${ }^{1} \mathrm{H}$ and $125 \mathrm{MHz}$ for ${ }^{13} \mathrm{C}$ ), or a Varian DirectDrive $600\left(600 \mathrm{MHz}\right.$ for ${ }^{1} \mathrm{H}$ and $150 \mathrm{MHz}$ for ${ }^{13} \mathrm{C}$ ). The $600 \mathrm{MHz}$ instrument was equipped with a Varian inverse-detected triple-resonance $\mathrm{HCN}$ cold probe operating at $25 \mathrm{~K}$. All ${ }^{1} \mathrm{H}$ and ${ }^{13} \mathrm{C}$ resonances were referenced to the residual solvent peak. All ${ }^{31} \mathrm{P}$ and ${ }^{19} \mathrm{~F}$ resonances were referenced 
to $\mathrm{H}_{3} \mathrm{PO}_{4}$ and $\mathrm{CFCl}_{3}$ respectively. NMR samples were prepared under an inert atmosphere unless otherwise stated. Infrared spectra were obtained with a PerkinElmer Spectrum One FT-IR spectrophotometer using pressed KBr discs. GCMS analysis was performed on a Shimadzu GC-2010 GCMS with a RTX-5sil stationary phase. Microanalyses were performed by the Campbell Microanalytical Laboratory at the University of Otago. Single-crystal X-ray diffraction data were obtained by the X-ray Crystallography Laboratory at the University of Canterbury. Electrospray ionisation mass spectra were performed by the GlycoSyn QC Laboratory at Industrial Research Limited using a Waters Q-TOF Premier Tandem mass spectrometer, or in-house using an Agilent 6530 Series Q-TOF mass spectrometer.

\section{Crystallography}

Diffraction data* (see appropriate tables for details) were collected using Bruker CCD diffractometers with Mo K $\alpha$ radiation (0.71073 $\AA$ ) from fine focus sealed tubes with graphite monochromators, using phi and omega scans. Multi-scan absorption corrections were applied. The structures were solved using Patterson methods, and refined using a full-matrix least squares method, ${ }^{\dagger}$ with anisotropic thermal motion parameters for all non-hydrogen atoms. ${ }^{237}$ Hydrogen atoms were placed in calculated positions and allowed to refine freely using a riding model. OLEx2 (Version 1.1.5) ${ }^{238}$ was used as a front-end for the SHELX executables during structure solution and refinement. All relevant bond distances and angles were calculated using MERCURY (Version 2.4.5), and molecular drawings were generated using ORTEP3 (Version 1.0.3).

\subsection{Transition Metal Precursors}

\section{$[\operatorname{PtClMe}($ hex $)]$}

Dichloroplatinum-1,5-hexadiene (1.01 g, $2.9 \mathrm{mmol})$ was suspended in diethyl ether $(20 \mathrm{~mL})$. To this was added a solution of $1 \mathrm{~mol} \mathrm{dm}^{-3}$ dimethylzinc in diethyl ether (3.5 mL, $3.5 \mathrm{mmol}$ ) portionwise with stirring over $20 \mathrm{~min}$. The dark brown solution was stirred for a further $45 \mathrm{~min}$, then washed with a saturated ammonium

*Bruker SMART (Version 5.054), SADABS (Version 2.03), and SAINT (Version 6.02A), Bruker AXS Inc., Madison, Wisconsin, USA, 1997.

${ }^{\dagger}$ G. M. Sheldrick, SHELX-97. Programmes for the Solution and Refinement of Crystal Structures, 1997. 
chloride solution $(15 \mathrm{~mL})$. The organic layer was decanted, and the aqueous layer was further extracted with diethyl ether $(5 \times 15 \mathrm{~mL})$. The combined organic extracts were dried over magnesium sulfate, then volatiles were removed in vacuo carefully (as the product may sublime), giving a yellow oil that crystallised into an off-white solid $\left(\left[\mathrm{PtMe}_{2}(\right.\right.$ hex $\left.\left.)\right]\right)$ at $-15{ }^{\circ} \mathrm{C}$. The solid was then dissolved in a 2:3 methanol/dichloromethane mixture $(15 \mathrm{~mL})$, and acetyl chloride $(160 \mu \mathrm{L}, 2.3 \mathrm{mmol})$ was added dropwise over 1 minute. The solution was stirred for a further $10 \mathrm{~min}$, and the solvent was reduced to about $2 \mathrm{~mL}$ in vacuo. The product was isolated as a cream-coloured solid by crystallisation from the reaction mixture at $-15{ }^{\circ} \mathrm{C}$ and washing with pentane $(435 \mathrm{mg}, 46 \%)$. The product could also be isolated as a minor impurity (typically c.a. $1 \%$ yield) in the synthesis of $\left[\mathrm{PtMe}_{2}(\right.$ hex $\left.)\right]$ during purification by sublimation.

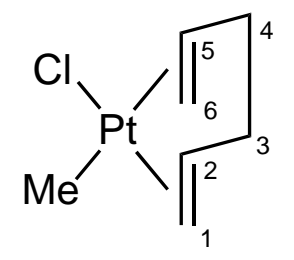

${ }^{1} \mathrm{H}-\mathrm{NMR}:\left(500 \mathrm{MHz}, \mathrm{CDCl}_{3}\right) \delta_{\mathrm{H}} 5.52\left(\mathrm{dd},{ }^{2} J_{\mathrm{Pt}-\mathrm{H}}=28.9 \mathrm{~Hz},{ }^{3} J_{\mathrm{H}-\mathrm{H}}=8.4 \mathrm{~Hz},{ }^{3} J_{\mathrm{H}-\mathrm{H}}\right.$ $\left.=1.8 \mathrm{~Hz}, 1 \mathrm{H}, \mathrm{C}=\mathrm{CH}_{2} \mathrm{H}-1\right), 5.38(\mathrm{~m}, 1 \mathrm{H}, \mathrm{C}=\mathrm{CH} \mathrm{H}-2), 4.64\left(\mathrm{~m},{ }^{2} J_{\mathrm{Pt}-\mathrm{H}}=65.2 \mathrm{~Hz}\right.$, $1 \mathrm{H}, \mathrm{C}=\mathrm{CH} \mathrm{H}-5), 4.37\left(\mathrm{~d},{ }^{2} J_{\mathrm{Pt}-\mathrm{H}}=29.4 \mathrm{~Hz},{ }^{3} J_{\mathrm{H}-\mathrm{H}}=14.4 \mathrm{~Hz}, 1 \mathrm{H}, \mathrm{C}=\mathrm{CH}_{2} \mathrm{H}-1\right)$, $3.46\left(\mathrm{~d},{ }^{2} J_{\mathrm{Pt}-\mathrm{H}}=72.3 \mathrm{~Hz},{ }^{3} J_{\mathrm{H}-\mathrm{H}}=7.8 \mathrm{~Hz}, 1 \mathrm{H}, \mathrm{C}=\mathrm{CH}_{2} \mathrm{H}-6\right), 3.31\left(\mathrm{~d},{ }^{2} J_{\mathrm{Pt}-\mathrm{H}}=\right.$ $\left.71.8 \mathrm{~Hz},{ }^{3} J_{\mathrm{H}-\mathrm{H}}=13.4 \mathrm{~Hz}, 1 \mathrm{H}, \mathrm{C}=\mathrm{CH}_{2} \mathrm{H}-6\right), 2.65\left(\mathrm{~m}, 1 \mathrm{H}, \mathrm{C}-\mathrm{CH}_{2} \mathrm{H}-4\right), 2.54(\mathrm{~m}$, $\left.1 \mathrm{H}, \mathrm{C}-\mathrm{CH}_{2} \mathrm{H}-4\right), 2.33\left(\mathrm{~m}, 1 \mathrm{H}, \mathrm{C}-\mathrm{CH}_{2} \mathrm{H}-3\right), 1.84$ (m, 1H, C- $\left.\mathrm{CH}_{2} \mathrm{H}-3\right), 0.99$ (s, $\left.{ }^{2} J_{\mathrm{Pt}-\mathrm{H}}=73.8 \mathrm{~Hz}, 3 \mathrm{H}, \mathrm{CH}_{3}\right) \cdot{ }^{13} \mathrm{C}-\mathrm{NMR}:\left(125 \mathrm{MHz} \mathrm{CDCl}_{3}\right) \delta_{\mathrm{C}} 118.9(\mathrm{~s}, 1 \mathrm{C}, \mathrm{C}-2)$, $103.3(\mathrm{~s}, 1 \mathrm{C}, \mathrm{C}-1), 86.4\left(\mathrm{~s},{ }^{1} J_{\mathrm{Pt}-\mathrm{C}}=210 \mathrm{~Hz}, 1 \mathrm{C}, \mathrm{C}-5\right), 58.5\left(\mathrm{~s},{ }^{1} J_{\mathrm{Pt}-\mathrm{C}}=224 \mathrm{~Hz}, 1 \mathrm{C}\right.$, C-6), $33.9\left(\mathrm{~s},{ }^{2} J_{\mathrm{Pt}-\mathrm{C}}=31.2 \mathrm{~Hz}, 1 \mathrm{C}, \mathrm{C}-4\right), 26.7(\mathrm{~s}, 1 \mathrm{C}, \mathrm{C}-3), 6.4\left(\mathrm{~s},{ }^{1} J_{\mathrm{Pt}-\mathrm{C}}=618 \mathrm{~Hz}\right.$, $\left.1 \mathrm{C}, \mathrm{CH}_{3}\right)$.

\section{$\mathrm{ZnMe}_{2}$}

Zinc powder (13.8 g, $0.21 \mathrm{~mol})$ and magnesium powder $(5.4 \mathrm{~g}, 0.22 \mathrm{~mol})$ were dry stirred under nitrogen for 3 hours, then suspended in dibutyl ether (90 mL). Methyl iodide $(26.1 \mathrm{~mL}, 0.42 \mathrm{~mol})$ was added to the stirred suspension at a rate that kept the reaction mixture around $60{ }^{\circ} \mathrm{C}$. The suspension was then heated to $70{ }^{\circ} \mathrm{C}$ for 18 hours, after which time the product was isolated by fractional distillation from the reaction mixture, taking care as the product is extremely pyrophoric (13.9 g, $70 \%$ ). Solutions of dimethylzinc in diethyl ether employed were prepared using 
diethyl ether in place of dibutyl ether, distilling the dimethylzinc across with the ether. Concentrations of diethyl etheric solutions of dimethylzinc were determined by back-titration with standard $1 \mathrm{~mol} \mathrm{dm}{ }^{-3}$ hydrochloric acid. ${ }^{1} \mathrm{H}-\mathrm{NMR}$ : (300 MHz, $\left.\mathrm{C}_{6} \mathrm{D}_{6}\right) \delta_{\mathrm{H}}-0.68\left(\right.$ br s, $6 \mathrm{H}, \mathrm{CH}_{3}$ ).

\subsection{Ligands}

\section{$1,3-\left[\left(\mathrm{C}_{6} \mathrm{~F}_{5}\right)_{2} \mathrm{PO}\right]_{2} \mathrm{C}_{6} \mathrm{H}_{4}(\mathrm{POCOPH})(1)$}

Resorcinol (1.00 g, $9.1 \mathrm{mmol})$ was suspended in a solution of triethylamine $(2.6 \mathrm{~mL}$, $18.6 \mathrm{mmol})$ in diethyl ether $(80 \mathrm{~mL})$. The reaction mixture was cooled on ice and a solution of $\operatorname{BrP}\left(\mathrm{C}_{6} \mathrm{~F}_{5}\right)_{2}(4.1 \mathrm{~mL}, 18.2 \mathrm{mmol})$ in diethyl ether $(10 \mathrm{~mL})$ was added dropwise. Stirring was continued for 1 hour on ice, then for 18 hours at room temperature. The reaction mixture was filtered thorough celite, washing with diethyl ether $(3 \times 14 \mathrm{~mL})$. The diethyl ether was removed in vacuo, and $\mathbf{1}$ was isolated by recrystallisation from 1:1 toluene/hexane $(25 \mathrm{~mL})$ at $-15^{\circ} \mathrm{C}$, washing the white needles obtained with hexane $(6.61 \mathrm{~g}, 87 \%)$.

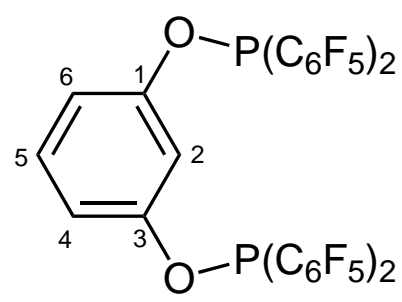

${ }^{1} \mathrm{H}-\mathrm{NMR}:\left(500 \mathrm{MHz}, \mathrm{CD}_{2} \mathrm{Cl}_{2}\right) \delta_{\mathrm{H}} 7.24\left(\mathrm{t},{ }^{3} J_{\mathrm{H}-\mathrm{H}}=7.8 \mathrm{~Hz}, 1 \mathrm{H}, \mathrm{H}-5\right), 6.86\left(\mathrm{dd},{ }^{3} J_{\mathrm{H}-\mathrm{H}}\right.$ $\left.=7.5 \mathrm{~Hz},{ }^{4} J_{\mathrm{H}-\mathrm{H}}=1.6 \mathrm{~Hz}, 2 \mathrm{H}, \mathrm{H}-4,6\right), 6.85$ (s, 1H, H-2). ${ }^{31} \mathrm{P}-\mathrm{NMR}:(121 \mathrm{MHz}$, $\left.\mathrm{CD}_{2} \mathrm{Cl}_{2}\right) \delta_{\mathrm{P}} 87.1$ (quint, ${ }^{3} J_{\mathrm{P}-\mathrm{F}}=35.0 \mathrm{~Hz}$ ). ${ }^{19} \mathrm{~F}-\mathrm{NMR}:\left(282 \mathrm{MHz}, \mathrm{CD}_{2} \mathrm{Cl}_{2}\right) \delta_{\mathrm{F}}-133.1$ $\left(\mathrm{m}, 2 \mathrm{~F}, o-\mathrm{C}_{6} \mathrm{~F}_{5}\right),-148.6\left(\mathrm{t},{ }^{3} J_{\mathrm{F}-\mathrm{F}}=19.7 \mathrm{~Hz}, 1 \mathrm{~F}, p-\mathrm{C}_{6} \mathrm{~F}_{5}\right),-160.6\left(\mathrm{~m}, 2 \mathrm{~F}, m-\mathrm{C}_{6} \mathrm{~F}_{5}\right)$. ${ }^{13} \mathrm{C}-\mathrm{NMR}:\left(125 \mathrm{MHz}, \mathrm{CD}_{2} \mathrm{Cl}_{2}\right) \delta_{\mathrm{C}} 156.5\left(\mathrm{~d},{ }^{2} J_{\mathrm{P}-\mathrm{C}}=16.3 \mathrm{~Hz}, 2 \mathrm{C}, \mathrm{C}-1,3\right) 146.7(\mathrm{~d}$, $\left.{ }^{1} J_{\mathrm{F}-\mathrm{C}}=256 \mathrm{~Hz}, 8 \mathrm{C}, o-\mathrm{C}_{6} \mathrm{~F}_{5}\right), 143.2\left(\mathrm{~d},{ }^{1} J_{\mathrm{F}-\mathrm{C}}=\mathrm{nr}, 4 \mathrm{C}, p-\mathrm{C}_{6} \mathrm{~F}_{5}\right), 137.7\left(\mathrm{~d},{ }^{1} J_{\mathrm{F}-\mathrm{C}}\right.$ $\left.=255 \mathrm{~Hz}, 8 \mathrm{C}, m-\mathrm{C}_{6} \mathrm{~F}_{5}\right), 130.8(\mathrm{~s}, 1 \mathrm{C}, \mathrm{C}-5), 113.6\left(\mathrm{~d},{ }^{3} J_{\mathrm{P}-\mathrm{C}}=13.4 \mathrm{~Hz}, 2 \mathrm{C}, \mathrm{C}-\right.$ 4,6), $111.1\left(\mathrm{~m}, 4 \mathrm{C}, i-\mathrm{C}_{6} \mathrm{~F}_{5}\right), 109.1\left(\mathrm{t},{ }^{3} J_{\mathrm{P}-\mathrm{C}}=10.9 \mathrm{~Hz}, 1 \mathrm{C}, \mathrm{C}-2\right)$. Anal. Calcd for $\mathrm{C}_{30} \mathrm{H}_{4} \mathrm{O}_{2} \mathrm{~F}_{20} \mathrm{P}_{2}$ : C, 42.98; H, 0.48. Found C, 42.86; H, 0.63. HRMS calcd for $\left(\mathrm{C}_{30} \mathrm{H}_{8} \mathrm{NO}_{4} \mathrm{~F}_{20} \mathrm{P}_{2}\right)\left[\mathrm{M}+2 \mathrm{O}+\mathrm{NH}_{4}\right]^{+}: m / z=887.9609$, found $=887.9618$. 
Method A: As per the method of Chase et al., ${ }^{57}$ half-scale (1.00 g, 39\%). Method B: Magnesium powder $(0.10 \mathrm{~g}, 4.1 \mathrm{mmol})$ in THF $(24 \mathrm{~mL})$ was activated by the addition of 1,2-dibromoethane $(0.1 \mathrm{~mL})$ followed by heating with a heat gun until rapid bubbling was observed. After bubbling had ceased, anthracene (1.4 g, $9.0 \mathrm{mmol}$ ) was added, and the reaction mixture was stirred at room temperature for 4 days. The supernatant was decanted and the orange solid isolated was washed with THF $(4 \times 10 \mathrm{~mL})$ and dried briefly in vacuo. The solid $\left[\mathrm{Mg}(\operatorname{anth})(\mathrm{THF})_{3}\right]$ was resuspended in THF and a solution of dichloro- $m$-xylene (350 mg, $2.0 \mathrm{mmol}$ ) in THF $(6 \mathrm{~mL})$ was added dropwise and stirred overnight. The supernatant was isolated by decantation and cooled to $-78^{\circ} \mathrm{C}$. To this cooled solution was added $\operatorname{BrP}\left(\mathrm{C}_{6} \mathrm{~F}_{5}\right)_{2}$ $(0.84 \mathrm{~mL}, 3.68 \mathrm{mmol})$ dropwise. The reaction mixture was allowed to warm to room temperature overnight, then the volatiles were removed in vacuo and the remaining solid extracted with hexane $(4 \times 50 \mathrm{~mL})$ and filtered through celite. Volatiles were again removed under vacuum, and chromatography on silica in air (petroleum ether eluent, $\mathrm{R}_{f}=0.1$ ) afforded 2 as a white solid (1.03 g, 67\%). Spectroscopic data matched that previously reported for $2 .{ }^{57}{ }^{1} \mathrm{H}-\mathrm{NMR}$ : $\left(300 \mathrm{MHz}, \mathrm{C}_{6} \mathrm{D}_{6}\right) \delta_{\mathrm{H}} 6.93$ $(\mathrm{s}, 1 \mathrm{H}, \mathrm{H}-2), 6.67\left(\mathrm{t},{ }^{3} J_{\mathrm{H}-\mathrm{H}}=7.7 \mathrm{~Hz}, 1 \mathrm{H}, \mathrm{H}-5\right), 6.54\left(\mathrm{dd},{ }^{3} J_{\mathrm{H}-\mathrm{H}}=8.0 \mathrm{~Hz},{ }^{4} J_{\mathrm{H}-\mathrm{H}}\right.$ $=1.5 \mathrm{~Hz}, 2 \mathrm{H}, \mathrm{H}-4,6), 3.47$ (d, $\left.{ }^{2} J_{\mathrm{P}-\mathrm{H}}=4.0 \mathrm{~Hz}, 4 \mathrm{H}, \mathrm{CH}_{2}\right) .{ }^{31} \mathrm{P}-\mathrm{NMR}:(121 \mathrm{MHz}$, $\left.\mathrm{C}_{6} \mathrm{D}_{6}\right) \delta_{\mathrm{P}}-46.3$ (quint, $\left.{ }^{3} J_{\mathrm{P}-\mathrm{F}}=25.0 \mathrm{~Hz}\right) .{ }^{19} \mathrm{~F}-\mathrm{NMR}:\left(282 \mathrm{MHz}, \mathrm{C}_{6} \mathrm{D}_{6}\right) \delta_{\mathrm{F}}-130.5$ $\left(\mathrm{m}, 8 \mathrm{~F}, o-\mathrm{C}_{6} \mathrm{~F}_{5}\right),-149.3\left(\mathrm{t},{ }^{3} J_{\mathrm{F}-\mathrm{F}}=21.2 \mathrm{~Hz}, 4 \mathrm{~F}, p-\mathrm{C}_{6} \mathrm{~F}_{5}\right),-160.4\left(\mathrm{~m}, 8 \mathrm{~F}, m-\mathrm{C}_{6} \mathrm{~F}_{5}\right)$.

\section{1- $\left[\left(\mathrm{C}_{6} \mathrm{~F}_{5}\right)_{2} \mathrm{PO}\right]-3-\left({ }^{t} \mathrm{Bu}_{2} \mathrm{PCH}_{2}\right) \mathrm{C}_{6} \mathrm{H}_{4}(\mathrm{POCCPH})$}

A solution of (3-hydroxybenzyl)di-tert-butyl phosphine (1.15 g, $4.56 \mathrm{mmol})$ and triethylamine $(1.4 \mathrm{~mL}, 10 \mathrm{mmol})$ in THF $(50 \mathrm{~mL})$ was cooled on ice, and a solution of $\operatorname{BrP}\left(\mathrm{C}_{6} \mathrm{~F}_{5}\right)_{2}(1.04 \mathrm{~mL}, 4.56 \mathrm{mmol})$ in THF $(15 \mathrm{~mL})$ was added over $10 \mathrm{~min}$. The solution was stirred on ice for a further $10 \mathrm{~min}$, then at room temperature overnight. The reaction mixture was filtered through celite, and the filter cake washed with THF $(5 \times 15 \mathrm{~mL})$. The crude material was dried in vacuo, and purified by extraction with hexane $(3 \times 12 \mathrm{~mL})$ at $-78^{\circ} \mathrm{C}$, followed by filtration through celite . Removal of hexane in vacuo gave 3 as a viscous, pale yellow oil (2.42 g, 86\%).

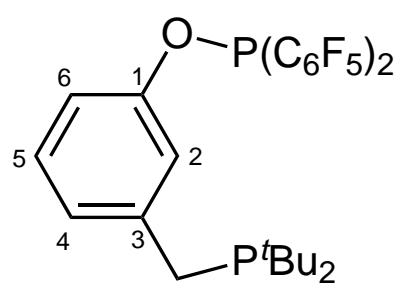


${ }^{1} \mathrm{H}-\mathrm{NMR}:\left(500 \mathrm{MHz}, \mathrm{C}_{6} \mathrm{D}_{6}\right) \delta_{\mathrm{H}} 7.47$ (s, 1H, H-2), $7.02(\mathrm{~m}, 3 \mathrm{H}, \mathrm{H}-4,5,6), 2.65$ (d, $\left.{ }^{2} J_{\mathrm{P}-\mathrm{H}}=2.6 \mathrm{~Hz}, 2 \mathrm{H}, \mathrm{CH}_{2}\right), 0.99\left(\mathrm{~d},{ }^{3} J_{\mathrm{P}-\mathrm{H}}=10.8 \mathrm{~Hz}, 18 \mathrm{H}, \mathrm{C}\left(\mathrm{CH}_{3}\right)_{3}\right) .{ }^{31} \mathrm{P}-\mathrm{NMR}$ : $\left(121 \mathrm{MHz}, \mathrm{C}_{6} \mathrm{D}_{6}\right) \delta_{\mathrm{P}} 82.9$ (quint, $\left.{ }^{3} J_{\mathrm{P}-\mathrm{F}}=35.5 \mathrm{~Hz}, 1 \mathrm{P}, \mathrm{P}\left(\mathrm{C}_{6} \mathrm{~F}_{5}\right)_{2}\right), 34.3(\mathrm{~s}, 1 \mathrm{P}$, $\left.\mathrm{P}^{t} \mathrm{Bu}_{2}\right) .{ }^{19} \mathrm{~F}-\mathrm{NMR}:\left(282 \mathrm{MHz}, \mathrm{C}_{6} \mathrm{D}_{6}\right) \delta_{\mathrm{F}}-133.2\left(\mathrm{~m}, 4 \mathrm{~F}, o-\mathrm{C}_{6} \mathrm{~F}_{5}\right),-148.5\left(\mathrm{t},{ }^{3} J_{\mathrm{F}-\mathrm{F}}\right.$ $\left.=21.3 \mathrm{~Hz}, 2 \mathrm{~F}, p-\mathrm{C}_{6} \mathrm{~F}_{5}\right),-160.3\left(\mathrm{tm},{ }^{3} J_{\mathrm{F}-\mathrm{F}}=21.5 \mathrm{~Hz}, 4 \mathrm{~F}, m-\mathrm{C}_{6} \mathrm{~F}_{5}\right) .{ }^{13} \mathrm{C}-\mathrm{NMR}$ : $\left(125 \mathrm{MHz}, \mathrm{C}_{6} \mathrm{D}_{6}\right) \delta_{\mathrm{C}} 155.5\left(\mathrm{~d},{ }^{2} J_{\mathrm{P}-\mathrm{C}}=15.3 \mathrm{~Hz}, 1 \mathrm{C}, \mathrm{C}-1\right), 146.6\left(\mathrm{dm},{ }^{1} J_{\mathrm{F}-\mathrm{C}}=\right.$ $\left.256 \mathrm{~Hz}, 4 \mathrm{C}, o-\mathrm{C}_{6} \mathrm{~F}_{5}\right), 144.8\left(\mathrm{~d},{ }^{2} J_{\mathrm{P}-\mathrm{C}}=13.4 \mathrm{~Hz}, 1 \mathrm{C}, \mathrm{C}-3\right), 142.8\left(\mathrm{dm},{ }^{1} J_{\mathrm{F}-\mathrm{C}}=\right.$ $\left.257 \mathrm{~Hz}, 2 \mathrm{~F}, p-\mathrm{C}_{6} \mathrm{~F}_{5}\right), 137.4\left(\mathrm{dm},{ }^{2} J_{\mathrm{F}-\mathrm{C}}=254 \mathrm{~Hz}, 4 \mathrm{~F}, m-\mathrm{C}_{6} \mathrm{~F}_{5}\right), 129.5(\mathrm{~s}, 1 \mathrm{C}, \mathrm{C}-5)$, $125.5\left(\mathrm{~d},{ }^{3} J_{\mathrm{P}-\mathrm{C}}=8.1 \mathrm{~Hz}, 1 \mathrm{C}, \mathrm{C}-4\right), 119.4\left(\mathrm{vt},{ }^{3} J_{\mathrm{P}-\mathrm{C}}=11.5 \mathrm{~Hz}, 1 \mathrm{C}, \mathrm{C}-2\right), 114.9(\mathrm{~d}$, $\left.{ }^{3} J_{\mathrm{P}-\mathrm{C}}=12.5 \mathrm{~Hz}, 1 \mathrm{C}, \mathrm{C}-6\right), 110.9\left(\mathrm{dt},{ }^{1} J_{\mathrm{P}-\mathrm{C}}=44.2 \mathrm{~Hz},{ }^{2} J_{\mathrm{F}-\mathrm{C}}=22.1 \mathrm{~Hz}, 2 \mathrm{C}, i-\mathrm{C}_{6} \mathrm{~F}_{5}\right)$, $31.3\left(\mathrm{~d},{ }^{1} J_{\mathrm{P}-\mathrm{C}}=24.0 \mathrm{~Hz}, 2 \mathrm{C}, C\left(\mathrm{CH}_{3}\right)_{3}\right), 29.3\left(\mathrm{~d},{ }^{2} J_{\mathrm{P}-\mathrm{C}}=13.4 \mathrm{~Hz}, 6 \mathrm{C}, \mathrm{C}\left(\mathrm{CH}_{3}\right)_{3}\right)$, $28.5\left(\mathrm{~d},{ }^{1} J_{\mathrm{P}-\mathrm{C}}=25.9 \mathrm{~Hz}, 1 \mathrm{C}, \mathrm{CH}_{2}\right)$. HRMS calcd for $\left(\mathrm{C}_{27} \mathrm{H}_{24} \mathrm{OF}_{10} \mathrm{P}_{2}\right)[\mathrm{M}+\mathrm{H}]^{+}: \mathrm{m} / z$ $=617.1215$, found $=617.1220$. HRMS calcd for $\left(\mathrm{C}_{15} \mathrm{H}_{25} \mathrm{OP}\right)\left[\mathrm{M}-\mathrm{P}\left(\mathrm{C}_{6} \mathrm{~F}_{5}\right)_{2}+2 \mathrm{H}\right]^{+}$: $m / z=253.1716$, found $=253.1756$.

\section{$1,3-\left[\left(\mathrm{C}_{6} \mathrm{~F}_{5}\right)_{2} \mathrm{PNH}\right]_{2} \mathrm{C}_{6} \mathrm{H}_{3} \mathrm{~N}(\mathrm{PNNNP})(10)$}

A solution of 2,6-diaminopyridine $(187 \mathrm{mg}, 1.71 \mathrm{mmol})$ and triethylamine $(0.96 \mathrm{~mL}$, $6.9 \mathrm{mmol})$ in THF $(15 \mathrm{~mL})$ was cooled on ice, and then a solution of $\operatorname{BrP}\left(\mathrm{C}_{6} \mathrm{~F}_{5}\right)_{2}$ $(0.77 \mathrm{~mL}, 3.39 \mathrm{mmol})$ in THF $(8 \mathrm{~mL})$ was added dropwise. The reaction mixture was stirred on ice for 1 hour, then at room temperature for a further 14 hours. The reaction mixture was filtered through celite, and the filter cake washed with toluene $(3 \times 5 \mathrm{~mL})$. Volatiles were removed in vacuo, and recrystallisation from 1:1 hexane/toluene $(7 \mathrm{~mL})$ at $-15{ }^{\circ} \mathrm{C}$ and subsequent washing with hexane gave 10 as an off-white, microcrystalline solid (948 mg, 66\%).

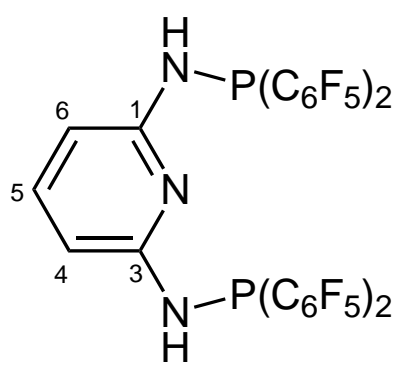

${ }^{1} \mathrm{H}-\mathrm{NMR}:\left(500 \mathrm{MHz}, \mathrm{C}_{6} \mathrm{D}_{6}\right) \delta_{\mathrm{H}} 7.03\left(\mathrm{t},{ }^{3} J_{\mathrm{H}-\mathrm{H}}=8.1 \mathrm{~Hz}, 1 \mathrm{H}, \mathrm{H}-5\right), 6.21\left(\mathrm{~d},{ }^{3} J_{\mathrm{H}-\mathrm{H}}=\right.$ $8.0 \mathrm{~Hz}, 2 \mathrm{H}, \mathrm{H}-4,6), 5.80\left(\mathrm{~d},{ }^{2} J_{\mathrm{P}-\mathrm{H}}=8.5 \mathrm{~Hz}, 2 \mathrm{H}, \mathrm{NH}\right) .{ }^{31} \mathrm{P}-\mathrm{NMR}:\left(121 \mathrm{MHz}, \mathrm{C}_{6} \mathrm{D}_{6}\right)$ $\delta_{\mathrm{P}}-10.8$ (quint, $\left.{ }^{3} J_{\mathrm{P}-\mathrm{F}}=38.2 \mathrm{~Hz}\right) .{ }^{19} \mathrm{~F}-\mathrm{NMR}:\left(282 \mathrm{MHz}, \mathrm{C}_{6} \mathrm{D}_{6}\right) \delta_{\mathrm{F}}-134.6(\mathrm{~m}, 8 \mathrm{~F}$, $\left.o-\mathrm{C}_{6} \mathrm{~F}_{5}\right),-149.4\left(\mathrm{t},{ }^{3} J_{\mathrm{F}-\mathrm{F}}=21.2 \mathrm{~Hz}, 4 \mathrm{~F}, p-\mathrm{C}_{6} \mathrm{~F}_{5}\right),-160.0\left(\mathrm{tm},{ }^{3} J_{\mathrm{F}-\mathrm{F}}=22.8 \mathrm{~Hz}, 8 \mathrm{~F}\right.$, $\left.m-\mathrm{C}_{6} \mathrm{~F}_{5}\right) .{ }^{13} \mathrm{C}-\mathrm{NMR}:\left(125 \mathrm{MHz}, \mathrm{C}_{6} \mathrm{D}_{6}\right) \delta_{\mathrm{C}} 155.4\left(\mathrm{~d},{ }^{2} J_{\mathrm{P}-\mathrm{C}}=25.5 \mathrm{~Hz}, 2 \mathrm{C}, \mathrm{C}-1,3\right)$, 
$146.8\left(\mathrm{~d},{ }^{1} J_{\mathrm{F}-\mathrm{C}}=244 \mathrm{~Hz}, 8 \mathrm{C}, o-\mathrm{C}_{6} \mathrm{~F}_{5}\right), 142.4\left(\mathrm{dm},{ }^{1} J_{\mathrm{F}-\mathrm{C}}=257 \mathrm{~Hz}, 4 \mathrm{C}, p-\mathrm{C}_{6} \mathrm{~F}_{5}\right)$, $140.3(\mathrm{~s}, 1 \mathrm{C}, \mathrm{C}-5), 137.3\left(\mathrm{dm},{ }^{1} J_{\mathrm{F}-\mathrm{C}}=255 \mathrm{~Hz}, 8 \mathrm{C}, m-\mathrm{C}_{6} \mathrm{~F}_{5}\right), 110.3\left(\mathrm{~m}, 4 \mathrm{C}, i-\mathrm{C}_{6} \mathrm{~F}_{5}\right)$, $101.6\left(\mathrm{~d},{ }^{3} J_{\mathrm{P}-\mathrm{C}}=11.5 \mathrm{~Hz}, 2 \mathrm{C}, \mathrm{C}-4,6\right)$. HRMS calcd for $\left(\mathrm{C}_{29} \mathrm{H}_{6} \mathrm{~N}_{3} \mathrm{~F}_{20} \mathrm{P}_{2}\right)[\mathrm{M}+\mathrm{H}]^{+}$: $m / z=837.9718$, found $=837.9738$.

\section{$1,3-\left[\left(\mathrm{C}_{6} \mathrm{~F}_{5}\right)_{2} \mathrm{PO}\right]_{2} \mathrm{C}_{6} \mathrm{H}_{3} \mathrm{~N}(\mathrm{PONOP})(11)$}

A solution of 2,6-dihydroxypyridine hydrochloride (250 $\mathrm{mg}, 1.69 \mathrm{mmol}$ ) and triethylamine $(0.96 \mathrm{~mL}, 6.9 \mathrm{mmol})$ in THF $(15 \mathrm{~mL})$ was cooled on ice, and then a solution of $\operatorname{BrP}\left(\mathrm{C}_{6} \mathrm{~F}_{5}\right)_{2}(0.77 \mathrm{~mL}, 3.39 \mathrm{mmol})$ in THF $(8 \mathrm{~mL})$ was added dropwise. The reaction mixture was stirred on ice for 1 hour, then at room temperature for a further 15 hours. The reaction mixture was filtered through celite, and the filter cake washed with THF $(3 \times 5 \mathrm{~mL})$. Volatiles were removed in vacuo, and recrystallisation from 1:1 hexane/dichloromethane $(15 \mathrm{~mL})$ at $-15{ }^{\circ} \mathrm{C}$ and subsequent washing with hexane gave 11 as white, crystalline solid (871 mg, 61\%). ${ }^{1} \mathrm{H}-\mathrm{NMR}$ : $\left(300 \mathrm{MHz}, \mathrm{C}_{6} \mathrm{D}_{6}\right) \delta_{\mathrm{H}}$ $6.87\left(\mathrm{t},{ }^{3} J_{\mathrm{H}-\mathrm{H}}=7.9 \mathrm{~Hz}, 1 \mathrm{H}, \mathrm{H}-5\right), 6.31\left(\mathrm{~d},{ }^{3} J_{\mathrm{H}-\mathrm{H}}=8.2 \mathrm{~Hz}, 2 \mathrm{H}, \mathrm{H}-4,6\right) .{ }^{31} \mathrm{P}-\mathrm{NMR}$ : $\left(121 \mathrm{MHz}, \mathrm{C}_{6} \mathrm{D}_{6}\right) \delta_{\mathrm{P}} 70.2$ (quint, ${ }^{3} J_{\mathrm{P}-\mathrm{F}}=42.8 \mathrm{~Hz}$ ). ${ }^{19} \mathrm{~F}-\mathrm{NMR}:\left(282 \mathrm{MHz}, \mathrm{C}_{6} \mathrm{D}_{6}\right)$ $\delta_{\mathrm{F}}-134.0\left(\mathrm{~m}, 8 \mathrm{~F}, o-\mathrm{C}_{6} \mathrm{~F}_{5}\right),-147.8\left(\mathrm{t},{ }^{3} J_{\mathrm{F}-\mathrm{F}}=21.0 \mathrm{~Hz}, 4 \mathrm{~F}, p-\mathrm{C}_{6} \mathrm{~F}_{5}\right),-160.0(\mathrm{~m}$, $\left.8 \mathrm{~F}, m-\mathrm{C}_{6} \mathrm{~F}_{5}\right) .{ }^{13} \mathrm{C}-\mathrm{NMR}:\left(125 \mathrm{MHz}, \mathrm{C}_{6} \mathrm{D}_{6}\right) \delta_{\mathrm{C}} 159.2\left(\mathrm{~d},{ }^{2} J_{\mathrm{P}-\mathrm{C}}=9.1 \mathrm{~Hz}, 2 \mathrm{C}, \mathrm{C}-1,3\right)$, $146.8\left(\mathrm{dm},{ }^{1} J_{\mathrm{F}-\mathrm{C}}=247 \mathrm{~Hz}, 8 \mathrm{C}, o_{o}-\mathrm{C}_{6} \mathrm{~F}_{5}\right), 143.2(\mathrm{~s}, 1 \mathrm{C}, \mathrm{C}-5), 143.1\left(\mathrm{dm},{ }^{1} J_{\mathrm{F}-\mathrm{C}}=\right.$ $\left.259 \mathrm{~Hz}, 4 \mathrm{C}, p-\mathrm{C}_{6} \mathrm{~F}_{5}\right), 137.5\left(\mathrm{dm},{ }^{1} J_{\mathrm{F}-\mathrm{C}}=255 \mathrm{~Hz}, 8 \mathrm{C}, m-\mathrm{C}_{6} \mathrm{~F}_{5}\right), 110.2(\mathrm{~m}, 4 \mathrm{C}, i-$ $\left.\mathrm{C}_{6} \mathrm{~F}_{5}\right), 105.8$ (s, 2C, C-4,6). HRMS calcd for $\left(\mathrm{C}_{29} \mathrm{H}_{3} \mathrm{NO}_{4} \mathrm{~F}_{20} \mathrm{P}_{2} \mathrm{Na}\right)[\mathrm{M}+2 \mathrm{O}+\mathrm{Na}]^{+}$: $m / z=893.9116$, found $=893.9142$.

\subsection{Platinum Complexes}

\section{$[(\mathrm{POCOPH}) \operatorname{Pt}(\mathrm{nb})]_{2}(14)$}

A solution of $\mathrm{Pt}(\mathrm{nb})_{3}(117 \mathrm{mg}, 0.25 \mathrm{mmol})$ and $1(204 \mathrm{mg}, 0.24 \mathrm{mmol})$ in dichloromethane $(7 \mathrm{~mL})$ was heated at $40{ }^{\circ} \mathrm{C}$ for 36 hours, at which point all volatiles were removed in vacuo. The oily solid was washed with ice-cold pentane, then redissolved in toluene $(5 \mathrm{~mL})$ and heated at $50{ }^{\circ} \mathrm{C}$ for 12 hours. The volume was reduced in vacuo to approximately $2 \mathrm{~mL}$, with the addition of hexane to the toluene solution causing the precipitation of $\mathbf{1 4}$ as a white solid, which was isolated by decantation of the supernatant and washing with pentane (107 mg, 39\%). Crystals suitable for single crystal X-ray diffraction were obtained by solvent diffusion at room temperature, 
with methanol layered above a dichloromethane solution of 14. ${ }^{1} \mathrm{H}-\mathrm{NMR}$ : $(500 \mathrm{MHz}$, $\left.\mathrm{CD}_{2} \mathrm{Cl}_{2}\right) \delta_{\mathrm{H}} 6.85\left(\mathrm{t},{ }^{3} J_{\mathrm{H}-\mathrm{H}}=8.1 \mathrm{~Hz}, 1 \mathrm{H}, \mathrm{H}-5\right), 6.69\left(\mathrm{~d},{ }^{3} J_{\mathrm{H}-\mathrm{H}}=8.1 \mathrm{~Hz}, 2 \mathrm{H}, \mathrm{H}-4,6\right)$, $6.12(\mathrm{~s}, 1 \mathrm{H}, \mathrm{H}-2), 2.75\left(\mathrm{~d},{ }^{2} J_{\mathrm{Pt}-\mathrm{H}}=70.8 \mathrm{~Hz},{ }^{3} J_{\mathrm{H}-\mathrm{H}}=10.7 \mathrm{~Hz}, 2 \mathrm{H}, \mathrm{nb} \mathrm{C}=\mathrm{CH}\right), 2.20$ $\left(\mathrm{d},{ }^{2} J_{\mathrm{H}-\mathrm{H}}=10.0 \mathrm{~Hz}, 2 \mathrm{H}, \mathrm{nb} \mathrm{C}-\mathrm{CH}\right), 1.44\left(\mathrm{~d},{ }^{2} J_{\mathrm{H}-\mathrm{H}}=7.3 \mathrm{~Hz}, 2 \mathrm{H}, \mathrm{nb} \mathrm{H}_{2} \mathrm{C}-\mathrm{CH}_{2}\right)$, $0.96\left(\mathrm{~d},{ }^{2} J_{\mathrm{H}-\mathrm{H}}=7.6 \mathrm{~Hz}, 2 \mathrm{H}, \mathrm{nb} \mathrm{H}_{2} \mathrm{C}-\mathrm{CH}_{2}\right), 0.42\left(\mathrm{~m}, 1 \mathrm{H}, \mathrm{nb} \mathrm{HC}-\mathrm{CH}_{2}-\mathrm{CH}\right), 0.15$ $\left(\mathrm{d},{ }^{2} J_{\mathrm{H}-\mathrm{H}}=8.5 \mathrm{~Hz}, 1 \mathrm{H}, \mathrm{nb} \mathrm{HC}-\mathrm{CH}_{2}-\mathrm{CH}\right) .{ }^{31} \mathrm{P}-\mathrm{NMR}:\left(121 \mathrm{MHz}, \mathrm{CD}_{2} \mathrm{Cl}_{2}\right) \delta_{\mathrm{P}} 100.5$ $\left(\mathrm{s},{ }^{1} J_{\mathrm{Pt}-\mathrm{P}}=4623 \mathrm{~Hz}\right) \cdot{ }^{19} \mathrm{~F}-\mathrm{NMR}:\left(282 \mathrm{MHz}, \mathrm{CD}_{2} \mathrm{Cl}_{2}\right) \delta_{\mathrm{F}}-131.5\left(\mathrm{~m}, 8 \mathrm{~F}, o-\mathrm{C}_{6} \mathrm{~F}_{5}\right)$, $-149.3\left(\mathrm{~m}, 4 \mathrm{~F}, p-\mathrm{C}_{6} \mathrm{~F}_{5}\right),-160.8\left(\mathrm{~m}, 8 \mathrm{~F}, m-\mathrm{C}_{6} \mathrm{~F}_{5}\right) .{ }^{13} \mathrm{C}-\mathrm{NMR}:\left(125 \mathrm{MHz}, \mathrm{CD}_{2} \mathrm{Cl}_{2}\right)$ $\delta_{\mathrm{C}} 152.5(\mathrm{~s}, 2 \mathrm{C}, \mathrm{C}-1,3), 145.0\left(\mathrm{~d},{ }^{3} J_{\mathrm{F}-\mathrm{C}}=244 \mathrm{~Hz}, 8 \mathrm{C}, o_{-}-\mathrm{C}_{6} \mathrm{~F}_{5}\right), \approx 142^{\ddagger}\left(\mathrm{d},{ }^{3} J_{\mathrm{F}-\mathrm{C}}=\right.$ nr, $\left.4 \mathrm{C}, p-\mathrm{C}_{6} \mathrm{~F}_{5}\right), 136.9\left(\mathrm{~d},{ }^{3} J_{\mathrm{F}-\mathrm{C}}=249 \mathrm{~Hz}, 8 \mathrm{C}, m-\mathrm{C}_{6} \mathrm{~F}_{5}\right), 128.5(\mathrm{~s}, 1 \mathrm{C}, \mathrm{C}-5), 113.4$ $(\mathrm{s}, 2 \mathrm{C}, \mathrm{C}-4,6), 112.1\left(\mathrm{~m}, 4 \mathrm{C}, i-\mathrm{C}_{6} \mathrm{~F}_{5}\right), 111.7(\mathrm{~s}, 1 \mathrm{C}, \mathrm{C}-2), 65.7\left(\mathrm{~m},{ }^{1} J_{\mathrm{Pt}-\mathrm{C}}=259 \mathrm{~Hz}\right.$, $2 \mathrm{C}, \mathrm{nb} \mathrm{C}=\mathrm{CH}), 42.0(\mathrm{~s}, 2 \mathrm{C}, \mathrm{nb} \mathrm{C}-\mathrm{CH}), 39.8$ (s, $\left.1 \mathrm{C}, \mathrm{nb} \mathrm{HC}-\mathrm{CH}_{2}-\mathrm{CH}\right), 27.7$ (s, $\left.{ }^{3} J_{\mathrm{Pt}-\mathrm{C}}=53.2 \mathrm{~Hz}, 2 \mathrm{C}, \mathrm{H}_{2} \mathrm{C}-\mathrm{CH}_{2}\right)$. Anal. Calcd for $\mathrm{C}_{74} \mathrm{H}_{28} \mathrm{O}_{4} \mathrm{~F}_{40} \mathrm{P}_{4} \mathrm{Pt}_{2} \cdot \mathrm{CH}_{2} \mathrm{Cl}_{2}: \mathrm{C}$, 38.50; H, 1.29. Found C, 38.80; H, 1.40. HRMS calcd for $\left(\mathrm{C}_{60} \mathrm{H}_{8} \mathrm{O}_{4} \mathrm{~F}_{40} \mathrm{NaP}_{4} \mathrm{Pt}_{2}\right)$ $[\mathrm{M}-2 \mathrm{nb}+\mathrm{Na}]^{+}: m / z=2086.7886$, found $=2086.7883$.

\section{$\left[(\mathrm{POCOPH}) \mathrm{PtMe}_{2}\right]_{2}$}

A solution of ligand 1 (200 mg, $0.24 \mathrm{mmol})$ and $\left[\mathrm{PtMe}_{2}(\mathrm{hex})\right]$ (73.3 mg, $0.24 \mathrm{mmol}$ ) in toluene $(15 \mathrm{~mL})$ was stirred at room temperature for 72 hours. The solution was concentrated in vacuo to about $3 \mathrm{~mL}$, and the oligomeric byproduct 16 precipitated out by the addition of $3 \mathrm{~mL}$ hexane to the solution. Decantation and isolation of the supernatant, followed by removal of the solvent in vacuo and washing with hexane afforded 15 as a yellow, microcrystalline solid (199 mg, 78\%). ${ }^{1} \mathrm{H}-\mathrm{NMR}$ : (300 MHz, $\left.\mathrm{CD}_{2} \mathrm{Cl}_{2}\right) \delta_{\mathrm{H}} 7.05\left(\mathrm{t},{ }^{3} J_{\mathrm{H}-\mathrm{H}}=8.3 \mathrm{~Hz}, 2 \mathrm{H}, \mathrm{H}-5\right), 6.82\left(\mathrm{~d},{ }^{3} J_{\mathrm{H}-\mathrm{H}}=\mathrm{H}-4,6 \mathrm{~Hz}, 4 \mathrm{H}, \mathrm{H}-4,6\right)$, $6.42(\mathrm{~s}, 2 \mathrm{H}, \mathrm{H}-2), 0.21\left(\mathrm{vt},{ }^{2} J_{\mathrm{Pt}-\mathrm{H}}=71.0 \mathrm{~Hz},{ }^{3} J_{\mathrm{P}-\mathrm{H}}=\mathrm{nr}, 12 \mathrm{H}, \mathrm{CH}_{3}\right) .{ }^{31} \mathrm{P}-\mathrm{NMR}$ : $\left(121 \mathrm{MHz}, \mathrm{CDCl}_{3}\right) \delta_{\mathrm{P}} 89.9\left(\mathrm{~s},{ }^{1} J_{\mathrm{Pt}-\mathrm{P}}=2202 \mathrm{~Hz}\right) .{ }^{19} \mathrm{~F}-\mathrm{NMR}:\left(282 \mathrm{MHz}, \mathrm{CDCl}_{3}\right)$ $\delta_{\mathrm{F}}-129.4\left(\mathrm{br} \mathrm{s}, 16 \mathrm{~F}, o-\mathrm{C}_{6} \mathrm{~F}_{5}\right.$ ), -145.7 (br s, 8F, $p-\mathrm{C}_{6} \mathrm{~F}_{5}$ ), -159.1 (br s, $18 \mathrm{~F}, m-$ $\left.\mathrm{C}_{6} \mathrm{~F}_{5}\right) \cdot{ }^{13} \mathrm{C}-\mathrm{NMR}:\left(125 \mathrm{MHz}, \mathrm{CD}_{2} \mathrm{Cl}_{2}\right) \delta_{\mathrm{C}} 153.4(\mathrm{~s}, 4 \mathrm{C}, \mathrm{C}-1,3), 146.4\left(\mathrm{dm},{ }^{1} J_{\mathrm{P}-\mathrm{F}}=\right.$ $\left.259 \mathrm{~Hz}, 16 \mathrm{C}, o-\mathrm{C}_{6} \mathrm{~F}_{5}\right), 143.7\left(\mathrm{dm},{ }^{1} J_{\mathrm{P}-\mathrm{F}}=262 \mathrm{~Hz}, 8 \mathrm{C}, p-\mathrm{C}_{6} \mathrm{~F}_{5}\right), 137.6\left(\mathrm{dm},{ }^{1} J_{\mathrm{P}-\mathrm{F}}\right.$ $\left.=259 \mathrm{~Hz}, 16 \mathrm{C}, m-\mathrm{C}_{6} \mathrm{~F}_{5}\right), 129.3(\mathrm{~s}, 2 \mathrm{C}, \mathrm{C}-5), 116.5(\mathrm{~s}, 4 \mathrm{C}, \mathrm{C}-4,6), 112.4(\mathrm{~s}, 2 \mathrm{C}$, $\mathrm{C}-2), 108.8\left(\mathrm{~m}, 8 \mathrm{C}, i-\mathrm{C}_{6} \mathrm{~F}_{5}\right), 1.7\left(\mathrm{dd},{ }^{2} J_{\mathrm{P}-\mathrm{C}}=116 \mathrm{~Hz}, 4 \mathrm{C}, \mathrm{CH}_{3}\right)$. HRMS calcd for $\left(\mathrm{C}_{64} \mathrm{H}_{20} \mathrm{O}_{4} \mathrm{~F}_{40} \mathrm{NaP}_{4} \mathrm{Pt}_{2}\right)[\mathrm{M}+\mathrm{Na}]^{+}: m / z=2146.8825$, found $=2146.8855$.

\footnotetext{
${ }^{\ddagger}$ The chemical shift $\delta_{\mathrm{C}}$ and coupling constants for the $p-\mathrm{C}_{6} \mathrm{~F}_{5}$ carbon environment could not be determined due to peak overlap with the adjacent $o-\mathrm{C}_{6} \mathrm{~F}_{5}$ environment.
} 
The oligomeric species $\mathbf{1 6}$ could be isolated as a byproduct during the synthesis of the dimer $\mathbf{1 5}$ as above, with shorter reaction times in dichloromethane were observed to increase the yield of 16. A solution of ligand 1 (50 mg, $60 \mu \mathrm{mol})$ and $\left[\mathrm{PtMe}_{2}(\mathrm{hex})\right](18.5 \mathrm{mg}, 60 \mu \mathrm{mol})$ in dichloromethane $(5 \mathrm{~mL})$ was stirred at room temperature for 24 hours. The solvent was reduced to about $0.5 \mathrm{~mL}$ in vacuo, and precipitation of the product with hexane followed be decantation of the supernatant and washing of the precipitate in situ with pentane afforded $\mathbf{1 6}$ as a white solid (48 mg, 76\%). Samples of $\mathbf{1 6}$ were found to be contaminated with small quantities of the dimer $15 .{ }^{1} \mathrm{H}-\mathrm{NMR}$ : $\left(300 \mathrm{MHz}, \mathrm{CD}_{2} \mathrm{Cl}_{2}\right) \delta_{\mathrm{H}} 7.14\left(\mathrm{t},{ }^{3} J_{\mathrm{H}-\mathrm{H}}=8.2 \mathrm{~Hz}, 1 \mathrm{H}\right.$, H-5), 6.94 (s, 1H, H-2), 6.77 (d, $\left.{ }^{3} J_{\mathrm{H}-\mathrm{H}}=\mathrm{H}-4,6 \mathrm{~Hz}, 2 \mathrm{H}, \mathrm{H}-4,6\right), 0.42$ (dd, ${ }^{2} J_{\mathrm{Pt}-\mathrm{H}}=$ $\left.70.6 \mathrm{~Hz},{ }^{3} J_{\mathrm{P}-\mathrm{H}}=3.0 \mathrm{~Hz}, \mathrm{nr}, 6 \mathrm{H}, \mathrm{CH}_{3}\right) .{ }^{31} \mathrm{P}-\mathrm{NMR}:\left(121 \mathrm{MHz}, \mathrm{CD}_{2} \mathrm{Cl}_{2}\right) \delta_{\mathrm{P}} 90.9(\mathrm{~s}$, $\left.{ }^{1} J_{\mathrm{Pt}-\mathrm{P}}=2150 \mathrm{~Hz}\right) \cdot{ }^{19} \mathrm{~F}-\mathrm{NMR}:\left(282 \mathrm{MHz}, \mathrm{CD}_{2} \mathrm{Cl}_{2}\right) \delta_{\mathrm{F}}-129.0\left(\mathrm{dm},{ }^{3} J_{\mathrm{P}-\mathrm{F}}=16.4 \mathrm{~Hz}\right.$, $\left.8 \mathrm{~F}, o-\mathrm{C}_{6} \mathrm{~F}_{5}\right),-146.5\left(\mathrm{tm},{ }^{3} J_{\mathrm{F}-\mathrm{F}}=20.8 \mathrm{~Hz}, 4 \mathrm{~F}, m-\mathrm{C}_{6} \mathrm{~F}_{5}\right),-159.7\left(\mathrm{~m}, 8 \mathrm{~F}, p-\mathrm{C}_{6} \mathrm{~F}_{5}\right)$. ${ }^{13} \mathrm{C}-\mathrm{NMR}:\left(125 \mathrm{MHz}, \mathrm{CD}_{2} \mathrm{Cl}_{2}\right) \delta_{\mathrm{C}} 153.4(\mathrm{~s}, 4 \mathrm{C}, \mathrm{C}-1,3), 146.4\left(\mathrm{dm},{ }^{1} J_{\mathrm{P}-\mathrm{F}}=259 \mathrm{~Hz}\right.$, $\left.8 \mathrm{C}, o-\mathrm{C}_{6} \mathrm{~F}_{5}\right), 143.7\left(\mathrm{dm},{ }^{1} J_{\mathrm{P}-\mathrm{F}}=262 \mathrm{~Hz}, 4 \mathrm{C}, p-\mathrm{C}_{6} \mathrm{~F}_{5}\right), 137.6\left(\mathrm{dm},{ }^{1} J_{\mathrm{P}-\mathrm{F}}=259 \mathrm{~Hz}\right.$, $8 \mathrm{C}, m-\mathrm{C}_{6} \mathrm{~F}_{5}$ ), 129.9 (s, 1C, C-5), 115.8 (s, 2C, C-4,6), 112.3 (s, 1C, C-2), 108.8 (m, $\left.4 \mathrm{C}, i-\mathrm{C}_{6} \mathrm{~F}_{5}\right), 4.0\left(\mathrm{dd},{ }^{2} J_{\mathrm{P}-\mathrm{C}}=115 \mathrm{~Hz}, 2 \mathrm{C}, \mathrm{CH}_{3}\right)$.

\section{Observation of cis- $\left[\left(\kappa^{1}-\mathrm{POCOPH}\right)_{2} \mathrm{PtCl}_{2}\right](18)$}

Ligand 1 (30 mg, $36 \mu \mathrm{mol})$ and $\left[\mathrm{PtCl}_{2}\right.$ (hex)] (12.5 mg, $\left.36 \mu \mathrm{mol}\right)$ were dissolved in benzene- $d_{6}$ in an NMR tube, and the course of the reaction monitored by NMR spectroscopy. After two hours at room temperature, NMR spectroscopy revealed the formation of $\mathbf{1 8}$ along with the presence of unreacted starting material. Heating of the reaction mixture to $60{ }^{\circ} \mathrm{C}$ for two minutes to encourage dissolution of the $\left[\mathrm{PtCl}_{2}(\right.$ hex $\left.)\right]$ resulted instead in the formation of significant quantities of the dimer 19. As such, compound $\mathbf{1 8}$ was not isolated for further analysis.

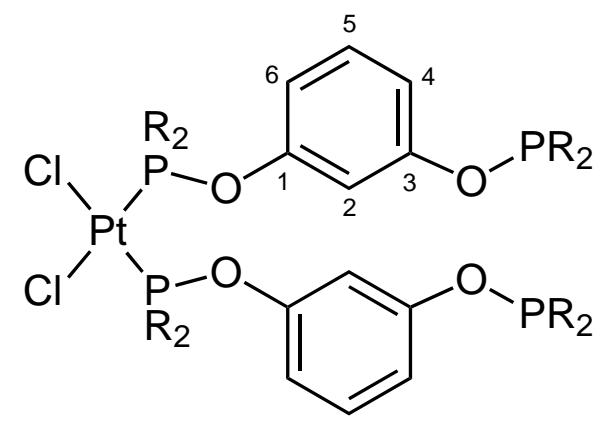

where $\mathrm{R}=\mathrm{C}_{6} \mathrm{~F}_{5}$ 
${ }^{1} \mathrm{H}-\mathrm{NMR}:\left(300 \mathrm{MHz}, \mathrm{C}_{6} \mathrm{D}_{6}\right) \delta_{\mathrm{H}} 6.91(\mathrm{~s}, 2 \mathrm{H}, \mathrm{H}-2), 6.79\left(\mathrm{~d},{ }^{3} J_{\mathrm{H}-\mathrm{H}}=7.9 \mathrm{~Hz}, 2 \mathrm{H}, \mathrm{H}-6\right)$, $6.73\left(\mathrm{vt},{ }^{3} J_{\mathrm{H}-\mathrm{H}}=8.2 \mathrm{~Hz}, 2 \mathrm{H}, \mathrm{H}-5\right), 6.57\left(\mathrm{~d},{ }^{3} J_{\mathrm{H}-\mathrm{H}}=7.7 \mathrm{~Hz}, 2 \mathrm{H}, \mathrm{H}-4\right) .{ }^{31} \mathrm{P}-\mathrm{NMR}$ : $\left(121 \mathrm{MHz}, \mathrm{C}_{6} \mathrm{D}_{6}\right) \delta_{\mathrm{P}} 85.4$ (quint, $\left.{ }^{3} J_{\mathrm{P}-\mathrm{F}}=36.8 \mathrm{~Hz}, 2 \mathrm{P}, \mathrm{P}\left(\mathrm{C}_{6} \mathrm{~F}_{5}\right)_{2}\right), 51.4\left(\mathrm{~s},{ }^{1} J_{\mathrm{Pt}-\mathrm{P}}\right.$ $=4531 \mathrm{~Hz}, 2 \mathrm{P}, \mathrm{P}-$ trans-Cl$) .{ }^{19} \mathrm{~F}-\mathrm{NMR}:\left(282 \mathrm{MHz}, \mathrm{C}_{6} \mathrm{D}_{6}\right) \delta_{\mathrm{F}}-127.5--130.7$ (br $\mathrm{m}, 16 \mathrm{~F}, o-\mathrm{C}_{6} \mathrm{~F}_{5}$ ), $-151.4--153.3$ (br m, 8F, p- $\mathrm{C}_{6} \mathrm{~F}_{5}$ ), -158.9--160.0 (br m, 16F, $\left.m-\mathrm{C}_{6} \mathrm{~F}_{5}\right)$.

cis, trans-[(POCOPH)PtCl $]_{2}(19)$

A solution of ligand $1(30 \mathrm{mg}, 36 \mu \mathrm{mol})$ and $\left[\mathrm{PtCl}_{2}(\mathrm{hex})\right](12.5 \mathrm{mg}, 36 \mu \mathrm{mol})$ in benzene- $d_{6}$ was heated to $90{ }^{\circ} \mathrm{C}$ for three minutes (to ensure dissolution of starting material), then left standing at room temperature for 12 hours. The reaction mixture was heated for a further 80 minutes at $90{ }^{\circ} \mathrm{C}$, at which point NMR spectroscopy revealed almost quantitative formation of $\mathbf{1 9}$. Removal of the volatiles in vacuo and repeated washing with hexane afforded the product 19 as a cream-coloured solid (8 $\mathrm{mg}, 20 \%)$.

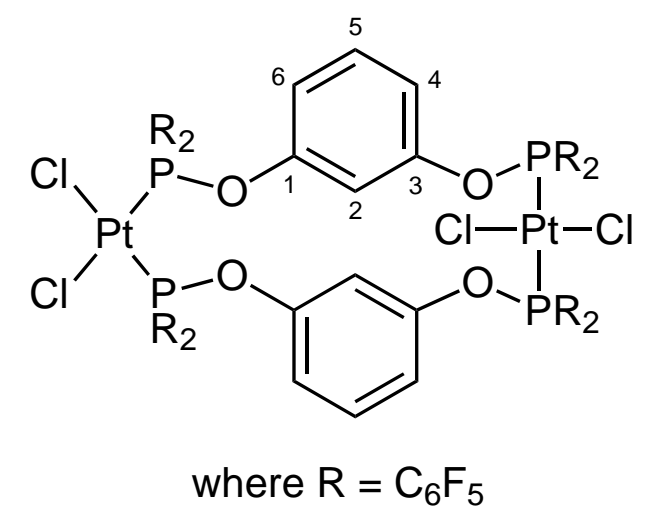

${ }^{1} \mathrm{H}-\mathrm{NMR}:\left(300 \mathrm{MHz}, \mathrm{C}_{6} \mathrm{D}_{6}\right) \delta_{\mathrm{H}} 7.38$ (s, 2H, H-2), 6.68 (d, 2H, H-6), $6.61(\mathrm{t}, 2 \mathrm{H}$, H-5), 6.53 (d, 2H, H-4). ${ }^{31} \mathrm{P}-\mathrm{NMR}:\left(121 \mathrm{MHz}, \mathrm{C}_{6} \mathrm{D}_{6}\right) \delta_{\mathrm{P}} 80.6\left(\mathrm{~s},{ }^{1} J_{\mathrm{Pt}-\mathrm{P}}=3357 \mathrm{~Hz}\right.$, 2P, P-trans-P), 55.5 (s, ${ }^{1} J_{\mathrm{Pt}-\mathrm{P}}=4582 \mathrm{~Hz}, 2 \mathrm{P}, \mathrm{P}$-trans-Cl). ${ }^{19} \mathrm{~F}-\mathrm{NMR}:(282 \mathrm{MHz}$, $\left.\mathrm{C}_{6} \mathrm{D}_{6}\right) \delta_{\mathrm{F}}-126.9--130.5$ (br m, 16F, o- $\mathrm{C}_{6} \mathrm{~F}_{5}$ ), -141.0--142.3 (br m, 8F, p- $\mathrm{C}_{6} \mathrm{~F}_{5}$ ), $-158.2-158.6$ (br m, 16F, $m-\mathrm{C}_{6} \mathrm{~F}_{5}$ ). HRMS calcd for $\left(\mathrm{C}_{60} \mathrm{H}_{8} \mathrm{O}_{4} \mathrm{~F}_{40} \mathrm{NaCl}_{4} \mathrm{P}_{4} \mathrm{Pt}_{2}\right)$ $[\mathrm{M}+\mathrm{Na}]^{+}: m / z=2230.6665$, found $=2230.6653$. Samples of $\mathbf{1 9}$ were contaminated with small amounts of $\mathbf{1 8}$, preventing satisfactory elemental analysis data from being obtained. 
A solution of [PtClMe(hex)] (101 mg, $0.30 \mathrm{mmol})$ and ligand 1 (249 mg, $0.31 \mathrm{mmol})$ in toluene $(5 \mathrm{~mL})$ was heated at $40{ }^{\circ} \mathrm{C}$ for 24 hours, then stirred at room temperature for a further 8 hours. The solution was filtered through a short column of alumina in air, washing the column with toluene $(3 \times 2 \mathrm{~mL})$. Volatiles were removed in vacuo, and the white solid redissolved in a 1:1 toluene/hexane solution, purifying the product by precipitation of byproducts from solution at $-15^{\circ} \mathrm{C}$. The supernatant was decanted and collected, and removal of the volatiles in vacuo followed by washing with pentane gave $\mathbf{2 1}$ as a white, microcrystalline solid (65 mg, 20\%).

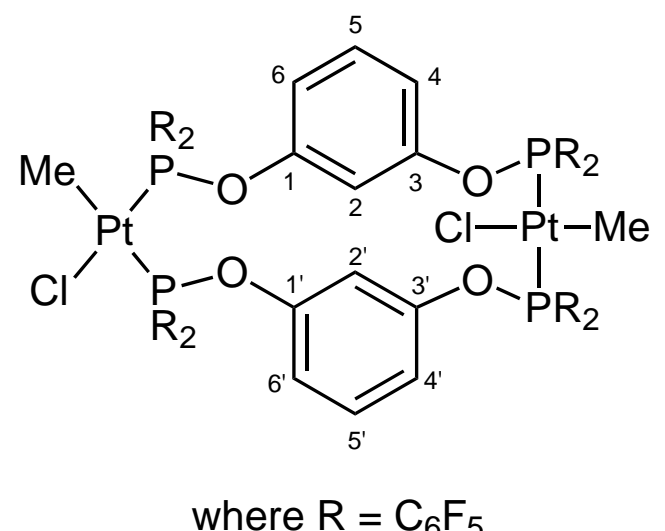

${ }^{1} \mathrm{H}-\mathrm{NMR}:\left(600 \mathrm{MHz}, \mathrm{C}_{6} \mathrm{D}_{6}\right) \delta_{\mathrm{H}} 7.54$ (s, 1H, H-2), 7.34 (s, 1H, H-2'), 6.68 (d, ${ }^{3} J_{\mathrm{H}-\mathrm{H}}$ $=7.9 \mathrm{~Hz}, 1 \mathrm{H}, \mathrm{H}-6), 6.62\left(\mathrm{~m}, 3 \mathrm{H}, \mathrm{H}-5,5^{\prime}, 6^{\prime}\right), 6.55\left(\mathrm{~d},{ }^{3} J_{\mathrm{H}-\mathrm{H}}=8.2 \mathrm{~Hz}, 1 \mathrm{H}, \mathrm{H}-4\right)$, $6.53\left(\mathrm{~d},{ }^{3} J_{\mathrm{H}-\mathrm{H}}=7.0 \mathrm{~Hz}, 1 \mathrm{H}, \mathrm{H}-4^{\prime}\right), 1.27$ (br m, 3H, CH -trans-P) 0.26 (br t, ${ }^{3} J_{\mathrm{P}-\mathrm{H}}$ $=6.6 \mathrm{~Hz}, 3 \mathrm{H}, \mathrm{CH}_{3}$-trans-Cl). ${ }^{31} \mathrm{P}-\mathrm{NMR}:\left(121 \mathrm{MHz}, \mathrm{C}_{6} \mathrm{D}_{6}\right) \delta_{\mathrm{P}} 94.4\left(\mathrm{~d},{ }^{2} J_{\mathrm{P}-\mathrm{P}} 15.4\right.$, ${ }^{1} J_{\mathrm{Pt}-\mathrm{P}}=2066 \mathrm{~Hz}, 1 \mathrm{P}, \mathrm{P}$-trans-CH$\left.{ }_{3}\right), 90.3\left(\mathrm{~s},{ }^{1} J_{\mathrm{Pt}-\mathrm{P}}=4028 \mathrm{~Hz}, 2 \mathrm{P}, \mathrm{P}\right.$-trans-P), 62.1 $\left(\mathrm{d},{ }^{2} J_{\mathrm{P}-\mathrm{P}} 15.4,{ }^{1} J_{\mathrm{Pt}-\mathrm{P}}=5494 \mathrm{~Hz}, 1 \mathrm{P}, \mathrm{P}\right.$-trans-Cl$) .{ }^{19} \mathrm{~F}-\mathrm{NMR}:\left(282 \mathrm{MHz}, \mathrm{C}_{6} \mathrm{D}_{6}\right) \delta_{\mathrm{F}}$ $-127.6--129.5\left(\mathrm{~m}, 16 \mathrm{~F}, o-\mathrm{C}_{6} \mathrm{~F}_{5}\right),-142.7--144.0\left(\mathrm{~m}, 8 \mathrm{~F}, p-\mathrm{C}_{6} \mathrm{~F}_{5}\right),-158.2--159.6$ $\left(\mathrm{m}, 16 \mathrm{~F}, m-\mathrm{C}_{6} \mathrm{~F}_{5}\right) .{ }^{13} \mathrm{C}-\mathrm{NMR}:\left(150 \mathrm{MHz}, \mathrm{C}_{6} \mathrm{D}_{6}\right) \delta_{\mathrm{C}} 153.9\left(\mathrm{~d},{ }^{2} J_{\mathrm{P}-\mathrm{C}}=\mathrm{nr}, 1 \mathrm{C}, \mathrm{C}-\right.$ $\left.1^{\prime}\right), 153.8\left(\mathrm{~d},{ }^{2} J_{\mathrm{P}-\mathrm{C}}=13.8 \mathrm{~Hz}, 2 \mathrm{C}, \mathrm{C}-3,3^{\prime}\right), 153.1$ (d, $\left.{ }^{2} J_{\mathrm{P}-\mathrm{C}}=17.9 \mathrm{~Hz}, 1 \mathrm{C}, \mathrm{C}-1\right)$, 148.2-144.3 (br m, 24C, o,p- $\mathrm{C}_{6} \mathrm{~F}_{5}$ ), 138.4 (br d, ${ }^{1} J_{\mathrm{F}-\mathrm{C}}=255 \mathrm{~Hz}, 16 \mathrm{C}, m-\mathrm{C}_{6} \mathrm{~F}_{5}$ ), 130.4 (s, 1C, C-5'), 130.3 (s, 1C, C-5), 118.5 (s, 1C, C-4), 118.3 (s, 1C, C-4'), 116.5 (s, 1C, C-6'), 116.2 (s, 1C, C-6), 114.5 (s, 1C, C-2), 114.3 (s, 1C, C-2'), 108.2-105.8 (br m, 8C, $i-\mathrm{C}_{6} \mathrm{~F}_{5}$ ), 5.9 (br d, ${ }^{2} J_{\mathrm{P}-\mathrm{C}}=116 \mathrm{~Hz}, 1 \mathrm{C}, \mathrm{CH}_{3}$-trans-P), -18.3 (br s, $1 \mathrm{C}$, $\mathrm{CH}_{3}$-trans-Cl). HRMS calcd for $\left(\mathrm{C}_{64} \mathrm{H}_{17} \mathrm{NO}_{4} \mathrm{~F}_{40} \mathrm{P}_{4} \mathrm{ClPt}_{2}\right)[\mathrm{M}-\mathrm{Cl}+\mathrm{NCMe}]^{+}: \mathrm{m} / z=$ 2170.8430 , found $=2170.8496$. Accurate elemental analysis data was not able to be obtained for 21. 


\section{$[(\mathrm{POCOP}) \mathrm{PtCl}](25)$}

A solution of [PtClMe(hex)] (79 mg, $0.24 \mathrm{mmol})$ and 1 (203 mg, $0.24 \mathrm{mmol})$ in toluene $(6 \mathrm{~mL})$ was heated at reflux for 48 hours. All volatiles were then removed in vacuo and the oily yellow residue was triturated with hexane to give the crude material as a pale yellow solid. Precipitation of $[(\mathrm{POCOPH}) \mathrm{PtClMe}]_{\mathrm{x}}$ species from a solution of $2: 1$ hexane/toluene at $-15^{\circ} \mathrm{C}$, followed by removal of volatiles in vacuo and washing with pentane at $-78^{\circ} \mathrm{C}$ gave 25 as a pale yellow solid (121 $\mathrm{mg}$, 47\%). ${ }^{1} \mathrm{H}-\mathrm{NMR}:\left(300 \mathrm{MHz}, \mathrm{C}_{6} \mathrm{D}_{6}\right) \delta_{\mathrm{H}} 6.87\left(\mathrm{t},{ }^{3} J_{\mathrm{H}-\mathrm{H}}=8.0 \mathrm{~Hz}, 1 \mathrm{H}, \mathrm{H}-5\right), 6.79(\mathrm{~d}$, $\left.{ }^{3} J_{\mathrm{H}-\mathrm{H}}=7.8 \mathrm{~Hz}, 2 \mathrm{H}, \mathrm{H}-4,6\right) .{ }^{31} \mathrm{P}-\mathrm{NMR}:\left(121 \mathrm{MHz}, \mathrm{C}_{6} \mathrm{D}_{6}\right) \delta_{\mathrm{P}} 107.8\left(\mathrm{~s},{ }^{1} J_{\mathrm{Pt}-\mathrm{P}}=\right.$ $3663 \mathrm{~Hz}) .{ }^{19} \mathrm{~F}-\mathrm{NMR}:\left(282 \mathrm{MHz}, \mathrm{C}_{6} \mathrm{D}_{6}\right) \delta_{\mathrm{F}}-128.4\left(\mathrm{dm},{ }^{3} J_{\mathrm{P}-\mathrm{F}}=22.2 \mathrm{~Hz}, 8 \mathrm{~F}, o-\right.$ $\left.\mathrm{C}_{6} \mathrm{~F}_{5}\right),-141.8\left(\mathrm{tt},{ }^{3} J_{\mathrm{F}-\mathrm{F}}=20.0 \mathrm{~Hz},{ }^{5} J_{\mathrm{F}-\mathrm{F}}=6.8 \mathrm{~Hz}, 4 \mathrm{~F}, p-\mathrm{C}_{6} \mathrm{~F}_{5}\right),-157.6(\mathrm{~m}, 8 \mathrm{~F}$, $\left.m-\mathrm{C}_{6} \mathrm{~F}_{5}\right) \cdot{ }^{13} \mathrm{C}-\mathrm{NMR}:\left(125 \mathrm{MHz}, \mathrm{C}_{6} \mathrm{D}_{6}\right) \delta_{\mathrm{C}} 161.2(\mathrm{~s}, 2 \mathrm{C}, \mathrm{C}-1,3), 146.6\left(\mathrm{dm},{ }^{1} J_{\mathrm{F}-\mathrm{C}}=\right.$ $\left.256 \mathrm{~Hz}, 8 \mathrm{C}, o-\mathrm{C}_{6} \mathrm{~F}_{5}\right), 144.6\left(\mathrm{dm},{ }^{1} J_{\mathrm{F}-\mathrm{C}}=264 \mathrm{~Hz}, 4 \mathrm{C}, p-\mathrm{C}_{6} \mathrm{~F}_{5}\right), 137.7\left(\mathrm{dm},{ }^{1} J_{\mathrm{F}-\mathrm{C}}=\right.$ $257 \mathrm{~Hz}, 8 \mathrm{C}, m-\mathrm{C}_{6} \mathrm{~F}_{5}$ ), $129.5(\mathrm{~s}, 1 \mathrm{C}, \mathrm{C}-5), 123.7$ (s, 1C, C-2), $108.2\left(\mathrm{t},{ }^{3} J_{\mathrm{PC}}=7.5 \mathrm{~Hz}\right.$, $2 \mathrm{C}, \mathrm{C}-4,6), 102.5\left(\mathrm{~m}, 4 \mathrm{C}, i-\mathrm{C}_{6} \mathrm{~F}_{5}\right)$. Anal. Calcd for $\mathrm{C}_{30} \mathrm{H}_{3} \mathrm{O}_{2} \mathrm{~F}_{20} \mathrm{P}_{2} \mathrm{ClPt} \cdot \mathrm{CH}_{2} \mathrm{Cl}_{2}: \mathrm{C}$, 32.30; H, 0.44. Found C, 32.45; H, 0.55. HRMS calcd for $\left(\mathrm{C}_{30} \mathrm{H}_{3} \mathrm{O}_{2} \mathrm{~F}_{20} \mathrm{NaP}_{2} \mathrm{ClPt}\right)$ $[\mathrm{M}+\mathrm{Na}]^{+}: m / z=1088.8502$, found $=1088.8513$.

\section{$[(\mathrm{PCCCP}) \mathrm{PtCl}](26)$}

A solution of [PtClMe(hex)] (82 $\mathrm{mg}, 0.25 \mathrm{mmol})$ and $2(206 \mathrm{mg}, 0.25 \mathrm{mmol})$ in toluene $(5 \mathrm{~mL})$ was heated at $100{ }^{\circ} \mathrm{C}$ for 18 hours. All volatiles were then removed in vacuo and the oily yellow residue was triturated with hexane to give a creamcoloured solid, which was washed with pentane to afford $\mathbf{2 6}$ as an off-white solid (252 mg, 96\%). ${ }^{1} \mathrm{H}-\mathrm{NMR}:\left(500 \mathrm{MHz}, \mathrm{CDCl}_{3}\right) \delta_{\mathrm{H}} 7.11$ (m, 3H, H-4,5,6), 4.26 (vt, $\left.{ }^{3} J_{\mathrm{Pt}-\mathrm{H}}=29.8 \mathrm{~Hz},{ }^{2} J_{\mathrm{P}-\mathrm{H}}=4.5 \mathrm{~Hz}, 4 \mathrm{H}, \mathrm{CH}_{2}\right) .{ }^{31} \mathrm{P}-\mathrm{NMR}:\left(121 \mathrm{MHz}, \mathrm{CDCl}_{3}\right) \delta_{\mathrm{P}} 11.5$ $\left(\mathrm{s},{ }^{1} J_{\mathrm{Pt}-\mathrm{P}}=3379 \mathrm{~Hz}\right) .{ }^{19} \mathrm{~F}-\mathrm{NMR}:\left(282 \mathrm{MHz}, \mathrm{CDCl}_{3}\right) \delta_{\mathrm{F}}-126.7\left(\mathrm{~m}, 8 \mathrm{~F}, o-\mathrm{C}_{6} \mathrm{~F}_{5}\right)$, $-144.6\left(\mathrm{t},{ }^{3} J_{\mathrm{F}-\mathrm{F}}=20.7 \mathrm{~Hz}, 4 \mathrm{~F}, p-\mathrm{C}_{6} \mathrm{~F}_{5}\right),-157.9\left(\mathrm{~m}, 8 \mathrm{~F}, m-\mathrm{C}_{6} \mathrm{~F}_{5} .{ }^{13} \mathrm{C}-\mathrm{NMR}\right.$ : $\left(125 \mathrm{MHz}, \mathrm{CDCl}_{3}\right) \delta_{\mathrm{C}} 147.2\left(\mathrm{dm},{ }^{1} J_{\mathrm{F}-\mathrm{C}}=255 \mathrm{~Hz}, 8 \mathrm{C}, o-\mathrm{C}_{6} \mathrm{~F}_{5}\right), 144.1\left(\mathrm{dm},{ }^{1} J_{\mathrm{F}-\mathrm{C}}=\right.$ $\left.262 \mathrm{~Hz}, 4 \mathrm{C}, p-\mathrm{C}_{6} \mathrm{~F}_{5}\right), 144.0\left(\mathrm{~s},{ }^{1} J_{\mathrm{Pt}-\mathrm{C}}=875 \mathrm{~Hz}, 1 \mathrm{C}, \mathrm{C}-2\right), 143.6\left(\mathrm{t},{ }^{2} J_{\mathrm{P}-\mathrm{C}}=9.4 \mathrm{~Hz}\right.$, 2C, C-1,3), $138.0\left(\mathrm{dm},{ }^{1} J_{\mathrm{F}-\mathrm{C}}=258 \mathrm{~Hz}, 8 \mathrm{C}, m-\mathrm{C}_{6} \mathrm{~F}_{5}\right), 126.6(\mathrm{~s}, 1 \mathrm{C}, \mathrm{C}-5), 123.8(\mathrm{t}$, $\left.{ }^{3} J_{\mathrm{P}-\mathrm{C}}=11.0 \mathrm{~Hz}, 2 \mathrm{C}, \mathrm{C}-4,6\right), 103.0\left(\mathrm{~m}, 4 \mathrm{C}, i-\mathrm{C}_{6} \mathrm{~F}_{5}\right), 43.4\left(\mathrm{t},{ }^{1} J_{\mathrm{P}-\mathrm{C}}=17.8 \mathrm{~Hz}, 2 \mathrm{C}\right.$, $\mathrm{CH}_{2}$ ). Anal. Calcd for $\mathrm{C}_{32} \mathrm{H}_{7} \mathrm{~F}_{20} \mathrm{P}_{2}$ ClPt: C, 36.13; H, 0.66. Found C, 36.13; H, 0.88. HRMS calcd for $\left(\mathrm{C}_{34} \mathrm{H}_{10} \mathrm{NF}_{20} \mathrm{P}_{2} \mathrm{Pt}\right)\left[\mathrm{M}-\mathrm{Cl}+\mathrm{CH}_{3} \mathrm{CN}\right]^{+}: \mathrm{m} / z=1063.9568$, found $=$ 1063.9564. 


\section{$[(\mathrm{POCCP}) \mathrm{PtCl}](27)$}

A solution of $\left[\mathrm{PtCl}_{2}\left(\mathrm{SEt}_{2}\right)_{2}\right](191 \mathrm{mg}, 0.43 \mathrm{mmol})$ and $3(265 \mathrm{mg}, 0.24 \mathrm{mmol})$ in toluene $(15 \mathrm{~mL})$ was heated at reflux for 64 hours. Removal of volatiles in vacuo gave a oily yellow residue, which was purified by recrystallisation from 3:1 hexane/dichloromethane at $-15{ }^{\circ} \mathrm{C}$ followed by washing with hexane, yielding 27 as a yellow microcrystalline solid $(181 \mathrm{mg}, 50 \%) .{ }^{1} \mathrm{H}-\mathrm{NMR}$ : $\left(500 \mathrm{MHz}, \mathrm{CDCl}_{3}\right) \delta_{\mathrm{H}} 7.04$ $\left(\mathrm{vt},{ }^{3} J_{\mathrm{H}-\mathrm{H}}=7.6 \mathrm{~Hz}, 1 \mathrm{H}, \mathrm{H}-5\right), 6.95\left(\mathrm{~d},{ }^{3} J_{\mathrm{H}-\mathrm{H}}=7.3 \mathrm{~Hz}, 1 \mathrm{H}, \mathrm{H}-4\right), 6.76\left(\mathrm{~d},{ }^{4} J_{\mathrm{Pt}-\mathrm{H}}\right.$ $\left.=16.7 \mathrm{~Hz},{ }^{3} J_{\mathrm{H}-\mathrm{H}}=7.9 \mathrm{~Hz}, 1 \mathrm{H}, \mathrm{H}-6\right), 3.42\left(\mathrm{~d},{ }^{3} J_{\mathrm{Pt}-\mathrm{H}}=22.8 \mathrm{~Hz},{ }^{2} J_{\mathrm{P}-\mathrm{H}}=10.3 \mathrm{~Hz}\right.$, $\left.2 \mathrm{H}, \mathrm{CH}_{2}\right), 1.45\left(\mathrm{~d},{ }^{3} J_{\mathrm{P}-\mathrm{H}}=14.3 \mathrm{~Hz}, 18 \mathrm{H}, \mathrm{C}\left(\mathrm{CH}_{3}\right)_{3}\right) .{ }^{31} \mathrm{P}-\mathrm{NMR}:\left(121 \mathrm{MHz}, \mathrm{CDCl}_{3}\right)$ $\delta_{\mathrm{P}} 107.8\left(\mathrm{~d},{ }^{1} J_{\mathrm{Pt}-\mathrm{P}}=3294 \mathrm{~Hz},{ }^{2} J_{\mathrm{P}-\mathrm{P}}=469 \mathrm{~Hz}, 1 \mathrm{P}, \mathrm{P}\left(\mathrm{C}_{6} \mathrm{~F}_{5}\right)_{2}\right), 72.1\left(\mathrm{~d},{ }^{1} J_{\mathrm{Pt}-\mathrm{P}}=\right.$ $\left.3088 \mathrm{~Hz},{ }^{2} J_{\mathrm{P}-\mathrm{P}}=468 \mathrm{~Hz}, 1 \mathrm{P}, \mathrm{P}^{t} \mathrm{Bu}_{2}\right) .{ }^{19} \mathrm{~F}-\mathrm{NMR}:\left(282 \mathrm{MHz}, \mathrm{CDCl}_{3}\right) \delta_{\mathrm{F}}-128.1(\mathrm{~m}$, $\left.4 \mathrm{~F}, o-\mathrm{C}_{6} \mathrm{~F}_{5}\right),-144.6\left(\mathrm{tm},{ }^{3} J_{\mathrm{F}-\mathrm{F}}=20.7 \mathrm{~Hz}, 2 \mathrm{~F}, p-\mathrm{C}_{6} \mathrm{~F}_{5}\right),-158.4\left(\mathrm{~m}, 4 \mathrm{~F}, m-\mathrm{C}_{6} \mathrm{~F}_{5}\right)$. ${ }^{13} \mathrm{C}-\mathrm{NMR}:\left(125 \mathrm{MHz}, \mathrm{CDCl}_{3}\right) \delta_{\mathrm{C}} 161.5\left(\mathrm{dd},{ }^{2} J_{\mathrm{P}-\mathrm{C}}=15.4 \mathrm{~Hz},{ }^{3} J_{\mathrm{P}-\mathrm{C}}=3.9 \mathrm{~Hz}, 1 \mathrm{C}\right.$, $\mathrm{C}-1), 149.1\left(\mathrm{dd},{ }^{2} J_{\mathrm{Pt}-\mathrm{C}}=77.8 \mathrm{~Hz},{ }^{2} J_{\mathrm{P}-\mathrm{C}}=10.4 \mathrm{~Hz},{ }^{3} J_{\mathrm{P}-\mathrm{C}}=5.1 \mathrm{~Hz}, 1 \mathrm{C}, \mathrm{C}-3\right), 146.7$ $\left(\mathrm{dm},{ }^{1} J_{\mathrm{F}-\mathrm{C}}=257 \mathrm{~Hz}, 4 \mathrm{C}, o-\mathrm{C}_{6} \mathrm{~F}_{5}\right), 144.4\left(\mathrm{dm},{ }^{1} J_{\mathrm{F}-\mathrm{C}}=262 \mathrm{~Hz}, 2 \mathrm{C}, p-\mathrm{C}_{6} \mathrm{~F}_{5}\right), 137.9$ $\left(\mathrm{dm},{ }^{1} J_{\mathrm{F}-\mathrm{C}}=252 \mathrm{~Hz}, 4 \mathrm{C}, m-\mathrm{C}_{6} \mathrm{~F}_{5}\right), 133.8\left(\mathrm{~s},{ }^{1} J_{\mathrm{Pt}-\mathrm{C}}=453 \mathrm{~Hz}, 1 \mathrm{C}, \mathrm{C}-2\right), 126.6(\mathrm{~s}$, $1 \mathrm{C}, \mathrm{C}-5), 119.6\left(\mathrm{~d},{ }^{3} J_{\mathrm{Pt}-\mathrm{C}}=39.4 \mathrm{~Hz},{ }^{3} J_{\mathrm{P}-\mathrm{C}}=16.3 \mathrm{~Hz}, 1 \mathrm{C}, \mathrm{C}-4\right), 110.4\left(\mathrm{~d},{ }^{3} J_{\mathrm{P}-\mathrm{C}}\right.$ $\left.=16.8 \mathrm{~Hz},{ }^{3} J_{\mathrm{Pt}-\mathrm{C}}=15.4 \mathrm{~Hz}, 1 \mathrm{C}, \mathrm{C}-6\right), 107.8\left(\mathrm{~m}, 2 \mathrm{C}, i-\mathrm{C}_{6} \mathrm{~F}_{5}\right), 36.3\left(\mathrm{dd},{ }^{2} J_{\mathrm{Pt}-\mathrm{C}}=\right.$ $\left.24.0 \mathrm{~Hz},{ }^{1} J_{\mathrm{P}-\mathrm{C}}=20.0 \mathrm{~Hz},{ }^{3} J_{\mathrm{P}-\mathrm{C}}=5.1 \mathrm{~Hz}, 2 \mathrm{C}, C\left(\mathrm{CH}_{3}\right)_{3}\right), 34.8\left(\mathrm{dd},{ }^{2} J_{\mathrm{Pt}-\mathrm{C}}=102 \mathrm{~Hz}\right.$, $\left.{ }^{1} J_{\mathrm{P}-\mathrm{C}}=29.8 \mathrm{~Hz},{ }^{3} J_{\mathrm{P}-\mathrm{C}}=2.4 \mathrm{~Hz}, 1 \mathrm{C}, \mathrm{CH}_{2}\right), 29.1\left(\mathrm{~s}, 6 \mathrm{C}, \mathrm{C}\left(\mathrm{CH}_{3}\right)_{3}\right)$. HRMS calcd for $\left(\mathrm{C}_{27} \mathrm{H}_{27} \mathrm{NOF}_{10} \mathrm{P}_{2} \mathrm{ClPt}\right)\left[\mathrm{M}+\mathrm{NH}_{4}\right]^{+}: \mathrm{m} / z=864.0737$, found $=864.0720$. HRMS calcd for $\left(\mathrm{C}_{23} \mathrm{H}_{27} \mathrm{NO}_{2} \mathrm{~F}_{5} \mathrm{P}_{2} \mathrm{Pt}\right)\left[\mathrm{M}-\mathrm{Cl}-\mathrm{C}_{6} \mathrm{~F}_{5}+\mathrm{OH}+\mathrm{NCMe}\right]^{+}: m / z=701.1036$, found $=701.1031$.

\section{$[(\mathrm{POCOP}) \mathrm{PtMe}](43)$}

A solution of $25(79 \mathrm{mg}, 74 \mu \mathrm{mol})$ in toluene $(5 \mathrm{~mL})$ was cooled to $-78{ }^{\circ} \mathrm{C}$ and treated with a $1.5 \mathrm{~mol} \mathrm{dm}{ }^{-3}$ dimethylzinc solution in toluene $(60 \mu \mathrm{L}, 90 \mu \mathrm{mol})$. The reaction mixture was then stirred at room temperature for five hours, at which point all volatiles were removed in vacuo. The resultant yellow oil was repeatedly extracted with a 1:1 mixture of hexane and toluene, with the extracts filtered through celite and collected. Removal of all volatiles in vacuo and washing with hexane at $-15{ }^{\circ} \mathrm{C}$ afforded compound 43 as a white solid (58.6 mg, 76\%). ${ }^{1} \mathrm{H}-\mathrm{NMR}$ : (600 MHz, $\left.\mathrm{C}_{6} \mathrm{D}_{6}\right) \delta_{\mathrm{H}} 7.05(\mathrm{~m}, 3 \mathrm{H}, \mathrm{H}-4,5,6) .1 .34$ (br vt, $\left.{ }^{2} J_{\mathrm{Pt}-\mathrm{H}}=57.4 \mathrm{~Hz},{ }^{2} J_{\mathrm{P}-\mathrm{H}}=\mathrm{nr}, 3 \mathrm{H}, \mathrm{CH}_{3}\right)$. ${ }^{31}$ P-NMR: $\left(121 \mathrm{MHz}, \mathrm{C}_{6} \mathrm{D}_{6}\right) \delta_{\mathrm{P}} 108.5\left(\mathrm{~s},{ }^{1} J_{\mathrm{Pt}-\mathrm{P}}=3752 \mathrm{~Hz}\right) \cdot{ }^{19} \mathrm{~F}-\mathrm{NMR}:(282 \mathrm{MHz}$, $\left.\mathrm{C}_{6} \mathrm{D}_{6}\right) \delta_{\mathrm{F}}-130.3\left(\mathrm{~m}, 8 \mathrm{~F}, o-\mathrm{C}_{6} \mathrm{~F}_{5}\right),-144.0\left(\mathrm{tt},{ }^{3} J_{\mathrm{P}-\mathrm{F}}=21.6 \mathrm{~Hz},{ }^{5} J_{\mathrm{P}-\mathrm{F}}=\mathrm{nr}, 4 \mathrm{~F}\right.$, $\left.p-\mathrm{C}_{6} \mathrm{~F}_{5}\right),-158.3\left(\mathrm{~m}, 8 \mathrm{~F}, m-\mathrm{C}_{6} \mathrm{~F}_{5}\right) .{ }^{13} \mathrm{C}-\mathrm{NMR}:\left(125 \mathrm{MHz}, \mathrm{C}_{6} \mathrm{D}_{6}\right) \delta_{\mathrm{C}} 160.4\left(\mathrm{vt},{ }^{2} J_{\mathrm{P}-\mathrm{C}}\right.$ $=8.2 \mathrm{~Hz}, 2 \mathrm{C}, \mathrm{C}-1,3), 146.6\left(\mathrm{~d},{ }^{1} J_{\mathrm{F}-\mathrm{C}}=253 \mathrm{~Hz}, 8 \mathrm{C}, o-\mathrm{C}_{6} \mathrm{~F}_{5}\right), 144.2\left(\mathrm{~d},{ }^{1} J_{\mathrm{F}-\mathrm{C}}=\right.$ 
$\left.262 \mathrm{~Hz}, 4 \mathrm{C}, p-\mathrm{C}_{6} \mathrm{~F}_{5}\right), 138.1\left(\mathrm{~d},{ }^{1} J_{\mathrm{F}-\mathrm{C}}=256 \mathrm{~Hz}, 8 \mathrm{C}, m-\mathrm{C}_{6} \mathrm{~F}_{5}\right), 129.5(\mathrm{~s}, 1 \mathrm{C}, \mathrm{C}-5)$, $107.7\left(\mathrm{vt},{ }^{3} J_{\mathrm{P}-\mathrm{C}}=7.5 \mathrm{~Hz}, 2 \mathrm{C}, \mathrm{C}-4,6\right), 107.4\left(\mathrm{~m}, 4 \mathrm{C}, i-\mathrm{C}_{6} \mathrm{~F}_{5}\right),-15.1\left(\mathrm{~s},{ }^{1} J_{\mathrm{Pt}-\mathrm{C}}=\right.$ $\left.522 \mathrm{~Hz}, 1 \mathrm{C}, \mathrm{CH}_{3}\right)$. The signal for $\mathrm{C}-2$ could not be conclusively assigned due to overlap with other resonances, but appears to be at $141.9 \mathrm{ppm}$. HRMS calcd for $\left(\mathrm{C}_{34} \mathrm{H}_{10} \mathrm{NF}_{20} \mathrm{P}_{2} \mathrm{Pt}\right)\left[\mathrm{M}-\mathrm{CH}_{3}+\mathrm{CH}_{3} \mathrm{CN}\right]^{+}: \mathrm{m} / z=1072.9154$, found $=1072.9200$.

\section{[(PCCCP)PtMe] (44)}

A solution of $26(102 \mathrm{mg}, 96 \mu \mathrm{mol})$ in toluene $(5 \mathrm{~mL})$ was cooled to $-78{ }^{\circ} \mathrm{C}$ and treated with a $1.5 \mathrm{~mol} \mathrm{dm}^{-3}$ dimethylzinc solution in toluene $(80 \mu \mathrm{L}, 120 \mu \mathrm{mol})$. The reaction mixture was then stirred at room temperature for two hours, at which point all volatiles were removed in vacuo. The resultant pale yellow residue was repeatedly extracted with a 1:1 mixture of hexane and toluene, with the extracts filtered through celite and collected. Removal of all volatiles in vacuo gave a pale yellow oil that was triturated with hexane to give compound 44 as a white solid, which was subsequently washed with hexane $(87.3 \mathrm{mg}, 87 \%) .{ }^{1} \mathrm{H}-\mathrm{NMR}$ : $(500 \mathrm{MHz}$, $\left.\mathrm{C}_{6} \mathrm{D}_{6}\right) \delta_{\mathrm{H}} 7.26(\mathrm{br} \mathrm{s}, 3 \mathrm{H}, \mathrm{H}-4,5,6), 4.20\left(\mathrm{vt},{ }^{3} J_{\mathrm{Pt}-\mathrm{H}}=28.5 \mathrm{~Hz},{ }^{2} J_{\mathrm{P}-\mathrm{H}}=4.5 \mathrm{~Hz}, 4 \mathrm{H}\right.$, $\mathrm{CH}_{2}$ ), 1.02 (br vt, $\left.{ }^{2} J_{\mathrm{Pt}-\mathrm{H}}=55.8 \mathrm{~Hz},{ }^{2} J_{\mathrm{P}-\mathrm{H}}=\mathrm{nr}, 3 \mathrm{H}, \mathrm{CH}_{3}\right) .{ }^{31} \mathrm{P}-\mathrm{NMR}$ : $(121 \mathrm{MHz}$, $\left.\mathrm{C}_{6} \mathrm{D}_{6}\right) \delta_{\mathrm{P}} 9.6\left(\mathrm{~s},{ }^{1} J_{\mathrm{Pt}-\mathrm{P}}=3496 \mathrm{~Hz}\right) .{ }^{19} \mathrm{~F}-\mathrm{NMR}:\left(282 \mathrm{MHz}, \mathrm{C}_{6} \mathrm{D}_{6}\right) \delta_{\mathrm{F}}-129.2(\mathrm{~m}$, $\left.8 \mathrm{~F}, o-\mathrm{C}_{6} \mathrm{~F}_{5}\right),-146.2\left(\mathrm{t},{ }^{3} J_{\mathrm{F}-\mathrm{F}}=21.8 \mathrm{~Hz}, 4 \mathrm{~F}, p-\mathrm{C}_{6} \mathrm{~F}_{5}\right),-158.9\left(\mathrm{~m}, 8 \mathrm{~F}, m-\mathrm{C}_{6} \mathrm{~F}_{5}\right)$. ${ }^{13} \mathrm{C}-\mathrm{NMR}:\left(150 \mathrm{MHz}, \mathrm{C}_{6} \mathrm{D}_{6}\right) \delta_{\mathrm{C}} 172.0\left(\mathrm{~s},{ }^{1} J_{\mathrm{Pt}-\mathrm{C}}=510 \mathrm{~Hz}, 1 \mathrm{C}, \mathrm{C}-2\right), 146.6(\mathrm{dm}$, $\left.{ }^{1} J_{\mathrm{F}-\mathrm{C}}=252 \mathrm{~Hz}, 8 \mathrm{C}, o-\mathrm{C}_{6} \mathrm{~F}_{5}\right), 144.8\left(\mathrm{t},{ }^{2} J_{\mathrm{Pt}-\mathrm{C}}=64 \mathrm{~Hz},{ }^{2} J_{\mathrm{P}-\mathrm{C}}=9.5 \mathrm{~Hz}, 2 \mathrm{C}, \mathrm{C}-1,3\right)$, $142.9\left(\mathrm{dm},{ }^{1} J_{\mathrm{F}-\mathrm{C}}=260 \mathrm{~Hz}, 4 \mathrm{C}, p-\mathrm{C}_{6} \mathrm{~F}_{5}\right), 137.6\left(\mathrm{dm},{ }^{1} J_{\mathrm{F}-\mathrm{C}}=255 \mathrm{~Hz}, 8 \mathrm{C}, m-\mathrm{C}_{6} \mathrm{~F}_{5}\right)$, $125.8(\mathrm{~s}, 1 \mathrm{C}, \mathrm{C}-5), 122.1$ (t, $\left.{ }^{3} J_{\mathrm{P}-\mathrm{C}}=10.7 \mathrm{~Hz}, 2 \mathrm{C}, \mathrm{C}-4,6\right), 104.5\left(\mathrm{~m}, 4 \mathrm{C}, i-\mathrm{C}_{6} \mathrm{~F}_{5}\right), 48.8$ $\left(\mathrm{t},{ }^{1} J_{\mathrm{P}-\mathrm{C}}=20.0 \mathrm{~Hz}, 2 \mathrm{C}, \mathrm{CH}_{2}\right),-12.3\left(\mathrm{~s},{ }^{1} J_{\mathrm{Pt}-\mathrm{C}}=470 \mathrm{~Hz}, 1 \mathrm{C}, \mathrm{CH}_{3}\right)$. Anal. Calcd for $\mathrm{C}_{33} \mathrm{H}_{10} \mathrm{~F}_{20} \mathrm{P}_{2} \mathrm{Pt}$ : C, 37.99; H, 0.97. Found $\mathrm{C}$, 38.06; H, 1.02. HRMS calcd for $\left(\mathrm{C}_{34} \mathrm{H}_{10} \mathrm{NF}_{20} \mathrm{P}{ }_{2} \mathrm{Pt}\right)\left[\mathrm{M}-\mathrm{CH}_{3}+\mathrm{CH}_{3} \mathrm{CN}\right]^{+}: \mathrm{m} / z=1068.9615$, found $=1068.9629$.

\section{[(POCCP)PtMe $](45)$}

A solution of complex $45(82.0 \mathrm{mg}, 97 \mu \mathrm{mol})$ in toluene $(4 \mathrm{~mL})$ was cooled to $-78{ }^{\circ} \mathrm{C}$, and $1.6 \mathrm{~mol} \mathrm{dm}{ }^{-3}$ dimethylzinc solution in toluene $(440 \mu \mathrm{L}, 0.71 \mathrm{mmol})$ was added, with the reaction mixture left stirring in the cold bath to gradually warm to room temperature over the course of 10 hours. The reaction was stirred at room temperature for a further 44 hours, at which point all volatiles were removed in vacuo. The residue was extracted with 1:1 hexane/toluene $(4 \times 2 \mathrm{~mL})$, with the extracts combined, filtered through celite and taken to dryness under vacuum. The yellow oil was triturated then washed with pentane at $-20{ }^{\circ} \mathrm{C}$, giving compound 45 
as a white solid (50.4 mg, 63\%). ${ }^{1} \mathrm{H}-\mathrm{NMR}$ : $\left(600 \mathrm{MHz}, \mathrm{C}_{6} \mathrm{D}_{6}\right) \delta_{\mathrm{H}} 7.04\left(\mathrm{vt},{ }^{3} J_{\mathrm{H}-\mathrm{H}}=\right.$ $7.5 \mathrm{~Hz}, 1 \mathrm{H}, \mathrm{H}-5), 7.01\left(\mathrm{~d},{ }^{3} J_{\mathrm{H}-\mathrm{H}}=7.7 \mathrm{~Hz}, 1 \mathrm{H}, \mathrm{H}-4\right), 6.81\left(\mathrm{~d},{ }^{3} J_{\mathrm{H}-\mathrm{H}}=7.6 \mathrm{~Hz}, 1 \mathrm{H}\right.$, $\mathrm{H}-6), 3.65\left(\mathrm{~d},{ }^{3} J_{\mathrm{Pt}-\mathrm{H}}=20.0 \mathrm{~Hz},{ }^{2} J_{\mathrm{P}-\mathrm{H}}=9.7 \mathrm{~Hz}, 2 \mathrm{H}, \mathrm{CH}_{2}\right), 1.33\left(\mathrm{~d},{ }^{3} J_{\mathrm{P}-\mathrm{H}}=13.8 \mathrm{~Hz}\right.$, $\left.18 \mathrm{H}, \mathrm{C}\left(\mathrm{CH}_{3}\right)_{3}\right), 0.53\left(\mathrm{~d},{ }^{2} J_{\mathrm{Pt}-\mathrm{H}}=57.1 \mathrm{~Hz},{ }^{3} J_{\mathrm{P}-\mathrm{H}}=7.6 \mathrm{~Hz}, 3 \mathrm{H}, \mathrm{Pt}-\mathrm{CH}_{3}{ }^{31} \mathrm{P}-\mathrm{NMR}\right.$ : $\left(121 \mathrm{MHz}, \mathrm{C}_{6} \mathrm{D}_{6}\right) \delta_{\mathrm{P}} 108.7\left(\mathrm{~d},{ }^{1} J_{\mathrm{Pt}-\mathrm{P}}=3464 \mathrm{~Hz},{ }^{2} J_{\mathrm{P}-\mathrm{P}}=489 \mathrm{~Hz}, 1 \mathrm{P}, \mathrm{P}\left(\mathrm{C}_{6} \mathrm{~F}_{5}\right)_{2}\right)$, $70.2\left(\mathrm{~d},{ }^{1} J_{\mathrm{Pt}-\mathrm{P}}=3102 \mathrm{~Hz},{ }^{2} J_{\mathrm{P}-\mathrm{P}}=489 \mathrm{~Hz}, 1 \mathrm{P}, \mathrm{P}^{t} \mathrm{Bu}_{2}\right) .{ }^{19} \mathrm{~F}-\mathrm{NMR}:\left(282 \mathrm{MHz}, \mathrm{C}_{6} \mathrm{D}_{6}\right)$ $\delta_{\mathrm{F}}-130.9\left(\mathrm{~m}, 4 \mathrm{~F}, o-\mathrm{C}_{6} \mathrm{~F}_{5}\right),-146.7\left(\mathrm{tm},{ }^{3} J_{\mathrm{F}-\mathrm{F}}=20.7 \mathrm{~Hz}, 2 \mathrm{~F}, p-\mathrm{C}_{6} \mathrm{~F}_{5}\right),-159.6(\mathrm{~m}$, $\left.4 \mathrm{~F}, m-\mathrm{C}_{6} \mathrm{~F}_{5}\right) .{ }^{13} \mathrm{C}-\mathrm{NMR}:\left(150 \mathrm{MHz}, \mathrm{C}_{6} \mathrm{D}_{6}\right) \delta_{\mathrm{C}} 160.6\left(\mathrm{dd},{ }^{2} J_{\mathrm{P}-\mathrm{C}}=14.3 \mathrm{~Hz},{ }^{3} J_{\mathrm{P}-\mathrm{C}}=\right.$ $4.8 \mathrm{~Hz}, 1 \mathrm{C}, \mathrm{C}-1), 158.3\left(\mathrm{~s},{ }^{1} J_{\mathrm{Pt}-\mathrm{C}}=509 \mathrm{~Hz}, 1 \mathrm{C}, \mathrm{C}-2\right), 149.8\left(\mathrm{dd},{ }^{2} J_{\mathrm{Pt}-\mathrm{C}}=40.3 \mathrm{~Hz}\right.$, $\left.{ }^{2} J_{\mathrm{P}-\mathrm{C}}=9.5 \mathrm{~Hz},{ }^{3} J_{\mathrm{P}-\mathrm{C}}=5.1 \mathrm{~Hz}, 1 \mathrm{C}, \mathrm{C}-3\right), 146.6\left(\mathrm{dm},{ }^{1} J_{\mathrm{F}-\mathrm{C}}=253 \mathrm{~Hz}, 4 \mathrm{C}, o-\mathrm{C}_{6} \mathrm{~F}_{5}\right)$, $143.9\left(\mathrm{dm},{ }^{1} J_{\mathrm{F}-\mathrm{C}}=259 \mathrm{~Hz}, 2 \mathrm{C}, p-\mathrm{C}_{6} \mathrm{~F}_{5}\right), 138.2\left(\mathrm{dm},{ }^{1} J_{\mathrm{F}-\mathrm{C}}=248 \mathrm{~Hz}, 4 \mathrm{C}, m-\mathrm{C}_{6} \mathrm{~F}_{5}\right)$, $125.9(\mathrm{~s}, 1 \mathrm{C}, \mathrm{C}-5), 120.0\left(\mathrm{~d},{ }^{3} J_{\mathrm{Pt}-\mathrm{C}}=17.6 \mathrm{~Hz},{ }^{3} J_{\mathrm{P}-\mathrm{C}}=16.1 \mathrm{~Hz}, 1 \mathrm{C}, \mathrm{C}-4\right), 109.8$ $\left(\mathrm{m}, 2 \mathrm{C}, i-\mathrm{C}_{6} \mathrm{~F}_{5}\right), 108.9\left(\mathrm{~d},{ }^{3} J_{\mathrm{P}-\mathrm{C}}=16.9 \mathrm{~Hz}, 1 \mathrm{C}, \mathrm{C}-6\right), 40.5\left(\mathrm{dd},{ }^{2} J_{\mathrm{Pt}-\mathrm{C}}=41.0 \mathrm{~Hz}\right.$, $\left.{ }^{1} J_{\mathrm{P}-\mathrm{C}}=19.9 \mathrm{~Hz},{ }^{3} J_{\mathrm{P}-\mathrm{C}}=5.2 \mathrm{~Hz}, 1 \mathrm{C}, \mathrm{CH}_{2}\right), 36.2\left(\mathrm{~d},{ }^{2} J_{\mathrm{Pt}-\mathrm{C}}=47.6 \mathrm{~Hz},{ }^{1} J_{\mathrm{P}-\mathrm{C}}\right.$ $\left.=33.1 \mathrm{~Hz}, 2 \mathrm{C}, \mathrm{C}\left(\mathrm{CH}_{3}\right)_{3}\right), 29.3\left(\mathrm{~s}, 6 \mathrm{C}, \mathrm{C}\left(\mathrm{CH}_{3}\right)_{3}\right)-16.5\left(\mathrm{~s},{ }^{1} J_{\mathrm{Pt}-\mathrm{C}}=492 \mathrm{~Hz}, 1 \mathrm{C}\right.$, $\mathrm{Pt}-\mathrm{CH}_{3}$. HRMS calcd for $\left(\mathrm{C}_{29} \mathrm{H}_{26} \mathrm{NOF}_{10} \mathrm{P}_{2} \mathrm{Pt}\right)[\mathrm{M}-\mathrm{Me}+\mathrm{MeCN}]^{+}: \mathrm{m} / z=850.0957$, found $=850.0993$. HRMS calcd for $\left(\mathrm{C}_{24} \mathrm{H}_{29} \mathrm{NOF}_{5} \mathrm{P}_{2} \mathrm{Pt}\right)\left[\mathrm{M}-\mathrm{C}_{6} \mathrm{~F}_{5}+\mathrm{MeCN}\right]^{+}: \mathrm{m} / z$ $=698.1271$, found $=698.1274$.

\section{Reaction of 25 with MeMgI: Formation of [(POCOP)PtI] (41)}

To a solution of $\mathbf{2 5}(95.0 \mathrm{mg}, 94 \mu \mathrm{mol})$ in diethyl ether $(5 \mathrm{~mL})$ was added a $0.5 \mathrm{~mol} \mathrm{dm}{ }^{-3}$ solution of MeMgI in diethyl ether $(0.2 \mathrm{~mL}, 100 \mu \mathrm{mol})$. The solution was stirred at room temperature for 4 hours, at which point all volatiles were removed in vacuo. The solid was extracted with 1:1 toluene/hexane, with the extracts filtered through celite under ambient conditions. Removal of the solvent under reduced pressure gave a yellow oil, which was dissolved in dichloromethane and purified by passage through a short alumina column. The dichloromethane was subsequently removed in vacuo, with recrystallisation of the residue from an acetone/hexane mixture yielded $\mathbf{4 1}$ as a yellow solid (43.2 $\mathrm{mg}, 42 \%)$. ${ }^{1} \mathrm{H}-\mathrm{NMR}$ : $\left(500 \mathrm{MHz}, \mathrm{C}_{6} \mathrm{D}_{6}\right) \delta_{\mathrm{H}} 6.96\left(\mathrm{t},{ }^{3} J_{\mathrm{H}-\mathrm{H}}=8.0 \mathrm{~Hz}, 1 \mathrm{H}, \mathrm{H}-5\right), 6.85\left(\mathrm{~d},{ }^{3} J_{\mathrm{H}-\mathrm{H}}=8.1 \mathrm{~Hz}\right.$, 2H, H-4,6). ${ }^{31}$ P-NMR: (121 MHz, $\left.\mathrm{C}_{6} \mathrm{D}_{6}\right) \delta_{\mathrm{P}} 109.2\left(\mathrm{~s},{ }^{1} J_{\mathrm{Pt}-\mathrm{P}}=3541 \mathrm{~Hz}\right) .{ }^{19} \mathrm{~F}-\mathrm{NMR}$ : $\left(282 \mathrm{MHz}, \mathrm{C}_{6} \mathrm{D}_{6}\right) \delta_{\mathrm{F}}-125.9\left(\mathrm{~m}, 8 \mathrm{~F}, o-\mathrm{C}_{6} \mathrm{~F}_{5}\right),-142.1\left(\mathrm{tm},{ }^{3} J_{\mathrm{F}-\mathrm{F}}=20.9 \mathrm{~Hz}, 4 \mathrm{~F}\right.$, $\left.p-\mathrm{C}_{6} \mathrm{~F}_{5}\right),-157.1\left(\mathrm{~m}, 4 \mathrm{~F}, m-\mathrm{C}_{6} \mathrm{~F}_{5}\right) .{ }^{13} \mathrm{C}-\mathrm{NMR}:\left(125 \mathrm{MHz}, \mathrm{C}_{6} \mathrm{D}_{6}\right) \delta_{\mathrm{C}} 160.6\left(\mathrm{vt},{ }^{2} J_{\mathrm{P}-\mathrm{C}}\right.$ $=\mathrm{Hz}, 2 \mathrm{C}, \mathrm{C}-1,3), 146.4\left(\mathrm{dm},{ }^{1} J_{\mathrm{F}-\mathrm{C}}=259 \mathrm{~Hz}, 8 \mathrm{C}, o_{-} \mathrm{C}_{6} \mathrm{~F}_{5}\right), \sim 144\left(\mathrm{~d},{ }^{3} J_{\mathrm{F}-\mathrm{C}} \approx\right.$ $\left.260 \mathrm{~Hz}, 4 \mathrm{C}, p-\mathrm{C}_{6} \mathrm{~F}_{5}\right), 137.7\left(\mathrm{dm},{ }^{1} J_{\mathrm{F}-\mathrm{C}}=257 \mathrm{~Hz}, 8 \mathrm{C}, m-\mathrm{C}_{6} \mathrm{~F}_{5}\right), 130.4(\mathrm{~s}, 1 \mathrm{C}, \mathrm{C}-2)$, $129.5(\mathrm{~s}, 1 \mathrm{C}, \mathrm{C}-5), 108.0\left(\mathrm{t},{ }^{3} J_{\mathrm{PC}}=7.6 \mathrm{~Hz}, 2 \mathrm{C}, \mathrm{C}-4,6\right), 105.1$ (m, 4C, $\left.i-\mathrm{C}_{6} \mathrm{~F}_{5}\right)$. Exact chemical shift and coupling constants for the $p-\mathrm{C}_{6} \mathrm{~F}_{5}$ environment could not be determined due to peak overlap with the adjacent $o-\mathrm{C}_{6} \mathrm{~F}_{5}$ environment. Analysis 
of $\mathbf{4 1}$ by HRMS was not able to confirm the presence of a Pt-I moiety.

\section{Reaction of 27 with MeMgI: Formation of [(POCCP)PtI] (42)}

To a solution of $27(15.6 \mathrm{mg}, 18 \mu \mathrm{mol})$ in diethyl ether $(0.5 \mathrm{~mL})$ was added a $0.5 \mathrm{~mol} \mathrm{dm}{ }^{-3}$ solution of MeMgI in diethyl ether $(50 \mu \mathrm{L}, 25 \mu \mathrm{mol})$. After 14 hours at room temperature all volatiles were removed in vacuo, and the product was repeatedly extracted into toluene. Removal of the toluene under vacuum and followed by washing of the residue with hexane at $-15{ }^{\circ} \mathrm{C}$ gave 42 as a red-brown solid (9.2 mg, 53\%). ${ }^{1} \mathrm{H}-\mathrm{NMR}:\left(500 \mathrm{MHz}, \mathrm{C}_{6} \mathrm{D}_{6}\right) \delta_{\mathrm{H}} 7.04(\mathrm{~m}, 2 \mathrm{H}, \mathrm{H}-4,5), 6.85\left(\mathrm{~d},{ }^{3} J_{\mathrm{H}-\mathrm{H}}\right.$ $=6.8 \mathrm{~Hz}, 1 \mathrm{H}, \mathrm{H}-6), 3.07\left(\mathrm{~d},{ }^{3} J_{\mathrm{Pt}-\mathrm{H}}=23.7 \mathrm{~Hz},{ }^{2} J_{\mathrm{P}-\mathrm{H}}=9.7 \mathrm{~Hz}, 2 \mathrm{H}, \mathrm{CH}_{2}\right), 1.22$ $\left(\mathrm{d},{ }^{3} J_{\mathrm{P}-\mathrm{H}}=14.1 \mathrm{~Hz}, 18 \mathrm{H}, \mathrm{C}\left(\mathrm{CH}_{3}\right)_{3}\right) .{ }^{31} \mathrm{P}-\mathrm{NMR}:\left(121 \mathrm{MHz}, \mathrm{C}_{6} \mathrm{D}_{6}\right) \delta_{\mathrm{P}} 108.6(\mathrm{~d}$, $\left.{ }^{1} J_{\mathrm{Pt}-\mathrm{P}}=3184 \mathrm{~Hz},{ }^{2} J_{\mathrm{P}-\mathrm{P}}=468 \mathrm{~Hz}, 1 \mathrm{P}, \mathrm{P}\left(\mathrm{C}_{6} \mathrm{~F}_{5}\right)_{2}\right), 72.9\left(\mathrm{~d},{ }^{1} J_{\mathrm{Pt}-\mathrm{P}}=3023 \mathrm{~Hz},{ }^{2} J_{\mathrm{P}-\mathrm{P}}\right.$ $\left.=468 \mathrm{~Hz}, 1 \mathrm{P}, \mathrm{P}^{t} \mathrm{Bu}_{2}\right) \cdot{ }^{19} \mathrm{~F}-\mathrm{NMR}:\left(282 \mathrm{MHz}, \mathrm{C}_{6} \mathrm{D}_{6}\right) \delta_{\mathrm{F}}-127.5\left(\mathrm{~m}, 4 \mathrm{~F}, o-\mathrm{C}_{6} \mathrm{~F}_{5}\right)$, $-144.4\left(\mathrm{tm},{ }^{3} J_{\mathrm{F}-\mathrm{F}}=20.7 \mathrm{~Hz}, 2 \mathrm{~F}, p-\mathrm{C}_{6} \mathrm{~F}_{5}\right),-159.0\left(\mathrm{~m}, 4 \mathrm{~F}, m-\mathrm{C}_{6} \mathrm{~F}_{5}\right)$. HRMS calcd for $\left(\mathrm{C}_{27} \mathrm{H}_{23} \mathrm{OF}_{10} \mathrm{NaP}_{2} \mathrm{IPt}\right)[\mathrm{M}+\mathrm{Na}]^{+}: \mathrm{m} / z=958.9647$, found $=958.9634$.

\section{Reaction of 27 with MeLi: Observation of $\left[\left(\mathrm{P}^{\mathrm{Me}} \mathrm{OCCP}\right) \operatorname{Pt}\left(\mathrm{C}_{6} \mathrm{~F}_{5}\right)\right]$ (46)}

A solution of complex $45(79.9 \mathrm{mg}, 94 \mu \mathrm{mol})$ in toluene $(4 \mathrm{~mL})$ was cooled to $-78^{\circ} \mathrm{C}$, and $1.3 \mathrm{~mol} \mathrm{dm}{ }^{-3}$ methyllithium solution in diethyl ether $(80 \mu \mathrm{L}, 104 \mu \mathrm{mol})$ was added, with the reaction mixture stirred cold for 5 minutes, then at room temperature for a further 90 minutes. All volatiles were then removed in vacuo and the residue extracted with 1:1 toluene/hexane $(3 \times 2 \mathrm{~mL})$, with the extracts combined, filtered through celite and taken to dryness under vacuum. This offwhite solid was further extracted with hot hexane $(3 \times 2 \mathrm{~mL})$, with the extracts dried and analysed by NMR spectroscopy. NMR data showed a mixture of $\mathbf{4 6}$ and starting material $\mathbf{2 7}$, as well as small quantities of unidentified products.

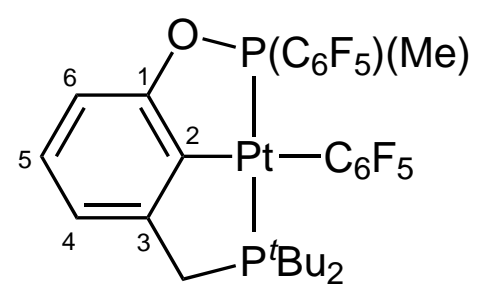

${ }^{1} \mathrm{H}-\mathrm{NMR}:\left(300 \mathrm{MHz}, \mathrm{C}_{6} \mathrm{D}_{6}\right) \delta_{\mathrm{H}} 6.97(\mathrm{~m}, 3 \mathrm{H}, \mathrm{H}-4,5,6), 3.21\left(\mathrm{~m}, 2 \mathrm{H}, \mathrm{CH}_{2}\right), 1.68(\mathrm{~d}$, $\left.{ }^{3} J_{\mathrm{Pt}-\mathrm{H}}=26.6 \mathrm{~Hz},{ }^{2} J_{\mathrm{P}-\mathrm{H}}=9.5 \mathrm{~Hz}, 3 \mathrm{H}, \mathrm{P}-\mathrm{CH}_{3}\right), 0.97\left(\mathrm{~d},{ }^{3} J_{\mathrm{P}-\mathrm{H}}=13.3 \mathrm{~Hz}, 9 \mathrm{H}\right.$, $\left.\mathrm{C}\left(\mathrm{CH}_{3}\right)_{3}\right), 0.92\left(\mathrm{~d},{ }^{3} J_{\mathrm{P}-\mathrm{H}}=13.1 \mathrm{~Hz}, 9 \mathrm{H}, \mathrm{C}\left(\mathrm{CH}_{3}\right)_{3}\right) .{ }^{31} \mathrm{P}-\mathrm{NMR}:\left(121 \mathrm{MHz}, \mathrm{C}_{6} \mathrm{D}_{6}\right) \delta_{\mathrm{P}}$ 
$132.0\left(\mathrm{~d},{ }^{1} J_{\mathrm{Pt}-\mathrm{P}}=3157 \mathrm{~Hz},{ }^{2} J_{\mathrm{P}-\mathrm{P}}=421 \mathrm{~Hz}, 1 \mathrm{P}, \mathrm{P}\left(\mathrm{C}_{6} \mathrm{~F}_{5}\right)\left(\mathrm{CH}_{3}\right)\right), 73.8\left(\mathrm{~d},{ }^{1} J_{\mathrm{Pt}-\mathrm{P}}=\right.$ $\left.3157 \mathrm{~Hz},{ }^{2} J_{\mathrm{P}-\mathrm{P}}=421 \mathrm{~Hz}, 1 \mathrm{P}, \mathrm{P}^{t} \mathrm{Bu}_{2}\right) .{ }^{19} \mathrm{~F}-\mathrm{NMR}:\left(282 \mathrm{MHz}, \mathrm{C}_{6} \mathrm{D}_{6}\right) \delta_{\mathrm{F}}-114.3(\mathrm{dm}$, $\left.{ }^{3} J_{\mathrm{Pt}-\mathrm{F}}=267 \mathrm{~Hz},{ }^{5} J_{\mathrm{F}-\mathrm{F}}=32.7 \mathrm{~Hz}, 1 \mathrm{~F}, \mathrm{Pt}-\mathrm{C}_{6} \mathrm{~F}_{5} o-\mathrm{F}\right),-115.5\left(\mathrm{dm},{ }^{3} J_{\mathrm{Pt}-\mathrm{F}}=230 \mathrm{~Hz}\right.$,

$\left.{ }^{5} J_{\mathrm{F}-\mathrm{F}}=32.0 \mathrm{~Hz}, 1 \mathrm{~F}, \mathrm{Pt}-\mathrm{C}_{6} \mathrm{~F}_{5} o-\mathrm{F}\right),-132.4\left(\mathrm{dm},{ }^{3} J_{\mathrm{F}-\mathrm{F}}=17.4 \mathrm{~Hz}, 2 \mathrm{~F}, \mathrm{P}-\mathrm{C}_{6} \mathrm{~F}_{5}\right.$ $o-\mathrm{F}),-147.8\left(\mathrm{tm},{ }^{3} J_{\mathrm{F}-\mathrm{F}}=20.9 \mathrm{~Hz}, 1 \mathrm{~F}, \mathrm{P}-\mathrm{C}_{6} \mathrm{~F}_{5} p-\mathrm{F}\right),-160.1\left(\mathrm{~m}, 2 \mathrm{~F}, \mathrm{P}-\mathrm{C}_{6} \mathrm{~F}_{5}\right.$ $o-\mathrm{F}),-161.1\left(\mathrm{vtm},{ }^{3} J_{\mathrm{F}-\mathrm{F}}=19.9 \mathrm{~Hz}, 2 \mathrm{~F}, \mathrm{Pt}-\mathrm{C}_{6} \mathrm{~F}_{5} p-\mathrm{F}\right),-163.1\left(\mathrm{~m}, 2 \mathrm{~F}, \mathrm{Pt}-\mathrm{C}_{6} \mathrm{~F}_{5}\right.$ $m-\mathrm{F}),-163.8\left(\mathrm{~m}, 2 \mathrm{~F}, \mathrm{Pt}-\mathrm{C}_{6} \mathrm{~F}_{5} m-\mathrm{F}\right)$.

\section{$[(\mathrm{POCOP}) \mathrm{Pt}(\mathrm{CO})]\left[\mathrm{SbF}_{6}\right](31)$}

A foil-wrapped flask containing $25(50 \mathrm{mg}, 0.047 \mathrm{mmol})$ and $\mathrm{AgSbF}_{6}(18 \mathrm{mg}$, $0.052 \mathrm{mmol})$ was cooled to $-78^{\circ} \mathrm{C}$ and dichloromethane $(10 \mathrm{~mL})$ was added. Carbon monoxide was then bubbled through solution as the reaction mixture was allowed to warm to room temperature. After 30 minutes the carbon monoxide addition was stopped and the foil removed from the flask. After stirring for a further 20 hours the reaction mixture was subsequently filtered through celite and all volatiles removed in vacuo. The oily pale yellow residue was washed with diethyl ether, and $\mathbf{3 1}$ was isolated as colourless rod-like crystals by slow evaporation of a dichloromethane solution (17.9 mg, 30\%). ${ }^{1} \mathrm{H}-\mathrm{NMR}$ : $\left(300 \mathrm{MHz}, \mathrm{CD}_{2} \mathrm{Cl}_{2}\right) \delta_{\mathrm{H}} 7.51\left(\mathrm{t},{ }^{3} J_{\mathrm{H}-\mathrm{H}}=8.2 \mathrm{~Hz}\right.$, 1H, H-5), 7.10 (m, 2H, H-4,6). ${ }^{31}$ P-NMR: (121 MHz, $\left.\mathrm{CD}_{2} \mathrm{Cl}_{2}\right) \delta_{\mathrm{P}} 104.4\left(\mathrm{~s},{ }^{1} J_{\mathrm{Pt}-\mathrm{P}}=\right.$ $3345 \mathrm{~Hz}) .{ }^{19} \mathrm{~F}-\mathrm{NMR}:\left(282 \mathrm{MHz}, \mathrm{CD}_{2} \mathrm{Cl}_{2}\right) \delta_{\mathrm{F}}-129.1\left(\mathrm{~m}, 8 \mathrm{~F},{ }_{o}-\mathrm{C}_{6} \mathrm{~F}_{5}\right),-139.3(\mathrm{~m}$, $\left.4 \mathrm{~F}, p-\mathrm{C}_{6} \mathrm{~F}_{5}\right),-156.6\left(\mathrm{~m}, 8 \mathrm{~F}, m-\mathrm{C}_{6} \mathrm{~F}_{5}\right) .{ }^{13} \mathrm{C}-\mathrm{NMR}:\left(125 \mathrm{MHz}, \mathrm{CD}_{2} \mathrm{Cl}_{2}\right) \delta_{\mathrm{C}} 177.5(\mathrm{~s}$, $\left.{ }^{1} J_{\mathrm{Pt}-\mathrm{C}}=\mathrm{nr}, 1 \mathrm{C}, \mathrm{CO}\right), 162.2(\mathrm{~s}, 2 \mathrm{C}, \mathrm{C}-1,3), 146.9\left(\mathrm{dm},{ }^{1} J_{\mathrm{F}-\mathrm{C}}=257 \mathrm{~Hz}, 8 \mathrm{C}, o-\mathrm{C}_{6} \mathrm{~F}_{5}\right)$, $146.5\left(\mathrm{dm},{ }^{1} J_{\mathrm{F}-\mathrm{C}}=264 \mathrm{~Hz}, 4 \mathrm{C}, p-\mathrm{C}_{6} \mathrm{~F}_{5}\right), 138.7\left(\mathrm{dm},{ }^{1} J_{\mathrm{F}-\mathrm{C}}=256 \mathrm{~Hz}, 8 \mathrm{C}, m-\mathrm{C}_{6} \mathrm{~F}_{5}\right)$, $135.0(\mathrm{~s}, 1 \mathrm{C}, \mathrm{C}-5), 131.0$ (s, $\left.{ }^{1} J_{\mathrm{PtC}}=\mathrm{nr}, 1 \mathrm{C}, \mathrm{C}-2\right), 109.4$ (s, 2C, C-4,6), $103.2(\mathrm{~m}, 4 \mathrm{C}$, $\left.i-\mathrm{C}_{6} \mathrm{~F}_{5}\right)$. IR $(\mathrm{KBr}): \nu_{\max }(\mathrm{CO})=2145 \mathrm{~cm}^{-1}$. Anal. Calcd for $\mathrm{C}_{31} \mathrm{H}_{3} \mathrm{O}_{3} \mathrm{~F}_{26} \mathrm{P}_{2} \mathrm{SbPt}$ : $\mathrm{C}, 28.67 ; \mathrm{H}, 0.38$. Found $\mathrm{C}, 28.73 ; \mathrm{H}, 0.23$. HRMS calcd for $\left(\mathrm{C}_{32} \mathrm{H}_{8} \mathrm{NO}_{3} \mathrm{~F}_{20} \mathrm{P}{ }_{2} \mathrm{Pt}\right)$ $\left[\mathrm{M}-\mathrm{CO}+\mathrm{CH}_{3} \mathrm{CN}+\mathrm{H}_{2} \mathrm{O}\right]^{+}: m / z=1090.9305$, found $=1090.9317$.

\section{$[(\mathrm{PCCCP}) \mathrm{Pt}(\mathrm{CO})]\left[\mathrm{SbF}_{6}\right](32)$}

A foil-wrapped flask containing $26(50 \mathrm{mg}, 0.047 \mathrm{mmol})$ and $\mathrm{AgSbF}_{6}(17 \mathrm{mg}$, $0.049 \mathrm{mmol})$ was cooled to $-78^{\circ} \mathrm{C}$ and dichloromethane $(10 \mathrm{~mL})$ was added. Carbon monoxide was then bubbled through solution as the reaction mixture was allowed to warm to room temperature. After 30 minutes the carbon monoxide addition was stopped and the foil removed from the flask. After stirring for a further 17 hours the reaction mixture was subsequently filtered through celite and all volatiles removed in vacuo. The oily residue was washed with diethyl ether, then twice recrystallised 
from dichloromethane/diethyl ether mixtures to give $\mathbf{3 2}$ as an off-white, microcrystalline solid (20.0 mg, 33\%). ${ }^{1} \mathrm{H}-\mathrm{NMR}$ : (300 MHz, acetone- $\left.d_{6}\right) \delta_{\mathrm{H}} 7.59(\mathrm{~m}, 2 \mathrm{H}$, $\mathrm{H}-4,6), 7.44(\mathrm{~m}, 1 \mathrm{H}, \mathrm{H}-5), 5.06\left(\mathrm{t},{ }^{3} J_{\mathrm{Pt}-\mathrm{H}}=36.6 \mathrm{~Hz},{ }^{2} J_{\mathrm{P}-\mathrm{H}}=5.3 \mathrm{~Hz}, 4 \mathrm{H}, \mathrm{CH}_{2}\right)$. ${ }^{31}$ P-NMR: $\left(121 \mathrm{MHz}\right.$, acetone- $\left.d_{6}\right) \delta_{\mathrm{P}} 6.2\left(\mathrm{~s},{ }^{1} J_{\mathrm{Pt}-\mathrm{P}}=3056 \mathrm{~Hz}\right) \cdot{ }^{19} \mathrm{~F}-\mathrm{NMR}:(282 \mathrm{MHz}$, acetone- $\left.d_{6}\right) \delta_{\mathrm{F}}-123.7\left(\mathrm{~m}, 8 \mathrm{~F}, o-\mathrm{C}_{6} \mathrm{~F}_{5}\right),-140.0\left(\mathrm{tt},{ }^{3} J_{\mathrm{F}-\mathrm{F}}=20.6 \mathrm{~Hz},{ }^{4} J_{\mathrm{F}-\mathrm{F}}=6.7 \mathrm{~Hz}\right.$, $\left.4 \mathrm{~F}, p-\mathrm{C}_{6} \mathrm{~F}_{5}\right),-154.5\left(\mathrm{~m}, 8 \mathrm{~F}, m-\mathrm{C}_{6} \mathrm{~F}_{5}\right) .{ }^{13} \mathrm{C}-\mathrm{NMR}:\left(150 \mathrm{MHz}\right.$, acetone- $\left.d_{6}\right) \delta_{\mathrm{C}} 180.6$ $\left(\mathrm{s},{ }^{1} J_{\mathrm{Pt}-\mathrm{C}}=1068 \mathrm{~Hz}, 1 \mathrm{C}, \mathrm{CO}\right), 153.6\left(\mathrm{~s},{ }^{1} J_{\mathrm{Pt}-\mathrm{C}}=723 \mathrm{~Hz}, 1 \mathrm{C}, \mathrm{C}-2\right), 148.2\left(\mathrm{dm},{ }^{1} J_{\mathrm{F}-\mathrm{C}}\right.$ $\left.=256 \mathrm{~Hz}, 8 \mathrm{C}, o-\mathrm{C}_{6} \mathrm{~F}_{5}\right), 147.2\left(\mathrm{t},{ }^{2} J_{\mathrm{Pt}-\mathrm{C}}=70.2 \mathrm{~Hz},{ }^{2} J_{\mathrm{P}-\mathrm{C}}=9.0 \mathrm{~Hz}, 2 \mathrm{C}, \mathrm{C}-1,3\right), 145.8$ $\left(\mathrm{dm},{ }^{1} J_{\mathrm{F}-\mathrm{C}}=261 \mathrm{~Hz}, 4 \mathrm{C}, p-\mathrm{C}_{6} \mathrm{~F}_{5}\right), 139.1\left(\mathrm{dm},{ }^{1} J_{\mathrm{F}-\mathrm{C}}=255 \mathrm{~Hz}, 8 \mathrm{C}, m-\mathrm{C}_{6} \mathrm{~F}_{5}\right), 131.6$ $(\mathrm{s}, 1 \mathrm{C}, 5-\mathrm{C}), 125.3(\mathrm{~m}, 2 \mathrm{C}, \mathrm{C}-4,6), 102.2\left(\mathrm{~m}, 4 \mathrm{C}, i-\mathrm{C}_{6} \mathrm{~F}_{5}\right), 43.3\left(\mathrm{t},{ }^{2} J_{\mathrm{Pt}-\mathrm{C}}=71.8 \mathrm{~Hz}\right.$, $\left.{ }^{1} J_{\mathrm{P}-\mathrm{C}}=18.2 \mathrm{~Hz}, 2 \mathrm{C}, \mathrm{CH}_{2}\right)$. IR $(\mathrm{KBr}): \nu_{\max }(\mathrm{CO})=2127 \mathrm{~cm}^{-1}$. HRMS calcd for $\left(\mathrm{C}_{33} \mathrm{H}_{7} \mathrm{OF}_{20} \mathrm{P}_{2} \mathrm{Pt}\right)[\mathrm{M}]^{+}: m / z=1055.9298$, found $=1055.9038$.

\section{$[(\mathrm{POCCP}) \mathrm{Pt}(\mathrm{CO})]\left[\mathrm{SbF}_{6}\right](33)$}

A foil-wrapped flask containing $27(50 \mathrm{mg}, 0.059 \mathrm{mmol})$ and $\mathrm{AgSbF}_{6}(22 \mathrm{mg}$, $0.064 \mathrm{mmol})$ was cooled to $-78^{\circ} \mathrm{C}$ and dichloromethane $(10 \mathrm{~mL})$ was added. Carbon monoxide was then bubbled through solution as the reaction mixture was allowed to warm to room temperature. After 30 minutes the carbon monoxide addition was stopped and the foil removed from the flask. After stirring for a further 19 hours the reaction mixture was filtered through celite and reduced to approximately $2 \mathrm{~mL}$ in vacuo. Addition of diethyl ether to the dichloromethane solution afforded $\mathbf{3 3}$ as a pale yellow solid, which was washed with diethyl ether and dried in vacuo (36.1 mg, 57\%). ${ }^{1} \mathrm{H}-\mathrm{NMR}:\left(300 \mathrm{MHz}, \mathrm{CD}_{2} \mathrm{Cl}_{2}\right) \delta_{\mathrm{H}} 7.38$ (vt, $\left.{ }^{3} J_{\mathrm{H}-\mathrm{H}}=7.9 \mathrm{~Hz}, 1 \mathrm{H}, \mathrm{H}-5\right), 7.29$ $\left(\mathrm{d},{ }^{3} J_{\mathrm{H}-\mathrm{H}}=7.5 \mathrm{~Hz}, 1 \mathrm{H}, \mathrm{H}-4\right), 7.13\left(\mathrm{~d},{ }^{4} J_{\mathrm{Pt}-\mathrm{H}}=11.6 \mathrm{~Hz},{ }^{3} J_{\mathrm{H}-\mathrm{H}}=7.8 \mathrm{~Hz}, 1 \mathrm{H}, \mathrm{H}-6\right)$, $3.96\left(\mathrm{~d},{ }^{3} J_{\mathrm{Pt}-\mathrm{H}}=24.0 \mathrm{~Hz},{ }^{2} J_{\mathrm{P}-\mathrm{H}}=10.1 \mathrm{~Hz}, 2 \mathrm{H}, \mathrm{CH}_{2}\right), 1.47\left(\mathrm{~d},{ }^{3} J_{\mathrm{P}-\mathrm{H}}=15.4 \mathrm{~Hz}\right.$, $\left.18 \mathrm{H}, \mathrm{C}\left(\mathrm{CH}_{3}\right)_{3}\right) .{ }^{31} \mathrm{P}-\mathrm{NMR}:\left(121 \mathrm{MHz}, \mathrm{CD}_{2} \mathrm{Cl}_{2}\right) \delta_{\mathrm{P}} 108.3\left(\mathrm{~d},{ }^{1} J_{\mathrm{Pt}-\mathrm{P}}=2800 \mathrm{~Hz},{ }^{2} J_{\mathrm{P}-\mathrm{P}}\right.$ $\left.=326 \mathrm{~Hz}, 1 \mathrm{P}, \mathrm{P}\left(\mathrm{C}_{6} \mathrm{~F}_{5}\right)_{2}\right), 91.4\left(\mathrm{~d},{ }^{1} J_{\mathrm{Pt}-\mathrm{P}}=2616 \mathrm{~Hz},{ }^{2} J_{\mathrm{P}-\mathrm{P}}=324 \mathrm{~Hz}, 1 \mathrm{P}, \mathrm{P}^{t} \mathrm{Bu}_{2}\right)$. ${ }^{19}$ F-NMR: $\left(282 \mathrm{MHz}, \mathrm{CD}_{2} \mathrm{Cl}_{2}\right) \delta_{\mathrm{F}}-129.7\left(\mathrm{~m}, 4 \mathrm{~F},{ }_{o}-\mathrm{C}_{6} \mathrm{~F}_{5}\right),-140.2\left(\mathrm{ttd},{ }^{3} J_{\mathrm{F}-\mathrm{F}}=\right.$ $\left.20.7 \mathrm{~Hz},{ }^{3} J_{\mathrm{F}-\mathrm{F}}=7.7 \mathrm{~Hz},{ }^{5} J_{\mathrm{P}-\mathrm{F}}=1.9 \mathrm{~Hz}, 2 \mathrm{~F}, p-\mathrm{C}_{6} \mathrm{~F}_{5}\right),-156.7\left(\mathrm{tm},{ }^{3} J_{\mathrm{F}-\mathrm{F}}=20.1 \mathrm{~Hz}\right.$, $\left.4 \mathrm{~F}, m-\mathrm{C}_{6} \mathrm{~F}_{5}\right) .{ }^{13} \mathrm{C}-\mathrm{NMR}:\left(150 \mathrm{MHz}, \mathrm{CD}_{2} \mathrm{Cl}_{2}\right) \delta_{\mathrm{C}} 180.1\left(\mathrm{~s},{ }^{1} J_{\mathrm{Pt}-\mathrm{C}}=1064 \mathrm{~Hz}, 1 \mathrm{C}\right.$, $\mathrm{CO}), 162.8\left(\mathrm{dd},{ }^{2} J_{\mathrm{Pt}-\mathrm{C}}=39 \mathrm{~Hz},{ }^{2} J_{\mathrm{P}-\mathrm{C}}=14 \mathrm{~Hz},{ }^{3} J_{\mathrm{P}-\mathrm{C}}=3.9 \mathrm{~Hz}, 1 \mathrm{C}, \mathrm{C}-1\right), 152.0$ $\left(\mathrm{dd},{ }^{2} J_{\mathrm{Pt}-\mathrm{C}}=87 \mathrm{~Hz},{ }^{2} J_{\mathrm{P}-\mathrm{C}}=8.5 \mathrm{~Hz},{ }^{3} J_{\mathrm{P}-\mathrm{C}}=5.9 \mathrm{~Hz}, 1 \mathrm{C}, \mathrm{C}-3\right), 146.1\left(\mathrm{dm},{ }^{1} J_{\mathrm{F}-\mathrm{C}}\right.$ $\left.=253 \mathrm{~Hz}, 4 \mathrm{~F}, o-\mathrm{C}_{6} \mathrm{~F}_{5}\right), 145.3\left(\mathrm{dm},{ }^{1} J_{\mathrm{F}-\mathrm{C}}=265 \mathrm{~Hz}, 2 \mathrm{~F}, p-\mathrm{C}_{6} \mathrm{~F}_{5}\right), 144.1\left(\mathrm{~d},{ }^{1} J_{\mathrm{Pt}-\mathrm{C}}\right.$ $\left.=746 \mathrm{~Hz},{ }^{2} J_{\mathrm{P}-\mathrm{C}}=6.5 \mathrm{~Hz}, 1 \mathrm{C}, \mathrm{C}-2\right), 137.9\left(\mathrm{dm},{ }^{1} J_{\mathrm{F}-\mathrm{C}}=258 \mathrm{~Hz}, 4 \mathrm{C}, m-\mathrm{C}_{6} \mathrm{~F}_{5}\right)$, $131.8(\mathrm{~s}, 1 \mathrm{C}, \mathrm{C}-5), 120.4\left(\mathrm{~d},{ }^{3} J_{\mathrm{Pt}-\mathrm{C}}=30.2 \mathrm{~Hz},{ }^{3} J_{\mathrm{P}-\mathrm{C}}=18.3 \mathrm{~Hz}, 1 \mathrm{C}, \mathrm{C}-4\right), 110.8$ (d, $\left.{ }^{3} J_{\mathrm{P}-\mathrm{C}}=18.2 \mathrm{~Hz},{ }^{3} J_{\mathrm{Pt}-\mathrm{C}}=11.2 \mathrm{~Hz}, 1 \mathrm{C}, \mathrm{C}-6\right), 103.7\left(\mathrm{dt},{ }^{1} J_{\mathrm{P}-\mathrm{C}}=62.8 \mathrm{~Hz},{ }^{2} J_{\mathrm{F}-\mathrm{C}}=\right.$ $\left.17.6 \mathrm{~Hz}, 2 \mathrm{C}, i-\mathrm{C}_{6} \mathrm{~F}_{5}\right), 36.9\left(\mathrm{dd},{ }^{2} J_{\mathrm{Pt}-\mathrm{C}}=25.2 \mathrm{~Hz},{ }^{1} J_{\mathrm{P}-\mathrm{C}}=21.5 \mathrm{~Hz},{ }^{3} J_{\mathrm{P}-\mathrm{C}}=3.0 \mathrm{~Hz}\right.$, $\left.2 \mathrm{C}, C\left(\mathrm{CH}_{3}\right)_{3}\right), 35.0\left(\mathrm{~d},{ }^{2} J_{\mathrm{Pt}-\mathrm{C}}=80.7 \mathrm{~Hz},{ }^{1} J_{\mathrm{P}-\mathrm{C}}=35.1 \mathrm{~Hz}, 1 \mathrm{C}, \mathrm{CH}_{2}\right), 28.1(\mathrm{~s}, 6 \mathrm{C}$, 
$\left.\mathrm{C}\left(\mathrm{CH}_{3}\right)_{3}\right) . \mathrm{IR}(\mathrm{KBr}): \nu_{\max }(\mathrm{CO})=2111 \mathrm{~cm}^{-1}$.

\section{[(PNNNP)PtCl $][\mathrm{Cl}](47)$}

A solution of 10 (200 mg, $0.24 \mathrm{mmol})$ and $\left[\mathrm{PtCl}_{2}(\mathrm{COD})\right](89 \mathrm{mg}, 0.24 \mathrm{mmol})$ in dichloromethane $(10 \mathrm{~mL})$ was stirred at room temperature for 48 hours. Volatiles were removed in vacuo and the residue washed with diethyl ether $(3 \times 10 \mathrm{~mL})$. Purification of the ether-washed solid by recrystallation from 1:1 dichloromethane/diethyl ether afforded 59 as a white, microcrystalline solid (97 mg, 37\%). ${ }^{1} \mathrm{H}-\mathrm{NMR}$ : (300 MHz, acetone- $\left.d_{6}\right) \delta_{\mathrm{H}} 12.48(\mathrm{br} \mathrm{s}, 2 \mathrm{H}, \mathrm{N}-\mathrm{H}), 7.90\left(\mathrm{tt},{ }^{3} J_{\mathrm{H}-\mathrm{H}}=8.1 \mathrm{~Hz},{ }^{5} J_{\mathrm{P}-\mathrm{H}}=2.0 \mathrm{~Hz}\right.$, $1 \mathrm{H}, \mathrm{H}-5), 7.22$ (d, $\left.{ }^{3} J_{\mathrm{H}-\mathrm{H}}=8.2 \mathrm{~Hz}, 2 \mathrm{H}, \mathrm{H}-4,6\right) .{ }^{31} \mathrm{P}-\mathrm{NMR}:\left(121 \mathrm{MHz}\right.$, acetone- $\left.d_{6}\right)$ $\delta_{\mathrm{P}} 30.6\left(\mathrm{~s},{ }^{1} J_{\mathrm{Pt}-\mathrm{P}}=3153 \mathrm{~Hz}\right) .{ }^{19} \mathrm{~F}-\mathrm{NMR}:\left(282 \mathrm{MHz}\right.$, acetone- $\left.d_{6}\right) \delta_{\mathrm{F}}-128.3(\mathrm{~d}$, $\left.{ }^{3} J_{\mathrm{F}-\mathrm{F}}=20.1 \mathrm{~Hz}, 8 \mathrm{~F}, o-\mathrm{C}_{6} \mathrm{~F}_{5}\right),-144.4\left(\mathrm{br} \mathrm{s}, 4 \mathrm{~F}, p-\mathrm{C}_{6} \mathrm{~F}_{5}\right),-160.0\left(\mathrm{~m}, 8 \mathrm{~F}, m-\mathrm{C}_{6} \mathrm{~F}_{5}\right)$. Anal. Calcd for $\mathrm{C}_{29} \mathrm{H}_{5} \mathrm{~N}_{3} \mathrm{~F}_{20} \mathrm{P}_{2} \mathrm{Cl}_{2} \mathrm{Pt} \cdot \frac{1}{2} \mathrm{CH}_{2} \mathrm{Cl}_{2}$ : C, 28.67; H, 0.38. Found $\mathrm{C}, 28.73$; $\mathrm{H}, 0.23$. HRMS calcd for $\left(\mathrm{C}_{29} \mathrm{H}_{5} \mathrm{~N}_{3} \mathrm{~F}_{20} \mathrm{P}_{2} \mathrm{ClPt}\right)[\mathrm{M}]^{+}: m / z=1065.8955$, found $=$ 1065.8970 .

\section{Observation of $\left[(\mathrm{PONOP}) \mathrm{PtCl}_{2}\right]_{\mathrm{x}}(48)$}

A solution of ligand $11(20 \mathrm{mg}, 24 \mu \mathrm{mol})$ and $\left[\mathrm{PtCl}_{2}\left(\mathrm{SEt}_{2}\right)_{2}\right](8.5 \mathrm{mg}, 24 \mu \mathrm{mol})$ in acetone- $d_{6}$ was monitored in situ by NMR spectroscopy. After 15 hours at room temperature almost quantitative formation of $\mathbf{4 8}$ in solution was indicated. No significant changes were observed in the reaction mixture after an additional 48 hours at $60{ }^{\circ} \mathrm{C}$. HRMS analysis of this reaction mixture did not reveal any further information about the nature of 48. ${ }^{1} \mathrm{H}-\mathrm{NMR}$ : $\left(300 \mathrm{MHz}\right.$, acetone- $\left.d_{6}\right) \delta_{\mathrm{H}} 8.23\left(\mathrm{t},{ }^{3} J_{\mathrm{H}-\mathrm{H}}\right.$ $=8.4 \mathrm{~Hz}, 1 \mathrm{H}, \mathrm{H}-5), 6.83\left(\mathrm{~d},{ }^{3} J_{\mathrm{H}-\mathrm{H}}=8.5 \mathrm{~Hz}, 2 \mathrm{H}, \mathrm{H}-4,6\right) .{ }^{31} \mathrm{P}-\mathrm{NMR}:(121 \mathrm{MHz}$, acetone- $\left.d_{6}\right) \delta_{\mathrm{P}} 29.0\left(\mathrm{~s},{ }^{1} J_{\mathrm{Pt}-\mathrm{P}}=4148 \mathrm{~Hz}\right) \cdot{ }^{19} \mathrm{~F}-\mathrm{NMR}:\left(282 \mathrm{MHz}\right.$, acetone- $\left.d_{6}\right) \delta_{\mathrm{F}}$ $-130.1\left(\mathrm{~m}, 8 \mathrm{~F}, o-\mathrm{C}_{6} \mathrm{~F}_{5}\right),-151.5\left(\mathrm{t},{ }^{3} J_{\mathrm{F}-\mathrm{F}}=20.4 \mathrm{~Hz}, 4 \mathrm{~F}, p-\mathrm{C}_{6} \mathrm{~F}_{5}\right),-163.8(\mathrm{~m}, 8 \mathrm{~F}$, $\left.m-\mathrm{C}_{6} \mathrm{~F}_{5}\right)$.

\subsection{Palladium Complexes}

\section{Observation of trans-[(POCOPH $\left.) \mathrm{PdCl}_{2}\right]_{2}(22)$}

Ligand $1(30 \mathrm{mg}, 36 \mu \mathrm{mol})$ and $\left[\mathrm{PdCl}_{2}(\mathrm{NCMe})_{2}\right](9.6 \mathrm{mg}, 37 \mu \mathrm{mol})$ were dissolved 
in dichloromethane- $d_{2}$ in an NMR tube, and analysed immediately by NMR spectroscopy, which revealed the quantitative formation of $\mathbf{6 0}$. However, isomerisation to compound $\mathbf{2 3}$ occurred readily at room temperature, and further characterisation of 60 was not able to be performed. ${ }^{1} \mathrm{H}-\mathrm{NMR}$ : $\left(300 \mathrm{MHz}, \mathrm{CD}_{2} \mathrm{Cl}_{2}\right) \delta_{\mathrm{H}} 7.42$ $\left(\mathrm{t},{ }^{3} J_{\mathrm{H}-\mathrm{H}}=8.5 \mathrm{~Hz}, 2 \mathrm{H}, \mathrm{H}-5\right), 7.24\left(\mathrm{~d},{ }^{3} J_{\mathrm{H}-\mathrm{H}}=8.5 \mathrm{~Hz}, 4 \mathrm{H}, \mathrm{H}-4,6\right), 7.02(\mathrm{~s}, 2 \mathrm{H}$, H-2). ${ }^{31} \mathrm{P}-\mathrm{NMR}:\left(121 \mathrm{MHz}, \mathrm{CD}_{2} \mathrm{Cl}_{2}\right) \delta_{\mathrm{P}} 87.7$ (s). ${ }^{19} \mathrm{~F}-\mathrm{NMR}:\left(282 \mathrm{MHz}, \mathrm{CD}_{2} \mathrm{Cl}_{2}\right)$ $\delta_{\mathrm{F}}-127.0\left(\mathrm{br} \mathrm{s}, 16 \mathrm{~F}, o-\mathrm{C}_{6} \mathrm{~F}_{5}\right),-144.5\left(\mathrm{~m}, 8 \mathrm{~F}, p-\mathrm{C}_{6} \mathrm{~F}_{5}\right),-159.7$ (br s, $\left.16 \mathrm{~F}, m-\mathrm{C}_{6} \mathrm{~F}_{5}\right)$.

\section{cis,trans- $\left[(\mathrm{POCOPH}) \mathrm{PdCl}_{2}\right]_{2}$}

A solution of ligand 1 (101 mg, $0.12 \mathrm{mmol})$ and $\left[\mathrm{PdCl}_{2}(\mathrm{NCMe})_{2}\right](32 \mathrm{mg}, 0.12 \mathrm{mmol})$ in dichloromethane $(15 \mathrm{~mL})$ was heated in an oil bath at $45^{\circ} \mathrm{C}$ for 12 hours, at which point the volume of the solution was reduced to approximately $2 \mathrm{~mL}$ in vacuo, and the supernatant decanted and retained. Addition of hexane to the supernatant gave a pale yellow precipitate, which was isolated and washed thoroughly with pentane, affording $\mathbf{2 3}$ as a pale yellow solid (101 $\mathrm{mg}$, 83\%). Samples of $\mathbf{2 3}$ were observed to undergo a degree of rearrangement to higher oligomers on standing in polar solvents such as chloroform or acetone.

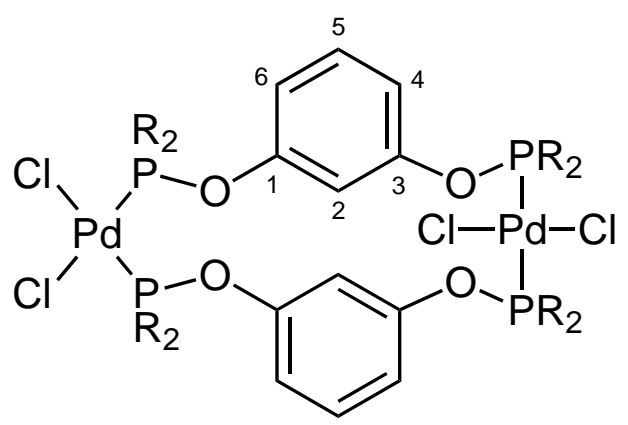

where $\mathrm{R}=\mathrm{C}_{6} \mathrm{~F}_{5}$

${ }^{1} \mathrm{H}-\mathrm{NMR}:\left(500 \mathrm{MHz}, \mathrm{CD}_{2} \mathrm{Cl}_{2}\right) \delta_{\mathrm{H}} 7.17(\mathrm{~s}, 2 \mathrm{H}, \mathrm{H}-2), 7.16\left(\mathrm{vt},{ }^{3} J_{\mathrm{H}-\mathrm{H}}=8.3 \mathrm{~Hz}, 2 \mathrm{H}\right.$, $\mathrm{H}-5), 6.85\left(\mathrm{dd},{ }^{3} J_{\mathrm{H}-\mathrm{H}}=8.3 \mathrm{~Hz},{ }^{4} J_{\mathrm{H}-\mathrm{H}}=2.0 \mathrm{~Hz}, 2 \mathrm{H}, \mathrm{H}-4\right), 6.55\left(\mathrm{~d},{ }^{3} J_{\mathrm{H}-\mathrm{H}}=8.1 \mathrm{~Hz}\right.$, 2H, H-6). ${ }^{31}$ P-NMR: (121 MHz, $\left.\mathrm{CD}_{2} \mathrm{Cl}_{2}\right) \delta_{\mathrm{P}} 88.7$ (s, 2P, P-trans-P), 78.5 (s, 2P, P-trans-Cl). ${ }^{19} \mathrm{~F}-\mathrm{NMR}:\left(282 \mathrm{MHz}, \mathrm{CD}_{2} \mathrm{Cl}_{2}\right) \delta_{\mathrm{F}}-126.4\left(\mathrm{~s}, 8 \mathrm{~F}, o-\mathrm{C}_{6} \mathrm{~F}_{5}\right),-126.8(\mathrm{~s}$, $\left.8 \mathrm{~F}, o-\mathrm{C}_{6} \mathrm{~F}_{5}\right),-141.7\left(\mathrm{~s}, 4 \mathrm{~F}, p-\mathrm{C}_{6} \mathrm{~F}_{5}\right),-143.1\left(\mathrm{~s}, 4 \mathrm{~F}, p-\mathrm{C}_{6} \mathrm{~F}_{5}\right),-158.5\left(\mathrm{t},{ }^{3} J_{\mathrm{F}-\mathrm{F}}=\right.$ $\left.19.3 \mathrm{~Hz}, 8 \mathrm{~F}, m-\mathrm{C}_{6} \mathrm{~F}_{5}\right),-158.9\left(\mathrm{td},{ }^{3} J_{\mathrm{F}-\mathrm{F}}=21.7,6.9 \mathrm{~Hz}, 8 \mathrm{~F}, m-\mathrm{C}_{6} \mathrm{~F}_{5}\right) .{ }^{13} \mathrm{C}-\mathrm{NMR}$ : $\left(125 \mathrm{MHz}, \mathrm{CD}_{2} \mathrm{Cl}_{2}\right) \delta_{\mathrm{C}} 153.2(\mathrm{~s}, 2 \mathrm{C}, \mathrm{C}-3), 152.1(\mathrm{~s}, 2 \mathrm{C}, \mathrm{C}-1), 146.8$ (dd, ${ }^{1} J_{\mathrm{F}-\mathrm{C}}=$ $\left.246 \mathrm{~Hz},{ }^{2} J_{\mathrm{C}-\mathrm{F}}=40.8 \mathrm{~Hz}, 16 \mathrm{C}, o-\mathrm{C}_{6} \mathrm{~F}_{5}\right), 145.3\left(\mathrm{dm},{ }^{1} J_{\mathrm{F}-\mathrm{C}}=255 \mathrm{~Hz}, 8 \mathrm{C}, p-\mathrm{C}_{6} \mathrm{~F}_{5}\right)$, $138.0\left(\mathrm{dm},{ }^{1} J_{\mathrm{F}-\mathrm{C}}=254 \mathrm{~Hz}, 16 \mathrm{C}, m-\mathrm{C}_{6} \mathrm{~F}_{5}\right), 130.9(\mathrm{~s}, 2 \mathrm{C}, \mathrm{C}-5), 119.5$ (s, 2C, C-6), $116.0(\mathrm{~s}, 2 \mathrm{C}, \mathrm{C}-4), 112.8$ ( $\mathrm{s}, 2 \mathrm{C}, \mathrm{C}-2)$. The resonance of the $i-\mathrm{C}_{6} \mathrm{~F}_{5}$ environment was 
unable to be identified. Anal. Calcd for $\mathrm{C}_{60} \mathrm{H}_{8} \mathrm{O}_{4} \mathrm{~F}_{40} \mathrm{P}_{4} \mathrm{Cl}_{4} \mathrm{Pd}_{2}$ : C, 35.48; H, 0.40. Found $\mathrm{C}$, 35.64; $\mathrm{H}, 0.38$. HRMS calcd for $\left(\mathrm{C}_{60} \mathrm{H}_{8} \mathrm{O}_{4} \mathrm{~F}_{40} \mathrm{P}_{4} \mathrm{Cl}_{3} \mathrm{Pd}_{2}\right)[\mathrm{M}-\mathrm{Cl}]^{+}: \mathrm{m} / z=$ 1994.5881, found $=1994.5869$.

\section{$[(\mathrm{POCOP}) \mathrm{PdCl}](28)$}

A solution of $\left[\mathrm{PdCl}_{2}(\mathrm{NCMe})_{2}\right](96.5 \mathrm{mg}, 0.37 \mathrm{mmol})$ and 1 (316 mg, $\left.0.38 \mathrm{mmol}\right)$ in toluene $(15 \mathrm{~mL})$ was heated at reflux for 80 hours. All volatiles were then removed in vacuo and the viscous yellow oil was triturated with hexane to give a pale yellow solid. Repeated washing with hexane at $-78^{\circ} \mathrm{C}$ gave 28 as a pale yellow solid (291 mg, 80\%). ${ }^{1} \mathrm{H}-\mathrm{NMR}$ : (300 MHz, $\left.\mathrm{CDCl}_{3}\right) \delta_{\mathrm{H}} 7.20\left(\mathrm{t},{ }^{3} J_{\mathrm{H}-\mathrm{H}}=8.3 \mathrm{~Hz}\right.$, $1 \mathrm{H}, \mathrm{H}-5), 6.78\left(\mathrm{~d},{ }^{3} J_{\mathrm{H}-\mathrm{H}}=7.9 \mathrm{~Hz}, 2 \mathrm{H}, \mathrm{H}-4,6\right) .{ }^{31} \mathrm{P}-\mathrm{NMR}:\left(121 \mathrm{MHz}, \mathrm{CDCl}_{3}\right) \delta_{\mathrm{P}}$ $114.4(\mathrm{~s}) .{ }^{19} \mathrm{~F}-\mathrm{NMR}:\left(282 \mathrm{MHz}, \mathrm{CDCl}_{3}\right) \delta_{\mathrm{F}}-127.0\left(\mathrm{~m}, 8 \mathrm{~F}, o_{-}-\mathrm{C}_{6} \mathrm{~F}_{5}\right),-141.9$ (tt, $\left.{ }^{3} J_{\mathrm{F}-\mathrm{F}}=20.9 \mathrm{~Hz},{ }^{4} J_{\mathrm{F}-\mathrm{F}}=6.4 \mathrm{~Hz}, 4 \mathrm{~F}, p-\mathrm{C}_{6} \mathrm{~F}_{5}\right),-157.0\left(\mathrm{~m}, 8 \mathrm{~F}, m-\mathrm{C}_{6} \mathrm{~F}_{5}\right) .{ }^{13} \mathrm{C}-\mathrm{NMR}$ : $\left(150 \mathrm{MHz}, \mathrm{C}_{6} \mathrm{D}_{6}\right) \delta_{\mathrm{C}} 162.3\left(\mathrm{t},{ }^{2} J_{\mathrm{PC}}=9.0 \mathrm{~Hz} 2 \mathrm{C}, \mathrm{C}-1,3\right), 145.9\left(\mathrm{dm},{ }^{1} J_{\mathrm{F}-\mathrm{C}}=258 \mathrm{~Hz}\right.$, $\left.8 \mathrm{C}, o-\mathrm{C}_{6} \mathrm{~F}_{5}\right), \sim 144\left(\mathrm{dm},{ }^{1} J_{\mathrm{F}-\mathrm{C}} \approx 260 \mathrm{~Hz}, 4 \mathrm{C}, p-\mathrm{C}_{6} \mathrm{~F}_{5}\right), 137.1\left(\mathrm{dm},{ }^{1} J_{\mathrm{F}-\mathrm{C}}=257 \mathrm{~Hz}\right.$, $8 \mathrm{C}, m-\mathrm{C}_{6} \mathrm{~F}_{5}$ ), 132.1 (s, 1C, C-2), 130.1 (s, 1C, C-5), 108.1 (t, ${ }^{3} J_{\mathrm{PC}}=9.3 \mathrm{~Hz}, 2 \mathrm{C}$, $\mathrm{C}-4,6), 104.1\left(\mathrm{~m}, 4 \mathrm{C}, i-\mathrm{C}_{6} \mathrm{~F}_{5}\right)$. Exact chemical shift and coupling constants for the $p-\mathrm{C}_{6} \mathrm{~F}_{5}$ environment could not be determined due to peak overlap with the adjacent $o-\mathrm{C}_{6} \mathrm{~F}_{5}$ environment. HRMS calcd for $\left(\mathrm{C}_{30} \mathrm{H}_{4} \mathrm{O}_{2} \mathrm{~F}_{20} \mathrm{P}_{2} \mathrm{ClPt}\right)[\mathrm{M}+\mathrm{H}]^{+}: \mathrm{m} / z=$ 976.8501, found $=976.8498$.

\section{[(PCCCP)PdCl] (29)}

A solution of $\mathrm{PdCl}_{2}(\mathrm{NCMe})_{2}(59 \mathrm{mg}, 0.23 \mathrm{mmol})$ and $2(189 \mathrm{mg}, 0.23 \mathrm{mmol})$ in toluene $(6 \mathrm{~mL})$ was heated at reflux for 48 hours. All volatiles were then removed in vacuo and the orange expanded oil obtained triturated with hexane. Precipitation of $\left[(\mathrm{PCCCPH}) \mathrm{PdCl}_{2}\right]_{\mathrm{x}}$ species from a solution of $1: 1$ hexane:toluene at $-15^{\circ} \mathrm{C}$, followed by recrystallisation from $3: 1$ hexane/toluene at $-15{ }^{\circ} \mathrm{C}$ gave $\mathbf{2 6}$ as a yellow, microcrystalline solid (142 mg, 64\%). ${ }^{1} \mathrm{H}-\mathrm{NMR}$ : (300 MHz, $\left.\mathrm{CDCl}_{3}\right) \delta_{\mathrm{H}} 7.08(\mathrm{~m}, 3 \mathrm{H}$, H-4,5,6). ${ }^{31} \mathrm{P}-\mathrm{NMR}:\left(121 \mathrm{MHz}, \mathrm{CDCl}_{3}\right) \delta_{\mathrm{P}} 5.9(\mathrm{~s}) .{ }^{19} \mathrm{~F}-\mathrm{NMR}:\left(282 \mathrm{MHz}, \mathrm{CDCl}_{3}\right) \delta_{\mathrm{F}}$ $-126.7\left(\mathrm{~d},{ }^{3} J_{\mathrm{P}-\mathrm{F}}=20.3 \mathrm{~Hz}, 8 \mathrm{~F}, o-\mathrm{C}_{6} \mathrm{~F}_{5}\right),-144.2\left(\mathrm{t},{ }^{3} J_{\mathrm{F}-\mathrm{F}}=20.6 \mathrm{~Hz}, 4 \mathrm{~F}, p-\mathrm{C}_{6} \mathrm{~F}_{5}\right)$, $-157.6\left(\mathrm{~m}, 8 \mathrm{~F}, m-\mathrm{C}_{6} \mathrm{~F}_{5}\right) .{ }^{13} \mathrm{C}-\mathrm{NMR}$ : $\left(150 \mathrm{MHz}, \mathrm{CDCl}_{3}\right) \delta_{\mathrm{C}} 154.9$ (s, 1C, C-2), 147.3 $\left(\mathrm{dm},{ }^{1} J_{\mathrm{F}-\mathrm{C}}=253 \mathrm{~Hz}, 8 \mathrm{C}, o-\mathrm{C}_{6} \mathrm{~F}_{5}\right), 144.8\left(\mathrm{t},{ }^{2} J_{\mathrm{P}-\mathrm{C}}=11.0 \mathrm{~Hz}, 2 \mathrm{C}, \mathrm{C}-1,3\right), 144.3(\mathrm{dm}$, $\left.{ }^{1} J_{\mathrm{F}-\mathrm{C}}=262 \mathrm{~Hz}, 4 \mathrm{C}, p-\mathrm{C}_{6} \mathrm{~F}_{5}\right), 138.2\left(\mathrm{dm},{ }^{1} J_{\mathrm{F}-\mathrm{C}}=257 \mathrm{~Hz}, 8 \mathrm{C}, m-\mathrm{C}_{6} \mathrm{~F}_{5}\right), 127.7(\mathrm{~s}$, $1 \mathrm{C}, \mathrm{C}-5), 124.7\left(\mathrm{t},{ }^{3} J_{\mathrm{P}-\mathrm{C}}=12.8 \mathrm{~Hz}, 2 \mathrm{C}, \mathrm{C}-4,6\right), 103.4\left(\mathrm{~m}, 4 \mathrm{C}, i-\mathrm{C}_{6} \mathrm{~F}_{5}\right), 42.7$ (br t,

$\left.{ }^{1} J_{\mathrm{P}-\mathrm{C}}=\mathrm{nr}, 2 \mathrm{C}, \mathrm{CH}_{2}\right)$. Anal. Calcd for $\mathrm{C}_{32} \mathrm{H}_{7} \mathrm{~F}_{20} \mathrm{P}_{2} \mathrm{ClPt} \cdot \frac{1}{2} \mathrm{C}_{7} \mathrm{H}_{8}: \mathrm{C}, 41.65 ; \mathrm{H}, 1.07$. Found $\mathrm{C}, 41.50 ; \mathrm{H}, 1.18$. HRMS calcd for $\left(\mathrm{C}_{34} \mathrm{H}_{10} \mathrm{NF}_{20} \mathrm{P} 2 \mathrm{Pt}\right)\left[\mathrm{M}-\mathrm{Cl}+\mathrm{CH}_{3} \mathrm{CN}\right]^{+}$: 
$m / z=975.9025$, found $=975.9022$.

\section{$[(\mathrm{POCCP}) \mathrm{PdCl}](30)$}

A solution of $\left[\mathrm{PdCl}_{2}(\mathrm{NCMe})_{2}\right](71.9 \mathrm{mg}, 0.28 \mathrm{mmol})$ and $3(172 \mathrm{mg}, 0.28 \mathrm{mmol})$ in toluene $(6 \mathrm{~mL})$ was heated at $100{ }^{\circ} \mathrm{C}$ for 20 hours, at which point all volatiles were removed in vacuo. The resultant oil was triturated with hexane and washed with pentane, affording 30 as a bright yellow solid (115 mg, 55\%). ${ }^{1} \mathrm{H}-\mathrm{NMR}$ : $(500 \mathrm{MHz}$, $\left.\mathrm{CDCl}_{3}\right) \delta_{\mathrm{H}} 7.04\left(\mathrm{vt},{ }^{3} J_{\mathrm{H}-\mathrm{H}}=7.7 \mathrm{~Hz}, 1 \mathrm{H}, \mathrm{H}-5\right), 6.95\left(\mathrm{~d},{ }^{3} J_{\mathrm{H}-\mathrm{H}}=7.6 \mathrm{~Hz}, 1 \mathrm{H}, \mathrm{H}-\right.$ 4), $6.76\left(\mathrm{~d},{ }^{3} J_{\mathrm{H}-\mathrm{H}}=7.8 \mathrm{~Hz}, 1 \mathrm{H}, \mathrm{H}-6\right), 3.47\left(\mathrm{~d},{ }^{2} J_{\mathrm{P}-\mathrm{H}}=9.5 \mathrm{~Hz}, 2 \mathrm{H}, \mathrm{CH}_{2}\right), 1.45$ $\left(\mathrm{d},{ }^{3} J_{\mathrm{P}-\mathrm{H}}=14.4 \mathrm{~Hz}, 18 \mathrm{H}, \mathrm{C}\left(\mathrm{CH}_{3}\right)_{3}\right) .{ }^{31} \mathrm{P}-\mathrm{NMR}:\left(121 \mathrm{MHz}, \mathrm{CDCl}_{3}\right) \delta_{\mathrm{P}} 111.5(\mathrm{~d}$, $\left.{ }^{2} J_{\mathrm{P}-\mathrm{P}}=460 \mathrm{~Hz}, 1 \mathrm{P}, \mathrm{P}\left(\mathrm{C}_{6} \mathrm{~F}_{5}\right)_{2}\right), 83.5\left(\mathrm{~d},{ }^{2} J_{\mathrm{P}-\mathrm{P}}=460 \mathrm{~Hz}, 1 \mathrm{P}, \mathrm{P}^{t} \mathrm{Bu}_{2}\right) .{ }^{19} \mathrm{~F}-\mathrm{NMR}$ : $\left(282 \mathrm{MHz}, \mathrm{CDCl}_{3}\right) \delta_{\mathrm{F}}-127.8\left(\mathrm{dm},{ }^{3} J_{\mathrm{P}-\mathrm{F}}=21.3 \mathrm{~Hz}, 4 \mathrm{~F}, o-\mathrm{C}_{6} \mathrm{~F}_{5}\right),-144.4\left(\mathrm{t},{ }^{3} J_{\mathrm{F}-\mathrm{F}}\right.$ $\left.=20.6 \mathrm{~Hz}, 2 \mathrm{~F}, p-\mathrm{C}_{6} \mathrm{~F}_{5}\right),-158.3\left(\mathrm{~m}, 4 \mathrm{~F}, m-\mathrm{C}_{6} \mathrm{~F}_{5}\right) .{ }^{13} \mathrm{C}-\mathrm{NMR}:\left(125 \mathrm{MHz}, \mathrm{CDCl}_{3}\right)$ $\delta_{\mathrm{C}} 162.8\left(\mathrm{dd},{ }^{2} J_{\mathrm{P}-\mathrm{C}}=21.1 \mathrm{~Hz},{ }^{4} J_{\mathrm{P}-\mathrm{C}}=\mathrm{nr}, 1 \mathrm{C}, \mathrm{C}-1\right), 151.4\left(\mathrm{dd},{ }^{2} J_{\mathrm{P}-\mathrm{C}}=15.4 \mathrm{~Hz}\right.$, $\left.{ }^{4} J_{\mathrm{P}-\mathrm{C}}=1.9 \mathrm{~Hz}, 1 \mathrm{C}, \mathrm{C}-3\right), 146.7\left(\mathrm{dm},{ }^{1} J_{\mathrm{F}-\mathrm{C}}=256 \mathrm{~Hz}, 4 \mathrm{C}, o-\mathrm{C}_{6} \mathrm{~F}_{5}\right), 144.5(\mathrm{dm}$, $\left.{ }^{1} J_{\mathrm{F}-\mathrm{C}}=267 \mathrm{~Hz}, 2 \mathrm{C}, p-\mathrm{C}_{6} \mathrm{~F}_{5}\right), 144.0(\mathrm{~s}, 1 \mathrm{C}, \mathrm{C}-2), 137.8\left(\mathrm{dm},{ }^{1} J_{\mathrm{F}-\mathrm{C}}=252 \mathrm{~Hz}\right.$, $\left.4 \mathrm{C}, m-\mathrm{C}_{6} \mathrm{~F}_{5}\right), 127.6(\mathrm{~s}, 1 \mathrm{C}, \mathrm{C}-5), 119.9\left(\mathrm{~d},{ }^{3} J_{\mathrm{P}-\mathrm{C}}=20.1 \mathrm{~Hz}, 1 \mathrm{C}, \mathrm{C}-4\right), 111.1$ (d, $\left.{ }^{3} J_{\mathrm{P}-\mathrm{C}}=19.2 \mathrm{~Hz}, 1 \mathrm{C}, \mathrm{C}-6\right), 106.8\left(\mathrm{~m}, 2 \mathrm{C}, i-\mathrm{C}_{6} \mathrm{~F}_{5}\right), 35.8\left(\mathrm{dd},{ }^{1} J_{\mathrm{P}-\mathrm{C}}=13.3 \mathrm{~Hz}\right.$, $\left.{ }^{3} J_{\mathrm{P}-\mathrm{C}}=5.1 \mathrm{~Hz}, 2 \mathrm{C}, C\left(\mathrm{CH}_{3}\right)_{3}\right), 35.0\left(\mathrm{dd},{ }^{1} J_{\mathrm{P}-\mathrm{C}}=26.4 \mathrm{~Hz},{ }^{3} J_{\mathrm{P}-\mathrm{C}}=3.4 \mathrm{~Hz}, 1 \mathrm{C}\right.$, $\left.\mathrm{CH}_{2}\right), 29.1\left(\mathrm{dd},{ }^{2} J_{\mathrm{P}-\mathrm{C}}=4.3 \mathrm{~Hz},{ }^{4} J_{\mathrm{P}-\mathrm{C}}=2.4 \mathrm{~Hz}, 6 \mathrm{C}, \mathrm{C}\left(\mathrm{CH}_{3}\right)_{3}\right.$. Anal. Calcd for $\mathrm{C}_{27} \mathrm{H}_{23} \mathrm{OF}_{10} \mathrm{P}_{2} \mathrm{ClPd} \cdot \frac{1}{2} \mathrm{CH}_{2} \mathrm{Cl}_{2}$ : C, 41.38; $\mathrm{H}, 3.03$. Found $\mathrm{C}, 41.28 ; \mathrm{H}, 3.08$. HRMS calcd for $\left(\mathrm{C}_{29} \mathrm{H}_{26} \mathrm{NOF}_{10} \mathrm{P}_{2} \mathrm{Pt}\right)\left[\mathrm{M}-\mathrm{Cl}+\mathrm{CH}_{3} \mathrm{CN}\right]^{+}: \mathrm{m} / z=758.0386$, found $=$ 758.0357. HRMS calcd for $\left(\mathrm{C}_{23} \mathrm{H}_{27} \mathrm{NO}_{2} \mathrm{~F}_{5} \mathrm{P}_{2} \mathrm{Pt}\right)\left[\mathrm{M}-\mathrm{Cl}-\mathrm{C}_{6} \mathrm{~F}_{5}+\mathrm{OH}+\mathrm{NCMe}\right]^{+}: \mathrm{m} / z$ $=612.0493$, found $=612.0456$.

\section{$[(\mathrm{POCOP}) \mathrm{Pd}(\mathrm{CO})]\left[\mathrm{SbF}_{6}\right](34)$}

A foil-wrapped flask containing 28 (64.1 mg, $66 \mu \mathrm{mol})$ and $\mathrm{AgSbF}_{6}(23.0 \mathrm{mg}$, $67 \mu \mathrm{mol})$ was cooled to $-78^{\circ} \mathrm{C}$ and dichloromethane $(9 \mathrm{~mL})$ was added. The solution was stirred at $-78{ }^{\circ} \mathrm{C}$ for 10 minutes, then carbon monoxide was bubbled through solution as the reaction mixture was allowed to warm to room temperature. After 60 minutes the reaction mixture was filtered through celite, and the filter cake washed with dichloromethane. The filtrate was subsequently reduced in volume to approximately $1 \mathrm{~mL}$ in vacuo, and the crude product was precipitated out by the addition of $6 \mathrm{~mL}$ hexane to the dichloromethane solution saturated with carbon monoxide. Decantation of the supernatant and washing the precipitate with hexane gave 34 as an off-white solid (61.5 mg, 78\%). ${ }^{1} \mathrm{H}-\mathrm{NMR}$ : $\left(300 \mathrm{MHz}, \mathrm{CD}_{2} \mathrm{Cl}_{2}\right) \delta_{\mathrm{H}}$ 
$7.46\left(\mathrm{t},{ }^{3} J_{\mathrm{H}-\mathrm{H}}=8.1 \mathrm{~Hz}, 1 \mathrm{H}, \mathrm{H}-5\right), 7.03\left(\mathrm{~d},{ }^{3} J_{\mathrm{H}-\mathrm{H}}=8.3 \mathrm{~Hz}, 1 \mathrm{H}, \mathrm{H}-4,6\right) .{ }^{31} \mathrm{P}-\mathrm{NMR}$ : $\left(121 \mathrm{MHz}, \mathrm{CD}_{2} \mathrm{Cl}_{2}\right) \delta_{\mathrm{P}} 119.4(\mathrm{~s}) .{ }^{19} \mathrm{~F}-\mathrm{NMR}:\left(282 \mathrm{MHz}, \mathrm{CD}_{2} \mathrm{Cl}_{2}\right) \delta_{\mathrm{F}}-129.5(\mathrm{~m}, 8 \mathrm{~F}$, $\left.o-\mathrm{C}_{6} \mathrm{~F}_{5}\right),-140.0\left(\mathrm{tt},{ }^{3} J_{\mathrm{F}-\mathrm{F}}=20.6 \mathrm{~Hz},{ }^{5} J_{\mathrm{F}-\mathrm{F}}=7.8 \mathrm{~Hz}, 4 \mathrm{~F}, p-\mathrm{C}_{6} \mathrm{~F}_{5}\right),-156.9(\mathrm{~m}, 8 \mathrm{~F}$, $\left.p-\mathrm{C}_{6} \mathrm{~F}_{5}\right) \cdot{ }^{13} \mathrm{C}-\mathrm{NMR}:\left(150 \mathrm{MHz}, \mathrm{CD}_{2} \mathrm{Cl}_{2}\right) \delta_{\mathrm{C}} 176.0(\mathrm{~s}, 1 \mathrm{C}, \mathrm{CO}), 163.1$ (t, ${ }^{2} J_{\mathrm{P}-\mathrm{C}}=$ $8.1 \mathrm{~Hz}, 2 \mathrm{C}, \mathrm{C}-1,3), 147.1\left(\mathrm{dm},{ }^{1} J_{\mathrm{F}-\mathrm{C}}=255 \mathrm{~Hz}, 8 \mathrm{C}, o^{o}-\mathrm{C}_{6} \mathrm{~F}_{5}\right), 146.7\left(\mathrm{dm},{ }^{1} J_{\mathrm{F}-\mathrm{C}}=\right.$ $\left.268 \mathrm{~Hz}, 4 \mathrm{C}, p-\mathrm{C}_{6} \mathrm{~F}_{5}\right), 139.0\left(\mathrm{dm},{ }^{1} J_{\mathrm{F}-\mathrm{C}}=258 \mathrm{~Hz}, 8 \mathrm{C}, m-\mathrm{C}_{6} \mathrm{~F}_{5}\right), 134.3(\mathrm{~s}, 1 \mathrm{C}, \mathrm{C}-5)$, 132.0 (br s, 1C, C-2), 110.4 (t, $\left.{ }^{3} J_{\mathrm{P}-\mathrm{C}}=9.9 \mathrm{~Hz}, 2 \mathrm{C}, \mathrm{C}-4,6\right), 103.7$ (m, 4C, $i-\mathrm{C}_{6} \mathrm{~F}_{5}$ ). IR $(\mathrm{KBr}): \nu_{\max }(\mathrm{CO})=2170 \mathrm{~cm}^{-1}$.

\section{$[(\mathrm{PCCCP}) \mathrm{Pd}(\mathrm{CO})]\left[\mathrm{SbF}_{6}\right]$}

A foil-wrapped flask containing 29 (75 mg, $77 \mu \mathrm{mol})$ and $\operatorname{AgSbF}_{6}(28 \mathrm{mg}, 81 \mu \mathrm{mol})$ was cooled to $-78{ }^{\circ} \mathrm{C}$ and dichloromethane $(10 \mathrm{~mL})$ was added. The solution was stirred at $-78{ }^{\circ} \mathrm{C}$ for 5 minutes, then carbon monoxide was bubbled through solution as it was allowed to warm to room temperature over the course of 15 minutes. The reaction mixture was stirred under a carbon monoxide atmosphere for a further 12 hours, then filtered through celite, washing the filter cake with dichloromethane. The filtrate was subsequently reduced in volume to approximately $2 \mathrm{~mL}$ in vacuo, and the crude product was precipitated out by the addition of $20 \mathrm{~mL}$ hexane into the dichloromethane solution saturated with carbon monoxide. Decantation of the supernatant and washing the precipitate with hexane gave $\mathbf{3 4}$ as a white microcrystalline solid (35 mg, 38\%).

An alternate synthesis of $\mathbf{3 5}$ containing the tetrafluoroborate anion was performed as follows, filtering the reaction mixture after halide abstraction but prior to carbonylation, owing to the lower than expected solubility of $\mathbf{3 5}$ in dichloromethane: A foil-wrapped flask containing $29(89.8 \mathrm{mg}, 0.092 \mathrm{mmol})$ and $\mathrm{AgBF}_{4}(23.9 \mathrm{mg}$, $0.123 \mathrm{~mol})$ was cooled to $-78{ }^{\circ} \mathrm{C}$ and dichloromethane $(9 \mathrm{~mL})$ was added. After stirring for 30 minutes at $-78{ }^{\circ} \mathrm{C}$ and 5 hours at room temperature, the reaction mixture was filtered through celite and then carbon monoxide was bubbled through solution for 15 minutes. Slow evaporation of a dichloromethane solution of $\mathbf{3 5}$ saturated with carbon monoxide yielded $[(\mathrm{PCCCP}) \mathrm{Pd}(\mathrm{CO})]\left[\mathrm{BF}_{4}\right]$ as colourless crystals (61.7 mg, 64\%). ${ }^{1} \mathrm{H}-\mathrm{NMR}:\left(300 \mathrm{MHz}, \mathrm{CD}_{2} \mathrm{Cl}_{2}\right) \delta_{\mathrm{H}} 7.34(\mathrm{~m}, 3 \mathrm{H}, \mathrm{H}-4,5,6), 4.63$ (t, $\left.{ }^{2} J_{\mathrm{P}-\mathrm{H}}=5.6 \mathrm{~Hz}, 4 \mathrm{H}, \mathrm{CH}_{2}\right) .{ }^{31} \mathrm{P}-\mathrm{NMR}:\left(121 \mathrm{MHz}, \mathrm{CD}_{2} \mathrm{Cl}_{2}\right) \delta_{\mathrm{P}} 7.1$ (s). ${ }^{19} \mathrm{~F}-\mathrm{NMR}$ : $\left(282 \mathrm{MHz}, \mathrm{CD}_{2} \mathrm{Cl}_{2}\right) \delta_{\mathrm{F}}-128.7\left(\mathrm{~m}, 8 \mathrm{~F}, o-\mathrm{C}_{6} \mathrm{~F}_{5}\right),-142.5\left(\mathrm{tt},{ }^{3} J_{\mathrm{F}-\mathrm{F}}=20.9 \mathrm{~Hz},{ }^{5} J_{\mathrm{F}-\mathrm{F}}\right.$ $\left.=6.3 \mathrm{~Hz}, 4 \mathrm{~F}, p-\mathrm{C}_{6} \mathrm{~F}_{5}\right),-157.1\left(\mathrm{~m}, 8 \mathrm{~F}, m-\mathrm{C}_{6} \mathrm{~F}_{5}\right) \cdot{ }^{13} \mathrm{C}-\mathrm{NMR}:\left(150 \mathrm{MHz}, \mathrm{CD}_{2} \mathrm{Cl}_{2}\right) \delta_{\mathrm{C}}$ 177.9 (br s, 1C, CO), $157.2(\mathrm{~s}, 1 \mathrm{C}, \mathrm{C}-2), 146.5\left(\mathrm{dm},{ }^{1} J_{\mathrm{F}-\mathrm{C}}=253 \mathrm{~Hz}, 8 \mathrm{C}, o-\mathrm{C}_{6} \mathrm{~F}_{5}\right.$ ), $144.5\left(\mathrm{t},{ }^{2} J_{\mathrm{P}-\mathrm{C}}=\mathrm{nr}, 2 \mathrm{C}, \mathrm{C}-1,3\right), 144.1\left(\mathrm{dm},{ }^{1} J_{\mathrm{F}-\mathrm{C}}=261 \mathrm{~Hz}, 4 \mathrm{C}, p-\mathrm{C}_{6} \mathrm{~F}_{5}\right), 137.6$ $\left(\mathrm{dm},{ }^{1} J_{\mathrm{F}-\mathrm{C}}=255 \mathrm{~Hz}, 8 \mathrm{C}, m-\mathrm{C}_{6} \mathrm{~F}_{5}\right), 129.3(\mathrm{~s}, 1 \mathrm{C}, 5-\mathrm{C}), 124.3\left(\mathrm{t},{ }^{3} J_{\mathrm{PC}}=14.2 \mathrm{~Hz}, 2 \mathrm{C}\right.$, 
$\mathrm{C}-4,6), 101.5\left(\mathrm{~m}, 4 \mathrm{C}, i-\mathrm{C}_{6} \mathrm{~F}_{5}\right), 41.6\left(\mathrm{t},{ }^{1} J_{\mathrm{P}-\mathrm{C}}=\mathrm{nr}, 2 \mathrm{C}, \mathrm{CH}_{2}\right) . \mathrm{IR}(\mathrm{KBr}): \nu_{\max }(\mathrm{CO})$ $=2148 \mathrm{~cm}^{-1}$.

\section{$[(\mathrm{POCCP}) \mathrm{Pd}(\mathrm{CO})]\left[\mathrm{SbF}_{6}\right](36)$}

A foil-wrapped flask containing $30(51.2 \mathrm{mg}, 67 \mu \mathrm{mol})$ and $\mathrm{AgSbF}_{6}(24.1 \mathrm{mg}$, $70 \mu \mathrm{mol})$ was cooled to $-78{ }^{\circ} \mathrm{C}$ and dichloromethane $(6 \mathrm{~mL})$ was added. Carbon monoxide was then bubbled through solution as the reaction mixture was allowed to warm to room temperature. After 20 minutes the carbon monoxide addition was stopped, the reaction mixture left to stir for a further hour, then subsequently filtered through celite, and the filter cake washed with dichloromethane. The solvent was reduced in vacuo and the resultant residue triturated and washed with hexane to give 36 as a yellow solid (41.0 mg, 62\%). ${ }^{1} \mathrm{H}-\mathrm{NMR}$ : $\left(300 \mathrm{MHz}, \mathrm{CD}_{2} \mathrm{Cl}_{2}\right) \delta_{\mathrm{H}} 7.33$ $\left(\mathrm{t},{ }^{3} J_{\mathrm{H}-\mathrm{H}}=7.7 \mathrm{~Hz}, 1 \mathrm{H}, \mathrm{H}-5\right), 7.19\left(\mathrm{~d},{ }^{3} J_{\mathrm{H}-\mathrm{H}}=7.6 \mathrm{~Hz}, 1 \mathrm{H}, \mathrm{H}-4\right), 7.06\left(\mathrm{~d},{ }^{3} J_{\mathrm{H}-\mathrm{H}}=\right.$ $7.9 \mathrm{~Hz}, 1 \mathrm{H}, \mathrm{H}-6), 3.85\left(\mathrm{~d},{ }^{2} J_{\mathrm{P}-\mathrm{H}}=10.0 \mathrm{~Hz}, 2 \mathrm{H}, \mathrm{CH}_{2}\right), 1.47\left(\mathrm{~d},{ }^{3} J_{\mathrm{P}-\mathrm{H}}=15.3 \mathrm{~Hz}\right.$, 18H, C $\left.\left(\mathrm{CH}_{3}\right)_{3}\right) .{ }^{31} \mathrm{P}-\mathrm{NMR}:\left(121 \mathrm{MHz}, \mathrm{CD}_{2} \mathrm{Cl}_{2}\right) \delta_{\mathrm{P}} 117.0$ (br dq, ${ }^{2} J_{\mathrm{P}-\mathrm{P}}=320 \mathrm{~Hz}$, $\left.{ }^{3} J_{\mathrm{P}-\mathrm{F}}=16.7 \mathrm{~Hz}, 1 \mathrm{P}, \mathrm{P}\left(\mathrm{C}_{6} \mathrm{~F}_{5}\right)_{2}\right), 109.1\left(\mathrm{~d},{ }^{2} J_{\mathrm{P}-\mathrm{P}}=320 \mathrm{~Hz}, 1 \mathrm{P}, \mathrm{P}^{t} \mathrm{Bu}_{2}\right) .{ }^{19} \mathrm{~F}-\mathrm{NMR}$ : $\left(282 \mathrm{MHz}, \mathrm{CD}_{2} \mathrm{Cl}_{2}\right) \delta_{\mathrm{F}}-130.0\left(\mathrm{~m}, 4 \mathrm{~F}, o-\mathrm{C}_{6} \mathrm{~F}_{5}\right),-141.0\left(\mathrm{tt},{ }^{3} J_{\mathrm{F}-\mathrm{F}}=20.6 \mathrm{~Hz},{ }^{5} J_{\mathrm{F}-\mathrm{F}}\right.$ $\left.=7.5 \mathrm{~Hz}, 2 \mathrm{~F}, p-\mathrm{C}_{6} \mathrm{~F}_{5}\right),-156.7\left(\mathrm{~m}, 4 \mathrm{~F}, m-\mathrm{C}_{6} \mathrm{~F}_{5}\right) .{ }^{13} \mathrm{C}-\mathrm{NMR}:\left(150 \mathrm{MHz}, \mathrm{CD}_{2} \mathrm{Cl}_{2}\right)$ $\delta_{\mathrm{C}} 179.7(\mathrm{br} s, 1 \mathrm{C}, \mathrm{CO}), 163.2\left(\mathrm{~d},{ }^{2} J_{\mathrm{P}-\mathrm{C}}=19.7 \mathrm{~Hz}, 1 \mathrm{C}, \mathrm{C}-1\right), 152.7\left(\mathrm{~d},{ }^{2} J_{\mathrm{P}-\mathrm{C}}=\right.$ $12.8 \mathrm{~Hz}, 1 \mathrm{C}, \mathrm{C}-3), 147.8$ ( $\mathrm{s}, 1 \mathrm{C}, \mathrm{C}-2), 146.7$ (dm, $\left.{ }^{1} J_{\mathrm{F}-\mathrm{C}}=252 \mathrm{~Hz}, 4 \mathrm{C}, o-\mathrm{C}_{6} \mathrm{~F}_{5}\right), 145.8$ $\left(\mathrm{dm},{ }^{1} J_{\mathrm{F}-\mathrm{C}}=264 \mathrm{~Hz}, 2 \mathrm{C}, p-\mathrm{C}_{6} \mathrm{~F}_{5}\right), 138.5\left(\mathrm{dm},{ }^{1} J_{\mathrm{F}-\mathrm{C}}=256 \mathrm{~Hz}, 4 \mathrm{C}, m-\mathrm{C}_{6} \mathrm{~F}_{5}\right), 131.5$ (s, 1C, C-5), $121.4\left(\mathrm{~d},{ }^{3} J_{\mathrm{P}-\mathrm{C}}=22.0 \mathrm{~Hz}, 1 \mathrm{C}, \mathrm{C}-4\right), 112.4\left(\mathrm{~d},{ }^{3} J_{\mathrm{P}-\mathrm{C}}=20.3 \mathrm{~Hz}, 1 \mathrm{C}\right.$, $\mathrm{C}-6), 104.5\left(\mathrm{dm},{ }^{1} J_{\mathrm{P}-\mathrm{C}}=43.9 \mathrm{~Hz}, 2 \mathrm{C}, i-\mathrm{C}_{6} \mathrm{~F}_{5}\right), 37.1\left(\mathrm{dd},{ }^{1} J_{\mathrm{P}-\mathrm{C}}=15.0 \mathrm{~Hz},{ }^{3} J_{\mathrm{P}-\mathrm{C}}=\right.$ $\left.3.5 \mathrm{~Hz}, 2 \mathrm{C}, \mathrm{C}\left(\mathrm{CH}_{3}\right)_{3}\right), 35.8\left(\mathrm{~d},{ }^{1} J_{\mathrm{P}-\mathrm{C}}=29.5 \mathrm{~Hz}, 1 \mathrm{C}, \mathrm{CH}_{2}\right), 28.9\left(\mathrm{~s}, 6 \mathrm{C}, \mathrm{C}\left(\mathrm{CH}_{3}\right)_{3}\right)$. IR (ATR film from $\mathrm{CH}_{2} \mathrm{Cl}_{2}$ ): $\nu_{\max }(\mathrm{CO})=2140 \mathrm{~cm}^{-1}$.

\section{Observation of $[(\mathrm{PCP}) \mathrm{Pd}(\mathrm{CO})]\left[\mathrm{SbF}_{6}\right]$ decarbonylation products $[(\mathrm{PCP}) \mathrm{Pd}]\left[\mathrm{SbF}_{6}\right](38,39$, and 40)}

Compounds 38, 39, and 40 were observed in solution arising from the decarbonylation of the parent palladium carbonyl compounds 34, 35, and 36 respectively, either by passage of inert gas through dichloromethane solutions, or upon prolonged standing under ambient conditions.

[(POCOP)Pd] $\left[\mathrm{SbF}_{6}\right]$ (38): ${ }^{1} \mathrm{H}-\mathrm{NMR}:\left(300 \mathrm{MHz}, \mathrm{CD}_{2} \mathrm{Cl}_{2}\right) \delta_{\mathrm{H}} 7.30\left(\mathrm{t},{ }^{3} J_{\mathrm{H}-\mathrm{H}}=\right.$ $7.8 \mathrm{~Hz}, 1 \mathrm{H}, \mathrm{H}-5), 8.85$ (d, $\left.{ }^{3} J_{\mathrm{H}-\mathrm{H}}=8.0 \mathrm{~Hz}, 2 \mathrm{H}, \mathrm{H}-4,6\right) .{ }^{31} \mathrm{P}-\mathrm{NMR}:\left(121 \mathrm{MHz}, \mathrm{CD}_{2} \mathrm{Cl}_{2}\right.$ ) $\delta_{\mathrm{P}} 112.6(\mathrm{~s}) .{ }^{19} \mathrm{~F}-\mathrm{NMR}:\left(282 \mathrm{MHz}, \mathrm{CD}_{2} \mathrm{Cl}_{2}\right) \delta_{\mathrm{F}}-129.9\left(\mathrm{br} \mathrm{s}, 8 \mathrm{~F}, o-\mathrm{C}_{6} \mathrm{~F}_{5}\right),-141.0$ 
$\left(\mathrm{tm},{ }^{3} J_{\mathrm{F}-\mathrm{F}}=20.5 \mathrm{~Hz}, 4 \mathrm{~F}, p-\mathrm{C}_{6} \mathrm{~F}_{5}\right),-157.5\left(\mathrm{tm},{ }^{3} J_{\mathrm{F}-\mathrm{F}}=18.9 \mathrm{~Hz}, 8 \mathrm{~F}, m-\mathrm{C}_{6} \mathrm{~F}_{5}\right)$.

[(PCCCP)Pd] $\left[\mathrm{SbF}_{6}\right]$ (39): ${ }^{1} \mathrm{H}-\mathrm{NMR}:\left(300 \mathrm{MHz}, \mathrm{CD}_{2} \mathrm{Cl}_{2}\right) \delta_{\mathrm{H}} 7.15$ (br s, 3H, H$4,5,6), 4.38\left(\mathrm{t},{ }^{2} J_{\mathrm{P}-\mathrm{H}}=4.7 \mathrm{~Hz}, 4 \mathrm{H}, \mathrm{CH}_{2}\right) .{ }^{31} \mathrm{P}-\mathrm{NMR}:\left(121 \mathrm{MHz}, \mathrm{CD}_{2} \mathrm{Cl}_{2}\right) \delta_{\mathrm{P}} 1.7$ (s). ${ }^{19} \mathrm{~F}-\mathrm{NMR}:\left(282 \mathrm{MHz}, \mathrm{CD}_{2} \mathrm{Cl}_{2}\right) \delta_{\mathrm{F}}-129.7\left(\mathrm{~m}, 8 \mathrm{~F}, o-\mathrm{C}_{6} \mathrm{~F}_{5}\right),-143.6\left(\mathrm{tm},{ }^{3} J_{\mathrm{F}-\mathrm{F}}\right.$ $\left.=20.4 \mathrm{~Hz}, 4 \mathrm{~F}, p-\mathrm{C}_{6} \mathrm{~F}_{5}\right),-157.8\left(\mathrm{tm},{ }^{3} J_{\mathrm{F}-\mathrm{F}}=20.2 \mathrm{~Hz}, 8 \mathrm{~F}, m-\mathrm{C}_{6} \mathrm{~F}_{5}\right) .{ }^{13} \mathrm{C}-\mathrm{NMR}$ : $\left(150 \mathrm{MHz}, \mathrm{CD}_{2} \mathrm{Cl}_{2}\right) \delta_{\mathrm{C}} 147.3\left(\mathrm{dm},{ }^{1} J_{\mathrm{F}-\mathrm{C}}=247 \mathrm{~Hz}, 8 \mathrm{C}, o-\mathrm{C}_{6} \mathrm{~F}_{5}\right), 144.9(\mathrm{~m}, 3 \mathrm{C}$, $\mathrm{C}-1,2,3), 144.7\left(\mathrm{dm},{ }^{1} J_{\mathrm{F}-\mathrm{C}}=264 \mathrm{~Hz}, 4 \mathrm{C}, p-\mathrm{C}_{6} \mathrm{~F}_{5}\right), 138.3\left(\mathrm{dm},{ }^{1} J_{\mathrm{F}-\mathrm{C}}=257 \mathrm{~Hz}, 8 \mathrm{C}\right.$, $\left.m-\mathrm{C}_{6} \mathrm{~F}_{5}\right), 128.7(\mathrm{~s}, 1 \mathrm{C}, 5-\mathrm{C}), 125.7\left(\mathrm{t},{ }^{3} J_{\mathrm{P}-\mathrm{H}}=13.0 \mathrm{~Hz}, 2 \mathrm{C}, \mathrm{C}-4,6\right), 102.0(\mathrm{~m}, 4 \mathrm{C}$, $i-\mathrm{C}_{6} \mathrm{~F}_{5}$ ), 39.5 (br s, $2 \mathrm{C}, \mathrm{CH}_{2}$ ).

[(POCCP)Pd $]\left[\mathrm{SbF}_{6}\right] \quad(\mathbf{4 0}):{ }^{1} \mathrm{H}-\mathrm{NMR}:\left(300 \mathrm{MHz}, \mathrm{CD}_{2} \mathrm{Cl}_{2}\right) \delta_{\mathrm{H}} 7.13\left(\mathrm{t},{ }^{3} J_{\mathrm{H}-\mathrm{H}}=\right.$ $7.6 \mathrm{~Hz}, 1 \mathrm{H}, \mathrm{H}-5), 7.03\left(\mathrm{~d},{ }^{3} J_{\mathrm{H}-\mathrm{H}}=7.3 \mathrm{~Hz}, 1 \mathrm{H}, \mathrm{H}-4\right), 6.81\left(\mathrm{~d},{ }^{3} J_{\mathrm{H}-\mathrm{H}}=8.0 \mathrm{~Hz}, 1 \mathrm{H}\right.$, $\left.\mathrm{H}-6), 3.47\left(\mathrm{~d},{ }^{2} J_{\mathrm{P}-\mathrm{H}}=9.9 \mathrm{~Hz}, 2 \mathrm{H}, \mathrm{CH}_{2}\right), 1.44\left(\mathrm{~d},{ }^{3} J_{\mathrm{P}-\mathrm{H}}=14.5 \mathrm{~Hz}, 18 \mathrm{H}, \mathrm{C}_{\left(\mathrm{CH}_{3}\right.}\right)_{3}\right)$. ${ }^{31}$ P-NMR: $\left(121 \mathrm{MHz}, \mathrm{CD}_{2} \mathrm{Cl}_{2}\right) \delta_{\mathrm{P}} 107.9\left(\mathrm{br} \mathrm{dq},{ }^{2} J_{\mathrm{P}-\mathrm{P}}=392 \mathrm{~Hz},{ }^{3} J_{\mathrm{P}-\mathrm{F}}=16.5 \mathrm{~Hz}\right.$, $\left.1 \mathrm{P}, \mathrm{P}\left(\mathrm{C}_{6} \mathrm{~F}_{5}\right)_{2}\right), 87.7\left(\mathrm{~d},{ }^{2} J_{\mathrm{P}-\mathrm{P}}=392 \mathrm{~Hz}, 1 \mathrm{P}, \mathrm{P}^{t} \mathrm{Bu}_{2}\right) .{ }^{19} \mathrm{~F}-\mathrm{NMR}:\left(282 \mathrm{MHz}, \mathrm{CD}_{2} \mathrm{Cl}_{2}\right)$ $\delta_{\mathrm{F}}-130.5\left(\mathrm{~m}, 4 \mathrm{~F}, o-\mathrm{C}_{6} \mathrm{~F}_{5}\right),-142.8\left(\mathrm{t},{ }^{3} J_{\mathrm{F}-\mathrm{F}}=20.2 \mathrm{~Hz}, 2 \mathrm{~F}, p-\mathrm{C}_{6} \mathrm{~F}_{5}\right),-158.3(\mathrm{t}$, $\left.{ }^{3} J_{\mathrm{F}-\mathrm{F}}=18.9 \mathrm{~Hz}, 4 \mathrm{~F}, m-\mathrm{C}_{6} \mathrm{~F}_{5}\right)$.

\subsection{Rhodium Complexes}

\section{[(PNNNP)RhCl $](49)$}

A solution of ligand $10(343 \mathrm{mg}, 0.41 \mathrm{mmol})$ and $[\mathrm{Rh}(\mathrm{COD}) \mathrm{Cl}]_{2}(93 \mathrm{mg}, 0.19 \mathrm{mmol})$ in toluene $(6 \mathrm{~mL})$ was heated gently with a heat gun to dissolve the starting material, then heated at $100{ }^{\circ} \mathrm{C}$ for 4 hours and $75{ }^{\circ} \mathrm{C}$ for 10 hours, without stirring. The formation of bright red crystals of the toluene solvate $[(\mathrm{PNNNP}) \mathrm{RhCl}] \cdot \mathrm{xC}_{7} \mathrm{H}_{8}$ on the walls of the reaction vessel was observed, with crystallisation completed by cooling the solution to $-15{ }^{\circ} \mathrm{C}$. Decantation of the supernatant and washing of the crystalline solid with ice-cold dichloromethane destroyed the crystals, and afforded 49 as an orange powder $(297.6 \mathrm{mg}, 71 \%) .{ }^{1} \mathrm{H}-\mathrm{NMR}$ : $\left(300 \mathrm{MHz}\right.$, acetone- $\left.d_{6}\right) \delta_{\mathrm{H}} 8.72$ (br s, 2H, NH), 7.56 (t, $\left.{ }^{3} J_{\mathrm{H}-\mathrm{H}}=8.1 \mathrm{~Hz}, 1 \mathrm{H}, \mathrm{H}-5\right), 6.70\left(\mathrm{~d},{ }^{3} J_{\mathrm{H}-\mathrm{H}}=8.2 \mathrm{~Hz}, 2 \mathrm{H}\right.$, H-4,6). ${ }^{31}$ P-NMR: $\left(121 \mathrm{MHz}\right.$, acetone- $\left.d_{6}\right) \delta_{\mathrm{P}} 34.0\left(\mathrm{~d},{ }^{1} J_{\mathrm{Rh}-\mathrm{P}}=180 \mathrm{~Hz}\right) .{ }^{19} \mathrm{~F}-\mathrm{NMR}$ : $\left(282 \mathrm{MHz}\right.$, acetone- $\left.d_{6}\right) \delta_{\mathrm{F}}-131.1\left(\mathrm{~d},{ }^{3} J_{\mathrm{P}-\mathrm{F}}=20.9 \mathrm{~Hz}, 8 \mathrm{~F}, o-\mathrm{C}_{6} \mathrm{~F}_{5}\right),-150.6\left(\mathrm{t},{ }^{3} J_{\mathrm{F}-\mathrm{F}}\right.$ $\left.=20.3 \mathrm{~Hz}, 4 \mathrm{~F}, p-\mathrm{C}_{6} \mathrm{~F}_{5}\right),-162.2\left(\mathrm{~m}, 8 \mathrm{~F}, m-\mathrm{C}_{6} \mathrm{~F}_{5}\right) .{ }^{13} \mathrm{C}-\mathrm{NMR}:\left(125 \mathrm{MHz}\right.$, acetone- $\left.d_{6}\right)$ $\delta_{\mathrm{C}} 158.9(\mathrm{~s}, 2 \mathrm{C}, \mathrm{C}-1,3), 146.8\left(\mathrm{dm},{ }^{1} J_{\mathrm{F}-\mathrm{C}}=256 \mathrm{~Hz}, 8 \mathrm{C}, o-\mathrm{C}_{6} \mathrm{~F}_{5}\right), 143.8\left(\mathrm{dm},{ }^{1} J_{\mathrm{F}-\mathrm{C}}=\right.$ $258 \mathrm{~Hz}, 4 \mathrm{C}, p-\mathrm{C}_{6} \mathrm{~F}_{5}$ ), $137.2(\mathrm{~s}, 1 \mathrm{C}, \mathrm{C}-5), 137.7\left(\mathrm{dm},{ }^{1} J_{\mathrm{F}-\mathrm{C}}=254 \mathrm{~Hz}, 8 \mathrm{C}, m-\mathrm{C}_{6} \mathrm{~F}_{5}\right.$ ), 
$105.3\left(\mathrm{~m}, 4 \mathrm{C}, i-\mathrm{C}_{6} \mathrm{~F}_{5}\right.$ ), 100.8 (s, 2C, C-4,6). Anal. Calcd for $\mathrm{C}_{29} \mathrm{H}_{5} \mathrm{~N}_{3} \mathrm{~F}_{20} \mathrm{P}_{2} \mathrm{ClRh}$ : C, 35.70; H, 0.52; N, 4.31. Found C, 35.56; H, 0.71; N, 4.25. HRMS calcd for $\left(\mathrm{C}_{29} \mathrm{H}_{5} \mathrm{~N}_{3} \mathrm{~F}_{20} \mathrm{P}_{2} \mathrm{Rh}\right)[\mathrm{M}-\mathrm{Cl}]^{+}: \mathrm{m} / z=939.8694$, found $=939.8701$.

\section{Methylation of 49 with dimethylzinc in toluene- $d_{8}$}

Typical procedure was as follows: To a solution of $49(15.0 \mathrm{mg}, 15 \mu \mathrm{mol})$ in toluene- $d_{8}(0.4 \mathrm{~mL})$, was added a $1.5 \mathrm{~mol} \mathrm{dm}{ }^{-3}$ dimethylzinc solution in toluene $(20 \mu \mathrm{L}, 30 \mu \mathrm{mol})$. The reaction mixture was agitated, and the progress of the reaction to form $\mathbf{5 0}$ was monitored in situ by NMR spectroscopy. The temperature of the dimethylzinc addition was also varied (at either room temperature, $0{ }^{\circ} \mathrm{C}$, or $-78{ }^{\circ} \mathrm{C}$ ) in order to determine whether the formation of the degradation product 51 was favoured at lower reaction temperatures.

Spectroscopic data for product 50, [(PNNNP)RhMe]: ${ }^{1} \mathrm{H}-\mathrm{NMR}$ : (300 MHz, toluene- $\left.d_{8}\right) \delta_{\mathrm{H}} 5.89(\mathrm{~s}, 2 \mathrm{H}, \mathrm{N}-\mathrm{H}), 5.69\left(\mathrm{~d},{ }^{3} J_{\mathrm{H}-\mathrm{H}}=7.9 \mathrm{~Hz}, 2 \mathrm{H}, \mathrm{H}-4,6\right), 1.09$ (br t, $\left.{ }^{3} J_{\mathrm{P}-\mathrm{H}}=6.3 \mathrm{~Hz}, 3 \mathrm{H}, \mathrm{Rh}-\mathrm{Me}\right) .{ }^{31} \mathrm{P}-\mathrm{NMR}:\left(121 \mathrm{MHz}\right.$, toluene- $\left.d_{8}\right) \delta_{\mathrm{P}} 36.3(\mathrm{~d}$, $\left.{ }^{1} J_{\mathrm{Rh}-\mathrm{P}}=222 \mathrm{~Hz}\right) .{ }^{19} \mathrm{~F}-\mathrm{NMR}:\left(282 \mathrm{MHz}\right.$, toluene- $\left.d_{8}\right) \delta_{\mathrm{F}}-133.4\left(\mathrm{~d},{ }^{3} J_{\mathrm{P}-\mathrm{F}}=22.3 \mathrm{~Hz}\right.$, $\left.8 \mathrm{~F}, o-\mathrm{C}_{6} \mathrm{~F}_{5}\right),-149.3\left(\mathrm{t},{ }^{3} J_{\mathrm{F}-\mathrm{F}}=20.5 \mathrm{~Hz}, 4 \mathrm{~F}, p-\mathrm{C}_{6} \mathrm{~F}_{5}\right),-159.8\left(\mathrm{~m}, 8 \mathrm{~F}, m-\mathrm{C}_{6} \mathrm{~F}_{5}\right)$. The resonance of the $\mathrm{H}-5$ environment was obscured by the presence of toluene. Approximate chemical shift $\left(\delta_{\mathrm{H}} \approx 7.0 \mathrm{ppm}\right)$ was confirmed by COSY experiment.

Spectroscopic data for degradation product 51, [(PN*NNP)RhMe $\cdot$ ZnMe $]$ : ${ }^{1} \mathrm{H}-\mathrm{NMR}:\left(300 \mathrm{MHz}\right.$, toluene- $\left.d_{8}\right) \delta_{\mathrm{H}} 5.98\left(\mathrm{~d},{ }^{3} J_{\mathrm{H}-\mathrm{H}}=7.7 \mathrm{~Hz}, 1 \mathrm{H}, \mathrm{H}-4\right.$ or H-6), $5.86\left(\mathrm{~d},{ }^{3} J_{\mathrm{H}-\mathrm{H}}=8.2 \mathrm{~Hz}, 1 \mathrm{H}, \mathrm{H}-4\right.$ or H-6), 5.39 (br s, $\left.1 \mathrm{H}, \mathrm{N}-\mathrm{H}\right), 1.21$ (br s, $3 \mathrm{H}$, Rh-Me), -0.18 (s, 3H, Zn-Me). ${ }^{31} \mathrm{P}-\mathrm{NMR}:\left(121 \mathrm{MHz}\right.$, toluene- $\left.d_{8}\right) \delta_{\mathrm{P}} 74.4$ (dd, $\left.{ }^{2} J_{\mathrm{P}-\mathrm{P}}=522 \mathrm{~Hz},{ }^{1} J_{\mathrm{Rh}-\mathrm{P}}=156 \mathrm{~Hz}, 1 \mathrm{P}, \mathrm{N}-\mathrm{P}\left(\mathrm{C}_{6} \mathrm{~F}_{5}\right)_{2}\right) 44.7\left(\mathrm{dd},{ }^{2} J_{\mathrm{P}-\mathrm{P}}=522 \mathrm{~Hz}\right.$, $\left.{ }^{1} J_{\mathrm{Rh}-\mathrm{P}}=158 \mathrm{~Hz}, 1 \mathrm{P}, \mathrm{N}-\mathrm{P}\left(\mathrm{C}_{6} \mathrm{~F}_{5}\right)_{2}\right) \cdot{ }^{19} \mathrm{~F}-\mathrm{NMR}:\left(282 \mathrm{MHz}\right.$, toluene- $\left.d_{8}\right) \delta_{\mathrm{F}}-118.1$ (br s, $1 \mathrm{~F}, o-\mathrm{C}_{6} \mathrm{~F}_{5}$ ), -124.2 (br s, $1 \mathrm{~F}, o-\mathrm{C}_{6} \mathrm{~F}_{5}$ ), -131.0 (br m, 2F, o- $\mathrm{C}_{6} \mathrm{~F}_{5}$ ), -131.8 (br m, 2F, o- $\mathrm{C}_{6} \mathrm{~F}_{5}$ ), -133.7 (br m, 2F, o- $\mathrm{C}_{6} \mathrm{~F}_{5}$ ), -144.1 (vt, ${ }^{3} J_{\mathrm{F}-\mathrm{F}}=21.7 \mathrm{~Hz}, 1 \mathrm{~F}$, $\left.p-\mathrm{C}_{6} \mathrm{~F}_{5}\right),-144.8\left(\mathrm{vt},{ }^{3} J_{\mathrm{F}-\mathrm{F}}=20.0 \mathrm{~Hz}, 1 \mathrm{~F}, p-\mathrm{C}_{6} \mathrm{~F}_{5}\right),-148.2\left(\mathrm{vt},{ }^{3} J_{\mathrm{F}-\mathrm{F}}=21.5 \mathrm{~Hz}\right.$, $\left.1 \mathrm{~F}, p-\mathrm{C}_{6} \mathrm{~F}_{5}\right),-149.6\left(\mathrm{vt},{ }^{3} J_{\mathrm{F}-\mathrm{F}}=21.0 \mathrm{~Hz}, 1 \mathrm{~F}, p-\mathrm{C}_{6} \mathrm{~F}_{5}\right),-157.8\left(\mathrm{~m}, 2 \mathrm{~F}, m-\mathrm{C}_{6} \mathrm{~F}_{5}\right)$, -158.0 (br m, $2 \mathrm{~F}, m-\mathrm{C}_{6} \mathrm{~F}_{5}$ ), -159.4 (m, $2 \mathrm{~F}, m-\mathrm{C}_{6} \mathrm{~F}_{5}$ ), -160.3 (br m, $2 \mathrm{~F}, m-\mathrm{C}_{6} \mathrm{~F}_{5}$ ). The resonance of the $\mathrm{H}-5$ environment was obscured by the presence of toluene. Approximate chemical shift $\left(\delta_{\mathrm{H}} \approx 7.1 \mathrm{ppm}\right)$ was confirmed by COSY experiment.

When traces of methyl iodide were present in the dimethylzinc solution used, reactions were observed to produce quantities of the N-methylated complex 53. 
Spectroscopic data for product 53, [(PN $\left.\left.{ }^{\mathrm{Me} N N P}\right) \mathrm{RhMe}_{2} \mathrm{Cl}\right]:{ }^{1} \mathrm{H}-\mathrm{NMR}$ : $(300 \mathrm{MHz}$, toluene- $\left.d_{8}\right) \delta_{\mathrm{H}} 6.84\left(\mathrm{vt},{ }^{3} J_{\mathrm{H}-\mathrm{H}}=7.8 \mathrm{~Hz}, 1 \mathrm{H}, \mathrm{H}-5\right), 6.21\left(\mathrm{~d},{ }^{3} J_{\mathrm{H}-\mathrm{H}}=8.2 \mathrm{~Hz}, 1 \mathrm{H}\right.$, H-4/6), 6.09 (br s, $1 \mathrm{H}, \mathrm{N}-\mathrm{H}), 5.65$ (d, $\left.{ }^{3} J_{\mathrm{H}-\mathrm{H}}=7.9 \mathrm{~Hz}, 1 \mathrm{H}, \mathrm{H}-4 / 6\right), 2.68$ (br d, ${ }^{3} J_{\mathrm{P}-\mathrm{H}}=9.7 \mathrm{~Hz}, 3 \mathrm{H}, \mathrm{N}-\mathrm{Me}$ ), 0.14 (br vt, ${ }^{3} J_{\mathrm{P}-\mathrm{H}}=6.5 \mathrm{~Hz}, 3 \mathrm{H}, \mathrm{Rh}-\mathrm{Me}$ ). ${ }^{31} \mathrm{P}-\mathrm{NMR}$ : $\left(121 \mathrm{MHz}\right.$, toluene- $\left.d_{8}\right) \delta_{\mathrm{P}} 85.9\left(\mathrm{dd},{ }^{2} J_{\mathrm{P}-\mathrm{P}}=615 \mathrm{~Hz},{ }^{1} J_{\mathrm{Rh}-\mathrm{P}}=150 \mathrm{~Hz}, 1 \mathrm{P}\right), 50.9(\mathrm{dd}$, $\left.{ }^{2} J_{\mathrm{P}-\mathrm{P}}=615 \mathrm{~Hz},{ }^{1} J_{\mathrm{Rh}-\mathrm{P}}=135 \mathrm{~Hz}, 1 \mathrm{P}\right)$. The sample of $\mathbf{5 3}$ was not sufficiently pure to allow unambiguous assignment of the ${ }^{19} \mathrm{~F}$ NMR spectrum.

\section{[(PNNNP)RhCIMeI] (54)}

To a solution of 49 (100 mg, $0.10 \mathrm{mmol})$ in THF (3 mL) was added methyl iodide in excess $(50 \mu \mathrm{L}, 0.80 \mathrm{mmol})$. After 10 minutes at room temperature the solution was reduced to about $1 \mathrm{~mL}$ in vacuo and $4 \mathrm{~mL}$ hexane added. The supernatant was isolated and dried in vacuo to give a pale orange precipitate. This crude product was purified by recrystallisation from a warm mixture of 1:2:2 acetone/hexane/diethyl ether followed by washing with diethyl ether, giving $\mathbf{5 4}$ as pale orange, microcrystalline blocks $(95 \mathrm{mg}, 83 \%) .{ }^{1} \mathrm{H}-\mathrm{NMR}$ : (300 MHz, acetone- $d_{6}$ ) $\delta_{\mathrm{H}} 8.52(\mathrm{br} \mathrm{s}, 2 \mathrm{H}, \mathrm{NH}), 7.69\left(\mathrm{tt},{ }^{3} J_{\mathrm{H}-\mathrm{H}}=8.1 \mathrm{~Hz},{ }^{5} J_{\mathrm{P}-\mathrm{H}}=1.8 \mathrm{~Hz}, 1 \mathrm{H}, \mathrm{H}-5\right), 7.05$ $\left(\mathrm{dm},{ }^{3} J_{\mathrm{H}-\mathrm{H}}=8.2 \mathrm{~Hz}, 2 \mathrm{H}, \mathrm{H}-4,6\right), 1.13\left(\mathrm{br} \mathrm{td},{ }^{3} J_{\mathrm{P}-\mathrm{H}}=6.8 \mathrm{~Hz},{ }^{2} J_{\mathrm{Rh}-\mathrm{H}}=1.7 \mathrm{~Hz}\right.$, $\left.3 \mathrm{H}, \mathrm{Rh}-\mathrm{CH}_{3}\right)$. ${ }^{31} \mathrm{P}-\mathrm{NMR}:\left(121 \mathrm{MHz}\right.$, acetone- $\left.d_{6}\right) \delta_{\mathrm{P}} 54.7\left(\mathrm{~d},{ }^{1} J_{\mathrm{Rh}-\mathrm{P}}=133 \mathrm{~Hz}\right)$. ${ }^{19} \mathrm{~F}-\mathrm{NMR}:\left(282 \mathrm{MHz}\right.$, acetone- $\left.d_{6}\right) \delta_{\mathrm{F}}-117.2\left(\mathrm{v}\right.$ br s, $2 \mathrm{~F}, o_{-}-\mathrm{C}_{6} \mathrm{~F}_{5}$ ), -127.6 (br s, 4F, $o-\mathrm{C}_{6} \mathrm{~F}_{5}$ ), $-132.3\left(\mathrm{v}\right.$ br s, $2 \mathrm{~F}, o-\mathrm{C}_{6} \mathrm{~F}_{5}$ ), -148.0 (tt, ${ }^{3} J_{\mathrm{P}-\mathrm{P}}=20.4 \mathrm{~Hz},{ }^{5} J_{\mathrm{P}-\mathrm{P}}=5.4 \mathrm{~Hz}$, $\left.2 \mathrm{~F}, p-\mathrm{C}_{6} \mathrm{~F}_{5}\right),-150.0\left(\mathrm{tt},{ }^{3} J_{\mathrm{P}-\mathrm{P}}=20.9 \mathrm{~Hz},{ }^{5} J_{\mathrm{P}-\mathrm{P}}=\mathrm{nr} \mathrm{Hz}, 2 \mathrm{~F}, p-\mathrm{C}_{6} \mathrm{~F}_{5}\right),-161.5(\mathrm{~m}$, $\left.4 \mathrm{~F}, m-\mathrm{C}_{6} \mathrm{~F}_{5}\right),-163.0\left(\mathrm{br} \mathrm{s}, 4 \mathrm{~F}, m-\mathrm{C}_{6} \mathrm{~F}_{5}\right) .{ }^{13} \mathrm{C}-\mathrm{NMR}$ : $\left(125 \mathrm{MHz}\right.$, acetone- $\left.d_{6}\right) \delta_{\mathrm{C}}$ $157.1\left(\mathrm{vt},{ }^{3} J_{\mathrm{P}-\mathrm{C}}=8.1 \mathrm{~Hz}, 2 \mathrm{C}, \mathrm{C}-1,3\right), 146.8\left(\mathrm{dm},{ }^{1} J_{\mathrm{F}-\mathrm{C}}=256 \mathrm{~Hz}, 8 \mathrm{C}, o-\mathrm{C}_{6} \mathrm{~F}_{5}\right)$, $143.8\left(\mathrm{dm},{ }^{1} J_{\mathrm{F}-\mathrm{C}}=258 \mathrm{~Hz}, 4 \mathrm{C}, p-\mathrm{C}_{6} \mathrm{~F}_{5}\right), 141.1(\mathrm{~s}, 1 \mathrm{C}, \mathrm{C}-5), 137.7\left(\mathrm{dm},{ }^{1} J_{\mathrm{F}-\mathrm{C}}=\right.$ $\left.254 \mathrm{~Hz}, 8 \mathrm{C}, m-\mathrm{C}_{6} \mathrm{~F}_{5}\right), 105.3\left(\mathrm{~m}, 4 \mathrm{C}, i-\mathrm{C}_{6} \mathrm{~F}_{5}\right), 103.3$ (s, 2C, C-4,6), 8.1 (d, ${ }^{1} J_{\mathrm{Rh}-\mathrm{C}}=$ $19.0 \mathrm{~Hz}, 1 \mathrm{C}, \mathrm{Rh}-\mathrm{CH}_{3}$ ). Anal. Calcd for $\mathrm{C}_{30} \mathrm{H}_{8} \mathrm{~N}_{3} \mathrm{~F}_{20} \mathrm{P}_{2}$ ClIRh: C, 32.24; H, 0.72; N, 3.76. Found $\mathrm{C}, 32.49 ; \mathrm{H}, 0.91 ; \mathrm{N}, 3.81$. HRMS calcd for $\left(\mathrm{C}_{30} \mathrm{H}_{8} \mathrm{~N}_{3} \mathrm{~F}_{20} \mathrm{NaP}_{2} \mathrm{ClIRh}\right)$ $[\mathrm{M}+\mathrm{Na}]^{+}: m / z=1139.7555$, found $=1139.7582$.

\section{Observation of $[(\mathrm{PNNNP}) \mathrm{RhI}](56)$}

To a solution of $49(17.3 \mathrm{mg}, 18 \mu \mathrm{mol})$ in benzene- $d_{6}$, was added an excess of sodium iodide $(21.0 \mathrm{mg}, 140 \mu \mathrm{mol})$. Analysis of the solution after 10 minutes by NMR spectroscopy revealed the formation of 56. ${ }^{1} \mathrm{H}-\mathrm{NMR}$ : (300 MHz, acetone- $d_{6}$ ) $\delta_{\mathrm{H}} 9.20(\mathrm{br} \mathrm{s}, 2 \mathrm{H}, \mathrm{NH}), 7.60\left(\mathrm{t},{ }^{3} J_{\mathrm{H}-\mathrm{H}}=8.1 \mathrm{~Hz}, 1 \mathrm{H}, \mathrm{H}-5\right), 6.93$ (m, 2H, H-4,6). ${ }^{31}$ P-NMR: $\left(121 \mathrm{MHz}\right.$, acetone- $\left.d_{6}\right) \delta_{\mathrm{P}} 40.5 \mathrm{~d},{ }^{1} J_{\mathrm{Rh}-\mathrm{P}}=170 \mathrm{~Hz} \cdot{ }^{19} \mathrm{~F}-\mathrm{NMR}:(282 \mathrm{MHz}$, 
acetone- $\left.d_{6}\right) \delta_{\mathrm{F}}-128.8\left(\mathrm{~m}, 8 \mathrm{~F}, o-\mathrm{C}_{6} \mathrm{~F}_{5}\right),-151.1\left(\mathrm{~s}, 4 \mathrm{~F}, p-\mathrm{C}_{6} \mathrm{~F}_{5}\right),-162.3(\mathrm{~m}, 8 \mathrm{~F}, m-$ $\left.\mathrm{C}_{6} \mathrm{~F}_{5}\right)$. HRMS calcd for $\left(\mathrm{C}_{31} \mathrm{H}_{8} \mathrm{~N}_{4} \mathrm{~F}_{20} \mathrm{P}_{2} \mathrm{I}_{2} \mathrm{Rh}\right)[\mathrm{M}+\mathrm{I}+\mathrm{NCMe}]^{+}: \mathrm{m} / z=1234.7044$, found $=1234.7053$.

\section{Observation of $\left[(\mathrm{PNNNP}) \mathrm{RhI}_{3}\right](55)$}

To the solution of $\mathbf{5 6}$ above was added toluene $(0.5 \mathrm{~mL})$, and the solution filtered through celite, washing with toluene $(3 \times 0.5 \mathrm{~mL})$. The filtrate had all volatiles removed in vacuo, and the residue was redissolved in benzene- $d_{6}$. To this was added one crystal of iodine. Analysis of the reaction mixture by NMR spectroscopy revealed immediate and quantitative formation of 55. ${ }^{1} \mathrm{H}-\mathrm{NMR}$ : $\left(300 \mathrm{MHz}, \mathrm{C}_{6} \mathrm{D}_{6}\right) \delta_{\mathrm{H}}$ $6.46\left(\mathrm{t},{ }^{3} J_{\mathrm{H}-\mathrm{H}}=8.2 \mathrm{~Hz}, 1 \mathrm{H}, \mathrm{H}-5\right), 6.27$ (br s, $\left.2 \mathrm{H}, \mathrm{H}-4,6\right), 5.37$ (d, ${ }^{2} J_{\mathrm{P}-\mathrm{H}}=8.0 \mathrm{~Hz}$, 2-H, NH). ${ }^{31}$ P-NMR: (121 MHz, $\left.\mathrm{C}_{6} \mathrm{D}_{6}\right) \delta_{\mathrm{P}} 36.6\left(\mathrm{~d},{ }^{1} J_{\mathrm{Rh}-\mathrm{P}}=115 \mathrm{~Hz}\right) .{ }^{19} \mathrm{~F}-\mathrm{NMR}$ : $\left(282 \mathrm{MHz}, \mathrm{C}_{6} \mathrm{D}_{6}\right) \delta_{\mathrm{F}}-112.0$ (br s, $4 \mathrm{~F}, o-\mathrm{C}_{6} \mathrm{~F}_{5}$ ), -134.2 (br s, $4 \mathrm{~F}, o-\mathrm{C}_{6} \mathrm{~F}_{5}$ ), -143.7 $\left(\mathrm{m}, 4 \mathrm{~F}, p-\mathrm{C}_{6} \mathrm{~F}_{5}\right.$ ), -156.7 (br s, $4 \mathrm{~F}, m-\mathrm{C}_{6} \mathrm{~F}_{5}$ ), -159.7 (br s, $4 \mathrm{~F}, m-\mathrm{C}_{6} \mathrm{~F}_{5}$ ), ${ }^{13} \mathrm{C}-\mathrm{NMR}$ : $\left(150 \mathrm{MHz}\right.$, acetone- $\left.d_{6}\right) \delta_{\mathrm{C}} 157.4\left(\mathrm{dt},{ }^{2} J_{\mathrm{Rh}-\mathrm{C}}=11.0 \mathrm{~Hz},{ }^{3} J_{\mathrm{P}-\mathrm{C}}=7.5 \mathrm{~Hz}, 2 \mathrm{C}, \mathrm{C}-1,3\right)$, $147.5\left(\mathrm{br} \mathrm{d},{ }^{1} J_{\mathrm{F}-\mathrm{C}}=252 \mathrm{~Hz}, 8 \mathrm{C}, o-\mathrm{C}_{6} \mathrm{~F}_{5}\right), 144.9\left(\mathrm{dm},{ }^{1} J_{\mathrm{F}-\mathrm{C}}=258 \mathrm{~Hz}, 4 \mathrm{C}, p-\mathrm{C}_{6} \mathrm{~F}_{5}\right)$, $142.9(\mathrm{~s}, 1 \mathrm{C}, \mathrm{C}-5), 138.4$ (br d, $\left.{ }^{1} J_{\mathrm{F}-\mathrm{C}}=254 \mathrm{~Hz}, 8 \mathrm{C}, m-\mathrm{C}_{6} \mathrm{~F}_{5}\right), 111.4(\mathrm{~m}, 4 \mathrm{C}, i$ $\mathrm{C}_{6} \mathrm{~F}_{5}$ ), 104.5 (s, 2C, C-4,6), HRMS calcd for $\left(\mathrm{C}_{29} \mathrm{H}_{9} \mathrm{~N}_{4} \mathrm{~F}_{20} \mathrm{P}_{2} \mathrm{I}_{3} \mathrm{Rh}\right)\left[\mathrm{M}+\mathrm{NH}_{4}\right]^{+}: \mathrm{m} / z$ $=1338.6167$, found $=1338.6207$.

\subsection{Catalytic Testing}

Standard solutions of $1 \mathrm{mmol} \mathrm{dm}{ }^{-3}$ [(POCOP)PdCl] (28), [(PCCCP)PdCl] (29), and [(POCCP) $\mathrm{PdCl}](\mathbf{3 0})$ were prepared by dissolving $9.8 \mathrm{mg}$ of $\mathbf{2 8}, 9.8 \mathrm{mg}$ of $\mathbf{2 9}$, or $7.6 \mathrm{mg}$ of $\mathbf{3 0} \mathrm{in} 10 \mathrm{~mL}$ toluene in a volumetric flask. Each reaction was carried out in a $10 \mathrm{~mL}$ Young's tube, heating to the desired temperature with stirring for 2 minutes to ensure adequate mixing. The appropriate amount of $1 \mathrm{mmol} \mathrm{dm}^{-3}$ catalyst solution was added by microsyringe and the reaction commenced. At the appropriate time interval, $0.1 \mathrm{~mL}$ aliquots of the reaction mixture were withdrawn by syringe, then stored in stoppered glass vials at $-15{ }^{\circ} \mathrm{C}$. Mercury poisoning reactions were carried out under standard catalytic conditions, with the addition of one drop (roughly $60 \mathrm{mg}$ ) of mercury prior to the mixing of reactants.

Samples withdrawn from reaction mixtures were diluted by taking $10 \mu \mathrm{L}$ and making up to $10 \mathrm{~mL}$ with dichloromethane. Each sample was spiked with $10 \mu \mathrm{L}$ of a 
$7.0 \mathrm{mmol} \mathrm{dm}{ }^{-3}$ solution of 2-methylnaphthalene, used as an internal standard, and analysed by GCMS. Standard solutions of 4-methoxybiphenyl and 4-bromoanisole were analysed over the desired concentration range to confirm a linear response of the detector to the analytes. Reaction progress was determined from peak integrations, looking at the disappearance of the starting material (bromobenzene in Heck reactions, 4-bromoanisole in Suzuki reactions) as well as the appearance of products.

\section{Typical Heck Reaction}

Performed with bromobenzene $(105 \mu \mathrm{L}, 1.0 \mathrm{mmol})$, styrene $(128 \mu \mathrm{L}, 1.1 \mathrm{mmol})$, and $\mathrm{K}_{2} \mathrm{CO}_{3}(152 \mathrm{mg}, 1.1 \mathrm{mmol})$ in $2.5 \mathrm{~mL}$ DMF under a nitrogen atmosphere. To this, $20 \mu \mathrm{L}$ of catalyst solution $(0.002 \mathrm{~mol} \%)$ was added, with the reaction carried out at $140{ }^{\circ} \mathrm{C}$ for 16 hours.

\section{Typical Suzuki Reaction}

Performed with phenylboronic acid (146 mg, $1.2 \mathrm{mmol})$, 4-bromoanisole $(100 \mu \mathrm{L}$, $0.80 \mathrm{mmol})$, and $\mathrm{K}_{3} \mathrm{PO}_{4} \cdot \mathrm{H}_{2} \mathrm{O}(368 \mathrm{mg}, 1.6 \mathrm{mmol})$ in $4 \mathrm{~mL}$ toluene under a nitrogen atmosphere. To this, $8 \mu \mathrm{L}$ of catalyst solution $(0.001 \mathrm{~mol} \%)$ was added, with the reaction carried out at $100{ }^{\circ} \mathrm{C}$ for 2 hours.

\section{Large-Scale Suzuki Reaction}

Phenylboronic acid (2.92 g, $24 \mathrm{mmol})$, 4-bromoanisole (2.00 mL, $16 \mathrm{mmol})$, and $\mathrm{K}_{3} \mathrm{PO}_{4} \cdot \mathrm{H}_{2} \mathrm{O}(7.36 \mathrm{~g}, 32 \mathrm{mmol})$ were combined in toluene $(80 \mathrm{~mL})$ under a nitrogen atmosphere. The reaction vessel was pre-heated to $100{ }^{\circ} \mathrm{C}$ with stirring, and a $1 \mathrm{mmol} \mathrm{dm}^{-3}$ solution of catalyst 28 was added $(160 \mu \mathrm{L}, 0.001 \mathrm{~mol} \%)$. Heating was stopped after 11 hours. The reaction was quenched with $2 \mathrm{~mol} \mathrm{dm}-3$ $\mathrm{HCl}(150 \mathrm{~mL})$ and extracted with ethyl acetate $(3 \times 50 \mathrm{~mL})$. The combined extracts were washed with saturated sodium bicarbonate solution $(3 \times 50 \mathrm{~mL})$ and water $(2 \times 50 \mathrm{~mL})$, and dried over magnesium sulfate. Volatiles were removed in vacuo, and the crude product purified by column chromatography on neutral alumina, eluting with toluene. Recrystallisation from a mixture of dichloromethane and hexane yielded 4-methoxybiphenyl as a white solid (2.90 g, 98\%). NMR characterisation data matches that known for 4-methoxybiphenyl. 


\section{Catalyst Stability Tests}

Palladium complexes 28 (15 mg, $0.015 \mathrm{mmol}$ ), 29 (15 mg, $0.015 \mathrm{mmol}$ ), or 30 (12 mg, $0.016 \mathrm{mmol})$ were combined with phenylboronic acid $(9.3 \mathrm{mg}, 0.077 \mathrm{mmol})$ and $\mathrm{K}_{3} \mathrm{PO}_{4} \cdot \mathrm{H}_{2} \mathrm{O}(18 \mathrm{mg}, 0.077 \mathrm{mmol})$ in benzene- $d_{6}$. Hexafluorobenzene $(10 \mu \mathrm{L})$ was added as an internal standard. Control experiments with 28 and phenylboronic acid, and 28 and $\mathrm{K}_{3} \mathrm{PO}_{4} \cdot \mathrm{H}_{2} \mathrm{O}$ were also undertaken. Reaction mixtures were heated at $80{ }^{\circ} \mathrm{C}$ for 3 hours, monitoring reaction progress by NMR spectroscopy at 0,5 , 30 and 180 minutes. The relative amounts of decafluorobiphenyl and 2,3,4,5,6pentafluorobiphenyl present were determined by integration of the ortho and para ${ }^{19} \mathrm{~F}$ NMR resonances against those of the starting material prior to degradation, normalised against the hexafluorobenzene internal standard. At the conclusion of heating, each reaction mixture had $2 \times 0.1 \mathrm{~mL}$ aliquots withdrawn and diluted with $1 \mathrm{~mL}$ dichloromethane. One of the aliquots was hydrolysed by stirring with $1 \mathrm{~mL}$ water for $10 \mathrm{~min}$, after which time the organic fraction was decanted and both the hydrolysed and non-hydrolysed aliquots were subjected to GCMS analysis.

\section{Cyclic Voltammetry}

Standard solutions of $\mathbf{2 8}(2.4 \mathrm{mg}, 2.5 \mu \mathrm{mol}), 29(2.4 \mathrm{mg}, 2.5 \mu \mathrm{mol})$, and $\mathbf{3 0}(1.9 \mathrm{mg}$, $2.5 \mu \mathrm{mol})$ were prepared with tetrabutylammonium hexafluorophosphate (190 mg, $0.5 \mu \mathrm{mol})$ in acetonitrile $(5 \mathrm{~mL})$. Solutions were analysed by cyclic voltammetry using a platinum working electrode and a $\mathrm{Ag} / \mathrm{AgCl}$ reference electrode, scanning from $-0.5-1.8 \mathrm{~V}$ at a rate of $100 \mathrm{mV} \mathrm{s}^{-1}$. No meaningful oxidation events were observed for any of the solutions analysed. 


\section{References}

1. Kostova, I. Recent Patents on Anti-Cancer Drug Discovery 2006, 1, 1-22.

2. Breslow, R.; Belvedere, S.; Gershell, L.; Leung, D. Pure Appl. Chem. 2000, 72, 333-342.

3. Jeffrey, J. C.; Rauchfuss, T. B. Inorg. Chem. 1979, 18, 2658-2666.

4. Tan, X.; Li, L.; Zhang, J.; Han, X.; Jiang, L.; Li, F.; Su, C.-Y. Chem. Mater. 2012, 24, 480-485.

5. Allendorf, M. D.; Bauer, C. A.; Bhakta, R. K.; Houk, R. J. T. Chem. Soc. Rev. 2009, 38, 1330-1352.

6. Nishiyama, H. Chem. Soc. Rev. 2007, 36, 1133-1141.

7. Fan, L.; Foxman, B. M.; Ozerov, O. V. Organometallics 2004, 23, 326-328.

8. Hill, A. F.; Lee, S. B.; Park, J.; Shang, R.; Willis, A. C. Organometallics 2010, 29, 5661-5669.

9. Haenel, M. W.; Oevers, S.; Angermund, K.; Kaska, W. C.; Fan, H.-J.; Hall, M. B. Angew. Chem., Int. Ed. 2001, 40, 3596-3600.

10. Pugh, D.; Danopoulos, A. A. Coord. Chem. Rev. 2007, 251, 610-641.

11. Koridze, A. A.; Kuklin, S. A.; Sheloumov, A. M.; Dolgushin, F. M.; Lagunova, V. Y.; Petukhova, I. I.; Ezernitskaya, M. G.; Peregudov, A. S.; Petrovskii, P. V.; Vorontsov, E. V.; Baya, M.; Poli, R. Organometallics 2004, 23, $4585-4593$.

12. Albrecht, M.; van Koten, G. Angew. Chem., Int. Ed. 2001, 40, 3750-3781.

13. Eberhard, M. R.; Matsukawa, S.; Yamamoto, Y.; Jensen, C. M. J. Organomet. Chem. 2003, 687, 185-189.

14. Gunanathan, C.; Ben-David, Y.; Milstein, D. Science 2007, 317, 790-792.

15. Choi, J.; MacArthur, A. H. R.; Brookhart, M.; Goldman, A. S. Chem. Rev. 2011, 111, 1761-1779.

16. Selander, N.; Szabó, K. J. Chem. Rev. 2011, 111, 2048-2076.

17. Serrano-Becerra, J. M.; Morales-Morales, D. Curr. Org. Synth. 2009, 6, 169192. 
18. Morales-Morales, D. Mini-Rev. Org. Chem. 2008, 5, 141-152.

19. Singleton, J. T. Tetrahedron 2003, 59, 1837-1857.

20. van der Boom, M. E.; Milstein, D. Chem. Rev. 2003, 103, 1759-1792.

21. Gupta, M.; Hagen, C.; Flesher, R. J.; Kaska, W. C.; Jensen, C. M. Chem. Commun. 1996, 2083-2084.

22. Haibach, M. C.; Kundu, S.; Brookhart, M.; Goldman, A. S. Acc. Chem. Res. 2012, 45, 947-958.

23. Goldman, A. S.; Roy, A. H.; Huang, Z.; Ahuja, R.; Schinski, W.; Brookhart, M. Science 2006, 312, 257-261.

24. Arndtsen, B. A.; Bergman, R. G.; Mobley, T. A.; Peterson, T. H. Acc. Chem. Res. 1995, 28, 154-162.

25. Bernskoetter, W. H.; Schauer, C. K.; Goldberg, K. I.; Brookhart, M. Science 2009, 326, 553-556.

26. Perutz, R. N.; Turner, J. J. J. Am. Chem. Soc. 1975, 97, 4791-4800.

27. Piers, W. E. Organometallics 2011, 30, 13-16.

28. Poverenov, E.; Efremenko, I.; Frenkel, A. I.; Ben-David, Y.; Shimon, L. J. W.; Leitus, G.; Konstantinovski, L.; Martin, J. M. L.; Milstein, D. Nature 2008, 455, 1093-1096.

29. Kohl, S. W.; Weiner, L.; Schwartsburd, L.; Konstantinovski, L.; Shimon, L. J. W.; Ben-David, Y.; Iron, M. A.; Milstein, D. Science 2009, 324, 74-77.

30. Mann, G.; Hartwig, J. F. Tetrahedron Lett. 1997, 38, 8005-8008.

31. Liu, S.; Saidi, O.; Berry, N.; Ruan, J.; Pettman, A.; Thomson, N.; Xiao, J. Lett. Org. Chem. 2009, 6, 60-64.

32. Banet Osuna, A. M.; Chen, W.; Hope, E. G.; Kemmitt, R. D. W.; Paige, D. R.; Stuart, A. M.; Xiao, J.; Xu, L. J. Chem. Soc., Dalton Trans. 2000, 4052-4055.

33. Ess, D. H.; Goddard, W. A.; Periana, R. A. Organometallics 2010, 29, 64596472 .

34. Sakaki, S.; Biswas, B.; Sugimoto, M. Organometallics 1998, 17, 1278-1289.

35. Moloy, K. G.; Petersen, J. L. J. Am. Chem. Soc. 1995, 117, 7696-7710.

36. Dias, P. B.; de Piedade, M. E.; Simes, J. A. Coord. Chem. Rev. 1994, 135-136, 737-807.

37. Tolman, C. A. Chem. Rev. 1977, 77, 313-348.

38. Fernandez, A. L.; Wilson, M. R.; Prock, A.; Giering, W. P. Organometallics 2001, 20, 3429-3435.

39. Pollock, C. L.; Saunders, G. C.; Smyth, E. S.; Sorokin, V. I. J. Fluorine Chem. 2008, 129, 142-166. 
40. Göttker-Schnetmann, I.; White, P.; Brookhart, M. J. Am. Chem. Soc. 2004, 126, 1804-1811.

41. Orpen, A. G.; Connelly, N. G. Organometallics 1990, 9, 1206-1210.

42. Göttker-Schnetmann, I.; Brookhart, M. J. Am. Chem. Soc. 2004, 126, 93309338.

43. Adams, J. J.; Arulsamy, N.; Roddick, D. M. Organometallics 2011, 30, 697711.

44. Kossoy, E.; Rybtchinski, B.; Diskin-Posner, Y.; Shimon, L. J. W.; Leitus, G.; Milstein, D. Organometallics 2009, 28, 523-533.

45. Kossoy, E.; Iron, M. A.; Rybtchinski, B.; Ben-David, Y.; Shimon, L. J. W.; Konstantinovski, L.; Martin, J. M. L.; Milstein, D. Chem. Eur. J. 2005, 11, 2319-2326.

46. Huang, Z.; White, P. S.; Brookhart, M. Nature 2010, 465, 598-601.

47. Dani, P.; Richter, B.; van Klink, G. P.; van Koten, G. Eur. J. Inorg. Chem. 2001, 2001, 125-131.

48. Aydin, J.; Kumar, K. S.; Eriksson, L.; Szabó, K. J. Adv. Synth. Catal. 2007, 349, 2585-2594.

49. Aydin, J.; Kumar, K. S.; Sayah, M. J.; Wallner, O. A.; Szabó, K. J. J. Org. Chem. 2007, 72, 4689-4697.

50. Schuster, E. M.; Botoshansky, M.; Gandelman, M. Angew. Chem. 2008, 120, 4631-4634.

51. Kimura, T.; Uozumi, Y. Organometallics 2006, 25, 4883-4887.

52. Bolliger, J. L.; Blacque, O.; Frech, C. M. Angew. Chem., Int. Ed. 2007, 46, 6514-6517.

53. Heck, R. F.; Nolley, J. P. J. Org. Chem. 1972, 37, 2320-2322.

54. Miyaura, N.; Yamada, K.; Suzuki, A. Tetrahedron Lett. 1979, 20, 3437-3440.

55. Ohff, M.; Ohff, A.; van der Boom, M. E.; Milstein, D. J. Am. Chem. Soc. 1997, 119, 11687-11688.

56. Gagliardo, M.; Chase, P. A.; Lutz, M.; Spek, A. L.; Hartl, F.; Havenith, R. W. A.; van Klink, G. P. M.; van Koten, G. Organometallics 2005, 24, 45534557 .

57. Chase, P. A.; Gagliardo, M.; Lutz, M.; Spek, A. L.; van Klink, G. P. M.; van Koten, G. Organometallics 2005, 24, 2016-2019.

58. Adams, J. J.; Lau, A.; Arulsamy, N.; Roddick, D. M. Inorg. Chem. 2007, 46, $11328-11334$.

59. Brinkmann, Y.; Madhushaw, R. J.; Jazzar, R.; Bernardinelli, G.; Kündig, E. P. Tetrahedron 2007, 63, 8413-8419. 
60. Alezra, V.; Bernardinelli, G.; Corminboeuf, C.; Frey, U.; Kündig, E. P.; Merbach, A. E.; Saudan, C. M.; Viton, F.; Weber, J. J. Am. Chem. Soc. 2004, 126, 4843-4853.

61. Morales-Morales, D.; Grause, C.; Kasaoka, K.; Redón, R.; Cramer, R. E.; Jensen, C. M. Inorg. Chim. Acta 2000, 300-302, 958-963.

62. Motoyama, Y.; Shimozono, K.; Nishiyama, H. Inorg. Chim. Acta 2006, 359, $1725-1730$.

63. Kündig, E. P.; Saudan, C. M.; Bernardinelli, G. Angew. Chem., Int. Ed. 1999, 38, 1219-1223.

64. RajanBabu, T. V.; Radetich, B.; You, K. K.; Ayers, T. A.; Casalnuovo, A. L.; Calabrese, J. C. J. Org. Chem. 1999, 64, 3429-3447.

65. Harvey, S.; Junk, P. C.; Raston, C. L.; Salem, G. J. Org. Chem. 1988, 53, $3134-3140$.

66. Hoge, B.; Thösen, C.; Herrmann, T.; Pantenburg, I. Inorg. Chem. 2002, 41, $2260-2265$.

67. Cooke, M.; Green, M.; Kirkpatrick, D. J. Chem. Soc. A 1968, 1507-1510.

68. Klein, J.; Medlik, A.; Meyer, A. Tetrahedron 1976, 32, 51-56.

69. Ahuja, R.; Punji, B.; Findlater, M.; Supplee, C.; Schinski, W.; Brookhart, M.; Goldman, A. S. Nat. Chem. 2011, 3, 167-171.

70. Guiso, M.; Betrow, A.; Marra, C. Eur. J. Org. Chem. 2008, 2008, 1967-1976.

71. Chand, S.; Banwell, M. G. Aust. J. Chem. 2007, 60, 243-250.

72. Yandulov, D. V.; Tran, N. T. J. Am. Chem. Soc. 2007, 129, 1342-1358.

73. Park, S.; Pontier-Johnson, M.; Roundhill, D. M. Inorg. Chem. 1990, 29, 26892697.

74. Mohr, W.; Stark, G.; Jiao, H.; Gladysz, J. Eur. J. Inorg. Chem. 2001, 2001, 925-933.

75. McGibbon, A.; Nieuwenhuyzen, M.; Saunders, G. C. J. Fluorine Chem. 2011, 132, 495-500.

76. Nycz, J. E. Phosphorus, Sulfur Silicon Relat. Elem. 2009, 184, 2605-2612.

77. Bernskoetter, W. H.; Hanson, S. K.; Buzak, S. K.; Davis, Z.; White, P. S.; Swartz, R.; Goldberg, K. I.; Brookhart, M. J. Am. Chem. Soc. 2009, 131, 8603-8613.

78. Benito-Garagorri, D.; Bocokić, V.; Mereiter, K.; Kirchner, K. Organometallics 2006, 25, 3817-3823.

79. Schirmer, W.; Flörke, U.; Haupt, H.-J. Z. Anorg. Allg. Chem. 1987, 545, 8397. 
80. Clarke, M. L.; Ellis, D.; Mason, K. L.; Orpen, A. G.; Pringle, P. G.; Wingad, R. L.; Zaher, D. A.; Baker, R. T. Dalton Trans. 2005, 1294-1300.

81. Casalnuovo, A. L.; RajanBabu, T. V.; Ayers, T. A.; Warren, T. H. J. Am. Chem. Soc. 1994, 116, 9869-9882.

82. Darcel, C.; Moulin, D.; Henry, J.-C.; Lagrelette, M.; Richard, P.; Harvey, P. D.; Jugé, S. Eur. J. Org. Chem. 2007, 200\%, 2078-2090.

83. Wolfe, B.; Livinghouse, T. J. Am. Chem. Soc. 1998, 120, 5116-5117.

84. Moulton, C. J.; Shaw, B. L. J. Chem. Soc., Dalton Trans. 1976, 1020-1024.

85. Al-Salem, N. A.; Empsall, H. D.; Markham, R.; Shaw, B. L.; Weeks, B. J. Chem. Soc., Dalton Trans. 1979, 1972-1982.

86. Rimml, H.; Venanzi, L. M. J. Organomet. Chem. 1983, 259, C6-C7.

87. Paganelli, S.; Matteoli, U.; Scrivanti, A.; Botteghi, C. J. Organomet. Chem. 1990, 397, 375-381.

88. Petzold, H.; Görls, H.; Weigand, W. J. Organomet. Chem. 2007, 692, 27362742 .

89. Carr, N.; Dunne, B. J.; Mole, L.; Orpen, A. G.; Spencer, J. L. J. Chem. Soc., Dalton Trans. 1991, 863-871.

90. Baker, M. J.; Harrison, K. N.; Orpen, A. G.; Pringle, P. G.; Shaw, G. J. Chem. Soc., Dalton Trans. 1992, 2607-2614.

91. van der Boom, M. E.; Gozin, M.; Ben-David, Y.; Shimon, L. J. W.; Frolow, F.; Kraatz, H.-B.; Milstein, D. Inorg. Chem. 1996, 35, 7068-7073.

92. Baber, R. A.; Bedford, R. B.; Betham, M.; Blake, M. E.; Coles, S. J.; Haddow, M. F.; Hursthouse, M. B.; Orpen, A. G.; Pilarski, L. T.; Pringle, P. G.; Wingad, R. L. Chem. Commun. 2006, 3880-3882.

93. Sinnokrot, M. O.; Valeev, E. F.; Sherrill, C. D. J. Am. Chem. Soc. 2002, 124, 10887-10893.

94. Pandarus, V.; Zargarian, D. Organometallics 2007, 26, 4321-4334.

95. Sjövall, S.; Andersson, C.; Wendt, O. F. Inorg. Chim. Acta 2001, 325, 182-186.

96. Sjövall, S.; Johansson, M.; Andersson, C. Eur. J. Inorg. Chem. 2001, 2001, 2907-2912.

97. Olsson, D.; Arunachalampillai, A.; Wendt, O. F. Dalton Trans. 2007, 54275433.

98. Nandy, R.; Subramoni, M.; Varghese, B.; Sankararaman, S. J. Org. Chem. 2007, 72, 938-944.

99. Fanjul, T.; Eastham, G.; Fey, N.; Hamilton, A.; Orpen, A. G.; Pringle, P. G.; Waugh, M. Organometallics 2010, 29, 2292-2305. 
100. Bennett, M. A.; Jin, H.; Willis, A. C. J. Organomet. Chem. 1993, 451, 249256.

101. Hill, W. E.; Minahan, D. M. A.; Taylor, J. G.; McAuliffe, C. A. J. Am. Chem. Soc. 1982, 104, 6001-6005.

102. Pearson, R. G. Inorg. Chem. 1973, 12, 712-713.

103. Harvey, J. N.; Heslop, K. M.; Orpen, A. G.; Pringle, P. G. Chem. Commun. 2003, 278-279.

104. Nifantyev, E. E.; Rasadkina, E. N.; Vasyanina, L. K.; Belsky, V. K.; Stash, A. I. J. Organomet. Chem. 1997, 529, 171-176.

105. Armstrong, S.; Cross, R.; Farrugia, L.; Nichols, D.; Perry, A. Eur. J. Inorg. Chem. 2002, 2002, 141-151.

106. Cooper, S. J.; Brown, M. P.; Puddephatt, R. J. Inorg. Chem. 1981, 20, 13741377.

107. Albrecht, M.; Dani, P.; Lutz, M.; Spek, A. L.; van Koten, G. J. Am. Chem. Soc. 2000, 122, 11822-11833.

108. Canty, A. J.; van Koten, G. Acc. Chem. Res. 1995, 28, 406-413.

109. Garrou, P. E. Chem. Rev. 1981, 81, 229-266.

110. Johnson, M. T.; Wendt, O. F. Inorg. Chim. Acta 2011, 367, 222-224.

111. Ozerov, O. V.; Guo, C.; Foxman, B. M. J. Organomet. Chem. 2006, 691, 4802-4806.

112. Clark, H.; Manzer, L. J. Organomet. Chem. 1973, 59, 411-428.

113. Even, T.; Genge, A. R. J.; Hill, A. M.; Holmes, N. J.; Levason, W.; Webster, M. J. Chem. Soc., Dalton Trans. 2000, 655-662.

114. Babai, A.; Deacon, G. B.; Meyer, G. Z. Anorg. Allg. Chem. 2004, 630, 399-402.

115. Flapper, J.; Wormald, P.; Lutz, M.; Spek, A. L.; van Leeuwen, P. W. N. M.; Elsevier, C. J.; Kamer, P. C. J. Eur. J. Inorg. Chem. 2008, 2008, 4968-4976.

116. Drahos, B.; Rohlik, Z.; Kotek, J.; Cisarova, I.; Hermann, P. Dalton Trans. 2009, 4942-4953.

117. Albrecht, M.; Gossage, R. A.; Lutz, M.; Spek, A. L.; van Koten, G. Chem. Eur. J. 2000, 6, 1431-1445.

118. Appleton, T. G.; Bennett, M. A. Inorg. Chem. 1978, 17, 738-747.

119. Abdullah, B. H.; Abdullah, M. A.; Al-Jibori, S. A.; Al-Allaf, T. A. K. Asian J. Chem. 2007, 19, 1334-1340.

120. Deacon, G.; Nelson-Reed, K. J. Organomet. Chem. 1987, 322, 257-268.

121. Espinet, P.; Martínez-Ilarduya, J. M.; Pérez-Briso, C.; Casado, A. L.; Alonso, M. J. Organomet. Chem. 1998, 551, 9-20. 
122. Kemmitt, R. D. W.; Nichols, D. I.; Peacock, R. D. J. Chem. Soc. A 1968, 2149-2152.

123. Cross, R.; Kennedy, A.; Muir, K. J. Organomet. Chem. 1995, 487, 227-233.

124. Arunachalampillai, A.; Johnson, M. T.; Wendt, O. F. Organometallics 2008, 27, 4541-4543.

125. Siegbahn, P. E. M.; Crabtree, R. H. J. Am. Chem. Soc. 1996, 118, 4442-4450.

126. Bruce, M. I. Angew. Chem., Int. Ed. 1977, 16, 73-86.

127. Omae, I. J. Organomet. Chem. 2011, 696, 1128-1145.

128. Brookhart, M.; Green, M. L. H.; Parkin, G. Proc. Natl. Acad. Sci. U.S.A. 2007, 104, 6908-6914.

129. Wang, Z.; Sugiarti, S.; Morales, C. M.; Jensen, C. M.; Morales-Morales, D. Inorg. Chim. Acta 2006, 359, 1923-1928.

130. Ryabov, A. D.; Sakodinskaya, I. K.; Yatsimirsky, A. K. J. Chem. Soc., Dalton Trans. 1985, 2629-2638.

131. Ryabov, A. D. Chem. Rev. 1990, 90, 403-424.

132. Albrecht, M. Chem. Rev. 2010, 110, 576-623, PMID: 20017477.

133. Davies, D. L.; Donald, S. M. A.; Macgregor, S. A. J. Am. Chem. Soc. 2005, 127, 13754-13755.

134. Goldman, A. S.; Krogh-Jespersen, K. J. Am. Chem. Soc. 1996, 118, 1215912166.

135. Bolliger, J. L.; Blacque, O.; Frech, C. M. Chem. Eur. J. 2008, 14, 7969-7977.

136. Gerber, R.; Fox, T.; Frech, C. M. Chem. Eur. J. 2010, 16, 6771-6775.

137. Vuzman, D.; Poverenov, E.; Diskin-Posner, Y.; Leitus, G.; Shimon, L. J. W.; Milstein, D. Dalton Trans. 2007, 5692-5700.

138. Horton, A. D.; de With, J.; van der Linden, A. J.; van de Weg, H. Organometallics 1996, 15, 2672-2674.

139. Schwartsburd, L.; Poverenov, E.; Shimon, L. J. W.; Milstein, D. Organometallics 2007, 26, 2931-2936.

140. Shelly, K.; Bartczak, T.; Scheidt, W. R.; Reed, C. A. Inorg. Chem. 1985, 24, 4325-4330.

141. Salem, H.; Ben-David, Y.; Shimon, L. J. W.; Milstein, D. Organometallics 2006, 25, 2292-2300.

142. Poverenov, E.; Leitus, G.; Shimon, L. J. W.; Milstein, D. Organometallics 2005, 24, 5937-5944.

143. Tong, J.; Liu, S.; Zhang, S.; Li, S. Z. Spectrochim. Acta, Part A 2007, 67, 837-846. 
144. Wawrzyniak, P.; Fuller, A. L.; Slawin, A. M. Z.; Kilian, P. Inorg. Chem. 2009, 48, 2500-2506, PMID: 19216557.

145. Baber, R. A.; Haddow, M. F.; Middleton, A. J.; Orpen, A. G.; Pringle, P. G.; Haynes, A.; Williams, G. L.; Papp, R. Organometallics 2007, 26, 713-725.

146. Schwartsburd, L.; Cohen, R.; Konstantinovski, L.; Milstein, D. Angew. Chem., Int. Ed. 2008, 47, 3603-3606.

147. Nakajima, Y.; Ozawa, F. Organometallics 2012, 31, 2009-2015.

148. Sánchez-Cabrera, G.; Leyva, M. A.; Zuno-Cruz, F. J.; Hernández-Cruz, M. G.; Rosales-Hoz, M. J. J. Organomet. Chem. 2009, 694, 1949-1958.

149. Zhu, H.; Ziegler, T. Organometallics 2009, 28, 2773-2777.

150. Zhu, H.; Ziegler, T. Organometallics 2008, 27, 1743-1749.

151. Hughes, E. D.; Ingold, C. K. J. Chem. Soc. 1935, 244-255.

152. Feller, M.; Ben-Ari, E.; Iron, M. A.; Diskin-Posner, Y.; Leitus, G.; Shimon, L. J. W.; Konstantinovski, L.; Milstein, D. Inorg. Chem. 2010, 49, 1615-1625.

153. Kundu, S.; Brennessel, W. W.; Jones, W. D. Inorg. Chem. 2011, 50, 94439453.

154. Feller, M.; Diskin-Posner, Y.; Shimon, L. J. W.; Ben-Ari, E.; Milstein, D. Organometallics 2012, 31, 4083-4101.

155. Gunanathan, C.; Milstein, D. Acc. Chem. Res. 2011, 44, 588-602.

156. Schwartsburd, L.; Iron, M. A.; Konstantinovski, L.; Ben-Ari, E.; Milstein, D. Organometallics 2011, 30, 2721-2729.

157. Benito-Garagorri, D.; Kirchner, K. Acc. Chem. Res. 2008, 41, 201-213.

158. Burkill, H. A.; Vilar, R.; White, A. J. Inorg. Chim. Acta 2006, 359, 3709-3722.

159. Hanson, S. K.; Heinekey, D. M.; Goldberg, K. I. Organometallics 2008, 27, $1454-1463$.

160. Hahn, C.; Spiegler, M.; Herdtweck, E.; Taube, R. Eur. J. Inorg. Chem. 1998, 1998, 1425-1432.

161. Huff, C. A.; Kampf, J. W.; Sanford, M. S. Organometallics 2012, 31, 46434645 .

162. Fryzuk, M. D.; McConville, D. H.; Rettig, S. J. Organometallics 1993, 12, $2152-2161$.

163. Geerts, R. L.; Huffman, J. C.; Caulton, K. G. Inorg. Chem. 1986, 25, 590-591.

164. Moszner, M.; Ciunik, Z.; Ziólkowski, J. J. Polyhedron 2003, 22, 3195-3203.

165. Blank, B.; Glatz, G.; Kempe, R. Chem. Asian J. 2009, 4, 321-327.

166. Feller, M.; Iron, M. A.; Shimon, L. J. W.; Diskin-Posner, Y.; Leitus, G.; Milstein, D. J. Am. Chem. Soc. 2008, 130, 14374-14375. 
167. Hahn, C.; Spiegler, M.; Herdtweck, E.; Taube, R. Eur. J. Inorg. Chem. 1999, 1999, 435-440.

168. Yang, L.; Kruger, A.; Neels, A.; Albrecht, M. Organometallics 2008, 27, 31613171 .

169. Wehmschulte, R. J.; Twamley, B.; Khan, M. A. Inorg. Chem. 2001, 40, 60046008 .

170. Seligson, A. L.; Trogler, W. C. Organometallics 1993, 12, 744-751.

171. Bedford, R. B.; Draper, S. M.; Noelle Scully, P.; Welch, S. L. New J. Chem. 2000, 24, 745-747.

172. Kjellgren, J.; Aydin, J.; Wallner, O. A.; Saltanova, I. V.; Szabó, K. J. Chem. Eur. J. 2005, 11, 5260-5268.

173. Sebelius, S.; Olsson, V. J.; Szabó, K. J. J. Am. Chem. Soc. 2005, 127, 1047810479 .

174. Crisp, G. T. Chem. Soc. Rev. 1998, 27, 427-436.

175. Amatore, C.; Jutand, A. Acc. Chem. Res. 2000, 33, 314-321.

176. Phan, N. T. S.; Van Der Sluys, M.; Jones, C. W. Adv. Synth. Catal. 2006, 348, 609-679.

177. Herrmann, W. A.; Brossmer, C.; Öfele, K.; Reisinger, C.-P.; Priermeier, T.; Beller, M.; Fischer, H. Angew. Chem., Int. Ed. 1995, 34, 1844-1848.

178. Beller, M.; Fischer, H.; Herrmann, W. A.; Öfele, K.; Brossmer, C. Angew. Chem., Int. Ed. 1995, 34, 1848-1849.

179. Shaw, B. L. New J. Chem. 1998, 22, 77-79.

180. Miyazaki, F.; Yamaguchi, K.; Shibasaki, M. Tetrahedron Lett. 1999, 40, 73797383.

181. Louie, J.; Hartwig, J. F. Angew. Chem., Int. Ed. 1996, 35, 2359-2361.

182. Reetz, M. T.; Westermann, E. Angew. Chem., Int. Ed. 2000, 39, 165-168.

183. Eberhard, M. R. Org. Lett. 2004, 6, 2125-2128.

184. Yu, K.; Sommer, W.; Richardson, J. M.; Weck, M.; Jones, C. W. Adv. Synth. Catal. 2005, 34\%, 161-171.

185. de Vries, J. G. Dalton Trans. 2006, 421-429.

186. Xu, L.-M.; Li, B.-J.; Yang, Z.; Shi, Z.-J. Chem. Soc. Rev. 2010, 39, 712-733.

187. Sehnal, P.; Taylor, R. J. K.; Fairlamb, I. J. S. Chem. Rev. 2010, 110, 824-889.

188. Aydin, J.; Larsson, J. M.; Selander, N.; Szabó, K. J. Org. Lett. 2009, 11, $2852-2854$.

189. Blacque, O.; Frech, C. M. Chem. Eur. J. 2010, 16, 1521-1531. 
190. Gerber, R.; Blacque, O.; Frech, C. M. Dalton Trans. 2011, 40, 8996-9003.

191. Vicente, J.; Arcas, A.; Juliá-Hernández, F.; Bautista, D. Angew. Chem., Int. Ed. 2011, 50, 6896-6899.

192. Duncan, D.; Hope, E. G.; Singh, K.; Stuart, A. M. Dalton Trans. 2011, 40, 1998-2005.

193. Jung, I. G.; Son, S. U.; Park, K. H.; Chung, K.-C.; Lee, J. W.; Chung, Y. K. Organometallics 2003, 22, 4715-4720.

194. Gruber, A. S.; Zim, D.; Ebeling, G.; Monteiro, A. L.; Dupont, J. Org. Lett. 2000, 2, 1287-1290.

195. Whitesides, G. M.; Hackett, M.; Brainard, R. L.; Lavalleye, J. P. P. M.; Sowinski, A. F.; Izumi, A. N.; Moore, S. S.; Brown, D. W.; Staudt, E. M. Organometallics 1985, 4, 1819-1830.

196. Widegren, J. A.; Finke, R. G. J. Mol. Catal. A: Chem. 2003, 198, 317-341.

197. van Asselt, R.; Elsevier, C. J. J. Mol. Catal. 1991, 65, L13-L19.

198. Stein, J.; Lewis, L. N.; Gao, Y.; Scott, R. A. J. Am. Chem. Soc. 1999, 121, 3693-3703.

199. Sommer, W. J.; Yu, K.; Sears, J. S.; Ji, Y.; Zheng, X.; Davis, R. J.; Sherrill, C. D.; Jones, C. W.; Weck, M. Organometallics 2005, 24, 4351-4361.

200. Beletskaya, I. P.; Kashin, A. N.; Karlstedt, N. B.; Mitin, A. V.; Cheprakov, A. V.; Kazankov, G. M. J. Organomet. Chem. 2001, 622, 89-96.

201. Suijkerbuijk, B. M. J. M.; Herreras Martínez, S. D.; Koten, G. v.; Klein Gebbink, R. J. M. Organometallics 2008, 27, 534-542.

202. Beletskaya, I. P.; Cheprakov, A. V. Chem. Rev. 2000, 100, 3009-3066.

203. Salem, H.; Shimon, L. J. W.; Diskin-Posner, Y.; Leitus, G.; Ben-David, Y.; Milstein, D. Organometallics 2009, 28, 4791-4806.

204. Benito-Garagorri, D.; Mereiter, K.; Kirchner, K. Eur. J. Inorg. Chem. 2006, 2006, 4374-4379.

205. Beletskaya, I. P.; Cheprakov, A. V. J. Organomet. Chem. 2004, 689, 40554082.

206. Hoge, B.; Neufeind, S.; Hettel, S.; Wiebe, W.; Thösen, C. J. Organomet. Chem. 2005, 690, 2382-2387.

207. Ali, R.; Dillon, K. B. J. Chem. Soc., Dalton Trans. 1990, 2593-2596.

208. von Schenck, H.; Åkermark, B.; Svensson, M. Organometallics 2002, 21, 22482253 .

209. Ariafard, A.; Yates, B. F. J. Organomet. Chem. 2009, 694, 2075-2084.

210. Amatore, C.; Jutand, A.; Le Duc, G. Chem. Eur. J. 2011, 17, 2492-2503. 
211. Braga, A. A. C.; Morgon, N. H.; Ujaque, G.; Maseras, F. J. Am. Chem. Soc. 2005, 127, 9298-9307.

212. Bedford, R. B.; Hazelwood, S. L.; Horton, P. N.; Hursthouse, M. B. Dalton Trans. 2003, 4164-4174.

213. Martin, R.; Buchwald, S. L. Acc. Chem. Res. 2008, 41, 1461-1473.

214. Crabtree, R. H. Chem. Rev. 2012, 112, 1536-1554.

215. Kozlov, V. A.; Aleksanyan, D. V.; Nelyubina, Y. V.; Lyssenko, K. A.; Petrovskii, P. V.; Vasil'ev, A. A.; Odinets, I. L. Organometallics 2011, 30, 29202932 .

216. Bolliger, J. L.; Frech, C. M. Chem. Eur. J. 2010, 16, 4075-4081.

217. Takemoto, T.; Iwasa, S.; Hamada, H.; Shibatomi, K.; Kameyama, M.; Motoyama, Y.; Nishiyama, H. Tetrahedron Lett. 2007, 48, 3397-3401.

218. Zhang, H.; Lei, A. Dalton Trans. 2011, 40, 8745-8754.

219. Polukeev, A. V.; Kuklin, S. A.; Petrovskii, P. V.; Peregudova, S. M.; Smol'yakov, A. F.; Dolgushin, F. M.; Koridze, A. A. Dalton Trans. 2011, 40, 7201-7209.

220. Ghosh, R.; Emge, T. J.; Krogh-Jespersen, K.; Goldman, A. S. J. Am. Chem. Soc. 2008, 130, 11317-11327.

221. Olsson, D.; Wendt, O. F. J. Organomet. Chem. 2009, 694, 3112-3115.

222. Gerber, R.; Blacque, O.; Frech, C. M. ChemCatChem 2009, 1, 393-400.

223. Lin, Z. Coord. Chem. Rev. 2007, 251, 2280-2291.

224. Fulmer, G. R.; Muller, R. P.; Kemp, R. A.; Goldberg, K. I. J. Am. Chem. Soc. 2009, 131, 1346-1347.

225. Johansson, R.; Wendt, O. F. Organometallics 2007, 26, 2426-2430.

226. Bonnet, S.; van Lenthe, J. H.; Siegler, M. A.; Spek, A. L.; van Koten, G.; Gebbink, R. J. M. K. Organometallics 2009, 28, 2325-2333.

227. Bonnet, S.; Lutz, M.; Spek, A. L.; van Koten, G.; Klein Gebbink, R. J. M. Organometallics 2010, 29, 1157-1167.

228. Widenhoefer, R. A.; Zhong, H. A.; Buchwald, S. L. J. Am. Chem. Soc. 1997, 119, 6787-6795.

229. Zhang, J.; Medley, C. M.; Krause, J. A.; Guan, H. Organometallics 2010, 29, 6393-6401.

230. Armarego, L., Wilfred; Chai, L., Christina Purification of Laboratory Chemicals (5th Edition); Elsevier, 2003.

231. Kündig, E. P.; Dupré, C.; Bourdin, B.; Cunningham, A.; Pons, D. Helv. Chim. Acta 1994, 7r, 421-428. 
232. Hartley, F. R.; Murray, S. G.; McAuliffe, C. A. Inorg. Chem. 1979, 18, 13941397.

233. Jensen, K. A. Acta Chem. Scand. 1953, 7, 868-70.

234. Vaughan, T. F.; Koedyk, D. J.; Spencer, J. L. Organometallics 2011, 30, $5170-5180$.

235. Giordano, G.; Crabtree, R. H. Inorg. Synth. 1979, 19, 218-20.

236. Drew, D.; Doyle, J. R. Inorg. Synth. 1990, 28, 346-9.

237. Sheldrick, G. M. Acta Crystallogr., Sect. A: Found. Crystallogr. 2008, 64, 112122.

238. Dolomanov, O. V.; Bourhis, L. J.; Gildea, R. J.; Howard, J. A. K.; Puschmann, H. J. Appl. Crystallogr. 2009, 42, 339-341. 